\title{
EARTH SCIENCES DIVISION RESEARCH SUMMARIES 2006-2007
}

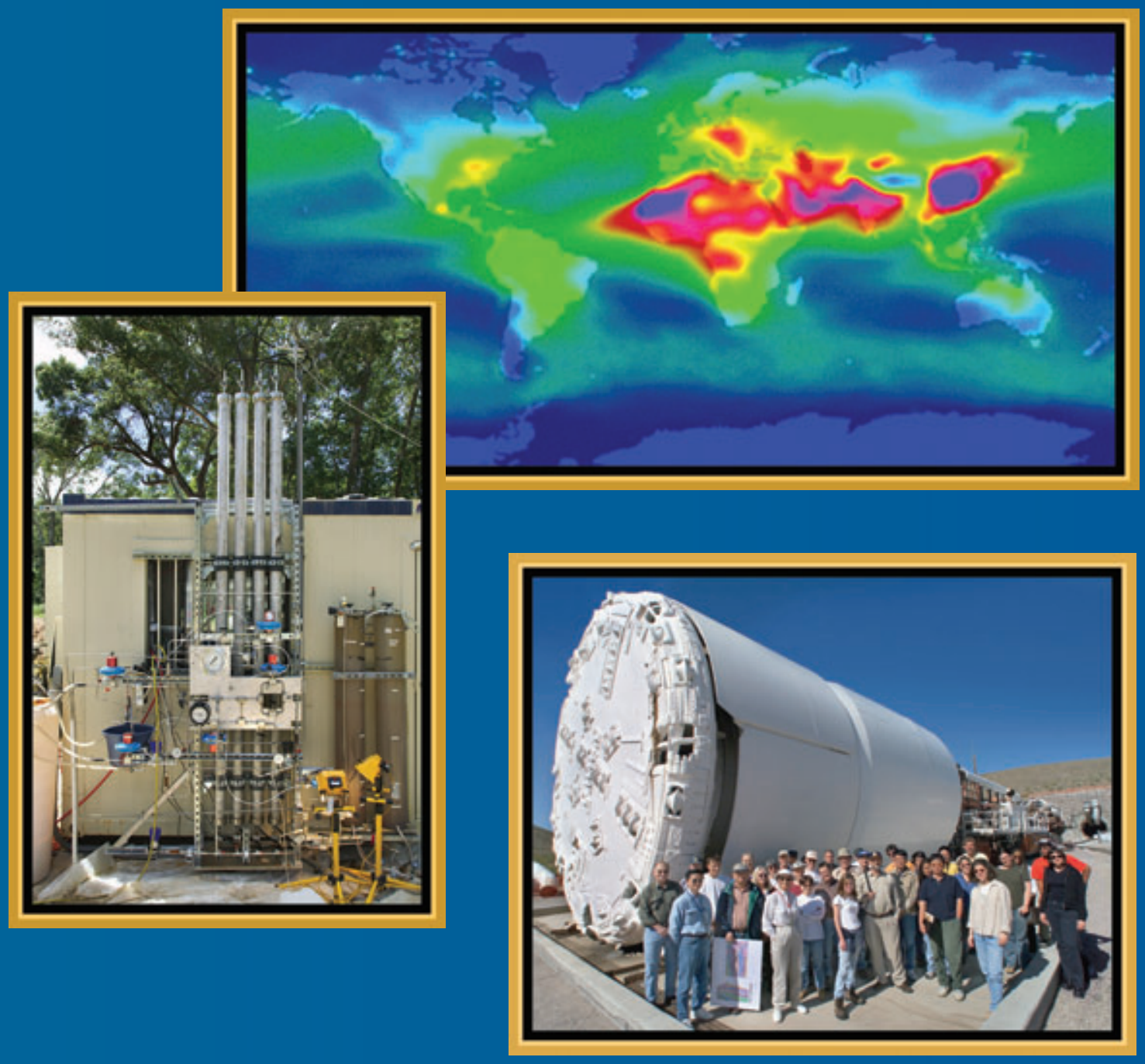

rerrert 


\section{Disclaimer}

This document was prepared as an account of work sponsored by the United States Government. While this document is believed to contain correct information, neither the United States Government nor any agency thereof, nor The Regents of the University of California, nor any of their employees, makes any warranty, express or implied, or assumes any legal responsibility for the accuracy, completeness, or usefulness of any information, apparatus, product, or process disclosed, or represents that its use would not infringe privately owned rights. Reference herein to any specific commercial product, process, or service by its trade name, trademark, manufacturer, or otherwise, does not necessarily constitute or imply its endorsement, recommendation, or favoring by the United States Government or any agency thereof, or The Regents of the University of California. The views and opinions of authors expressed herein do not necessarily state or reflect those of the United States Government or any agency thereof or The Regents of the University of California.

Ernest Orlando Lawrence Berkeley National Laboratory is an equal opportunity employer. 


\section{EARTH SCIENCES Division}
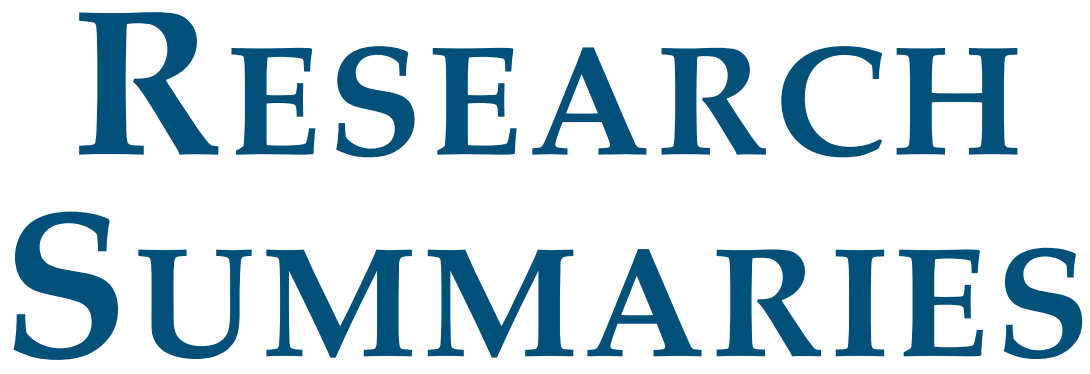

\section{6-2007}

This publication is dedicated to the memory of

Gudmundur "Bo" Bodvarsson

November 11, 1952 - November 29, 2006

Earth Sciences Division Family

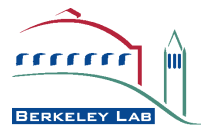

ERNEST $\square$ RLANDI LAWRENCE

BERKELEY NATIONAL LABDRATIRY

UNIVERSITY LF CALIFLRNIA

BERKELEY, CALIFIRNIA $9472 \square$

Prepared for the U.S. Department of Energy under Contract Number DE-AC02-05CH11231 


\section{EarTh Sciences Division Organization Chart}

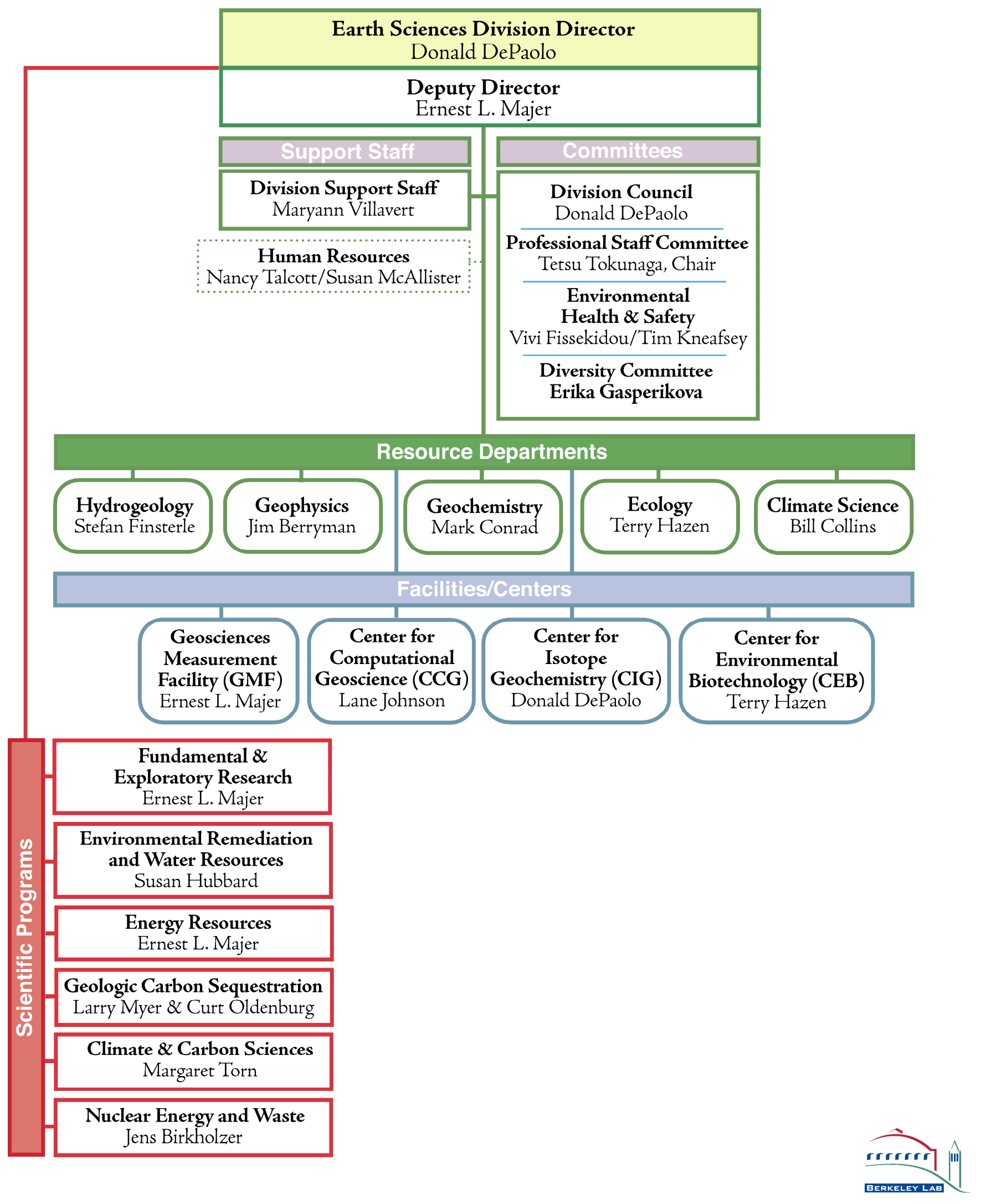




\section{TABle of CONTENTS}

A Perspective from the Division Director

RESOURCE DEPARTMENTS

HYDROGEOLOGY

GEOPHYSICS

GEOCHEMISTRY

13

ECOLOGY

15

Climate Science

Uncertainty Analysis Using Stochastic Rock-Physics Models and Markov Chain Monte Carlo Methods

Jinsong Chen

Measurements and Observations of Water, Gas, and Heat Flow in Hydrate-Bearing Sands

T.J. Kneafsey, L. Tomutsa, Y. Seol, R.C. Trautz, G.J. Moridis, and T.A. Ghezzehei

Frequency-Dependent Seismic Response of Underground Reservoirs

Valeri Korneev

Comparison of Kinetic and Equilibrium Reaction Models

in Simulating Gas Hydrate Behavior in Porous Media

Michael B. Kowalsky and George J. Moridis

Gas Production from Oceanic Class 2 Hydrate Accumulations

George J. Moridis and Matthew T. Reagan

A Strategy for Gas Production from Oceanic Class 3 Hydrate Deposits

George J. Moridis and Matthew T. Reagan

Shear-Stress Monitoring of a Fracture Using Seismic Waves

Seiji Nakagawa

Seismic Boundary Conditions for a Fluid-Filled Fracture

Seiji Nakagawa and Michael A. Schoenberg

Large Scale 3-D Electromagnetic Inversion Problems

Gregory A Newman and Michael Commer

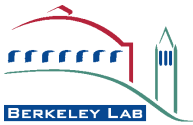


Enhanced Geothermal Systems (EGS) Using $\mathrm{CO}_{2}$ as Working Fluid

A Numerical Model for Analysis of the Geomechanical Performance of Hydrate-Bearing Sediments 
Nanoparticle Reactivity, Structure, and Growth Studies

Glenn Waychunas, Christopher Kim, Christopher Lentini, Ben Gilbert, Hengzhang Zhong, and Jillian Banfield

Incorporation of Aqueous Reaction Kinetics and Biodegradation into TOUGHREACT

Tianfu Xu and Curtis M. Oldenburg

Environmental Remediation and Water Resources Program

Comparative Analysis of Microbial Composition During Field-Scale Chromium Bioremediation

Eoin Brodie, Terry Hazen, Boris Faybishenko, Romy Chakraborty, Mark Conrad, Sharon Borglin,

Dominique Joyner, Jizhong Zhou, Joy Van Nostrand, Richard Phan, Gary Andersen

Influence of Electron Donor Type and Concentration on Microbial Population Dynamics

During Uranium Reduction and Remobilization

Eoin Brodie, Rebecca Daly, Yongman Kim, Jiamin Wan, Tetsu Tokunaga,

Gary Andersen, Terry Hazen, and Mary Firestone

Spatial Characterization of the Bacterial and Archaeal Community Structure in the Passalid Beetle Gut

Eoin Brodie, Nhu Nyugen, Todd DeSantis, Stephanie Gross, Sung-Oui Suh, James Nardi,

Tom Bruns, Meredith Blackwell, and Gary Andersen

Large-Scale Production of Anaerobic Sulfate Reducers

Jil T. Geller, Mary E. Singer, Tamas Torock, and Terry C. Hazen

Greengenes: Gene Database and Web Application for Microbial Ecologists

Todd DeSantis, Eoin Brodie, Yvette Piceno, Phillip Hugenholtz, and Gary Andersen

Development of a New Tool to Study Nucleation and Growth of Nanoparticles on Mineral Surfaces Young-Shin Jun, Glenn A. Waychunas, Byeongdu Lee, and Mike F. Toney

Environmental Science Program at the Advanced Light Source

Peter Nico

Development of Highly Heterogeneous Zones of Microbial Activity During Enhanced Bioremediation

Mark Conrad, Eoin Brodie, and Markus Bill

Sulfur Isotopes as Indicators of Bacterial Sulfate-Reduction Processes

During Field-Scale Uranium Bioremediation

Jennifer L. Druhan, Mark E. Conrad, Kenneth Hurst Williams, Lucie N'Guessan,

Philip E. Long, and Susan S. Hubbard

Tracing ${ }^{99} \mathrm{Tc}$ Contamination at the Hanford Site Using the Isotopic Composition of Nitrate

John N. Christensen, Mark Conrad, P. Evan Dresel, and Donald J. DePaolo

Induced Polarization Monitoring of Microbial Activity During Stimulated Subsurface Bioremediation Kenneth H. Williams, Susan S. Hubbard, and Jillian F. Banfield

Geophysical Monitoring of Stimulated Subsurface Bioremediation Using the Self-Potential Technique

Kenneth H. Williams, Susan S. Hubbard, and Jillian F. Banfield

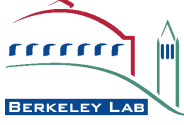


A Statistical Framework for Monitoring Biogeochemical Transformations

Using Time-Lapse Geophysical Data

Jinsong Chen and Susan Hubbard

Development and Application of an Approach for Interpreting Breakthrough Curves

Associated with Complex Tracer Injections

Andreas Englert and Susan Hubbard

Exploring the Impact of Stimulated Bioremediation on Flow Characteristics

Andreas Englert, Michael Kowalsky, Li Li, and Susan Hubbard

Inversion of Tracer Data to Quantify Bioremediation-Induced Changes in a Contaminated Aquifer

Michael B. Kowalsky, Andreas Englert, Li Li, and Susan S. Hubbard

Multiscale Hydrogeophysical Data Integration for Parameterization of

Dual-Domain Transport Model at Savannah River Site

Michael B. Kowalsky, Jinsong Chen, John Peterson, Jens Birkholzer, and Susan S. Hubbard

Berkeley UXO Discriminator (BUD) for the Detection and Characterization of UXO

E. Gasperikova, J. T. Smith, H. F. Morrison, and A. Becker

Quantifying and Predicting Reactive Transport of Uranium in Hanford Waste Plumes

Jiamin Wan, Tetsu Tokunaga, and Yongman Kim

Mesoscale Biotransformation of Uranium

Tetsu Tokunaga, Yongman Kim, Jiamin Wan, Rebecca Daly, Eoin Brodie, Mary K. Firestone,

Terry C. Hazen, Steve Sutton, Matt Newville, and Antonio Lanzirotti

In Situ Long-Term Bioimmobilization of CR(VI) in Groundwater Using

$\delta^{13}$ C-Labeled Slow-Release Lactate

B. Faybishenko, T.C. Hazen, E.L. Brodie, M.S. Conrad, S.S. Hubbard, D. Joyner, S. Borglin, R. Chakraborty, K.H. Williams, J.E. Peterson, J. Chen, T.K. Tokunaga, J. Wan, and M. Firestone

Modeling Biogeochemical Transport of Metals in Mining-Impacted Lake Sediments

N. Spycher, S. Sengor, and T. Ginn

Mobility of Tritium in Engineered and Earth Materials

Stefan Finsterle, Mark Conrad, Quanlin Zhou, Grace Su, and Karsten Pruess

The Berkeley Water Center's Digital Watershed

N. L. Miller, D.A. Agarwal, B.E. Bargmeyer, C. Gu, G.M. Hornberger, S.S. Hubbard, J.R. Hunt, J. Jin,

F. Maggi, C.M. Oldenburg, L. Pan, W.J. Riley, N. Spycher, C.I. Steefel, C. Van Ingen, and T. Xu

Coupled Modeling of Hydrology, Nutrient Cycling, and Vegetation: Applications to Agricultural Fields

F. Maggi, C. Gu, W.J. Riley, T. Xu, C.I. Steefel, N.L. Miller, and C.M. Oldenburg

Comparison of Two State-of-the-Art Remote-Sensing Techniques

for Development of Improved Wetland-Drainage Management Practices

Nigel W.T. Quinn and Josephine R. Burns

Impacts of Delayed Drawdown on Aquatic Biota and Water Quality in Seasonal Wetlands 
Toward Understanding the Relationship Between Groundwater

and Hydroclimate over the California Merced River Basin

Jiming Jin and Norman L. Miller

Drought Analysis of the

California Central Valley Surface-Groundwater Conveyance Sysytem

Norman L. Miller, Larry L. Dale, Sebastian Vicuna, Charles Brush, John Dogrul, and Tariq Kadir

Climate and Carbon Sciences Program

Understanding the Role of Land-Surface Processes

in the Regional Climate System: A WRF Modeling Study

Jiming Jin, Norman L. Miller, and Nicole J. Schegel

Regional Climate Simulations To Quantify the Range of Land-Use Change

and Impacts on Hydroclimate in the California Central Valley

Jiming Jin and Norman L. Miller

Climate Change Projected Fire Weather Sensitivity: California Santa Ana Wind Occurrence

Norman L. Miller and Nicole J. Schlegel

Climate-Change-Related Extreme Heat, Mortality, and Energy Demand

Norman L. Miller, Jiming Jin, Katharine Hayhoe, and Max Auffhammer

The California Regional Climate Modeling Intercomparison and Climate Change Sensitivity Study

N.L. Miller, J. Jin, N. Schlegel, M. Synder, L.C. Sloan, M. Kanamitsu,

H. Kanamaru, H. Hildago, P.B. Duffy, M.D. Dettinger, and D. Cayan

Developing a Coupled Land Surface and Subsurface Model: CLMT2

Lehua Pan, Jiming Jin, Norman Miller, Yu-Shu Wu, and Gudmundur Bodvarsson

Where Do Fossil Fuel Carbon Dioxide Emissions from California Go?

W.J. Riley, D.Y. Hsueh, M.L. Fischer, J.G. Hatch, D.E. Pataki, S. Cheong, M.L. Goulden, and J.T. Randerson

Interannual Regional $\mathrm{CO}_{2}$ and Latent Heat Surface Fluxes in the

Southern Great Plains: Measurements, Modeling, and Scaling

W.J. Riley, S.C. Biraud, M.S. Torn, M.L. Fischer, J.A. Berry, and D. Billesbach

Modelng of Hydrology, The Nitrogen Cycle, and

Vegetation Dynamics in Natural and Managed Ecosystems

W.J. Riley, D.Y. Hsueh, F. Maggi, C. Gu, T. Xu, C.I. Steefel, N.L. Miller, and C.M. Oldenburg

Carbon Cycling in the Southern Great Plains During the

ARM Cumulus-Land Surface Interaction Campaign (CLASIC)

Margaret S. Torn, Marc L. Fischer, William J. Riley, Sébastien Biraud, and J. Berry

Missing Feedbacks, Asymmetric Uncertainties, and the Underestimation of Future Warming

Characterizing Root Dynamics in Forests: Estimates Using Radiocarbon Data and Numerical Modeling 
Large-Scale Hydrological Evaluation of $\mathrm{CO}_{2}$ Injection-Storage

and Modeling of the Impact on Groundwater Systems

Jens Birkholzer, Quanlin Zhou, Jonny Rutqvist, Keni Zhang, and Chin-Fu Tsang

Geochemical Investigations on the Vulnerability of Groundwater Resources

in Case of $\mathrm{CO}_{2}$ Leakage from Deep Geological Storage

Jens T. Birkholzer, John Apps, Tianfu Xu, Yingqi Zhang, Liange Zheng, and Chin-Fu Tsang

Optimality in Characterizing $\mathrm{CO}_{2}$ Seepage from Geological Carbon Sequestration Sites

Andrea Cortis, Curtis M. Oldenburg, and Sally M. Benson

Continuous Active-Source Seismic Monitoring (CASSM) for $\mathrm{CO}_{2}$ Injection in a Brine Aquifer

Thomas M. Daley, Ray D. Solbau, Jonathan B. Ajo-Franklin, and Sally M. Benson

Modeling Geologic Storage of Carbon Dioxide:

Comparison of Nonhysteretic and Hysteretic Characteristic Curves

Christine Doughty

Site Characterization for $\mathrm{CO}_{2}$ Geologic Storage and Vice-Versa-

The Frio Brine Pilot, Texas, as a Case Study

Christine Doughty, Barry M. Freifeld, and Robert C. Trautz

Eddy Covriance Measurements of $\mathrm{CO}_{2}$ Emissions at Mammoth Mountain, California

Jennifer L. Lewicki, Marc L. Fischer, and George E. Hilley

Natural Analogues for $\mathrm{CO}_{2}$ Leakage from Geologic Storage Sites

Jennifer L. Lewicki, Jens Birkholzer, and Chin-Fu Tsang

Certification Framework (CF) for Geological $\mathrm{CO}_{2}$ Storage

Curtis M. Oldenburg, Steven L. Bryant, Jean-Philippe Nicot, Yingqi Zhang, and Preston Jordan

Simulations of $\mathrm{CO}_{2}$ Release for The ZERT Montana Shallow Release Experiment

Curtis M. Oldenburg

Joule-Thomson Cooling due to $\mathrm{CO}_{2}$ Injection into Natural Gas Reservoirs

Curtis M. Oldenburg

ECO2N: A TOUGH2 Fluid Property Module for Mixtures of Water, $\mathrm{NaCl}$, and $\mathrm{CO}_{2}$

Karsten Pruess

Analysis of Fault Rupture and $\mathrm{CO}_{2}$ Upwelling during the 1960s Matsushiro Earthquake Swarm as a Natural Analogue of $\mathrm{CO}_{2}$ Storage and Leakage

Jonny Rutqvist, Frederic Cappa, Jennifer Lewicki, and Sally Benson

Coupled Reservoir-Geomechanical Analysis of Geomechanical Damage

Associated with $\mathrm{CO}_{2}$ Geological Storage

Jonny Rutqvist, Jens Birkholzer, and Chin-Fu Tsang

A Model of Buoyancy-Driven Evolution of a Carbon Dioxide Plume

Dmitriy Silin, Tad Patzek, and Sally M. Benson 
Development of an Efficient Parallel Simulator for Modeling

Keni Zhang, Christine Doughty, Yu-Shu Wu, and Karsten Pruess

System-Level Modeling for Economic Evaluation of Geological $\mathrm{CO}_{2}$ Storage in Gas Reservoirs

Yingqi Zhang, Curtis M. Oldenburg, Stefan Finsterle, and Gudmundur S. Bodvarsson

Nuclear Energy and Waste Program

Geomechanical/Geochemical Modeling Studies Conducted

with the International Cooperative Decovalex-THMC Project

Jens Birkholzer, Jonny Rutqvist, Eric Sonnenthal, and Chin-Fu Tsang

Continuous-Time Random-Walk Analysis of Dual-Permeability Fractured Media

Andrea Cortis and Jens Birkholzer

Heterogeneous Seepage at the Nopal I Uranium Mine, Chihuahua, Mexico

Patrick F. Dobson, Paul J. Cook, Teamrat Ghezzehei, J. Alfredo Rodríguez, and Rodrigo de la Garza

Evaluation of Uncertainties due to Hydrogeological Modeling and Groundwater Flow Analysis:

Strategy for Characterizing a New Site

Christine Doughty and Kenzi Karasaki

Analysis of Flowing Fluid-Electric-Conductivity Logs under Nonideal Conditions

Christine Doughty and Chin-Fu Tsang

Climatic Forecasting of Net Infiltration at Yucca Mountain Using Analogue Meteorological Data

Boris Faybishenko

A Modeling Study on Natural Convection in Emplacement Drifts and Its Impact on Drift Seepage

N. Halecky, J. Birkholzer, G. Danko1, and D. Bahrami

Examining the Drift Shadow in Nature

Timothy J. Kneafsey, Teamrat Ghezzehei, Paul Cook, and John Peterson

Sensitivity Analysis for Joint Inversion of Ground-Penetrating Radar and

Thermal-Hydrological Data from a Large-Scale Underground Heater Test

Michael B. Kowalsky, Jens Birkholzer, John E. Peterson, Stefan Finsterle,

Sumit Mukhopadhyay, and Yvonne Tsang

Mechanisms for Scale Dependence of the Effective Matrix Diffusion Coefficient

Hui-Hai Liu, Yingqi Zhang, Quanlin Zhou, and Fred J. Molz

Feedback of Coupled Thermal-Hydrological-Chemical Processes on

Seepage at Yucca Mountain, Nevada: Role of Permeability and Capillarity Heterogeneity

Sumit Mukhopadhyay, Eric L. Sonnenthal, and Nicolas Spycher

Simple Model Representations of Transport in a Complex Fracture

and Their Use in Long-Term Predictions

Chin-Fu Tsang and Christine Doughty

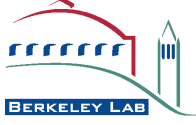


Estimating the Large-Scale Fracture Permeability of Unsaturated Rock Using Barometric Pressure Data Yu-Shu Wu, Guoping Lu, Keni Zhang, and Lehua Pan

Coupling Seepage and Radionuclide Fate/Transport in and around 


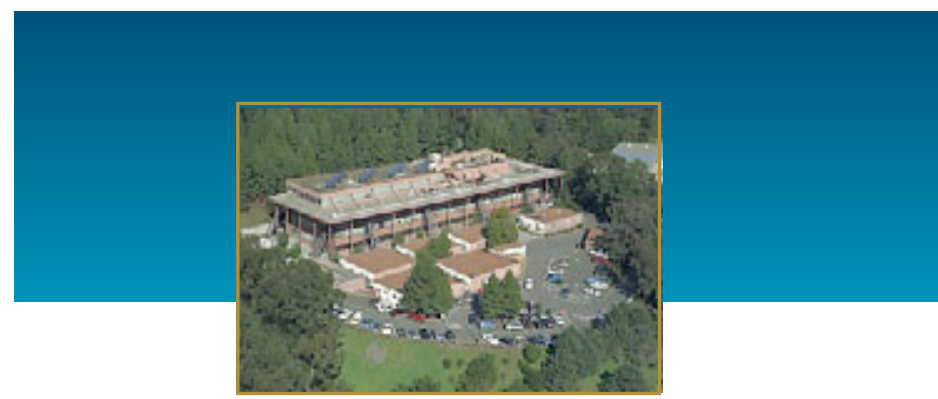

Ernest Orlando Lawrence Berkeley National Laboratory

\section{EARTH SCIENCES DIVISION RESEARCH SUMMARIES 2006-2007}

A Perspective from the Division Director

\section{Donald J. DePaolo}

(510)486-7560

DJDepaolo@lbl.gov

Research in earth and atmospheric sciences has become increasingly important in light of the energy, climate change, and other environmental issues facing the United States and the world. The development of new energy resources other than fossil hydrocarbons, the safe disposal of nuclear waste and greenhouse gases, and a detailed understanding of the climatic consequences of our energy choices are all critical to meeting energy needs while ensuring environmental safety. The cleanup of underground contamination and the preservation and management of water supplies continue to provide challenges, as they will for generations into the future.

To address the critical energy and environmental issues requires continuing advances in our knowledge of Earth systems and our ability to translate that knowledge into new technologies. The fundamental Earth science research common to energy and environmental issues largely involves the physics, chemistry, and biology of fluids in and on the Earth. To manage Earth fluids requires the ability to understand their properties and behavior at the most fundamental molecular level, as well as prediction, characterization, imaging, and manipulation of those fluids and their behavior in real Earth reservoirs. The broad range of disciplinary expertise, the huge range of spatial and time scales, and the need to integrate theoretical, computational, laboratory and field research, represent both the challenge and the excitement of Earth science research.

The Earth Sciences Division (ESD) of the Ernest Orlando Lawrence Berkeley National Laboratory (Berkeley Lab) is committed to addressing the key scientific and technical challenges that are needed to secure our energy future in an environmentally responsibly way. Our staff of over 200 scientists, UC Berkeley faculty, support staff and guests perform worldacclaimed fundamental research in hydrogeology and reservoir engineering, geophysics and geomechanics, geochemistry, microbial ecology, climate systems, and environmental engineering. Building on this scientific foundation, we also perform applied earth science research and technology development to support DOE in a number of its program areas. We currently organize our efforts in the following Division Programs:
- Fundamental and Exploratory Research-fundamental research in geochemistry, geophysics, and hydrology to provide a basis for new and improved energy and environmental technologies

- Climate and Carbon Sciences_carbon cycling in the terrestrial biosphere and oceans, and global and regional climate modeling, are the cornerstones of a major developing divisional research thrust related to understanding and mitigating the effects of increased greenhouse gas concentrations in the atmosphere

- Energy Resources-collaborative projects with industry to develop or improve technologies for the exploration and production of oil, gas, and geothermal reservoirs, and for the development of bioenergy.

- Environmental Remediation and Water Resources-innovative technologies for locating, containing, and remediating metals, radionuclides, chlorinated solvents, and energy-related contaminants in soils and groundwaters

- Geologic Carbon Sequestration-development and testing of methods for introducing carbon dioxide to subsurface geologic reservoirs, and predicting and monitoring its subsequent migration.

- Nuclear Waste and Energy-theoretical, experimental, and simulation studies of the unsaturated zone at Yucca Mountain, Nevada.

These programs draw from each of ESD's disciplinary departments: Climate Science, Ecology, Geochemistry, Geophysics, and Hydrogeology. Short descriptions of these departments are provided as introductory material.

In this document, we present summaries of selected current research projects. While it is not a complete accounting, the projects described here are representative of the nature and breadth of the ESD research effort. We are proud of our scientific accomplishments and we hope that you will find this material useful and exciting. A list of publications for the period from January 2006 to June 2007, along with a listing of our personnel, are also appended. Any comments on our research are appreciated and can be sent to me personally.

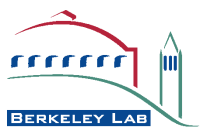




\section{Acknowledgments}

We gratefully acknowledge the support of our major sponsors in the Department of Energy, which include the Office of Science, the Office of Fossil Energy, the Office of Energy Efficiency and Renewable Energy, and the Office of Civilian Radioactive Waste Management. We also appreciate the support received from other state and federal agencies such as the Bureau of Reclamation, the Department of Defense, the
Environmental Protection Agency, NASA, and others. Lastly, we must also acknowledge and thank our industrial collaborators, universities, and other National Laboratories, who provide both financial and in-kind support through various partnership projects, and who bring additional ideas, data, and expertise to ESD.

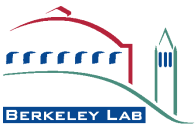




\section{OUR ORGANIZATION}

Management (and Name) Changes

In November 2007, the Earth Sciences Division (ESD) welcomed its new Director, Don DePaolo. A professor at UC Berkeley in the Earth and Planetary Science Department, Don is also the long-time head of the Center for Isotope Geochemistry, as well as ESD's former Geochemistry Department Manager. Consequently, he was no stranger to ESD and knew what was in store for him. To quote his words at his first Town Hall meeting with the entire ESD staff, "So far it's been fun!" and we hope the fun continues. Ernie Majer, who had stepped in as the Acting Division Director in November 2006, gladly handed over the reigns (and a new management team) to Don.

In early 2006, in keeping with the rotational nature of the Department Head role, Curt Oldenburg was succeeded by Stefan Finsterle for the Hydrogeology Department, and Mark Conrad took over the Geochemistry Department from Don DePaolo. In the summer of 2006, Jim Berryman was hired to assume the Geophysics Department Head role from Ernie Majer. By April 2007, Bill Collins was hired to lead the new Climate Science Department. In addition, the Geologic Carbon Sequestration Program was formed, led by Larry Myer and Curt Oldenburg. Finally, in the fall of 2007, Ernie Majer assumed full Program Head responsibilities for Energy Resources. Also note that since the beginning of 2006, there have been slight changes to the Division Program names, which are reflected in the Organizational Chart (p. ii).

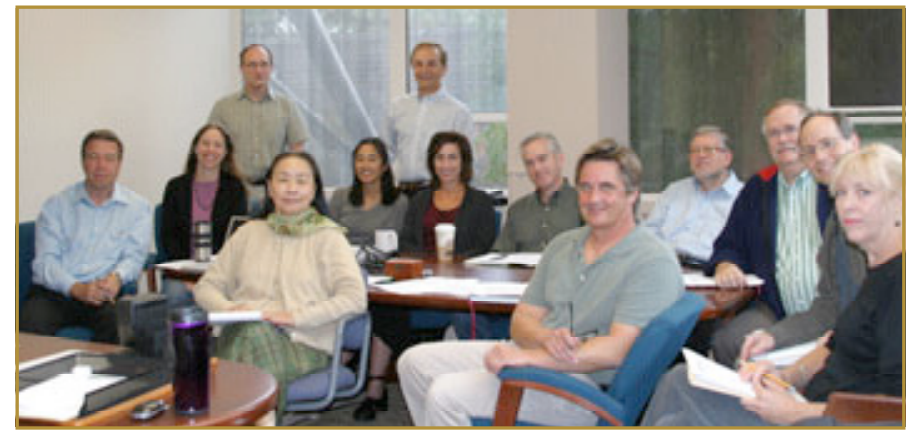

For more information on Berkeley Lab's organization, please visit http:/ / www.lbl.gov.
Maryann Villavert

(510)486-7357

MVillavert@lbl.gov

\section{OUR SAFETY CONSCIOUS WORK ENVIRONMENT}

The ESD approach to safety includes Berkeley Lab's Integrated Safety Management model. This model identifies the individual employee as the first person accountable for his or her own health and safety, assisted by a Division Safety Coordinator, who in turn works with a Berkeley Lab Environmental Health and Safety Liaison, to maintain an open line of communication with Berkeley Lab's Environmental Health and Safety Division. Because ESD has a matrixed structure, the employee works with both supervisors and principal investigators to identify, manage, and elevate issues to the ESD Safety Coordinator, Department Head, and Division Director. Additionally, the ESD Safety Committee meets regularly to discuss ESD and Berkeley Lab issues.

In November 2007, Vassiliki "Vivi" Fissekidou was appointed as the new ESD Safety Coordinator and the Chair of the ESD Safety Committee. The ESD Safety Committee also implemented a 3-year-service rotation for its members. The members include representatives from each ESD department, as well as additional members that represent specific work environments (laboratories, field work on land and at sea, computational activities, etc.).

Safety will always be a top priority for ESD. We attribute the success of our safety program to all our staff, which has been very cooperative and pro-active in resolving safety issues, providing suggestions on improvements to safety practices, and implementing changes that result in a safer work environment. For more information about ESD's Health and Safety program, visit http: / / www-esd.lbl.gov/ESDEHS / index.html

\section{TECHNOLOGY TRANSFER}

Over the past two years, ESD has partnered effectively with Berkeley Lab's Technology Transfer Department to license software/ codes and secure patents (as appropriate) for ESD-developed technologies. We have also earned accolades!

Licenses-The Division successfully licensed several of its software codes, such as Tough-Fx and Tough-Fx / Hydrate Software and EM2D_INV. These codes and others are available for purchase by contacting Berkeley Lab's Technology Transfer

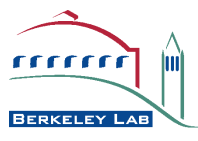


Department. The Tough Family of Codes and information on purchasing these codes can be found at http://wwwesd.lbl.gov/TOUGHPLUS/. ESD is continuing to identify and develop more technologies that can be licensed. To learn more about Earth Sciences and Berkeley Lab's available licensed technology, go to http: / / www.lbl.gov/Tech-Transfer /

Accolades - In 2007, the $R$ E $D$ Magazine's 100 Top Technologies award, also known as the "R\&D 100 Awards" went to the Berkeley Unexploded Ordnance Discriminator, developed by (among others) ESD's Frank Morrison, Erika Gasperikova, Alex Becker, and J. Torquil Smith. In 2006, the award was given to The Carbon Explorer, developed principally by ESD's Jim Bishop.

\section{A DIVERSE WORKFORCE}

One of ESD's primary goals is to create a supportive environment to attract, nurture, and retain the most qualified and diverse workforce, including under-represented group members. Along with Berkeley Lab's Workforce Diversity Office and the Center for Science and Engineering Education (CSEE), we continually evaluate and identify the necessary tools to support and enrich our workforce. Erika Gasperikova serves as the Chair of the ESD Diversity Committee and is the Representative of LBNL's Best Practices Diversity Council.

\section{WRITING TRAINING AND DEVELOPMENT}

In support of employee development and training, ESD was very fortunate to have in-house experts who have volunteered to share their experience and knowledge regarding writing in a scientific environment. One of these courses, was presented by fellow scientists, Karsten Pruess and Yu-Shu Wu several years ago, with much interest. In 2007, we presented the return of Karsten and Yu-Shu for "Science Writing" and added another course offered by ESD's Technical Writer/Editor, Dan Hawkes on "The Art of Science Writing."

\section{FACILITIES/CENTERS}

\section{Center for Computational Seismology}

The Center for Computational Seismology (CCS), which focuses on geophysical computing research, maintains a state-ofthe-art computing environment in support of various seismological and geophysical researdh programs, in particular the development of new methods for imaging the subsurface and its processes, and methods for visualizing results. A wide variety of modern software and hardware is developed and maintained to support this high-level research. In addition to the many "inhouse"-developed codes (three-dimensional modeling, forward and inverse codes, etc.), we use a wide variety of commercially supported packages, including CogniSeis Focus (interactive 3-D seismic processing), Baker-Atlas SEISLINK (VSP and crosswell imaging), GeoQuest GXII (interactive raytrace modeling for surface and borehole data), Lynx (geologic modeling), Earthvision (Dynamic Graphics) AVS (3-D visualization), and the complete Promax/Landmark processing and modeling software. These packages provide a powerful modeling base upon which we build our specialized codes.

Our facilities support researdh focused on subsurface imaging, using active and passive sources at scales ranging from meters to whole-Earth dimensions. Research activities include the processing and interpretation of vertical seismic profiles (VSP) for fracture detection and fault delineation, induced seismicity associated with energy resources, seismic reflection imaging, single well and crosshole seismic profiling for 2-D and 3-D imaging, fracture detection between wells, and processing/analysis of micro-earthquake data for imaging of geothermal fields. The hardware facilities include multiple Exabyte tape drives, over 8000 Gbytes of hard drive storage, a 24-inch color Versatec plotter, a 36-inch HP color plotter, and multiple $\mathrm{X}$-terminals and workstations. We have recently upgraded our computer system to the new SUN series 4-CPU server, with10 GByte of memory and 4 Tera bytes of disk. We also recently acquired a "pc cluster" with 256 nodes ( 2 cpu per node) at 3.6 $\mathrm{GHz}$ and 4 Gbyte memory per node. In addition, the CCS facility is linked to the National Energy Research Supercomputing Center at Berkeley Lab, which hosts a variety of supercomputers available for use.

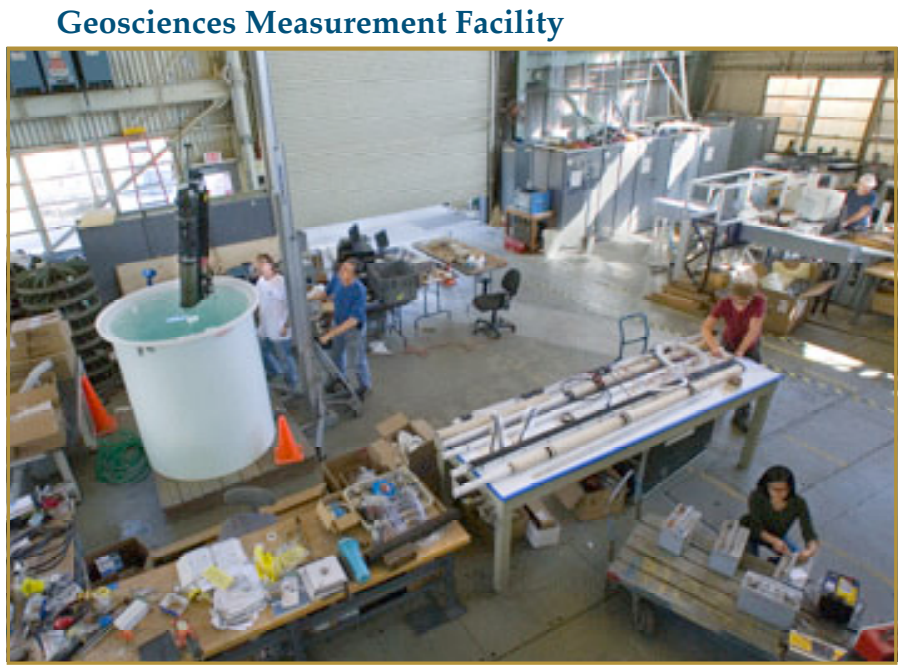

ESD field work is supported by the Geophysical Measurements Facility (GMF), a DOE-supported facility designed to develop and maintain a variety of geophysical and geoscience instrumentation and measurement equipment. For example, research on piezoelectric sources and borehole sensor arrays, as well as high-frequency seismic recording, has been supported by GMF for over 10 years. GMF is the focal point for an extensive inventory of complex scientific equipment used for Berkeley Lab projects, with responsibility for the maintenance,

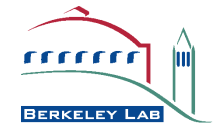


upgrading, training, and field operations of this hardware. GMF will allow for management of the full complement of sophisticated field instrumentation and associated support vehicles necessary to test and develop a piezoelectric multisource phased array. GMF maintains a state-of-the-art multidisciplinary field instrumentation facility in support of various environmental, geophysical, and hydrogeological researdh programs at Berkeley Lab. This facility also assists in development of new instrumentation and field methods for investigating the subsurface and its processes by providing professional in-field technical support for scientific staff and management of the complex and varied field studies required in scientific research programs. The GMF includes electronic and mechanical technicians and shop facilities, field support vehicles, (including wireline and recording trucks), and a three borehole test facility.

\section{Center for Environmental Biotechnology}

The core microbiology facility of LBNL is in the Center for Environmental Biotechnology, located in Building 70 and 70A. The seven-laboratory unit occupies a total area of 5,290 $\mathrm{ft}^{2}$. The laboratories are set up for Class II, Type A/B3 molecular- and microbiology work. Level 1 quality and safety assurance procedures are in place. The following work-specific equipment and instruments are available in the laboratories:

- Lietz Laser Con Focal Microscope with digital imaging.

- 5 SterilGARD II 6-foot vertical laminar-flow, biological safety cabinet (Baker);

- 4 Fermantic 5L Extreomophile bioreactors;

- 2 Fairmentec 3L Extreomophile bioreactors

- 2 Avanti J-25 high performance centrifuge (Beckman);

- DU 640 UV/VIS scanning spectrophotometer (Beckman);

- 4 Ultra-low temperature freezer (Revco);

- 2 Axioskop RLF for DIC, phase contrast, epifluorescence, microphotography (Zeiss);

- Integrated SpeedVac (Savant);

- Electroporator;

- Affymetrix Microarray Systems;

- BioRad MyiQ RT-PCR system

- 2 GeneAmp PCR system 9700 (Applied Biosystems)

- Model 600 PCR Workstation Hood (AirClean)

- QuantityOne Gel Documentation System (BioRad)

- Nanodrop model ND 1000 Spectrophotometer

- Nanodrop model ND 3300 Fluorospectrophotometer

- Model 5415-R refrigerated bench top centrifuge (Epindorf)

- FastPrep 120 Bead mill homogenizer (Bio101)

- Dual 96-well tissue homogenizer (Qiagen)

- 3 Coy double wide anaerobic chambers with incubators;

- GeneAmp PCR system 9600 (Perkin-Elmer);

- Expedite 8909 DNA synthesizer (PerSeptive Biosystems);

- Model 377 ABI Prism automated DNA sequencer (Perkin Elmer);
- CHEF DRII pulsed field electrophoresis equipment (Bio-Rad)

- OmniLog Phenotypic Microarray Systems

- MIDI identification system (Hewlett Packard);

- High sensitivity MSD mainframe for the HP 6890 GC (Hewlett Packard)

- BIOLOG microbial identification system (BIOLOG);

- Environmental shakers with photosynthetic light banks (New Brunswick);

- Columbus Instruments Microrespirometer $\left(\mathrm{H}, \mathrm{H}_{2} \mathrm{~S}, \mathrm{CO}_{2}, \mathrm{CH}_{4}, \mathrm{O}_{2}\right)$

- Alliance HPLC system with a 996 photodiodphotodiode array detector and a 474 scanning fluorescence detector (Waters).

- Dionex 2000 Ion Chromatograph

- Sonicator with microprobe

- 1D Gel electrophoresis set up

Other necessary support equipment and installations are also available, such as autoclave, DI-water, refrigerators, freezers for low temperature storage of temperature sensitive materials, balance, ice-maker, shakers, incubators, magnetic stirrer, hotplates, microcentrifuges, computers, different electrophoresis boxes and power supplies, chemical fume hoods. The core facility has access to the Environmental Measurement Laboratory at LBNL.

For more information concerning the Center for Environmental Biotechnology Core Facility, please contact Terry Hazen, phone: 510-486-6223, e-mail: TCHazen@lbl.gov

\section{Soil and Rock Properties Laboratory}

At Berkeley Lab's Soil and Rock Properties Lab, electrical resistivity, ultrasonic wave propagation, and hydraulic conductivity can be measured in a triaxial cell equipped to measure all these parameters simultaneously. Confining and axial stresses are set independently to represent in situ states of stress. The cell is designed to handle samples from 3-inchdiameter Shelby tubes, using sample transfer techniques developed from geotechnical practice. The sample is jacketed with a flexible membrane, either latex, viton or teflon, depending on the sample texture and fluid composition. Sample length is determined by considerations of ultrasonic wave attenuation and the extent of stratification of the core. Typically, samples of approximately $5 \mathrm{~cm}$ lengths are used, although different lengths can be accommodated. The advantage of making these measurements simultaneously on the same sample is that disturbance from sample transfer between test cells, a particular concern for unconsolidated samples, is avoided. The endcaps of the test cell contain $1 \mathrm{MHz}$ piezo-electric crystals for $\mathrm{P}$ - and S-wave transmission and receiving, flow ports, and pressure ports. Porous aluminum plates between the sample and the endcaps provide even flow distribution over the sample cross section. Both faces of the aluminum plates are gold coated.

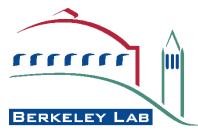


Electrical resistivity is measured by the four-electrode technique. Electrical current is driven through the outside faces, and voltage drop is measured from the inside faces; a GenRad 1692 RLC Digibridge supplies current and measures voltage drops at five test frequencies varying from $100 \mathrm{~Hz}$ to $100 \mathrm{kHz}$. $\mathrm{P}$-wave and S-wave propagation (velocity and attenuation) is measured by the pulse-transmission technique.

Voltage pulses are generated by Cober Model 605P High Power Pulse Generator (Cober Electronics, Stamford, Connecticut), and data are acquired via a $40 \mathrm{MHz}$ Gagescope data acquisition board (Gage Applied Sciences Inc., Montreal, Quebec) installed in a PC. Hydraulic conductivity is measured either by the constant head method for more permeable samples, or by the falling head method for tighter samples. Differential pressure across the column is measured with variable reluctance transducers (Validyne, Northridge, CA). When possible, site water is used for the hydraulic conductivity measurements to avoid dispersion of clays. Otherwise, test water is generated based upon a chemical analysis of the site water. In addition to the above facilities, a highresolution x-ray facility (linear x-ray and CAT scan) and an NMR imaging facility enables detailed core studies and simultaneous flow and transport studies. Further capabilities are provided by linking these studies with ultra-high-resolution tomographic work at Berkeley Lab's Advanced Light Source.

For more information on CCS, GMF, or the Rock Lab, please contact Ernest L. Majer, phone: 510-486-6709, elmajer@lbl.gov

\section{Center for Isotope Geochemistry}

The Center for Isotope Geochemistry (CIG), Lawrence Berkeley National Laboratory, and Department of Geology and Geophysics, University of California, Berkeley, is directed by Prof. Donald J. DePaolo. The Center includes six analytical facilities: stable isotope and noble gas isotope laboratories; a soil carbon laboratory; an analytical chemistry laboratory; the Inductively Coupled Plasma Multi-Collector Magnetic Sector mass spectrometry laboratory, and a thermal-ionization mass spectrometry laboratory located on the UC Berkeley campus. We also have an affiliation with the cosmogenic isotope laboratory in UC Berkeley's Space Sciences Laboratory. These facilities provide state-of-the-art characterization of all types of earth materials for research throughout the department and elsewhere in ESD. The instrumentation and laboratories are an integral part of the Center's focus on new ways to use isotopic ratio methods to study fundamental earth processes and environmental and energy problems of national interest.

\section{Summary of Research Areas}

and Programmatic Applicability

The research we pursue is ultimately guided not only by the needs of advancing basic energy science (BES), but by applicability to other basic and applied programs within the DOE mission-nuclear waste management (NW), environmental remediation science (ERSP), fossil and geothermal energy resources (FE, GE), climate change and carbon management (CCC), and security.

1. Basic geochemistry (BES)

- Dissolution, precipitation, ion exchange and transport in natural systems

- Isotopic probes of micro- and nanoscale material properties

- Geochronology

- Isotopic variations in nature due to chemical and nuclear processes

- Volcanic processes and volcano monitoring

- Reactive transport: conceptual and numerical models, isotopic effects

\section{Groundwater processes}

- Field-scale characterization of groundwater systemsflow velocities, effective permeabilities, infiltration rates (ERSP, NW)

- Mineral-fluid reaction rates in fluid-rock systems (ERSP, NW, FE, GE)

- Isotopic monitoring of subsurface remediation (ERSP)

- Isotopic diagnostics for subsurface chemical processes (ERSP, NW, FE, GE)

- Isotopic diagnostics and phase partitioning tracers related to geologic sequestration of carbon (FE, CCC)

\section{Hydrothermal systems}

- Reservoir characteristics, water and heat sources (GE, NW)

- Relations between volcanism and geothermal potential, volcanic hazards (GE)

4. Sedimentary basin hydrology

- Characterizing geological structure and reservoir properties (FE, ERSP)

- Tracing fluid flow (FE, CCC)

- Sourcing hydrocarbons (FE)

\section{Isotopic climate records (GCC, NW)}

For more information concerning the Center for Isotope Geochemistry, please contact: B. Mack Kennedy, Center for Isotope Geochemistry, phone: 510-486-6451, e-mail: bmkennedy@lbl.gov

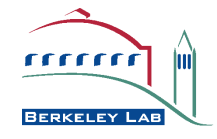




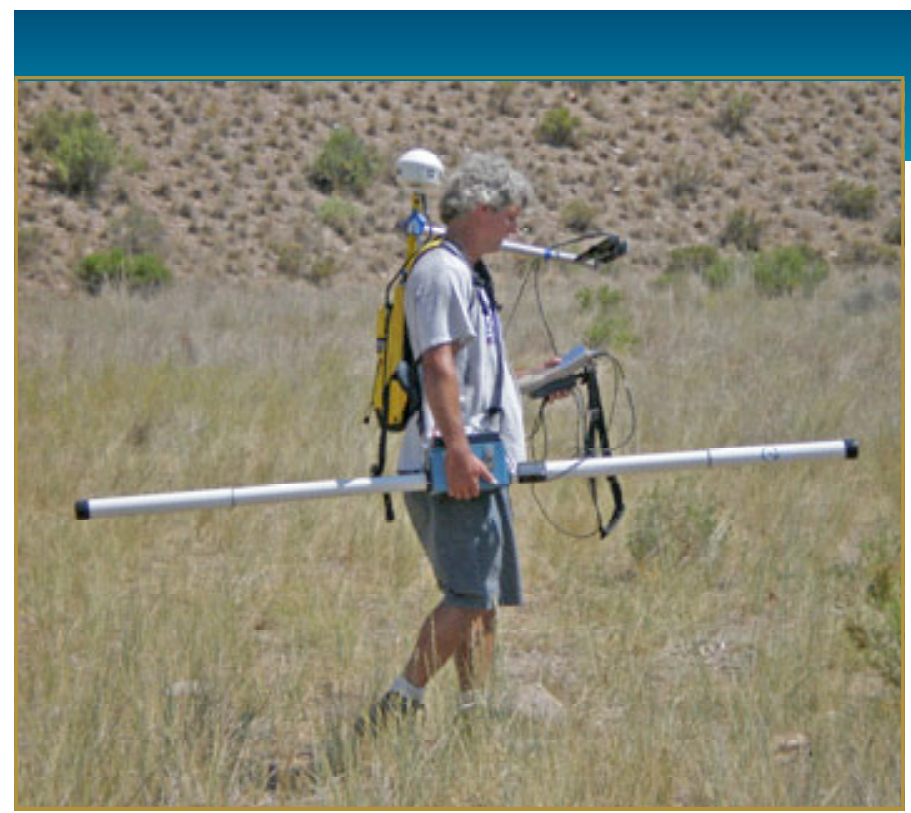

Earth Sciences Division Research Summaries 2006-2007 Resource DepartMents

\section{Hydrogeology Department}

GEOPHYSICS DEPARTMENT

GeOCHEMistry DepartMent

ECOLOGY DEPARTMENT

Climate Science Department 


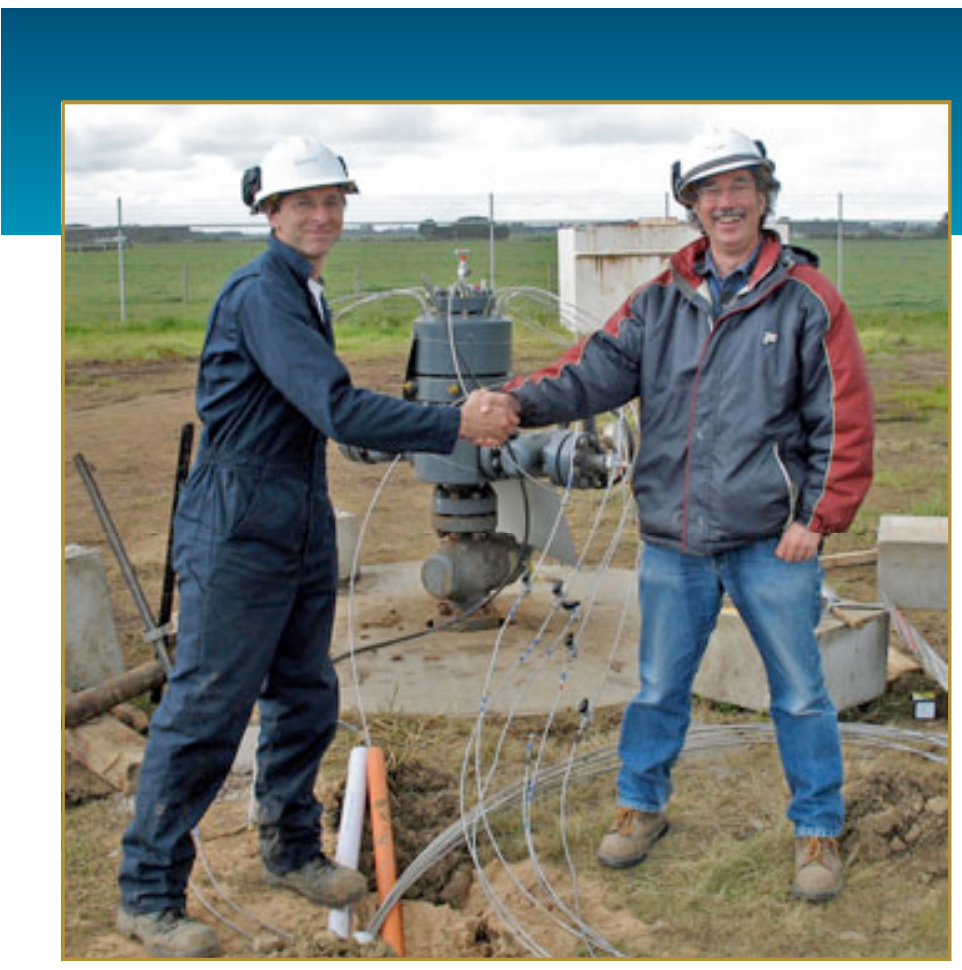

The Hydrogeology Department (HD) consists of more than 50 scientists, postdocs, research associates, and graduate students carrying out a broad range of cutting-edge research in fundamental and applied hydrogeology. HD has expertise in theoretical, experimental, field, and modeling approaches in a variety of research areas, among which are unsaturated zone and fractured rock hydrology, reservoir engineering (geothermal, gas, oil, and hydrates), $\mathrm{CO}_{2}$ sequestration and nuclear waste isolation, contaminant hydrology, and coupled nonisothermal, geochemical, and geomechanical processes. Highlights of research efforts in these areas over the last two years include the following:

\section{SUBSURFACE ENERGY RESOURCE RECOVERY}

Researchers in HD are studying ways to enhance production of energy from subsurface reservoirs containing methane gas hydrates, geothermal energy, and traditional oil and gas resources. In the area of methane hydrates, HD scientists are carrying out a sustained laboratory campaign to uncover fundamental properties of methane hydrates, such as dissociation kinetics and constitutive models, which can be incorporated into the world's leading methane hydrate simulator, TOUGH+Hydrate. Moreover, numerical simulation studies and laboratory experiments were conducted to examine the geomechanical behavior of hydrate-bearing sediments. The potential for significant methane releases from oceanic sediments caused by hydrate dissociation in response to climate change has also been investigated.
Resource Department

Hydrogeology DePARTMENT
Stefan Finsterle

510-486-5205

SAFinsterle@lbl.gov

Continuing the long tradition of geothermal research in the ESD, staff members in HD are investigating reactive geochemistry in geothermal reservoirs, to devise ways to avoid mineral scaling and maintain injectivity without inducing short-circuiting flow paths. This effort is undertaken using TOUGHREACT, the reactive geochemical simulator developed by HD researchers. HD scientists performed the first quantitative analysis of a novel concept for producing geothermal energy that would use carbon dioxide instead of water as heat transmission fluid, and would accomplish geologic storage of $\mathrm{CO}_{2}$ as an ancillary benefit. $\mathrm{CO}_{2}$ was shown to achieve approximately $50 \%$ larger heat extraction rates than water.

In collaboration with researchers in the ESD Geophysics Department, HD staff confirmed that viscous fluid flow creates anomalies at low seismic frequencies that can be used to image oil reservoirs. By this method, re-analysis of 3-D seismic data, using frequency-dependent approaches, has revealed hydrocarbon-rich layers where none was detected by standard analysis.

\section{SUBSURFACE REMEDIATION}

HD researchers address national and international needs for subsurface contaminant characterization and remediation across the spectrum of approaches. In the lab, HD researchers are investigating some of the nation's most critical subsurface contamination issues, including the chemical evolution of highly alkaline Hanford tank waste, reduction, re-oxidation, and diffusion of uranium (U)VI in sediments, hydraulic properties of unsaturated gravels, and the natural production of transport-enhancing mobile nanoparticles in the subsurface.

Advances in subsurface characterization are expected to follow from HD's work on joint hydrologic and geophysical inversion. Specifically, the approach aims at identifying soil structure, flow and transport properties, and the system state

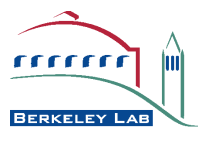


by jointly estimating hydrogeological, petrophysical, and geostatistical parameters, using hydrological, thermal, biogeochemical, and geophysical data. Geophysical models for simulating ground penetrating radar and electrical resistivity tomography have been implemented in the iTOUGH2 nonisothermal multiphase flow and transport simulation and optimization code, enabling us to jointly invert complementary data sets. The approach has been studied extensively using synthetic data, and has been applied to field data at contaminated sites, including those at Hanford and the Savannah River Site

\section{GEOLOGIC $\mathrm{CO}_{2}$ STORAGE}

HD researchers are involved in a wide range of efforts involving field pilot tests, laboratory work, simulation and modeling of coupled $\mathrm{CO}_{2}$ flow and transport processes, and risk assessment. Theoretical and modeling accomplishments aim at answering fundamental questions about the effects of $\mathrm{CO}_{2}$ injection on hydrological, geochemical, and geomechanical conditions in a potential storage formation. Field pilot tests of $\mathrm{CO}_{2}$ injection were performed that included tracer tests, sampling, and geophysical surveys. Numerical simulations were used to design and analyze these tests. Field measurements of $\mathrm{CO}_{2}$ flux using eddy covariance and accumulation chamber methods were employed to examine the possibility of detecting potential leakage from $\mathrm{CO}_{2}$ storage sites. These field tests were complemented with theoretical and modeling studies. Systemlevel modeling tools were employed to evaluate the feasibility of carbon sequestration with enhanced gas recovery.

\section{NUCLEAR WASTE DISPOSAL IN THE} UNSATURATED ZONE

The motivation for HD's extensive effort in unsaturated zone hydrology and coupled processes is stimulated by the need to understand flow and transport in the unsaturated zone at Yucca Mountain, Nevada. Research by HD scientists in this p rominent area includes infiltration and seepage, coupled nonisothermal and geomechanical effects, and transport of radionuclides. The decade-long contributions of ESD and HD staff to the understanding and characterization of the hydrological, thermal, and geochemical conditions at Yucca Mountain (both before and after waste emplacement) are reflected in the technical reports and publications that support the license application to the Nuclear Regulatory Commission. The expertise gained in conceptualizing complex systems and phenomena in unsaturated fractured rock, and the related modeling, laboratory, and field testing, is increasingly applied to nuclear waste p rograms in other countries, as well as to other characterization and radionuclide transport problems in the U.S.

\section{FUNDING}

Funding for HD comes primarily from the U.S. Department of Energy, including: the Director, Office of Science, Office of Basic Energy Sciences, Division of Chemical Sciences, Geosciences, and Biosciences; the Director, Office of Science, Office of Biological and Environmental Research; the Assistant Secretary for Energy Efficiency and Renewable Energy, Office of Technology Development, Office of Geothermal Technologies; the Assistant Secretary for Fossil Energy, Office of Coal and Power Systems and Office of Natural Gas and Petroleum Technology, through the National Energy Technology Laboratory; the Director, Office of Civilian Radioactive Waste Management; and the Assistant Secretary of the Office of Environmental Management, Office of Science and Technology, Environmental Management Science Program. HD also receives funding support from the U.S. Environmental Protection Agency. Other funding is provided through the Laboratory Directed Research and Development Program at Berkeley Lab.

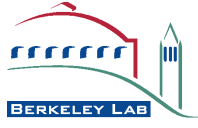




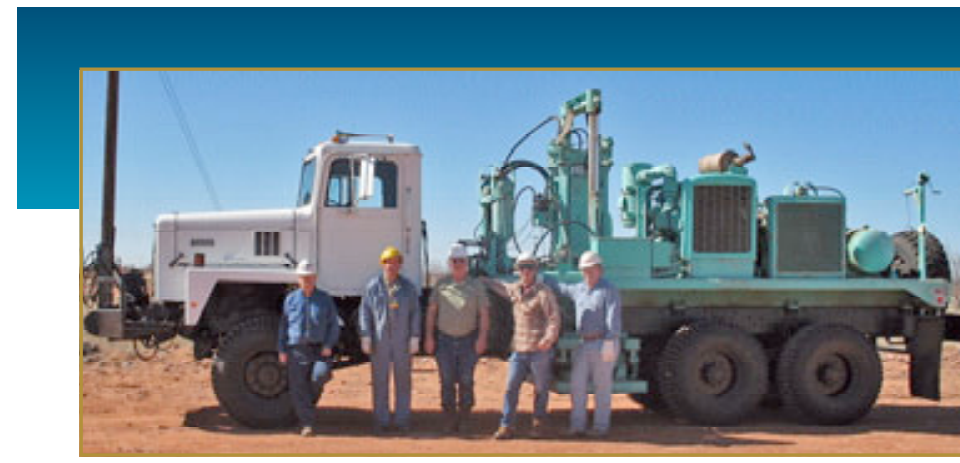

Resource Department

\section{GEOPHYSICS DEPARTMENT}

The ESD Geophysics Department is driven by two primary objectives. One is to advance the state of the art in new technologies for extracting subsurface properties, including fluid properties, saturation, porosity, pore pressure, permeability, and in situ stress from laboratory and/or field measurements at the earth surface or in boreholes. These new methodologies incorporate information obtained using a variety of data, including geophysical data such as seismic, electromagnetic (EM), electrical, seismo-electric, gravity, ground-penetrating radar (GPR), geomechanical (tilt, deformation), fluid flow (pressure), and satellite (GPS). The other, equally important objective is the development of technologies for subsurface resource extraction, including seismically enhanced oil recovery, geomechanics-based subsurface permeability enhancement, and unconventional hydrocarbon resource development, such as gas hydrate and tight gas.

Fundamental and applied research carried out in support of these objectives include laboratory rock physics and porescale imaging studies, field geophysical-imaging hardware development, theory development, computational geophysics as well as geomechanical modeling and analysis, together with imaging and inversion (both deterministic and stochastic) algorithm and code development. Motivating this research is the increasing need for direct imaging of fluid saturations, pore pressures, and permeability in the subsurface for energy production, environmental remediation, carbon management, and nuclear waste disposal purposes, and to do so in the presence of anisotropy and multiscale heterogeneities.

\section{SCIENTIFIC RESEARCH AREAS}

The department is organized into five scientific research areas, led by the indicated Research Area Leaders:

- Computational Geophysics (Don Vasco)

- Rock Physics and Coupled Dynamics (Seiji Nakagawa)

- Hydrogeophysics \& Biogeophysics (Susan Hubbard)

- Advanced Geophysical Instrumentation (Tom Daley)

- Geomechanics (Jim Berryman)
The primary purpose of these research areas is to advance the science that will enable new high-resolution methods for extracting subsurface properties and process-related information from geophysical, geomechanical, fluid-flow, and satellite data.

\section{Computational Geophysics (Don Vasco)}

The focus of this research area is to develop efficient 3-D numerical codes for modeling seismic wave propagation and electromagnetic wave propagation and diffusion. The challenge is to develop accurate and efficient computer codes capable of modeling the seismic and electromagnetic response of complex geologic structures (i.e., structures that may contain anisotropy or multiscale heterogeneities in the form of fractures, faults, and / or patchy saturation). A variety of methods, including boundary integral equation, global matrix, finite difference, spectral element, discrete element, and asymptotic ray methods, are currently being developed for high-performance parallel computing frameworks. These codes will serve as the computational engines for the next generation of modeling-based deterministic and stochastic inversion algorithms. This research is performed using the supercomputers at the National Energy Research Scientific Computing Center (NERSC) at Berkeley Lab, and the PC cluster maintained by the Center for Computational Seismology (CCS) within ESD.

\section{Rock Physics and Coupled Dynamics (Seiji Nakagawa)}

The connections between a geophysical observable (such as seismic velocities and attenuation, electrical conductivity, and dielectric constant) and rock properties (such as porosity, permeability, and fluid saturation) are provided by rock-physics measurements and/or theoretical understanding. Rock-properties measurement efforts are carried out at our Rock and Soil Physics Lab. This facility has electronics instrumentation and mechanical equipment needed to perform a variety of geophysical measurements, including seismic, electrical, electromagnetic, and fluid flow, under low to moderate confining pressures. Experiments requiring detailed information about the porous microstructure and fluid saturations at the pore

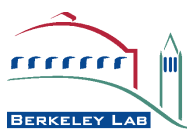


level are carried out using our x-ray computed tomography (CT) scanner in the Rock Imaging Lab, or using the focused ion beam (FIB) located at Berkeley Lab's National Center for Electron Microscopy. Other facilities at the Berkeley Lab Advanced Light Source (ALS) are also used for microtomography of geologic materials, including fluid-infiltrated porous media.

The primary focus of our laboratory efforts is towards increasing our experimental knowledge base for geophysical properties of rocks and sediments that are either not well described by conventional rock-physics understanding (e.g., poorly consolidated sands and clays, gas hydrates, fractured rock) or have yet to be fully exploited (e.g., seismic attenuation, seismo-electric response). Complementary theoretical efforts are also under way to explore the dynamics of poroelastic and seismoelectric responses in rock that contains one or multiple fluid phases.

\section{Hydrogeophysics \& Biogeophysics (Susan Hubbard)}

Research in this area combines the disciplines of geophysics, hydrogeology, and biogeochemistry to develop new approaches for characterizing shallow subsurface properties and for monitoring complex processes associated with natural or induced subsurface perturbations. This interdisciplinary field is unique in the level of fusion among hydrobiogeochemical-geophysical data sets, the incorporation of complex petrophysical models, and the application of emerging stochastic inversion techniques geared toward shallow subsurface systems. The majority of the research performed in this area is focused on developing and testing methods that will improve our ability to manage and monitor water resources and environmental contaminants.

\section{Advanced Geophysical Instrumentation (Tom Daley)}

The focus of this research area is the development of innovative geophysical hardware and methodologies for subsurface imaging and monitoring. Efforts that are currently under way include the development of passive and active seismi systems for utilizing microhole technology, an optimum electro- magnetic system for detecting and identifying unexploded ordnance, and a novel electromagnetic imaging system (for environmental applications) that operates in the frequency band between electromagnetic diffusion and wave propagation. Additionally, development continues on a small-diameter high-frequency orbital-vibrator shear source for crosswell and single-well seismic imaging applications, high-resolution tomographic (radar and seismic) tools, and micro-earthquake monitoring systems.

\section{Geomechanics (Jim Berryman)}

This research area is concerned with the development of new computational and analytical tools for predicting stressinduced changes in transport properties, rock-formation fracturing, and fault slip resulting from fluid injection, fluid withdrawal, and thermal loading. Of particular interest is the development of new computational geomechanics-based inverse methods for estimating subsurface fracturing and fluid movement, for predicting the seismic response resulting from fluid injection into fractured rock, and for relating seismic wave propagation velocities to these properties for purposes of reservoir monitoring and/or hydrocarbon resource localization.

\section{FUNDING}

The Geophysics Department derives its funding from a variety of U.S. Department of Energy (DOE) and non-DOE sources. The primary sources of DOE funding are the Director, Office of Science (Basic Energy Sciences and Biological and Environmental Research), Office of Environmental Management, Fossil Energy, Office of Geothermal Technologies, Office of Civilian Radioactive Waste Management, the Environmental Protection Agency, and the Berkeley Lab Laboratory Directed Research and Development (LDRD) Program. Non-DOE funding sources include the U.S. Department of Defense (SERDP and ESTCP), Shell Oil Company, Exxon Oil Company, Chevron Energy Research and Technology, and California Water Agencies. 


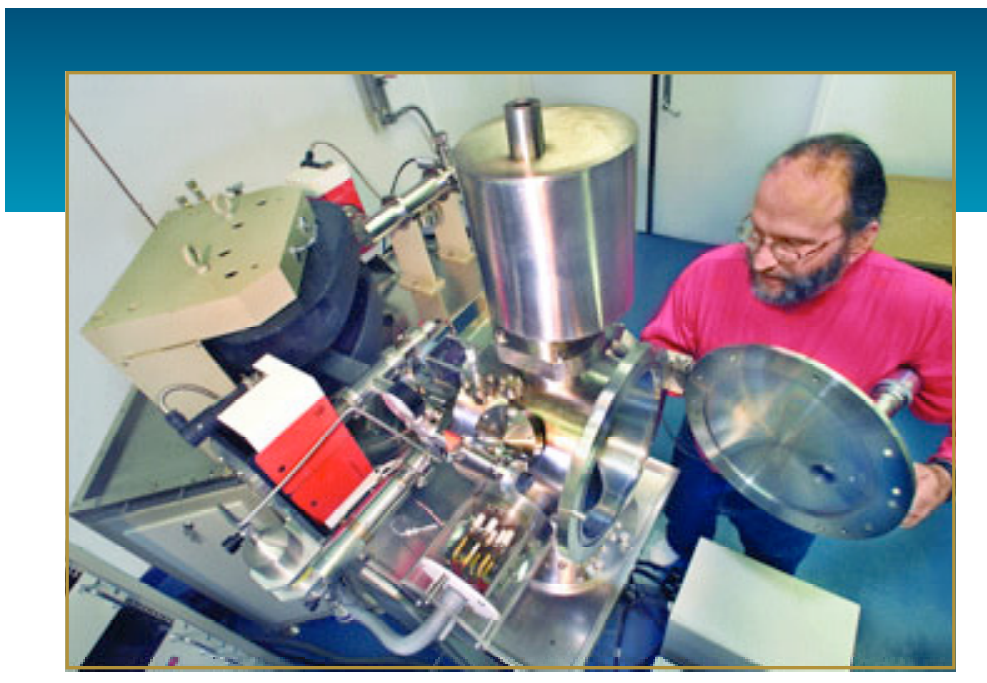

Resource Department

GEOCHEMISTRY DEPARTMENT

ESD's Geochemistry Department has expertise in isotopic geochemistry, reactive transport modeling, experimental mineral-water kinetics, molecular geochemistry and nanogeoscience, soil geochemistry, marine geochemistry, global and regional climate modeling, and mineralogy. The department has four major thrust areas, as described below.

\section{MOLECULAR GEOCHEMISTRY AND NANOGEOSCIENCE}

This effort involves fundamental studies on the nature of the aqueous solution/mineral interface, the structure of solvated ions and colloids down to the nanometer scale, and the properties and aggregation behavior of nanoparticles. Current work includes: molecular-dynamics modeling of the interlayersolvated cations in clays, the aggregation dynamics of nanoparticle iron oxides, and the structure of mineral interface water; studies of the solvation environment of contaminant and nutrient molecules in aqueous solution; determination of the molecular identity and kinetics of formation of iron oxide precipitates on quartz surfaces; and characterization of the surface chemistry and structure of environmentally important minerals using simulation, x-ray scattering, and x-ray spectroscopy. Many of the studies employ newly developed capabilities such as synchrotron x-ray grazing-incidence methods, and laser-based phase-sensitive nonlinear optical spectroscopy. Studies of the aqueous behavior of organic species on mineral surfaces and in solution, and on nanoparticle structure, are carried out at Berkeley Lab's Advanced Light Source as well as other synchrotron sites. The group also does extensive collaborative research using the National Center for Electron Microscopy (NCEM) at Berkeley Lab and takes advantage of the National Energy Research Scientific Computing (NERSC) facility (also at Berkeley Lab) as well as other computational sources for large-scale molecular dynamics and $a b$ initio simulations. Kinetics studies, mainly focusing on heterogeneous precipitation reactions, are also conducted

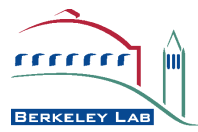
collaboratively with the National Science
Foundation Environmental Molecular Science Institute (NSF EMSI) at Pennsylvania State University.

\section{ISOTOPE GEOCHEMISTRY}

The Center for Isotope Geochemistry (CIG) includes stable isotope and noble gas isotope laboratories; a soil carbon laboratory; an analytical chemistry laboratory; the Inductively Coupled Plasma Multi-Collector Magnetic Sector mass spectrometry laboratory; and a thermal-ionization mass spectrometry laboratory located on the UC Berkeley campus. There is also an affiliation with the cosmogenic isotope laboratory in UC Berkeley's Space Sciences Laboratory. The CIG facilities enable state-of-the-art characterization of all types of earth materials. The instrumentation and laboratories are an integral part of the Center's focus on new ways to use isotopic ratio methods to study fundamental earth processes, and environmental and energy problems of national interest.

Examples of current research programs are: (1) development of models that use isotopic composition data from element pairs in fluids to constrain fluid flow rates, water-mineral reaction rates, and the geometry and spacing of fractures in rock matrices; (2) development and application of noble gas isotopes as natural tracers for fluid source and movement in hydrocarbon and geothermal systems, and as induced phase-partitioning tracers for monitoring geologic sequestration of $\mathrm{CO}_{2}$; (3) development of techniques for dating Quaternary geological events using U-Th-He; (4) use of U, N, and $\mathrm{O}$ isotopes to understand subsurface contamination sources; (5) geochemical monitoring and analysis of large-scale experiments simulating the effects of nuclear-waste heat generation within the proposed repository in Yucca Mountain, Nevada; (6) the use of C, N, and O isotopes to quantify in situ bioremediation and monitor remediation; (7) the use of carbon isotopes to quantify rates of organic carbon cycling and storage efficiency in soils, the impact of climate change on carbon cycling, and linkages between carbon, water, and nitrogen cycles; and (8) applications of hydrogen and oxygen isotopes to issues concerning the water cycle. 


\section{GEOCHEMICAL TRANSPORT}

This effort involves simulation and study of coupled mineral-water-gas reactive transport in unsaturated porous media. The work covers infiltration/evaporation processes in the soil zone, reaction-transport processes in fractured rock under boiling conditions, injection of $\mathrm{CO}_{2}$ in deep aquifers, hydrothermal alteration in geothermal systems, the controls on the rates of chemical weathering, and biogeochemical reaction networks in low-temperature environments. Although reaction-transport modeling and code development are the predominant activities, the group is also active in planning the analysis and drilling activities for underground thermal experiments, laboratory experiments focusing on the rates of water-mineral interaction, and field studies of geothermal systems and natural analogues for nuclear waste isolation. A new effort in this regard applies Lattice-Boltzmann models to reaction-transport processes at the microscopic scale. Efforts are also under way to understand the scale dependence of mineral-water reaction kinetics using pore network models. These new modeling efforts are being combined with the world's first experimental studies using engineered microfluidic reactors to determine mineral-water reaction rates directly at the pore $(\mathrm{mm})$ scale.

Much of the geochemical transport work is focused on predicting thermally driven processes accompanying the proposed emplacement of high-level nuclear waste at Yucca Mountain, Nevada, and on the understanding of the evolution of the natural hydrogeochemical system. One focus of this work is on integrating the thermal-hydrologic-chemical environment in the near field of the proposed Yucca Mountain repository with THC processes occurring inside emplacement drifts, including on the surface of the waste package (where corrosion is the main issue) and inside the waste packages (where dissolution of spent fuel is the main issue). Another focus is on understanding the controls on chemical weathering. One such effort involves integration of uranium-series isotopic disequilibria with major element profiles to determine in situ reaction rates in deep-sea marine sediments. Another involves understanding the controls on, and rates of, formation of weathering rinds; this work has demonstrated the key role of reaction-induced porosity change in controlling the weathering rate. Collaboration with other departments in ESD brings together essential pieces of the pro blem, including hydrological processes in the unsaturated zone, thermodynamics and kinetics of geochemical processes, and isotopic effects.

Current projects include:

(1) Analysis of geochemical and isotopic data from Yucca Mountain to constrain models of flow and transport in the unsaturated zone
(2) Development of models for reactive-transport in unsaturated systems and co-development of the reactive-transport code TOUGHREACT

(3) Improved thermodynamic and kinetic databases for water-rock interaction modeling

(4) Research on natural analogue sites, including the Yellowstone geothermal system, Peña Blanca, Mexico, and the Idaho National Engineering and Environmental Laboratory subsurface conditions

(5) Modeling of $\mathrm{CO}_{2}$ sequestration in saline aquifers

(6) Modeling hydrothermal alteration in geothermal systems

(7) Development of a Pitzer-type geochemical reactive transport model and simulation of high-ionic-strength groundwater contamination

(8) Prediction of the rate of strontium migration at the Hanford site

(9) Experimental and modeling studies on the scale dependence of mineral reaction kinetics

(10) Modeling of bioremediation field tests at the Hanford site

(11) Study of long-term benthic biogeochemical dynamics of heavy metal cycling and benthic fluxes in lake sediments at Lake Coeur d'Alene, Idaho, including the development of a dynamic numerical biogeochemical model of heavy metal fate and transport in benthic sediments.

\section{FUNDING}

Funding for the Geochemistry Department comes from the U.S. Department of Energy, Office of Science, Office of Basic Energy Sciences, Chemical Sciences, Geosciences and Biosciences Division; DOE Office of Biological and Environmental Research; DOE Office of Environmental Management, Office of Science and Technology; DOE Office of Energy Efficiency and Renewable Energy, Office of Utility Technologies and Office of Geothermal Technologies; DOE Office of Civilian Radioactive Waste Management; U.S. Environmental Protection Agency; U.S. Navy; NASA Space and Earth Sciences Program; National Science Foundation, Office of Polar Programs; U.S. Department of Agriculture; the University of California Campus-Laboratory Collaboration Hydrology Project; National Oceanographic Partnership Program (administered by the Office of Naval Research); National Oceanic and Atmospheric Administration, Office of Global Programs of the U.S. Department of Commerce, California Environmental Protection Agency, the California Energy Commission, National Institutes of Health, National Institute of Environmental Health Sciences program, the Hanford Science and Technology Program, and the Berkeley Lab Laboratory-Directed Research and Development Program.

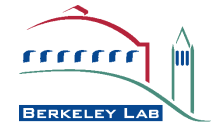




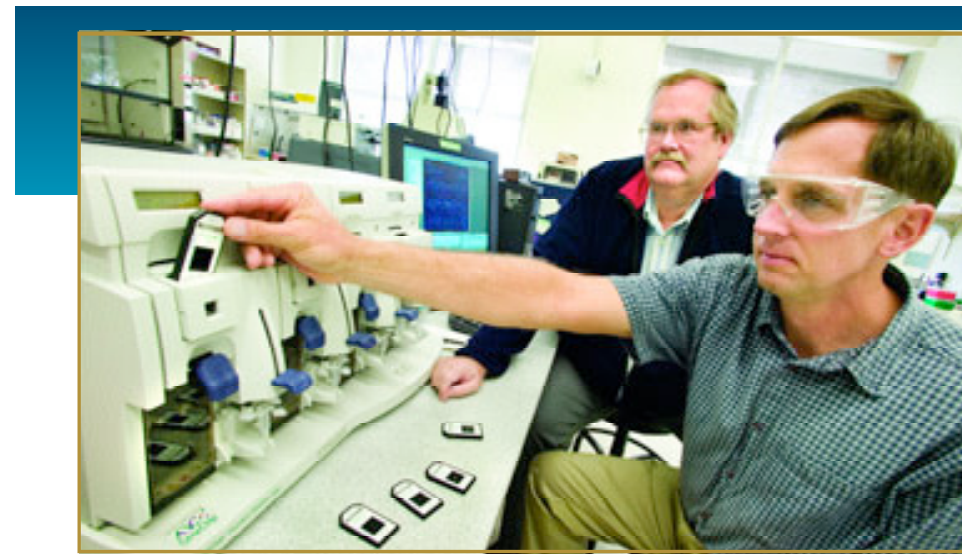

Resource Department

ECOLOGY DEPARTMENT

\section{SCIENTIFIC FOCUS AREAS}

The Ecology Department (ED) intends to maintain the highest quality and highest visibility for its research and development in five areas:

- Bioenergy

- Hydroecological engineering advanced decision support

- Molecular microbial ecology

- Real-time assessment of bioavailability and biokinetics

- Bioremediation and natural attenuation

These four R\&D areas are largely integrated, but contain some domains that are not inclusive. They are considered ED's core competencies.

\section{Bioenergy}

Concerns about global warming, dependence on foreign oil, and rising gasoline prices have spurred a strong interest in bioenergy in the United States. ED scientists will be contributing their expertise in microbiology, molecular microbial ecology, and bacterial metabolism to a scientific challenge of great national importance: the efficient production of biofuels. LBNL has been a key strategic partner in large research initiatives for developing bioenergy, including the Joint Bioenergy Institute (JBEI), a Department of Energy-funded research center that will receive $\$ 125$ million over five years, and the Energy Biosciences Institute (EBI), which is being funded by the energy company BP at a level of $\$ 500$ million over ten years. Both JBEI and EBI began operations in late 2007. The Ecology department will play an important role in both JBEI and EBI. For example, two ED personnel will serve as directors in JBEI: the director of microbial communities (responsible for studying the structural and functional dynamics of microbial communities that efficiently degrade lignocellulosic biomass) and the director of biofuels pathways (responsible for discovering genes that can be engineered into metabolic pathways, resulting in efficient production of various transportation fuels from cellulose degradation products). In EBI, ED scientists will be involved in the study of microbially enhanced hydrocarbon recovery and will co-lead a systems biology program, which will entail the intensive, genome-enabled study of certain bioenergy-related microorganisms ("omics" studies, including transcriptomics, proteomics, and metabolomics).

\section{Hydroecological Engineering Advanced Decision Support (HEADS)}

The HEADS research focus area has established a strong track record in the rapidly growing, new subject area of Ecological Engineering. The term "hydroecological engineering" signifies the group's concentration on water resources and wastewater engineering. Recognizing the growth in the field of environmental informatics and the application of computerbased models in the development of decision support systems, the group is active in this niche area. Interest in decision support interfaces well with the group's expertise in the deployment of real-time flow and water quality sensors, rapid laboratory assessment techniques, and mathematical models to develop an early warning system for contaminant management and containment.

\section{Molecular Microbial Ecology (MME)}

Understanding microbial interactions is key to the study of global warming, biodegradation of harmful compounds, and the exploration of complex microbial communities in their natural environment. The DOE has placed an increased emphasis on the role microbes play in modifying their environment and their impact on energy security. The MME group has responded to these needs by aggressively seeking out new projects and expanding its staff to develop new core capabilities. One of the key challenges has been to harness the explosion of microbial DNA sequence information to accurately measure the microbial dynamics in extreme environments. Since less than one percent of the microbial species can be cultured from these environments, our knowledge of what these organisms may be doing is limited to where they are observed and the similarity of their genomes to studied organisms. By understanding the ecological structure of

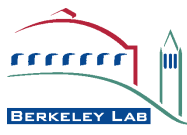


microbial communities and the fine-scale dynamics resulting from subtle perturbations, it may be possible to identify novel functional pathways and use the diverse microbial capabilities to assist in key DOE missions. Molecular tools being developed in the ESD Center for Environmental Biotechnology will position us to be leaders in this area.

\section{(RABB)}

Real-Time Assesment of Bioavailability and Biokinetics

Interactions between environmental pollutants and ecological receptors begin when the pollutants become available to the target sites of a live ecological receptor. The ability to characterize the dynamics of pollutant bioavailability, their transformation kinetics, and the subsequent ecological response is a keystone to advancing the science in relevant DOE areas, including biogeochemistry, bioremediation, and exposure and risk assessment. Since 1999, the RABB research group at ED has pioneered the development and application of several cuttingedge technologies, such as synchrotron radiation-based spectromicroscopy, in vitro human gastrointestinal mimetic reactors, and in vivo mouse protocols that allow for the real-time assessment of bioavailability and biokinetics of environmental pollutants. The RABB group intends to establish this capability further by seeking out new projects and new collaborators, as well as expanding our staff, to position us as leaders in the areas of biological and environmental sciences.

\section{Bioremediation and Natural Attenuation}

Bioremediation and natural attenuation have been rapidly growing areas of scientific study over the past decade. The acceptance of natural attenuation as a solution for cleaning up contaminated sites, and DOE's recognition that they will have long-term stewardship concerns to address at the most contaminated sites, has greatly increased the urgency for research related to microbial ecology, biogeochemistry, biochemistry, and molecular detection techniques. This type of research is truly enabling for natural attenuation, since characterization, transport-and-fate prediction, and verification monitoring require a strong scientific basis. Natural attenuation is viewed as the best solution for cleaning up many waste sites and will save billions of dollars in cleanup costs.

ED scientists and engineers are recognized leaders in the field of bioremediation and natural attenuation. The Center for
Environmental Biotechnology provides the primary facilities used by ED, including state-of-the-art equipment for microbiology and environmental engineering. ED investigators have extensive experience in both water treatment and aquifer bioremediation. In addition to basic research, ED investigators have been involved in various aspects of more than 100 field demonstrations and deployments, and have five patents in this area that are licensed to more than 30 companies. The types of contaminants in which ED investigators have expertise include chlorinated solvents, petroleum hydrocarbons (including monoaromatic and polynuclear aromatic hydrocarbons), methyl tert-butyl ether (MTBE), high explosives (especially TNT and RDX), nitrate, plutonium, neptunium, chromium, and uranium. The Bioremediation and Natural Attenuation area has both basic research and field application foci for the ED. The basic research foci are metabolism and physiology (including the genetics and biochemistry underlying pollutant metabolism), biotransformation kinetics, and modeling of biogeochemical processes. Field-application foci are in situ monitoring techniques (including molecular, mass spectrometric, and biogeochemical approaches), co-metabolic techniques, and modeling of attenuation and environmental fate.

\section{FUNDING}

ED personnel are funded by DOE Programs in (1) the Office of Science, Office of Biological and Environmental Research (OBER) (Environmental Remediation Sciences Program, Genomics:GTL, Bioenergy, and Medical Sciences); (2) the Office of Environmental Management, Environmental Restoration Programs; (3) the Office of Fossil Research, the Petroleum Environmental Research Forum; and (4) the National Nuclear Security Administration, Office of Nonproliferation Research and Engineering (NN20). In addition, support is provided by the U.S. Department of Homeland Security, National Institutes of Health, U. S. Environmental Protection Agency, the Department of Agriculture; the Department of the Interior, Bureau of Land Management and Bureau of Reclamation under the CALFED program, NASA Astrobiology Institutes, as well as several projects with remediation companies using DOE-patented technologies for in situ bioremediation. ED personnel are also funded by Berkeley Lab's Laboratory Directed Research and Development (LDRD) Program in the area of microbial fuel cells, fungal rDNA arrays, and FTIR biokinetic analaysis.

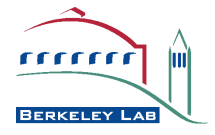




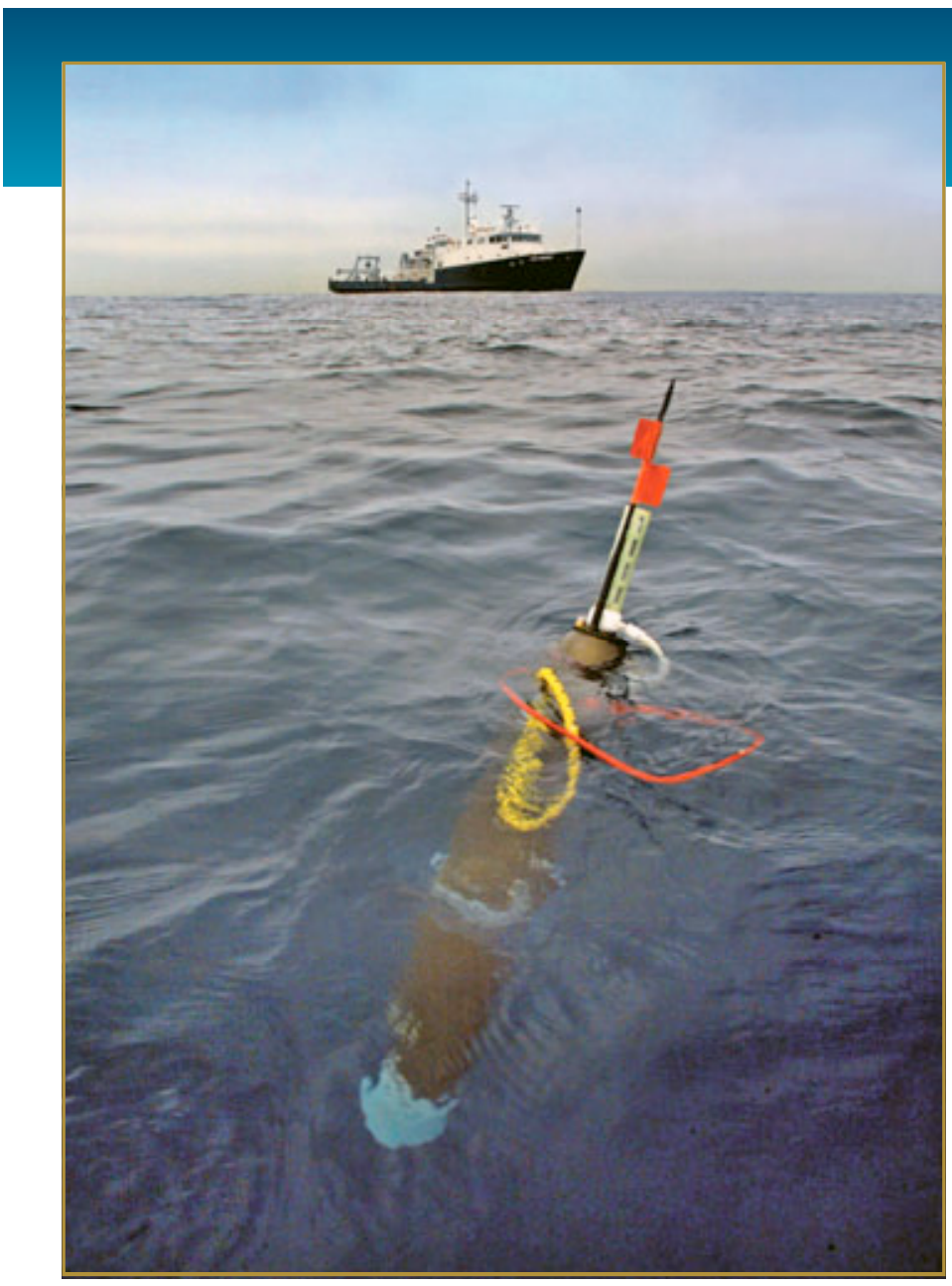

The Climate Science Department, ESD's newest department (started in April 2007), is dedicated to atmospheric and climate science. With roughly a dozen distinguished scientists, specialists, and technicians, this world-class team will lead the ESD towards the creation of a new kind of climate model, integrating cutting-edge climate science, such as the pioneering work on the carbon cycle conducted at Berkeley Lab, and drawing on work by scientists at UC Berkeley and other universities and national laboratories. The goal is not to predict climate alone, but to facilitate interactions among climate, water, and energy on a global scale. Wholly interactive, it will be able to incorporate fresh data and generate new scenarios at any point-energy demand and carbon emissions, changes in the composition of the atmosphere and the heat entering and leaving it, impacts on ecosystems and human well-being-and develop different strategies to mitigate or adapt to change.

The department's major areas of scientific focus will include climate change forcing, climate change modeling, and climate change mitigation.
Resource Department

\section{Climate Science Department}

\section{William (Bill) Collins}

$510 / 495-2407$

WDCollins@lbl.gov

\section{CLIMATE CHANGE FORCING}

The goal of Berkeley Lab's terrestrial carbon research is to support the development, testing, and application of Integrated Terrestrial Carbon Models (ITCMs) that will be used to simulate carbon fluxes in North America in the near term, and coupled with global climate models in the long term. This work is a multi-institution collaboration under the coordination of the lead lab in this SFA, Oak Ridge. Berkeley Lab is pursuing five areas of research relevant to improving carbon cycle understanding: (1) better characterization of ecosystem $\mathrm{CO}_{2}$ fluxes and resulting atmospheric concentrations; (2) spatially and temporally resolved measurements of fossil $\mathrm{CO}_{2}$ emissions; (3) better understanding of soil carbon cycling; (4) simulation of feedbacks between carbon dynamics and climate change in global carbon-climate models; and (5) diagnosis of carbon modules in global climate models using AmeriFlux, North American Carbon Program (NACP), and other carbon system observations.

The Climate Science Department is also carrying out innovative observations of the ocean carbon cycle that would contribute to removing a major gap in coupled carbon-climate modeling. Oceans contain more carbon than any other dynamic reservoir on earth. They pose a great observational challenge because the pulses of biological productivity are episodic and cover vast areas. The Climate Science Department has developed the Carbon Explorer (see figure), an autonomous float that uses satellite telemetry to report its observations from distant oceans. Twelve of these low-cost robots have achieved the equivalent of 8 years of continuous observations of particulate organic carbon in remote and biologically dynamic ocean regions, observations that would not have been possible with conventional research ships. Seagoing work to prove and enhance new sensors for the Carbon Explorer is ongoing. The Department's new sensor for particulate inorganic carbon was operationally deployed to full ocean depth during a pole-topole survey transects of the Atlantic Ocean in July 2003 and January 2005. The data it reported allow the first comprehensive examination of the spatial variability of particulate organic and inorganic carbon. The Department's optical carbon sedimentation recorder was most recently deployed in Oyshio waters near Japan.

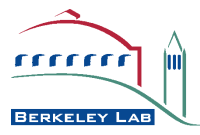




\section{CLIMATE CHANGE MODELING}

Simulations from global models provide critical information required to attribute past climate change and ameliorate future climate change. Despite the sophistication of current coupled climate models, these generally do not include the biogeochemical feedbacks, the spatial resolution, and the understanding of abrupt change required for comprehensive projections. To understand the role of these processes in regional and global climate change, the climate community should develop Earth System Models (ESMs). ESMs will be designed to simulate the coupled physical, chemical, and biogeochemical evolution of the environment. It is increasingly critical to project local extremes in precipitation and other weather conditions forced by climate change. However, these projections are subject to large uncertainties governed by model physics and model resolution. Uncertainty reduction hinges in part on site-toregional scale process-based studies leading to new parameterizations in ESMs, analysis of model-simulated atmospheric physics and dynamics with observational evaluations, and high-resolution studies of the space-time evolution of extremes and anomalous weather and climate states. New research is needed to understand whether projections of extremes can converge with better process fidelity and higher spatial resolution.

\section{CLIMATE CHANGE MITIGATION}

Limitations in current soil carbon models cripple scientists' ability to predict climate effects on $\mathrm{CO}_{2}$ fluxes or to evaluate carbon sequestration and land management strategies. Four major gaps in the understanding of soil carbon dynamics have been identified (e.g., by BERAC, DOE, and USDA) that are important for both coupled climate-carbon modeling and carbon management, and that can be addressed in the next 5 years. Specifically, the priority areas for soil carbon research are (1) the effect of plant allocation and species on carbon residence time; (2) physical protection of soil organic matter, by minerals and aggregation; (3) temperature and moisture interactions; and (4) testing and improvement of model performance. We have in hand sufficient understanding and data to begin developing much improved model parameterizations for several of these areas.

\section{FUNDING}

Funding for the Climate Science Department comes from the U.S. Department of Energy, Office of Science, Office of Biological and Environmental Research, and the Berkeley Lab Laboratory-Directed Research and Development Program. 


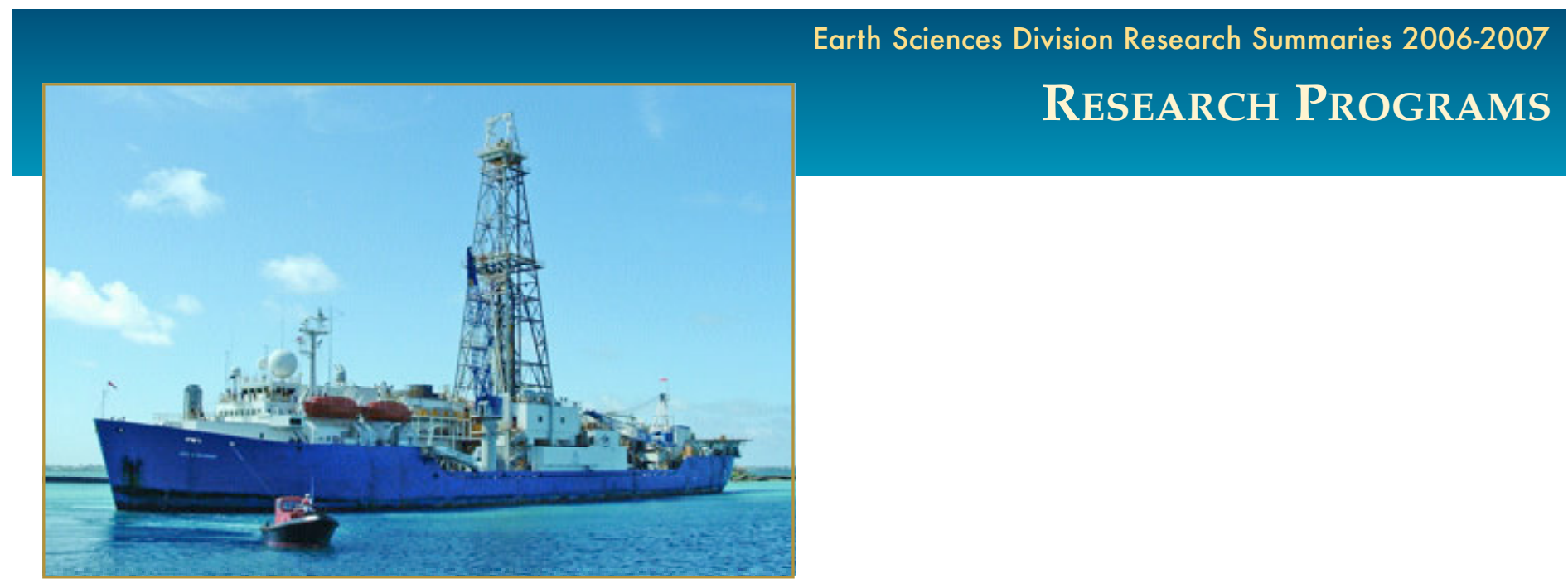

Energy Resources Program

Fundamental and Exploratory Research Program

ENVIRONMENTAL REMEDIATION AND

Water Resources Program

Climate and Carbon Sciences Program

Geologic Carbon Sequestration Program

Nuclear Energy and Waste Program 


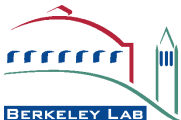




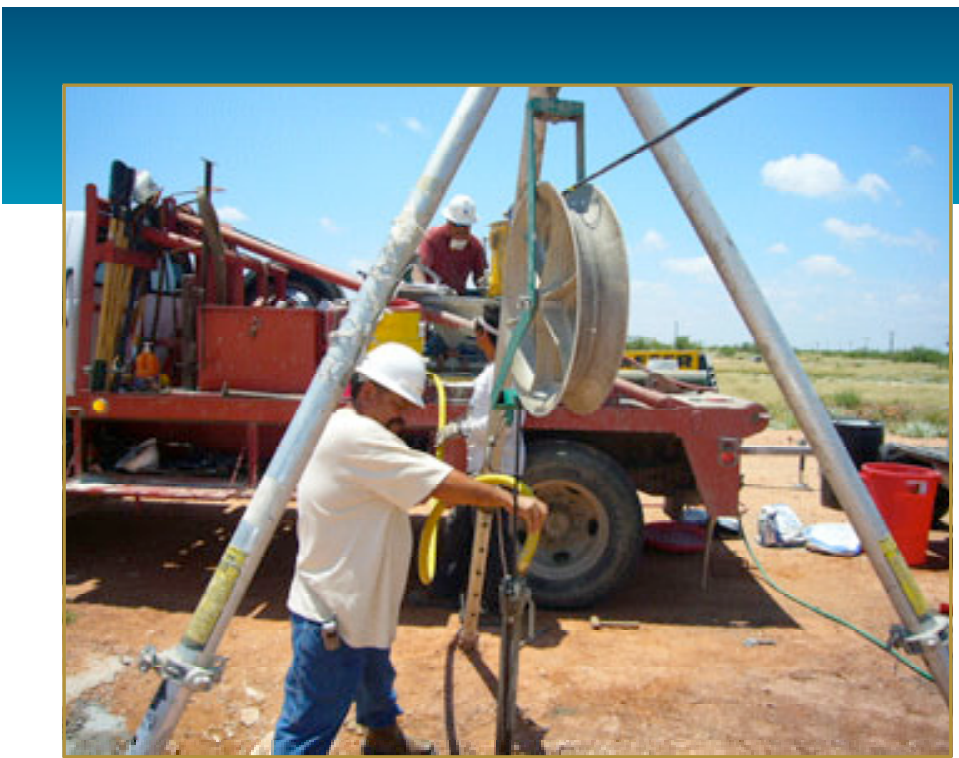

The Energy Resources Program (ER) within ESD is responsible for two major program areas: Oil and Gas Exploration and Development, and Geothermal Energy Development.

\section{OIL AND GAS EXPLORATION AND DEVELOPMENT}

Multidisciplinary research is being conducted in reservoir characterization and monitoring, optimization of reservoir performance, and environmental protection. Using basic research studies as a source of innovative concepts, ER researchers seek to transform these concepts into tangible products of use to industry within a time frame consistent with today's rapid growth in technology. Reservoir characterization and monitoring involve development of new seismic and electromagnetic techniques focused at the interwell scale. Field acquisition, laboratory measurements, and numerical simulation play important roles in the development activities. Optimization of reservoir performance is focused on simulation-based methods for enhancing reservoir management strategies. Emphasis is placed on the integration of geophysical data, production data, and reservoir simulation. The next major step in research will focus on methods to optimize performance through integration of monitored geophysical data, production data, and reservoir simulation.

International and national concern about the variable climatic effects of greenhouse gases produced by burning of fossil fuels is increasing, while it is also recognized that these fuels will remain a significant energy source for the indefinite future. In response to these concerns, ER has initiated research focused on development of technologies that will minimize the impact of fossil-fuel usage on the environment. Methane hydrates constitute a huge potential fuel source, with lower carbon emissions than coal or oil. ER researchers are developing and evaluating possible methods for

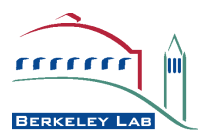
producing gas from such deposits.
Research Program

\section{Energy Resources Program}

Geophysical data acquisition and inversion methods developed in the ER program are also being applied in a new ESD program, the Geologic Carbon Sequestration Program, described elsewhere in this volume.

Principal research activities include:

- Development of 3-D electromagnetic processing methods for oil and gas recovery

- Development of microwell seismic technology, including instrumentation, acquisition, and processing

- Applications of seismic methods for characterization of fractured reservoirs

- Development of joint electrical-seismic inversion methods for oil and gas exploration and monitoring

- Use of passive (microearthquake) and active seismic imaging for understanding and controlling enhanced geothermal systems (EGS)

- Laboratory measurement of the seismic properties of poorly consolidated sands

- Evaluation of seismic stimulations methods and their application to different classes of oil reservoirs

- Improved inversion methods for reservoir characterization, with a focus on combining production and geophysical data

- Application of x-ray computed tomography (CT) and nuclear magnetic resonance (NMR) imaging to study multiphase flow processes

- Pore-to-laboratory-scale study of physical properties and processes, with a focus on controlling phase mobility, predicting multiphase flow properties, and increasing drilling efficiency

- Development of new methods to mitigate environmental effects of petroleum refining and use

- Enhancement of refining processes using biological technologies

- Numerical simulation of subsurface methane hydrate systems

Since 1994, the major part of the Oil and Gas Exploration and Development program has been funded through the Natural Gas and Oil Technology Partnership Program. Begun in 1989, the partnership was expanded in 1994 and again in 1995 to include all nine Department of Energy multiprogram laboratories, and has grown over the years to become an important part of the DOE Oil and Gas Technologies program for the national 
laboratories. Partnership goals are to develop and transfer to the domestic oil industry the new technologies needed to produce more oil and gas from the nation's aging, mature domestic oil fields, while safeguarding the environment.

Partnership technology areas are:

- Oil and gas recovery technology

- Diagnostics and imaging technology

- Drilling, completion and stimulation

- Environmental technologies

- Downstream technologies

\section{GEOTHERMAL ENERGY DEVELOPMENT}

There are two main objectives of ER's geothermal energy development program: (1) to reduce uncertainties associated with finding, characterizing, and evaluating geothermal resources, and (2) to develop and understand the enhancement of current geothermal systems to significantly increase production, i.e., Enhanced Geothermal System (EGS). The ultimate purpose is to lower the cost of geothermal energy for electrical generation or direct uses (e.g., agricultural and industrial applications, aquaculture, balneology). The program encompasses theoretical, laboratory, and field studies, with an emphasis on multidisciplinary approaches to solving the problems at hand. Existing tools and methodologies are upgraded, and new techniques and instrumentation are developed for use in the areas of geology, geophysics, geochemistry, and reservoir engineering. Cooperative work with industry, universities, and government agencies draws from Berkeley Lab's 25 years of experience in the area of geothermal research and development.

In recent years, DOE's geothermal program has become more industry-driven, and the Berkeley Lab effort has been directed toward technology transfer and furthering our understanding of the nature and dynamics of geothermal resources under production.

At present, the main research activities of the program include:

- Geothermal Reservoir Dynamics: development and enhancement of computer codes for modeling heat and mass transfer in porous and fractured rocks, with specific projects such as modeling the migration of phase-partitioning tracers in boiling geothermal systems; modeling of mineral dissolution and precipitation during natural evolution, production, and injection operations; and geophysical-signature prediction of reservoir conditions and processes

- Isotope Geochemistry: identification of past and present heat and fluid sources, development of natural tracers for monitoring fluids re-injected into geothermal reservoirs, better understanding of the transition from magmatic to geothermal production fluids, and enhancement of reservoir-simulation methods and models by providing isotopic and chemical constraints on fluid source, mixing, and flow paths

- Geochemical Baseline Studies: documentation of geothermal-fluid behavior under commercial production and injection operations (e.g., field case studies), with specific emphasis on The Geysers field in Northern California

- Electromagnetic Methods for Geothermal Exploration: development of efficient numerical codes for mapping high-permeability zones, using single-hole electromagnetic data and surface magnetotelluric data.

Future research will concentrate on the development of innovative techniques for geothermal exploration and assisting in a reassessment of geothermal power potential in the U.S. The emphasis will be on expanding existing fields, prolonging their productive life, and finding new "blind" geothermal systems, i.e., those that do not have any surface manifestations, such as hot springs or fumaroles, that suggest the presence of deeper hydrothermal systems.

\section{FUNDING}

Within ER, The Oil and Gas Exploration and Development program receives support from the Assistant Secretary for Fossil Energy, Office of Natural Gas and Petroleum Technology, through the National Energy Technology Laboratory, the National Petroleum Technology Office, and the Natural Gas and Oil Technology Partnership, under U.S Department of Energy Contract No. DE-AC02-05CH11231. Support is also provided from industry and other sources through the Berkeley Lab Work for Others program. Industrial collaboration is an important component of DOE Fossil Energy projects.

The Geothermal Energy Development program receives support from the Assistant Secretary for Energy Efficiency and Renewable Energy, Office of Power Technologies, Office of Wind and Geothermal Technologies, of the U.S. Department of Energy.

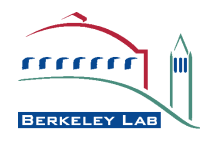




\title{
Uncertainty Analysis Using Stochastic Rock-Physics Models and Markov Chain Monte Carlo Methods
}

\author{
Jinsong Chen \\ Contact: 510/486-6842, jchen@lbl.gov
}

\section{RESEARCH OBJECTIVES}

Rock-physics models are needed for reservoir parameter estimation using marine seismic amplitude-versus-angles (AVA) and controlled source electromagnetic (CSEM) data. They are typically derived in practice from suitable nearby borehole logs. However, since relationships between reservoir parameters and geophysical attributes are often nonlinear and non-unique, the derived rock-physics models are inevitably subject to uncertainty. Traditional methods for analyzing uncertainty in rock-physics models are performed by varying a small subset of the rock-physics parameters while keeping others unchanged. In essence, those methods explore the marginal effects of the parameters on reservoir parameter estimation and are valid only when the parameters being investigated are independent of those kept unchanged. Since rock-physics parameters often depend on each other, the utility of such approaches is limited. Additionally, the above methods only analyze the effects of uncertainty in the estimated rock-physics parameters without considering uncertainty in the rockphysics model outputs. The goal of this study is to develop a flexible, robust, and integrated approach for analysis of uncertainty in rock-physics models.

\section{APPROACH}

A Bayesian framework and stochastic rock-physics models are used to combine marine seismic AVA and CSEM data for reservoir parameter estimation. The outputs of rock-physics models are considered as random functions of reservoir parameters, and the parameters of rock-physics models are considered as random variables. Markov chain Monte Carlo (MCMC) methods are used to draw many samples from the joint posterior probability density functions. The obtained samples from MCMC methods are used to estimate reservoir parameters as well as uncertainty information in the estimation.

\section{ACCOMPLISHMENTS}

A Bayesian model based on a layered reservoir model has been developed to jointly invert seismic AVA and CSEM data, and the model has been applied to a synthetic reservoir with high gas saturation. Figure 1 shows the estimated posterior probability density functions (pdfs) of water saturation, shale content, and porosity using stochastic rock-physics models at various noise levels.

\section{SIGNIFICANCE OF FINDINGS}

Uncertainty in both the outputs and the parameters of rockphysics models could have significant effects on the estimates of reservoir parameters in joint inversion of marine seismic AVA and CSEM data. The effects of uncertainty in rock-physics model parameters are generally less significant than those of uncertainty in rock-physics model outputs. The study also shows that an integrated approach is important for uncertainty analysis in reservoir parameter estimation, and that the developed Bayesian model in this study provides a consistent and effective method for uncertainty analysis.

\section{RELATED PUBLICATIONS}

Chen, J., and T. Dickens, Effects of uncertainty in rock-physics models on reservoir parameter estimation using marine seismic AVA and CSEM data. SEG Expanded Abstracts (in press), 2007.

\section{ACKNOWLEDGMENTS}

This work was supported by the ExxonMobil Upstream Research Company and by the U.S. DOE Assistant Secretary for Fossil Energy, Office of Oil and Natural Gas, under Contract No. DE-AC02-05CH11231.
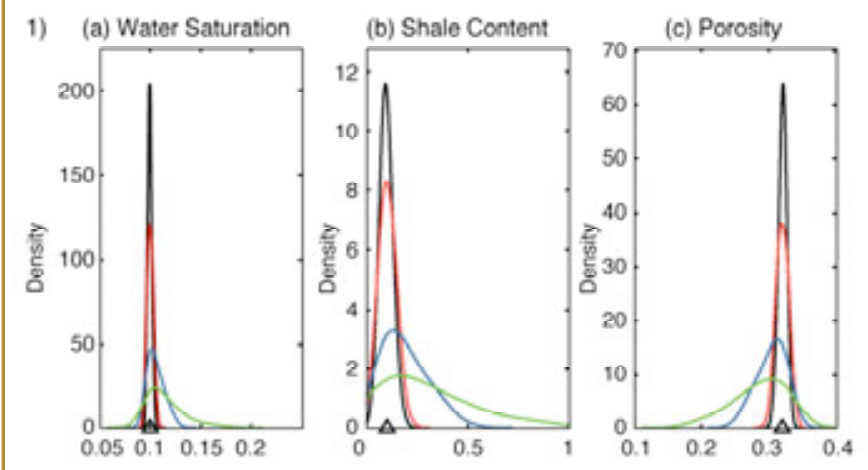

2)
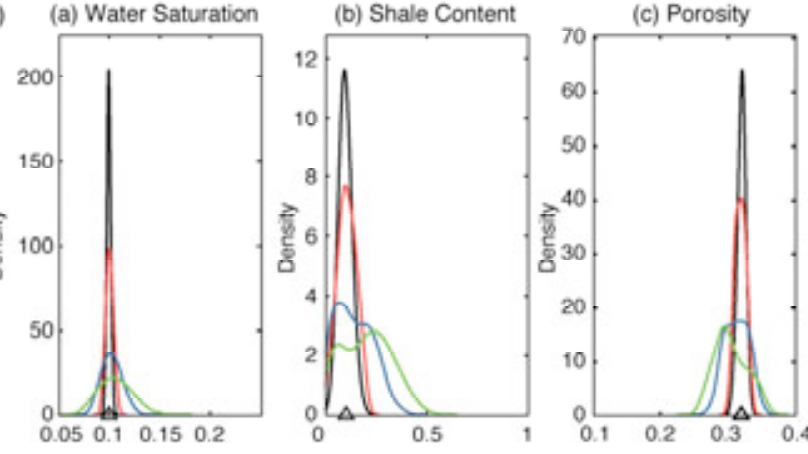

Figure 1. Estimated posterior pdfs of water saturation, shale content, and porosity, where the black, red, blue, and green curves are the estimated posterior pdfs when the outputs of rock-physics models are exact, and have errors equivalent to the coefficients of variations of $1 \%, 3 \%$, and $5 \%$, respectively.

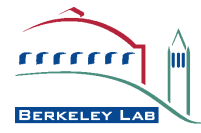




\section{Measurements and Observations of Water, Gas, and Heat Flow in Hydrate-Bearing SANDS}

T.J. Kneafsey, L. Tomutsa, Y. Seol, R.C. Trautz, G.J. Moridis, and T.A. Ghezzehei

Contact: T.J. Kneafsey, 510/486-4414, tjkneafsey@lbl.gov

\section{RESEARCH OBJECTIVES}

Natural deposits of gas hydrates (i.e., methane molecules enclosed within a solid water lattice at elevated pressure and cool temperatures), thought to contain vast amounts of recoverable natural gas, are present in the subsurface in permafrost areas and at or below the ocean floor. To capture this gas, the solid hydrate must be destabilized (by lowering pressure, increasing temperature, or adding an inhibitor), causing it to dissociate into methane gas and water, and extracted from the earth using a production well. Modeling of these processes is needed to estimate the economics of gas production, but the modeling must be based on correct physics. Our objectives are to perform experiments that provide visualizations and quantifications of water and gas flow through hydrate-bearing samples to improve conceptual and numerical models of gas production from hydrates.

\section{APPROACH}

We form methane hydrate in samples contained in x-ray transparent vessels, while controlling the water content, pressure, and temperature. We then quantify water and gas flow through the resulting system using pressure, temperature, and flow measurements, and use x-ray computed tomography to visualize changes and water flow.

\section{ACCOMPLISHMENTS}

We have performed a number of flow tests for sands having different characteristics at several hydrate saturations and have estimated relative permeabilities based on our measurements. We have performed and monitored dissociation tests by depressurization and thermal stimulation, and have performed flow tests through samples that had heterogeneities in hydrate saturation and prescribed heterogeneities. Also, we are developing a procedure for measuring the characteristic curve of hydrate-bearing sand.

\section{SIGNIFICANCE OF FINDINGS}

Our measurements are being incorporated into numerical simulations of gas production from hydrates, adding confidence to the modeling. Visualizations have taught us about the roles of heat and mass transfer in the technique used to make hydrate samples and their influence on resulting measurements.
Additionally, the impact of parameter changes caused by the presence of hydrate in a porous medium can be seen directly in our visualizations, allowing better understanding and modeling of the processes.

\section{RELATED PUBLICATIONS}

Kneafsey, T.J., L. Tomutsa, G.J. Moridis, Y. Seol, B.M. Freifeld, C.E. Taylor, and A. Gupta, Methane hydrate formation and dissociation in a core-scale partially saturated sand sample. LBNL-59087. Journal of Petroleum Science and Engineering, 56, 108-126. 2007.

Gupta, A., T.J. Kneafsey, G.J. Moridis, Y. Seol, M.B. Kowalsky, and E.D. Sloan Jr., Methane hydrate thermal conductivity in a large heterogeneous porous sample. LBNL-59088. J. Phys. Chem. B; ASAP Web Release Date: August 2, 2006; DOI: 10.1021/jp0619639, 2006.

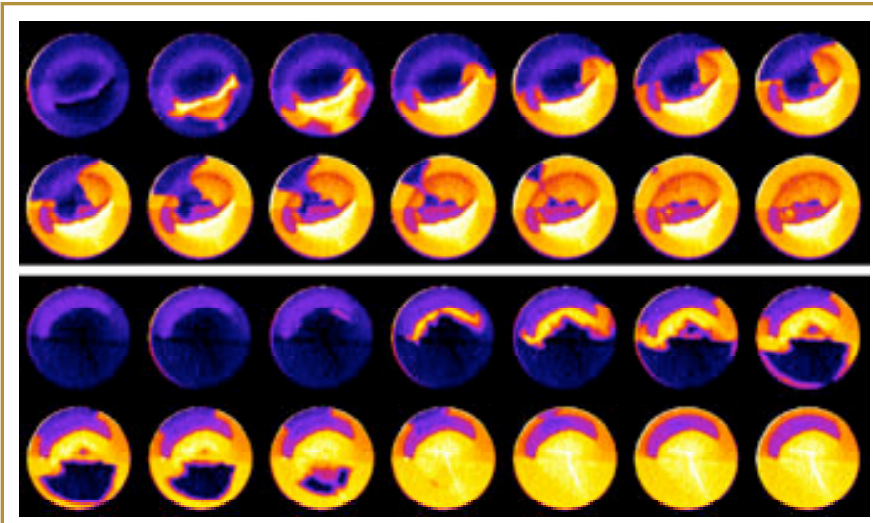

Figure 1. Sequence of CT scans showing water flow through a sample having coarse sand on the top and fine sand on the bottom. The brighter regions in the first scan show higher saturations of hydrate. In later scans, the brighter regions show the initial hydrate saturation with increasing saturations of water.

\section{ACKNOWLEDGMENTS}

This work was supported by the Assistant Secretary for Fossil Energy, Office of Natural Gas and Petroleum Technology, through the National Energy Technology Laboratory, under the U.S. DOE, Contract No. DE-AC02-05CH11231.

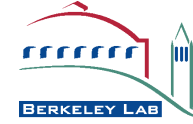




\title{
FreQuency-Dependent SeIsMic Response OF UNDERGROUND RESERVOIRS
}

\author{
Valeri Korneev \\ Contact: 510/486-7214, vakorneev@lbl.gov
}

\section{RESEARCH OBJECTIVES}

The main objective of this project is the development and application of the new advanced technology of hydrocarbon reservoir imaging, supported by a frequency-dependent reflectivity model. Reaching this objective requires an understanding of how seismic waves interact with fluid-saturated rocks.

\section{APPROACH}

This project involves development of theory and processing algorithms, laboratory experiments, and verification of results, using field data provided by industrial partners. For theory development with respect to frequency-dependent reflections, a computer code was developed that models (in two and three layers) the reflections caused by $\mathrm{P}$ - wave incidence. Fluid flow effects are modeled by application of Biot's porous rock theory and generalized to the double porosity theory.

\section{ACCOMPLISHMENTS}

Modeling results were compared with observations in laboratory data and field experiments. It was determined that the modeled effects are too small to explain the data. On the other hand, analyses of permeability values consistently demonstrate that measurements at field scale (hundreds of meters to kilometers) show an increase of several orders of magnitude compared to values obtained at laboratory scales. This behavior finds an explanation in the self-similar distribution of fractures in rock and implies that fluid-related effects in fractures have to be considered for realistical models of rock. An analytical solution has been obtained with respect to the phase velocity of a Stoneley guided wave for an infinite fracture filled with viscous fluid. The solution suggests differentiating between oil and water fluids at low frequencies. It also suggests that at seismic frequencies, resonances are possible at reservoir scales (Figure 1). Resonant frequencies depend on fluid parameters and fracture dimensions.

\section{SIGNIFICANCE OF FINDINGS}

The Stoneley wave (slow wave) effect, which is not a part of any existing poroelastic theory, needs to be taken into account at field scales. For proper extraction of permeability information, the scattering of seismic waves caused by fracturing within heterogeneous systems needs to be included in reservoir models. Resonant characteristics of fluid-filled fractures are likely to cause nonlinear effects when seismic waves interact with reservoirs.

\section{RELATED PUBLICATIONS}

Korneev, V.A, Slow waves in fractures filled with viscous fluid. Geophysics (in press), 2007.

Silin, D.B., V.A. Korneev, G.M. Goloshubin, and T.W. Patzek, Low-frequency asymptotic analysis of seismic reflection from a fluid-saturated medium. LBNL-54955. Transport in Porous Media, 62, 283-305, 2006.

Goloshubin, G.M., C. VanChuyver, V.A. Korneev, D.B. Silin, and V. Vingalov, Reservoir imaging using low frequencies of seismic reflections. LBNL-60853. The Leading Edge, 25 (5), 527-531, 2006.

Korneev, V., A. Bakulin, T. Watanabe, and S. Ziatdinov, Timelapse changes in tube and guided waves in cross-well Mallik experiment. SEG Annual Meeting, Expanded Abstracts, 2006.

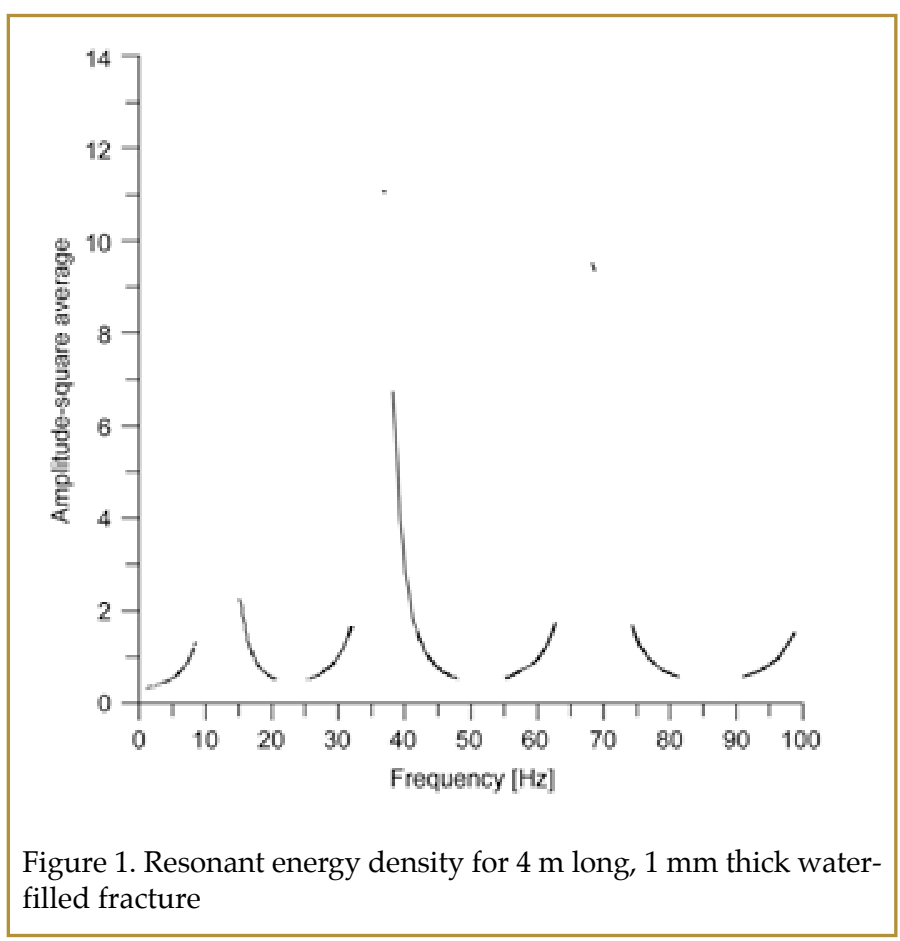

\section{ACKNOWLEDGMENTS}

This work was supported by the Assistant Secretary for Fossil Energy, Office of Natural Gas and Petroleum Technology, through the National Energy Technology Laboratory, of the U.S. Department of Energy under Contract No. DE-AC02$05 \mathrm{CH} 11231$.

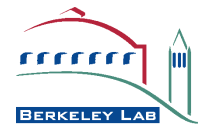




\title{
Comparison of Kinetic and Equilibrium ReACTION MODELS IN Simulating Gas Hydrate Behavior in Porous Media
}

\author{
Michael B. Kowalsky and George J. Moridis \\ Contact: Michael B. Kowalsky, 510/486-7314, mbkowalsky@lbl.gov
}

\section{RESEARCH OBJECTIVES}

Production of natural gas from hydrate accumulations may proceed by inducing dissociation, an endothermic reaction which results in the production of gas and water, using one or a combination of the following mechanisms: (1) depressurization; (2) thermal stimulation; and (3) inhibitors (such as salts and alcohols). The objective in this work is to evaluate, through numerical simulation, the importance of employing kinetic versus equilibrium reaction models to describe the hydrate dissociation reaction and thus predict the response of (methane) hydrate-bearing systems to external stimuli, such as changes in pressure and temperature.

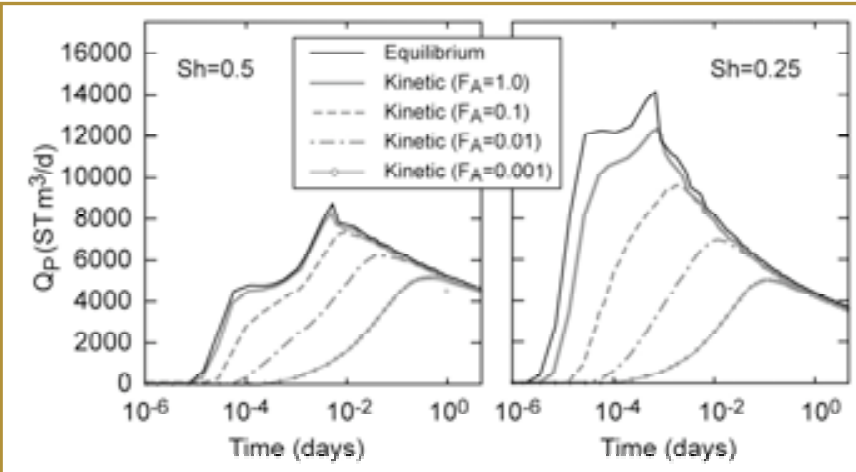

Figure 1. Effect of reaction area on early-time $\mathrm{CH}_{4}$ production in Class 3 hydrate accumulation undergoing depressurization. The responses for two different initial hydrate saturations are shown in (a) and (b), respectively, for the equilibrium reaction model and for the kinetic reaction model with a decreasing amount of surface area available in the hydrate dissociation reaction.

\section{APPROACH}

Our numerical studies are conducted using TOUGH+HYDRATE (formerly TOUGH-Fx/HYDRATE), which models the nonisothermal hydration reaction, phase behavior, and flow of fluids and heat under conditions typical of natural hydrate deposits in complex formations. It includes both equilibrium and kinetic models of hydrate formation and dissociation, and can handle any combination of the possible hydrate dissociation mechanisms mentioned above. The code accounts for heat and up to four mass components (i.e., water, $\mathrm{CH}_{4}$, hydrate, and water-soluble inhibitors such as salts or alcohols) that are partitioned among four possible phases (gas, liquid, ice, or hydrate phases, which may exist individually or in any of 12 possible combinations).

\section{ACCOMPLISHMENTS}

In this study, we (1) analyze and compare the responses simulated using both reaction models for natural gas production from hydrates in various settings, and for the case of depressurization in a hydrate-bearing core during extraction; and (2) examine the sensitivity to factors such as initial hydrate saturation, hydrate reaction surface area, and numerical discretization. An example of production in a Class 3 hydrate accumulation undergoing depressurization is shown in Figure 1 for different initial values of hydrate saturation, and for a decreasing amount of surface area available in the dissociation reaction for both kinetic and equilibrium reaction models. We find that for large-scale systems undergoing thermal stimulation and depressurization, the long-term predictions for both reaction models are remarkably similar, though some differences are observed at early times. However, for modeling short-term processes, such as the rapid recovery of a hydratebearing core, kinetic limitations can be important, and neglecting them may lead to significant underprediction of recoverable hydrate.

\section{SIGNIFICANCE OF FINDINGS}

Assuming validity of the most accurate kinetic reaction model that is currently available, use of the equilibrium reaction model appears to be justified in many cases and preferred for simulating the behavior of gas hydrates, given that the computational demands for the kinetic reaction model far exceed those for the equilibrium reaction model.

\section{RELATED PUBLICATIONS}

Kowalsky, M. B., and G. J. Moridis, Comparison of kinetic and equilibrium reactions in simulating the behavior of gas hydrates. LBNL-63357. Energy Conversion and Management, 48, 1850-1863, doi:10.1016/j.enconman.2007.01.017, 2007.

Moridis, G. J., and M. B. Kowalsky, Response of oceanic hydratebearing sediments to thermal stresses. LBNL-60150. SPE Journal, 12(2), 253-268, doi:10.2118/111572-PA, 2007.

\section{ACKNOWLEDGMENTS}

This work was supported by the Assistant Secretary for Fossil Energy, Office of Natural Gas and Petroleum Technology, through the National Energy Technology Laboratory (NETL), under the U.S. Department of Energy, Contract No. DE-AC0205CH11231.

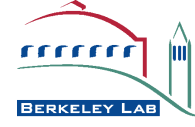




\title{
Gas Production from Oceanic Class 2 Hydrate Accumulations
}

\author{
George J. Moridis and Matthew T. Reagan
}

Contact: George J. Moridis, 510/486-4746, gjmoridis@lbl.gov:

\section{RESEARCH OBJECTIVES}

The objective of this study is to evaluate the production potential of marine Class 2 methane hydrate accumulations by means of numerical simulation, and to develop appropriate strategies for gas production.

\section{APPROACH}

Class 2 hydrate deposits comprise two zones: a hydrate-bearing layer (zone) overlying a zone of mobile water. The simulated system is based in the "Tigershark" area, located in the Alaminos Canyon Block 818 of the Gulf of Mexico. Data from an exploration well in 2,750 m of water indicated the presence of a thick, sandy hydrate layer, and preliminary calculations suggested that the base of the gas hydrate stability zone occurs at or slightly below the base of the hydrate-bearing layer. A zone of mobile water underneath the hydrate zone was assumed.

Depressurization-induced dissociation, enhanced by the near-incompressibility of water, was selected as the most promising gas production strategy because of its simplicity and its technical and economic effective-

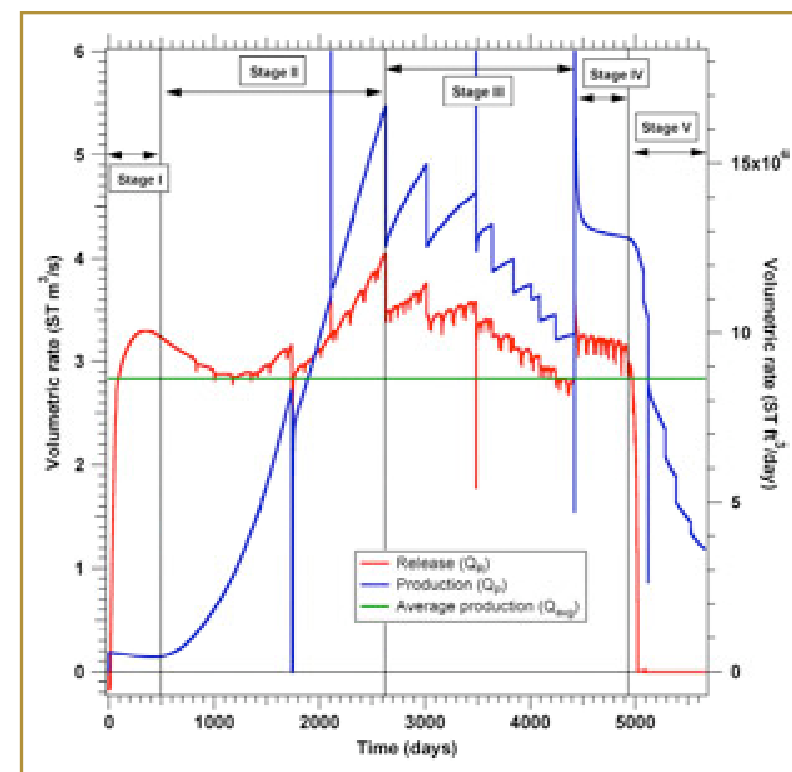

Figure 1. Rates of (a) hydrate-originating $\mathrm{CH}_{4}$ release in the reservoir (red) and (b) $\mathrm{CH}_{4}$ production at the well (blue) during production from a Class 2 oceanic hydrate deposit using a novel wellbore design. The stages of production and the average production rate $\left(\mathrm{Q}_{\mathrm{avg}}\right.$, in green) over the simulation period are also shown. through the hydrate layer and into the zone of mobile water, with the entire interval perforated for production. This method resulted in the highest gas production rates, but was subject to flow blockages by secondary hydrate and ice formation. The second involved a well with a production interval below the base of the hydrate. This strategy was also subject to secondary hydrate formation, and production rates were limited because of a well design that did not access gas accumulated at the top of the formation. The third method combined features of the first two cases, using a combination of electrical heating to initiate production, warm seawater circulation during continued production, and finally the application of a novel well design that combines warm seawater injection with a long production interval to access the remaining hydrate and avoid blockages and cavitation (see the related publication). Production with this process proceeds in five stages (see figure): initiation, increasing production, depletion, enhanced recovery ness. The code used in this study is TOUGH+HYDRATE, developed by Berkeley Lab staff with support from DOE-NETL. The code can describe any combination of hydrate dissociation mechanisms and can account for four mass components partitioned among four possible phases (gas, liquid, ice, hydrate), with a total of 15 unique phase states. Well configurations and the rates of mass production evolved during the course of production in response to system behavior.

\section{ACCOMPLISHMENTS}

For all strategies, production at the well is characterized by distinct cycles, with the end of each cycle marked by cavitation (rapid pressure drop at the well), caused by either secondary hydrate blockages near the well or by the increasing participation of lower-density gas. This is remedied by warm seawater injection to destroy any barrier (if it exists) and a reduction in the total mass production rate. Gas production continuously increases during each cycle, with a corresponding reduction in the water production.

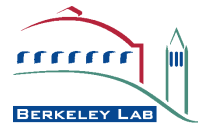

Three productions strategies were evaluated. The first involved a well completed (through the novel well design), and exhaustion. This approach is clearly superior to conventional production techniques for production under the Tigershark reference conditions.

\section{SIGNIFICANCE OF FINDINGS}

This study demonstrates that gas can be produced at high rates over long periods from Class 2 oceanic hydrate deposits, using conventional technology, and can be further enhanced through the use of novel production methods.

\section{RELATED PUBLICATION}

Moridis, G.J., and M.T. Reagan, Gas production from Oceanic Class 2 hydrate accumulations. LBNL-62757. OTC 18866, Offshore Technology Conference, Houston, Texas, USA, April 30-May 3, 2007.

\section{ACKNOWLEDGMENTS}

This work was supported by the Assistant Secretary for Fossil Energy, Office of Natural Gas and Petroleum Technology, through the National Energy Technology Laboratory (NETL), under the U.S. Department of Energy, Contract No. DE-AC02-05CH11231. 


\section{A Strategy for Gas Production from Oceanic Class 3 Hydrate Deposits}

\section{RESEARCH OBJECTIVES}

The main objective of this study is to evaluate the natural gas production potential of marine Class 3 accumulations and to determine the factors and conditions affecting it. Class 3 accumulations are composed of a single zone bounded by confining strata, the hydrate-bearing interval, with no underlying zone of mobile fluids. Previous studies have dismissed such formations as targets for gas production, but an appropriate choice of production techniques allows us to reexamine their viability.

\section{APPROACH}

The code used in this study is TOUGH+HYDRATE, developed by Berkeley Lab staff with support from DOE-NETL. The model system is based on the "Tigershark" area of the Gulf of Mexico, where an exploration well in 2,750 $\mathrm{m}$ of water has indicated the presence of an $18.25 \mathrm{~m}$ thick sandy hydrate layer. Previous studies have used thermal stimulation, via electrical heating or warm water injection, to induce dissociation in Class 3 deposits, but with disappointing results. A new strategy uses constant-pressure production to overcome the very low initial effective permeability of the deposit, the uncertainty over the evolution of effective permeability over time, and the lack of a mobile water zone.

\section{ACCOMPLISHMENTS}

The simulated gas production occurs in a cyclical pattern. Each cycle consists of a long stage of increasing gas production, followed by sharp production decline, followed by a recovery. This pattern is repeated until the cessation of production. Peak production regularly exceeds $5 \mathrm{ST} \mathrm{m} 3 / \mathrm{s}$ of $\mathrm{CH}_{4}(15 \mathrm{MMSCFD})$, and the average gas production during the 6,000-day simulation period is $2.61 \mathrm{ST}$ $\mathrm{m}^{3} / \mathrm{s}\left(8.10\right.$ MMSCFD), with a total of $1.37 \times 10^{9} \mathrm{ST} \mathrm{m}^{3}\left(4.84 \times 10^{10}\right.$ ST $\mathrm{ft}^{3}$ ) produced, all of which originated from the hydrate. Recovery from each production decline occurs naturally, without heating or fluid injection. Unlike conventional natural gas reservoirs, water production decreases continuously with time.

The figure shows the evolution of the spatial distribution of hydrate saturation over time and provides an explanation for

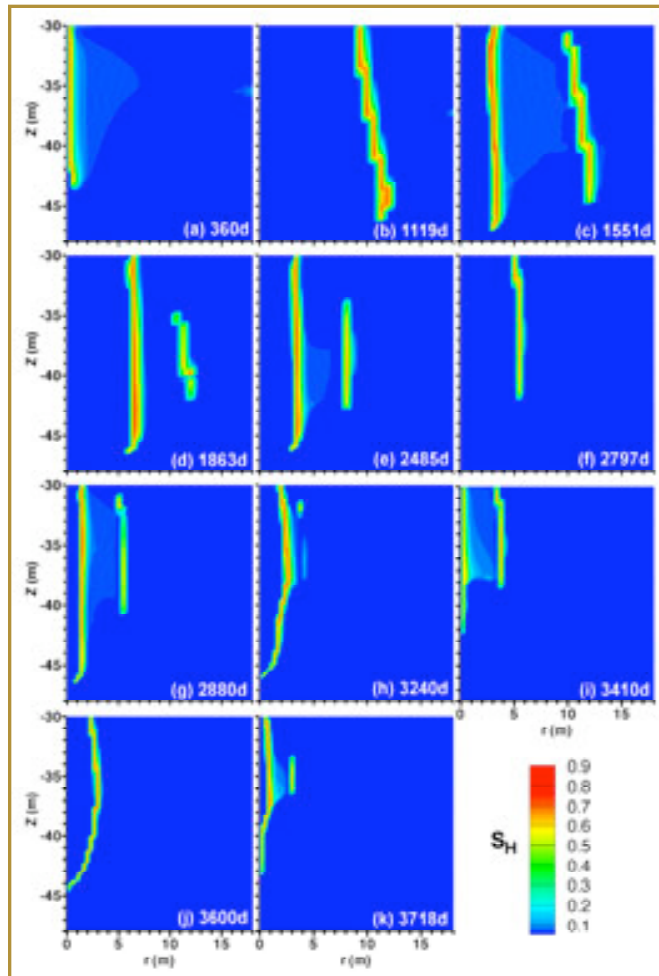

Figure 1. Evolution of spatial distribution of hydrate saturation, $S_{H}$, during gas production from a Class 3 oceanic hydrate deposit, showing the traveling barriers of secondary hydrate. the cyclical pattern. The precipitous drops in production are caused by the evolution of traveling dual barriers of secondary hydrate around the well. As gas flows to the well, a cylindrical sheath of secondary hydrate forms next to the wellbore (Panel a). Because the inner radius of the barrier is exposed to intense depressurization, it dissociates, but additional hydrate forms on its outer radius, resulting in the appearance of a traveling barrier (b). A second barrier forms (c) and moves outward through the previously described process (d). The outer barrier is now shielded from steep pressure gradients and begins to dissociate through contact with the warmer fluids behind it (d through $\mathrm{f}$ ). As time advances, new barriers appear and disappear ( $\mathrm{g}$ though $\mathrm{k}$ ). Each of the temporary drops in production occurs when a new inner secondary hydrate barrier is formed.

\section{SIGNIFICANCE OF FINDINGS}

This study demonstrates that large volumes of hydrate-originating natural gas can be produced at high rates over long periods from Class 3 oceanic hydrate deposits. With straightforward depressurization-induced dissociation and gas production at constant pressure, secondary hydrate blockages can be "self-healing" without additional heating or fluid injection. Hence, these deposits may be viable candidates for future exploitation and development.

\section{RELATED PUBLICATIONS}

Moridis, G.J. and M.T. Reagan, Strategies for gas production from Oceanic Class 3 hydrate accumulations. LBNL-62758. OTC 18866, 2007 Offshore Technology Conference, Houston, Texas, U.S.A., April 30-May 3, 2007.

\section{ACKNOWLEDGMENTS}

This work was supported by the Assistant Secretary for Fossil Energy, Office of Natural Gas and Petroleum Technology, through the National Energy Technology Laboratory (NETL), under the U.S. Department of Energy, Contract No. DE-AC0205CH11231.

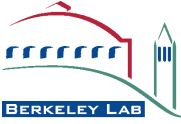




\section{Shear-Stress Monitoring of a Fracture Using Seismic Waves \\ Seiji Nakagawa \\ Contact: 510/486-7894, snakagawa@lbl.gov}

\section{RESEARCH OBJECTIVES}

Transmission and reflection of seismic waves can be used to monitor changes in in situ geological stresses on a fracture. For example, an increase in normal stress (compression) can be measured from an amplitude increase and a phase delay of the transmitted wave. For monitoring shear stress, a less wellknown effect can be used: when an incoming wave is normally incident on a fracture, the amplitude and particle motions of the transmitted and reflected waves with mode conversions (compressional [P] waves to shear [S] waves, and vice versa) change as a function of the magnitude and direction of shearing on the fracture (Nakagawa et al., 2000). However, several questions need to be answered before this effect can be used as a practical tool for stress monitoring. These include: What is the quantitative relationship between the magnitude of shear stress and the conversions of the waves? What is the impact of normal stress acting together with the shear stress? What is the effect of nonlinear elastic and inelastic (frictional slips and failure) deformations of the fracture? And is there a precursor in the converted waves before a slip along a fracture?

\section{APPROACH}

A series of laboratory seismic measurements was conducted on both a natural rock sample and synthetic (aluminum and steel) samples. (Sample diameter $=10.16 \mathrm{~cm}$, height $=5.08 \mathrm{~cm}$.) A single, through-going fracture was induced by tension in the rock sample. The metal samples contained a pair of sine-wave fracture surfaces (period=1.25 mm, peak-trough amplitude=250 $\mathrm{mm}$ ). The fractures were quasi-statically sheared under a constant normal stress. The stresses were controlled using a small, bi-axial loading machine capable of applying up to $5.5 \mathrm{MPa}$ of both normal and shear stresses on the fracture surface. For each combination of normal and shear stresses, converted S-waves and unconverted Pwaves transmitted through a fracture from an incident $\mathrm{P}$-wave (central frequency $=500 \mathrm{kHz}$ ) were measured.

\section{ACCOMPLISHMENTSC}

From an experiment using a natural rock sample (granite), the amplitude of converted S-waves was found to increase nonlinearly with shear stress (Figure 1). Also, the magnitude of normal stress did not have a strong effect on the amplitude. At low normal stresses, P-waves also showed small increases in amplitude. However, at high stresses, the rate of increase reduced, and the amplitude decreased for large shear stresses. A metal sample exhibited a somewhat diffe rent behavior, because stable, large slips occurred during shearing, owing to the smooth (although not flat) surface of the machined fracture. Further, a cyclic shearing of the fracture resulted in hys-

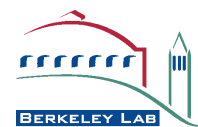

waves, which exhibited larger changes when the fracture surface was sliding.

\section{SIGNIFICANCE OF FINDINGS}

The experiments indicate that the relationship between the shear stress on a fracture and the amplitude of shear-induced, mode-converted waves depends strongly on the mechanical properties of the fracture.

\section{RELATED PUBLICATION}

Nakagawa, S., K. T. Nihei, and L. R. Myer, Shear-induced conversion of seismic waves across single fractures. Int. J. Rock Mech. Min. Sci. \& Geomech. Abstr., 37(1-2), 203-218, 2000.

\section{ACKNOWLEDGMENTS}

This work was supported by the Director, Office of Science, Office of Basic Energy Sciences, Division of Chemical Sciences, Geosciences, and Biosciences, of the U.S. Department of Energy under Contract No. DE-AC02-05CH11231.

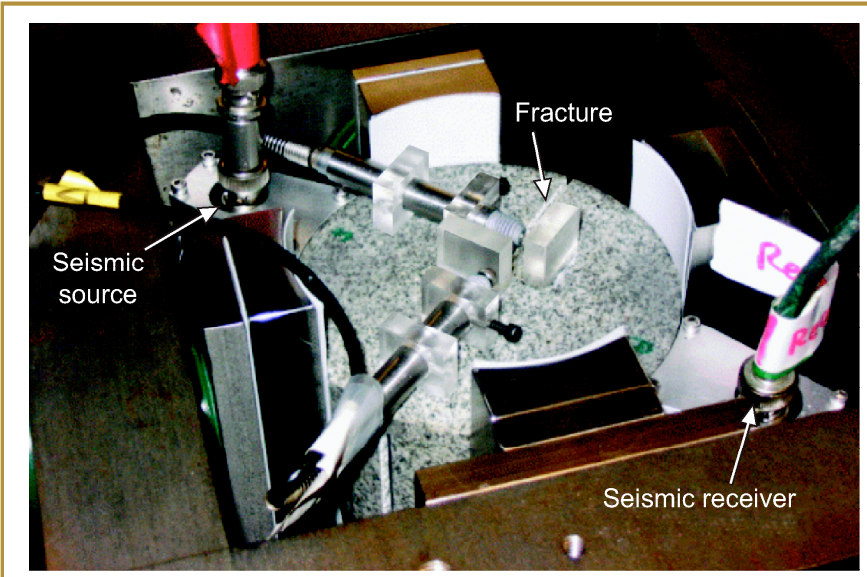

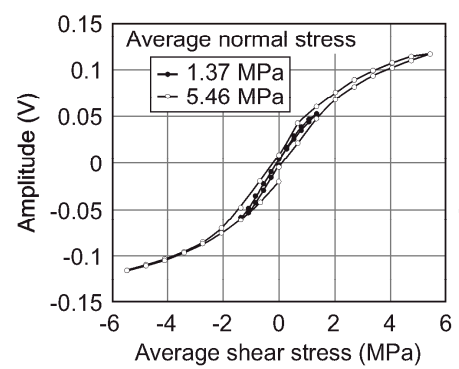

(a) Converted S-waves

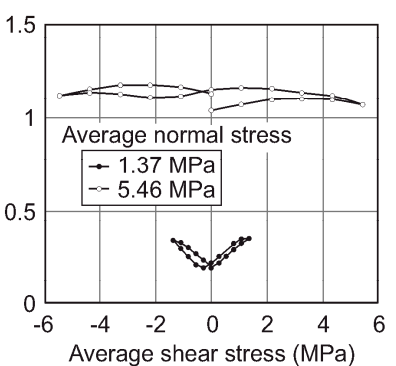

(b) Unconverted P-waves
Figure 1. Laboratory seismic wave transmission experiment during bi-axial shear loading. Both normal and shear stresses were applied to a granite cylinder containing a single, through-going tensile fracture. Static deformations normal and parallel to the fracture were also monitored during the experiment. 


\section{Seismic Boundary Conditions for a Fluid-Filled Fracture}

Seiji Nakagawa and Michael A. Schoenberg

Contact: Seiji Nakagawa, 510/486-7894, snakagawa@lbl.gov

\section{RESEARCH OBJECTIVES}

Rock is often permeated by compliant plane discontinuities (such as fractures and faults) that, depending on their permeability relative to the background, serve as either conduits or barriers to subsurface fluid. The fluid permeability of a fracture is often a key parameter, yet the quantitative relationship between permeability and its effect on seismic wave scattering is not fully understood. Strong scattering of seismic waves by a fracture is usually related to large permeability, because an open fracture with partial surface contacts has increased mechanical compliance (deformability). However, if a fluidcontaining fracture is filled with debris, or a single fracture consists of a large number of microcracks, complex interactions between rock and pore fluid in the fracture result. In this research, a simple mathematical model that captures the essential nature of solid-fluid interaction within a fracture is developed, to predict the effect of hydraulic permeability and other fracture properties on seismic wave scattering.

\section{APPROACH}

We envision a fracture as a thin, flat, homogeneous poroelastic layer surrounded by homogeneous halfspaces. Starting from the governing equations of poroelastic wave propagation, the spatial gradient in the fracture-normal direction of the displacement (or particle velocity), stress, and fluid pressure induced by seismic waves are expressed using other wave field variables. When accumulated (integrated) over the thickness of the fracture, this gradient produces a finite jump (discontinuity) in the field quantities. If this jump can be expressed using only the wave-induced displacement and stress (and pressure) on the surfaces of a fracture, we obtain a seismic boundary condition.

\section{ACCOMPLISHMENTS}

The derivation of the jump conditions resulted in a pair of compact, coupled matrix equations for fast and slow compressional $(\mathrm{P})$ waves and a shear $(\mathrm{S})$ wave. For a fracture thickness much smaller than the wavelength of propagating seismic waves, these jump conditions correctly capture frequencydependent changes in the poroelastic behavior of a fracture. A further simplification of the equations resulted in a set of boundary conditions that are a function of five fracture characteristic parameters defined for a fluid-filled fracture (for a dry fracture, there are only two-normal and shear fracture compliances). One of the five parameters represents the fracture- normal hydraulic permeability, but none is related to the fracture-parallel permeability.

\section{SIGNIFICANCE OF FINDINGS}

The five characteristic parameters identified in the boundary conditions govern the scattering of seismic waves by a fluid-filled homogeneous fracture. Conversely, these are the only quantities that can possibly be determined from seismic measurements alone. An interesting and surprising finding is that the scattering of seismic waves is insensitive to the fracture-parallel permeability. (This was also confirmed using an independent numerical modeling.) A further investigation is under way to see if this is also true when the material properties within a fracture are heterogeneously distributed.

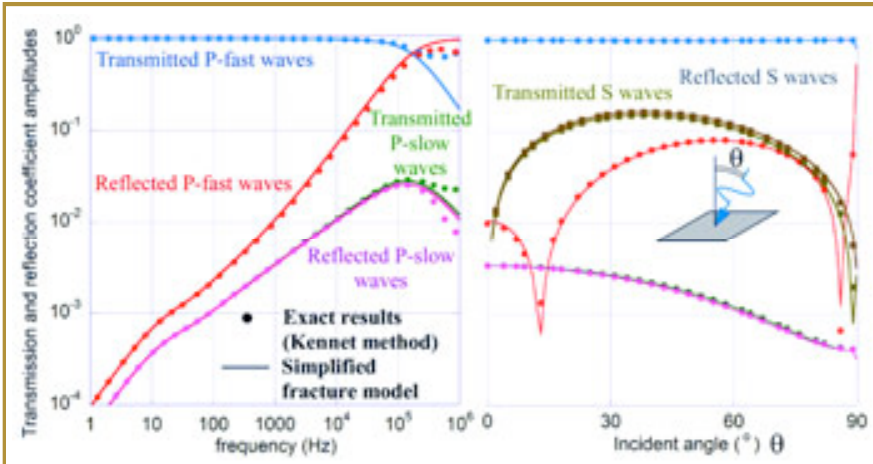

(a) Normal incidence frequency response. $k_{0}=10^{-16} \mathrm{~m}$.

(b) $1 \mathrm{kHz}$ angle-of-incidence response. $k_{0}=10^{-16} \mathrm{~m}^{2}$

Figure 1. Transmission and reflection coefficients of a thin $(1 \mathrm{~mm})$, homogeneous fracture for incident Biot's fast P-waves. The coefficients were computed using both an exact method and the simplified poroelastic fracture boundary conditions with the five characteristic fracture parameters.

\section{RELATED PUBLICATION}

Nakagawa, S., and M.A. Schoenberg, Poroelastic modeling of seismic boundary conditions across a fracture. LBNL-60862. J. Acoust. Soc. Am, 122(2), 831-847, 2007.

\section{ACKNOWLEDGMENTS}

This work was supported by the Director, Office of Science, Office of Basic Energy Sciences, Division of Chemical Sciences, Geosciences, and Biosciences, of the U.S. Department of Energy under Contract No. DE-AC02-05CH11231.

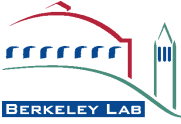




\section{Large Scale 3-D Electromagnetic Inversion Problems \\ Gregory A Newman and Michael Commer \\ Contact: Gregory A. Newman, 510/486-6887, ganewman@lbl.gov}

\section{RESEARCH OBJECTIVES}

Large-scale controlled source electromagnetic (CSEM) 3-D geophysical imaging is now receiving considerable attention for mapping complex geological systems, with emphasis on mapping fluids associated with potential oil and gas and geothermal reservoirs and subsurface contamination. When combined with established seismic methods, direct imaging of reservoir fluids is possible. While modeling in 1-D is relatively easy, and trialand-error 3-D forward modeling is straightforward, 3-D imaging is necessary in highly complex and subtle geological environments. Faster 2-D CSEM imaging technology has some relevance to this problem, but because of its assumption of 2-D geology, it cannot be relied upon for a consistent treatment of the 3-D imaging problem, especially when data are acquired specifically for a 3-D imaging experiment. Because of the size of the 3-D CSEM imaging, problem strategies are required that exploit computational parallelism and optimal meshing.

\section{APPROACH}

New techniques for improving both the computational and imaging performance for 3-D electromagnetic (EM) inversion have been developed. A nonlinear conjugate gradient algorithm is the framework of the inversion scheme. Full wave equation modeling for controlled sources is utilized for the data simulation and efficient gradient computation needed for the iterative model update. Improving the modeling efficiency of the 3-D finite difference method involves the separation of the potentially large model mesh, defining the set of model parameters, from the computational finite-difference meshes used for field solution. Meshing and thus overall mesh sizes can be reduced and optimized according to source, frequencies, and sourcereceiver offsets of a given input data set. Further computational efficiency is obtained by combining different levels of parallelization over the model and data spaces, avoiding the performance loss caused by computationally idle message-passing when increasing the number of parallel tasks. Image enhancement is achieved by model parameter transformation functions, which enforce bounds on the conductivity parameters and thus p revent parameter overshoots. Further, we have developed a remedy for treating distorted data within the inversion process. Data distortions simulated here include positioning errors and a highly conductive overburden, hiding the desired target signal. The methods are demonstrated using synthetic and field data.

\section{ACCOMPLISHMENTS}

In a recent imaging experiment, we have demonstrated the power of our 3-D imaging approach, where 32,768 tasks/processors were utilized on the IBM Watson Research Blue Gene/L supercomputer. Over a 24-hour period, we were able to image a large-scale field data set that previously required over four months of processing time on distributed clusters, based on Intel or AMD processors utilizing 1024 tasks on an Infiniband fabric. Results of the Blue Gene/L experiment showed that the broadside inline component data displays a systematic bias that could not be fit to a degree that is within the anticipated noise level of the measurements; other field components were satisfactorily fit. Modeling confirms that a likely explanation for this outcome is the need to incorporate conductivity that exhibits transverse anisotropy within the 3-D model. The speed at which the Blue Gene/L platform delivered this result is consistent with time frames required by practical exploration problems.

\section{SIGNIFICANCE OF FINDINGS}

We have made significant progress in reducing computational demands for solving large-scale, 3-D, electromagnetic imaging problems.

\section{ACKNOWLEDGMENTS}

Funding for this work was provided by the ExxonMobil Corporation, and by the Director, Office of Science, Office of Basic Energy Sciences, Division of Chemical Sciences, Geosciences, and Biosciences, of the U.S. Department of Energy under Contract No. DE-AC02-05CH11231.

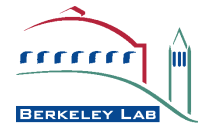




\section{Enhanced Geothermal Systems (EGS) Using $\mathrm{CO}_{2}$ as Working Fluid \\ Karsten Pruess \\ Contact: 510/486-6732, K_Pruess@lbl.gov}

\section{RESEARCH OBJECTIVES}

The resource base for geothermal energy is enormous, but commercial production of geothermal energy is currently limited to high-grade hydrothermal systems, in which naturally present fracture networks permit fluid circulation, and allow geothermal heat to be produced by tapping these hot fluids through wellbores. The concept of "enhanced geothermal systems" (EGS) aims to extract geothermal energy from lowergrade resoures by stimulating fracture permeability and extracting energy through a system of injection and production boreholes. Previous attempts to develop EGS in the U.S. and other countries have all employed water as heat transmission fluid and have met with limited success. At geothermal temperatures, water is a powerful solvent for many rock minerals, which poses great difficulties in achieving and maintaining water circulation at adequate rates.

The purpose of this research was to explore an alternative concept, first proposed by Donald Brown (2000), that would use carbon dioxide $\left(\mathrm{CO}_{2}\right)$ instead of water as a heat-transmission fluid.

\section{APPROACH}

Mathematical modeling capabilities for flow of water and $\mathrm{CO}_{2}$ previously developed in the DOE geothermal and carbon management programs were enhanced to permit application to temperatures of up to $250^{\circ} \mathrm{C}$. These capabilities were used to evaluate and compare flow of water and $\mathrm{CO}_{2}$ in injection and production wells, and to analyze heat sweep in fractured reservoirs.

\section{ACCOMPLISHMENTS}

At temperature and pressure conditions of interest, typically $200^{\circ} \mathrm{C}$ and 500 bar, $\mathrm{CO}_{2}$ has far greater compressibility and expansivity than water. As a consequence, for comparable injection conditions, a $\mathrm{CO}_{2}$ production well would have far greater wellhead pressure than a water production well. This finding is very favorable, as it implies that EGS operated with $\mathrm{CO}_{2}$ would require less pumping power than water to maintain fluid circulation, and may in fact achieve commercially adequate flow rates without artificial pumping. $\mathrm{CO}_{2}$ also has very favorable heat extraction characteristics. Figure 1 compares numerically simulated heat extraction for a typical EGS that would be operated either with water or with $\mathrm{CO}_{2}$. Heat extraction rates with $\mathrm{CO}_{2}$ are seen to be approximately $50 \%$ larger than with water.

\section{SIGNIFICANCE OF FINDINGS}

Initial evaluation of this novel concept-operating enhanced geothermal systems with $\mathrm{CO}_{2}$ - has shown very promising results. In addition to favorable wellbore hydraulics and reservoir heat extraction, inevitable fluid losses during
EGS operation provide opportunities for carbon storage in $\mathrm{CO}_{2}$-driven systems, whereas in water systems, fluid losses present a severe economic liability.

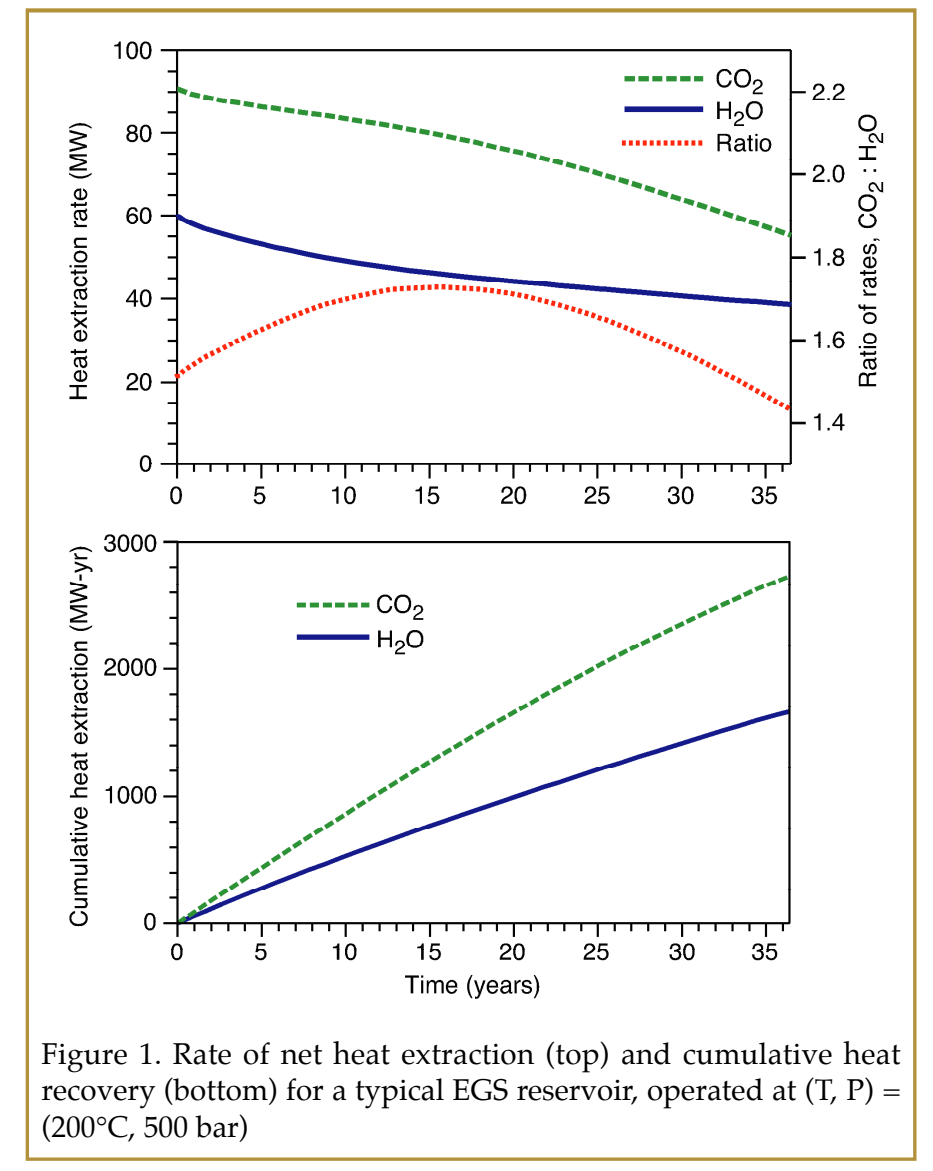

\section{RELATED PUBLICATIONS}

Pruess, K. and M. Azaroual, On the feasibility of using supercritical $\mathrm{CO}_{2}$ as heat transmission fluid in an engineered hot dry rock geothermal system. Proceedings, 31st Workshop on Geothermal Reservoir Engineering, Stanford University, Stanford, CA, January 30-February 1, 2006.

Pruess, K. Enhanced geothermal systems (EGS) using $\mathrm{CO}_{2}$ as working fluid-A novel approach for generating renewable energy with simultaneous sequestration of carbon. Geothermics, 35(4), 351-367, August 2006.

\section{ACKNOWLEDGMENTS}

This work was supported by the Assistant Secretary for Energy Efficiency and Renewable Energy, Office of Geothermal Technologies, of the U.S. Department of Energy under Contract No. DE-AC02$05 \mathrm{CH} 11231$.

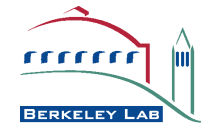




\section{OCEANIC GAS-HYdRATE INSTABILITY AND Dissociation under Climate Change Scenarios}

Matthew T. Reagan and George J. Moridis

Contact: Matthew T. Reagan, 510/486-6517, mtreagan@lbl.gov

\section{RESEARCH OBJECTIVES}

The dissociation of accumulated oceanic gas-hydrate deposits and the release of large quantities of methane-a powerful greenhouse gas-over a short period of time could have dramatic climatic consequences, leading to further atmospheric and oceanic warming and accelerated decomposition of the remaining hydrates. This positive-feedback mechanism has been proposed as a significant contributor to rapid and significant climate changes in the past. However, the behavior of contemporary oceanic methane-hydrate deposits subjected to rapid temperature changes, like those predicted under future climate change scenarios, is poorly understood. In this study, we simulated the dynamic response of several types of oceanic gas-hydrate accumulations to temperature changes at the seafloor and assessed the potential for methane release into the ecosystem.

\section{APPROACH}

The amount of methane hydrate currently residing in the deep ocean and along continental margins is estimated to be from 500 billion to over 3,000 billion tons. In oceanic deposits, hydrate stability depends on the pressure (imposed by the water depth) and temperature. Figure 1 presents a general schematic of the hydrate-phase boundary (red) and gashydrate stability zone (GHSZ) for oceanic hydrates (shaded area). An increase in water temperature at the seafloor (a shift from Temperature Profile 1 to Profile 2) lowers the position of the top of the GHSZ (A) and raises the position of the bottom of the GHSZ (B). Such a shift could induce hydrate dissociation and lead to the release of methane into the ocean and atmosphere.

We modeled oceanic hydrate deposits under a range of depths and temperatures, using the TOUGH+HYDRATE code. We simulated temperature increases of $1^{\circ} \mathrm{C}$ to $5^{\circ} \mathrm{C}$ over a $100 \mathrm{yr}$ simulation period, representing possible changes in seafloor temperature as predicted by advanced climate simulators, and determined the dynamic evolution of dissociating hydrates and the transport of released methane through benthic sediments to the seafloor.

\section{ACCOMPLISHMENTS}

The simulations found that deep, cold hydrates at $1,000 \mathrm{~m}$ or greater depth are stable under all anticipated temperaturechange scenarios, and methane release was insignificant. Warmer, shallower hydrate deposits, representative of Gulf of Mexico formations, exhibited a stronger response, producing significant aqueous and gaseous fluxes of methane. These fluxes, however, were within the range of anaerobic

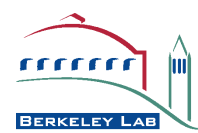

A shallow, cold hydrate deposit, representative of the arctic continental shelf, exhibited the strongest response, with explosive releases of methane, primarily in the gaseous phase, at rates that greatly exceed possible chemical or biological consumption.

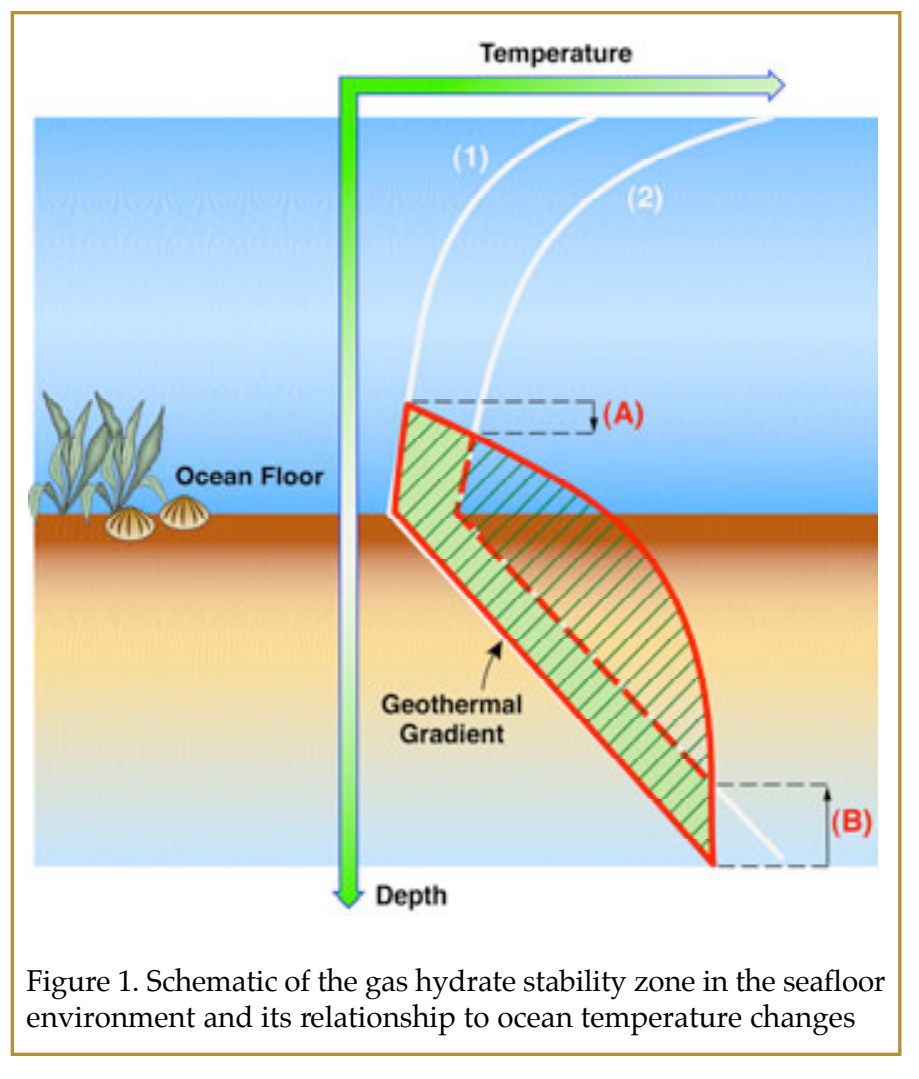

\section{SIGNIFICANCE OF FINDINGS}

These results indicate that while many deep oceanic hydrate deposits are indeed stable under the influence of significant temperature variations, shallow deposits, such as those found in arctic regions or in the Gulf of Mexico, can undergo rapid dissociation and release large quantities of carbon. In arctic regions in particular, temperature changes are expected to be more pronounced, increasing the risk of hydrate destabilization even further. These simulations can provide a source term to global climate models, yielding a prediction of the possible effects of oceanic hydrate decomposition on global climate.

\section{ACKNOWLEDGMENTS}

This work was supported by Laboratory Directed Research and Development (LDRD) funding from Berkeley Lab, provided by the Director, Office of Science, of the U.S. Department of Energy under Contract No. DE-AC02-05CH11231. 


\title{
A Numerical Model for Analysis of the Geomechanical Performance of HydRATE-BEARING SEDIMENTS
}

\author{
Jonny Rutqvist and George Moridis \\ Contact: Jonny Rutqvist, 510/486-5432, jrutqvist@lbl.gov
}

\section{RESEARCH OBJECTIVES}

Several methods are being considered for production of gas from hydrate-bearing sediments (HBS), including depressurization, thermal methods, and inhibitor injection. However, methane hydrate drilling and production operation may pose a significant hazard, because thermal and mechanical loading can result in hydrate dissociation and a significant pressure increase, with potentially adverse consequences on wellbore stability and the structural integrity and stability of the HBS. The objective of this research project is to build a simulator that can be used for scientific and engineering analyses of hydrate stability, including well bore and seafloor stability during production from oceanic hydrate formations.

\section{APPROACH}

The starting point of our approach is the TOUGH+HYDRATE simulator, which is an advanced code currently available for the simulation of multiphase flow system behavior in geological media containing gas hydrates. To consider geomechanical effects, the TOUGH+HYDRATE has been coupled with FLAC3D, a code widely used for soil and rock mechanics engineering, and for scientific research in academia. The two codes, TOUGH+HYDRATE and FLAC3D, are linked through a coupled thermal-hydrological-mechanical (THM) model of the HBS (Figure 1). Based on the coupled THM model, coupling functions are developed that serve to pass relevant parameters between the multiphase heat and transport analysis in TOUGH+HYDRATE and the geomechanical stress-strain analysis in FLAC3D.

\section{ACCOMPLISHMENTS}

The first version of the simulator is now fully operational and is currently being tested on a suite of problems related to geomechanical behavior of the HBS (Rutqvist and Moridis, 2007). The first involves hydrate heating as warm fluids from deeper conventional reservoirs ascend to the ocean floor, through uninsulated pipes intersecting the HBS. The second case describes system response during gas production from a hydrate deposit, and the third involves mechanical loading caused by the weight of structures placed on hydrate-bearing sediments at the ocean floor. Our simulation results indicate that the stability of HBS in the vicinity of warm pipes may be significantly affected, especially if the sediments are unconsolidated and more compressible. Gas production from oceanic deposits may also affect the geomechanical stability of HBS under the conditions that are deemed desirable for production. Conversely, the increased pressure caused by the weight of structures on the ocean floor increases the stability of hydrates.

\section{SIGNIFICANCE OF FINDINGS}

Methane hydrates occur naturally offshore in shallow depths below the ocean floor and onshore beneath the permafrost. They contain enormous quantities of methane gas, which if economically producible will have significant implications for U.S. energy security. The developed numerical model provides a state-of-the-art numerical tool for analyzing geomechanical hazards and optimizing production from hydratebearing sediments.

\section{RELATED PUBLICATIONS}

Rutqvist, J. and G. Moridis, Numerical studies on the geomechanical stability of hydrate-bearing sediments. OTC-18860, Presented at the 2007 Offshore Technology Conference held in Houston, Texas, U.S.A., April 30-May 3, 2007.

\section{ACKNOWLEDGMENTS}

This work was supported by the Assistant Secretary for Fossil Energy, Office of Natural Gas and Petroleum Technology, through the National Energy Technology Laboratory, under the U.S. Department of Energy, Contract No. DE-AC0205CH11231.

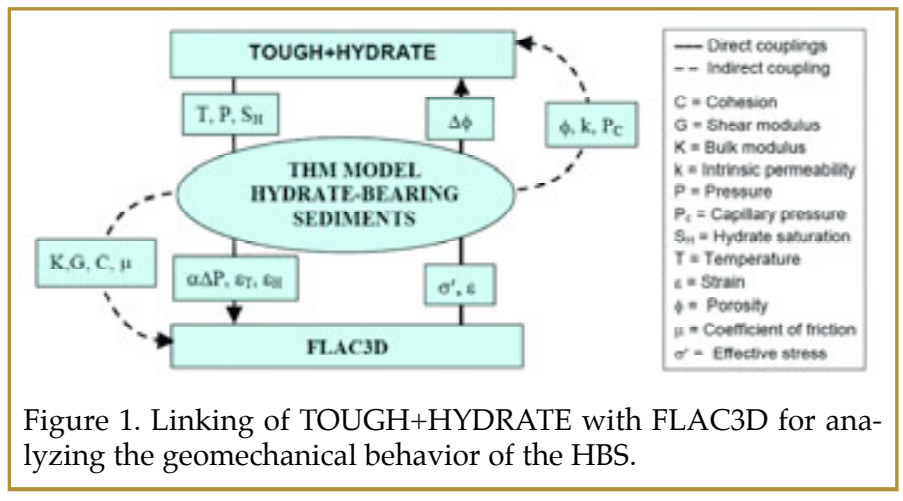

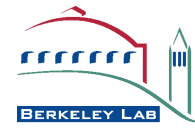




\section{Simulations of Induced Seismicity by Injection and Production AT The Geysers Geothermal Field}

Jonny Rutqvist and Curtis M. Oldenburg

Contact: Jonny Rutqvist, 510/486-5432, jrutqvist@lbl.gov

\section{RESEARCH OBJECTIVES}

The Geysers Geothermal Field in Northern California is the site of the largest geothermal electricity generating operation in the world. It is also one of the most seismically active regions in California. At The Geysers, water injection into the steam-dominated reservoir is necessary to maintain reservoir pressure and economical production. However, an increasing injection rate over the years has also resulted in an increased level of seismicity, which has raised concern in the local communities. The purpose of this study is to investigate the causes and mechanisms of seismicity at the Geysers and to develop injection and production strategies that minimize induced seismicity while maximizing energy recovery.

\section{APPROACH}

The approach we take is numerical simulation, using coupled hydrogeomechanical models for the reservoir based on TOUGH2 and TOUGH-FLAC. Simulation results are integrated with observations from expanded seismic arrays and satellite-based surface strain at The Geysers. The coupled-reservoir geomechanical analysis described herein is used to calculate the time evolution of the three-dimensional stress field during steam production and cold-water injection, and to evaluate the potential evolution and distribution of microearthquakes (MEQs) using various failure criteria. An important aspect of the analysis is the concept of a rock mass that is critically stressed for shear failure, conditions under which very small perturbations of the stress field can trigger seismicity.

\section{ACCOMPLISHMENTS}

We have conducted numerical simulations to analyze the potential for induced seismicity during both steam production and cold-water injection. Figure 1 shows injection-induced high potential for failure indicated by orange and yellow contours occurring near the injection well and in a plume at a distance several kilometers below in the injection well. This is consistent

\section{RELATED PUBLICATIONS}

Rutqvist, J., E. Majer, C. Oldenburg, J. Peterson J., and D. Vasco, Integrated modeling and field study of potential mechanisms for induced seismicity at The Geysers Geothermal Field, California. GRC Transactions, 30, 629-633, 2006.

Rutqvist, J. and C. Oldenburg, Analysis of cause and mechanism for injection-induced seismicity at the Geysers Geothermal Field, California Geothermal Research Council, Annual Meeting, Sparks, Nevada, September 30-October 3, 2007.

\section{ACKNOWLEDGMENTS}

This work was conducted with funding from the California Energy Commission (CEC) with matching funds from the Assistant Secretary for Energy Efficiency and Renewable Energy, Geothermal Technologies Program, of the U.S. Department of Energy under Contract No. DE-AC02$05 \mathrm{CH} 11231$.

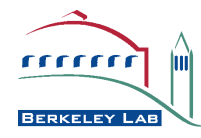




\title{
ImAging EleCtronic AND Atomic Redistribution DuRing Redox ReActions at SuRfaces
}

\author{
Glenn Waychunas, Ben Gilbert, Roger Falcone, Jillian Banfield, Klaus Attenkofer, and Robert Schoenlein \\ Contact: Glenn Waychunas, 510/495-2224, gawaychunas@lbl.gov, or Ben Gilbert, 510/495-2748, bgilbert@lbl.gov
}

\section{RESEARCH OBJECTIVES}

A number of important geochemical reactions are activated by electron transfer at mineral surfaces. Processes include reductive and oxidative dissolution, coupled redox-sorption of complexes, respiration of surface-associated biota, photoactivated reactions, and electrochemical cell reactions. Our research focuses on electron-transfer-initiated dissolution reactions, which are particularly important processes in the generation of acid mine drainage and resultant pollutant transfer in the environment. The crucial electron transfer step is faster than can be studied by currently available means, but the series of succeeding relaxation steps can be probed to elucidate much of the reaction mechanism.

\section{APPROACH}

In reductive dissolution processes, electrons are transferred from solution species via shared surface ligands to surface metal ions, like Fe(III), producing surface Fe(II) species. The $\mathrm{Fe}(\mathrm{II})-\mathrm{O}$ bonds at the surface must lengthen from this reaction, introducing surface vibrations and a general expansion of the surface. This localized strain may act to limit the density of Fe(II) that can be produced at the surface, depending on the rate of electron transfer within the mineral. We wish to measure the rate of Fe(II) buildup on Fe oxide surfaces and the ultimate concentration limit, as well as surface and bulk conduction rates. We are conducting such experiments at the Advanced Photon Source at Argonne National Laboratory (and soon also at the Advanced Light Source at Berkeley Lab), starting with observations of $\mathrm{Fe}(\mathrm{II})$ creation from a photostimulated ligand attached to Fe oxide nanoparticles and single crystal surfaces. A femtosecond laser system activates electron transfer from the ligand, and the synchrotron x-rays detect the result at variable delay times after the excitation. This is a "pumpprobe" experiment.

\section{ACCOMPLISHMENTS}

Our first static experiments at the APS beamline 13ID showed proof of concept with respect to electron injection from the organic ligand alizarin red into the (1-102) hematite surface. A significant amount of surface Fe(II) (Figure 1) is produced within minutes of exposure to the dye. We observe the Fe(II) by collecting surface diffraction data via the (10L) crystal truncation rod. Collection of complete data sets will enable refinement of the electronic configuration of the excited hematite surface, and this process can be done as a function of timedelay to detect the time-resolved progress of charge migration and relaxation. Our first time-resolved experiments at the APS beamline 11-ID showed measurable Fe(II) creation in $2 \mathrm{~nm}$ maghemite nanoparticles coated with eosin $\mathrm{Y}$ surface ligands with a time delay of 154 ns. In these experiments, the nanoparticles were studied in recycled suspension, and the near Fe Kedge $\mathrm{x}$-ray absorption spectra was collected.

\section{SIGNIFICANCE OF FINDINGS}

The static results on large single crystal hematite surfaces demonstrate the sensitivity of the CTR method to the presence of reduced surface atoms. We observe that significant concentrations of Fe(II) are stable for long time periods, but that control of the injection process may be difficult. The time-resolved nanoparticle results show that varied ligands can have much different injection probability, and that beam damage (either from laser or x-rays) may have a significant effect on nanoparticle structure or chemistry. Both issues will be investigated in the next series of experiments.

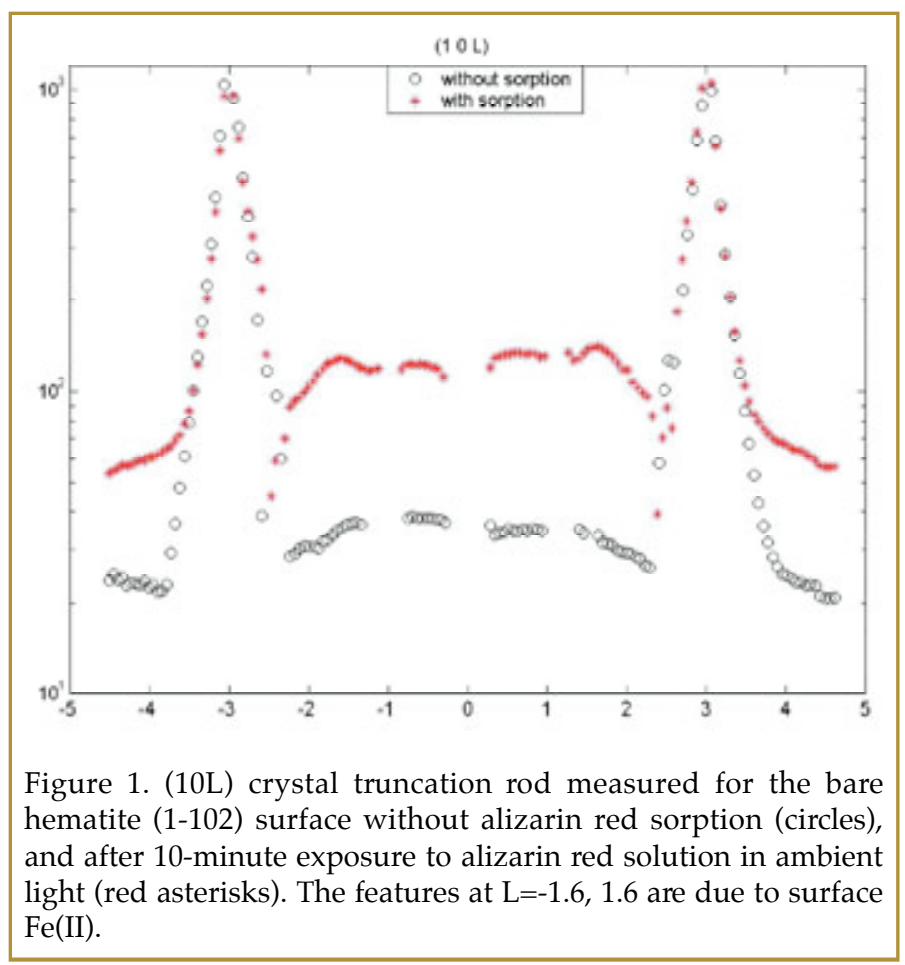

\section{ACKNOWLEDGMENTS}

This work was supported by the Director, Office of Science, Office of Basic Energy Sciences, Division of Chemical Sciences, Geosciences, and Biosciences, of the U.S. Department of Energy under Contract No. DE-AC02-05CH11231. We thank Dr. Lin Chen (Argonne National Laboratory Chemistry Division) for the use of the LITR-XAS facility at the Advanced Photon Source.

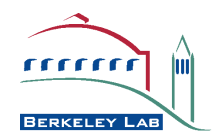




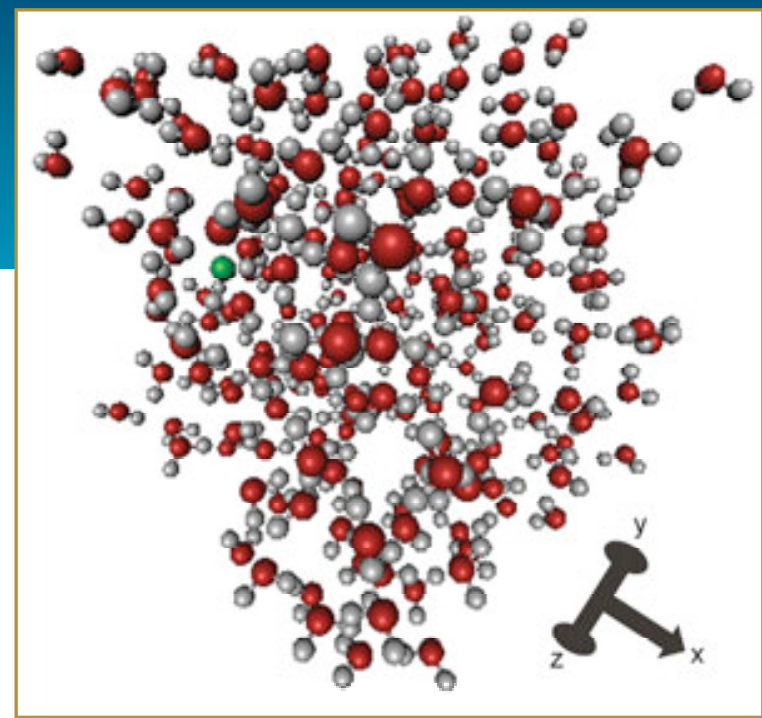

The Fundamental and Exploratory Research Program (FERP) within ESD covers fundamental earth sciences research conducted in support of the Department of Energy's science mission. This mission includes research in the natural sciences to provide a basis for new and improved energy technologies and for understanding and mitigating the environmental impacts of energy development and use. FERP also includes exploratory research in important new energy and environmental topics conducted under the Laboratory Directed Research and Development (LDRD) program. The scientific insights and breakthroughs achieved in FERP often become the underpinnings for projects that support DOE's applied research and development program offices.

The basic earth sciences research program at Berkeley Lab has focused on four broad earth sciences problems:

1. Fundamental studies of chemical and mass transport in geologic media-with special reference to predictive modeling of multiphase, multicomponent, nonisothermal fluid flow in saturated and unsaturated fractured rock

2. The development of new isotopic techniques for understanding the nature of a broad range of global processes-from the relatively short-term effects of natural fluid migration in the crust to longer-term (i.e., 10-20 thousand years) global climate variations

3. Imaging the subsurface-fundamental studies in the propagation of seismic/acoustic and broadband electromagnetic waves through geologic media, with emphasis on new computational techniques for high-resolution imaging of near-surface and crustal structures (such as possible fracture flow paths) and for inferring the types of fluids present in pores and fractures

4. The development of a nanogeoscience center-The defining aspect of nanogeoscience is its interconnecting chemical
Research Program FUNDAMENTAL AND EXPLORATORY RESEARCH Program

Ernest L. Majer

510/486-6709

elmajer @lbl.gov

pathways, uniting aspects of natural inorganic, organic, and biological processes with specific interactions and mechanisms common to mineral surfaces, small particles, and particle aggregates. The focus is on various combinations of several key topics, defined with respect to important specific problems within the DOE complex, and aimed at elucidation of fundamental scientific problems and reaction pathways. Work in this field is necessarily diverse and complex, and requires the close collaborations of investigators having both a wide knowledge base-encompassing geochemistry, colloid chemistry, surface chemistry, materials science, biochemistry and physical chemistry-and a strong interest in shared goals, utilizing a team effort. In bringing separate tasks together under one umbrella, efforts can now be combined with a grand synergy that would be difficult to achieve with separate subprojects operated in different locations without temporal congruence. The new laboratory and task structure will function as a focus for all relevant environmentally related nanoscience at LBNL, and also provide a superb location for the development of top-flight scientists in this field.

Results from these research endeavors have had a major impact on applied energy, carbon management/climate change, environmental, and radioactive waste management programs. Current research projects are briefly described here.

\section{CHEMICAL AND MASS TRANSPORT INVESTIGATIONS}

Current research in this area is focused on nanoparticle and colloid transport in unsaturated porous media and rock fractures, chemical transport in structure porous media, unsaturated fast flow in fractured rock, and production and evaluation of coupled processes for $\mathrm{CO}_{2}$ in aquifers. The nanoparticle research focuses on the mobile fractions and natural abundance in soils. Also studied is the rate of infiltration in the unsaturated zone, an important issue in studying and defining the driving forces and boundary conditions in the containment and cleanup of contaminants in the subsurface. Also being researched are interfacial reactions such as at quartz/water

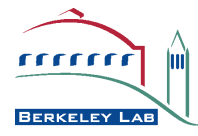


interfaces and the intergranular diffusion in uranium and in sand. Another study has focused on the use of x-ray absorption and diffraction methods to study sorption processes.

\section{ISOTOPE GEOCHEMISTRY}

The Center for Isotope Geochemistry (CIG) is a state-of-theart analytical facility established in 1988 to measure the concentrations and isotopic compositions of elements in rocks, minerals, and fluids in the earth's crust, atmosphere, and oceans. Fundamental research conducted at this center is directed at finding new ways to use isotopic information to study earth processes, such as long-term climate changes, and at predicting the chemical transport of mantle-derived or deep crustal fluids as they move through the crust.

One of the major problems being studied at CIG is how to estimate fluid-solid reaction rates in natural-groundwater highertemperature geothermal conditions, particularly as these rates affect mineral dissolution and secondary mineral precipitation. ESD researchers are developing novel ways of estimating reaction rates by using isotopic tracers (primarily strontium, but also uranium and neodymium) to determine solid-fluid exchange rates in various natural situations. Scientists are able to derive the "reaction length," a parameter that depends on the ratio of isotope transport by diffusion and advection to the reaction rate. The ultimate objective is to understand the microscopic (as well as porescale and mesoscale) characteristics of natural systems that have been characterized in terms of "field scale" reaction-rate measures. An intermediate goal is to establish empirically the natural range of fluid-solid reaction rates. Examples are the $\mathrm{U}-\mathrm{Th} / \mathrm{He}$ geochronology of young volcanic rock, understanding and predicting the microbial degradation of chlorinated solvents with carbon isotopes, and using xenon isotopes to study buried waste.

\section{ADVANCED COMPUTATION FOR EARTH IMAGING}

The Center for Computational Seismology (CCS) was created in 1983 as the Berkeley Lab and UC Berkeley nucleus for seismic research related to data processing, advanced imaging, and visualization. In recent years, a great deal of cross-fertilization between seismologists and other geophysicists and hydrogeologists has developed within the division, resulting in collaborations on a wide variety of fundamental imaging problems. A primary thrust in this research has been to jointly develop seismic and electrical methods for understanding fluid flow and properties within the subsurface. In addition, fundamental studies on improved inversion and modeling of complex media in 3-D are being carried out to analyze such effects as matrix heterogeneity fluid flow and anisotropy. Applications range from small-scale environmental problems to oil and gas reservoirs.

\section{ROCK PHYSICS}

A variety of rock and soil science experiments are being conducted through ESD's Geoscience Measurements Facility, which supports both field and laboratory work. In one new laboratory project, researchers are studying the compaction and fracturing of weakly cemented granular rocks. This study examines the effect of micromechanical properties of weak granular rock on macroscopic properties such as load-displacement response, ultimate strength, and failure mode. In a second study, a fundamental investigation of scattering and intrinsic attenuation of seismic waves in rock with heterogeneous distributions of fluids and gas is being conducted. This research represents a departure from past rock-physics studies on seismic attenuation, in that the emphasis here is not a detailed study of a specific attenuation mechanism, but rather to investigate theoretical and laboratory methods for obtaining separate estimates of scattering and intrinsic attenuation in rock with heterogeneous pore-fluid distributions.

\section{FUNDING}

Funding for research in the Fundamental and Exploratory Research Program comes from a variety of sources. These include (primarily) the U.S. Department of Energy, through the Director, Office of Science, Office of Basic Energy Sciences, Division of Chemical Sciences, Geosciences, and Biosciences; the Office of Biological and Environmental Research; the Assistant Secretary for Fossil Energy, Office of Natural Gas and Petroleum Technology, National Petroleum Technology Office, Natural Gas and Oil Technology Partnership; and the Office of Environmental Management Science Program. Funding is also provided by the Laboratory Directed Research and Development Program (LDRD) at Berkeley Lab. 


\section{ISOTOPE FractionAtion BY DIFFUSION IN LIQUID WATER \\ Ian C. Bourg and Garrison Sposito \\ Contact: Ian C. Bourg, 510/643-3172, ibourg@nature.berkeley.edu}

\section{RESEARCH OBJECTIVES}

Solute isotopic compositions have emerged as important indicators of geophysical and biogeochemical processes. The best-known isotopic geochemistry methods use various isotopic ratios as "signatures" of biological processes, geological time, and paleoclimatic conditions. Less widely recognized is the fact that diffusion in liquid water, a transport process ubiquitous in the biogeosphere, has its own isotopic signature, which results from the isotopic mass dependence of solute diffusion coefficients. We have applied molecular dynamics simulations to investigate this mass dependence for both neutral and ionic solutes in liquid water (Bourg and Sposito, 2007a,b), to determine its potential influence on solute isotopic compositions.

\section{APPROACH}

We carried out 8 ns molecular dynamics simulations of the motions of a single solute ion or molecule, immersed in 215 to 550 water molecules and moving in a periodically replicated simulation cell (Figure 1) maintained at the appropriate density of liquid water. In such simulations, molecular positions and velocities are calculated from the Newton-Euler equations of motion for a set of molecules (with freely chosen atomic masses) and interatomic potentials. We repeated each simulation with the solute isotopic mass varied systematically over a broad range (2 to $132 \mathrm{Da}$ ) to determine the mass dependence of the solute diffusion coefficient, which was calculated by a standard quadrature involving the velocity autocorrelation function.

\section{ACCOMPLISHMENTS}

We determined the isotopic mass dependence for the diffusion coefficients of five noble gases ( $\mathrm{He}, \mathrm{Ne}, \mathrm{Ar}, \mathrm{Kr}, \mathrm{Xe})$, four monovalent cations $\left(\mathrm{Li}^{+}, \mathrm{Na}^{+}, \mathrm{K}^{+}, \mathrm{Cs}^{+}\right)$, a monovalent anion $\left(\mathrm{Cl}^{-}\right)$and a divalent cation $\left(\mathrm{Mg}^{2+}\right)$. All solutes exhibited an inverse power-law relation between their diffusion coefficients and isotopic mass, as proposed recently by our collaborator Frank M. Richter (University of Chicago) on empirical grounds. The solute isotopic mass dependence increased in the o rder: divalent ion < monovalent ions < noble gases. Simulation results obtained for $\mathrm{Li}^{+}, \mathrm{Cl}^{-}$and $\mathrm{Mg}^{2+}$ and for the major isotopes of the noble gases $\mathrm{He}, \mathrm{Ne}, \mathrm{Kr}$, and $\mathrm{Xe}$ agreed with available experimental data, thus confirming the accuracy of our simulation methods (Bourg and Sposito, 2007a,b).

\section{SIGNIFICANCE OF FINDINGS}

Our results for the noble gases have particularly important geochemical implications, because of the paucity of experimen-

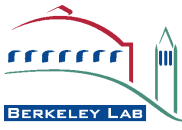
tal data on noble gas diffusion in liquid water and because of the widespread use of dissolved noble gases in paleoclimatic and groundwater hydrology studies. More specifically, we found that the isotopic mass dependence of noble gas diffusion coefficients in water is significantly smaller than that predicted by a kinetic-theory model used extensively-but never tested directly-in noble gas geochemistry. This finding offers a major challenge to the conclusions reached in many recent publications on paleoclimatic reconst ructions and groundwater hydrology.

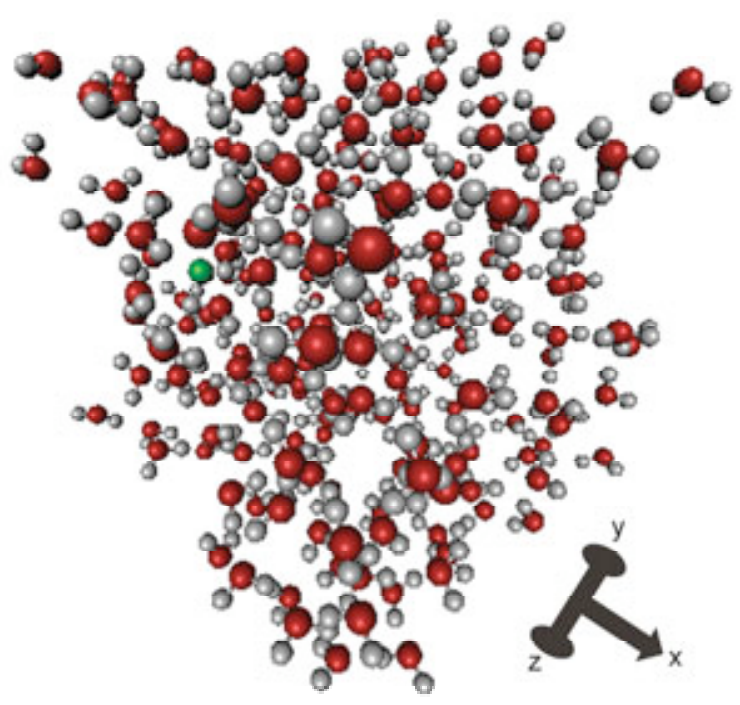

Figure 1. Eight-nanosecond molecular dynamics simulations of the motions of a single solute ion or molecule, immersed in 215 to 550 water molecules and moving in a periodically replicated simulation cell maintained at the appropriate density of liquid water.

\section{RELATED PUBLICATION}

Bourg, I.C., and G. Sposito, Molecular dynamics simulations of kinetic isotope fractionation during the diffusion of ionic species in liquid water. LBNL-61833. Geochimica et Cosmochimica Acta (in press), 2007a.

Bourg, I.C., and G. Sposito, Isotopic mass-dependence of noble gas diffusion coefficients in water. Physical Review Letters (submitted), 2007b.

\section{ACKNOWLEDGMENTS}

This research was supported by the Director, Office of Science, Office of Basic Energy Sciences, Division of Chemical Sciences, Geosciences, and Biosciences, of the U.S. Department of Energy under Contract No. DE-AC02-05CH11231. Our computations used resources of the National Energy Research Scientific Computing Center (NERSC) at Berkeley Lab, which is also supported by DOE under Contract No. DE-AC0205CH11231. 


\section{Using Isotopes to Track Spring 2002 Dust Storms over Hefei, China}

John N. Christensen, Steven S. Cliff ${ }^{1}$, Richard A. VanCuren ${ }^{1,2}$, Kevin D. Perry ${ }^{3}$, and Donald J. DePaolo

${ }^{1}$ U.C. Davis, Dept. of Applied Science, ${ }^{2}$ Research Div., California Air Resources Board, ${ }^{3}$ Univ. of Utah, Dept. of Meteorology Contact: John N. Christensen, 510/486-6735, jnchristensen@lbl.gov

\section{RESEARCH OBJECTIVES}

Aerosols-small particles less than 50 microns lofted into the atmosphere-can travel on winds vast distances across the globe. Such fine particulates can include soil-derived dust, biogenic material, sea spray, and industrial pollutants. Far-traveled aerosols can affect air quality, the way the atmosphere absorbs or reflects the sun's heat, and cloud formation. Analysis of elemental data for aerosols collected over California has identified a persistent Asian soil dust component that peaks with Asian dust storm events. Such Asianderived aerosols can also bring with them industrial pollutants that can affect air quality in California. To further understanding of intercontinental transport of aerosols, we undertook a study of the isotopic composition of aerosols as a fingerprint for their sources and evolution. As a basis for our study of aerosols collected in California, we have analyzed samples from Hefei, China, as a representation of Asian sources.

\section{APPROACH}

We use isotopic fingerprinting based on the isotopic composition of $\mathrm{Sr}$, represented by the ${ }^{87} \mathrm{Sr} / 86 \mathrm{Sr}$ ratio. Through the decay of ${ }^{87} \mathrm{Rb}$ over geologic time, the ${ }^{87} \mathrm{Sr} /{ }^{86} \mathrm{Sr}$ of earth materials differs, depending on their geologic history and ${ }^{87} \mathrm{Rb} /{ }^{86} \mathrm{Sr}$. We combined Sr isotopic data with elemental data both determined from daily 24-hour samples of PM2.5 (particles $<2.5$ microns in diameter) collected in Hefei, China. The collection period was during spring 2002, concurrent with changing weather conditions, including a series of major dust storms arising from deserts in western China. Prior to destructive isotopic analysis, the elemental composition of the particulate matter (PM) was determined by synchrotron-XRF analysis performed at the ALS at Lawrence Berkeley National Laboratory. The PM was then dissolved off the filters and analyzed for $\mathrm{Sr}$ isotopic composition and concentrations of $\mathrm{K}, \mathrm{Rb}$, $\mathrm{Sr}$, and Nd. We used a modeling program HYSPLIT provided by the National Oceanographic and Atmospheric Administration to calculate air-mass back trajectories to support interpretations of aerosol sources.

\section{ACCOMPLISHMENTS}

We analyzed filters covering April to the beginning of May 2002. Comparison of the time series for ${ }^{87} \mathrm{Sr} / 86 \mathrm{Sr}$ (Figure 1, top) with calcium and silicon concentrations (not shown) indicates a correspondence between high-dust days and highs in ${ }^{87} \mathrm{Sr} /{ }^{86} \mathrm{Sr}$, while lows are associated with precipitation events. Plotting $\mathrm{Sr}$ isotopic composition against various elemental ratios provides identification of end-member components in the mixture of particulates in the sample. For example, Figure 1 (bottom) shows ${ }^{87} \mathrm{Sr} /{ }^{86} \mathrm{Sr}$ vs. $\mathrm{Ca} / \mathrm{Sr}$. This plot indicates that the PM2.5 samples represent variable mixing between a high ${ }^{87} \mathrm{Sr} /{ }^{86} \mathrm{Sr}$ end-member (desert dust), and at least two different low ${ }^{87} \mathrm{Sr} /{ }^{86} \mathrm{Sr}$ components, one representing marine aerosol and another representing "local" China sources influenced by urban pollution. Samples labeled with dates have a significant component of marine aerosol $\mathrm{Sr}$, consistent with weather and air mass back-trajectories.

\section{SIGNIFICANCE OF FINDINGS}

This study demonstrates that the components of aerosol mixtures can be traced to their sources using a combination of Sr isotopic data and elemental data. The results we presented here yield constraints on the isotopic signatures of aerosols derived from China that include both desert dust and pollution sources. This provides us a starting-point compositional signature for determining the contribution of Asian-sourced materials to aerosols in California and evaluating their role in air quality.

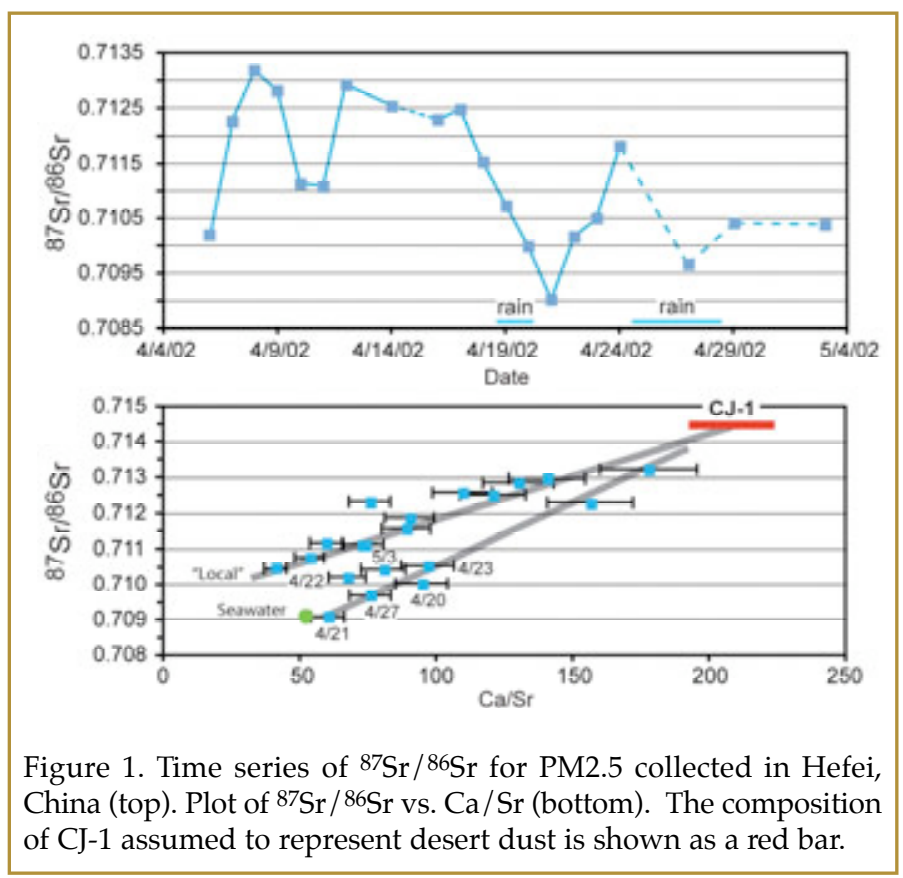

\section{RELATED PUBLICATION}

Christensen, J.N., S.S. Cliff, R.A. Van Curen, K.D. Perry, and D.J. DePaolo, Tracking intercontinental dust transport with radiogenic isotopes: Hefei, China to California, Spring 2002. AGU 2006 Fall Meeting. Eos Transactions American Geophysical Union, Abstract A43B-0138, 2006.

\section{ACKNOWLEDGMENTS}

This work was supported by Laboratory Directed Research and Development (LDRD) funding from Berkeley Lab, provided by the Director, Office of Science, of the U.S. Department of Energy under Contract No. DEAC02-05CH11231.

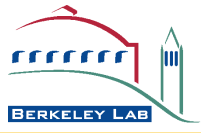




\section{Donald J. DePaolo and Matthew S. Fantle \\ Contact: Donald DePaolo, 510/643-5064, djdepaolo@lbl.gov6}

Investigating Mineral Precipitation Mechanisms with Ca Isotopes

\section{RESEARCH OBJECTIVES}

Precipitation of minerals from aqueous solution is often assumed to be a well-understood phenomenon. But the precise mechanisms by which dissolved ions are attached to mineral surfaces to allow solid crystals to grow from solution are both complex and poorly characterized, even for familiar minerals. Recent advances have been made using micro-imaging techniques such as atomic force microscopy and synchrotron x-rays, and insights are being gained from molecular dynamics and $a b$ initio calculations. The results of these studies tend to emphasize the complexity of the precipitation process, the variability as a function of conditions such as oversaturation and fluid chemistry, and the evidence that chemical equilibrium is not maintained during the process. In the geosciences, the departures from equilibrium attending mineral precipitation are particularly important, because mineral properties such as isotopic composition and trace-element content are used to reconstruct past Earth climate and ocean chemistry, and the assumption is commonly made that the minerals (such as calcite) form at equilibrium or near-equilibrium conditions.

\section{APPROACH}

We are investigating the use of isotopes to study processes at mineral surfaces. This approach is based on chemical differences between isotopic species-their mobility in solution, rates of attachment and detachment from mineral surfaces, and thermodynamic affinity in different chemical bonding environments. We measure $\mathrm{Ca}$ isotope ratios in the minerals calcite $\left(\mathrm{CaCO}_{3}\right)$ and gypsum $\left(\mathrm{CaSO}_{4}\right)$, which are formed by precipitation from aqueous solutions in soils, lakes, and the oceans. Ca isotopes are ideal because it appears that all of the fractionation produced in the precipitation process is caused by transport at the mineral-water interface.

\section{ACCOMPLISHMENTS}

To determine the equilibrium $\mathrm{Ca}$ isotope fractionation between calcite and aqueous $\mathrm{Ca}$, we measured the $\mathrm{Ca}$ isotopic composition of pore fluids and calcite from sedimentary carbonate ooze deposits on the ocean floor. The results indicate that at equilibrium, the $\mathrm{Ca}$ isotopic ratios of calcite and coexisting dissolved $\mathrm{Ca}$ are identical to within $0.002 \%$ per mass unit. When combined with observations from experiments and marine biogenic calcite, we find that this zero-fractionation condition occurs at both extremely slow and extremely fast rates of calcite precipitation. At intermediate rates, especially those applicable to biogenic calcite precipitation, there is fractionation that favors the incorporation of light isotopes into the precipitating solid. This behavior implies that the isotopic fractionation is heavily dependent on the kinetics of adsorptive attachment and detachment.

\section{SIGNIFICANCE OF FINDINGS}

The inference that isotopic fractionation is affected by attachment kinetics, in addition to equilibrium partitioning and diffusive transport, has far-reaching implications for understanding the chemical and isotopic composition of all natural materials. Not only is it important to be able to accurately describe the fractionations, but observed fractionations in natural materials may help to distinguish which processes are most active in their formation. A further challenge is to develop models that can predict isotopic fractionation based on the conditions of mineral growth from solution.

\section{RELATED PUBLICATIONS}

Fantle, M.S., and D.J. DePaolo, Ca isotopes in carbonate sediment and pore fluid from ODP Site 807A: The $\mathrm{Ca}^{2+}(\mathrm{aq})$-calcite equilibrium fractionation factor and calcite recrystalliza tion rates in Pleistocene sediments. Geochim. Cosmochim. Acta, doi: 10.1016/j.gca.2007.03.006, 2007.

\section{ACKNOWLEDGMENT}

This research was supported by the Director, Office of Science, Basic Energy Sciences, Chemical Sciences, Geosciences and Biosciences Division of the U.S. Department of Energy under Contract No. DE-AC02-05CH11231 to LBNL. Deep sea samples were obtained from the Ocean Drilling Program (ODP), which is sponsored by the U.S. National Science Foundation (NSF) and participating countries under management of Joint Oceanographic Institutions (JOI), Inc. 


\section{Lattice Boltzmann Simulations of Snow Crystal Growth \\ Donald J. DePaolo and Guoping Lu \\ Contact: Donald J. DePaolo, 510/643-5064, depaolo@eps.berkeley.edu}

\section{RESEARCH OBJECTIVES}

This study aims at simulating snow crystal growth-a vapor deposition process in clouds involving physics such as vapor diffusion, and reaction. Although it is known that crystal growth rate, which depends largely on the degree of vapor oversaturation, determines crystal morphology, there is no general model by which to relate morphology to vapor saturation. A numerical method capable of simulating the shape evolution of snow crystals is essential for interpreting relevant physics, effect of pollutants on snow formation, and isotope behaviors.

\section{APPROACH}

We use a 2-D lattice Boltzmann (LB) model to simulate diffusion-controlled snow crystal growth from vapor-oversaturated air. The vapor depositions were accommodated through both diffusion and reaction rates. The two-dimensional, ninespeed model of Kang et al. (2004) was modified to accommodate the specific boundary conditions needed. In the model, "crystals" grow solely according to the diffusive fluxes just above the crystal surfaces, and hence morphology arises only from the initial and boundary conditions. The effect of reaction relative to that of diffusion was described with the Damkohler number $D a=k_{r} h / D$, where $k_{r}$ is the local reaction-rate constant, $\mathrm{h}$ is length dimension of the model domain, and $D$ is the diffusivity.

\section{ACCOMPLISHMENTS}

We have successfully simulated the hexagonal symmetry of the snow growth (Figure 1). Our calculations demonstrate that crystal is a compact plate, and fractionation approaches the equilibrium value as the saturation factor approaches unity (S $=1.0$ ). Also, at higher oversaturation (e.g., $\mathrm{S}=1.2$ ), millimetersized dendritic crystals develop over time scales appropriate to cloud processes. The results help clarify the controls on dendritic crystal growth, and the relationships between saturation state, growth rate, crystal morphology, and isotopic fractionation. They also show the extent to which crystals are likely to be isotopically heterogeneous.

\section{SIGNIFICANCE OF FINDINGS}

LB simulation connects the crystal growth processes and isotopic kinetic effects. The simulation results explain the isotopic variation in evaporation/condensation, snow and ice formation, and crystal growth. LB simulation provides a method for evaluating the fractionation of isotopes in natural systems and extends the simulation capability for investigating the physics, bridging the molecular dynamics and conventional continuum domain.

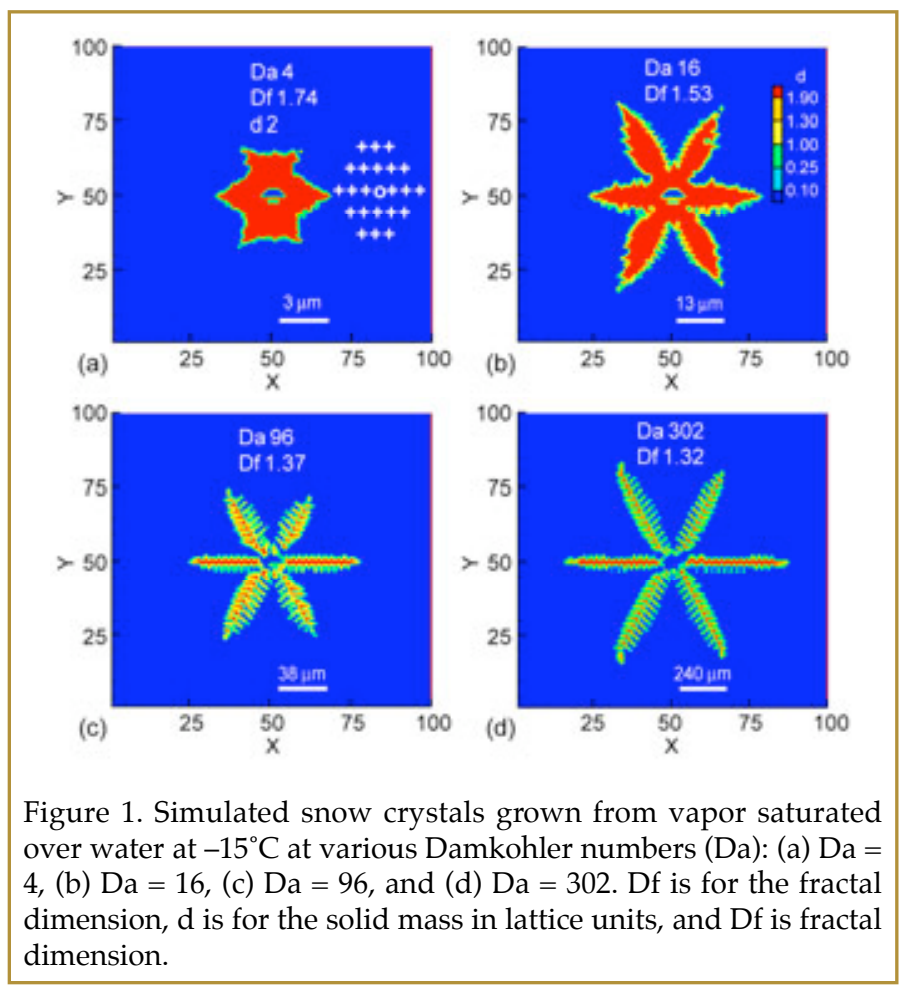

\section{RELATED PUBLICATION}

Kang et al., Lattice Boltzmann model for crystal growth from supersaturated solution. Geophys. Res. Lett. 31, L21604, 2004.

Lu, G., D.J. DePaolo, Q. Kang, and D. Zhang, Lattice Boltzmann simulation of snow crystal growth. Water Resour. Res., 2007.

DePaolo, D.J., and G. Lu, Kinetic isotope effects in snow crystal growth: Lattice Boltzmann approach. J. Geophys. Res., 2007.

\section{ACKNOWLEDGMENTS}

This work was supported in part by the Laboratory Directed Research and Development (LDRD) from Berkeley Lab, provided by the Director, Office of Science, of the U.S. Department of Energy under Contract No. DE-AC03-76SF0098. We would like to thank Qinjun Kang (Los Alamos National Laboratory) and Dongxiao Zhang (University of Southern California) for their support. 


\title{
DEVELOPMENT OF JOINT HYDROLOGICAL-GEOPHYSICAL DATA INVERSION METHODOLOGY
}

\author{
Stefan Finsterle and Michael B. Kowalsky \\ Contact: Stefan Finsterle, 510/486-5205, safinsterle@lbl.gov
}

\section{RESEARCH OBJECTIVES}

The ultimate goal of most site characterization efforts is to understand and describe the main subsurface features in sufficient detail, so that a reliable prediction of fluid movement, contaminant transport, and biogeochemical reactions can be made. The challenge of characterizing the subsurface at a specific site lies in the fact that the soil structure is unknown and exhibits both random and nonrandom features-i.e., it cannot be adequately described using purely stochastic or purely deterministic approaches. In addition to identifying the geometry of the stratal soil architecture, hydrologic and geochemical parameters need to be assigned. The specific objective of this research is to develop a data analysis method that allows one to determine both the soil structure and related hydrologic parameters with adequate resolution for a given site.

\section{APPROACH}

The basic concept is to perform a joint analysis of geophysical data, which provide a high-resolution image of the subsurface, and hydrological data, which contain information about the flow and transport properties of interest. Moreover, geostatistical simulation methods are used to flexibly describe a heterogeneous soil structure with a limited number of parameters. Finally, a petrophysical model is needed to relate, for example, the fluid distribution in the subsurface to the geophysical signal. The parameters of such a petrophysical model are also considered unknown or uncertain, and are thus concurrently estimated. In summary, we simultaneously estimate hydrological, petrophysical, and geostatistical parameters by jointly inverting time-lapse hydrological and geophysical data.

\section{ACCOMPLISHMENTS}

The joint inversion approach as been implemented into the iTOUGH2 code (Finsterle, 2004; http:/ / www-esd.lbl.gov/iTOUGH2) by linking geophysical modeling of ground penetrating radar (GPR) signals and geostatistical simulation to the already available nonlinear parameter estimation methods. An example of a synthetic infiltration experiment is shown in Figure 1, where a highly heterogeneous, anisotropic soil structure and its related properties are determined by the joint inversion of hydrological data (infiltration rates and water content data derived from neutron logging) and geophysical data (arrival times from a crosshole GPR survey).

\section{SIGNIFICANCE OF FINDINGS}

By dynamically adjusting the soil structure during the optimization process, the proposed approach significantly improves the model representation of subsurface heterogeneity, thus

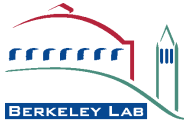
reducing the risk of substantial estimation and prediction errors. Moreover, the joint analysis of geophysical and hydrological data is a means to characterize both the key features and relevant processes that affect subsurface flow and transport. The approach is expected to be valuable for analyzing other geophysical data types, such as electrical resistance tomography (ERT).

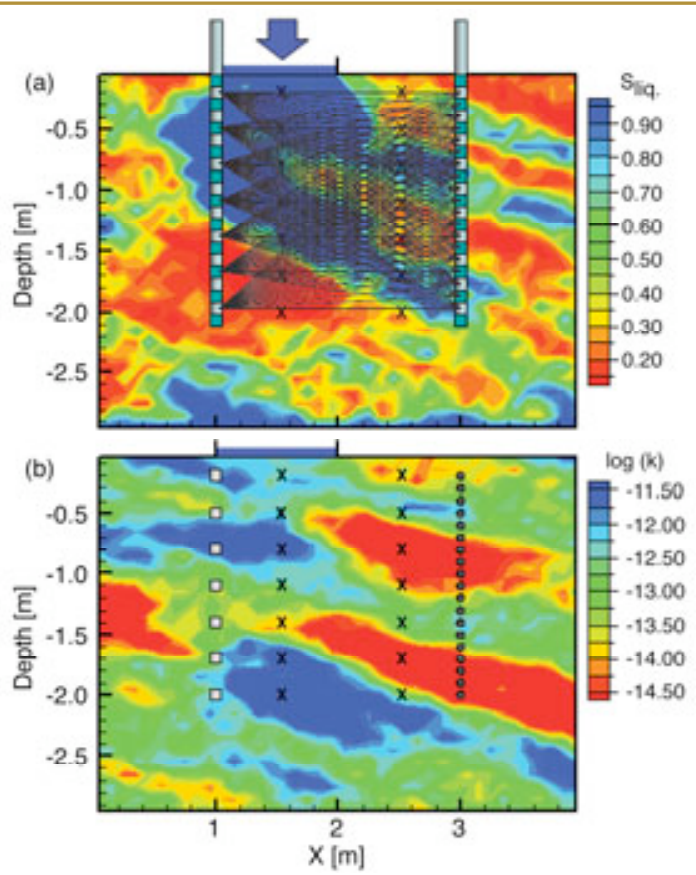

Figure 1. Demonstration of the joint hydrological-geophysical inversion approach for soil structure identification: (a) Liquid saturation distribution after one day of water release, locations of neutron probes in boreholes (squares), and GPR straight-ray paths used for inversion; (b) site-specific permeability field obtained by the joint estimation of geostatistical, hydrological, and petrophysical parameters.

\section{RELATED PUBLICATIONS}

Finsterle, S., Multiphase inverse modeling: Review and iTOUGH2 applications. Vadose Zone J., 3, 747-762, 2004.

Finsterle, S., and M.B. Kowalsky, Joint hydrological-geophysical inversion for soil structure identification. Vadose Zone J. (in press), 2007.

Kowalsky, M., S. Finsterle, J. Peterson, S. Hubbard, Y. Rubin, E. Majer, A. Ward, and G. Gee, Estimation of field-scale soil hydraulic parameters and dielectric parameters through joint inversion of GPR and hydrological data. Water Resour. Res., 41, W11425, doi:10.1029/2005WR004237, 2005.

\section{ACKNOWLEDGMENTS}

This work was supported by Laboratory Directed Research and Development (LDRD) funding from Berkeley Lab, provided by the Director, Office of Science, of the U.S. Department of Energy under Contract No. DE-AC02-05CH11231. 


\section{Effects of Nanoparticle Aggregation on Metal Ion Adsorption \\ B. Gilbert, K.A. Ching ${ }^{1}$, R.K. Ono ${ }^{1}$, C. Lentini ${ }^{1}$, and C. S. Kim ${ }^{1}$ \\ ${ }^{1}$ Chapman University \\ Contact: Benjamin Gilbert, 510/495-2748, bgilbert@lbl.gov}

\section{RESEARCH OBJECTIVES}

Nanoscale oxide and oxyhydroxide minerals are commonly found in the natural environment and play important roles in adsorbing and sequestering aqueous ions, including nutrients such as phosphates and contaminants such as heavy metals. Upon formation, these materials are typically subjected to natural flocculation events that reduce the nanoparticle surface area accessible by aqueous ions. Our goal is to assess the effects of different kinds of aggregation processes on the morphology of nanoparticle aggregates and metal uptake by the mineral surfaces.

\section{APPROACH}

We synthesized a suspension of $\sim 6 \mathrm{~nm}$ iron oxyhydroxide nanoparticles and subjected portions of this suspension to analogues of natural aggregation processes. These included: $\mathrm{pH}$ variation around the point of zero surface charge (simulating the neutralization of acid mine drainage), ionic strength elevation (simulating mixing of aquifer and brackish water), drying, and freezing. Following aggregation, each sample was retuned

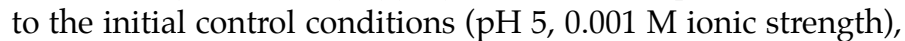
without significant change in aggregation state. The effect of aggregation on metal ion uptake was studied by exposing batches of aggregated and control samples to $0.5 \mathrm{mM}$ $\mathrm{Cu}\left(\mathrm{NO}_{3}\right)_{2}$ at $\mathrm{pH} 6.0$ for 24 hours.

\section{ACCOMPLISHMENTS}

While all aggregation processes caused visual flocculation and settling, there were large quantitative differences in the impact on $\mathrm{Cu}$ uptake. Aggregation via $\mathrm{pH}$ or ionic strength variation caused a modest drop in uptake relative to nonaggregated nanoparticles, while freezing and drying processes resulted in significant reductions in uptake. Small-angle x-ray scattering (SAXS) data provide insight into aggregate morphology and permit the batch uptake experiments to be interpreted. In water, the nanoparticles form open fractal-like networks, characterized by relatively low values of geometric fractal dimension (Df 1.4). By contrast, drying or freezing force the nanoparticles into densely packed clumps of porous material. SAXS-based simulation techniques can generate representative morphologies of the aggregates.

\section{SIGNIFICANCE OF FINDINGS}

These findings illustrate the diverse nanoporous structures that can be generated by natural nanoparticle aggregation processes. In particular, they highlight the important role of water in preserving hydrated channels among aggregated nanoparticles that are permeable to aqueous ions.

\section{RELATED PUBLICATIONS}

Gilbert, B., G. Lu and C.S. Kim, Stable cluster formation in aqueous suspensions of iron oxyhydroxide nanoparticles. J. Colloid Interf. Sci., 313, 152-159, 2007.

\section{ACKNOWLEDGMENTS}

Use of the Stanford Synchrotron Radiation Laboratory (SSRP) is supported by the U.S. Department of Energy, Office of Basic Energy Sciences, under Contract No. DE-AC0205CH11231. Financial support for this work came from the Director, Office of Science, Office of Basic Energy Sciences, of the U.S. Department of Energy under Contract No. DE-AC0205CH11231.

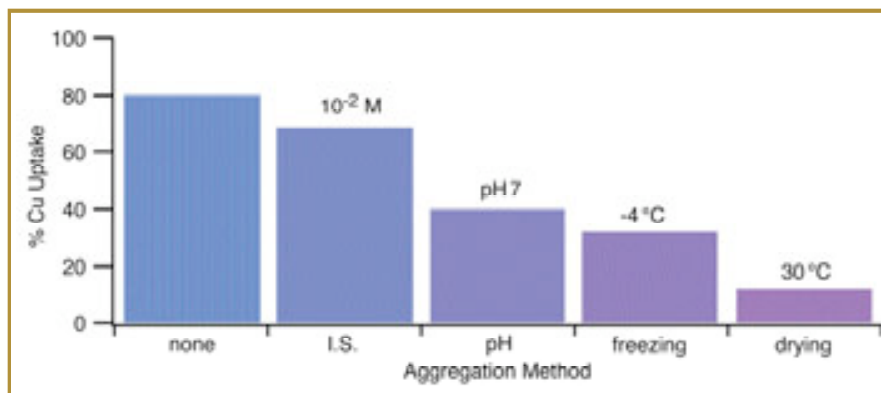

Figure 1. The proportion of $\mathrm{Cu}^{2+}$ (aq.) adsorbed from solution onto $\mathrm{FeOOH}$ nanoparticles that were either fully suspended or aggregated via different mechanisms. I.S. = "ionic strength."

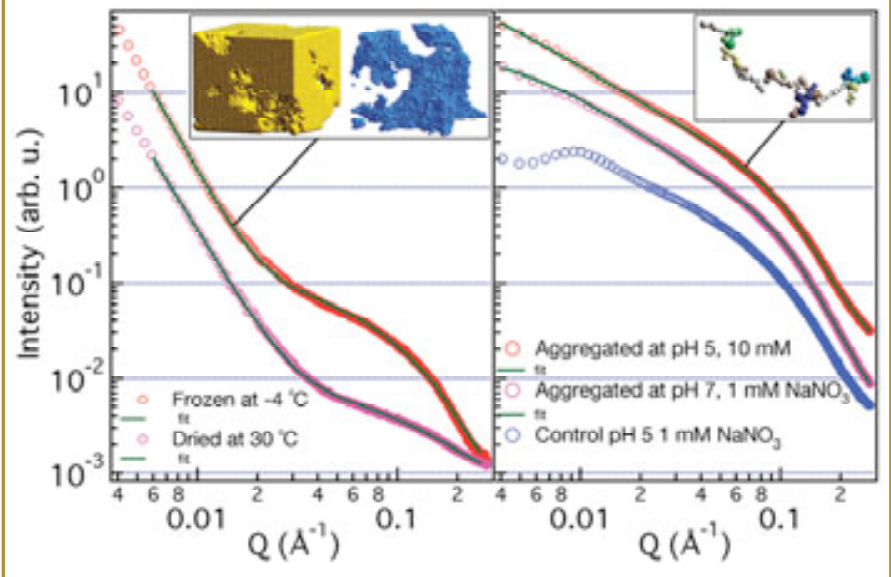

Figure 2. In situ small-angle x-ray scattering (SAXS) analysis of $\mathrm{FeOOH}$ nanoparticle aggregates in suspension (control), or aggregated. Fits to the experimental SAXS patterns permit representative aggregate morphologies to be generated (inset). Left inset: Pore structure of a compact nanoparticle aggregate generated by freezing. Brown $=$ particle structure, blue $=$ pore structure . Right inset: Structure of a fractal aggregate generated by increasing ionic strength. 


\title{
NATURE'S SEMICONDUCTOR'S: Electronic Structure of Biogenic Manganese Oxides
}

\author{
Kideok D. Kwon, Keith Refson¹, and Garrison Sposito \\ ${ }^{1}$ STFC Rutherford Appleton Laboratory, Didcot, Oxfordshire OX11 0QX, UK \\ Contact: Kideok Kwon, 510/643-3172, kkwon@lbl.gov
}

\section{RESEARCH OBJECTIVES}

Many bacteria produce $\mathrm{Mn}(\mathrm{IV})$ oxides $\left(\mathrm{MnO}_{2}\right)$, which are environmental nanoparticles having a layered structure. Biogenic Mn oxides are known to be key participants in both redox reactions and metal-scavenging in soil and aquatic environments. An important structural characteristic of the oxides is the presence of $\mathrm{Mn}(\mathrm{IV})$ cation vacancies, whose charge deficit is typically compensated by metal cations or protons (Villalobos et al., 2006). These vacancies have long been identified as strong adsorption sites for heavy metals, but they may also play an important role in redox biogeochemistry, particularly in photo-induced redox reactions occurring in natural waters. Because electronic structure is the key to understanding (photo) redox transformations, the objective of our research is to theoretically examine the electronic structures of Mn oxides, both with and without $\mathrm{Mn}(\mathrm{IV})$ vacancies.

\section{APPROACH}

Quantum mechanical (QM) calculations have advanced to the point where they can provide highly accurate electronic-structure information about solids without adjustable parameters. In our study, the electronic structures of $\mathrm{Mn}$ oxides were calculated for model $\mathrm{MnO}_{2}$ nanoparticles using a version of CASTEP, one of the best known and most reliable QM codes in solid-state physics. These extensive calculations required the massively parallel supercomputing resources of the National Energy Research Scientific Computing Center (NERSC).

\section{ACCOMPLISHMENTS}

We investigated $\mathrm{MnO}_{2}$ nanosheets containing $\mathrm{Mn}(\mathrm{IV})$ vacancies compensated by protons. Negative charge was found to be localized at the vacancy sites. Our results also demonstrated that increasing the Mn(IV) vacancy concentration effectively reduced the band-gap energy between the occupied valence band and the unoccupied conduction band of electrons in the Mn oxides (Figure 1). Thus, a $\mathrm{Mn}(\mathrm{IV})$ vacancy can facilitate photo-induced electron transfer

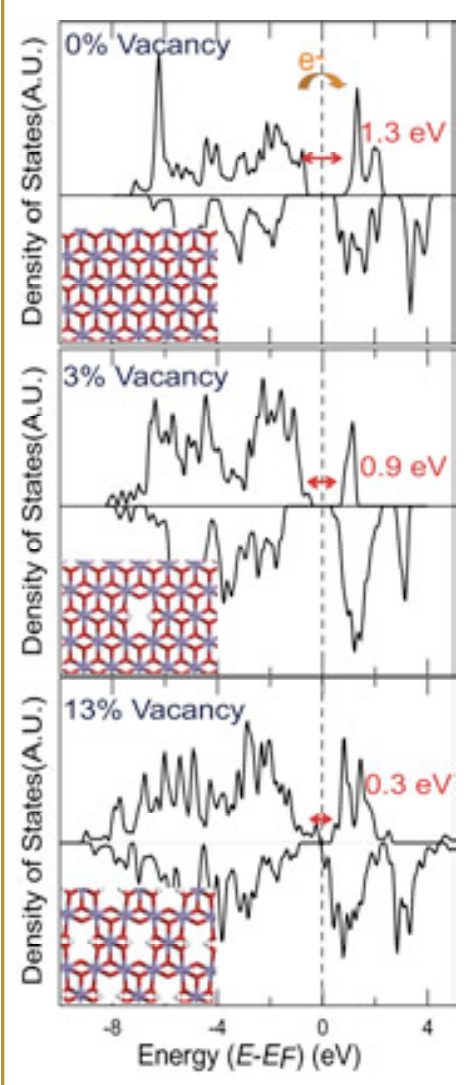

Figure 1 "Density of states" plots calculated for nanoparticle $\mathrm{MnO}$ 2 showing reduction of the band gap energy (denoted by a double arrow) resulting from an increase in $\mathrm{Mn}(\mathrm{IV})$ vacancy concentration. The inset figures are $a-b$ plane views of model Mn oxide structu res whose negatively-charged vacancies are compensated by protons (so-called Ruetschi defects). (redox reactions) between the oxide mineral and inorganic or organic compounds.

\section{SIGNIFICANCE OF FINDINGS}

Biogenic $\mathrm{MnO}_{2}$ nanoparticles can serve as design templates for new materials. Recent research in materials science indicates that synthetic Mn oxide nanoparticles with layer structures and $\mathrm{Mn}(\mathrm{IV})$ vacancies like those in biogenic $\mathrm{MnO}_{2}$ are semiconductors that produce photocurrents under visible light stimulation, thus making them attractive for applications in energy storage, solar cell fabrication, and catalysis. Understanding their electronic structure is essential to developing their full potential, and our $\mathrm{QM}$ results strongly imply that nanoparticle $\mathrm{MnO}_{2}$ with $\mathrm{Mn}(\mathrm{IV})$ vacancies offers an effective new material for generating and transferring photo-induced electrons and holes. More specifically, the finding of substantial band-gap energy reduction by a vacancy suggests that photocurrent production by layer type $\mathrm{MnO}_{2}$ can be optimized by the control of vacancy rates during synthesis.

\section{RELATED PUBLICATIONS}

Villalobos, M., B. Lanson, A. Manceau, B. Toner, and G. Sposito, Structural model for the biogenic Mn oxide produced by Pseudomonas putida. American Mineralogist, 91, 489-502, 2006.

Kwon, K.D., K. Refson, and G. Sposito, Ruetschi defects decrease the band gap energy in $\mathrm{MnO}_{2}$ nanosheets. Journal of American Chemical Society (in press), 2007.

\section{ACKNOWLEDGMENTS}

This research was supported by the Director, Office of Science, Office of Basic Energy Sciences, Division of Chemical Sciences, Geosciences, and Biosciences, of the U.S. Department of Energy under Contract No. DE-AC02-05CH11231. Our computations used resources of the National Energy Research Scientific Computing Center (NERSC) at Berkeley Lab, which is also supported by DOE Contract No. DE-AC02-05CH11231. 


\section{$\mathrm{CO}_{2}-\mathrm{H}_{2} \mathrm{O}$ Multiple Phase and Component Simulation: A LatTice BoltzManN Approach}

Guoping Lu and Donald J. DePaolo

Contact: Guoping Lu, 510/495-2359, gplu@lbl.gov

\section{RESEARCH OBJECTIVES}

Global warming from burning of fossil fuels has generated an increasing interest in the study of carbon dioxide's geological sequestration. Sequestration of $\mathrm{CO}_{2}$ into deep aquifers has been identified as a suitable route for mitigating $\mathrm{CO}_{2}$ released to the atmosphere. However, the permanency of such storage is a concern. When compressed $\mathrm{CO}_{2}$ is injected into aquifers, it is not in chemical equilibrium with the subsurface environment; it is buoyant, and therefore has the potential to migrate back to the surface through fissures in formation cap rock or along well bores. Compressed carbon dioxide-a nonwetting fluid of limited solubility in water-evolves as it interacts with water and with solid matrix in the subsurface. The objective of this study is to investigate the interaction between $\mathrm{CO}_{2}$ and water.

\section{APPROACH}

We used 2-D and 3-D lattice-Boltzmann (LB) models to simulate dissolution of $\mathrm{CO}_{2}$ in water. The two-dimensional, ninespeed model of Kang et al. (2002) was modified to accommodate multiple components and specific boundary conditions. In the model, the phase transition and solid interactions in porous media were accommodated through interfacial tensions.

\section{ACCOMPLISHMENTS}

We have successfully simulated the mutual dissolution of $\mathrm{CO}_{2}$ in water (Figure 1) under different supercritical conditions-with interfacial tension dropping under increased pressures. The model was calibrated with laboratory-measured interfacial tension data from the relevant literature. Results show that both $\mathrm{CO}_{2}$ and water might exist as isolated phases trapped within pores.

\section{SIGNIFICANCE OF FINDINGS}

LB simulation of $\mathrm{CO}_{2}-\mathrm{H}_{2} \mathrm{O}$-solid interfacing is a new approach for the study of $\mathrm{CO}_{2}$ behavior in geological sequestration. Our microscale simulation provides insight into $\mathrm{CO}_{2}$ behavior, which can be validated by micro-imaging of the $\mathrm{CO}_{2}-$ water-rock system. The multiphase flow problem in porous media is applicable to a wide range of practical problems, including heterogeneity and biopolymer surfactant issues in oil recovery, and the transport of nonaqueous-phase liquid contamination. The LB model can also be extended to study isotopic fractionation associated with storage of $\mathrm{CO}_{2}$ in aquifers.

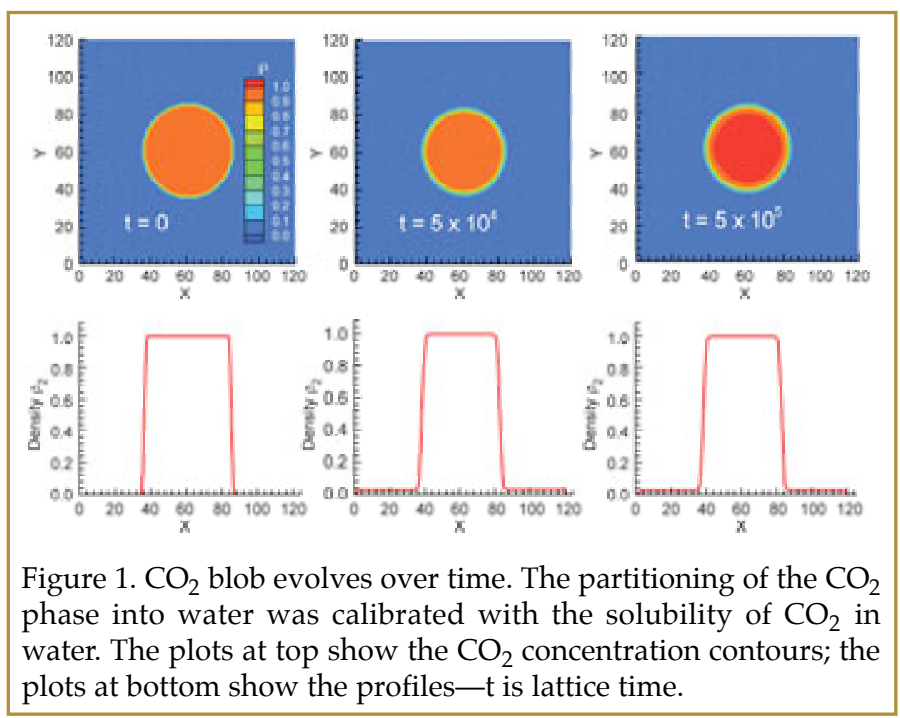

\section{RELATED PUBLICATION}

Kang et al., Displacement of a 2-D immiscible droplet. Physics of Fluids, 14, 2002.

Lu, G., D.J. DePaolo, Q. Kang, and D. Zhang, Lattice-Boltzmann simulation of $\mathrm{CO}_{2}$-water interaction in porous media. Water Resources Research (submitted), 2007.

\section{ACKNOWLEDGMENTS}

This work was supported by in part by Laboratory Directed Research and Development (LDRD) funds from Berkeley Lab, provided by the Director, Office of Science, of the U.S. Department of Energy under Contract No. DE-AC0205CH11231. We would like to thank Qinjun Kang (Los Alamos National Laboratory) and Dongxiao Zhang (University of Southern California) for their support of this work. 


\section{Shaped-Core Uniaxial CoMpression Test For STUdying BOREHOLE BREAKOUT IN ROCK \\ Seiji Nakagawa \\ Contact: 510/486-7894, snakagawa@lbl.gov}

\section{RESEARCH OBJECTIVES}

Borehole breakout in rock occurs as a result of stress concentration on a borehole wall subjected to far-field stresses. If the far-field stress is anisotropic, the highest stress concentration results in the directions corresponding to the minimum principal stress within a plane perpendicular to the borehole axis. To examine the effect of stress concentration on the formation of a borehole breakout, a "hollow-cylinder" test is conducted routinely, which applies an axisymmetric confining stress and an axial stress on a cylindrical rock sample containing a single hole along its axis. For more general, anisotropic stress states, a cube-shaped rock sample containing an analogue borehole is subjected to polyaxial stresses in the laboratory. The objective of this research is to develop an even simpler testing method for studying borehole breakout-as simple as a uniaxial compression test.

\section{APPROACH}

The shaped-core uniaxial compression (SCUC) test developed in this research potentially provides a very simple means by which to examine borehole breakout in rock. This method employs a cylindrical rock core with a narrowed cross section at the center (i.e., a "shaped" core), which gives the core an appearance of an

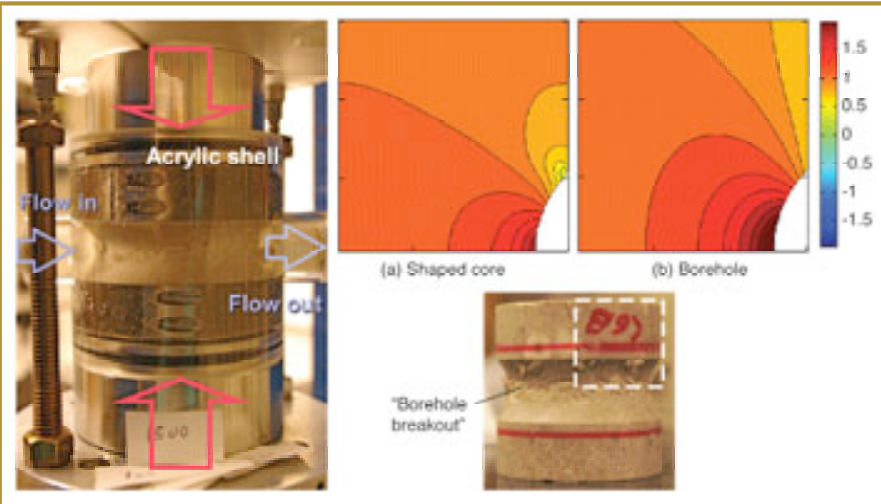

Figure 1. A shaped-core uniaxial compression test. An hourglassshaped rock core is compressed to produce a stress concentration on the core wall, resulting in a local failure analogous to a borehole breakout. Vertical compressional stresses are compared in the plots (Normalized for the axial stress (a) and far-field vertical stress (b). The ratio between the far-field vertical and horizontal stresses in (b) is 2:1.). hourglass or dogbone (Figure 1). The same sample geometry has been used to test rock and metal samples for their tensile and extensile strengths. When a shaped core is subjected to axial compression, the curved surface results in a stress concentration at the core wall, analogous to a real borehole subjected to far-field stresses. Laboratory experiments and numerical simulations using finite element models were conducted for a range of core geometry and material properties to examine the resulting stress distribution and borehole breakout.

\section{ACCOMPLISHMENTS}

SCUC tests were conduced on a variety of small rock samples (diameter $=5.08 \mathrm{~cm}$, height $=5.08 \mathrm{~cm}$ ), ranging from very friable, weakly cemented sand to extremely strong carbonate rock. This method was also used extensively to examine how fluid flow around a borehole affects sand production from a borehole breakout. Initial experiments and elastic stress analyses indicated that to model the behavior of a borehole adequately, a formation of tensile zone within a shaped core must be avoided by applying constraints at the top and bottom of a core.

\section{SIGNIFICANCE OF FINDINGS}

Unique advantages of the method were identified through the development of the SCUC test. These are (1) only a small sample is needed for the test, (2) both very weak and very strong samples can be tested easily, (3) an initiation and development of a borehole breakout can be visually and continuously observed, and (4) since no confining cell is needed, fluid can be circulated around the breakout within a transparent shell, to study sand production. However, it was also found that the stress concentration within a sample varies as a function of the core geometry and material properties.

\section{ACKNOWLEDGMENTS}

This work was supported by the Assistant Secretary for Fossil Energy, Office of Oil and Natural Gas, National Energy Technology Laboratory, and by Laboratory Directed Research and Development (LDRD) funding from Berkeley Lab, provided by the Director, Office of Science, Office of Basic Energy Sciences, of the U.S. Department of Energy under Contract No. DE-AC02-05CH11231. 


\title{
Physicochemical Controls on Unsaturated Flow and Transport in Geological Media
}

\author{
Tetsu K. Tokunaga, Jiamin Wan, Steve Sutton ${ }^{1}$, Matt Newville ${ }^{1}$, and Antonio Lanzirotti ${ }^{1}$ \\ ${ }^{1}$ University of Chicago \\ Contact: Tetsu K. Tokunaga, 510/486-7176, tktokunaga@lbl.gov
}

\section{RESEARCH OBJECTIVES}

The flow of water and transport of chemicals through soils and fractured rocks is strongly dependent on the water content (saturation), with very low flow and transport rates occurring at low saturation levels. Under conditions in which both air and water occupy pores (fractures), water films largely control macroscopic flow and chemical transport. However, some basic properties of water films in unsaturated media remain poorly understood. The environmentally important characteristics being investigated in this study are the dependence of water film thickness on capillary (matric) potential and water film's possible dependence on major ion chemistry.

\section{APPROACH}

Capillary potentials on water films coating mineral surfaces (smooth as well as rough) are controlled by equilibration with aqueous salt solutions, under regulated subatmospheric pressures in a small suction plate chamber. The chamber is placed in front of an x-ray beam (National Synchrotron Light Source X26A, and Advanced Photon Source 13-IDC) used to determine film thicknesses through $x$-ray fluorescence of monovalent $(\mathrm{Rb}+$ and $\mathrm{Br}-)$ and divalent $\left(\mathrm{Ca}^{2+}\right.$ and $\left.\mathrm{SeO}_{4}{ }^{2-}\right)$ ions in the water films. These hard $x$-ray synchrotron sources are necessary for efficient $x$-ray fluorescence monitoring of ionic tracers in water films within sealed environmental control chambers. The measurements on flat mineral surfaces provide information on limiting conditions for unsaturated flow and transport, and also test various equilibrium models for adsorbed water film thickness. The experiments on microscopically rough surfaces, including natural fracture surfaces, test the capillary film flow models.

\section{ACCOMPLISHMENTS}

Measurements of average film thicknesses and film transmissivities have been completed on several natural rough fracture surfaces. The most recent experiments have focused on basic measurements on flat quartz glass surfaces at very-near-zero matric potentials. We have successfully measured water films as thin as $90 \mathrm{~nm}$ using this method, and our calibration tests indicate that resolution of about $20 \mathrm{~nm}$ is achievable. The measured film thickness-potential trends (Figure 1) are qualitatively in agreement with an early model derived by Irving Langmuir, but the data are indicative of generally thicker films. A predicted ion-valence film-thickness dependence is being tested through comparisons of systems containing $\mathrm{RbBr}$ versus $\mathrm{CaSeO}_{4}$ and $\mathrm{MgSeO}_{4}$ solutions.

\section{SIGNIFICANCE OF FINDINGS}

Water films in the range of tens to hundreds of nanometer thickness are common in unsaturated soils and rocks, yet have previously been inaccessible to direct measurements. This new approach is allowing direct quantification of unsaturated transport properties within water films under environmentally relevant conditions, including physicochemical influences on unsaturated hydraulic properties.

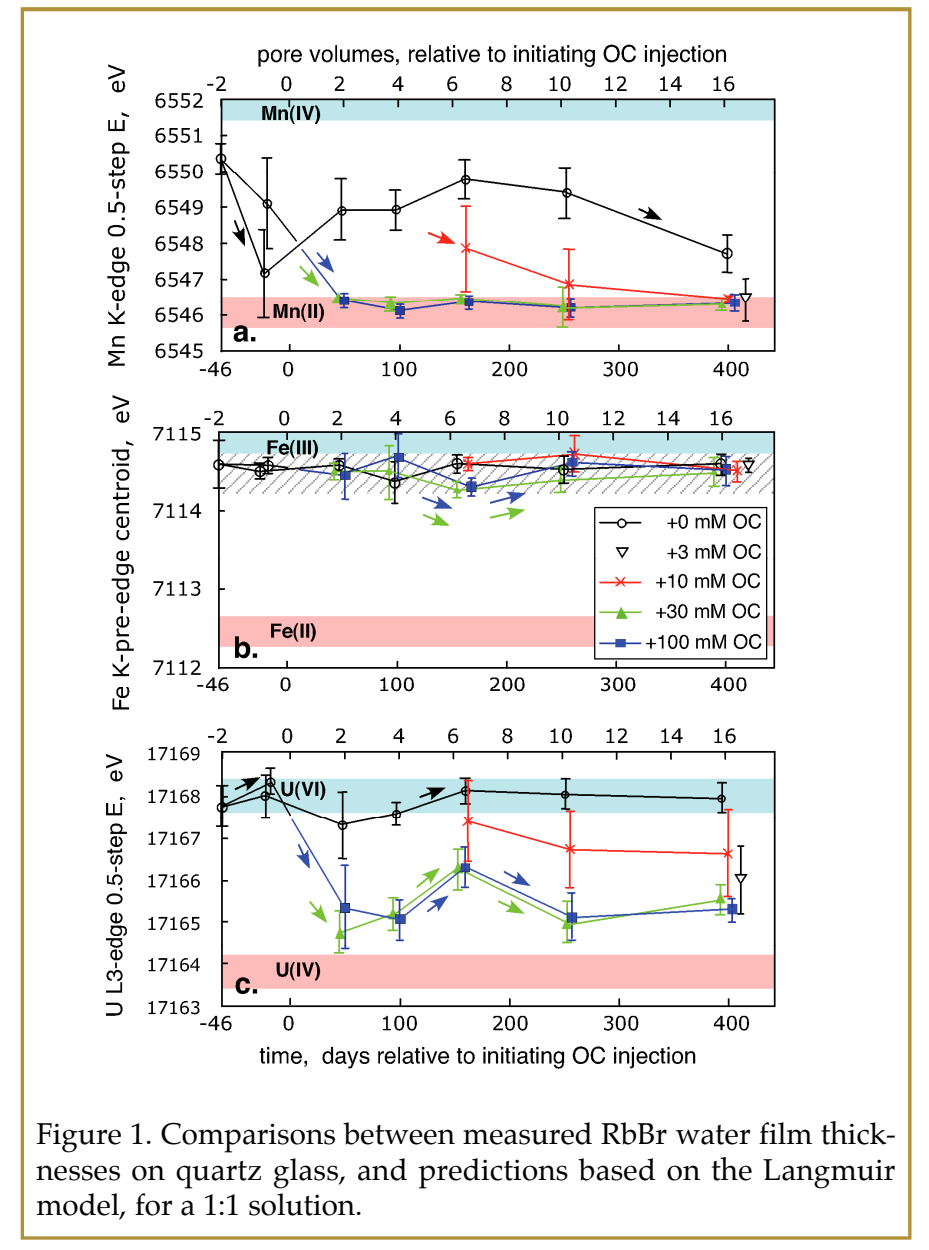

\section{RELATED PUBLICATION}

Tokunaga, T.K., K.R. Olson, and J. Wan, Infiltration flux distributions in unsaturated rock deposits and their potential implications for fractured rock formations. LBNL-57399. Geophys. Res. Lett., 32, L05405, doi:10.1029/2004GL022203., 2005.

\section{ACKNOWLEDGMENTS}

This project is supported by the Director, Office of Science, Office of Basic Energy Sciences, Division of Chemical Sciences, Geosciences, and Biosciences, of the U. S. Department of Energy under Contract No. DE-AC02-05CH11231. Use of the Advanced Photon Source and National Synchrotron Light Soure was also provided by DOE under Contract No. DE-AC02-05CH11231.

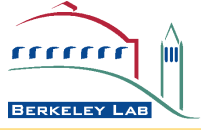




\section{Molecular-Level Studies of Mineral-Water Interface Structure and Chemistry}

Glenn Waychunas, Young-Shin Jun, Chuanshan Tian, Y. Ron Shen, Peter Eng, and Sanjit Ghose

Contact: Glenn Waychunas, 510/495-2224, gawaychunas@lbl.gov

\section{RESEARCH OBJECTIVES}

We wish to define the specific molecular geometries of mineral surfaces equilibrated with aqueous solutions, including the nature of surface relaxations or reorganizations, the attachment sites and type of binding of sorbates, and the structure of interfacial water. This type of information is important for the testing of molecular reaction models and simulation techniques that model the sorption/desorption and incorporation of migrant species in natural fluids, and hence determine the fate and transport of both toxic and nutrient species in the environment.

\section{APPROACH}

Our studies are conducted using a combination of molecular probes, largely synchrotron-based, with relatively simplified model systems. The chief synchrotron methods are: surface diffraction, by which we can refine the position and occupation of surface atoms at an interface; surface x-ray spectroscopy, by which we can obtain specific structural information about the local molecular environment of a sorbed molecule at an interface; and nonlinear optical spectroscopy, whereby we can probe the infrared vibrational spectrum of water molecules only at the solid-aqueous solution interface. Variations on these techniques also yield specific information such as the redox state of surface atoms, or the lifetimes of particular surface species.

\section{ACCOMPLISHMENTS}

Our most recent work focuses on the hematite, goethite, diaspore and corundum surfaces. For hematite, we are interested in the surface produced by acids or bases, as well as the geometry of sorbed species like arsenate or silicate. Results have shown that the geometry of monomeric silicate sorption is as a bidentate complex with strong surface attachment. The topology is such that silicate polymerization on the hematite surface (we investigated the R-plane) is likely, thus rendering the surface passivated to further reaction. For the goethite surface we were able to collect x-ray surface scattering data from submillimeter cleaved crystal fragments, and refine the surface structure for the first time. The surface is relaxed from the bulk, but also has two distinct layers of ordered water at the termination. Analogous surface refinements are in progress for the aluminum-analog of goethite-diaspore. Our surface water studies show that the $\mathrm{pH}$ point of zero charge (PZC) for clean corundum (0001) faces is ca. 5.5, rather than the ca. 9.0 measured for corundum powders. This points to a basic misunderstanding of the aluminate-water surface interaction, which we are addressing with further studies of the corundumwater surface structure as a function of $\mathrm{pH}$, as well as with MD simulations.

\section{SIGNIFICANCE OF FINDINGS}

The silicate/hematite work is vital to an understanding of how the reactivity of natural Fe oxide surfaces changes in varied environments, and whether effective remediation approaches for particular pollutants are efficacious. The goethite and diaspore work are crucial to the application of surface complexation modeling with accurate molecular parametrization. For example, current models assume bulkgoethite-like structure for the interface, which we show is not accurate. The PZC work shows fundamental problems with our solid-aqueous interface modeling assumptions, even in simple systems, but does yield new information by which we may be able to improve future molecular models.

\section{RELATED PUBLICATION}

Waychunas, G.A., Y.-S. Jun, P. J. Eng, S. Ghose, and T. P. Trainor, Anion sorption topology on hematite: Comparison of arsenate and silicate. LBNL-61150. Geomaterials II (chapter) (in press), 2007.

\section{ACKNOWLEDGMENTS}

This work was supported by the Director, Office of Science, Office of Basic Energy Sciences, Division of Chemical Sciences, Geosciences, and Biosciences, of the U.S. Department of Energy under Contract No. DE-AC02-05CH11231. 


\section{Nanoparticle Reactivity, Structure, and Growth Studies}

Glenn Waychunas, Christopher Kim, Christopher Lentini, Ben Gilbert, Hengzhang Zhong, and Jillian Banfield

Contact: Glenn Waychunas, 510/495-2224, gawaychunas@lbl.gov

\section{RESEARCH OBJECTIVES}

Our past work has shown that nanoparticles differ in structure and strain, depending on their precise chemical environment. It is also anticipated that structure, strain, and reactivity may change as a function of size. Our investigations aim to characterize possible differences in sorption mechanisms as a function of nanoparticle size, growth rate, and degree of aggregation.

\section{APPROACH}

Our studies are done using a combination of molecular probes, bulk measurements, and thermodynamic measurements. Some of the analysis is done in concert with molecular dynamic simulations. For nanoparticle size and shape, we employ small angle $x$-ray scattering (SAXS) and dynamic light scattering (DLS), while the specific molecular nature of surface species is determined with extended x-ray absorption fine structure spectroscopy (EXAFS). Other aspects of nanoparticle structure are determined via determination of pair correlation functions with high-energy wide angle $x$-ray scattering (WAXS). The x-ray measurements are done at the APS (Advanced Photon Source) and SSRL (Stanford Synchrotron Radiation Laboratory) synchrotron sources.

\section{ACCOMPLISHMENTS}

Recent work shows that nanoparticle uptake of many types of sorbants is very high, initially, reflecting the large surface-tovolume ratio. In general, the numbers of active surface sites that sorb aqueous species do not seem to markedly increase in nanoparticles relative to larger particles, and in some cases are observed to decrease. This may result from a high degree of surface strain, manifested by surface site distortion, and thus somewhat disfavored geometries for strong covalent binding of sorbates. Growth of nanoparticles is also affected by sorption, with effects proportional to the strength of the binding and hence the degree to which nanoparticles can aggregate or grow by Ostwald ripening. Nanoparticle growth mechanisms in iron oxyhydroxides appear to occur by both oriented attachment $(\mathrm{OA})$ and ripening mechanisms.

\section{SIGNIFICANCE OF FINDINGS}

An important mechanism for the uptake of natural pollutants by nanoparticles is incorporation by growing surfaces or via aggregation mechanisms. We have shown that growth is highly sensitive to sorption of particular species, and hence that the fate and transport of environmental toxics is related in a complex manner to the size distribution of nanoparticles and the precise chemistry of sorbing species. For example, nanoparticles at smallest sizes may transport sorbed species for long distances without aggregation or growth if sorption densities are high. However the same particles would tend to aggregate or continue growth at low sorption densities, thus encapsulating the pollutants but with very limited transport.
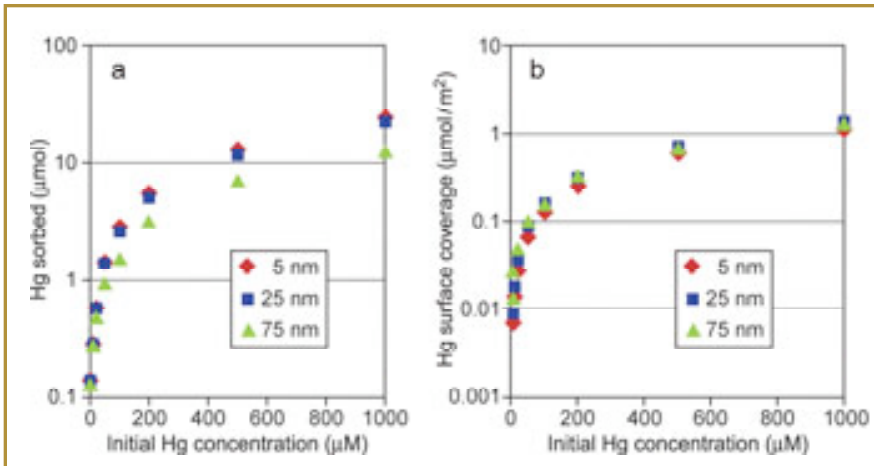

Figure 1. Uptake of solution $\mathrm{Hg}$ (II) by goethite nanoparticles of varying size: a) scaled via gross uptake reflecting surface area effect; b) scaled by surface area showing little effect of size over the investigated range.

\section{RELATED PUBLICATIONS}

Kim, C.S., C.J. Lentini, and G.A. Waychunas, Associations between iron oxyhydroxide nanoparticle growth and metal adsorption/structural incorporation. In: Geomaterials II, D. Kent and M. Barnett, eds., Elsevier (in press), 2007.

Waychunas, G.A., C. Kim, and J.F. Banfield, Nanoparticulate iron oxide minerals in soils and sediments: Unique properties and contaminant scavenging mechanisms. J. Nanoparticulate Res., 7, 409-433, 2005.

\section{ACKNOWLEDGEMENTS}

This work was supported by the Director, Office of Science, Office of Basic Energy Sciences, Division of Chemical Sciences, Geosciences, and Biosciences, of the U.S. Department of Energy under Contract No. DE-AC02-05CH11231. 


\title{
Incorporation of Aqueous Reaction Kinetics and Biodegradation into TOUGHREACT
}

\author{
Tianfu Xu and Curtis M. Oldenburg \\ Contact: Tianfu Xu, 510/486-7057, Tianfu_Xu@lbl.gov
}

\section{RESEARCH OBJECTIVES}

The need to consider aqueous and sorption reaction kinetics and microbiological processes arises in many subsurface problems. By adding these process-modeling capabilities to the TOUGH family of codes, complex problems can be addressed that involve multiphase fluid and heat flow, and biologically mediated geochemical interactions.

\section{APPROACH}

A general formulation for kinetic rates among chemical and microbial species has been incorporated into the reactive geochemical transport simulator TOUGHREACT. The space discretization used is based on a flexible integral finite difference approach that uses irregular gridding to model small-scale biogeologic regions and structures. A general multiregion model for hydrological transport coupled with microbiological and geochemical processes is used (Figure 1).

\section{ACCOMPLISHMENTS}

A 1-D reactive transport problem with kinetic biodegradation and sorption was used to test the enhanced simulator, which involves the processes that occur when a pulse of water containing NTA (nitrylotriacetate) and cobalt is injected into a column. The current simulation results agree very well with those obtained with other simulators. The applicability of this general multiregion model was validated by results from a published column experiment of denitrification and sulfate reduction. The matches with measured nitrate and sulfate concentrations were adjusted with the interfacial area between mobile and immobile regions (Figure 1). Results suggest that TOUGHREACT not only can be a useful interpretative tool for biogeochemical experiments, but also can produce insight into processes and parameters of coupled advective and diffusive transport, and their interplay with biogeochemical reactions.

\section{SIGNIFICANCE OF FINDINGS}

The resulting biogeochemical-transport-simulation capabilities may be useful for both deep and shallow subsurface problems, including acidic mine drainage remediation, biologically enhanced $\mathrm{CO}_{2}$ sequestration, microbially enhanced oil recovery, and environmental remediation of groundwater contamination. This new capability is finding immediate application to studies of nitrogen cycling in the shallow subsurface and its coupling to hydrologic and climatologic forcings through a joint Earth Sciences Division and Berkeley Water Center project.

\section{RELATED PUBLICATIONS}

$\mathrm{Xu}, \mathrm{T}$., Incorporation of aqueous reaction kinetics and biodegradation into TOUGHREACT: Application of a multi-region model to hydrobiogeochemical transport of denitrification and sulfate reduction. Vadose Zone Journal (in press), 2007.

Xu, T., E.L. Sonnenthal, N. Spycher, and K. Pruess, TOUGHREACT-A simulation program for non-isothermal multiphase reactive geochemical transport in variably saturated geologic media: Applications to geothermal injectivity and $\mathrm{CO}_{2}$ geological sequestration. Computers \& Geoscience, 32, 145-165. 2006.

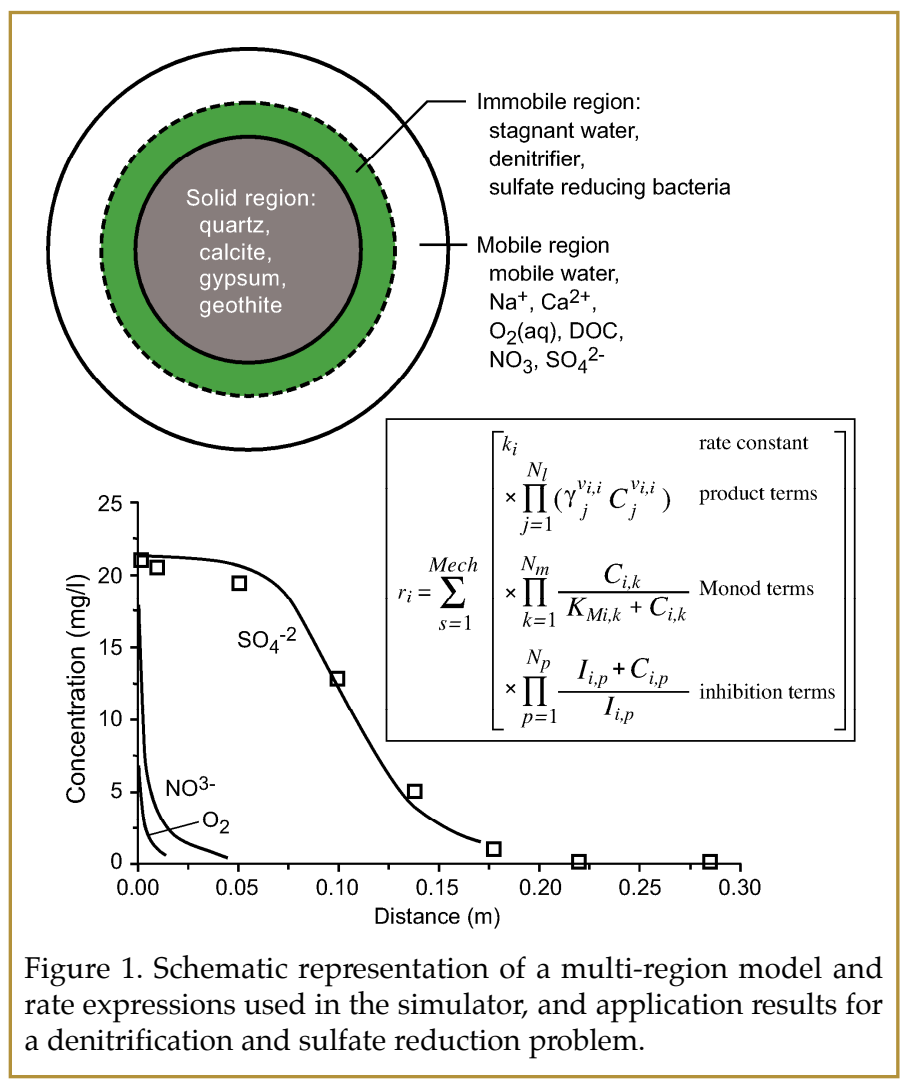

\section{ACKNOWLEDGMENTS}

This work was supported by Laboratory Directed Research and Development (LDRD) funding from Berkeley Lab, provided by the Director, Office of Science, Office of Basic Energy Science, of the U.S. Department of Energy under Contract No. DE-AC02-05CH11231. 


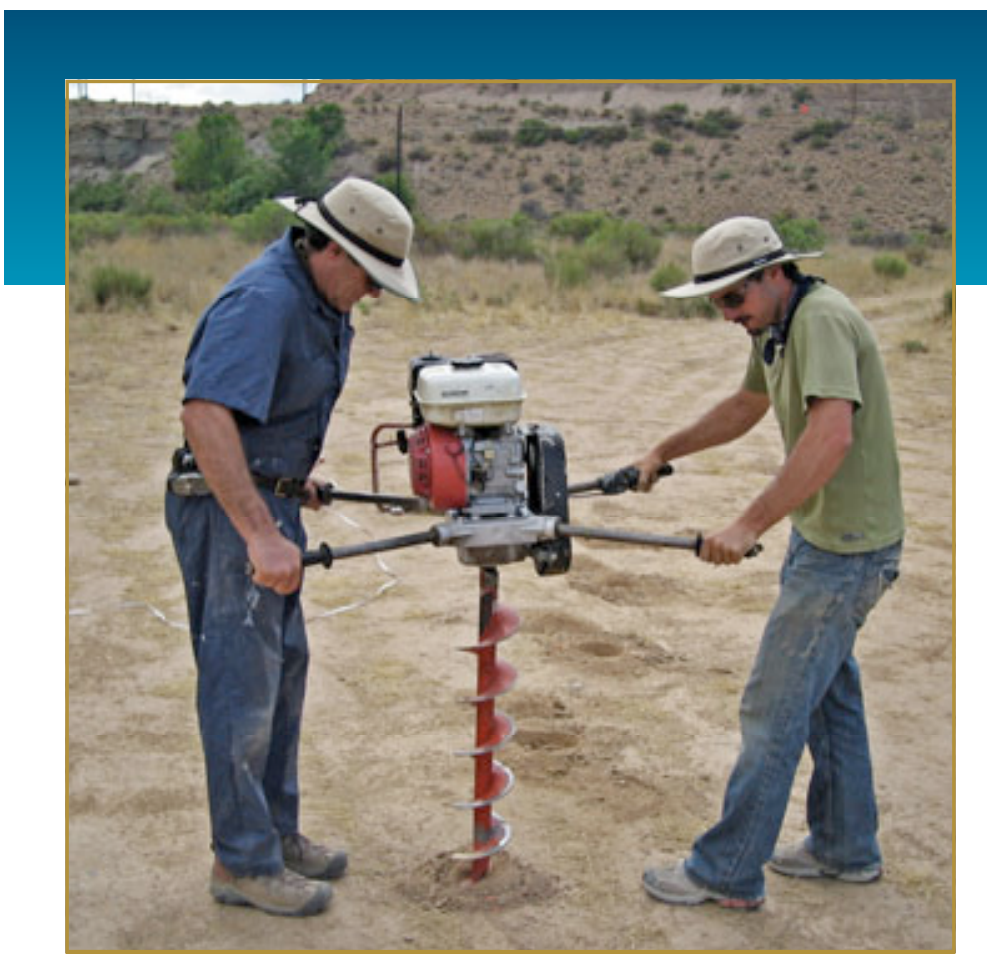

The key driver for the Environmental Remediation and Water Resources Program (ERWR) within ESD is to provide the scientific foundation needed to guide the management of our shallow subsurface contaminants and resources. Over the last decade, it has become increasingly clear that if we are to face the environmental and water challenges of the future, we must view the earth as a complex system, one that includes many components that are coupled and highly dynamic over various spatial and temporal scales.

The DOE is responsible for the environmental health of over 140 waste sites across the United States, created from the production and testing of nuclear weapons. Metals and many radionuclides pose particularly daunting remediation challenges for DOE at these sites because, unlike organic contaminants, they do not irreversibly degrade to benign products (or only do so through very slow radioactive decay). Because understanding the complexity of the subsurface systems is a prerequisite to successful stewardship at these waste sites, a significant fraction of the research within the ERWR program focuses on investigating, measuring, and predicting coupled physical, geochemical, and microbiological processes that govern contaminant fate and transport and that impact remediation efficacy.

The inability to accurately measure and predict water cycle phenomena hinders our ability to effectively inform water resources management and policy. Because adequate and clean water resources are particularly important for the vitality of water-stressed regions such as California, much of the waterresources research performed in the ERWR focuses on development of techniques and insights that will lead to better management of California water resources. In addition to State
Research Program

ENVIRONMENTAL REMEDIATION AND WATER RESOURCES Program

Susan Hubbard

510-486-5266

sshubbard@lbl.gov

water issues, the DOE is responsible for promoting America's energy security, while at the same time protecting the environment-this necessitates water-related research. For example, as worries over the world energy crisis and climate change intensify, the link between such issues and water is becoming ever more apparent and is leading to various challenges. The energy demand for generating and distributing clean water is tremendous; so too is the water demand for generating conventional and sustainable energy and biofuels. Although water issues are currently only a small fraction of the ERWR research portfolio, we anticipate more emphasis on research in this area, as both energy and water needs continue to increase.

Brief descriptions of research advances within the Environmental Remediation and Water Resources Program are given below.

\section{ENVIRONMENTAL REMEDIATION}

ESD scientists participating in the ERWR conduct multidisciplinary environmental research using theoretical, characterization, modeling, and experimental approaches that range from the molecular to the field scale. The synergy offered by the ensemble of competencies within the ESD facilitates investigation of complex natural systems. Many of the projects within the ERWR are associated with one of the following five themes:

- Development of advanced approaches for characterizing and predicting subsurface biological, hydrological, and geochemical properties and processes at the molecular to field scales

- Improved understanding of mechanisms and rates associated with complex subsurface system processes

- Improved understanding of the impact of subsurface system complexity on contaminant distribution and remediation efficacy

- Development of a basis for in situ remediation, especially for metals and radionuclides that respond to redox manipulation and microbial community stimulation.

- Development of remote approaches for detecting and discriminating unexploded ordnance (UXOs)

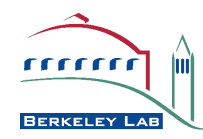


ESD scientists within the ERWR program have recently developed novel approaches that can be used to characterize microbial properties and processes, and to monitor how they change in response to environmental perturbations. ERWR scientists have combined phylogenetic and functional gene arrays to identify mechanisms of chromium immobilization via biostimulation, and have demonstrated the impact of electron-donor supply on microbial community development. ERWR researchers have developed techniques for large-scale extremophile production needed for protein complex analysis. They have developed a community database and web application that permits exploration of microbial community structure relationships to disease states and environmental factors. Many of these studies were performed in collaboration with the Virtual Institute of Microbial Stress and Survival (VIMSS), which is based at Berkeley Lab and which seeks to identify stressresponse pathways of microbes important for environmental remediation.

Through development of novel synchrotron and isotopic methods, ERWR scientists have quantified mechanisms and rates associated with contaminant transport and cleanup processes. Grazing incidence small angle x-ray scattering approaches have been used to elucidate the mechanism and kinetics of early environmental nanoparticle growth; synchrotron Fourier transform infrared methods have been used to explore iron biomineralization processes. Carbon isotopic compositions of dissolved methane and inorganic carbon were used to indicate the biodegradation potential in regions downgradient from a trichlorethene plume at the Idaho National Laboratory. ERWR researchers have used time-lapse sulfur isotopes to elucidate the timing and mechanism of sulfate reduction that occurred during bioremediation of a uranium-contaminated aquifer, and have used isotopic signatures to infer the origin of 99Tc in Hanford groundwater.

ERWR researchers have advanced the use of geophysical approaches for characterizing subsurface hydrogeological properties and for monitoring biogeochemical processes associated with remediation efforts. Spectral induced polarization and spontaneous potential methods were used to track the production of iron sulfides and the subsurface regions of active metabolism, respectively, during a local field-scale uranium biostimulation experiment. A stochastic framework was developed to permit the quantitative estimation of biogeochemical end-products using time-lapse geophysical datasets and petrophysical relationships. Development of analytical and numerical inversion approaches for interpreting complex, time-lapse conservative tracer breakthrough datasets both highlighted how biogeochemical products associated with remedial treatments can alter flow characteristics at the local field scale. Recognizing the need to provide quantitative information about subsurface properties and objects over plume-relevant scales, ERWR scientists have developed a framework for integration of multiscale hydrological data and have developed the Berkeley Unexploded-ordnance (UXO) Discriminator (BUD) field instrument that uses electromagnetic approaches. Their studies describe how BUD can differentiate (in real time) buried UXO from harmless metal and determine the location, size, and shape of such potential explosives. This instrument has won several accolades, including the 2007 "R\&D 100" award for technology advances from RED Magazine.

Laboratory and field studies conducted by ERWR scientists have revealed phenomena that have significant implications for contaminant transport and development of sustainable remediation strategies at many contaminated DOE sites. Column-profiling studies, designed to mimic the leakage of highly saline and alkaline radioactive waste solutions from Hanford storage tanks into sediments, revealed that the transport of uranium was strongly dependent on flow rate, and that conventional predictions based on equilibrium $\mathrm{Kd}$ partitioning of uranium would greatly underestimate the extent of plume migration. Column studies on sediments from the Oak Ridge National Laboratory Integrated Field-Scale Subsurface Research Challenges (IFC) site revealed that organic carbon-induced uranium reduction in sediments can exhibit rapid early stage reduction followed by transient uranium oxidation, and that the reoxidation can be facilitated by high concentrations of Fe(III). ERWR scientists have recently assessed the potential for immobilizing and detoxifying chromium-Cr(VI)-contaminated groundwater at the Hanford Site using lactate-stimulated bioreduction. Microbial, geophysical, and geochemical analysis of groundwater, coupled with stable isotope monitoring, permitted accurate tracking of microbial processes during this field treatabilty study. This study also confirmed that $\mathrm{Cr}(\mathrm{VI})$ was successfully removed from groundwater at the site and that concentrations of $\mathrm{Cr}(\mathrm{VI})$ remained below drinking water standards for several years after injection. Such studies reveal the benefits of interdisciplinary approaches for investigating and manipulating complex earth systems.

Several conceptual and numerical models have been developed to investigate contaminant transport and reactivity in natural and engineered environments. ERWR scientists have integrated the coupled effects of microbial Fe-oxide reductive dissolution, biogeneic sulfide production, and metal sorption

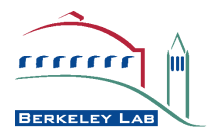


through the use of a full-surface-complexation model under redox disequilibrium to develop insights about important biogeochemical processes that affect the cycling of metals in lacustrine environments. The fate and transport of tritium within a constructed underground beamline facility was documented using numerical solutions supported by laboratory studies and data analysis. This study identified the main mechanisms of tritium transport through the engineered and surrounding fractured rock environment, and can be used to implement beamline design changes or mitigation measures, if necessary.

\section{WATER RESOURCES AND QUALITY}

To optimally manage our water resources, ERWR scientists are developing insights about the flow and transport of water and nutrients through natural systems. With a population of over 30 million people, an agricultural economy based on intensive irrigation, and large urban industrial areas, California is highly dependent on water for its vitality and productivity. Although the majority of the water research performed in ERWR is focused on California systems, the insights and approaches developed are expected to be generally transferable.

The Berkeley Water Center has been developed to bring together expertise at LBNL and UCB to tackle complex water problems and their impacts on biological, economic, agricultural, and other systems. The Berkeley Water Center Digital Watershed is a portfolio of projects that combine engineering, computer science, ecological, and hydrometerological expertise to advance our ability to interrogate and optimally manage water systems. A Microsoft-sponsored eScience project within this portfolio has created the cyberinfrastructure necessary to connect disparate databases and analysis tools, facilitating the synthesis of data acquired across the Ameriflux and FLUXNET observatories to address carbon science questions.

ERWR scientists have also developed a mechanistic model that couples hydrology, nutrient cycling, and vegetation responses. They have used this model to investigate $\mathrm{N}$ cycling and losses from agricultural fields, as well as to explore the effects on $\mathrm{N}$ cycling of irrigation and fertilizer parameters. Understanding $\mathrm{NO}, \mathrm{N}_{2} \mathrm{O}$, and $\mathrm{CO}_{2}$ emissions from the soil surface to the atmosphere is a necessary prerequisite to controlling greenhouse gas emissions. Development of such insights and modeling capabilities are increasingly necessary in light of increasing production of biofuels, food, and fiber production.

System-level studies have been carried out to characterize and understand complex hydrological and ecological phenom- ena that impact water resources and quality. Using spectral and shape characteristics from high-resolution remote sensing imagery and statistical discrimination approaches, ERWR scientists created maps of moist-soil wetland vegetation in the San Joaquin Basin, which can be used to assess seasonal evapotranspiration as needed for improving wetland-drainage management approaches. The Grassland Ecological Area within the San Joaquin Basin provide significant habitat to water fowl, although the water quality of the San Joaquin River is threatened by drainage from the salt, boron, and nutrient-rich wetlands. Through studying three matched pairs of wetland basins within a California Ecological Area, the impact of delayed wetland drainage on aquatic biota and water quality was established. Such studies can be used to guide the timing (and thus dilution) of wetland drainage to the river, with a goal of minimizing the impact on ducks and shorebirds.

The impact of climate on groundwater level was numerically investigated through two studies. Using $15 \mathrm{~km}$ grid cell resolution, an ERWR study documented the correlations between the water table within the Merced River Basin and precipitation, snow water equivalent, temperature, runoff, and evaporation as a function of season. The study illustrated that the correlations varied as a function of season, and that the strongest correlations were in the spring, when the interaction of landsurface and atmospheric processes were most active. Another study was performed to assess the impact of drought within the entire Central Valley of California from the period of 2010-2070. The study found that under the most severe drought scenarios, stream flow to major California reservoirs was reduced to $70 \%$ below average and that surface irrigation deliveries to agriculture fell to 50\%. Geographical comparisons of the simulations suggested that the northern Central Valley is better protected from drought than the southern region.

\section{ERWR TECHNICAL ASSISTANCE}

ERWR continues to be the Environmental Remediation Sciences Program (ERSP) Office for the Office of Science. The ERSP Office maintains the dynamic ERSP Web home page (www.lbl.gov/ERSP/) with links to investigators, program element managers, science team leaders, recent publications, annual meeting registration, calls for proposals, review documents, and other Web sites. In addition, the ERSP Office also organizes the ERSP annual investigators meeting, with more than 150 participants and sessions for posters, presentations, and breakout sessions. 
ERWR scientists also manage the Environmental Program at the Advanced Light Source (ALS), as described by http:/ / esd.lbl.gov/ALS_environmental/index.html. This program is designed to assist environmental researchers in gaining familiarity with and access to the ALS, and in assisting with environmental investigations at the ALS. Many of the environmental investigations in this program focus on understanding how and what microbiological and geochemical species are distributed relative to contaminants and within natural geological materials, which processes occur, and the rates at which they occur. With funding from ERSP, this program has provided support across four beamlines, providing a range of measurement support scales from nanometers to centimeters.

\section{SPONSORSHIP}

ERWR receives the majority of its support for environmental research from DOE programs in the Office of Science, Office of Biological and Environmental Research. These programs include the Environmental Remediation Sciences Program (ERSP) and the Genomics: GTL Program. DOE's Office of Environmental Management has supported some of the DOE site-specific studies. Research associated with unexploded ordnance is supported by the Environmental Security Technology Certification Program (ESTCP) of the U.S. Department of Defense. NSF supports the collaboration of ERWR scientists with university researchers. Many of the water quality projects were supported by the State Water Resources Control Board, the U.S. Bureau of Reclamation, the UC Salinity/Drainage Program, and LBNL Laboratory Directed funds. Studies exploring the impact of climate and groundwater were supported by the California Energy Commission. Support for ERP projects is also provided by NASA, SERDP, Cal-EPA, other DOE Labs, the Sonoma County Water Agency, U.S. Army, and the U.S. Bureau of Land Management.

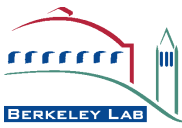




\title{
Comparative Analysis of Microbial Composition DuRing Field-Scale CHROMIUM BioREMEDiATION
}

\author{
Eoin Brodie, Terry Hazen, Boris Faybishenko, Romy Chakraborty, Mark Conrad, Sharon Borglin, Dominique Joyner, \\ Jizhong Zhou ${ }^{1}$, Joy Van Nostrand ${ }^{1}$, Richard Phan, Gary Andersen \\ ${ }^{1}$ Institute for Environmental Genomics, University of Oklahoma, Norman, OK \\ Contact: Eoin Brodie, 510/486-6584, elbrodie@lbl.gov
}

\section{RESEARCH OBJECTIVES}

During a field-scale chromium treatability study at the Department of Energy Hanford 100-H site, WA, a single dose of a slow-release electron-donor hydrogen-release compound (HRC) was applied to a contaminated aquifer to stimulate microbial reductive precipitation of hexavalent chromium $\mathrm{Cr}(\mathrm{VI})$. Here, we present an analysis of microarray-based prokaryotic population dynamics and correlations with geochemical observations following this application over two years.

\section{APPROACH}

A high-density $16 \mathrm{~S}$ rRNA phylogenetic microarray (PhyloChip) and a functional gene array (FGA) were used to analyze groundwater samples from multiple depths in injection and monitoring wells taken at intervals pre- and postHRC injection. Following filtration, genomic DNA was extracted and polymerase chain reaction (PCR) amplicons or MDA-amplified community-DNA were analyzed by microarray hybridization. A range of geochemical and geophysical parameters were also monitored.

\section{ACCOMPLISHMENTS}

Following HRC injection, reducing conditions had rapidly established with a corresponding decline in $\mathrm{DO}$, Eh, and nitrate. $\mathrm{Cr}(\mathrm{VI})$ concentrations declined steadily over 6 weeks and remained below upgradient concentrations. Microbial biomass was stimulated by two orders of magnitude (Figure 1a). PhyloChip data demonstrated depthstratified microbial communities with temporal shifts in composition corresponding with observed geochemistry. A sustained enrichment of iron (Figure 1c) and sulfate reducing bacteria (Figure 1b)
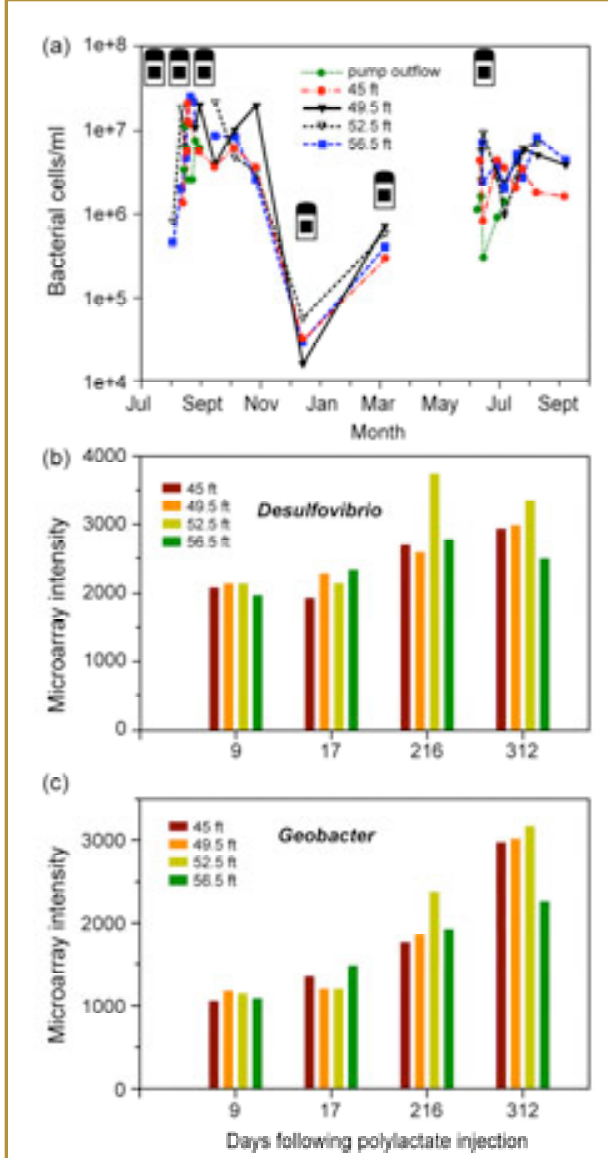

Figure 1. (a) Enrichment of microbial cells in Hanford groundwater following polylactate injection; (b) PhyloChip microarray intensity for sulfate-reducing Desulfovibrio spp. following polylactate injection; (c) PhyloChip microarray intensity for iron-reducing Geobacter spp. following polylactate injection. over the two years, and FGA array data demonstrated a susined enrichment of Pseudomonas chromate reductase genes, gesting that direct reduction of chromate may also be significant in chromium immobilization. Based on this data, organisms representing each of these functional groups have been isolated and characterized.

\section{SIGNIFICANCE OF FINDINGS}

The combination of phylogenetic and functional gene arrays represents a complementary high-throughput approach to elucidating mechanisms responsible for contaminant immobilization in the subsurface. In this study, we used this approach to identify the microorganisms contributing to both direct and indirect mechanisms of chromium immobilization at the Hanford $100-\mathrm{H}$ site, following a single-injection of polylactate electron donor.

\section{RELATED PUBLICATIONS}

DeSantis, T.Z., E.L. Brodie, J.P. Moberg, I.X. Zubieta, Y.M. Piceno, and G.L. Andersen, High-density universal $16 \mathrm{~S}$ rRNA microarray analysis reveals broader diversity than typical clone library when sampling the environment. Microb. Ecol. 53, 371-383, 2007.

Brodie, E.L., T.Z. DeSantis, Y.M. Piceno, and G. L. Andersen, High-density DNA microarray analysis for monitoring microbial community composition and dynamics. In: Molecular Microbial Ecology Manual. 3rd edition, G.A.Kowalchuk, ed., Kluwer Academic Publishers, Amsterdam, The Netherlands, 2007.

\section{ACKNOWLEDGEMENTS}

This work was supported by the Director, Office of Science, Office of Biological and Environmental Research, was observed, suggesting indirect chromium immobilization through interaction with reactive iron or sulfide by-products. Nitrate reducers such as Pseudomonas also remained elevated
Environmental Remediation Sciences Division, of the U.S. Department of Energy under Contract No. DE-AC02$05 \mathrm{CH} 11231$.

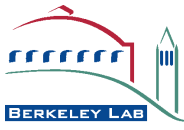




\section{Influence of Electron Donor TyPe ANd Concentration on Microbial Population DyNamics During URANIUM REDUCTION AND REMObILIZATION}

Eoin Brodie, Rebecca Daly ${ }^{1}$, Yongman Kim, Jiamin Wan, Tetsu Tokunaga, Gary Andersen, Terry Hazen, and Mary Firestone ${ }^{2}$

${ }^{1}$ Department of Plant and Microbial Biology, University of California, Berkeley, CA

${ }^{2}$ Department of Environmental Sciences Policy and Management, University of California, Berkeley, CA

Contact: Eoin Brodie, 510-486-6584, elbrodie@lbl.gov

\section{RESEARCH OBJECTIVES}

Enhanced reductive precipitation of $\mathrm{U}(\mathrm{VI})$ through stimulation of indigenous microorganisms is an attractive, low-cost strategy for in situ remediation of contaminated groundwaters and sediments. However, our previous long-term column studies demonstrated that after an initial period of $\mathrm{U}(\mathrm{VI})$ reduction and immobilization, reoxidation of $\mathrm{U}(\mathrm{IV})$ and remobilization of $\mathrm{U}(\mathrm{VI})$ occurred. The rate of organic carbon (OC) supply determines not only the amount of electron donor available for bioreduction of $\mathrm{U}(\mathrm{VI})$, but also affects the resulting concentration of aqueous (bi)carbonate generated by microbial respiration. Increased (bi)carbonate concentrations drive aqueous $\mathrm{U}(\mathrm{VI})$ concentrations to higher levels through formation of stable U(VI) carbonato complexes, including $\mathrm{Ca}_{2} \mathrm{UO}_{2}\left(\mathrm{CO}_{3}\right)_{3}(\mathrm{aq})$, and make U(IV) oxidation under reducing conditions favorable. We are currently investigating the effects of various OC forms and supply rates on the long-term stability of bioreduced $\mathrm{U}$, and on the dynamics of the resulting microbial communities in relation to $\mathrm{U}$ redox changes.

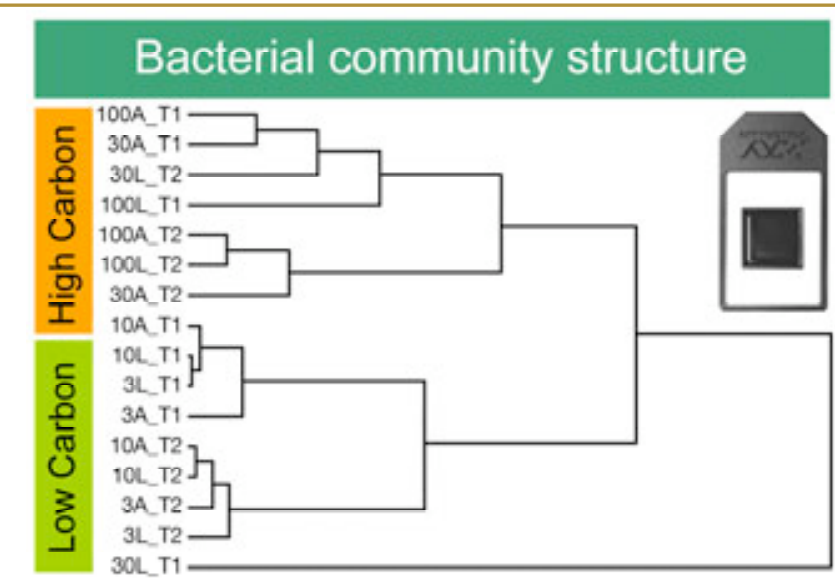

Figure 1. Hierarchical cluster analysis of PhyloChip microarray data showing electron donor (OC) concentration as the major factor distinguishing bacterial community composition. Numbers correspond to carbon concentration $(3,10,30,100 \mathrm{mM}), \mathrm{L}=$ lactate, $\mathrm{A}=$ acetate, $\mathrm{T} 1=$ sample taken during reduction phase in high OC columns, T2 = sample taken during re-oxidation phase in high OC columns.

\section{APPROACH}

Our current studies are being conducted using historically U-contaminated sediments from Area 2 of the Field Research Center, Oak Ridge National Laboratory, in flow-through columns. Microbial communities were stimulated by the addition of OC supplied as either acetate or lactate at four different concentrations $(100 \mathrm{mM}, 30 \mathrm{mM}, 10 \mathrm{mM}, 3 \mathrm{mM}$ OC equivalents). Columns were sampled at two time points. In the columns receiving 100 or $30 \mathrm{mM} \mathrm{OC}$, the time points corre- spond to a phase of net U-reduction and a later phase of U(IV) reoxidation and $\mathrm{U}(\mathrm{VI})$ remobilization. DNA was extracted from columns, 16S rRNA genes were amplified, and the microbial composition was analyzed using a high-density phylogenetic microarray (PhyloChip).

\section{ACCOMPLISHMENTS}

It was observed that lactate and acetate supplied at equivalent rates have a similar impact on uranium mobility with higher OC, resulting in re-oxidation of U(IV) after an initial period of $\mathrm{U}(\mathrm{VI})$ reduction. Similarly, it was demonstrated that organic carbon (OC) supply rate, not $\mathrm{OC}$ form, had the largest impact on microbial community structure (Figure 1). The diversity (richness) of bacterial and archaeal communities increased over time, with those receiving the fermentable OC form (lactate) having higher initial richness. Closer examination of the bacterial community composition demonstrated the presence of known metal/U-reducing bacteria in all columns at all time points; however, the dynamics of these organisms varied with both organic carbon supply rate and form.

\section{SIGNIFICANCE OF FINDINGS}

This data demonstrates that the initial rate of electron donor supply during heavy metal remediation strongly impacts microbial community development. Uranium remobilization occurred irrespective of electron donor form, and occurred despite the presence of multiple species of metalreducing bacteria.

\section{RELATED PUBLICATIONS}

Brodie, E.L., T.Z. DeSantis, D.C. Joyner, S. Baek, J.T. Larsen, G.L. Andersen, T.C. Hazen, D.J. Herman, T.K. Tokunaga, J.M. Wan, and M.K. Firestone,Application of a high-density oligonucleotide microarray approach to study bacterial population dynamics during uranium reduction and reoxidation. Appl. Environ. Microbiol. 72, 6288-6298, 2006.

Wan, J. M., T.K.Tokunaga, E. Brodie, Z.M. Wang, Z.P. Zheng, D. Herman, T.C. Hazen, M.K. Firestone, and S.R. Sutton, Reoxidation of bioreduced uranium under reducing conditions. Environ. Sci. Technol., 39, 6162-6169, 2005.

\section{ACKNOWLEDGEMENTS}

This work was supported by the Director, Office of Science, Office of Biological and Environmental Research, Environmental Remediation Sciences Division, Environmental Remediation Sciences Program (ERSP), of the U.S. Department of Energy under Contract No. DE-AC02-05CH11231.

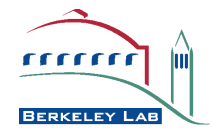




\title{
Spatial Characterization of the Bacterial and Archaeal Community Structure in the Passalid Beetle Gut
}

\author{
Eoin Brodie, Nhu Nyugen', Todd DeSantis, Stephanie Gross ${ }^{2}$, Sung-Oui Suh², James Nardi ${ }^{3}$, Tom Bruns ${ }^{1}$, Meredith Blackwell ${ }^{2}$, and Gary Andersen \\ ${ }^{1}$ Department of Plant and Microbial Biology, University of California, Berkeley, CA \\ ${ }^{2}$ Louisiana State University, Baton Rouge, LA 70803. ${ }^{3}$ University of Illinois at Urbana-Champaign, Urbana, IL \\ Contact: Eoin Brodie, 510/486-6584, elbrodie@lbl.gov
}

\section{RESEARCH OBJECTIVES}

Understanding the microbial processes by which woodingesting insects derive energy may aid large-scale conversion of lignocellulosic biomass into biofuel. We examined the prokaryotic biome of the wood-eating passalid beetle, Odontotaenius dis junctus, with a 500,000-probe PhyloChip targeting multiple unique regions of the $16 \mathrm{~S}$ rRNA gene. This passalid beetle has developed a symbiotic relationship with microbes to survive on a low-nitrogen diet that requires microbial-community-derived enzymes to digest the complex polysaccharides and lignins of plant cell walls. The adult gut of this approximately $3 \mathrm{~cm}$ beetle is over $10 \mathrm{~cm}$ in length and consists of four morphologically distinct sections, the foregut $(\mathrm{FG})$, midgut $(\mathrm{MG})$, anterior hindgut (AHG), and posterior hindgut (PHG). The fungal composition of these gut regions has been extensively studied, and microscopy has demonstrated a morphologically diverse bacterial population in the hindgut. Relatively little, however, is known about the bacterial or archaeal diversity, which likely contributes key enzymes for lignocellulose processing, in addition to fixing atmospheric nitrogen for host nutrition.

\section{APPROACH}

To perform an in-depth census of the prokaryotic composition of each gut region, we used a high-density (500,000-probe) $16 \mathrm{~S}$ rRNA microarray (PhyloChip) to screen for the presence and relative abundance of most known prokaryotes in a massively parallel assay. Living passalid adults were fed fresh hardwood chips for over one month before aseptic dissection of the gut and separation into the four sections. Sections were preserved and nucleic acids extracted from gut homogenates. Bacterial and archaeal $16 \mathrm{~S}$ rRNA genes were amplified separately. The amplified products for each sample were quantified, pooled, fragmented, biotinlabeled, and hybridized for $16 \mathrm{~h}$ to the PhyloChip.

\section{ACCOMPLISHMENTS}

Analysis of bacterial composition demonstrated that across the same gut region, composition was highly similar across multiple individuals, while most gut regions were distinct. The AHG had the most unique composition, with higher relative abundance of clostridia, Bacteroidetes, sulfate reducers, and potentially nitrogen-fixing spirochetes. The AHG was the only location where methanogens were detected. Earlier microscopic work has shown that bacteria in this section form a solid surface biofilm. By contrast, the MG and PHG from individual beetles clustered tightly and contained higher proportions of alpha-proteobacteria and lactobacilli.

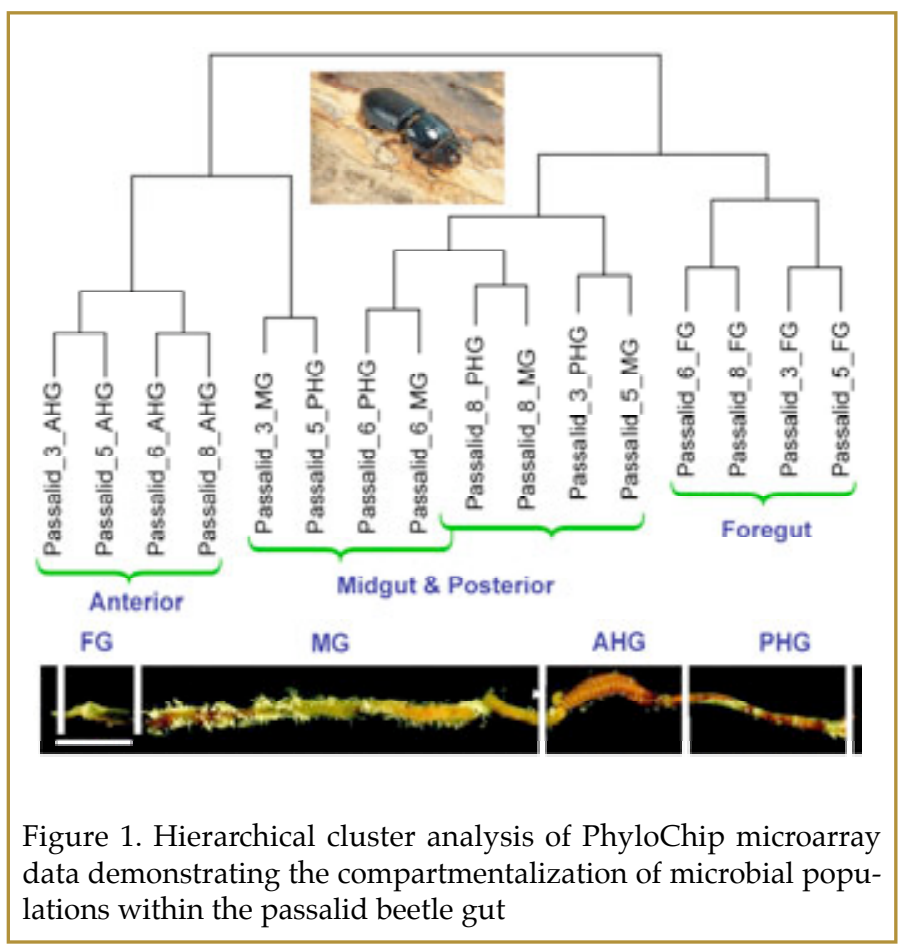

\section{SIGNIFICANCE OF FINDINGS}

This study represents the first comprehensive view of the passalid beetle gut bacterial and archaeal population. It has revealed a diverse community that is compartmentalized in terms of composition and likely function. The co-occurrence of anaerobic and aerobic processes within gut regions suggests existence of oxygen and/or hydrogen gradients along which microbial species partition themselves. This study represents the first steps to understand how these isolated populations, in one of nature's most efficient biorefineries, interact to deconstruct cellulosic materials.

\section{ACKNOWLEDGMENTS}

This work was partially supported by the National Science Foundation, Biodiversity Surveys and Inventories Program (DEB-0072741 and DEB-0417180) and REU supplements (to $\mathrm{MB}$ ) and use of the LSU DNA sequencing facility was supported by a NSF Multi-user Equipment Grant (DBI-0400797). Part of this work was performed under the auspices of the U.S. Department of Energy by the University of California, Lawrence Berkeley National Laboratory, under Contract No. DE-AC02-05CH11231.

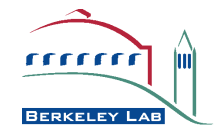




\title{
Large-Scale Production of Anaerobic Sulfate Reducers
}

\author{
Jil T. Geller, Mary E. Singer, Tamas Torok, and Terry C. Hazen \\ Contact: Jil Geller, 510/486-7313, jtgeller@lbl.gov
}

\section{RESEARCH OBJECTIVES}

The Virtual Institute for Microbial Stress and Survival (VIMSS, http: / /vimss.lbl.gov/) investigates stress response pathways in model organisms for the remediation of heavy metals and radionuclides in groundwater at DOE sites. The Protein Complex Analysis Project (PCAP, http://pcap.lbl.gov/), a subproject of VIMSS, focuses on high-throughput analysis of microbial protein complexes in the anaerobic, sulfate-reducing organism, Desulfovibrio vulgaris Hildenborough (DvH). Large volumes of culture of consistent quality are needed because of the relatively low cell density of DvH cultures (one order of magnitude lower than E. coli, for example) and PCAP's challenge to characterize low-abundance membrane proteins. In this summary, we describe our unique application of continuous flow fermentors to produce the large quantities of extremophile biomass required for PCAP.

\section{APPROACH}

Large-scale production of DvH presents several challenges. The organism is a metal reducer, and produces hydrogen sulfide, which is corrosive to the stainless steel parts of standard fermentors. Because the production volumes preclude working either in an anaerobic chamber or in a biosafety cabinet, we designed a benchtop system that could maintain anaerobic conditions and prevent contamination. Our facility has four 5liter volume fermentors, which were custom-manufactured with nonmetallic wetted parts by Electrolab, UK. The fermentors are equipped with temperature and $\mathrm{pH}$ control, and redoxpotential monitoring. To maintain anaerobic conditions, the fermentors are continuously sparged with nitrogen.

To improve culture consistency, we operate the fermentors in continuous flow mode. $\mathrm{DvH}$ is grown to late-mid-log phase (about $5 \times 10^{8}$ cells $/ \mathrm{mL}$ ) in batch mode, and then a lactate and sulfate-defined medium is applied at a dilution rate of $0.15 \mathrm{~h}^{-1}$ $(0.6 \mathrm{~L} / \mathrm{h})$ to maintain the culture at a constant cell density. The prevailing redox potential during culture growth ranges from -500 to $-700 \mathrm{mV}$. Once flow is initiated, the collected effluent is chilled to $4^{\circ} \mathrm{C}$ and held until the biomass is harvested by centrifugation. Cell density, which is directly correlated to optical density, is continuously monitored by pumping a recycle line through a cuvette in a small spectrophotometer (Ocean Optics, FL).

Figure 1 shows the optical density increase during batch phase, followed by its approach to steady state during continuous-flow phase. For reference, the OD of stationary phase DvH is 1 . The total protein concentrations throughout the production range from 110 to $200 \mu \mathrm{g} / \mathrm{mL}$. During continuous flow, approximately 35 to 55 $\mathrm{mM}$ of lactate and 5 to $15 \mathrm{mM}$ of sulfate are consumed.

\section{ACCOMPLISHMENTS}

We have developed and optimized fermentor operation to produce large volumes of $\mathrm{DvH}$, yielding reproducible, highquality biomass for protein complex analysis. Running two fermentors in parallel, we are able to produce $100 \mathrm{~L}$ of culture per week. We have also developed analytical protocols to monitor consistency of production.

\section{SIGNIFICANCE OF FINDINGS}

Our methodology can be applied to produce a range of extremophile microorganisms under controlled conditions. Extremophiles have the greatest potential for remediating the most recalcitrant contaminants, and for the production of biofuels.

\section{ACKNOWLEDGMENTS}

We are grateful to Lauren Camp and Megan Shelby for assistance in the production runs and sample analysis. This work was supported by the Director, Office of Science, Office of Biological and Environmental Research, Genomics:GTL Program, of the U.S. Department of Energy, under Contract No. DE-AC02-05CH11231.

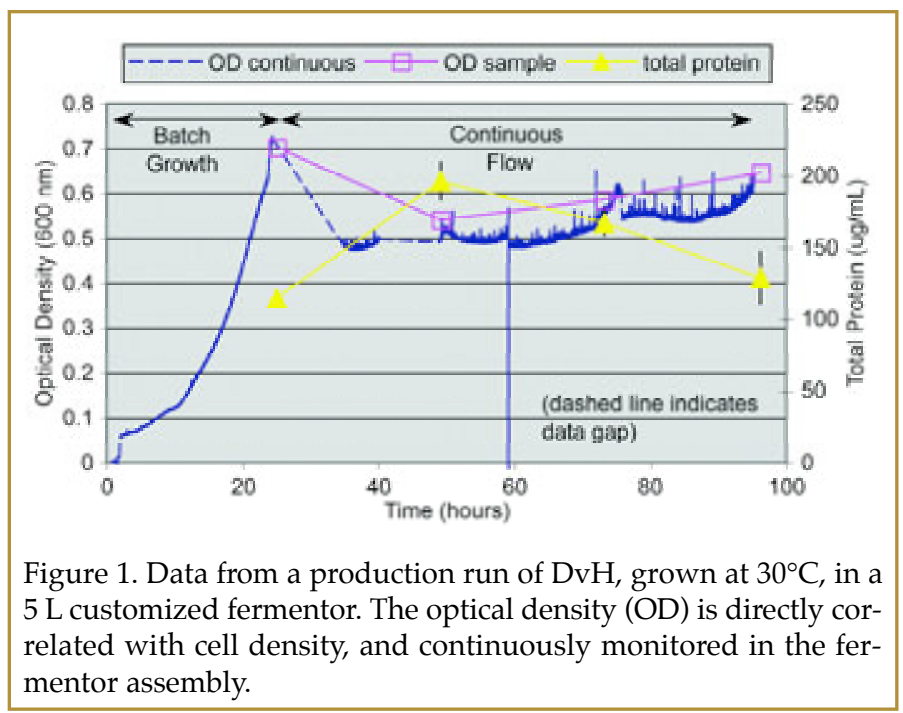

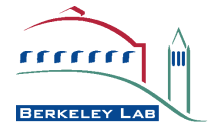




\title{
Greengenes: Gene Database and Web Application for Microbial Ecologists
}

\author{
Todd DeSantis, Eoin Brodie, Yvette Piceno, Phillip Hugenholtz ${ }^{1}$, and Gary Andersen \\ ${ }^{1}$ Microbial Ecology Program, DOE Joint Genome Institute, LBNL, Berkeley, CA 94598 \\ Contact: Todd DeSantis, 510/486-, TDeSantis@lbl.gov
}

\section{RESEARCH OBJECTIVES}

Diverse microorganisms living in the environment or within human tissues often act in con cert to affect natural chemical cycling in the environment, as well as human health. In contrast to identifying single species' infections or searching for a single organism correlating with an environmental change, ecologists and medical researchers now analyze the entire microbial community structure and dynamics. This new research field has provided insights into medical problems such as obesity, inflammatory bowel disease, allergen sensitivity, periodontitis, vaginosis, pneumonia, and viral infection susceptibility. Environmental microbiologists have likewise found trends between microbial communities and the corresponding atmospheric conditions, groundwater redox potential, and soil moisture, as examples. The objective of the Greengenes project is to efficiently relate microbial community structure to disease states and environmental factors.

\section{APPROACH}

Presently, the bacterial and archaeal communities of the human biome and earth's biosphere remain mostly undefined, because most microor-

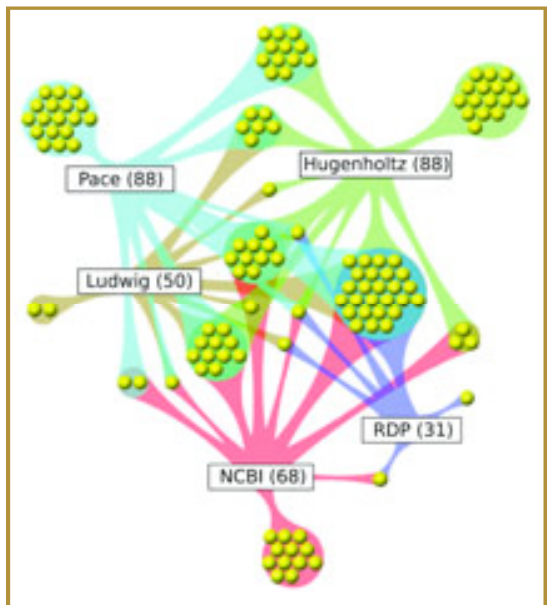

Figure 1. Classification terms shared among independent curators represented as a five-way Venn diagram. Yellow spheres represent the 126 Phyla or Candidate Division names encountered in at least one of the five taxonomy systems (Pace, Hugenholtz, Ludwig, RDP, or NCBI). Numbers in parentheses are the count of Phyla or Candidate Division names recognized by an individual curator. Clusters of yellow spheres connected by more than one colored web symbolize names recognized by multiple curators.

\section{ACCOMPLISHMENTS}

Greengenes has been implemented as a web application at http://greengenes. lbl.gov. It is the first resource to deconvolute the classification of microorganisms from both the environment and clinical material (Figure 1). It has become an internationally recognized resource, as evidenced by its frequent citations. Recently, it was used to demonstrate the effects of atmospheric pressure and temperature on urban bioaerosols at inhalation height, and to discover that salinity is the major factor influencing bacterial populations in aqueous environments.

\section{SIGNIFICANCE OF FINDINGS}

The Greengenes standardized methods for the analysis of community DNA data now allows research labs without dedicated bioinformatics support to determine factors affecting the types and quantities of microorganisms living in an environment. Continued usage of this service will be necessary for accurately analyzing the massive influx of $16 \mathrm{~S}$ rRNA and ITS data and for sharing and comparganisms have not been successfully grown (cultured) and studied in a laboratory. The advent of gene-based, culture-independent surveys and the availability and falling cost of high-throughput sequencing now allows inventories of all microbial community members. This has been accomplished most commonly using the $16 \mathrm{~S}$ rRNA gene, a highly conserved and reliable marker for archaeal and bacterial species identification, and more recently using the ITS region of fungal genomes. Unfortunately, the ability to analyze and compare these data, and thereby make insights into the associated diseases, is impeded by a lack of high-quality, comprehensive databases and tools. Currently, researchers must independently surmount the same post-sequencing obstacles, namely: (1) making a reliable multiple sequence alignment (MSA) in order to compare sequences, (2) filtering out low-quality reference sequences from the public databases, (3) determining the identity of their sequences in the face of limited taxonomic classification of public sequences, (4) handling increasingly large datasets in an efficient manner, and (5) comparing overall community structure of their samples to public datasets. This entire process has been simplified and standardized in the Greengenes web application. ing community structures from diverse samples. We anticipate this work will soon benefit clinicians making point-of-care decisions.

\section{RELATED PUBLICATIONS}

DeSantis, T.Z., P. Hugenholtz, N. Larsen, M. Rojas, E.L. Brodie, K. Keller, T. Huber, D. Dalevi, P. Hu, and G.L. Andersen, Greengenes: A chimera-checked 16S rRNA gene database and workbench compatible with ARB. Appl Environ Microbiol, 72, 5069-5072, 2006.

DeSantis, T.Z., P. Hugenholtz, K. Keller, E.L. Brodie, N. Larsen, Y.M. Piceno, R. Phan, and G.L. Andersen, NAST: A multiple sequence alignment server for comparative analysis of $16 \mathrm{~S}$ rRNA genes. Nucleic Acids Res, 34, W394-399, 2006.

\section{ACKNOWLEDGMENTS}

This work was supported by the Director, Office of Science, Office of Biological and Environmental Research, Genomics:GTL Program, of the U.S. Department of Energy, under Contract No. DE-AC02-05CH11231.

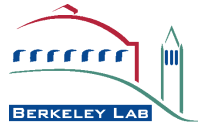




\section{Development of a New Tool to Study Nucleation and Growth of Nanoparticles on Mineral Surfaces}

Young-Shin Jun, Glenn A. Waychunas, Byeongdu Lee, and Mike F. Toney

Contact: Glenn A. Waychunas, 510/495-2224, GAWaychunas@lbl.gov

\section{RESEARCH OBJECTIVES}

The sorption, nucleation, and growth of nanoparticles on mineral surfaces may control the mineral surface's reactivity, and hence markedly affect aqueous metal contaminant transport and other surface-controlled reactions. Recently, grazing incidence small angle $x$-ray scattering (GISAXS) has been used to analyze the size, shape, and distribution of quantum dots and polymers on substrates. However, no work has been attempted thus far using GISAXS for in situ observations of environmental interfacial processes-such as the nucleation and growth or aggregation of nanoparticles on mineral surfaces. In this study, we (1) developed an in situ time-resolved GISAXS capability to allow real-time geochemical kinetics analysis of nanoparticle reactions at mineralwater interfaces; and (2) elucidated the mechanisms and kinetics of early nucleation and growth of important environmental nanoparticles.

\section{APPROACH}

We developed a new in situ time-

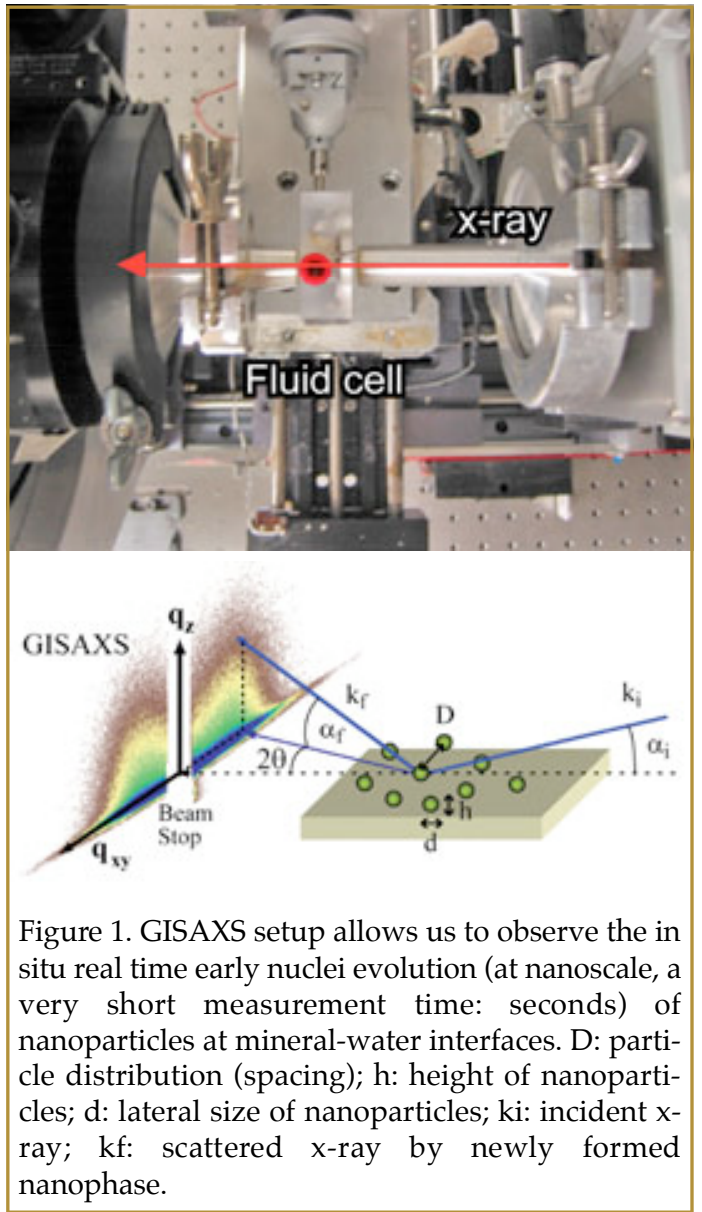

quantitative contribution between homogeneous and heterogeneous nucleation under different conditions. We found that ionic strength affects interparticle distances and particle sizes and shapes. At $\left[\mathrm{Fe}^{3+}\right]=10^{-4} \mathrm{M}$, heterogeneous nucleation was found to be more dominant and faster than homogeneous nucleation, whereas homogeneous nucleation is more important at higher $\left[\mathrm{Fe}^{3+}\right]$ concentrations. This result is the first direct experimental evidence showing quantitative contributions caused by each mechanism at aqueous interfaces.

\section{SIGNIFICANCE OF FINDINGS}

In situ observations of the early nucleation and growth of nanoparticles in aqueous systems have been a challenging issue, because of (1) difficulties in distinguishing between homogeneous and heterogeneous nucleation processes; (2) difficulties in measuring the early stage kinetics, as a result of slow data acquisition and disturbance of the reaction system; and (3) small observation areas. By using an in situ timeresolved simultaneous SAXS/GISAXS, we were able to distinguish the quantitative contribution between homogeneous and heterogeneous nucleation without interresolved GISAXS capability that can be used either under dry or aqueous conditions. Using this setup, we investigated the kinetics and mechanisms of early nucleation and growth of iron oxide nanoparticles at water-mineral interfaces. In the experiments, the samples consisted of quartz substrates exposed to freshly prepared ferric solutions. By analyzing the simultaneous small angle $x$-ray scattering (SAXS) from the overlying solution and the GISAXS from the interface, we could extract the initial kinetics of nucleation and growth (numbers of nuclei and volume) and the morphological parameters (size, shape, and distribution) for both homogeneously (solution) and heterogeneously (interface) nucleated particles.

\section{ACCOMPLISHMENTS}

We investigated the initial nucleation and growth of iron oxide nanoparticles on quartz surfaces under different ionic strengths and aqueous iron concentrations (Figure 1). Utilizing simultaneous SAXS/GISAXS, we were able to distinguish the rupting surface reactions. This technique also allows statistically improved real-time geochemical kinetics analysis of nanoparticle formation compared to other methods, and is highly complementary to imaging techniques such as atomic force microscopy or transmission electron microscopy.

\section{RELATED PUBLICATION}

Jun, Y.S., G.A. Waychunas, and B. Lee, Kinetic study of nucleation and growth of environmental nanoparticles at watermineral interfaces using in situ time-resolved GISAXS. American Crystallography Association Series 2, 34, 52, 2007.

This work was supported by the Director, Office of Science, Office of Biological and Environmental Research, Environmental Remediation Sciences Division, of the U.S. Department of Energy under Contract No. DE-AC02$05 \mathrm{CH} 11231$.

\section{ACKNOWLEDGMENTS}




\section{Environmental Science Program at the Advanced Light Source \\ Peter Nico \\ Contact Petr Nico, 510/486-7118, psnico@lbl.gov}

\section{RESEARCH OBJECTIVES}

The goal of the Environmental Science Program at the Advanced Light Source (ALS) is to further the research mission of OBER's Environmental Remediation Science Program (ERSP), by improving utilization of the ALS in the ERSP research effort, and generally increasing the quantity and quality of environmental research conducted at the ALS.

\section{APPROACH}

The ALS is a DOE national user facility with many unique spectroscopic and microscopic capabilities suited to application in environmental science. The program supports the research mission of ERSP by supporting ERSP PIs on research projects that can benefit from the resources at the ALS. These collaborations take many different forms, including participation in experimental design, novel sample cell development, data interpretation, beam time proposal writing, and data collection. The program specifically focuses on four beam lines at the ALS. These beam lines include BL 1.4.3-infrared spectromicroscopy, BL 8.3.2-x-ray micro-tomography, BL 10.3.2-x-ray microprobe and micro-x-ray diffraction, and BL 11.0.2-scanning transmission $x$-ray microscopy (STXM).

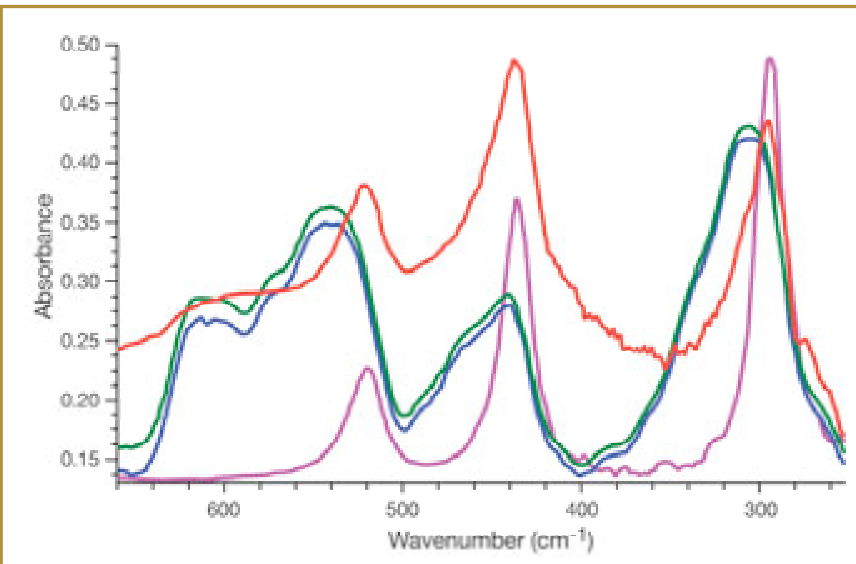

Figure 1. Synchrotron FTIR spectra of hematite with and without ferrihydrite before and after exposure to DIRB

\section{ACCOMPLISHMENTS AND SIGNIFICANCE}

In the past year, the program has worked with many different research groups for over 600 hours of beam time. Examples of these projects include: utilizing the Fourier transform infrared (FTIR) beamline (1.4.3) to study in situ iron biomineralization processes; imaging the pore-size distribution within synthetic aggregates using microtomography (8.3.2); examining the iron mineral transformations within those aggregates, using the micro-XAS capabilities of beamline 10.3.2; and investigating natural organic carbon interactions with mineral grains using the STXM (11.0.2).

The program also serves the general environmental synchrotron user community through coordination of resources across the four DOE-supported synchrotron user facilities (ALS, NSLS, and SSRL). By frequent communication with environmental scientists at the other three sources, the ALS program is able to help identify the most appropriate facility for a given investigation across the entire DOE synchrotron complex, and therefore maximize the scientific output of these facilities.

In addition, the program is also working to increase the total amount of beam time available at the ALS through leading the design and construction of a new FTIR beamline that will have greater photon flux $(\sim 2 \mathrm{x})$ compared to the existing FTIR beamline.

Using the newly developed FTIR environmental bioreactor, we were able to directly observe the alterations in two common iron oxides under microbial-induced reduction. Figure 1 shows the FTIR spectrum of a hematite film, which is originally very crystalline, as represented by the sharp distinct peaks seen in the magneta spectrum. This film is then coated with ferrihydrite, which adds a broad diffuse baseline to the spectrum but does not alter the position of the original hematite peaks (red). After 17 days of reaction with dissimilatory iron-reducing bacteria (DIRB), the signal from ferrihydrite has been significantly reduced, as a result of both flow-mediated erosion of the ferrihydrite layer and also of recrystallization of the ferrihydrite. A shoulder peak at $\sim 615 \mathrm{~cm}^{-1}$ appears, which is consistent with the formation of goethite, and there is a significant shift and broadening of the hematite peaks at $\sim 543 \mathrm{~cm}^{-1}, \sim 444 \mathrm{~cm}^{-1}$, and $\sim 305 \mathrm{~cm}^{-1}$, which is indicative of an increase in disorder in the hematite as a result of hydration and/or reductive dissolution.

\section{RELATED PUBLICATION}

Neiss, J.; B.D. Stewart, P.S. Nico, and S.E. Fendorf, Speciationdependent microbial reduction of uranium within ironcoated sands under dynamic flow. Environ. Sci. and Technol. (in press), 2007.

\section{ACKNOWLEDGMENTS}

This work was supported by the Director, Office of Science, Office of Biological and Environmental Research, Environmental Remediation Sciences Program, of the U.S. Department of Energy under Contract No. DE-AC02$05 \mathrm{CH} 11231$.

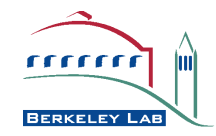




\title{
Development of Highly Heterogeneous Zones of Microbial Activity DURING ENHANCED BIOREMEDIATION
}

\author{
Mark Conrad, Eoin Brodie, and Markus Bill
}

Contact: Mark Conrad, 510/486-6141, msconrad@lbl.gov

\section{RESEARCH OBJECTIVES}

In situ bioremediation of contaminated aquifers through the addition of a carbon source to stimulate biological activity has been demonstrated to be a viable method for cleaning up groundwater. Enhanced microbial activity in the area of the injected carbon source creates conditions that have been shown to lead to biodegradation of chlorinated compounds and immobilization of metals. Not well understood, however, is what occurs in the downstream portions of these plumes.

For more than 8 years, organic carbon (initially Na-lactate, followed by whey) has been added to the groundwater at the source of a $3 \mathrm{~km}$ long trichloroethene (TCE) plume at the Test Area North (TAN) site of the Idaho National Laboratory. This activity has led to high levels of microbial activity, resulting in anaerobic conditions that have successfully stimulated complete reductive dechlorination of the TCE in the source area of the plume. The purpose of this project is to characterize biological activity in the downstream areas and assess the potential for additional bioremediation of the groundwater in the distal sections of the plume.

\section{APPROACH}

As a follow-up to a study of bioremediation conducted during the initial phases of enhanced bioremediation (Song et al., 2002), groundwater samples were collected in a series of monitoring wells extending from an injection well down-gradient in the plume. The compositions of the microbial communities in these samples were analyzed using high-density DNA microarrays (PhyloChips). The isotopic composition of dissolved gases (methane and inorganic carbon) and chlorinated solvents in the samples were also analyzed to assess the level of relevant microbial metabolic processes (methanogenesis, methane oxidation, reductive dechlorination of solvents, aerobic biodegradation of solvents).

\section{ACCOMPLISHMENTS}

Anaerobic conditions in the groundwater extend to greater than $150 \mathrm{~m}$ down-gradient from the injection well. Within this zone, concentrations of dissolved methane are high ( $>3$ micromolar), whereas nitrate and sulfate concentrations are depleted. Phylochip analyses of microbial communities, in groundwater samples from the anaerobic zone of the plume, have identified a range of aerobic microorganisms, including methane-oxidizing bacteria and anaerobic organisms such as methanogens, sulfate reducers, and anaerobic methane-oxidizing archaea. Large shifts in the carbon isotope ratios of the methane (from $-55 \%$ in the source area to $-28 \%$ in down-gradient wells) confirm that significant microbial methane oxidation is occurring within the largely anaerobic core of the TCE plume (Figure 1). In addition, the isotope compositions of dissolved inorganic carbon (DIC) decreased from values typical of methanogenic activity $(>8 \%$ o) in the source area to much lower values $(<-13 \%$ ) in the down-gradient anaerobic wells, as would be expected for high levels of methane oxidation. Downgradient from the anaerobic core of the plume, where the dissolved oxygen contents of the groundwater have rebounded, there is molecular evidence for methanogenic microorganisms.

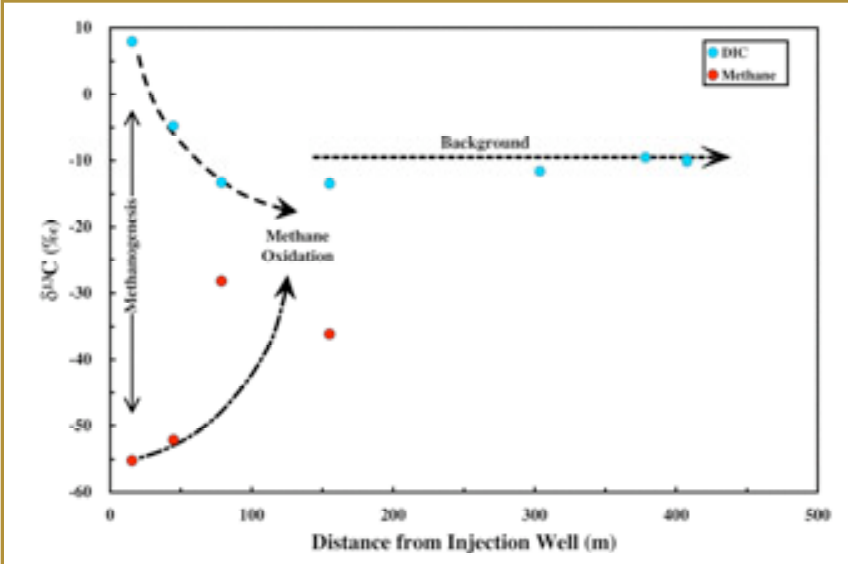

Figure 1. Carbon isotopic compositions of dissolved methane and inorganic carbon in samples from the TAN site plotted versus distance from the injection well. (Both the source area for the TCE and the well into which organic carbon has been added to stimulate microbial activity.) The large increase in the $\delta^{13} \mathrm{C}$ of the methane coupled with the drop in the $\delta^{13} \mathrm{C}$ of inorganic carbon with distance from the injection well indicate significant methane oxidation occurring within the $150 \mathrm{~m}$ of the injection well, despite anaerobic conditions. In the distal portions of the plume, the $\delta^{13} \mathrm{C}$ of inorganic carbon returns to background levels.

\section{SIGNIFICANCE OF FINDINGS}

These data indicate that diverse microbial communities capable of biodegrading contaminants exist in groundwater, where the groundwater geochemistry may not reflect the conditions thought to be required for some of these communities to be active. This implies that significant biodegradation of contaminants may be occurring in these portions of the plumes.

\section{RELATED PUBLICATION}

Song, D.L., M.E. Conrad, K.S. Sorenson, and L. Alvarez-Cohen, Stable carbon isotope fractionation during enhanced in situ bioremediation of trichloroethene. Environ. Sci. Technol. 36, 2262-2268, 2002.

\section{ACKNOWLEDGMENTS}

This work was supported by the Director, Office of Science, Office of Biological and Environmental Research, Environmental Remediation Sciences Division, of the U.S. Department of Energy under Contract No. DE-AC02-05CH11231.

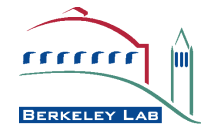




\section{Sulfur Isotopes as Indicators of Bacterial Sulfate-Reduction Processes DURING FIELD-SCALE URANIUM BIOREMEDIATION}

Jennifer L. Druhan, Mark E. Conrad, Kenneth Hurst Williams, Lucie N'Guessan, Philip E. Long, and Susan S. Hubbard Contact: Jennifer L. Druhan, jennydruhan@berkeley.edu

\section{RESEARCH OBJECTIVES}

Aqueous uranium concentrations in a contaminated aquifer in Rifle, Colorado, have been successfully lowered through enzymatic reduction to less soluble U(IV) by acetateamended microbial activity. The highest $\mathrm{U}(\mathrm{VI})$ removal rates are noted during iron reduction and decrease with the onset of sulfate reduction. However, sustained U(IV) attenuation is observed following sulfate reduction and subsequent termination of the acetate amendment. These findings indicate the transition between iron- and sulfate-reducing conditions is an important aspect of the remediation process. Samples collected during a 2006 acetate amendment were analyzed for $\delta^{34} S$ of sulfate and sulfide to explore the utility of sulfur isotopes as indicators of these processes. Objectives of this study include the use of these data to refine the timeline of iron- and sulfatereduction phases and to determine the stability of sulfide precipitates post-amendment.

\section{APPROACH}

Sulfate and sulfide isotopic samples were collected throughout the course of an in situ acetate amendment at the DOE Old Rifle Site near Rifle, Colorado. Samples were taken in one background well and three monitoring wells down-gradient of the injection gallery, in conjunction with aqueous geochemical measurements of sulfate, ferrous iron, $\mathrm{U}(\mathrm{VI})$, and acetate.

\section{ACCOMPLISHMENTS}

Results show an increase of up to $7 \% 0 \delta^{34} \mathrm{~S}$ in residual sulfate at the onset of sulfate reduction, followed by a return to background $\delta^{34} S$ values of $-8 \%$ with time. Sulfide $\delta^{34} S$ values of roughly $-20 \%$ at the onset of sulfate reduction increase to background sulfate $\delta^{34} \mathrm{~S}$ values as sulfate removal approaches $100 \%$ and subsequently return to depleted levels following cessation of acetate amendment (Figure 1). Comparable sulfate and sulfide $\delta^{34} S$ values at the height of sulfate reduction suggest total removal of all sulfate accessible to the microbial population. In addition, a steady decline in $\delta^{34} S$ of sulfide, concurrent with increased sulfide concentrations following cessation of acetate amendment, suggests that this increase is not a result of reoxidation of precipitated FeS species. Thus, FeS precipitates formed during the height of sulfate reduction appear to be stable following acetate amendment and may lend to the stability and long-term sequestration of precipitated U(IV).

\section{SIGNIFICANCE OF FINDINGS}

This study demonstrates the additional information regarding the transition to sulfate reduction and the fate of sulfide species gained through the use of sulfur isotopes beyond what is obtainable using aqueous geochemical measurements alone. The apparent stability of precipitated sulfide species, indicated by the isotopic data following acetate amendment, is particularly significant in that these species may influence long-term $\mathrm{U}(\mathrm{VI})$ sequestration.

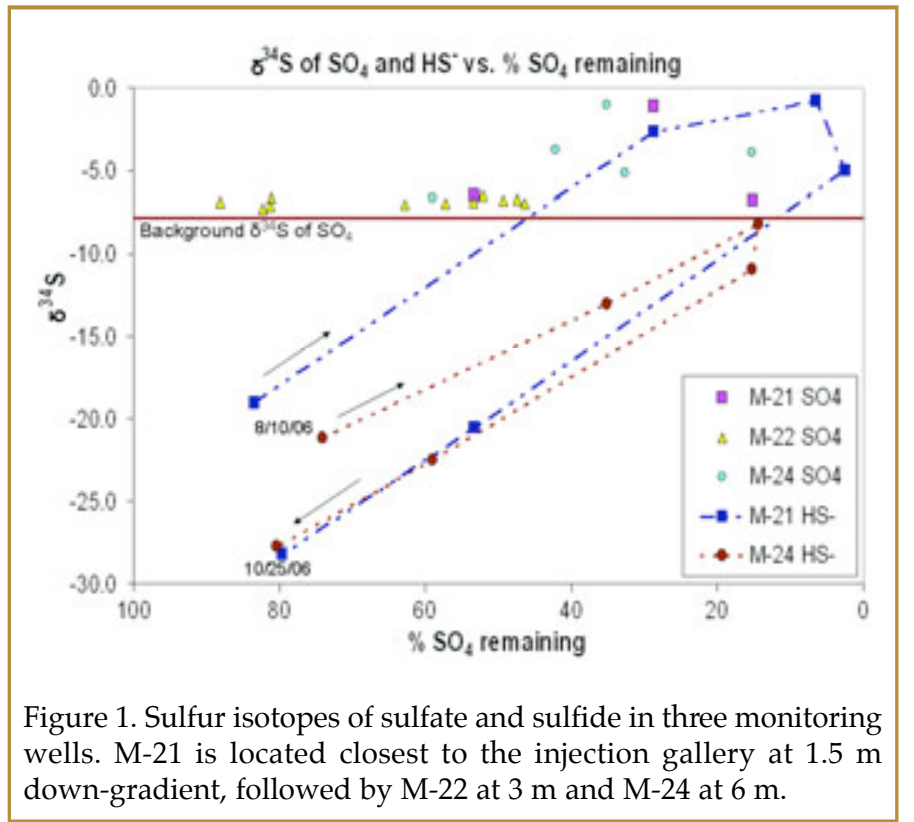

\section{RELATED PUBLICATION}

Druhan, J.L., M.E. Conrad, K.H. Williams, L. N'Guesson, P.E. Long, and S.S. Hubbard, Sulfur isotopes as indicators of bacterial sulfate reduction processes influencing field-scale uranium bioremediation. Env. Science and Technology (submitted), 2008.

\section{ACKNOWLEDGMENTS}

This work was supported by the Director, Office of Science, Office of Biological and Environmental Research, Environmental Remediation Sciences Division, of the U.S. Department of Energy under Contract No. DE-AC0205CH11231.

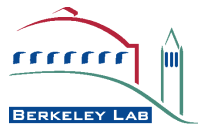




\title{
Tracing 99Tc Contamination at the Hanford Site UsING THE IsOTOPIC COMPOSITION OF NITRATE
}

\author{
John N. Christensen, Mark Conrad, P. Evan Dresel ${ }^{1}$, and Donald J. DePaolo \\ ${ }^{1}$ Pacific Northwest National Laboratory (PNNL) \\ Contact: John N. Christensen, 510/486-6735, jnchristensen@lbl.gov
}

\section{RESEARCH OBJECTIVES}

The Hanford Site in Washington State was used for decades (1940s to the late 1980s) for the production of weapons-grade plutonium, which resulted in significant local radioactive and nonradioactive contamination of the vadose zone and groundwater. Nitrate is a widespread groundwater contaminant at the Hanford Site, with $75 \mathrm{~km}^{2}$ of the aquifer above the $45 \mathrm{ppm}$ EPA drinking water limit, and locally reaching concentrations of over 1,000 $\mathrm{ppm}$. This contamination came from a variety of sources, including radioactive waste leaking from storage tanks, low-activity waste from site operations released to disposal structures in the ground, and rinsing of naturally occurring nitrate from vadose zone soils. Commonly, groundwater nitrate contamination at Hanford is accompanied by ${ }^{99} \mathrm{Tc}$ (technetium 99), a man-made radioactive isotope with a half-life of 214,000 years. In 2005, during the emplacement of a new groundwater monitoring well (W11-25B) near the WMA-T, high ${ }^{99} \mathrm{Tc}$ and nitrate concentrations were found just below the water table. The ${ }^{99} \mathrm{Tc}$ concentrations, up to $180,000 \mathrm{pCi} / \mathrm{L}$, were much higher than previously observed in nearby wells. Because both nitrate and ${ }^{99} \mathrm{Tc}$ have similar mobility in groundwater, we may also, by placing constraints on the source of nitrate contamination, constrain the source of ${ }^{99} \mathrm{Tc}$ contamination. We have developed an isotopic technique for tracing contaminant nitrate, providing such constraints on the source of the otherwise untraceable mono-isotopic technetium.

\section{APPROACH}

The isotopic composition of the nitrogen $\left(\delta^{15} \mathrm{~N}\right)$ and the oxygen $(\delta 18 \mathrm{O})$ in nitrate varies depending on the origin of the nitrate. Our previous research has shown that nitrate associated with synthetic nitric acid, nitrate associated with high-activity tank-related waste, and naturally occurring nitrate in the vadose zone each have their own characteristic isotopic signatures, which can be used to trace sources of nitrate contamination. We analyzed groundwater samples taken at discrete depths below the groundwater table in the well with the very high ${ }^{99} \mathrm{Tc}$ concentrations, as well as groundwater samples from other monitoring wells in the general vicinity, for the isotopic composition of nitrate.

\section{ACCOMPLISHMENTS}

The results of our groundwater nitrate isotopic analyses are presented in Figure 1. Most of the WMA-T groundwater samples (purple circles) form an array stretching from the field representing synthetic nitrate to the field representing natural background nitrate. The high $\delta 18 \mathrm{O}$ end of this array is represented by samples with very high (>900 ppm) nitrate concentrations. Samples at the low $\delta 18 \mathrm{O}$ end of the array have the lowest nitrate concentrations. These observations suggest that samples on this array represent mixing between synthetic nitrate and natural nitrate rinsed from the vadose zone. The depth discrete samples from Well W11-25B form a separate array (green squares), indicating mixing between synthetic nitrate and tank-related waste (with high ${ }^{99} \mathrm{Tc}$ content), possibly associated with a major tank leak in 1973.

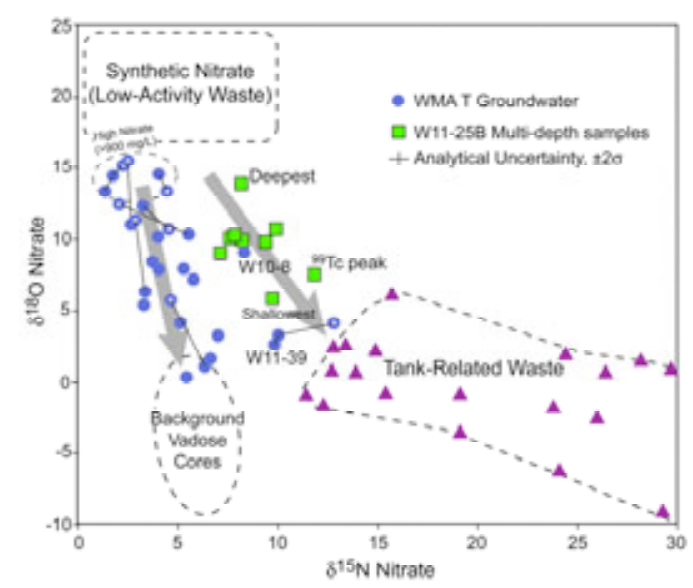

Figure 1. Plot of $\delta^{15} \mathrm{~N}$ vs. $\delta^{18} \mathrm{O}$ for nitrate in groundwater samples from the vicinity of the WMA-T and for the sub-water table depth discrete samples from W11-25B. Additional data, the field for background cores, and data for tank-related waste from contaminated cores from Singleton et al. (2005). The field for synthetic nitrate (representing low-activity waste) is from Kendall (1998) and Amberger and Schmidt (1987). Lines connect samples from the same well taken at different times, with the more recent sample indicated by a white dot.

\section{SIGNIFICANCE OF FINDINGS}

Our results demonstrate the power of isotopic tracking of contaminant nitrate and its co-contaminants, which have provided valuable constraints on the origin of significant ${ }^{99} \mathrm{Tc}$ contamination of groundwater near the WMA-T at the Hanford Site.

\section{RELATED PUBLICATION}

Christensen, J.N., M.E. Conrad, D.J. DePaolo, and P.E. Dresel, Isotopic studies of contaminant transport at the Hanford Site, Washington. LBNL-61935. Vadose Zone Journal 6, 1018-1030, 2007.

\section{ACKNOWLEDGMENTS}

This work was supported through the Hanford Remediation and Closure Science Project, funded through DOE Richland and by the Assistant Secretary of the Office of Environmental Management, Office of Science and Technology, under the Environmental Remediation Sciences Division, of the Department of Energy, Contract No. DEAC02-05CH11231 (Berkeley Lab) and Contract No. DE-AC06-76RL01830 (PNNL).

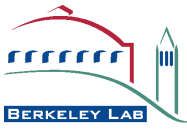




\title{
Induced Polarization Monitoring of Microbial Activity During STIMUlATED SubSURFACE BiOREMEDiATION
}

\author{
Kenneth H. Williams, Susan S. Hubbard, and Jillian F. Banfield
}

Contact: Kenneth H. Williams, 510/701-1089, khwilliams@lbl.gov

\section{RESEARCH OBJECTIVES}

Understanding how microorganisms alter their physical and chemical environment during bioremediation is hindered by our inability to resolve subsurface microbial activity with high spatial resolution. Geophysical methods, such as the induced polarization (IP) technique, have shown promise as sensitive means for delineating regions of stimulated biomineralization. Ongoing research suggests that the IP method may also be sensitive to variations in subsurface geochemical conditions resulting from biological processes, especially when multiple frequencies are utilized. Using an IP monitoring approach, spatiotemporal variations in the development of phase anomalies should reflect the location of active metabolism within aquifer sediments following organic carbon amendment, with the magnitude of the phase response at a given frequency indicative of characteristic metabolic end products, such as aqueous iron and sulfide, or insoluble mineral precipitates.

\section{APPROACH}

Surface IP datasets were acquired at multiple frequencies $(0.125,1$, and $10 \mathrm{~Hz})$ prior to and at multiple times after the injection of acetate into a uranium-contaminated aquifer near Rifle, Colorado. Differences between the phase and magnitude of the measured potentials relative to the injected current determine the frequency-dependent electrical resistivity of the subsurface. Regions exhibiting a strong frequency-dependence (i.e., those exhibiting a large phase shift) are found to correspond to areas where charge migration by electrolytic transfer in the pore fluid is impeded because of a variety of interfacial conduction mechanisms. These include regions where charge transfer changes from electrolytic to electronic (such as in poreblocking mineralized rocks) or where grain surface features impede the normal flow of current carrying ions (such as in clay-bearing zones or other areas of high surface charge density). As a result, reactions that alter the way in which electric charge is conducted through the pore space should lead to observable changes in the measured phase response of sediments accumulating electroactive ions and precipitates.

\section{ACCOMPLISHMENTS}

We have demonstrated the ability of the IP method to monitor microbe-mediated iron and sulfate reduction during acetate amendment of a uranium-contaminated aquifer near Rifle, CO. During IP measurements, spatiotemporal variations in the phase response between applied and measured voltages correlated with changes in groundwater geochemistry indicative of microbial iron and sulfate reduction and sulfide mineral precip-

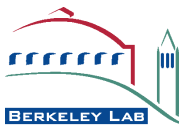
itation. The enhanced sensitivity of the high and low frequency phase responses to accumulated aqueous iron and sulfide, respectively, provide the ability to discriminate the dominant subsurface biogeochemical process. The spectral effect was verified and calibrated using a biostimulated column experiment containing Rifle sediments and groundwater. Sediments and fluids recovered from regions of the field site exhibiting an anomalous phase response were enriched in sorbed $\mathrm{Fe}^{2+}$ and cell-associated 2-4 $\mathrm{nm}$ diameter $\mathrm{FeS}$ nanoparticles. These mineral precipitates and accumulated electroactive ions altered the ability of pore fluids to conduct electrical charge, accounting for the IP response. The results reveal the usefulness of multi-frequency IP measurements for discriminating mineralogical and geochemical changes during stimulated subsurface bioremediation.

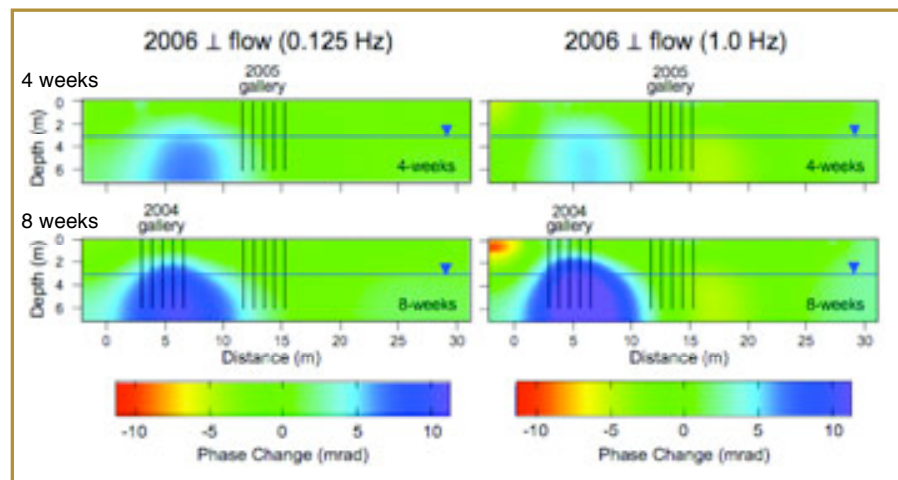

Figure 1. Differential comparison of 2006 surface IP inversion results acquired perpendicular to flow at two frequencies. The results were obtained by subtracting the data obtained after four and eight weeks from the baseline data. The magnitude of the anomalous-phase response was greater at 0.125 than $1 \mathrm{~Hz}$ after four weeks, with the opposite trend observed after eight weeks.

\section{SIGNIFICANCE OF FINDINGS}

These findings suggest that the spectral IP method represents a minimally invasive means for monitoring stimulated microbial activity within aquifer sediments, with frequency-dependent phase anomalies characteristic of iron and sulfate reduction.

\section{RELATED PUBLICATION}

Williams, K.H., A. Kemna, M. Wilkins, J. Druhan, E. Arntzen, L. N'Guessan, P.E. Long, S.S. Hubbard, and J.F. Banfield, Geophysical monitoring of microbial activity during stimulated subsurface bioremediation. Environmental Science \& Technology (in preparation), 2008.

\section{ACKNOWLEDGMENTS}

This work was supported by the Director, Office of Science, Office of Biological and Environmental Research, Environmental Remediation Sciences Division, of the U.S. Department of Energy under Contract No. DE-AC0205CH11231. 


\section{Geophysical Monitoring of Stimulated Subsurface Bioremediation Using the Self-Potential TechniQue}

Kenneth H. Williams, Susan S. Hubbard, and Jillian F. Banfield

Contact: Kenneth H. Williams, 510/701-1089, khwilliams@lbl.gov

\section{RESEARCH OBJECTIVES}

There is growing interest in the use of geophysical methods to track the products of subsurface microbial activity during stimulated subsurface bioremediation. Among such methods, the self-potential (SP) technique has shown promise as an inexpensive yet sensitive means for delineating variations in subsurface geochemical conditions resulting from biological processes. Interpreting the SP response within the context of a galvanic model makes it possible to use time-varying voltage anomalies to monitor geochemical changes induced by the stimulation of microbial activity. As a result, spatiotemporal variations in the onset, sustenance, and relaxation of the SP anomalies should reflect the location of active metabolism within aquifer sediments following organic carbon amendment. Such variations thus offer an indirect means for verifying geochemical conditions conducive to the removal of soluble contaminants, such as uranium.

\section{APPROACH}

Under conditions in which the measurement and reference electrodes are located in geochemically distinct redox environments, SP anomalies have been shown to be the result of electrochemical reactions involving the electrodes themselves. When bridged through a measuring voltmeter, electrodes located in the electrochemically distinct regions constitute a galvanic cell, generating a voltage potential that persists as long as the concentration gradient is maintained. Charge balance is maintained via electrolytic conduction through the pore space, which also acts to complete the overall circuit. Under conditions in which the geochemical environment directly interacts with the electrode surface, the electrode composition will determine the nature of the measured half-cell reaction. As a result, the magnitude of SP anomalies may be quantitatively interpreted in the same manner as voltages that result when using an ion-selective electrode, albeit over a scale of meters rather than millimeters.

\section{ACCOMPLISHMENTS}

We used the SP method to track the onset and persistence of the activity of sulfate-reducing bacteria in a uranium-contaminated aquifer following acetate amendment. Anomalous voltages approaching $900 \mathrm{mV}$ were measured between copper electrodes emplaced within the aquifer sediments and a single $\mathrm{Cu} / \mathrm{CuSO}_{4}$ reference electrode located at the ground surface.
Onset of the voltage anomalies correlated in time with both the accumulation of dissolved sulfide and the removal of uranium from groundwater. The anomalies persisted for 45 days after acetate injection had ceased, indicative of ongoing sulfate reduction. Geochemical data confirm that anomalous SP voltages and continued sulfate reduction correlated with the sustained removal of uranium. Currentvoltage and current-power relationships between measurement and reference electrodes exhibited a galvanic response, with current flow between the two electrodes yielding a steady-state power density of $10 \mathrm{~mW} / \mathrm{m}^{2}$ during the period of sulfate-reduction. Thus, we infer that the SP anomalies resulted from electrochemical differences that developed between geochemically reduced regions and areas having higher oxidation potential. Following the period of sulfate reduction, SP voltages ranged from 500 to $600 \mathrm{mV}$ and were associated with elevated concentrations of ferrous iron in the vicinity of the measurement electrodes. Within 10 days of the voltage decrease following the depletion of sulfide, uranium concentrations rebounded from 0.2 to $0.8 \mu \mathrm{M}$, a level still below the background value of 1.5 $\mu \mathrm{M}$.

\section{SIGNIFICANCE OF FINDINGS}

These findings demonstrate that SP measurements provide a minimally invasive means for monitoring stimulated microbial activity within aquifer sediments and verify that redox conditions remain favorable for the stability of bioimmobilized contaminants, such as uranium.

\section{RELATED PUBLICATION}

Williams, K.H., S.S. Hubbard, and J.F. Banfield, Galvanic interpretation of self-potential signals associated with microbial sulfate-reduction. LBNL-63396. Journal of Geophysical Research-Biogeosciences, 112, 2007.

\section{ACKNOWLEDGMENTS}

This work was supported by the Director, Office of Science, Office of Biological and Environmental Research, Environmental Remediation Sciences Division, of the U.S. Department of Energy under Contract No. DE-AC02-05CH11231.

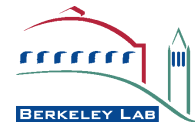




\title{
A Statistical Framework for Monitoring Biogeochemical Transformations Using Time-Lapse GeOPHysical Data
}

\author{
Jinsong Chen and Susan Hubbard \\ Contact: Jinsong Chen, 510/486-6842, jchen@lbl.gov
}

\section{RESEARCH OBJECTIVES}

Bioremediation treatments are used to facilitate reactions that degrade or immobilize contaminants in situ, so that they become less hazardous to human and ecological health. These treatments typically result in biogeochemical transformations, such as the formation of precipitates, gas bubbles, and biofilms. Conventional borehole methods limit our ability to monitor such transformations at sufficiently high spatial resolution at field-relevant scales. Although time-lapse geophysical methods have the potential to provide information at the scale and resolution that help us to better understand bioremediation processes, several obstacles hinder the quantitative use of timelapse geophysical techniques for such purposes. Examples of those challenges include the incomplete understanding of how geophysical attributes respond to biogeochemical transformations, non-uniqueness of relations between the produced biogeochemical transformation products and the recorded geophysical attributes, and scale discrepancies among geophysical and biogeochemical measurements. The goal of this research is to develop a general framework that tackles some of these challenges, and that can be used to provide quantitative estimates of biogeochemical transformations associated with remedial treatments using time-lapse geophysical data. Here, we focus on formulating the framework to estimate a particular transformation that occurs during sulfate reduction processes, using time-lapse seismic and induced polarization datasets.

\section{APPROACH}

We develop state-space Bayesian models and use Markov chain Monte Carlo sampling methods to quantitatively estimate the volume fraction and mean grain-size of iron and zinc sulfides commonly formed as a system is reduced via biostimulation. We use a patchy saturation model to relate the seismic data and the Cole-Cole model, together with empirical relations to relate the induced polarization (IP) data to the precipitate properties. The developed framework is applied to a geophysical dataset collected during a laboratory-scale biostimulation experiment (Williams et al., 2005). Figure 1 illustrates the use of the developed method with the laboratory seismic and IP time-lapse datasets, to estimate the evolution of the iron sulfide precipitates as a function of time after biostimulation was initiated.

\section{ACCOMPLISHMENTS}

The developed methodology has several favorable characteristics: (1) it is integrated, so that we can combine multiple types of geophysical data sets simultaneously; (2) it is flexible, so that we can explore the effects of different types of prior

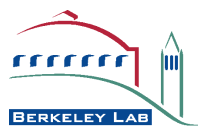
information for the estimation; and (3) it is
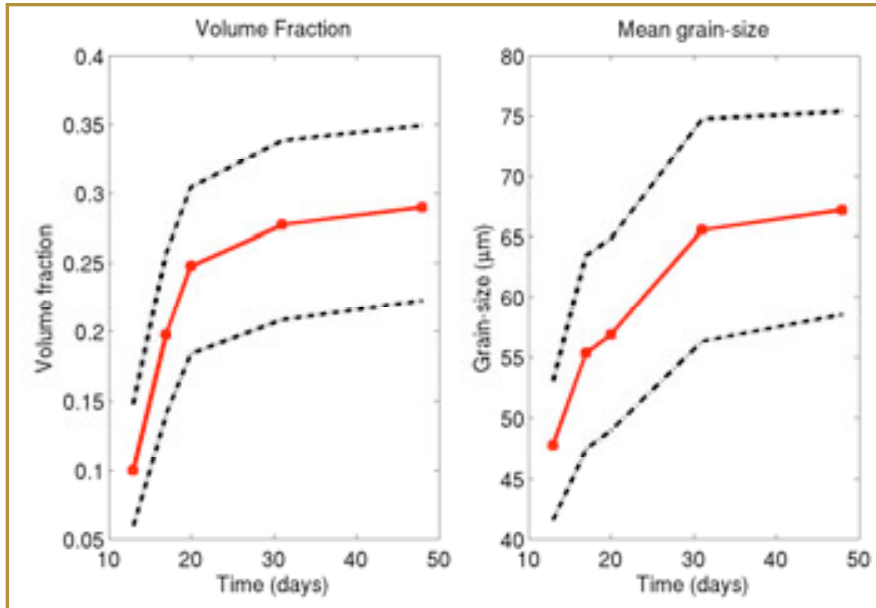

Figure 1. Estimated volume fraction and mean grain-size of precipitates using induced polarization and seismic data. The red lines are the medians of the estimated posterior probability density functions, and the black dashed lines are their corresponding 95\% predictive intervals.

robust, so that we can obtain uncertainty information as well as the estimates of quantities of interest.

\section{SIGNIFICANCE OF FINDINGS}

We have developed the first estimation framework that permits quantitative estimation of biogeochemical transformations using time-lapse geophysical datasets. This advance is expected to significantly improve our ability to remotely monitor changes that occur as a system is remediating, which can be used to guide and assess the efficacy of remedial treatments.

\section{RELATED PUBLICATIONS}

Chen, J., S. Hubbard, K. Williams, S. Pride, and L. Slater, A statistical framework for monitoring the evolution of precipitates associated with contaminant remediation using timelapse geophysical data. Water Resources Research (in preparation), 2008.

Chen, J., S. Hubbard, and A. Kemna, A comparison between deterministic and stochastic inversion methods for ColeCole model parameters using induced polarization data. Geophysics (in press), 2008.

\section{ACKNOWLEDGMENTS}

This work was supported by the Director, Office of Science, Office of Biological and Environmental Research, Environmental Remediation Sciences Division, of the U.S. Department of Energy under Contract No. DE-AC02$05 \mathrm{CH} 11231$. 


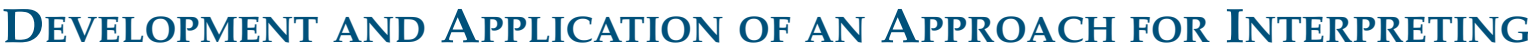 Breakthrough Curves Associated With Complex Tracer Injections \\ Andreas Englert and Susan Hubbard \\ Contact: Andreas Englert, 510/486-6596, alenglert@lbl.gov}

\section{RESEARCH OBJECTIVES}

Bromide breakth rough data were collected in conjunction with a biostimulation experiment that was performed at the $\mathrm{U}(\mathrm{VI})$ contaminated DOE Rifle Site in Colorado. Several factors contributed to a temporal variability in the bromide injection rate (Figure 1a), including changes in injection rate, injection concentrations, and the injection area. To interpret the bromide breakth rough data in terms of hydrological heterogeneity, we needed to account for the temporal variations in the injection rate. The objective of this study was to develop a method for
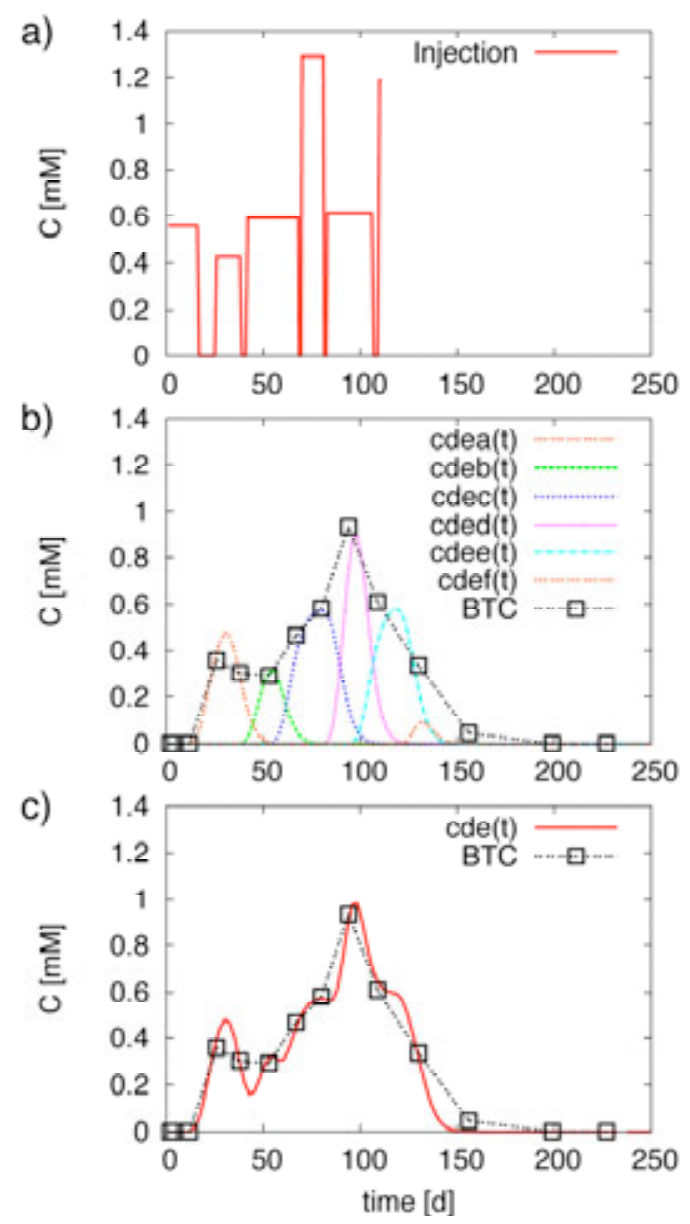

Figure 1. Application of analytical solutions of the one dimensional convection dispersion equation in the presence of a complex injection function: (a) complex injection function, (b) breakthrough curve (BTC) together with CDEs for every step in the injection function, and (c) fit of the superimposed individual CDEs together with the BTC. interpreting bre a th roughcurves associated with complex tracer injection functions, and to apply the methodology to the Rifle $b$ romide tracer test datasets.

\section{APPROACH}

Transport processes in the subsurface can be described by the convection dispersion equation (CDE). For one-dimensional transport processes, the CDE can be solved analytically. Although transport in the subsurface is a three-dimensional process, it can be represented as a bundle of 1-D transport processes. This representation permits the 1-D analytical solution of the CDE to be applied to 3-D field tracer tests. Here, we expand this approach to permit interpretation of complex, temporally variable injection functions based on superposition of several 1-D dimensional convection dispersion equations.

\section{ACCOMPLISHMENTS}

We applied our approach to the Rifle tracer test datasets. For each of the six steps in the injection function (Figure 1a), we invoked a 1-D convection dispersion equation. Figure $1 \mathrm{~b}$ illustrates the application of six different CDEs and their relationship to the measured breakthrough curve (BTC). As is shown in Figure 1c, the superposition approach enabled us to capture the complex features of the breakthrough curve at the Rifle site.

\section{SIGNIFICANCE OF FINDINGS}

Although tracer tests are often accompanied by complex injection functions, these variations are often not taken into account, which can confound interpretation of the hydrological heterogeneity. The developed approach is significant in that it permits the quantitative characterization of transport processes in the presence of complex injection functions.

\section{RELATED PUBLICATION}

Englert, A., S. Hubbard, K. Williams, J. Chen, J. Peterson, A. Kemna, F. Spane, D. Newcomer, and P. Long, Hydrogeophysical field characterization at the DOE Old Rifle site, CO. Eos Trans. AGU, 88(23), Jt. Assem. Suppl., Abstract H31F-05, 2007.

\section{ACKNOWLEDGMENTS}

This work was supported by the Director, Office of Science, Office of Biological and Environmental Research, Environmental Remediation Sciences Division, of the U.S. Department of Energy under Contract No. DE-AC0205CH11231.

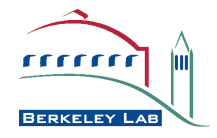




\title{
EXPLORING THE IMPACT OF STIMUlated BioremEdiation ON Flow ChaRACTERISTics
}

\author{
Andreas Englert, Michael Kowalsky, Li Li, and Susan Hubbard \\ Contact: Andreas Englert, 510/486-6596, alenglert@lbl.gov
}

\section{RESEARCH OBJECTIVES}

Many remediation approaches that are being implemented at contaminated subsurface sites induce biogeochemical transformations. Although not yet well quantified at the field scale, these transformations have the potential to alter flow characteristics, which could in turn impact the ability to introduce treatment into the subsurface (via injection) or to withdraw groundwater (via pumping). Here, we interpret bromide tracer test datasets associated with two biostimulation experiments, which were performed in 2002 and 2003 in a flow cell at the DOE Old Rifle Site in Colorado. The biostimulation experiments were intended to facilitate iron and sulfate reduction, which at this site was expected to result in the formation of FeS precipitates. We interpret the datasets in terms of flow velocities, and explore how the velocities vary in space and change over time in response to biogeochemical transformations, such as the generation of precipitates.

\section{APPROACH}

Natural gradient bromide tracer test data were collected during the 2002 and 2003 stimulated bioremediation experiments, using 20 injection wells and 15 monitoring wells. To estimate the heterogeneity of transport characteristics using the collected datasets, we invoked a stream tube model, which assumes that the 3-D transport process can be represented by of a bundle of 1-D convective dispersive processes. This approach permits characterization of the transport process in terms of averaged velocity and dispersivity along individual stream tubes.

\section{ACCOMPLISHMENTS}

The results of the stream tube analysis, shown in Figure 1, indicate that there is clear spatial variability in the velocity fields, and that the field transitions over time towards lower velocities close to the injection wells and higher velocities further away from the injection wells. The changes in velocity appear to be related to the potential biogeochemical transformations associated with the stimulation, such as iron sulfide and calcite precipitation. Comparison of the velocity field with aqueous geochemical data, collected during the stimulation experiments, in turn suggests that the flow characteristics influenced the spatiotemporal distribution of the biogeochemical transformations.

\section{SIGNIFICANCE OF FINDINGS}

The present study is, to our knowledge, one of the first field experiments to illustrate changes in flow characteristics associated with a remedial treatment. Since the heterogeneity of the flow velocity controlled the supply of the biostimulation amendments, the hydrological heterogeneity (and changes thereof) appears to have influenced the spatiotemporal distribution of the biogeochemical transformations. This study suggests that more research is warranted to examine the feedbacks between hydrological heterogeneity and biogeochemical transformations under dynamic conditions.
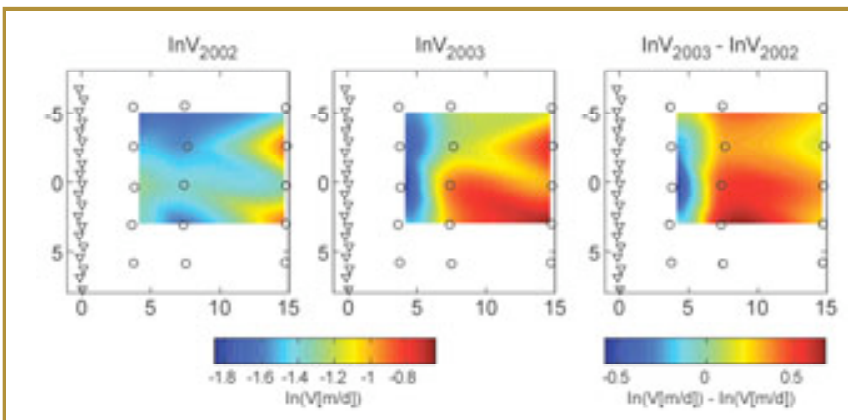

Figure 1. Flow velocity fields associated with the 2002 (left) and 2003 (middle) biostimulation experiment, and the difference between these velocity fields (right), inferred from breakthrough curves at the Old Rifle Site in Colorado. In each of the figures, the injection wells are shown as open triangles, while the downstream injection wells are shown as open circles.

\section{RELATED PUBLICATION}

Englert, A., S. Hubbard, K. Williams, J. Chen, J. Peterson, A. Kemna, F. Spane, D. Newcomer, and P. Long, Hydrogeophysical field characterization at the DOE Old Rifle Site, Colorado. Eos Trans. AGU, 88(23), Jt. Assem. Suppl., Abstract H31F-05, 2007.

\section{ACKNOWLEDGMENTS}

This work was supported by the Director, Office of Science, Office of Biological and Environmental Research, Environmental Remediation Sciences Division, of the U.S. Department of Energy under Contract No. DE-AC0205CH11231.

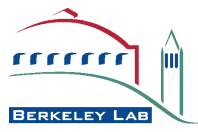




\title{
INVERSION OF TRACER DATA TO QUANTIFY BIOREMEDIATION- INDUCED CHANGES IN A CONTAMINATED AQUifER
}

\author{
Michael B. Kowalsky, Andreas Englert, Li Li, and Susan S. Hubbard
}

Contact: Michael B. Kowalsky, 510/486-7314, mbkowalsky

\section{RESEARCH OBJECTIVES}

Bioremediation techniques aiming to immobilize groundwater contaminants or reduce their toxicity appear to have great potential. There remains, however, a great deal of uncertainty in their application at the field scale, owing to the presence of heterogeneity, both hydrological and geochemical. Hydrological properties are especially important to characterize because they affect how amendments are delivered to contaminated regions of an aquifer. Furthermore, hydrological properties may be in turn affected by biogeochemical transformations during the bioremediation process itself. For example, a remediation technique for immobilizing hexavalent uranium in groundwater involves the stimulation of an iron-reducing bacteria species with an acetate amendment. By-products of the associated reactions, such as precipitation of iron sulfide, could conceivably reduce porosity and permeability. The overall objective of this research is to better understand how transformations associated with bioremediation affect hydrological properties, and vice versa, and whether such changes may be quantified using time-lapse hydrological data.
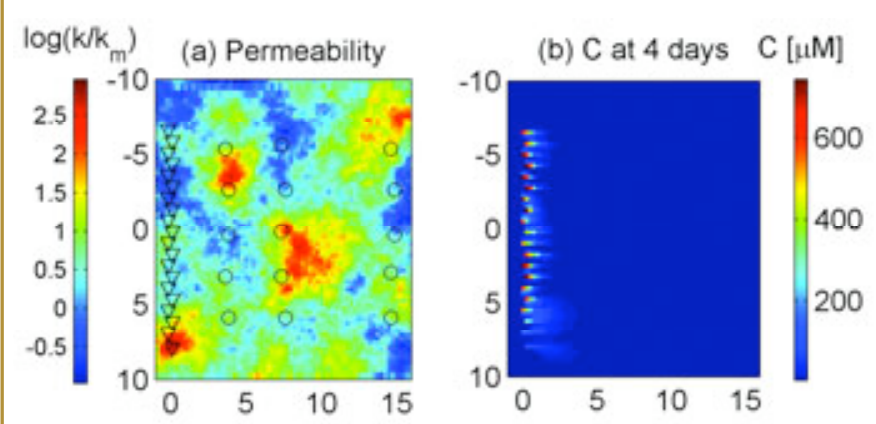

(c) $\mathrm{C}$ at 80 days

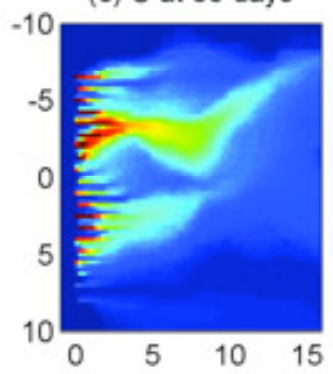

(d) $\mathrm{C}$ at 109 days

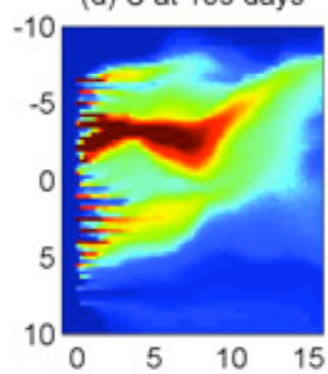

Figure 1. (a) Example permeability distribution obtained by inversion of the 2003 data, and the corresponding simulated distribution of the bromide tracer at several times $(b-d)$. Injection and monitoring wells are represented by triangles and circles, respectively, in (a).

\section{APPROACH}

The Old Rifle UMTRA field site in Western Colorado has been the subject of a number of bioremediation experiments that were performed in conjunction with hydrological, geochemical, and geophysical measurements. We consider data from two experiments that were conducted in 2002 and 2003, respectively, in which an acetate amendment, along with a bromide tracer, were delivered into the shallow unconfined aquifer through an injection gallery consisting of 20 closely spaced wells. For each experiment, bromide concentrations were measured in 15 down-gradient monitoring wells as a function of time. Here we aim to use the measured bromide concentrations to estimate the permeability distributions for both experiments using iTOUGH2, a code that provides inverse modeling capabilities to the flow simulator TOUGH2.

\section{ACCOMPLISHMENTS}

We developed a depth-averaged 2-D model that allows for inverse modeling of the bromide tracer data at the site. Bromide and water are injected into 20 wells according to the measured time-varying rates and accounting for the fluctuating aquifer thickness. A geostatistical parameterization of the permeability field was implemented using parameters that were inferred from borehole data, reducing the number of unknowns in the inverse problem (to 25 permeability values at selected pilot point locations). A permeability distribution obtained by inversion of the 2003 data and the corresponding simulated distribution of bromide at several times are shown in Figure 1, revealing complex tracer transport in the aquifer. Preliminary results indicate that permeability reduction occurred from 2002 to 2003 in some regions, suggesting that the stimulated bioremediation influenced the hydrological properties at the site.

\section{SIGNIFICANCE OF FINDINGS}

The proposed approach is able to capture the spatial heterogeneity of hydrological properties (without requiring direct hydraulic testing), allowing for better understanding of how hydrological heterogeneity changes in response to biogeochemical transformations in an aquifer undergoing bioremediation.

\section{ACKNOWLEDGMENTS}

This work was supported by the Director, Office of Science, Office of Biological and Environmental Research, Environmental Remediation Sciences Division, of the U.S. Department of the U.S. Department of Energy under Contract No. DE-AC02-05CH11231.

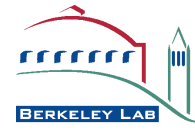




\section{Multiscale Hydrogeophysical Data Integration for Parameterization of Dual-Domain Transport Model at Savannah River Site}

Michael B. Kowalsky, Jinsong Chen, John Peterson, Jens Birkholzer, and Susan S. Hubbard

Contact: Michael B. Kowalsky, 7314, mbkowalsky@lbl.gov

\section{RESEARCH OBJECTIVES}

We are developing a multiscale characterization approach that integrates various data types, collected at differing measu rement scales, to improve transport predictions at the Savannah River Site. The approach uses surface-based and cross-borehole-based geophysical data, and wellbore data to provide input for a site-wide dual-domain transport model. The dual-domain transport model, also being developed in the characterization effort, incorporates the key interactions between mobile and immobile regions that are expected to play a role in long-term plume evolution.

\section{APPROACH}

Analysis of existing characterization data suggests that the study site can be described by two hydrofacies, with one that is on average more mobile than the other, and that these hydrofacies are related to two lithofacies ( $\mathrm{L}=0$ and $\mathrm{L}=1$, respectively). A statistical model is used to estimate the unknown proportion of lithofacies $\left(\mathrm{F}_{\mathrm{I}}\right)$ in each pixel I and the unknown lithofacies type $\left(L_{i}\right)$ in each pixel $i$, given the following data sets: (1) large-scale surface-based geophysical data; (2) small-scale cross-borehole geophysical data; and (3) small-scale wellbore data, such as from geophysical logs, flowmeter logs, or core samples. An example of the data sets and unknowns that we consider is depicted in Figure 1. Using a Bayesian framework, we derive a conditional probability distribution of the unknown variables $\left(\mathrm{F}_{\mathrm{I}}\right.$ and $\left.\mathrm{L}_{\mathrm{i}}\right)$ for all of their respective pixel locations. The resulting distribution depends on (for the example shown in Figure 1) the probability distribution of the large-scale geophysical data given the unknown values of $\mathrm{F}_{\mathrm{I}}$; the probability distribution of $\mathrm{F}_{\mathrm{I}}$ given the unknown values of $\mathrm{L}_{\mathrm{i}}$; and the probability distribution of unknown $L_{i}$ given the small-scale cross-borehole and wellbore data. The Markov-Chain Monte Carlo (MCMC) sampling method is used to efficiently draw samples from the conditional probability distribution, so that the probability distributions of the unknowns can be inferred.

\section{ACCOMPLISHMENTS}

We are currently applying the approach to synthetic data and petrophysical relationships that are representative of site conditions. The approach is being modified, as needed, based on ongoing collection and reduction of multiscale hydrological and geophysical data, and on the evolving characterization objectives that are identified during development of the sitewide transport model. Once refined, the multiscale approach will be used to characterize the relevant field-scale properties at the study site and to parameterize the transport model.

\section{SIGNIFICANCE OF FINDINGS}

This approach expands the scale of hydrogeophysical investigations, traditionally restricted to local-scale regions between closely spaced boreholes ( 10 m), to site-wide scales that are relevant for modeling plume fate and transport.

\section{RELATED PUBLICATIONS}

Chen, J., S. Hubbard, Y. Rubin, C. Murray, E. Roden, and E. Majer, Geochemical characterization using geophysical data and Markov Chain Monte Carlo methods: A case study at the South Oyster Bacterial Transport Site in Virginia. Water Resources Research, 40, W12412, doi: 10.1029/2003WR002883, 2004.

\section{ACKNOWLEDGMENTS}

This work was supported by the Director, Office of Science, Office of Biological and Environmental Research, Environmental Remediation Sciences Division of the U.S. Department of the U.S. Department of Energy under Contract No. DE-AC02-05CH11231.

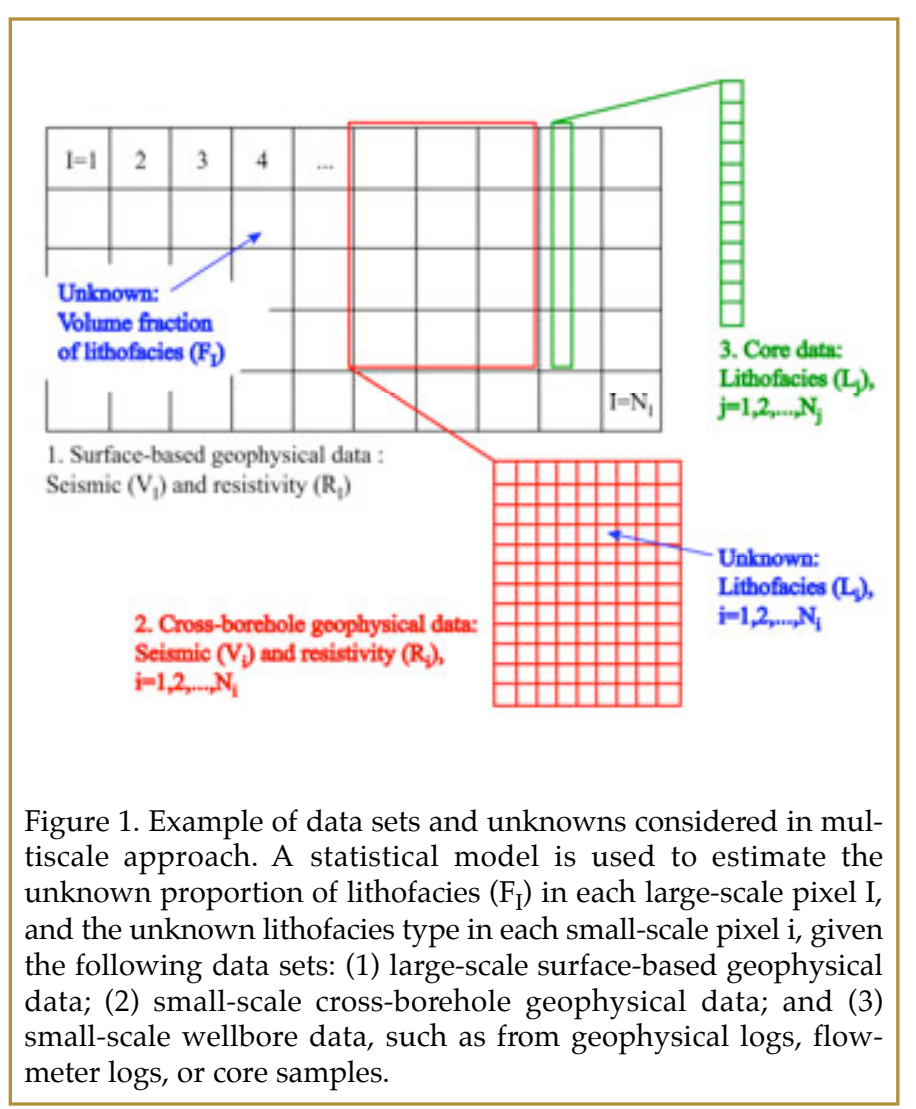

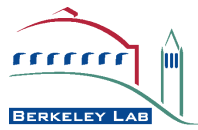




\title{
BERKELEY UXO DISCRIMINATOR (BUD) FOR THE DETECTION AND CHARACTERIZATION OF UXO
}

\author{
E. Gasperikova, J. T. Smith, H. F. Morrison, and A. Becker \\ Contact: Erika Gasperikova, 510-486-4930, egasperikova@lbl.gov
}

\section{RESEARCH OBJECTIVES}

The Berkeley Unexploded-ordnance Discriminator (BUD) is an optimally designed, active electromagnetic system that not only detects but also characterizes unexploded ordnance (UXO). Performance of the BUD system is governed by a target size-depth curve. BUD was designed to detect UXO in the 20 to $155 \mathrm{~mm}$ size range for depths between 0 and $1.5 \mathrm{~m}$, and to characterize them in a depth range from 0 to $1.1 \mathrm{~m}$.

\section{APPROACH}

The system incorporates three orthogonal transmitters and eight pairs of differenced receivers. BUD is mounted on a small cart to assure system mobility. System positioning is provided by state-of-the-art RTK GPS receiver. The system has two modes of operation: (1) search mode, in which BUD moves along a profile and exclusively detects targets in its vicinity, providing target depth and horizontal location, and (2) discrimination mode, in which BUD, stationary above a target, determines (from a single position) three discriminating polarizability responses together with the object location and orientation.

While UXO objects have a single major polarizability coincident with the long axis of the object and two equal transverse polarizabilities, scrap metal has three different principal polarizabilities. This description of the inherent polarizabilities of a target is a major advance in discriminating UXO from irregular scrap metal. Our results clearly show that BUD can resolve the intrinsic polarizabilities of a target, and that there are very clear distinctions between symmetric intact UXO and irregular scrap metal. Moreover, UXO have unique polarizability signatures, such that distinctions can be made between various UXO.

\section{ACCOMPLISHMENTS}

The field surveys at the Yuma Proving Ground in Arizona and Camp Sibert in Alabama showed excellent detection and characterization results within the predicted size-depth range. "Initial blind testing at a controlled site has shown that the BUD performance is significantly above any other system," said Dr. Jeffrey Marqusee, director of the DoD's Environmental Security Technology Certification Program, which administered the Yuma field test.

\section{SIGNIFICANCE OF FINDINGS}

BUD has shown that it can differentiate (in real time) buried UXO from harmless metal, as well as determine the location, size, and shape of such potential explosives. This technology can greatly reduce the time and cost of remediation, and potentially provides a valuable, worldwide humanitarian service. Note that BUD received a 2007 R\&D100 award for technology advances from RED Magazine.

\section{RELATED PUBLICATIONS}

Gasperikova, E., J.T. Smith, H.F. Morrison, and A. Becker, Berkeley UXO discriminator (BUD). LBNL-62263. SAGEEP Proceedings, 1049-1055, 2007.

Smith, J.T., H.F. Morrison, and A. Becker, Optimizing receiver configurations for resolution of equivalent dipole polarizabilities in situ: IEEE Trans. Geosci. Remote Sensing, 43, 1490-1498, 2005.

Smith, J.T., H.F. Morrison, L.R. Doolittle, and H.-W. Tseng, Multi-transmitter null coupled systems for inductive detection and characterization of metallic objects: Journal of Applied Geophysics, 61, 227-234, 2007.

\section{ACKNOWLEDGMENTS}

This research was supported by the Office of Management, Budget, and Evaluation, of the U.S. Department of Energy under Contract No. DE-AC0205CH11231, and the U.S. Department of Defense under the Environmental Security Technology Certification Program Project No. MM-0437 and Contract No. W74RDV61639913.

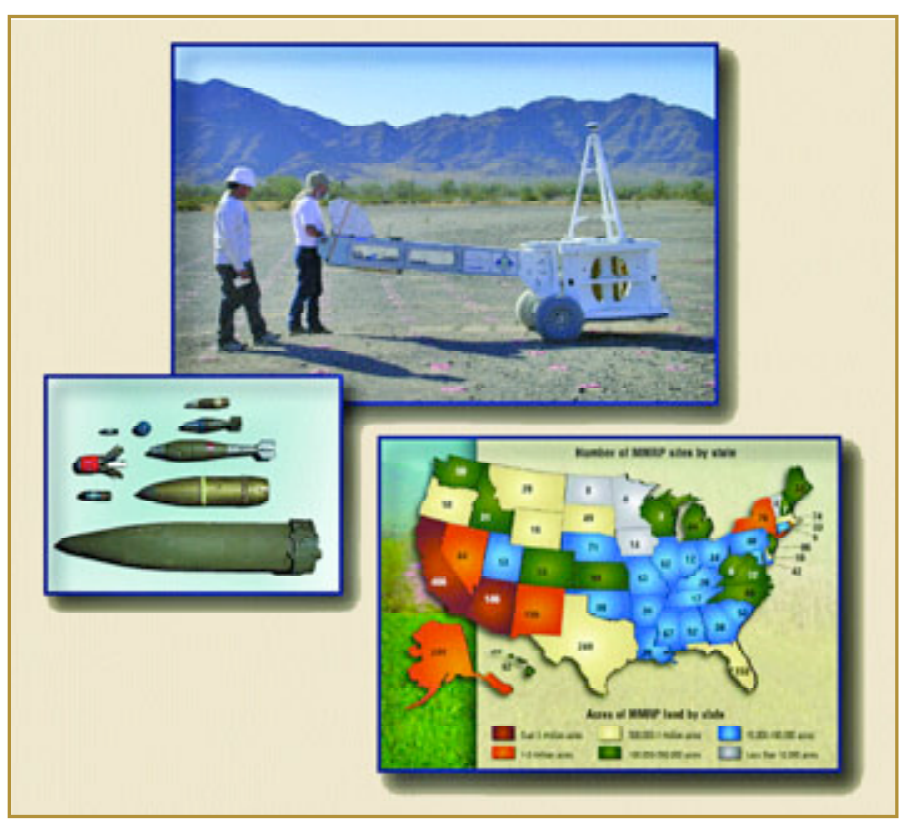

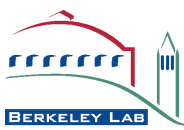




\section{Quantifying and Predicting Reactive Transport of Uranium in Hanford Waste Plumes}

Jiamin Wan, Tetsu Tokunaga, and Yongman Kim

Contact: Jiamin Wan, 510-486-6004, jwan@lbl.gov

\section{RESEARCH OBJECTIVES}

Massive quantities (exceeding 85 tons) of $U$ currently residing in the Hanford vadose zone will threaten groundwater and the accessible environment for generations to come. Understanding the status and mobility of this potentially contaminant $U$ in the vadose zone is extremely difficult because of insufficient historical records, sediment heterogeneity, and complexity of the strongly coupled hydrological and geochemical processes. The overall objective of this research is to identify the dominant geochemical reactions and transport processes between waste streams and sediments occurring during seepage and upon aging. This knowledge is important, because the major reactions and transport processes largely determine the current spatial distribution, speciation, and mobility of $U$ within the Hanford plumes.
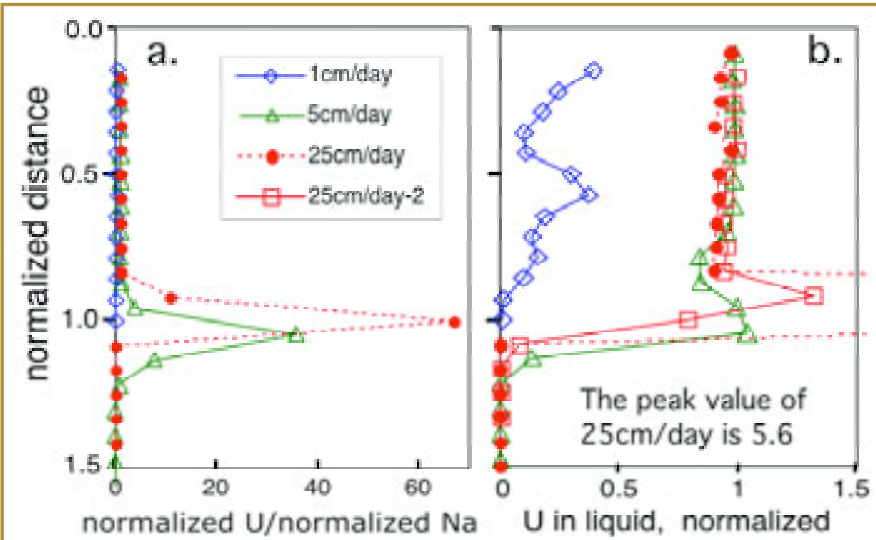

Figure 1. Accelerated U transport and kinetic limitations: (a) Profiles of normalized U:normalized $\mathrm{Na}$, showing $\mathrm{U}$ moved faster than $\mathrm{Na}$ up to 60 times, and $\mathrm{U}$ accumulation at the plume fronts; (b) U profiles normalized to the influent U concentration, showing $\mathrm{U}$ concentrations exceeding their source levels by up to 5-fold at the plume front region.

\section{APPROACH}

To achieve these goals, we constructed a field-processbased column-profiling method. Using this method, we can study flow and geochemical processes simultaneously. We simulated the Tank BX-102 over-filling event: We synthesized the historical tank waste solution containing extremely high levels of $U$ in alkaline brine, and conducted infiltration experiments, under well-defined conditions, as relevant to the field-release event as possible. Flow rate and aging time were chosen as the main variables in these experiments. Analyses of aqueous and solid phases from these experiments were used to obtain profiles of $\mathrm{U}$ concentrations and speciation along the plume paths.

\section{ACCOMPLISHMENTS}

Several processes demonstrated in these laboratory experiments have direct relevance to U-contaminated sediments such as those at the Hanford 200 Area. We found that dramatic pH reduction occurred at the plume fronts. Large peak values of $\mathrm{Ca}^{2+}$ and $\mathrm{Mg}^{2+}$ concentrations were found within plume profiles, resulting from rapid displacement of cation exchange sites by high concentrations of $\mathrm{Na}^{+}$. Maxima in concentrations of these divalent cations, along with $\mathrm{pH}$ minima, are indicators of the distance of waste-plume migration. We observed accelerated U transport (faster than the flow); the measured U concentration maxima at plume fronts exceed their source levels by up to 5 -fold. Thus, peaks in $\mathrm{U}$ concentrations in contaminated sediments can be expected from fast $\mathrm{U}$ transport and accumulation at plume fronts. We also found that transport of $\mathrm{U}$ in waste plumes was strongly dependent on flow rate. Kinetic limitations on sorption and precipitation permitted practically unretarded U transport at higher flow rates.

\section{SIGNIFICANCE OF FINDINGS}

In general, this laboratory study shows that the rate of waste infiltration must have been a primary factor behind the observed deep transport of $U$ in the Hanford vadose zone, and that predictions based on equilibrium $K_{d}$ partitioning of $U$ would greatly underestimate the extent of $U$ migration. This new understanding of $U$ fate and transport within alkaline waste plumes is important for predicting U current spatial distribution, speciation, and future mobility in the Hanford vadose zone.

\section{RELATED PUBLICATIONS}

Wan, J., T. K. Tokunaga, Y. Kim, Z. Wang, R. J. Serne, E. Saiz, and A. Lanzirotti, Effect of saline waste solution infiltration rates on uranium retention and spatial distribution in Hanford sediments. LBNL-63501. Environ. Sci. Technol., 42, 1973-1978, 2008.

\section{ACKNOWLEDGMENTS}

This work was supported by the Director, Office of Science, Office of Biological and Environmental Research, Environmental Remediation Sciences Division, of the U.S. Department of En e rgy under Contract No. DE-AC02-05CH11231.

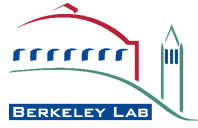




\title{
Mesoscale Biotransformation of UraniUm
}

\author{
Tetsu K. Tokunaga, Yongman Kim, Jiamin Wan, Rebecca Daly, Eoin Brodie, Mary K. Firestone, Terry C. Hazen, \\ Steve Sutton ${ }^{1}$, Matt Newville ${ }^{1}$, and Antonio Lanzirotti ${ }^{1}$ \\ ${ }^{1}$ University of Chicago \\ Contact: Tetsu K. Tokunaga, 510/486-7176, tktokunaga@lbl.gov
}

\section{RESEARCH OBJECTIVES}

Remediation and long-term stewardship of uranium-contaminated sediments and groundwaters are critical problems at a number of DOE facilities and mining sites. Some remediation strategies based on in situ bioreduction of uranium (U) are potentially effective in decreasing $U$ concentrations in g roundwaters. Our current research targets three basic issues concerning $\mathrm{U}$ bioreduction: (1) effects of organic carbon (OC) forms and supply rates on stability of bioreduced $\mathrm{U}$, (2) roles of iron $(\mathrm{Fe})$ and manganese $(\mathrm{Mn})$ oxides as potential $\mathrm{U}$ oxidants in sediments, and (3) microbial community changes in relation to $\mathrm{U}$ redox changes.

\section{APPROACH}

Our studies are being conducted on U-contaminated sediments from the Field Research Center (FRC), Oak Ridge National Laboratory, in flow-through columns. Transformations of $U$ are being tested in FRC sediments supplied with lactate or acetate at rates ranging from 0 to $580 \mathrm{mM}$ (kg sediment) ${ }^{-1}$ year $^{-1}$. Micro$x$-ray absorption spectroscopy is being used for directly determining distributions of $\mathrm{Mn}, \mathrm{Fe}$, and $\mathrm{U}$ oxidation states in sediments at various stages of OC-stimulated bioreduction. Chemical methods are being used for determining concentrations of $\mathrm{Fe}(\mathrm{II}), \mathrm{Fe}(\mathrm{III})$, and $\mathrm{U}$ in sediments and pore waters. We analyzed the structure of the stimulated microbial

supply rate. We identified several factors that point to a residual reactive $\mathrm{Fe}(\mathrm{III})$ fraction in these sediments that likely serves as the terminal electron acceptor for $U$ reoxidation.

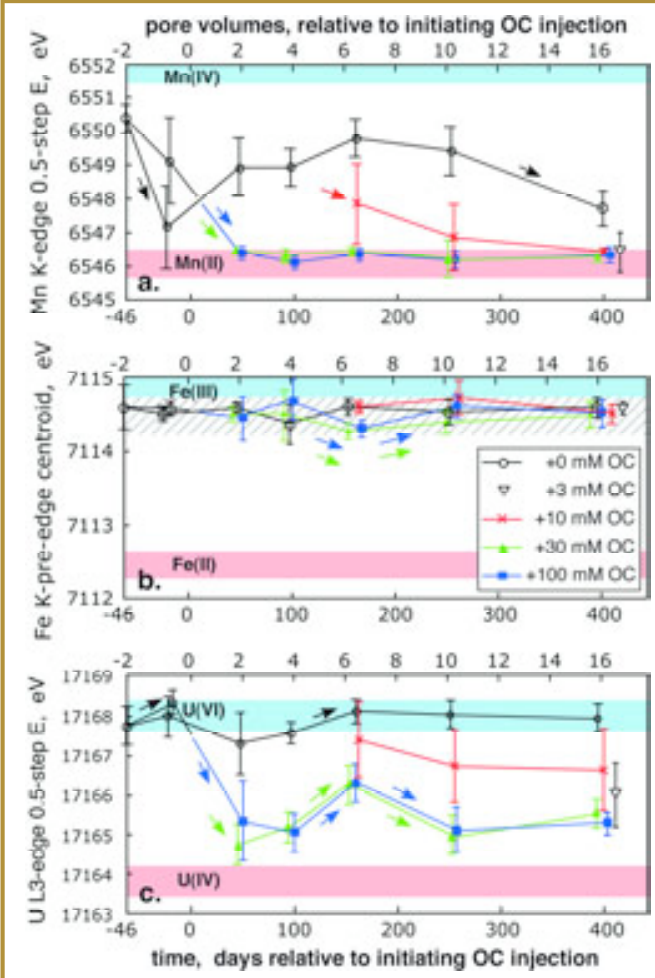

Figure 1. Time trends in average oxidation states, determined by $\mathrm{x}$-ray absorption spectroscopy, of (a) manganese, (b) iron, and (c) uranium in sediments supplied with different concentrations of organic carbon (OC). These results show that, unlike $\mathrm{Mn}$, a large Fe fraction remains unreduced after significant $U$ reduction has occurred. Community analysis using a high-density 16S microarray (16S Phylochip) indicates that OC supply rate is the primary determinant of the bacterial community composition, and that significant shifts in community dynamics occur between the U-reduction and remobilization phases.

\section{SIGNIFICANCE OF FINDINGS}

Organic-carbon-induced $U$ reduction in sediments can exhibit rapid early stage reduction followed by transient $U$ reoxidation. The persistence of high concentrations of Fe(III) supports the possibility that a reactive $\mathrm{Fe}(\mathrm{III})$ fraction can drive $U$ reoxidation under reducing conditions. This work also shows that although reoxidized $U$ is eventually further reduced under a continuous supply of OC, a U(VI) fraction remained after over 400 days of reduction.

\section{RELATED PUBLICATION}

Wan, J., T.K. Tokunaga, E. Brodie, Z. Wang, Z. Zheng, D. Herman, T.C. Hazen, M.K. Firestone, and S.R. Sutton. Reoxidation of bioreduced uranium under reducing conditions. LBNL56058. Environ. Sci. Technol. 39, 6162-6169. 2005. communities in columns receiving ten different OC supply treatments at two time points: first during a phase of net U-reduction, and later during a phase of U-reoxidation and remobilization.

\section{ACCOMPLISHMENTS}

Effluent $U$ concentrations show complex but reproducible dependence on the OC supply rate, consistent with OC oxidation having dual impacts of driving $U$ reduction as well as formation of soluble U(VI)-carbonato complexes. We also found that lactate and acetate have the same geochemical impact on effluent $\mathrm{U}$ concentrations, when compared on the basis of $\mathrm{C}$

\section{ACKNOWLEDGMENTS}

This project is supported by the Director, Office of Science, Office of Biological and Environmental Research, Environmental Remediation Science Program, and Office of Basic Energy Sciences, Division of Chemical Sciences, Geosciences, and Biosciences, of the U. S. Department of Energy under Contract No. DE-AC03-76-SF00098. Use of the Advanced Photon Source and National Synchrotron Light Source was supported by the DOE, Basic Energy Sciences, Office of Science.

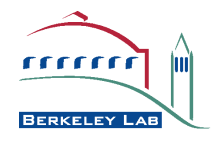




\title{
IN Situ Long-Term Bioimmobilization of CR(VI) IN GROUNDWATER USING $\delta^{13}$ C-LABElED SlOW-REleAse LACTATE
}

\author{
B. Faybishenko, T.C. Hazen, E.L. Brodie, M.S. Conrad, S.S. Hubbard, D. Joyner, S. Borglin, R. Chakraborty, \\ K.H. Williams, J.E. Peterson, J. Chen, T.K. Tokunaga, J. Wan, and M. Firestone \\ Contact: Boris Faybishenko, 510/486-4852, bafaybishenko@lbl.gov
}

\section{RESEARCH OBJECTIVES}

Our objective in this work is to develop a novel approach and provide evidence of long-term, in situ bioimmobilization of $\mathrm{Cr}(\mathrm{VI})$ in groundwater, by transformation of toxic and soluble $\mathrm{Cr}(\mathrm{VI})$ into nontoxic and insoluble $\mathrm{Cr}(\mathrm{III})$ complexes, using a ${ }^{13} \mathrm{C}$-labeled slow release polylactate.

\section{APPROACH AND METHODS}

A $\delta^{13} \mathrm{C}$-labeled slow-release polylactate was injected in the Hanford aquifer through the injection well in August 2004. Monitoring was conducted in one upgradient and three downgradient observation wells, using hydrogeological and geochemical measurements (including stable isotope analysis), geophysical measurements, and microbiological analyses of water samples and sediments.

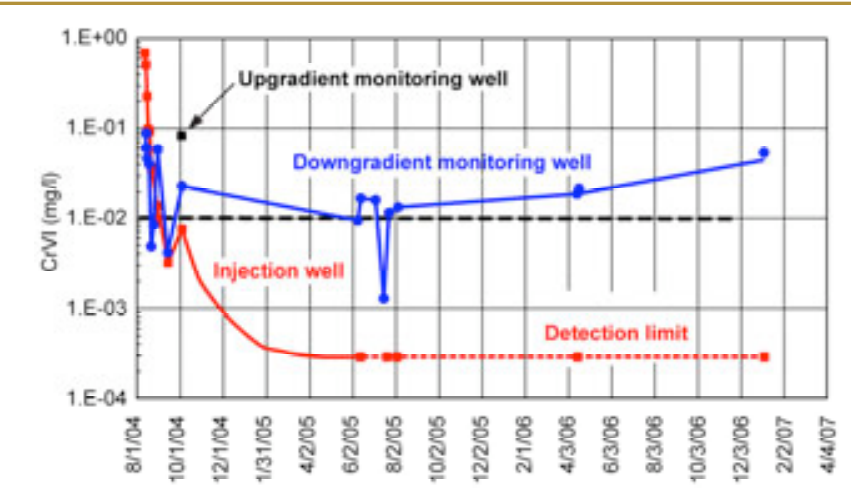

Figure 1. Average $\mathrm{Cr}(\mathrm{VI})$ concentrations in water from the injection and monitoring wells.

\section{ACCOMPLISHMENTS}

A single lactate injection into $\mathrm{Cr}(\mathrm{VI})$-contaminated groundwater stimulated a 2-3 order of magnitude increase in biomass-up to $10^{7}-10^{8}$ cells $\mathrm{ml}^{-1}$. The carbon isotope composition of DIC confirmed the presence of lactate metabolism byproducts. Depletion of competing terminal electron acceptors $\mathrm{O}^{2}$, $\mathrm{NO}^{3-}$, and $\mathrm{SO}_{4}{ }^{2-}$ occurred sequentially, resulting in the creation of dissolved ferrous ion. Sulfate and iron microbial reducers a p parently maintain the presence of $\mathrm{Fe}$ (II) and hydrogen sulfide, subsequently maintaining $\mathrm{Cr}(\mathrm{VI})$ reduction for about 3 years (a) below the drinking water standards in the injection well, and (b) below up-gradient (background) concentration. P revailing mechanisms of $\mathrm{Cr}(\mathrm{VI})$ reduction are direct enzymatic chromate reduction and/or abiotic geochemical processes involving the formation of insoluble complexes of $\mathrm{Cr}(\mathrm{III})$ with Fe(II) or $\mathrm{S}^{-2}$.

\section{SIGNIFICANCE OF FINDINGS}

Using naturally occurring microorganisms to reduce $\mathrm{Cr}(\mathrm{VI})$ is very important for reducing risk of groundwater pollution at contaminated sites. Adding lactate to a contaminated aquifer may offer a low-cost and effective approach to the control of $\mathrm{Cr}(\mathrm{VI})$-contaminated aquifers.

\section{RELATED PUBLICATION}

Faybishenko, B., et, el., In situ long-term bioimmobilization of $\mathrm{CR}(\mathrm{VI})$ in groundwater using slow-release lactate. Environmental Science and Technology (submitted), 2008.

\section{ACKNOWLEDGMENTS}

The project has been conducted jointly with PNNL (P.E. Long, D.R. Newcomer, C.T. Resch, K.J. Cantrell) and Regenesis, LLC. This work was supported by the Director, Office of Science, Office of Biological and Environmental Research, Environmental Remediation Sciences Division, of the U.S. Department of Energy under Contract No. DE-AC0205CH11231.

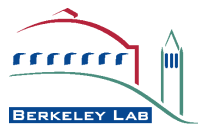




\title{
Modeling Biogeochemical Transport of Metals in Mining-IMPACTED LAKE SEDIMENTS
}

\author{
N. Spycher, S. Sengor ${ }^{1}$, and T. Ginn ${ }^{1}$ \\ ${ }^{1}$ U.C. Davis, Civil and Environmental Engineering Department \\ Contact: N. Spycher, 510-495-2388, nspycher@lbl.gov
}

\section{RESEARCH OBJECTIVES}

The mobility of metals in riverine, estuarine, and lacustrine sediments is affected by complex coupled biotic and abiotic geochemical processes that reflect redox disequilibrium and cannot be modeled using conventional equilibrium-based models. Competing mechanisms in these environments include the mobilization of sorbed metals by reductive dissolution of hydrous ferric oxides and the precipitation of metal sulfides upon reaction with biogenic sulfide. In addition, the toxicity of metals in polluted environments can have a significant effect on microbial activity and thus indirectly affect metal biogeochemical behavior. The objective of this project was to investigate these processes in the sediments of Lake Coeur d'Alene, Idaho, which have been heavily impacted by upstream mining activities, with emphasis on the reactive-diffusive transport of zinc, lead, and copper.

\section{APPROACH}

A conceptual model was developed based primarily on field data and observations reported in the literature. A numerical model was then implemented based upon the PHREEQC code. The model incorporates a multicomponent biotic reaction network, representing multiple terminal electron-accepting processes by a consortium of anaerobic microbial species, via nonlinear kinetics, to capture the electron-acceptor limitations on degradation rates, as well as the inhibition by presence of alternative electron acceptors (Figure 1). In addition, we have constructed and applied "dose-structured" kinetics to quantify the dynamics of microbial populations when their degradation rate is affected by the presence of toxic metals.

\section{ACCOMPLISHMENTS}

The model captures the mobilization of metals initially sorbed onto hydrous ferric oxides, through bacterial reduction of ferric iron near the top of the sediment column, coupled with the precipitation of metal sulfides at depth caused by biogenic sulfide production. Key reactions involve the dissolution of ferrihydrite and precipitation of siderite and iron sulfide. The model captures the observed trends of increased alkalinity, sulfide, iron, and heavy metal concentrations below the sediment-water interface, together with decreasing terminal electron-acceptor concentrations with depth, including the development of anoxic conditions within about a centimeter into the sediments.

\section{SIGNIFICANCE OF FINDINGS}

This effort provides insights on important biogeochemical processes affecting the cycling of metals in Lake Coeur d'Alene and similar metal-impacted lacustrine environments. In particular, simulation results indicate that the relative rates of ferric iron versus sulfate reduction may be an important factor controlling $\mathrm{pH}$ and types of ferrous minerals precipitation at depth. Upon reductive dissolution of hydrous ferric oxides, numerical experiments indicate that a delicate balance takes places between $\mathrm{FeS}$ and $\mathrm{FeCO}_{3}$ precipitation, which compete for aqueous ferrous iron, and the formation of aqueous (bi)sulfide complexes, which compete with the precipitation of FeS and other metal sulfides for biogenic sulfide. This study represents a first attempt, to our knowledge, at modeling the reactive transport of heavy metals in sediments by integrating the coupled effects of microbial Fe-oxide reductive dissolution, biogenic sulfide production, and metal sorption through the use of a full surface complexation model under redox disequilibrium.

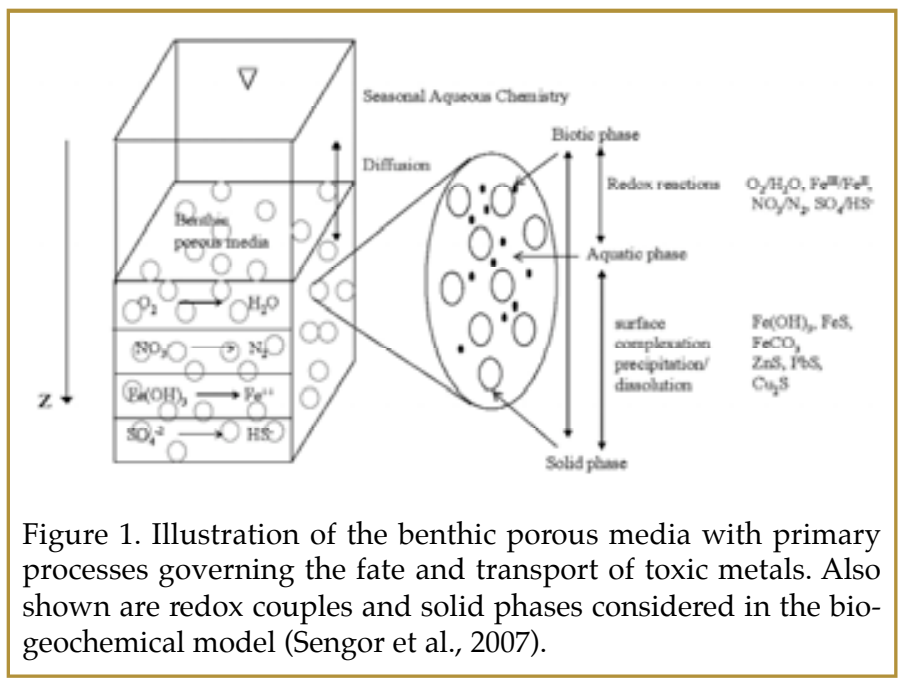

\section{RELATED PUBLICATIONS}

Sengor, S., N. Spycher, T.R. Ginn, R.K. Sani, and B. Peyton B., Biogeochemical reactive-diffusive transport of heavy metals in Lake Coeur d'Alene sediments. Applied Geochemistry, 22, 2569-2594, 2007.

\section{ACKNOWLEDGMENTS}

This work was supported by the National Science Foundation under Grant No. 0420374, "Metal Toxicity and Microbial Consortia: Response to Acid-Mine Drainage at Lake Coeur d'Alene, Idaho" and by the University of California Toxic Substances Research and Training Program.

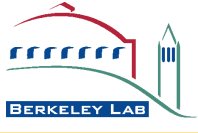




\title{
Mobility of Tritium in EngineERED AND EARTH MATERIAls
}

\author{
Stefan Finsterle, Mark Conrad, Quanlin Zhou, Grace Su, and Karsten Pruess \\ Contact: Stefan Finsterle, 510/486-5205, safinsterle@lbl.gov
}

\section{RESEARCH OBJECTIVES}

Fermi National Accelerator Laboratory (Fermilab) operates a neutrino beamline in an underground facility that includes several research halls, a vacuum decay pipe between the beam target and absorber, and various maintenance structures (drainage and ventilation systems, and a walkway). As a byproduct of beamline operation, tritium is produced in engineered materials (i.e., concrete and steel used for shielding) and the surrounding rock. Once the tritium is generated, it may be contained at the source locations or migrate (in the liquid phase or as tritium vapor) to other regions within the facility. The tritium is collected by the drainage system, aired to the atmosphere, contained in engineered materials, or released to the surrounding subsurface environment. The purpose of the study is to better understand the fate and transport of tritium generated in the facility, in order to be able to (1) evaluate potential environmental impacts, (2) determine tritium sources after the beam is shut off, (3) estimate the effects of the planned increase in beam intensity on tritium production and releases, and (4) help design and optimize mitigation measures.

\section{APPROACH}

The amount and distribution of tritium present in the facility-and its movement through engineered materials, the fractured rock, and the ventilation and drainage systems-are examined by (1) a detailed analysis of the tritium data collected by Fermilab, (2) laboratory diffusion tests on concrete and rock cores, (3) isotopic analyses of pore-water samples, and (4) numerical simulations of water and air flow and tritium transport.

\section{ACCOMPLISHMENTS}

Tritium concentrations in the concrete shielding and the rock formation were measured to determine whether these materials contain sufficient tritium to act as a long-term source. Tritium diffusion coefficients were determined, since they affect the long-term release and uptake of tritium. Using the information from the laboratory experiments and other available data, we developed a model that simulated (1) groundwater flow and tritium transport towards the drainage system, (2) air and water flow through the ventilated walkway and surrounding unsaturated concrete/rock, and (3) tritium vapor transport with the ventilated air and its transfer to liquid water in the surrounding concrete/rock. The main tritium transport mechanisms were identified as (1) advective transport with airflow and mass transfer between gaseous and aqueous phases in underground openings, (2) advective and diffusive transport in concrete and fractured rock, and (3) mass
rorrorrof (III) transfer between tritium vapor in the under-

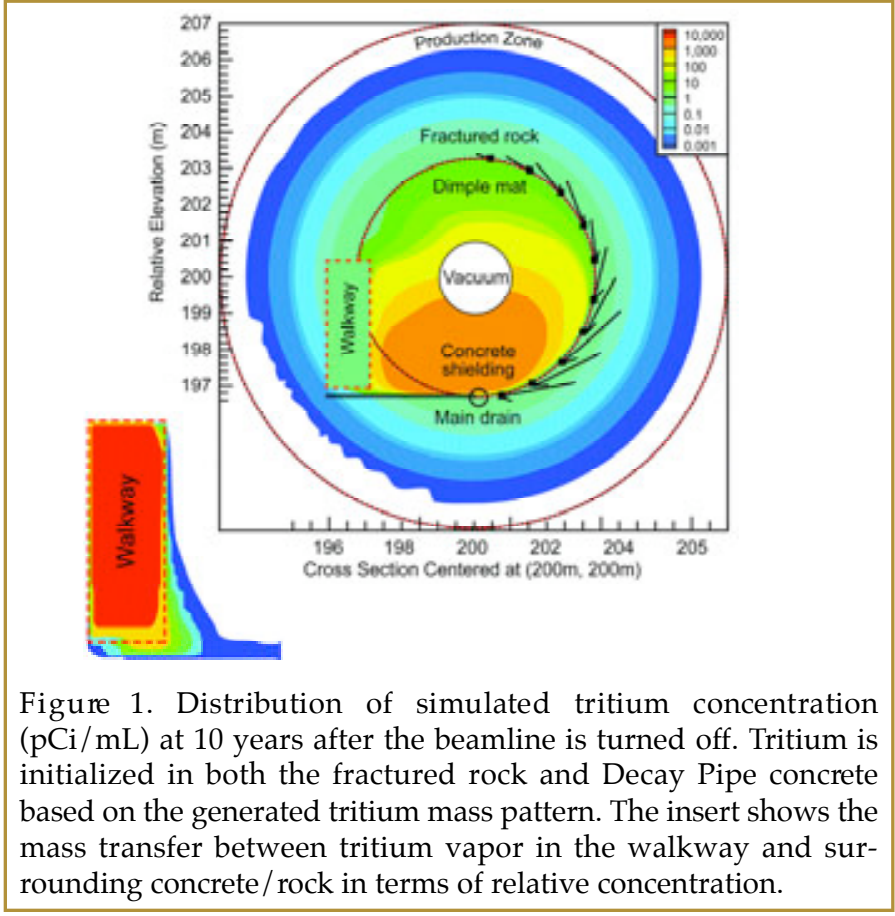

ground openings and concrete or rock walls. The model reproduced the measured tritium concentrations in the drainage water reasonably well.

\section{SIGNIFICANCE OF FINDINGS}

The combination of laboratory experiments, data analyses, and numerical modeling provided a good understanding of the tritium inventory and transport mechanisms that govern the short- and long-term behavior of this radionuclide. The impact of operational changes on tritium accumulation and release from the facility can be studied, allowing Fermilab to implement appropriate design changes and mitigation measures if necessary.

\section{RELATED PUBLICATION}

Finsterle, S., M. Conrad, M. Kennedy, T. Kneafsey, K. Pruess, R. Salve, G. Su., and Q. Zhou, Mobility of tritium in engineered and earth materials at the NuMI Facility, Fermilab. LBNL-61798, Lawrence Berkeley National Laboratory, Berkeley, California, March 2007.

\section{ACKNOWLEDGMENT}

This work was supported by the Fermi National Accelerator Laboratory (Fermilab). The support is provided to Berkeley Lab through the U.S. Department of Energy Contract No. DE-AC02-05CH11231. 


\title{
The Berkeley Water Center's Digital Watershed
}

\author{
N. L. Miller, D.A. Agarwal, B.E. Bargmeyer, C. Gu, G.M. Hornberger, S.S. Hubbard, J.R. Hunt, J. Jin, \\ F. Maggi, C.M. Oldenburg, L. Pan, W.J. Riley, N. Spycher, C.I. Steefel, C. Van Ingen, and T. Xu \\ Contact: Norman L. Miller; 510/495.2374; nlmiller@lbl.gov
}

\section{RESEARCH OBJECTIVES}

It has become increasingly clear that hydrological, meteorological, and biogeochemical processes are coupled and highly dynamic over a wide range of spatial and temporal scales. Understanding these processes with sufficient accuracy, and in the face of anthropogenic and global changes, is a prerequisite to successful management of water resources and ecosystems. Developing such an understanding is predicated on our ability to advance and synthesize relevant but disparate modeling, measurement, analysis, and visualization tools. The Digital Watershed brings together integrative projects that combine engineering, computer science, and ecologic and hydrometeorologic expertise to advance our ability to understand and optimally manage water systems. The Digital Watershed Research Thrust Area currently has three significant projects in its portfolio: Microsoft eScience, NSF/EPA EcoInformatics, and Modeling Framework for Nutrient Cycling.

\section{APPROACH AND ACCOMPLISHMENTS}

Our eScience project is a partnership between computer and physical science leaders that has developed key infrastructure. This infrastructure includes semi-automated data ingest tools, database and associated schema for data storage, data cubes with pre-defined multidimensional viewing, data browsing, and support for multiple data versions. The project has resulted in an Ameriflux and FLUXNET Scientific Data Server that has received high marks from the biogeochemistry community. The server is being applied to the Russian River watershed as part of an analysis of fish sustainability, in partnership with the National Marine Fisheries Service.

The EcoInformatics project is advancing information-system technology in support of environmental action, science, decision making, and program management. Emerging semantic technologies are enabling us to focus on environmental and health concepts, and the relations between concepts, in ways not previously possible. The research, development, demonstration, and education related to this project serves to advance semantics management and semantics services as needed, to improve the integration of - and the interaction with - the enormous and diverse store of structured, semi-structured, and unstructured data related to the environment and health.

The Modeling Framework for nutrient cycling is a joint BWCLBNL Earth Sciences Division project supported through the Laboratory Directed Research and Development program. It focuses on enhancing the ability to simulate local-to-regional-scale water, $\underset{-\cos }{\cos }$ - $\cos 2$
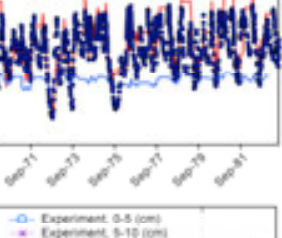

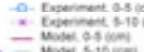
$d-7$ i $\quad$ i i. is: i कर का is 2 Figure 1. CLM and CLMOUGH comparison of the tal and modeled time-evolving average soil water saturation of solutes at $0-5 \mathrm{~cm}$ and $5-10 \mathrm{~cm}$ (Maggi et al. 2007) fif nutrient, and elemental fluxes. The motivation for this effort is the need to predict and interpret the effects of climate, hydrology, and land-use change in areas where such predictions are critical, such as the California Central Valley. The technical objective of the project is the development of a coupled modeling framework (using TOUGH2, TOUGHREACT, and CLM3.0) with reactive biogeochemical transport capabilities (see Figure 1).

\section{SIGNIFICANCE OF FINDINGS}

The eScience and EcoInformatics hydrologyecology-IT projects demonstrate advanced approaches for tackling complex water challenges through integration of advanced data services and IT expertise with hydrological concepts and analysis tools-to efficiently integrate disparate waterrelated datasets distributed over various scales and forms, in order to test hypotheses regarding water balance, water quality, and ecosystem science questions.

The Modeling Framework for Nutrient Cycling is being used to address the effects of regional climate change on the dynamics of the nitrogen cycle in the Central Valley. This includes simulations of climate forcing on infiltration, vegetation dynamics, microbial processes, chemical reactions, and chemical transport within the near-surface, vadose, and saturated zones. The expected outcome of simulation studies with the new code is a better understanding of water, energy, and nutrient cycling and their changes under various future climate, landuse, and hydrologic scenarios.

\section{RELATED PUBLICATIONS}

Gu, C., F. Maggi, G.M. Hornberger, W.J. Riley, R.T. Venterea, C.M. Oldenburg, N.L. Miller, N. Spycher, and T. Xu, Nitrogen losses from different $\mathrm{N}$ sources induced by fertilizer application. Water Resources Research (in review), 2008.

Maggi, F., C. Gu, W.J. Riley, R.T. Venterea, G. M. Hornberger, T. Xu, N. Spycher, C.I. Steefel, N.L. Miller, and C.M. Oldenburg, Mechanistic modeling of biogeochemical nitrogen cycling: Model development and application in an agricultural system. Journal of Geo. Res. - Biosciences, 113, 2008.

Pan, L., J. Jin, N.L. Miller, Y-S Wu, and G.S. Bodvarsson, Coupling TOUGH2 with CLM3: Developing a coupled land surface and subsurface model. LBNL61018, Vadose Zone Journal (in press), 2007.

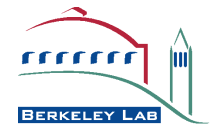




\title{
Coupled Modeling of Hydrology, Nutrient Cycling, ANd Vegetation: Applications to Agricultural Fields
}

\author{
F. Maggi, C. Gu, W.J. Riley, T. Xu, C.I. Steefel, N.L. Miller, and C.M. Oldenburg \\ Contact: C.M. Oldenburg, 510/486-7419, CMOldenburg@lbl.gov
}

\section{RESEARCH OBJECTIVES}

The biogeochemical nitrogen cycle and $\mathrm{NO}, \mathrm{N}_{2} \mathrm{O}$, and $\mathrm{CO}_{2}$ gas production in nitrogen-enriched agricultural fields are mediated by several soil microbial populations, the hydrological cycle, plant dynamics, and climate. Understanding the release of $\mathrm{NO}, \mathrm{N}_{2} \mathrm{O}$, and $\mathrm{CO}_{2}$ from the soil surface to the atmosphere is a key factor in controlling greenhouse gas emissions, and assumes ever greater importance in view of the expected increase in biofuel, food, and fiber production. The objective of this research is to develop a mechanistic model for $\mathrm{N}$ cycling and losses from agricultural fields, and to investigate the effects on $\mathrm{N}$ cycling of irrigation and fertilizer amount, type, and depth of application.

\section{APPROACH}

We have developed a mechanistic model (TOUGHREACT-N) based on TOUGH2 and TOUGHREACT for various nitrification and denitrification pathways, multiple microbial biomass dynamics, hydrological dynamics, and chemical reactions at local equilibrium and under kinetic control. The soil column is represented in a 1-D framework, with hydraulic properties described by a water tension/saturation model. Biotic and abiotic reactions are assumed to follow Michaelis-Menten kinetics, while biomass is assumed to follow multiple Monod growth kinetics accounting for electron donor, electron acceptor, and inhibitor concentrations. Water flow is modeled with the Darcy-Richards equation, and advective and diffusive tracer transport is modeled in both gaseous and liquid phases.

\section{ACCOMPLISHMENTS}

We have applied TOUGHREACT-N to an agricultural field and tested its performance against measurements of soil moisture, $\mathrm{pH}, \mathrm{NH}_{4}^{+}, \mathrm{NO}_{2}{ }^{-}$, and $\mathrm{NO}_{3}{ }^{-}$ion concentrations, and $\mathrm{NO}$, $\mathrm{N}_{2} \mathrm{O}$, and $\mathrm{CO}_{2}$ gas emissions from the soil. Good agreement between field observations and model predictions was found for all these quantities. We have been able to estimate gas emissions, solute leaching of several nitrogen species, and the vertical distribution of nitrifier and denitrifier populations. Finally, we have carried out a comprehensive analysis of gaseous emissions and solute-leaching fractionation and losses for various fertilization and irrigation practices.

\section{SIGNIFICANCE OF FINDINGS}

In contrast to the predictions of nonmechanistic, coarse-scale models, all losses nonlinearly increase with fertilizer and water application amount, and with fertilizer application depth (Figure 1). Different fertilizer types have resulted in substantially different
N-loss patterns over time, and these patterns depend strongly on soil type. Our results suggest revision of the general assessment of soil $\mathrm{N}$ balances and $\mathrm{N}$ loss estimates from field to watershed scales.

\section{RELATED PUBLICATIONS}

Maggi, F., C. Gu, W.J. Riley, G.M. Hornberger, R.T. Venterea, T. Xu, N. Spycher, C. Steefel, N.L. Miller, and C.M. Oldenburg, Mechanistic modeling of biogeochemical nitrogen cycling: model development and application in an agricultural system, JGR - Biogeosciences, 13, 2008.

Gu, C., F. Maggi, W. J. Riley, G.M. Hornberger, R.T. Venterea, T. $\mathrm{Xu}, \mathrm{N}$. Spycher, C. Steefel, N.L. Miller, and C.M. Oldenburg, Nitrogen losses from different $\mathrm{N}$ sources induced by fertilizer application. Water Resources Research (in review), 2008.

\section{ACKNOWLEDGMENT}

This work was supported by Laboratory Directed Research and Development (LDRD) funding from Berkeley Lab, provided by the Director, Office of Science, of the U.S. Department of Energy under Contract No. DE-AC02-05CH11231. This research was carried out in collaboration with the Berkeley Water Center.
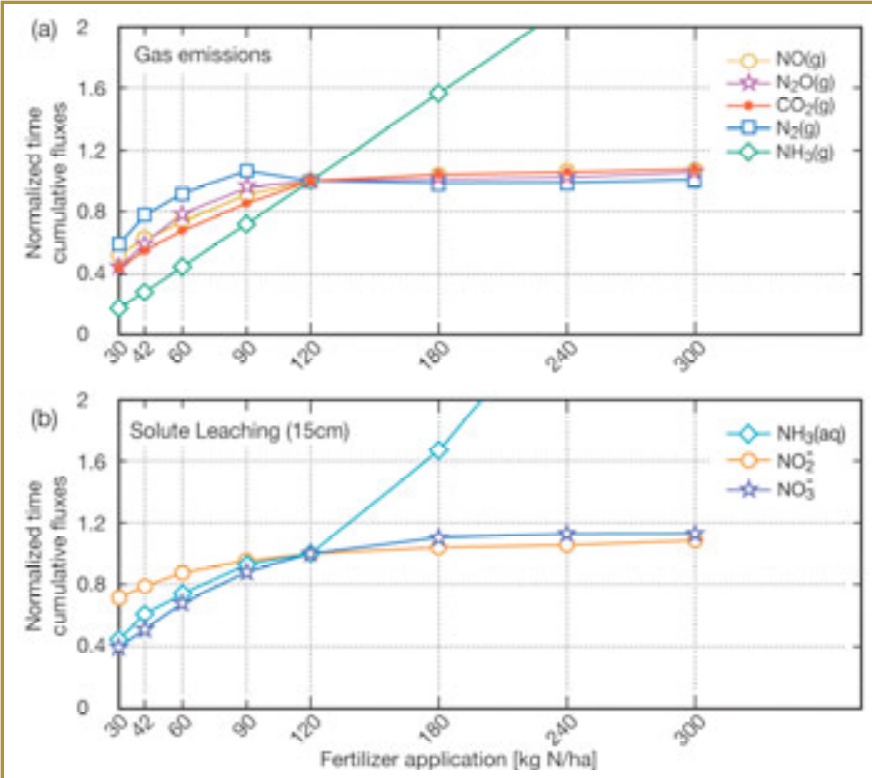

Figure 1. (a) Cumulative normalized gas fluxes and (b) leachate fluxes measured at $15 \mathrm{~cm}$ depth as functions of fertilizer amount. Fluxes are normalized to those corresponding to a fertilizer application of $120 \mathrm{~kg} \mathrm{~N} \mathrm{ha}^{-1}$.

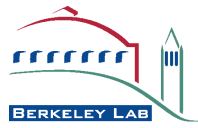




\title{
Comparison of Two State-OF-The-Art Remote-Sensing TechniQues for Development of Improved Wetland-Drainage Management Practices
}

\author{
Nigel W.T. Quinn and Josephine R. Burns \\ Contact: Nigel W.T. Quinn, 510/486-7056, nwquinn@lbl.gov
}

\section{RESEARCH OBJECTIVES}

In response to the looming threat of future water shortages in California, the Department of Interior has required that water contractors develop water conservation plans. The criteria for agricultural and urban water contractors are well established, but there are no equivalent criteria available for seasonally managed wetlands. The ability to estimate water and salinity balances from the 170,000 acres of managed seasonal wetlands in the San Joaquin Basin of California is also critical to management of salt loads in wetland drainage, to improve compliance with stringent Environmental Protection Agency (EPA) mandated water quality objectives for salinity. There is a dearth of data and of analytical tools to quantify the relationships between wetland water and drainage management and ecological health.

\section{APPROACH}

The most important factors in developing wetland water and drainage management practices are estimates of the composition of wetland moist-soil-plant vegetation, and the water required to meet evap-

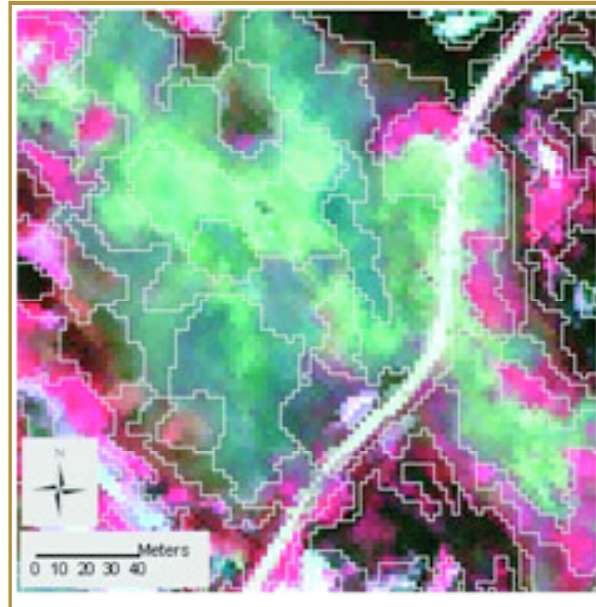

Figure 1. Polygon-based segmentation of high-resolution multispectral imagery of seasonal wetland vegetation into self-similar objects classes. An overall mapping accuracy of $60 \%$ was achieved, with slightly better overall accuracy achieved using the pixel-based methodology. However, future improved utilization of the information present in high-resolution imagery obtained at different dates, and improved stratification of data collection across land-cover classes, will likely favor the polygon-based method. Characteristic of the complexity inherent in these wetlands, landcover classes, such as the important waterfowl food source Crypsis schoenoides (swamp timothy), were mapped at an accuracy approaching $80 \%$ when combined with a spectrally similar land-cover class Scirpus marititimus (alkali bulrush).

\section{SIGNIFICANCE OF FINDINGS}

The results of this research are encouraging for future efforts to map seasonal wetlands throughout the United States. Remote-sensing techniques, such as those compared in this study, make it possible to develop quantitative vegetation assessments from previously unmapped areas that would otherwise be inaccessible or otranspiration losses of the various species of moist soil plants. Polygon-based E-Cognition software-which uses spectral and shape characteristics of the raw high-resolution imagery to separate pixels into self-similar landscape objects-and pixelbased ERDAS imaging software were compared in their ability to accurately map wetland vegetation. A maximum likelihood classifier was selected for analysis of the images. The technique requires the input of training data used to define statistically based spectral bounds for each class. Training areas were derived from ground-truth points by incorporating field data that described the size and shape of the patch within each wetland impoundment, using a modified version of the California Native Plant Society's Rapid Assessment Protocol. Accuracy assessment was performed using $50 \%$ of the ground-truth data not used for the development of spectral signatures.

\section{ACCOMPLISHMENTS}

Maps of seasonal wetland moist-soil plants in the San Joaquin Basin were developed with a combination of pixel- and polygonbased image processing techniques, using 2-meter Quickbird imagery flown during April, May, and June 2005. Twenty-six different plant communities were represented in a total of 20 land cover prohibitively costly to survey. Accurate maps of wetland vegetation will lead to the first realistic estimates of regional wetland seasonal evaporation and evapotranspiration-which are important first steps in developing improved water and drainage management practices. Future improvements to the methods developed in this research, using even finer resolution imagery and greater overflight frequency, will be sought through collaborative ventures with the Grassland Water District, the California Department of Fish and Game, and the California Waterfowl Association.

\section{RELATED PUBLICATIONS}

Quinn, N.W.T., and W. Mark Hanna, Real-time adaptive management of seasonal wetlands to improve water quality in the San Joaquin River. Advances in Environmental Research, 5, 309-317, Elsevier Science Ltd., 2002.

\section{ACKNOWLEDGMENTS}

We are grateful for the support of the Grassland Water District and the California Department of Fish and Game. Funding was provided by the State Water Resources Control Board and the U.S. Bureau of Reclamation.

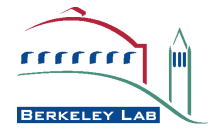




\title{
IMPACTS OF Delayed DraWdown ON AQUATIC Biota AND Water Quality in Seasonal Wetlands
}

\author{
Tryg J. Lundquist, Kyle N. Poole, and Nigel W.T. Quinn
}

Contact: Tryg J. Lundquist, 510/486-4715, tjlundquist@lbl.gov

\section{RESEARCH OBJECTIVES}

The 178,000-acre Grassland Ecological Area in California's San Joaquin Valley provides overwintering habitat to waterfowl on the Pacific Flyway. The major management activity is the fall flooding and spring drawdown of wetlands, timed to optimize the availability of forage vegetation and invertebrates for ducks and shorebirds. Wetland drainage contains elevated concentrations of salt, boron, and nutrients, increasing the frequency of water-quality violations in the San Joaquin River (SJR) during dry years. Compliance with SJR water-quality objectives can be achieved by timing wetland drawdown to coincide with the increased assimilative capacity in the river during mid-March to mid-April, when reservoir releases are increased to aid salmon migration.

The present research is part of a large-scale, multiyear investigation by several institutions on the impacts of delayed drawdown on wetland ecology and water quality. The focus of the present research is the algae and invertebrate populations and their effects on water quality. The unknowns addressed are (1) the concentrations of suspended microalgae resulting from delayed drawdown; (2) the relative importance of factors that limit microalgae concentrations, such as invertebrate grazing, nutrients, and turbidity; (3) changes in nutrient and organic matter concentrations in the drainage; and (4) changes in planktonic and benthic invertebrate densities.

\section{APPROACH}

To determine the effects of delayed drawdown, three matched pairs of wetland basins (20-100 acres each) were selected for study within the Grassland Ecological Area. In each pair, one wetland has been operated with the traditional March drawdown, while for the other, drawdown has been delayed a month. Water and soil samples have been collected during the flooded period at the inlet and outlet weirs and along transects. The water quality tests have included suspended solids, nitrogen and phosphorus, total organic carbon, alkalinity, and turbidity. Algae and invertebrates have been enumerated and identified to the genus level.

\section{ACCOMPLISHMENTS}

The sampling for the first year of the study was conducted during February-April 2007. Suspended microalgae were predominantly chorophytes and diatoms rather than cyanobacteria, and zooplankton were predominantly algae-grazing water fleas (Daphnia). Preliminary analysis shows that despite a general increase in the density of Daphnia during the delayed drawdown, microalgae concentrations still increased substantially in two of the three wetlands $(150 \%$ and $330 \%$ increases reaching up to $39 \mathrm{mg} / \mathrm{L}$ volatile suspended solids), whereas the concentration declined $47 \%$ in the third wetland. The reason for this decline has yet to be determined. Additional analysis and a second year of sampling is planned.

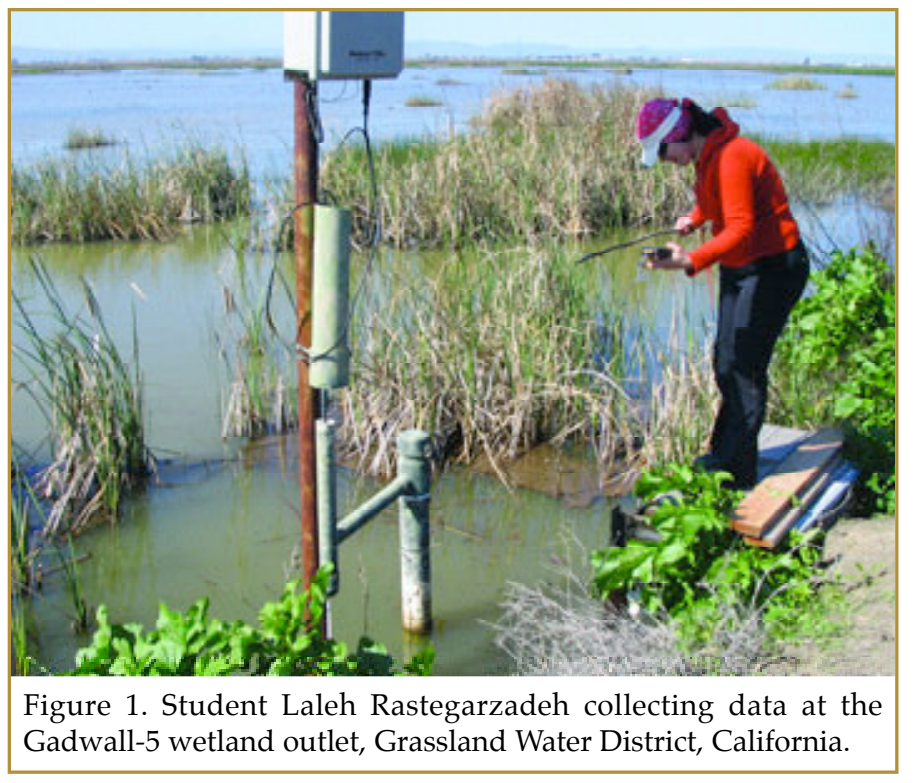

\section{SIGNIFICANCE OF FINDINGS}

The main purpose of delayed drawdown is to increase the dilution of the wetland drainage salinity in the SJR. The apparent secondary effect of increased invertebrate densities should improve shorebird forage opportunities, but concomitant increases in suspended algae biomass lead to greater organic matter discharges. These competing factors will be considered by wetland managers and regulators as they work to protect wildlife and societal uses of the San Joaquin Valley's water resources.

\section{RELATED PUBLICATIONS}

Stringfellow, W. T., and N.W.T. Quinn, Discriminating between west-side sources of nutrients and organic carbon contributing to algal growth and oxygen demand in the San Joaquin River. LBNL-51166, Lawrence Berkeley National Laboratory, Berkeley, Calif, 2002.

\section{ACKNOWLEDGMENTS}

We are grateful for the assistance of Refuge Supervisor John Beam and Ricardo Ortega, both of the California Department of Fish and Game. Funding is provided by the UC Salinity/Drainage Program.

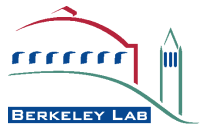




\section{Toward Understanding the Relationship Between Groundwater and Hydroclimate over the California Merced River Basin}

Jiming Jin and Norman L. Miller

Contact: Jiming Jin, 510/486-7551, JimingJin@lbl.gov

\section{RESEARCH OBJECTIVES}

Groundwater is a major source of fresh water resources in the western United States. Understanding and forecasting groundwater level changes are essential to the region's well being. The objective of this study is to quantitatively investigate the relationship between the groundwater level and climate variation over the Merced watershed.

\section{APPROACH}

This study was performed using the Single Column Climate Model (SCCM) developed by the National Center for

$\begin{array}{lcccc} & \text { Winter } & \text { Spring } & \text { Summer } & \text { Fall } \\ \text { P } & 0.38 & 0.39 & 0.45 & 0.16 \\ \text { E } & 0.10 & -0.42 & 0.45 & 0.48 \\ \text { R } & 0.30 & 0.52 & 0.15 & 0.58 \\ \text { SWE } & 0.54 & 0.38 & -0.23 & -0.22 \\ \text { TG } & 0.12 & -0.51 & -0.36 & 0.05\end{array}$

Table 1. Correlations coefficients between water table and precipitation $(\mathrm{P})$, evaporation $(\mathrm{E})$, surface temperature (TG), streamflow (R), and SWE for the 55-year period of 1948-2002. The numbers in italics indicate that the correlation coefficients do not pass the $99 \%$ significance test. (lower) water table produces stronger (weaker) evaporation, which significantly increases (decreases) precipitation. Meanwhile, the stronger (weaker) evaporation results in colder (warmer) surface. The water table in the fall has the same influence on evaporation as in summer, but it is also positively correlated with runoff. However, a relationship between precipitation and the water table is not found in this season, because the impact of surface and shallow subsurface runoff on the water table is very dominant.
Atmospheric Research (NCAR). In SCCM, 18 vertical sigma layers are configured from the surface to the top of the atmosphere, at which the pressure is $1.8 \mathrm{hPa}$. The study domain has 24 grid cells at approximately $15 \mathrm{~km}$ resolution, which covers the entire Merced watershed, and SCCM ran independently at each grid cell. Reanalysis data developed by the National Centers for Environmental Prediction (NCEP)/NCAR were used in the SSCM for the initial and lateral conditions, with the lateral conditions updated monthly. A 55-year simulation was performed over the period of 1948-2002 at each grid cell.

\section{ACCOMPLISHMENTS}

The statistical analysis based on the SCCM simulations (Table 1) indicates that the water table is positively correlated with precipitation and snow water equivalent (SWE) during winter. A similar correlation for precipitation and SWE is also seen in spring. In addition, the colder surface is found to lift the water table by suppressing evaporation in this season, while the warmer surface lowers the water table by increasing evaporation. Meanwhile, a positive correlation between water table and runoff is very significant in spring. In summer, the higher

\section{FINDINGS}

It is shown that the water table is highly correlated to the climate change in all four seasons, but the processes with high correlations are different in different seasons (Table 1). The strongest relationship between the water table and climate change is found in the spring season for this region, indicating that the land-surface processes are most actively interacting with the atmosphere during the this season, as compared to the other three seasons.

\section{RELATED PUBLICATIONS}

Pan, L., J. Jin, N.L. Miller, Y.-S. Wu, and G. Bodvarsson, Modeling hydraulic responses to meteorological forcing: From canopy to aquifer. Vadose Zone Journal, 7 (1), 2008.

\section{ACKNOWLEDGMENTS}

Support for this work is provided by the California Energy Commission under Grant 500-02-004. Work performed at Lawrence Berkeley National Laboratory is supported under Contract No. DE-AC02-05CH11231.

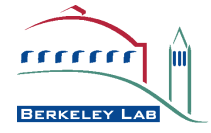




\title{
Drought Analysis of THE \\ California Central Valley Surface-Groundwater Conveyance Sysytem
}

\author{
Norman L. Miller, Larry L. Dale, Sebastian Vicuna, Charles Brush1, John Dogrul1, and Tariq Kadir ${ }^{1}$ \\ ${ }^{1}$ California Department of Water Resources, Modeling Branch \\ Contact: Norman L. Miller; 510/495.2374; nlmiller@lbl.gov
}

\section{RESEARCH OBJECTIVES}

To provide water-resources decision makers with a better understanding of the consequences of persistent droughts, we have developed a series of numerical investigations to determine system behavior and economic impacts under a range of prescribed drought conditions. The objectives of these investigations are to quantify the impacts of long-term droughts-an analogue for snowpack reduction-on water storage and hydropower generation, and to illustrate the potential for conjunctive use of surface and subsurface storage, in order to limit the adverse impacts of drought and snowpack reduction on water supply and hydropower generation. This includes how groundwater pumping compensates for reductions in surface inflow, the extent to which the water table is reduced, and when this system recovers or reaches a new equilibrium.

\section{APPROACH}

Analysis of California Central Valley impacts of sustained droughts are based on a series of specified reductions of $10-70 \%$ in net surface flows for periods ranging from 10 to 60 years, and applied to the California Department of Water Resources (CDWR) California Central Valley Simulation Model (C2VSIM). This simplified methodology represents a means by which to evaluate the impact of reductions in net surface inflow to reservoirs. Rather than focus on causes of global climate change, the CDWR Water Plan looks at potential impacts of climate change on water resources in California and strategies for adapting to these changes.

\section{ACCOMPLISHMENTS}

Evaluation of the range of reservoir-operating and groundwater-pumping scenarios show how the water table responds to these prescribed droughts, including the rate of groundwater drawdown during drought and the rate of groundwater recovery after drought. Figure 1 (upper) illustrates the relative change in Central Valley water table levels (delwt) in response to the most severe prescribed drought scenario for 60 years. Here, stream flow to major California reservoirs is reduced to $70 \%$ below average from 2010 to 2070. Surface irrigation deliveries to agriculture fall to roughly $50 \%$ of average deliveries, with farmers assumed to increase groundwater pumping to offset the decline in their surface deliveries. The uppermost panel illustrates groundwater levels with an assumed constant amount of irrigation water applied to agricultural crops. The lower panel assumes that low value crops are removed from production when groundwater pumping ceases to be profitable.

Constant crop-water demand in the Sacramento Basin

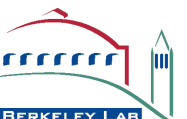
results in a groundwater decline of 77 feet during the imposed drought, but recovers rapidly. In the Tulare basin (SR17), groundwater drops $390 \mathrm{ft}$ during the drought period, recovering slightly after the drought. The northern Central Valley is better protected from drought than the southern region. The trend of falling groundwater is maintained even after accounting for a decline in high-cost groundwater pumping.

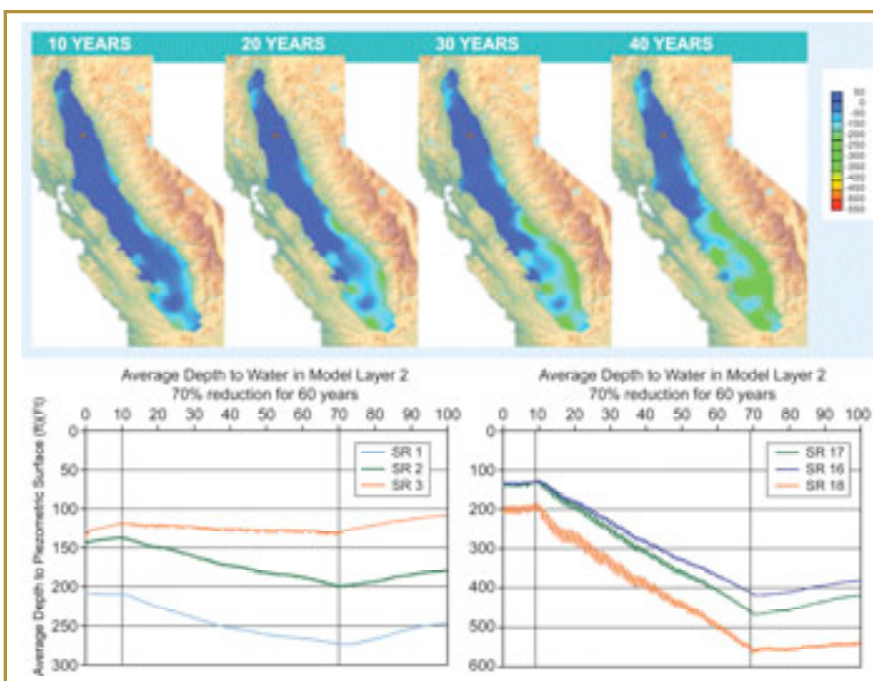

Figure 1. The upper panel shows California Central Valley water table response to a prescribed $70 \%$ reduction in surface flow for $10,20,30$, and 60 years. The 10-year spin-up, surface flow reduction, and 30-year recovery time series for Sacramento Basin subregions, SR1, SR2, SR3, and San Joaquin subregions SR16, SR17, and SR18.

\section{SIGNIFICANCE OF FINDINGS}

Our partial, preliminary results show that drought causes groundwater levels to decline throughout the California Central Valley, but the impact on the groundwater table is less severe in the northern than in the southern Central Valley (and groundwater recovery is much quicker). The impact is not severe enough to cause dramatic changes in crop-water demands in either the northern or southern basins.

\section{RELATED PUBLICATIONS}

Miller, N.L., L.L. Dale, S. Vicuna, C. Brush, J. Dogrul, and T. Kadir, Drought analysis of the California Central Valley surfacegroundwater-conveyance system. Report to the Department of Water Resources, Presentation at the Fall 2006 AGU, J. American Water Resources Association. (in review), 2007.

\section{ACKNOWLEDGMENTS}

Support for this work is provided by the California Energy Commission Public Interest Energy Research (PIER) Program. Work performed for the Department of Energy at Berkeley Lab is under Contract No. DE-AC02-05CH11231. 


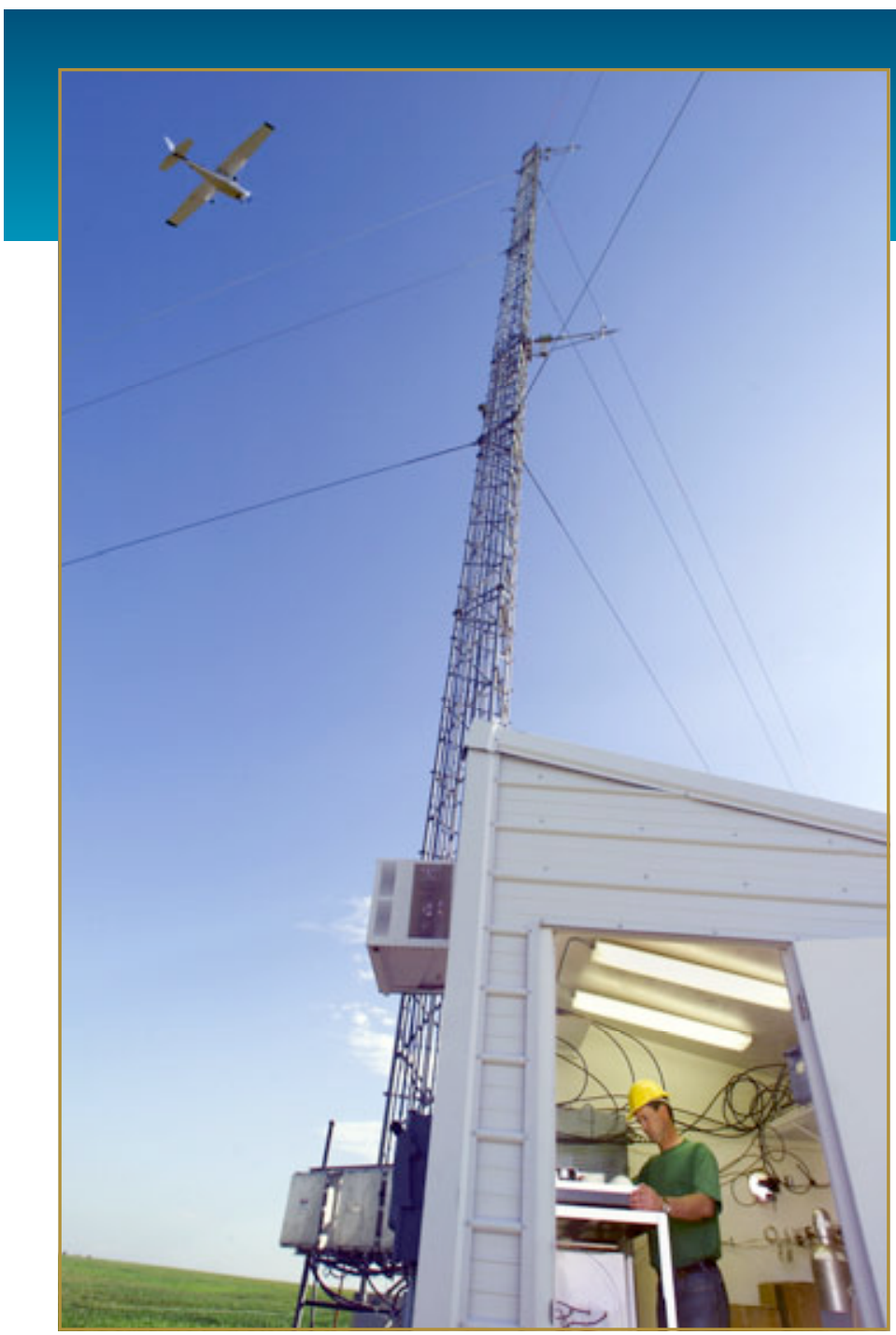

ESD's Climate and Carbon Sciences Program (CCS) is an integrated program that seeks to understand the physical, chemical, and biological processes affecting the Earth's atmosphere, land, and oceans. CCS conducts research into how Earth's processes may be affected, either directly or indirectly, by changes in radiative forcing of climate resulting from energy production and use. In addition, program research on biogeochemical cycles and climate also addresses other pressing issues such as stewardship of water resources and the environmental effects of biofuels. To that end, we have active projects on climate and hydrology, climate change, terrestrial and marine biogeochemistry, and carbon sequestration by terrestrial ecosystems. Note that after seven years of successful expansion, in 2007 the Climate Change and Carbon Management Program was divided into two programs, Climate and Carbon Sciences (summarized below) and one for Geologic Carbon Sequestration (summarized elsewhere in this volume).
Research Program

Climate AND Carbon Sciences Program

Margaret S. Torn

510/486-2223

mstorn@lbl.gov

CCS is also poised to develop new areas of climate science, including research in biofuels and alternative energy, subsurface hydrology, computational mathematics, and other fields. CCS has several significant research initiatives in these areas, with demonstrated potential to make vital contributions to BER's climate change portfolio. These include:

- The conceptual design of a Center for Integrated Earth System Modeling

- New methods for measuring and understanding the stabilization of soil organic matter and ecosystem feedbacks to climate change

- Regional hydrometeorology and the hydrological impacts of climate change

In the fall of 2007, the Program hosted a multinational lab workshop on abrupt climate change. The product of this workshop was the beginning of a large, long-term, interdisciplinary DOE-funded project that will greatly improve our ability to predict the probability of abrupt climate change-using a set of five different mechanisms, from ice-shelf disintegration to high latitude ecosystem response.

\section{SCIENTIFIC FOCUS AREAS}

CCS investigations span and integrate all four DOE Basic Energy Research Office (BER) Scientific Focus Areas (SFAs) in climate change:

1. Climate Change Forcing

2. Climate Change Modeling

3. Climate Change Response

4. Climate Change Mitigation

Each of these SFA's is discussed in greater detail below.

\section{Climate Change Forcing}

The goals of Berkeley Lab's terrestrial carbon research include supporting the development, testing, and application of Integrated Terrestrial Carbon Models (ITCMs) that will be used to simulate carbon fluxes in North America in the near term, and coupled with global climate models in the long term.

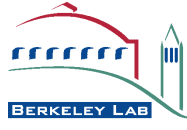


This work is a multi-institution collaboration under the coordination of the lead lab in this SFA, Oak Ridge. Berkeley Lab is pursuing five areas of research relevant to improving carbon cycle understanding: (1) better characterization of ecosystem $\mathrm{CO}_{2}$ fluxes and resulting atmospheric concentrations; (2) spatially and temporally resolved measurements of fossil $\mathrm{CO}_{2}$ emissions; (3) better understanding of soil carbon cycling; (4) simulation of feedbacks between carbon dynamics and climate change in global carbon-climate models; and (5) diagnosis of carbon modules in global climate models using AmeriFlux, North American Carbon Program (NACP), and other carbon system observations.

CCS is also carrying out innovative observations of the ocean carbon cycle that would contribute to removing a major gap in coupled carbon-climate modeling. Oceans contain more carbon than any other dynamic reservoir on earth. They pose a great observational challenge because the pulses of biological productivity are episodic and cover vast areas. CCS scientists have developed the Carbon Explorer, an autonomous float that uses satellite telemetry to report its observations from distant oceans. Twelve of these low-cost robots have achieved the equivalent of 8 years of continuous observations of particulate organic carbon in remote and biologically dynamic ocean regions, observations that would not have been possible with conventional research ships. Seagoing work to prove and enhance new sensors for the Carbon Explorer is ongoing. CCS's new sensor for particulate inorganic carbon was operationally deployed to full ocean depth during a pole-to-pole survey transects of the Atlantic Ocean in July 2003 and January 2005. The data it reported allow the first comprehensive examination of the spatial variability of particulate organic and inorganic carbon. CCS's optical carbon sedimentation recorder was most recently deployed in Oyshio waters near Japan.

\section{Climate Change Modeling}

Simulations from global models provide critical information required to attribute past climate change and ameliorate future climate change. Despite the sophistication of current coupled climate models, they will benefit from inclusion of biogeochemical feedbacks, improved spatial resolution, and an understanding of abrupt climate changes. To understand the role of these processes in regional and global climate change, the climate community should develop Earth System Models (ESMs) designed to simulate the coupled physical, chemical, and biogeochemical evolution of the environment. It is increasingly critical to project local extremes in precipitation and other weather conditions forced by climate change. However, these projections are subject to large uncertainties, driven by specific uncertainties in model physics and restricted model resolution. Uncertainty reduction hinges, in part, on site-to-regional-scale process-based studies leading to new parameterizations in
ESMs, analysis of model-simulated atmospheric physics and dynamics with observational evaluations, and high-resolution studies of the space-time evolution of extremes and anomalous weather and climate states. New research is needed to understand whether projections of extremes can converge with better process fidelity and higher spatial resolution.

\section{Climate Change Response}

To guide DOE energy policy decisions, we need integrated economic analyses of climate change, based on both projections of climate impacts and mitigation/adaptation analyses for biofuel and fossil fuel emissions (for GHGs and aerosols). Integrated models that address both the socio-economic and environmental impacts of energy and land-use systems, at a spatially disaggregated scale with temporal feedbacks, are lacking in current analyses.

Toward meeting this need, the physical impacts of climate variability and change (floods, droughts, heat waves, electricity demand) are a major modeling and analysis component at Berkeley Lab. Work within CCS on these impacts (by Miller and Jin) has appeared in the $2^{\text {nd }}, 3^{\text {rd }}$, and $4^{\text {th }}$ Intergovernmental Panel on Climate Change (IPCC) Assessment Reports, as well as the U.S. Global Change Research Program Assessments. Their research is focused on understanding regional impacts and reducing uncertainty across scales, and includes important studies of climate change impacts on alpine hydrology and snowpack. These activities, along with the DOE Water-Energy Nexus, have helped to develop the important physical-economic linkage for integrated assessment. Miller and Jin have quantified the range of possible extreme heat days and coupled energy demand, projected demand, and supply availability. New work includes development of heat day simulations and new building codes for cooling/heating.

\section{Climate Change Mitigation}

Limitations in current soil carbon models cripple scientists' ability to predict climate effects on $\mathrm{CO}_{2}$ fluxes or to evaluate carbon sequestration and land management strategies. Four major gaps in the understanding of soil carbon dynamics have been identified (e.g., by BERAC, DOE, and USDA) that are important for both coupled climate-carbon modeling and carbon management, and that can be addressed in the next 5 years. Specifically, the priority areas for soil carbon research are (1) the effect of plant allocation and species on carbon residence time; (2) physical protection of soil organic matter, by minerals and aggregation; (3) temperature and moisture interactions; and (4) testing and improvement of model performance. We have in hand sufficient understanding and data to begin developing much improved model parameterizations for several of these areas.

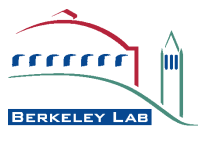




\section{Partnerships and Funding}

Underlying all this work are CCS's active partnerships with universities, industry, and other research laboratories. Key among these is our strong partnership with UC Berkeley, which includes collaboration with faculty, sharing research facilities, teaching, advising and mentoring UC students, and interaction with the Berkeley Atmospheric Sciences Center, Berkeley Water Center, and Berkeley Institute of the Environment. We have exciting new research projects on biofuels starting under the aegis of the Energy Biosciences Institute.
The Climate and Carbon Science Program is funded by a variety of federal and state agencies, and international collaborations. The most important sponsor is the U.S. Department of Energy, through the Office of Biological and Environmental Research, Office of Basic Energy Sciences, and Office of Fossil Energy. Valuable support also comes from the National Aeronautics and Space Administration; National Science Foundation; National Oceanographic and Atmospheric Administration, as well as the California Energy Commission, CAL-FED, and the Energy Biosciences Institute.

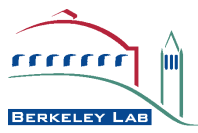




\title{
Understanding the Role of Land-surface Processes in the Regional Climate System: A WRF Modeling Study
}

\author{
Jiming Jin, Norman L. Miller, and Nicole J. Schegel \\ Contact: Jiming Jin, 510/486-7551, JimingJin@lbl.gov
}

\section{RESEARCH OBJECTIVES}

The state-of-the-art Weather Research and Forecasting (WRF) model, developed by the National Center for Atmospheric Research (NCAR), is a limited-area, nonhydrostatic, terrain-following sigma-coordinate model designed to simulate or predict regional weather and climate. The current-release version (2.2) of the WRF model includes three land-surface schemes-the simple soil thermal diffusion (STD) scheme, the Noah scheme, and the Rapid Update Cycle (RUC) scheme. We have recently coupled the sophisticated Community Land Model version 3 (CLM3) to WRF to better simulate and predict snow, vegetation dynamics, and related processes. The objective of this study is to quantify the role of varying levels of complexity in land-surface processes in the regional climate system, by performing a series of WRF runs with these four land-surface-scheme options. Among these four schemes, the STD scheme is the simplest one (both in structure and physics), CLM3 is the most sophisticated, and the complexity level of RUC is comparable with that of Noah. The modeling analysis shown in this study also gives insight into how the land-surface-scheme complexity level affects the accuracy of regional climate simulations.

\section{APPROACH}

Four WRF simulations, one for each land-surface scheme, were generated with a $30 \mathrm{~km}$ resolution domain that focuses on the western United States. Each simulation period was for 1 October 1, 1995, through September 30, 1996. The National Centers for Environmental Prediction-Department of Energy (DOE) Reanalysis (NCEP-2) data were used for the WRF initial and lateral boundary conditions, with the boundary forcing, including sea surface temperature (SST), updated every six hours.

\section{ACCOMPLISHMENTS}

The WRF simulations indicate that the most sophisticated land-surface model, CLM3, generated the best temperature simulations when compared to the results from the other three land surface models. The simplest model (STD), without a snow and

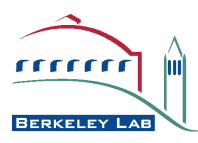
vegetation component, produces the worst results. Figure 1 shows the winter daily maximum temperature observations and simulations. The domain-wide averaged value for the observation is $5.5^{\circ} \mathrm{C}$, and for WRF-CLM3 is $5.0^{\circ} \mathrm{C}$. The daily maximum temperatures for RUC, $\mathrm{NOAH}$, and STD with WRF are $4.9^{\circ} \mathrm{C}$, $6.2^{\circ} \mathrm{C}$, and $10.4^{\circ} \mathrm{C}$, respectively. The simulated geographic distributions of temperature for all these models are quite similar, implying that the temperature distribution is more closely related to the atmospheric forcings than to the land-surface processes, whereas the temperature magnitude is dominated by land-surface characterizations. In addition, WRF dramatically overestimates precipitation, especially over complex topography in the western U.S. no matter which land-surface model is chosen, indicating that precipitation simulations are strongly connected with atmospheric processes over this region.

\section{SIGNIFICANCE OF FINDINGS}

The results from WRF with these land-surface schemes show that land-surface processes strongly affect temperature simulations; the WRF-CLM3 with the highest complexity level produces the best results. Precipitation is dramatically overestimated by WRF for all of the land-surface schemes over the western U.S. analyzed here, and does not show a close relationship to land-surface processes.

\section{RELATED PUBLICATION}

Jin, J., N. L. Miller, and N. Schegel, Understanding the role of land-surface processes in the Regional Climate System: A WRF modeling study. Proceedings of the Annual WRF Workshop, Boulder, Colorado, 2007.

\section{ACKNOWLEDGMENTS}

Support for this work provided by the California Energy Commission under Grant 500-02-004. Work performed at Lawrence Berkeley National Laboratory was supported by the Director, Office of Science, Office of Biological and Environmental Research, Climate Change Research Division, Atmospheric and Radiation Measurement Program, of the U.S. Department of Energy under Contract No. DE-AC03-765F0098. 


\title{
Regional Climate Simulations to Quantify the Range of Land-Use Change AND IMPACTS ON HydROClimate in the California Central Valley
}

\author{
Jiming Jin ${ }^{1}$ and Norman L. Miller \\ ${ }^{1}$ Utah State University, Logan, Utah \\ Contact: Jiming Jin, 435-797-8175, jiming.jin@usu.edu
}

\section{RESEARCH OBJECTIVES}

Over the last century, more than half of the California Central Valley area has been converted to agricultural area since the presettlement period. Understanding how and to what extent such land-use change affects local climate and ecosystems is critical to the economy of California, because the Central Valley produces one-quarter of the agricultural products in the United States, with an annual income exceeding $\$ 26$ billion and an export revenue exceeding $\$ 6.7$ billion. In this study, we use an advanced regional climate model to quantify the range of land-use change its impact on the local climate.

\section{APPROACH}

The regional climate model used here is the nonhydrostatic version of the fifthgeneration mesoscale model (MM5), version 3.7, developed by the Pennsylvania State University/National Center for Atmospheric Research (NCAR). We have coupled MM5 with the advanced NCAR Community Land Model version 3 (CLM3) (Jin and Miller 2007) to improve land-surface process simulations and forecasts. Two runs were performed with MM5-CLM3. The first run was forced with the modern-time land-use types that are the 24 U. S. Geological Survey (USGS) land-use types. The second run was forced with the presettlement-time land types that were created from the Olson Global Ecosystems data.

\section{ACCOMPLISHMENTS}

The observed temperature trends for the Central Valley indicate that daily maximum temperature (Tmax) has a negative (cooling) trend in all seasons (blue bars in Figure 1a) over the last century, with the strongest negative trend in the summer $\left(0.30^{\circ} \mathrm{C} /\right.$ decade), and the weakest trend in the winter $\left(-0.01^{\circ} \mathrm{C} /\right.$ decade). The daily minimum temperature (Tmin) has a significant positive trend for all seasons (red bars in Figure 1a). The simulated temperature changes (Figure $1 \mathrm{~b}$ ) agree well with the observations. Tmax decreases in the Central Valley over all four seasons in the modern time run, with the strongest decrease $\left(-0.55^{\circ} \mathrm{C}\right)$ occurring in winter, as compared with that in the presettlement run. In the mean time, the modern time Tmin increases by more than $0.40^{\circ} \mathrm{C}$ in the fall, and by a p proximately $1^{\circ} \mathrm{C}$ in the winter and spring; whereas in the summer, Tmin shows little change $\left(\sim 0.08^{\circ} \mathrm{C}\right)$. Detailed analysis shows that the temperature changes are caused by the sensible heat flux variations, in turn caused by the lower roughness length in the modern time than in the presettlement time over the Central Valley, where the grassland and trees have been converted to cropland and bare soil over the last hundred or so years.

\section{SIGNIFICANCE OF FINDINGS}

Our modeling results are consistent with the observations. Temperature variations are found to be related to the sensible heat flux changes caused by the lower surface-roughness length in the modern time than in the presettlement time. This study indicates that during the day, land-use change in the Central Valley overcomes the global warming signal and dominates the temperature change, whereas at night, land-use change strengthens such greenhouse-gas related warming.

\section{RELATED PUBLICATION}

Jin, J., and N. L. Miller, Regional climate simulations to quantify the range of land-use change and irrigation impacts on hydroclimate in the California Central Valley. Journal of Geophysical Research (in review), 2007.

\section{ACKNOWLEDGMENTS}

Support for this work is provided by the California Energy Commission under Grant 500-02-004. Work performed at Lawrence Berkeley National Laboratory was performed under Contract No. DE-AC02-05CH11231.

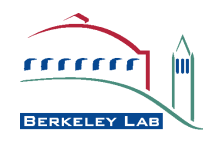




\title{
Climate Change Projected Fire Weather Sensitivity: California Santa Ana Wind Occurrence
}

\author{
Norman L. Miller and Nicole J. Schlege ${ }^{1}$ \\ Contact: Norman L. Miller; 510.495.2374/nlmiller@lbl.gov
}

\section{RESEARCH OBJECTIVES}

California coastal-region, wildfire-weather conditions typically occur during the fall prior to winter rains, when an inland high pressure and an offshore low pressure set up a strong pressure gradient, resulting in high offshore winds, heated air mass, and low humidity. These conditions are known locally as Santa Ana winds in southern California and Diablo winds in northern California, but are more generally defined as foehns. Such weather conditions have a long history of being associated with high winds that spread fires. The objectives of this study are to determine climate-change-related shifts in the occurrence of Santa Ana wind conditions.

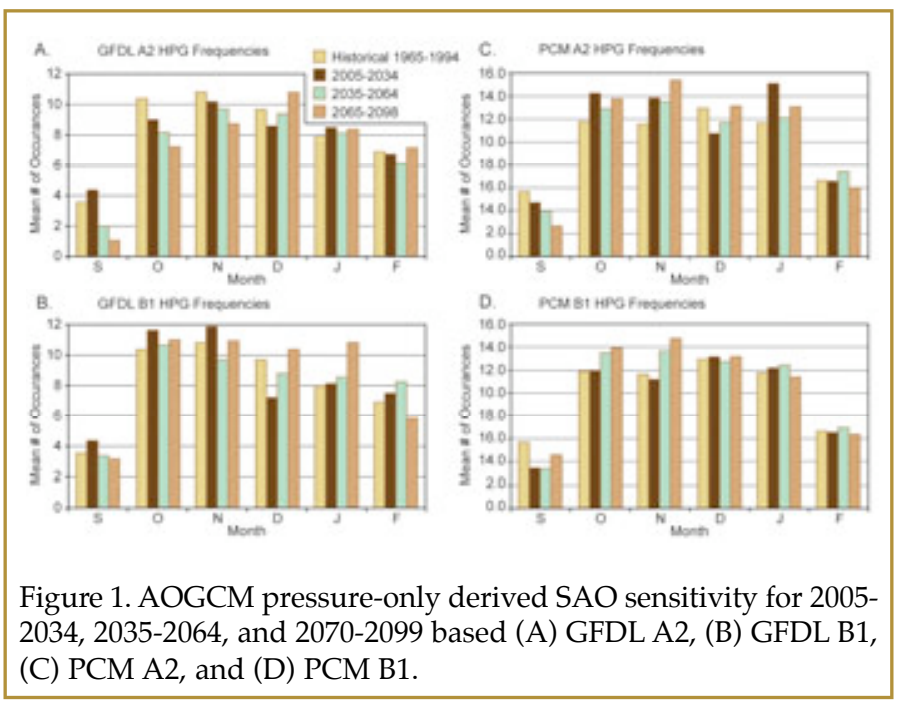

\section{APPROACH}

A new method based on atmosphere-ocean general circulation model (AOGCM) surface pressure gradients was developed for identifying coastal high-wind fire-weather conditions, such as the Santa Ana Occurrence (SAO). We use the low- and mid-range temperature-sensitivity National Center for Atmospheric Research (NCAR) Parallel Climate Model (PCM) and the National Oceanic \& Atmospheric Administration (NOAA) Geophysical Fluid Dynamics Laboratory version 2.1 (GFDL) climate models, forced by the Intergovernmental Panel on Climate Change (IPCC) Special Report on Emission Scenarios (SRES) high (A2) and low (B1) emissions for three 30year mean-monthly climatologies-2005-2034, 2035-2064, and 2070-2099. These scenarios represent the range of IPCC nonintervention emissions futures, with atmospheric $\mathrm{CO}_{2}$ approaching $550 \mathrm{ppm}$ (B1) to $830 \mathrm{ppm}$ (A2) by 2100. The surface-pressure difference criterion was applied to AOGCM historical and projected output to determine the percent change in occurrence

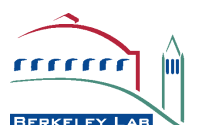
of simulated SAOs. AGCM-simulated 30-year mean-monthly climatologies for 1965-1994 were compared to SAO climatologies based on the observed wind direction and humidity, and showed good correlation.

\section{ACCOMPLISHMENTS}

The sensitivity of climate-change-projected SAOs was analyzed using AOGCM output from the low-temperature sensitivity PCM and middle-temperature sensitivity GFDL, with low-to-high emission scenarios. The trends match well, with September having the lowest number of SAO days, an increasing trend toward the maximum in December, and a decreasing trend for the period during January and February.

This climate change analysis shows consistent shifts in the maximum number of SAO events from September-October to November-December, suggesting SAOs may significantly increase the extent of California coastal areas burned by wildfires, loss of life, and property.

More research is required to fully establish the sensitivity of this mechanism under greenhouse gas forcing. Pressure and sea surface temperature (SST) variability are teleconnected to SAOs, and this remains poorly understood. The role of natural variability and climate change in ocean temperatures plays a significant role in the strength and increase or decrease of the number of SAOs. Further analyses of natural climate modes and variability will need to be carried out to fully understand this sensitivity.

\section{SIGNIFICANCE OF FINDINGS}

This study suggests there may be SAO increases during critical dry periods, especially late in the season, leading to more extensive wildfires. These initial findings indicate striking differences between early century and late century, and high-emission and lower-emission SAO sensitivities, with shifts in SAOs from earlier to later in the year that are greater under A2 relative to $\mathrm{B} 1$ and GFDL relative to PCM, and with no clear changes in the January to February SAOs.

\section{RELATED PUBLICATION}

Miller, N.L. and N.J. Schlegel, Climate change projected fire weather sensitivity: California Santa Ana wind occurrence. LBNL-61004. Geophysical Research Letters, 33, L15711, doi:101029/2006GL25808, 2006.

\section{ACKNOWLEDGMENTS}

Support for this work is provided by the California Energy Commission and by the California Environmental Protection Agency as a contribution to the Governor's Climate Science Report. Work performed for the Department of Energy at Berkeley National Laboratory is under Contract No. DE-AC02$05 \mathrm{CH} 11231$. 


\title{
Climate-Change-Related Extreme Heat, Mortality, and Energy Demand
}

\author{
Norman L. Miller, Jiming Jin, Katharine Hayhoe, and Max Auffhammer
}

Contact: Norman L. Miller; 510.495.2374/nlmiller@lbl.gov

\section{RESEARCH OBJECTIVES}

As the southwestern U.S. becomes more populated, and extreme heat days more frequent, electricity demand for cooling will continue to rise. Electricity supply failures have occurre d during recent extreme summer heat events. In 2006, Sacramento exceeded $110^{\circ} \mathrm{F}$ for 10 days. The objectives of this study are to quantify the projected increases in the frequency, intensity, and duration of extreme heat days and evaluate the impact on the energy sector from increased demand for cooling.

\section{APPROACH}

Extreme heat days are defined here as the warmest June to September (JJAS) days for 1961-1990 exceeding 90 percent (T90) of the summertime daily maximum temperatures at a given location. A heat wave is defined as 5 consecutive T90 days. We calculate the number of projected JJAS days at or above the historical T90s, an important metric used in California energy capacity analysis also referred to as the 1-in-10 high-temperature days. We also calculate standardized JJAS cooling degree-days as defined by the National Climatic Data Center; CDD $=$ (Ta-Tac) days, where Ta is the daily mean near-surface air temperature, Tac is an average daily-mean temperature threshold for human thermal comfort, and days is the number of days with temperatures exceeding Tac.

\section{ACCOMPLISHMENTS}

We evaluated the number of maximum temperatures days projected to exceed the 1960-1990 T90 threshold, at the California state level and for the five urban centers within California. California's historical $\mathrm{T} 90\left(35^{\circ} \mathrm{C}\right)$, is projected to double from 12 days to about 24 days by 2005-2034, regardless of the emissions scenario. By 2035-2064, this increases to 27-39 days (B1), 29-47 days (A2), and 32-66 days (A1fi). By 2070-2099, the statewide T90 days are projected to increase an average of 4 times (B1), 5.5 times (A2), and 6.5 times (A1fi) relative to 1960-1990 (Figure 1). City-specific T90 values are for San Francisco, Los Angeles, Sacramento, Fresno, and San Bernadino. The period 2070-2099 is projected to increase by 3.5 to 4 times under B1, 5.5 to 6 times higher under A2, and 6 to 7 times under the higher A1fi scenario, depending on the city. Historical California CDD values average $400-500^{\circ} \mathrm{C}$-days / year, are projected to increase to $600-1000^{\circ} \mathrm{C}$-days by $2035-2064$, and by 2070-2099 increase to $650-1000^{\circ} \mathrm{C}$-days under the B1 scenario; and up to $800-1250^{\circ} \mathrm{C}$-days and $1000-1500^{\circ} \mathrm{C}$-days under the higher $\mathrm{A} 2$ and A1fi scenarios, respectively (Table).
Together, the impact of projected increases in T90 frequencies and duration will significantly increase peak electricity demand. Peak electricity demand increases under all climate change scenarios, with residential peak electricity demand at mid-century increasing by 3.4-10.0 percent under the A1fi and A2 scenarios and by 2.8-7.7 percent under the B1 scenario.

\section{SIGNIFICANCE OF FINDINGS}

Projected increases in extreme temperatures and direct estimates of electricity demand suggest that electricity demand in California is likely to continue to rise over this century, even without considering likely population growth. Although California's installed electricity capacity will also continue to grow over time, its current rates of growth suggest summer electricity shortages may occur.

\begin{tabular}{|c|c|c|c|c|c|c|c|}
\hline & \multirow{2}{*}{$\begin{array}{c}1961-1990 \\
\text { (absoluse value) }\end{array}$} & \multicolumn{3}{|c|}{$2035-2065$} & \multicolumn{3}{|c|}{$2070-2099$} \\
\hline & & AIf & $A 2$ & BI & Alfi & $\mathrm{A} 2$ & BI \\
\hline San Francisco & 60 & $+90-150$ & $+90-100$ & +60.90 & $+260-340$ & $+140-220$ & $+110-15$ \\
\hline Los Angeles & 570 & $+190-340$ & $+210-310$ & $+150-200$ & $+410-590$ & $+260-550$ & $+230-316$ \\
\hline Sacramento & 690 & $+310-400$ & $+270-360$ & +220.280 & $+630-720$ & $+310 \cdot 630$ & $+330-418$ \\
\hline San Bemardino-Rivenside & 800 & $+250-430$ & $+200-410$ & +190.270 & $+520-750$ & +240.750 & $+290 \cdot 390$ \\
\hline Fresne & 900 & $+320-410$ & $+200-370$ & +220.310 & $+640-730$ & +250.670 & $+340 \cdot 416$ \\
\hline
\end{tabular}

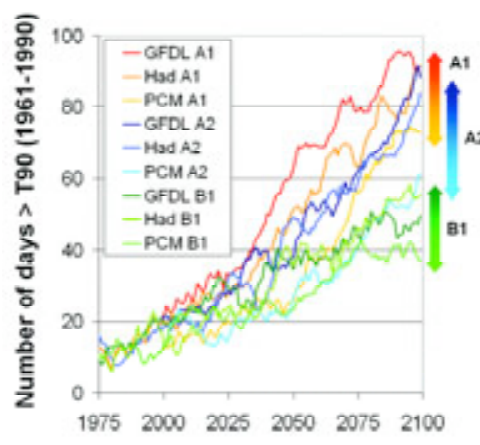

Figure 1. State-wide average number of JJAS T90 days/year from 1975 to 2100 for the HadCM3, GFDL and PCM models. Shaded arrows indicate the end-of-century range for simulations corresponding to the IPCC SRES A1fi (higher, red/orange), A2 (mid-high, blue) and B1 (lower, green) emission scenarios. Table 1. CDD relative to the historical average for five California cities, A1fi (higher), A2 (midhigh) and B1 (lower) using the HadCM3, GFDL2.1 and PCM downscaled output.

\section{RELATED PUBLICATION}

Miller, N.L., K. Hayhoe, J. Jin, and M. Auffhammer, Climate, extreme heat, and electricity demand in California. Journal of Applied Meteorology and Climatology, 47(6), 1834-1844), 2007.

\section{ACKNOWLEDGMENTS}

Support for this work is provided by the California Energy Commission and by the California Environmental Protection Agency as a contribution to the Governor's Climate Science Report. Work performed for the Department of Energy at Berkeley National Laboratory is under Contract No. DE-AC02-05CH11231.

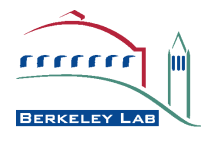




\title{
The California Regional Climate Modeling InTERCOMPARISON AND Climate Change Sensitivity STUdy
}

\author{
N.L. Miller, J. Jin, N. Schlegel, M. Synder, L.C. Sloan, M. Kanamitsu, \\ H. Kanamaru, H. Hildago, P.B. Duffy, M.D. Dettinger, and D. Cayan \\ Contact: Norman L. Miller; 510/495.2374; nlmiller@lbl.gov
}

\section{RESEARCH OBJECTIVES}

The California Energy Commission (CEC) Public Interest Energy Research (PIER) Program is supporting research to develop probabilistic climate change scenarios for California. The objectives of this study are to develop climate scenarios with reduced uncertainties for applications by State planning agencies and impacts research. This project has two phases: (1) an enhancement and intercomparison of regional climate models (RCMs), and (2) a climate change sensitivity analysis for impact studies as part of the California Governors response to AB32.

\section{APPROACH}

Three RCMs-LBNL's version of the National Center for Atmospheric Research (NCAR) Weather Forecast and Prediction model version 2 coupled to the

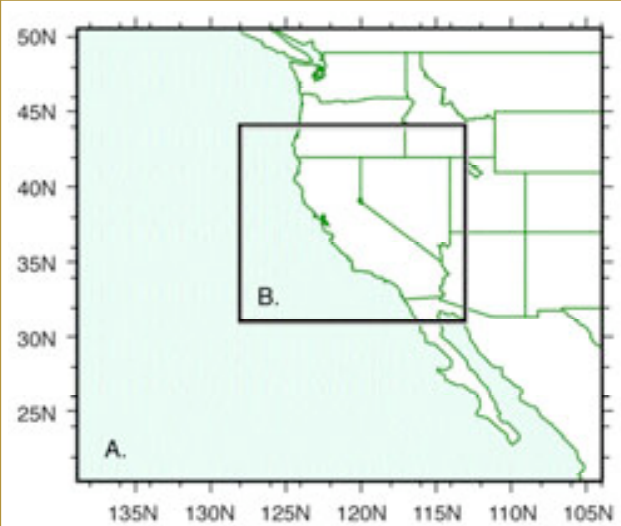

Figure 1. REBI domains and resolutions are (A) western U.S. and eastern Pacific Ocean, $30 \mathrm{~km}$ resolution, with coordinates of approximately [139W21N $\times 104 \mathrm{~W} 51 \mathrm{~N}]$ and (B) California Nevada, and eastern Pacific Ocean, $10 \mathrm{~km}$ resolution, with coordinates of approximately [128W31N $\times 113 \mathrm{~W} 44 \mathrm{~N}]$.
We have also completed two 10 -year simulations, one with the newly coupled WRF-CLM3 and one with the off the shelf WRF-RUC (Rapid Update Cycle) landsurface scheme.

\section{SIGNIFICANCE OF FINDINGS}

This RCM enhancement and intercomparison study is preparing the modeling groups for a comprehensive climate model bias and sensitivity analysis of projected climate change in California at fine scale. Understanding the details of model errors and how each model has propagated such errors further advances our probabilistic understanding of the potential consequences of climate change in California.

\section{RELATED PUBLICATIONS}

Community Land Model version 3 (LBNL/WRF-CLM3); UCSC's version of the Regional Climate Model version 3 (UCSC/RegCM3); and UCSD's version of the NOAA Regional Spectral Model (UCSD/RSM)—-have been selected for model enhancements and intercomparison. The goal of the model enhancement is to implement key improvements prior to the January 2007 start of the model intercomparison aspect of this project. While the RCM codes were frozen for the comparison runs, each group continued to improve their model.

Each modeling group set up two nested domains, a western U.S. $30 \mathrm{~km}$ resolution domain, and a California $10 \mathrm{~km}$ resolution domain (Figure 1). The NCAR/NCEP Reanalysis II is the common input data for RCM initial and lateral boundary conditions, and model output variables and fluxes were mapped onto a common grid for intercomparison analysis.

\section{ACCOMPLISHMENTS}

During the enhancement period, we completed the WRF and CLM3 coupling and evaluation, as well as a series of one-year simulations of WRF using three land-surface schemes, for presentation at the WRF User's Workshop in May 2007 (Jin et al., 2007).
Kueppers, L.M, M.A. Snyder, L.C. Sloan, D Cayan, J. Jin, H. Kanamaru, M. Kanamitsu, N.L. Miller, Mary Tyree, H. Du, and B. Weare, Regional climate effects of irrigation and urbanization in the Western United States: A model intercomparison. CEC-500-2006-031, Global and Planetary Change (in press), 2006.

Jin, J., N.L. Miller, and N.J. Schlegel, Understanding the role of land surface processes in the Regional Climate System: A WRF modeling experiment. The 2007 WRF Workshop Proceedings, 2007.

Miller, N., et al., An analysis of simulated California climate using multiple dynamical and statistical techniques. CEC REBI Report, 49pp. The 2008 California Assessment Scenarios Project, Governor's California Assessment.

\section{ACKNOWLEDGMENTS}

Support for this work provided by the California Energy Commission and by the California Environmental Protection Agency as a contribution to the Governor's Climate Science Report. Work performed for the Department of Energy at Berkeley National Laboratory is under Contract No. DE-AC02-05CH11231. 


\section{Developing a Coupled Land Surface and Subsurface Model: CLMT2}

Lehua Pan, Jiming Jin, Norman Miller, Yu-Shu Wu, and Gudmundur Bodvarsson

Contact: Lehua Pan, 510-495-2360, 1pan@lbl.gov

\section{RESEARCH OBJECTIVES}

An understanding of the hydrologic interactions among atmosphere, land surface, and subsurface is one of the keys to understanding the water cycling system that supports our life system on Earth. Properly modeling such interactions is a diff icult task because of the inherent coupled processes and complex feedback structures among subsystems. The objectives of this study are (1) to develop a new model of atmosphere-land-subsurface hydraulic interactions at watershed or regional scales by combining the best aspects of both CLM3 and TOUGH2; and (2) to show the importance of realistically modeling both surface and subsurface processes, as well as their interactions, in predicting the hydrologic responses to meteorological forces, by applying the new model to a watershed in Russia over an 18-year period.

\section{APPROACH}

The new model was developed by combining a state-of-theart land-surface model, the NCAR Community Land Model version 3 (CLM3), with a variably saturated groundwater model, the TOUGH2, through an internal interface that includes flux and state variables shared by the two submodels. Specifically, TOUGH2, in its simulation, uses infiltration, evaporation, and root-uptake rates, calculated by CLM3, as source/sink terms; CLM3, in its simulation, uses saturation and capillary pressure profiles, calculated by TOUGH2, as state variables.

From the perspective of CLM3, the new model no longer simulates the subsurface moisture movement as a one-dimensional process by explicit scheme. Instead, the 3-D Richards equation is solved implicitly by TOUGH2. In particular, the assumption that the permeability decreases exponentially from top to bottom of the soil is no longer used, and the groundwater depth is no longer a parameter calculated as saturation-weighted depth. From the perspective of TOUGH2, the new model no longer takes the net infiltration or root uptake as a prescribed boundary condition or source/sink term. Instead, the net infiltration and root uptake result from simulations of coupled energy, wind, vegetation, and hydraulic processes by CLM3. As a result, CLMT2 expands the scope of TOUGH2 such that more realistic modeling of land-surface conditions is possible.

The 18 years of observation data from Usadievsky Watershed, Valdai, Russia, were used to evaluate the performance of this new model, as compared to the CLM3 model.

\section{ACCOMPLISHMENTS}

A model that combines the ability to simulate the land-surface and subsurface hydrologic responses with meteorological forcing, CLMT2, has been developed, by combining a state-ofthe-art land-surface model, the NCAR Community Land Model version 3 (CLM3), and a variably saturated groundwater model, TOUGH2, through an internal interface that includes flux and state variables shared by the two submodels. The preliminary simulation results show that the coupled model greatly improves the predictions of the water table, evapotranspiration, surface temperature, and moisture in the top $20 \mathrm{~cm}$ of soil at a real watershed, as evaluated from 18 years of observed data.

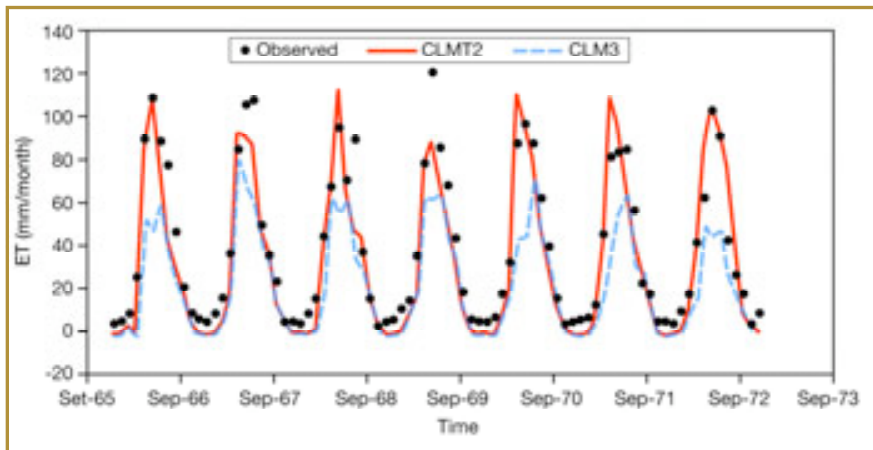

Figure 1. The observed (the dots) and simulated using CLMT2 (the red line) or CLM3 (the blue line) monthly ET.

\section{SIGNIFICANCE OF FINDINGS}

The results indicate that correct simulation of subsurface flow (including the water table) is very important, not only in assessing subsurface water resource itself, but also in simulating surface processes such as evapotranspiration or land-surface temperature, the two most important feedback factors for regional climate. The new model is a useful tool for simulating hydrologic systems at watershed or regional scale, especially the surface and subsurface responses to the climate changes.

\section{RELATED PUBLICATION}

Pan, Lehua, Jiming Jin, Norman Miller, Yu-Shu $\mathrm{Wu}$, and Gudmundur Bodvarsson, 2006. Modeling hydraulic responses to meteorological force: From canopy to aquifer. LBNL-61018. Vadose Zone Journal (in press), 2007.

\section{ACKNOWLEDGMENTS}

This work was supported by Laboratory Directed Research and Development (LDRD) funding from Berkeley Lab, provided by the Director, Office of Science, of the U.S. Department of Energy under Contract No. DE-AC02-05CH11231.

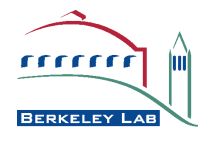




\section{Where Do Fossil Fuel Carbon Dioxide Emissions from California Go? \\ W.J. Riley, D.Y. Hsueh, M.L. Fischer, J.G. Hatch, D.E. Pataki, S. Cheong, M.L. Goulden, and J.T. Randerson Contact: W.J. Riley, 510/486-5036, wjriley@lbl.gov}

\section{RESEARCH OBJECTIVES}

It is widely recognized that climate is being impacted by anthropogenic fossil fuel $\mathrm{CO}_{2}$ emissions (IPCC, 2001). Accurate quantification of fossil fuel $\mathrm{CO}_{2}$ emissions is needed to properly account for climate change impacts, aid in policy development, improve climate prediction and climate change attribution, and aid in atmospheric inversion approaches to quantifying ecosystem $C$ fluxes. Further, other primary atmospheric pollutants of interest (e.g., $\mathrm{CO}$ ) are produced concurrently with $\mathrm{CO}_{2}$, and emission estimates for these gases can be improved using accurate fossil fuel $\mathrm{CO}_{2}$ emission and concentration $\left(C_{f}\right)$ estimates.

\section{APPROACH}

We applied radiocarbon $\left({ }^{14} \mathrm{C}\right)$ measurements in annual $\mathrm{C}_{3}$ grasses across California to test a regional model (Riley et al., 2003; Riley et al., 2005) that simulates surface anthropogenic and ecosystem $\mathrm{CO}_{2}$ fluxes, transport in the atmosphere, and the resulting $\Delta^{14} \mathrm{C}$ value of annual grasses $\left(\Delta_{\mathrm{g}}\right)$. The model was used to predict $\mathrm{CO}_{2}$ transport patterns within and outside of California, (Riley et al., 2008).

\section{ACCOMPLISHMENTS}

The model accurately predicted statewide patterns of $\Delta_{\mathrm{g}}$. Predicted annual-averaged $C f$ were $14.0 \mathrm{ppm}, 6.1 \mathrm{ppm}, 4.8$ ppm, and 0.3 ppm in Los Angeles, San Francisco, the Central Valley, and the North Coast, respectively. $\mathrm{CO}_{2}$ emitted in Los Angeles and San Francisco was predicted to move into the Central Valley, raising $C f$ above that expected from local emissions alone. Annually, about 21\%, 39\%, 35\%, and 5\% of Cf leaves the California airspace to the north, east, south, and west, respectively (Figure 1), with large seasonal variations in the proportions. Correlations between Santa Ana wind conditions and both eastward and westward fluxes were observed.

\section{SIGNIFICANCE OF FINDINGS}

Our results indicate that state and continental scale atmospheric inversions need to consider fluxes in areas where measurements are sparse (e.g., over the ocean), transport within and across the marine boundary layer, and terrestrial boundary layer dynamics. Proposals have been made to use $\mathrm{CO}_{2}$ measurements on the U.S. coastal boundaries to infer continental $\mathrm{CO}_{2}$ emissions and exchanges. Our estimate- that a substantial portion of California's fossil fuel $\mathrm{CO}_{2}$ emissions exits California in a relatively low elevation southward plume-implies that inversions need to accurately characterize these flows. Further, sampling protocols need to be designed to constrain estimates of these southward fluxes.

Other pollutants generated concurrently with $\mathrm{CO}_{2}$ or from atmospheric photochemical reactions will also be impacted by the transport patterns described here. Model predictions indicated that portions of the Central Valley and northern Mexico had higher near-surface $C f$ than would be expected from local emissions alone. The additional loading resulted from transport from the San Francisco Bay, mento, and Los Angeles air basins. Further research is required to characterize the impact of these transport patterns on ozone, $\mathrm{NO}_{\mathrm{x}}$, particulate matter, and $\mathrm{N}$ acid levels, and subsequent impact on local ecosystems, visibility, and human health.

\section{RELATED PUBLICATIONS}

Riley, W.J., C.J. Still, B.R. Helliker, M. Ribas-Carbo, and J.A. Berry, ${ }^{18} \mathrm{O}$ composition of $\mathrm{CO}_{2}$ and $\mathrm{H}_{2} \mathrm{O}$ ecosystem pools and fluxes in a tallgrass prairie: Simulations and comparisons to measurements. Global Change Biology, 9, 1567-1581, 2003.

Riley, W.J., J.T. Randerson, P.N. Foster, and T.J. Lueker, The influence of terrestrial ecosystems and topography on coastal $\mathrm{CO}_{2}$ measurements: A case study at Trinidad Head, California. JGR-Biogeosciences, 110, 2005.

Riley, W.J., D.Y. Hsueh, J.T. Randerson, M.L. Fischer, J.G. Hatch, D.E. Pataki, W.W. Wang, and M.L. Goulden, Where do fossil fuel carbon dioxide emissions from California go? An analysis based on radiocarbon observations and an atmospheric transport model. JGR-Biogeosciences (in press), 2008.

\section{ACKNOWLEDGMENTS}

We gratefully acknowledge support from NASA (NNG05GD126), the Office of Science, U.S. Department of Energy (DE-AC02-05CH11231), and the National Science Foundation (0620176).

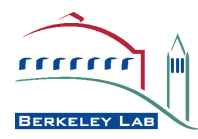




\title{
Interannual Regional $\mathrm{CO}_{2}$ and Latent Heat Surface FluXes in the Southern Great Plains: Measurements, Modeling, and scaling
}

\author{
W.J. Riley, S.C. Biraud, M.S. Torn, M.L. Fischer, J.A. Berry, and D. Billesbach \\ Contact: W.J. Riley, 510/486-5036, wjriley@lbl.gov
}

\section{RESEARCH OBJECTIVES}

Characterizing net ecosystem exchanges of $\mathrm{CO}_{2}$ (NEE) and energy exchanges in heterogeneous landscapes is notoriously difficult, yet critical given expected climate change and rapid development. We report here a three-year measurement and modeling study, conducted as part of the DOE Atmospheric Radiation Measurement Program and Berkeley Lab Carbon project, to improve our understanding of surface-to-atmosphere gas exchanges under highly heterogeneous land cover in the mostly agricultural U.S. Southern Great Plains Atmospheric Radiation Measurement Climate Research Facility (ACRF).

\section{APPROACH}

We combined multiyear site-level eddy covariance measurements in several of the dominant land-cover types with regional-scale climate data from the distributed Mesonet sampling stations and NEXRAD precipitation measurements, to calibrate a land-surface model of trace-gas and energy exchanges (ISOLSM; Cooley et al., [2005]; Riley et al., [2003]). Yearly variations in distributed vegetation cover type were estimated from archetypal phenology profiles and $250 \mathrm{~m}$ MODIS NDVI measurements. Soil hydraulic characteristics were determined from the USGS STATSGO $1 \mathrm{~km}$ resolution soil map. We first applied the model at a $250 \mathrm{~m}$ spatial scale to account for vegetation cover type and leaf area variations that occur on hundred-meter scales. Because of computational constraints, we developed a subsampling scheme within $10 \mathrm{~km}$ "macrocells" to perform these high-resolution simulations. The impact of spatial scale on predictions was determined using both a "dominant" vegetation cover approach and an approach that used the mean leaf area index (LAI) and fractional cover of each cover type.

\section{ACCOMPLISHMENTS}

Regional and subregional vegetation cover type estimates matched USDA census data well. Our results suggest that the ACRF region can be a net $\mathrm{CO}_{2}$ source or sink to the atmosphere, depending on variations in climate and agricultural practices; large seasonal variations in $\mathrm{CO}_{2}$ exchanges were also predicted (Figure 1). Predicted regional latent heat fluxes were largely independent of spatial scale of the model. However, both scaling approaches led to poor regional NEE estimates at all larger scales, and there was no quantifiable pattern in regional NEE between scales.

\section{SIGNIFICANCE OF FINDINGS}

Typical midday NEE variations across the ARM-SGP domain can be large (up to $25 \mu \mathrm{mol} \mathrm{m}^{-2} \mathrm{~s}^{-1}$ ). Our results show that large errors in predicted NEE result when cover type and status variations are not explicitly accounted for at the scale of spatial variation. Further, the current approach of assuming a uniform crop cover and properties across the region leads to large errors in predicted regional NEE. Our approach allows us to quantify uncertainty in regional flux estimates associated with uncertainties in vegetation type, soil types, and spatial and temporal scaling of surface characterization and meteorological forcing. This work will benefit both "bottom-up" and "top-down" approaches to quantifying regional-scale surface $\mathrm{CO}_{2}$ exchanges, as well as improving latent and sensible heat exchange estimates critical for boundary layer and cloud modeling.

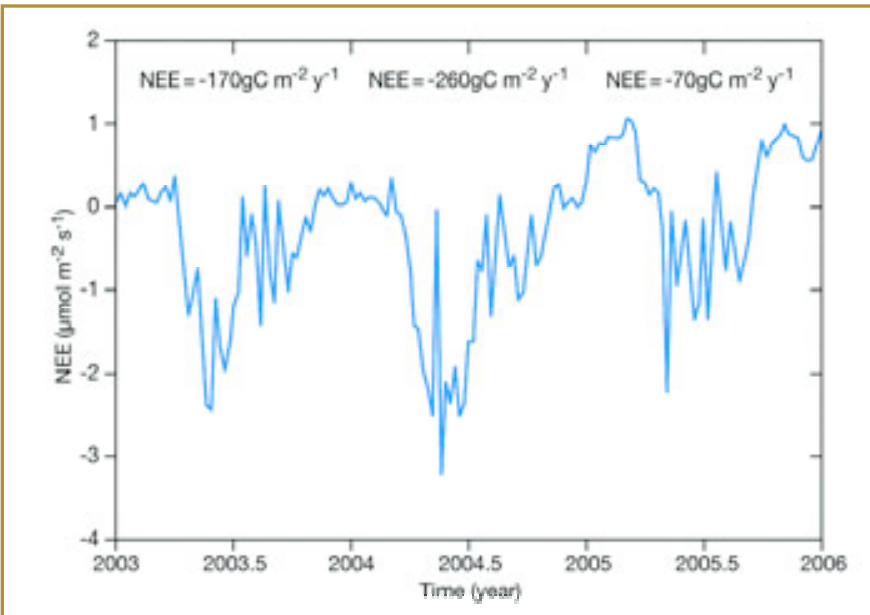

Figure 1. Predicted weekly NEE for the ACRF region. The region was predicted to be a net $\mathrm{CO}_{2}$ sink in 2003 and 2004 and a net source in 2005.

\section{RELATED PUBLICATIONS}

Cooley, H.S., W.J. Riley, M.S. Torn, and Y. He, Impact of agricultural practice on regional climate in a coupled land surface mesoscale model. Journal of Geophysical ResearchAtmospheres, 110, 2005.

Riley, W. J., C. J. Still, B. R. Helliker, M. Ribas-Carbo, and J. A. Berry, $18 \mathrm{O}$ composition of $\mathrm{CO}_{2}$ and $\mathrm{H}_{2} \mathrm{O}$ ecosystem pools and fluxes in a tallgrass prairie: Simulations and comparisons to measurements. Global Change Biology, 9, 1567-1581, 2003.

\section{ACKNOWLEDGMENTS}

This work was supported by the Director, Office of Science, O ffice of Biological and Environmental Research, Climate Change Research Division, Atmospheric and Radiation Measurements Program, of the U.S. Department of Energy under Contract No. DE-AC02-05CH11231.

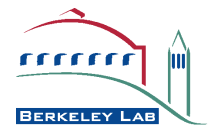




\title{
Modeling of Hydrology, The Nitrogen Cycle, and Vegetation Dynamics in Natural and Managed Ecosystems
}

\author{
W.J. Riley, F. Maggi, C. Gu, T. Xu, C.I. Steefel, N.L. Miller, and C.M. Oldenburg \\ Contact: W.J. Riley, 510/486-5036, wjriley@lbl.gov
}

\section{RESEARCH OBJECTIVES}

The biogeochemical nitrogen cycle and $\mathrm{NO}, \mathrm{N}_{2} \mathrm{O}$, and $\mathrm{CO}_{2}$ gas production in natural and agricultural ecosystems are impacted by several soil microbial populations, the hydrological cycle, plant dynamics, and climate. Understanding the release of $\mathrm{NO}, \mathrm{N}_{2} \mathrm{O}$, and $\mathrm{CO}_{2}$ from the soil surface to the atmosphere is a key factor in controlling greenhouse gas emissions, and assumes ever-greater importance in view of the foreseen increase in biofuel, food, and fiber production. The objective of this research is to develop a mechanistic analysis tool to investigate the effects of climate, irrigation, fertilizer application, and plant dynamics on $\mathrm{N}$ cycling and losses at site, watershed, and regional scales.

\section{APPROACH}

We have developed a coupled mechanistic modeling framework based on TOUGH2, TOUGHREACT, the CERES crop model, and the land-surface model CLM (the land-surface model used in the latest generation of the NCAR GCM). The framework includes various nitrification and denitrification pathways, multiple microbial biomass dynamics, hydrological dynamics, equilibrium and kinetic chemical reactions, landscape-scale hydrology, crop-specific plant growth and nutrient requirements, and natural ecosystem plant dynamics. Biotic and abiotic reactions are assumed to follow Michaelis-Menten kinetics, while biomass is assumed to follow multiple Monod growth kinetics accounting for electron donor, electron acceptor, and inhibitor concentrations. Water flow is modeled with the Darcy-Richards equation and advective, and diffusive tracer transport is modeled in both gaseous and liquid phases. The CERES crop model simulates plant biomass, $\mathrm{N}$ and water demand, and seed production (among others) across a range of crop phenotypes. Finally, CLM simulates the surface energy and radiation balance, natural ecosystem $\mathrm{CO}_{2}$ exchanges, and distributed surface hydrology.

\section{ACCOMPLISHMENTS}

We have applied the model in two different agricultural systems and tested its performance against measurements of soil moisture, $\mathrm{pH}, \mathrm{NH}_{4}{ }^{+}, \mathrm{NO}_{2}{ }^{-}$, and $\mathrm{NO}_{3}{ }^{-}$ion concentrations, and $\mathrm{NO}, \mathrm{N}_{2} \mathrm{O}$, and $\mathrm{CO}_{2}$ gas emissions (Gu et al., 2008; Maggi, et al., 2008). Good agreement between field observations and model predictions was found for all these quantities, as were variations associated with fertilizer type. We used the model to characterize gas emissions, solute leaching of several nitrogen species, and the vertical distribution of nitrifier and denitrifier populations associated with variations in fertilizer type and amount and irrigation practices. Preliminary analyses using

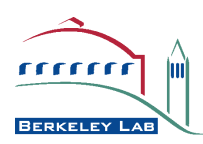
the coupled model to analyze watershed scale behavior have been encouraging.

\section{SIGNIFICANCE OF FINDINGS}

In contrast to the predictions of most current coarse-scale models, all $\mathrm{N}$ losses increased nonlinearly with fertilizer and water application amount, and with fertilizer application depth (Figure 1). Further, different fertilizer types resulted in substantially different $\mathrm{N}$-loss patterns over time, and these patterns depend strongly on soil properties. Our results imply that typical field, watershed, and regional-scale assessment approaches for ecosystem $\mathrm{N}$ balance and losses need to be revised.

\section{RELATED PUBLICATIONS}

$\mathrm{Gu}, \mathrm{C}$. , et al., Aqeuous and gaseous nitrogen losses induced by fertilizer application. JGR-Biogeosciences (submitted), 2008.

Maggi, F., et al., A mechanistic treatment of the dominant soil nitrogen cycling processes: Model development, testing, and application. Journal of Geophysical ResearchBiogeosciences, 113, 2008.

\section{ACKNOWLEDGMENT}

This work was supported by Laboratory Directed Research and Development (LDRD) funding from Berkeley Lab, provided by the Director, Office of Science, of the U.S. Department of Energy under Contract No. DE-AC02-05CH11231.

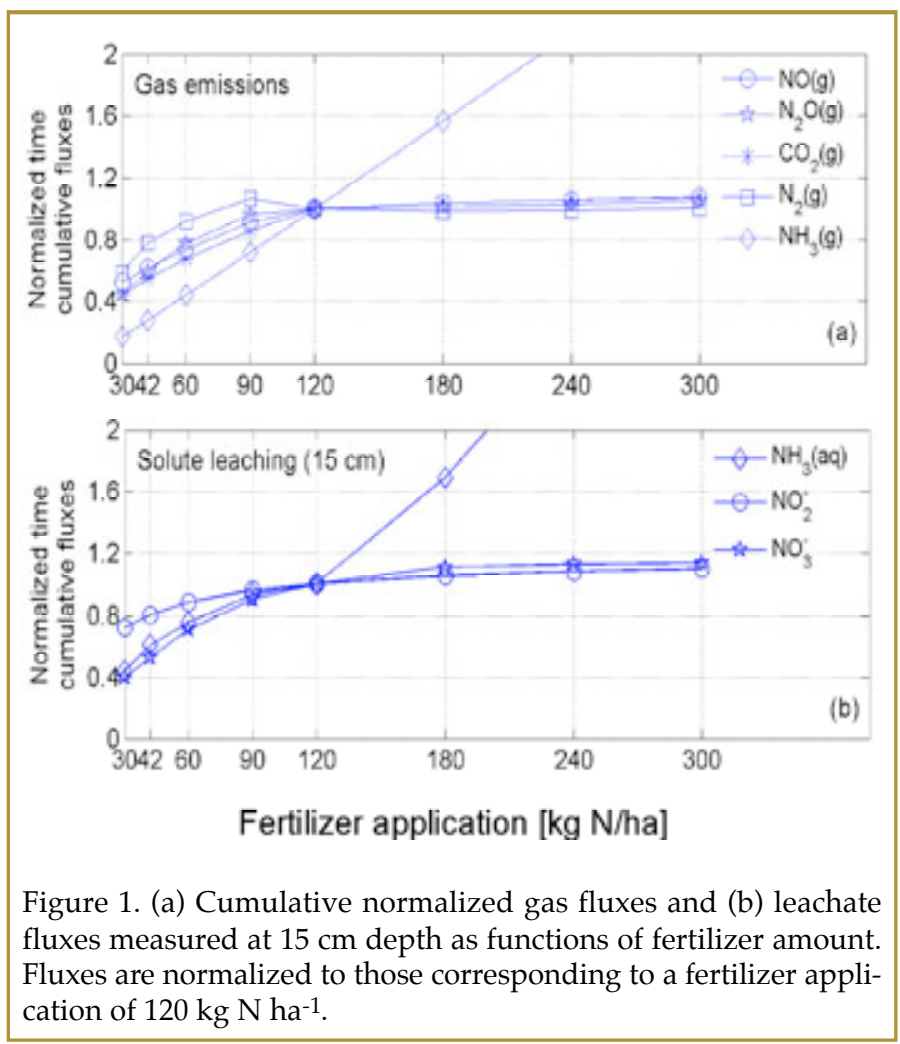




\title{
Carbon Cycling in the Southern Great Plains During the ARM Cumulus-LAND SuRfaCe INTERACTION CAMPAIGN (CLASIC)
}

\author{
Margaret S. Torn, Marc L. Fischer, William J. Riley, Sébastien Biraud, and J. Berry \\ Contact: Margaret S. Torn, 510/495-2223, MSTorn@lbl.gov
}

\section{RESEARCH OBJECTIVES}

The DOE Atmospheric and Radiation Measurement (ARM)/Berkeley Lab Carbon Project is making a coordinated suite of carbon concentration, isotope, and flux measurements to support climate modeling studies and the North American Carbon Program, including quantifying regional atmospheric $\mathrm{CO}_{2}$ sources and sinks, and implementing land-surface models for estimating regional carbon, water, and energy fluxes. During the ARM Cumulus-Land Surface Interaction Campaign (CLASIC), we collected airborne measurements for $\mathrm{CO}_{2}$ source attribution, which included (for example) characterizing the chemical fingerprint of anthropogenic greenhouse gas emissions.

\section{APPROACH}

CLASIC brought together eight aircraft, one helicopter, and over thirty scientists in June 2007, to study the influence of the land surface on cloud development and atmospheric dynamics, including greenhouse gas fluxes and concentrations. We planned carbon-centered flight paths, integrated instrumentation on a Cessna 206 and the CIRPAS Twin Otter, and helped coordinate deployment of 10 eddy flux towers by DOE, USDA, and NASA for land-surface studies.

\section{ACCOMPLISHMENTS}

We flew 22 flights with continuous $\mathrm{CO}_{2}$, NOAA flasks and ${ }^{14} \mathrm{CO}_{2}$ flasks, nine of which also had continuous $\mathrm{CO}$ and $\mathrm{CH}_{4}$ concentration measurements. All of these data sets have been processed and submitted to the CLASIC data archives. Results from the campaign have been presented at the CLASIC workshop and other scientific meetings, and are being integrated into the NACP Mid-Continent Intensive that took place in summer 2007, and other NACP synthesis activities. Here, we give results for anthropogenic source characterization.

On June 22, 2007, the CIRPAS Twin Otter flew an anthropogenic source characterization mission around Oklahoma City, Oklahoma (OKC), flying most of the time at $4,000 \mathrm{ft}$ above ground level (Figure 1a). Figure $1 \mathrm{~b}$ shows continuous $\mathrm{CO}_{2}$ (black dots), and flask $\mathrm{CO}$ (red dots) concentrations during the flight. The blue line shows background $\mathrm{CO}_{2}$ concentrations measured continuously at the $\mathrm{CF} 60 \mathrm{~m}$ tower (green square shown on Figure 1a). Note that these background $\mathrm{CO}_{2}$ concentrations decrease during the day as a result of plant uptake, while fossil sources in OKC elevate $\mathrm{CO}_{2}$ and $\mathrm{CO}$ concentrations. The NOAA flasks will provide concentrations of multiple hydrocarbon species to relate to the $\mathrm{CO}_{2}$ signal.

\section{SIGNIFICANCE OF FINDINGS}

The results demonstrate that we can use simultaneous analysis of multiple species to differentiate anthropogenic versus natural influences on atmospheric $\mathrm{CO}_{2}$ (and other species), (a)

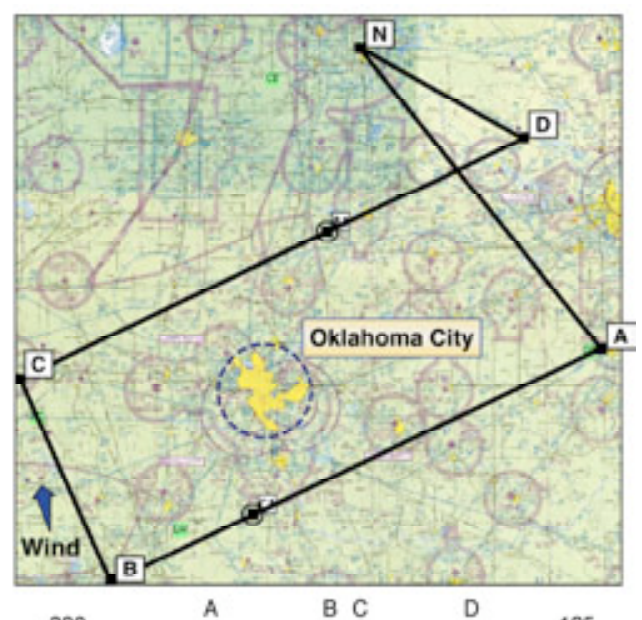

(b)

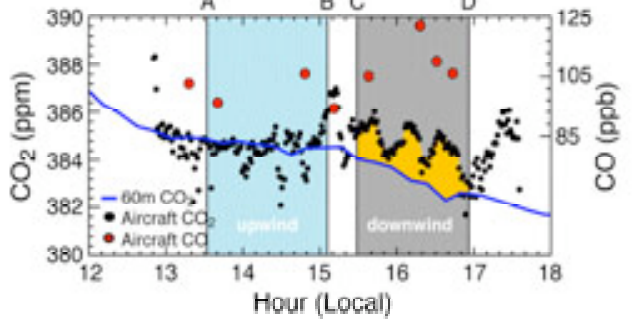

Figure 1. (a) Map showing the flight pattern flown by the CIRPAS Twin Otter on June 22, 2007; (b) Time series of continuous $\mathrm{CO}_{2}$ and flask $\mathrm{CO}$ concentrations measured during the mission. The prevailing wind was from the south.

upwind and downwind of OKC. Further analysis of $\mathrm{CO}, \mathrm{CH}_{4}$, ${ }^{13} \mathrm{CO}_{2}$, and ${ }^{14} \mathrm{CO}_{2}$ will allow quantitative partitioning of anthropogenic emissions and biosphere fluxes. Developing a quantitative fingerprint for anthropogenic emissions is important for verifying fossil fuel emissions inventories, estimating biosphere $\mathrm{CO}_{2}$ fluxes, and closing the North American carbon budget.

\section{RELATED PUBLICATIONS AND RESOURCES}

CLASIC Science Plan, 2007. http://www.arm.gov/publications/programdocs/doe-sc-arm-0703.pdf

ARM Carbon Web Site: http:/ / esd.lbl.gov/ARMCarbon/

\section{ACKNOWLEDGMENTS}

This work was supported by the Director, Office of Science, Office of Biological and Environmental Research, Climate Change Research Division, Atmospheric and Radiation Measurements Program, of the U.S. Department of Energy under Contract No. DE-AC02-05CH11231, and in-kind contributions from NOAA-ESRL, Lawrence Livermore National Laboratory, and Carnegie Institution of Washington.

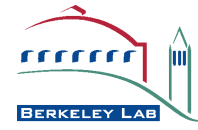




\section{Missing FeEdBACKS, AsymMetric UnCERTAinties, AND THE UNDERESTIMATION OF FUTURE WARMING \\ Margaret S. Torn and John Harte \\ Contact: Margaret S. Torn, 510/495-2223, MSTorn@lbl.gov}

\section{RESEARCH OBJECTIVES}

Historical evidence shows that atmospheric greenhouse gas (GhG) concentrations increase during periods of warming, implying a positive feedback to climate change. However, current general circulation models (GCMs) include only a subset of the possible GhG feedback processes-for example, methane processes are not included at all. We quantified the potential $\mathrm{CO}_{2}$ and $\mathrm{CH}_{4}$ feedback and explored the implications of positive feedback for climate risk.

\section{APPROACH}

To achieve a comprehensive accounting, we used a simple model combined with ice core data, which integrate over all active processes, rather than a detailed simulation model that could only capture a few processes. Specifically, we quantified climate-GhG feedbacks by combining the mathematics of feedback from engineering literature with (1) empirical ice-core data from the past 320,000 years and (2) general circulation model climate sensitivity from nine $\mathrm{GCMs}\left(1.2^{\circ} \mathrm{C} / 275 \mathrm{ppm} \mathrm{CO}_{2}\right.$ equivalent, in the absence of other feedbacks).

The strength of GhG feedbacks was estimated by the overall gain in the system-how much the final temperature change, $\Delta T_{F}$ was amplified or dampened from an initial perturbation, $\Delta T_{0}$. The feedbacks currently in GCMs - mainly water vapor, cloud, and ice-albedo processes-amplify the direct effect of doubled- $\mathrm{CO}_{2}$ $\left(1.2^{\circ} \mathrm{C}\right)$ to a total warming of $1.5-4.5^{\circ} \mathrm{C}$, meaning they have a gain of $0.20-0.73$ without GhG feedbacks (see equation in Figure 1). When we add the GhG gain to the existing climate model gain, the total gain is $0.27-0.8$.

\section{ACCOMPLISHMENTS/FINDINGS}

The feedback gain from greenhouse gases alone is fairly small. Each new increment of feedback, however, has an increasingly larger effect on the final temperature (see Figure 1). As a result, adding the GhG to the existing feedbacks greatly amplifies any climate perturbation. In fact, an emissions scenario that would currently be predicted to result in $1.5-4.5^{\circ} \mathrm{C}$ warming would actually result in $1.6-6.0^{\circ} \mathrm{C}$ warming at equilibrium. In other words, adding the small GhG gain results in up to $1.5^{\circ} \mathrm{C}$ warmer temperatures than currently predicted for emissions that would double atmospheric $\mathrm{CO}_{2}$ concentrations. That is because instead of merely doubling concentrations, those anthropogenic emissions would be matched by a kind of natural "matching fund." This additional feedback thus takes place because anthropogenic GhG emissions cause warming, which alters earth system processes, resulting in additional atmospheric greenhouse gas loading and additional warming.

\section{SIGNIFICANCE OF FINDINGS}

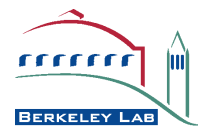

The GhG feedbacks greatly increase the warming commitment engendered for any given anthropogenic emissions scenario. Moreover, systems with positive feedbacks have a higher probability of experiencing the upper range of predicted change rather than the lower end. In other words, the presence of positive feedbacks means that there is a higher risk that we will experience more severe, not less severe, climate change than is currently forecast.

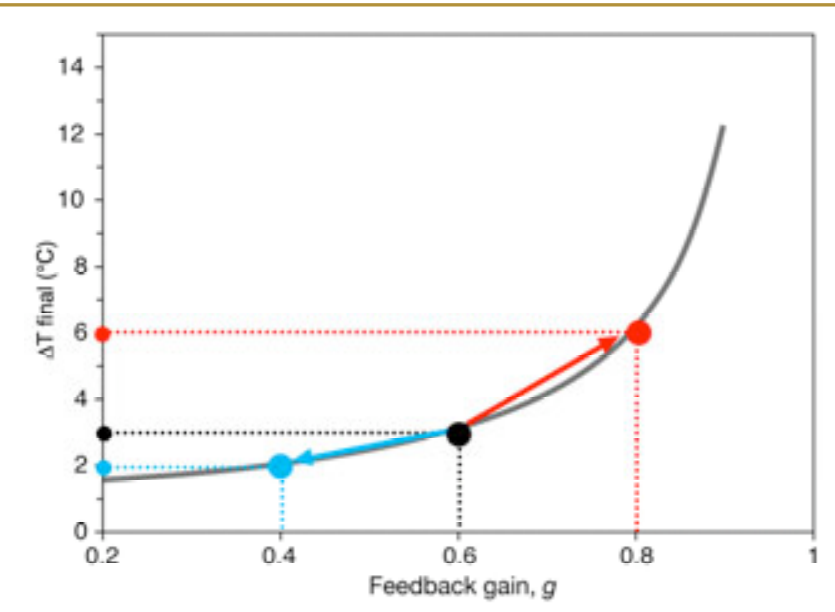

Figure 1. This graph of the feedback equation (inset in Figure) illustrates that a change or uncertainty in the feedback has an asymmetric effect, meaning that a +0.1 increase in feedback gain, $g$, has much bigger effect on temperature than does a -0.1 decrease. In the example shown here, the direct warming $(\Delta \mathrm{T} 0)$ is $1^{\circ} \mathrm{C}$, nominal $g=0.7$ and nominal final warming $(\Delta \mathrm{TF})=3.3^{\circ} \mathrm{C}$. An additional 0.1 negative feedback $(g=0.7-0.1=0.6)$ results in a warming of $2.5^{\circ} \mathrm{C}\left(0.8^{\circ} \mathrm{C}\right.$ lower than the nominal case), whereas the same magnitude of change or uncertainty in the positive direction $(g=$ $0.7+0.1=0.8)$ results in a $5^{\circ} \mathrm{C}$ warming $\left(1.7^{\circ} \mathrm{C}\right.$ higher $)$.

\section{RELATED PUBLICATIONS}

Torn, M.S. and J. Harte, Missing feedbacks, asymmetric uncertainties, and the underestimation of future warming. Geophys. Res. Lett., 33, L10703, doi:10.1029/2005GL025540, 2006.

Intergovernmental Panel on Climate Change, Climate Change 2007-The physical science basis. Contribution of Working Group I to the Fourth Assessment Report of the Intergovernmental Panel on Climate Change, 996 pp., Cambridge University Press, Cambridge, UK, 2007.

\section{ACKNOWLEDGMENTS}

This work was supported by the Director, Office of Science, Office of Biological and Environmental Research, Climate Change Research Division, of the U.S. Department of Energy under Contract No. DE-AC02-05CH11231, and the National Science Foundation under Contract No. DEB0211025. We thank $\mathrm{K}$. Cuffey for access to the ice core data. 


\title{
Characterizing RoOT DyNAMICS IN Forests: Estimates Using Radiocarbon Data AND Numerical Modeling
}

\author{
M. S. Torn, W. J. Riley, J. B. Gaudinski, John D. Joslin, Paul Hanson \\ Contact: Margaret S. Torn, 510/495-2223, MSTorn@lbl.gov
}

\section{RESEARCH OBJECTIVES}

In a typical year, terrestrial plants assimilate about twenty times as much $\mathrm{CO}_{2}$ as is produced through fossil fuel combustion. Of the assimilated carbon, most is rapidly respired back to the atmosphere, but a substantial fraction is used to build plant tissues. Root carbon (C) dynamics represent an important and poorly characterized component of these ecosystem C exchanges. Our goal in this research was to improve the conceptual representation of fine root $(<2 \mathrm{~mm}$ diameter) $\mathrm{C}$ flows in a numerical model applicable to site, regional, and global analyses.

\section{APPROACH}

We used a whole-ecosystem ${ }^{14} \mathrm{C}$ label to explore fundamental concepts of how carbon cycles through roots, and to develop, parameterize, and test a model (Radix1) of fine-root mortality and decomposition. Carbon-14 measurements from two root size classes at three soil depths, tree rings, and soil respiration, along with ancillary data on belowground primary production and specific root respiration rates at a temperate forest in Oak Ridge, Tennessee, were used in the development of Radix.

\section{ACCOMPLISHMENTS}

In contrast to previous models of fine root $C$ dynamics, the $14 \mathrm{C}$ measurements (Figure 1) from the Oak Ridge site clearly indicated the need for a storage pool to support root growth in some seasons and at least two $\mathrm{C}$ pools each for live and dead fine roots (Riley et al., 2008). We developed Radix to simulate two live root pools (one with structural and nonstructural $\mathrm{C}$ components), two dead root pools, non-normally distributed root mortality turnover times, a stored $\mathrm{C}$ pool, and seasonal growth and respiration patterns. The turnover time through the stored $\mathrm{C}$ pool was estimated to be $\sim 0.7$ years (Gaudinski et al., 2008), while root lifetimes were $\sim 1$ and 10 years for the fast and slow live-root pools, respectively (and similar for the fast and slow dead-root-pool decomposition turnover times). Interpretation of root ${ }^{14} \mathrm{C}$ measurements was strongly affected by root respiration estimates.

\section{SIGNIFICANCE OF FINDINGS}

We conclude that accurate characterization of $\mathrm{C}$ flows through fine roots requires a model with two live fine-root pools, two dead fine-root pools, storage, and root respiration. Root turnover times on the order of a decade imply substantially different response times in biomass and growth than are currently predicted by models with a single, relatively fast turnover pool. These results have a substantial impact on predictions for the amount of $\mathrm{C}$ that moves into soil organic matter, and hence on overall ecosystem $\mathrm{C}$ dynamics in forested ecosystems.

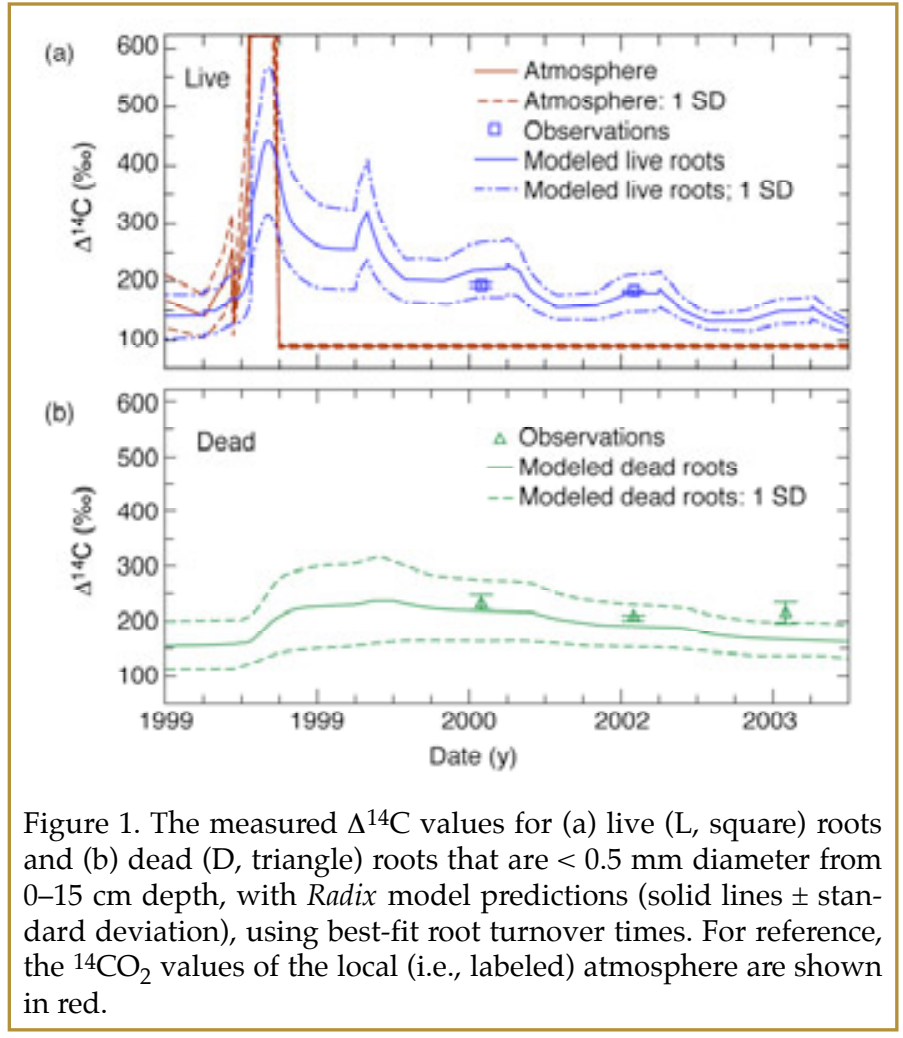

\section{RELATED PUBLICATIONS}

Gaudinski, J.B., W.J. Riley, M.S. Torn, J.D. Joslin, and P.J. Hanson, Quantification of root lifespan and stored carbon reserves to new root growth using radiocarbon data and a multi-compartment model. Global Change Biology (in press), 2008.

Riley, W.J., J.B.Gaudinski, M.S. Torn, J.D. Joslin, and P.J. Hanson, Fine-root mortality in a temperate forest: Estimates using radiocarbon data and numerical modeling. Global Change Biology (submitted), 2008.

\section{ACKNOWLEDGMENTS}

This work was supported by the Terrestrial Carbon Processes and Carbon Sequestration Programs, Office of Science, U.S. Department of Energy under Contract No. DEAC02-05CH11231.

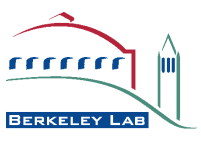




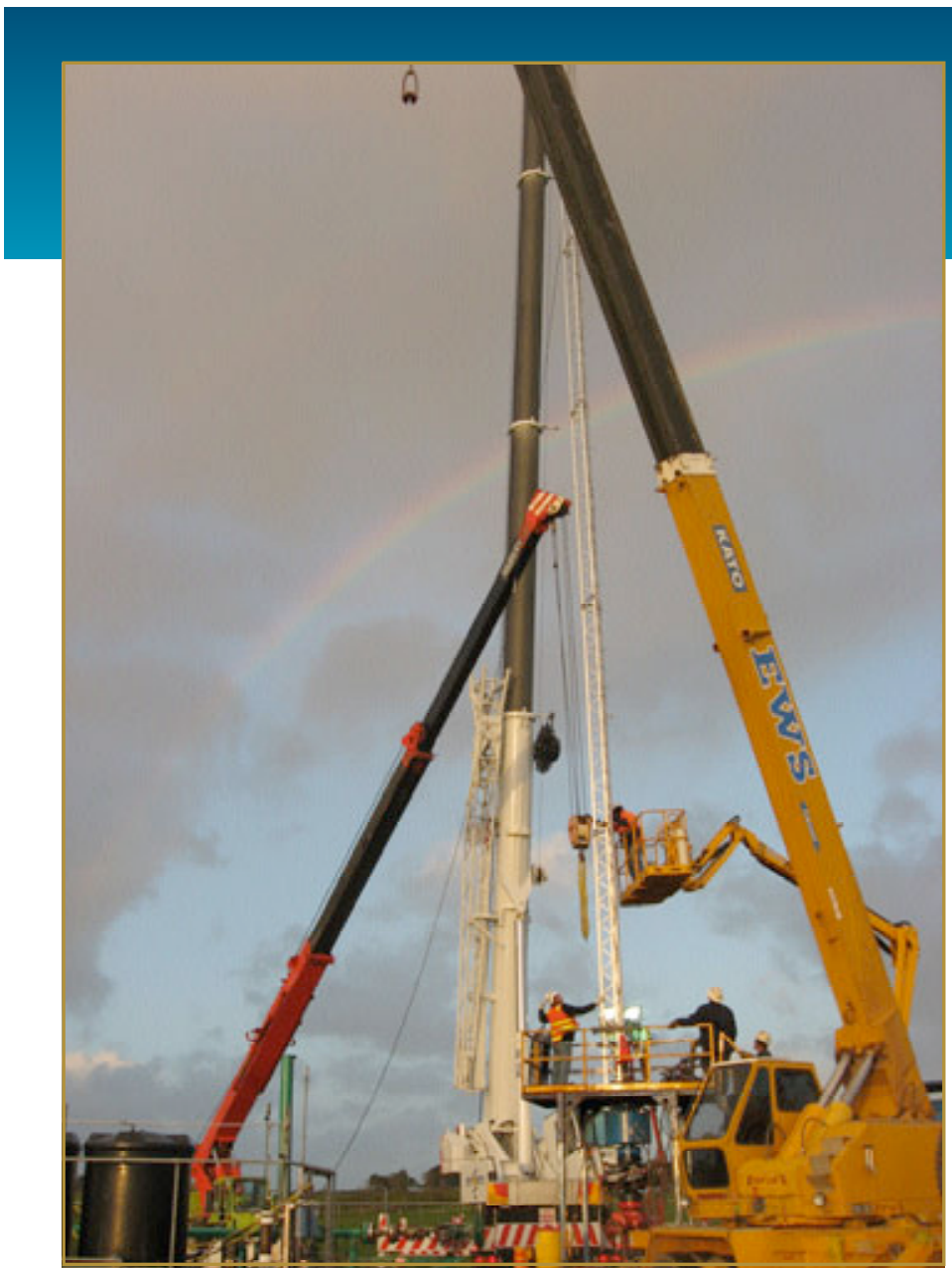

There is now broad agreement among scientists, scientific societies, and government agencies that growing atmospheric $\mathrm{CO}_{2}$ concentrations resulting from the burning of fossil fuels are causing increases in global atmospheric temperature. These temperature increases are projected to disrupt climate, warm the oceans, and melt continental ice sheets, leading to sea level rise. Avoiding the widespread and potentially disastrous consequences of climate change and sea-level rise depends on the ability of mankind to rapidly reduce $\mathrm{CO}_{2}$ emissions.

Experts believe that to avoid significant disruption of the climate system and ecosystems, $\mathrm{CO}_{2}$ concentrations must be stabilized within the next several decades. At today's emission rates of approximately $30 \mathrm{Gt} \mathrm{CO}_{2}$ /year from the burning of fossil fuels, $\mathrm{CO}_{2}$ concentrations will continue to grow and, within 50 years, may exceed the levels needed to protect sensitive agricultural and ecosystems and avoid flooding in lowlying coastal areas. This situation is even more urgent when we consider that, over the next 50 years, $\mathrm{CO}_{2}$ emissions are expected to double as the developing world's economies grow and the global standard of living increases.
Research Program Geologic Carbon Sequestration Program

\section{Curt Oldenburg}

(510) 486-7419 cmoldenburg@lbl.gov

\section{Larry Myer}

(510)486-6456 lrmyer@lbl.gov
One strategy to reduce effective $\mathrm{CO}_{2}$ emissions that has quickly advanced from a mere concept to a reality is the geologic storage of $\mathrm{CO}_{2}$ in underground formations. Significant progress has been made in the critical areas of geologic storage security and integrity, storage optimization, monitoring and verification, and risk assessment and mitigation. While additional research and testing remain to be accomplished before the technology can be applied widely, results of research conducted in various projects at Berkeley Lab and elsewhere demonstrate that this technology can make large contributions to the problem of reducing point-source $\mathrm{CO}_{2}$ emissions for climate-change mitigation.

On April 7, 2007, following nearly ten years of pioneering involvement in geologic carbon sequestration, the Earth Sciences Division added a new program, the Geologic Carbon Sequestration (GCS) Program. The GCS program is dedicated to addressing the pressing need for drastic reductions in $\mathrm{CO}_{2}$ emissions, while recognizing that fossil fuels will continue to be used for decades if not centuries. The GCS currently includes four main projects:

- GeoSeq

- West Coast Regional Carbon Sequestration Partnership (WESTCARB)

- Zero Emissions Research and Technology (ZERT)

- $\mathrm{CO}_{2}$ Geological Storage and Groundwater Resources

Each of these projects is focused on specific aspects of GCS. For example, GeoSeq focuses on scientific field testing and analysis of geologic storage including projects overseas. WESTCARB is a partnership for the purpose of pilot testing in western North America to demonstrate the potential for $\mathrm{CO}_{2}$ storage in deep geological formations and to enable deployment of CCS technology. ZERT performs fundamental research on geologic storage. The potential impact of large-scale geologic sequestration of $\mathrm{CO}_{2}$ on groundwater quality and hydrology is investigated in the fourth project.

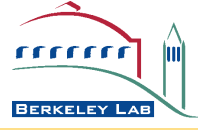




\section{GEOSEQ}

The GeoSeq Project has two primary goals:

(1) To develop ways to improve predictions of injectivity and effective capacity of saline formations and depleted gas reservoirs, and

(2) To develop and test innovative high-resolution methods for monitoring $\mathrm{CO}_{2}$ in the subsurface.

The GeoSeq project leverages scientific understanding and technology development from three highly visible, ongoing world-class (Carbon Sequestration Leadership Forum (CSLF)recognized) geologic $\mathrm{CO}_{2}$ storage projects, through leadership and collaboration in the scientific and engineering objectives. The three projects are the:

(1) Frio Brine Pilot Tests

(2) Otway Basin Pilot Project

(3) In Salah Industrial-Scale $\mathrm{CO}_{2}$ Storage Project

Within these projects, GeoSeq has led the development of downhole fluid and gas sampling by U-tube, downhole continuous active source seismic monitoring (CASSM), and reservoir simulation capabilities, including reactive geochemistry, multicomponent gas mixtures, and geomechanical coupling.

We continue to advance understanding of $\mathrm{CO}_{2}$ migration in brine formations and depleted gas reservoirs, and to investigate the geomechanical effects of industrial-scale $\mathrm{CO}_{2}$ injection. Although the three projects are carried out in distinct geological environments, the scope of GeoSeq is integrative, with strong cross-task communication and application of common tools and approaches in related projects. The overall objective of the GeoSeq project is to gain knowledge of geologic $\mathrm{CO}_{2}$ storage processes and mechanisms, and how to monitor and simulate them while making results available through publications and conference participation. Advances derived from GeoSeq efforts also support the DOE Regional Partnership Projects through the involvement of the investigators in various Partnership projects, and will likely be used in commercialscale $\mathrm{CO}_{2}$ operations in the future.

\section{WESTCARB}

The West Coast Regional Carbon Sequestration Partnership (WESTCARB) is a public-private partnership aimed at assessing carbon sequestration opportunities in the Western United States and Canada. The effort is led by the California Energy
Commission, which is responsible for the overall conduct of the work. The effort involves numerous private sector and public sector partners, who will be responsible for carrying out various aspects of the project. Overarching goals of the WESTCARB Phase III effort recently announced include:

(1) Promoting a better understanding of injectivity, capacity, and storativity of $\mathrm{CO}_{2}$ in typical West Coast formations

(2) Demonstrating the security and commercial viability of industrial-scale $\mathrm{CO}_{2}$ storage in a deep saline formation to pave the way for future widespread West Coast deployment of $\mathrm{CO}_{2}$ capture and geologic storage.

Lawrence Berkeley National Laboratory's contribution to the proposed work for the WESTCARB Phase III effort will cover the following:

(1) Site characterization efforts in support of local and regional reservoir model development, evaluation of formation capacity and injectivity, and regulatory permitting

(2) Risk assessment and mitigation planning in support of site selection, public safety, environmental protection, storage effectiveness, and regulatory permitting

(3) Performance monitoring in support of public safety, environmental protection, regulatory oversight, and demonstration of storage effectiveness.

ZERT

Carbon dioxide capture and storage in deep geologic formations has quickly emerged as one of the most promising options for reducing carbon dioxide emissions from combustion of fossil fuels. Potential storage formations include depleted oil and gas reservoirs, deep salt-water filled formations (saline formations), and unminable coal beds. ZERT focuses on developing fundamental knowledge needed to ensure successful storage in saline formations and hydrocarbon reservoirs. Topics of this study include the following:

(1) Performance prediction for underground fate and transport of $\mathrm{CO}_{2}$

(2) Measurement and monitoring techniques to verify storage and track migration of $\mathrm{CO}_{2}$

(3) Fundamental geochemical and hydrological investigations of $\mathrm{CO}_{2}$ storage 
The approach will include a combination of theoretical investigations, field studies, computer simulation, and laboratory experiments. Where appropriate, the applicability of these results to other types of storage formations will be identified.

\section{$\mathrm{CO}_{2}$ GEOLOGIC STORAGE \& GROUNDWATER RESOURCES (EPA-NETL)}

This project addresses concerns about the nation's groundwater resources and the potential impacts of large-scale deployment of geologic carbon sequestration on groundwater. For example, leakage of $\mathrm{CO}_{2}$ from depth and subsequent migration into shallow aquifers could result in water quality changes. Furthermore, the displacement of native brines caused by injection of large volumes of $\mathrm{CO}_{2}$ could impact the basin-scale hydrology of shallower groundwater systems, depending on the level of hydraulic communication between them. Two primary research tasks address these concerns, jointly coordinated by the U.S. Environmental Protection Agency (EPA) and the National Energy Technology Laboratory (NETL):
1. Understanding groundwater quality changes in case of $\mathrm{CO}_{2}$ intrusion (Task $\mathrm{A}$ )

2. Large-scale hydrological evaluation and modeling of impact on groundwater systems (Task B)

Both tasks are currently in the first year of a three-year research effort.

\section{FUNDING}

The GCS Program receives the bulk of its funding both directly and indirectly (e.g., indirectly through collaboration with regional partnership groups) from the Assistant Secretary for Fossil Energy, Office of Sequestration, Hydrogen, and Clean Coal Fuels, National Energy Technology Laboratory, of the U.S. Department of Energy under U.S. Department of Energy Contract No. DE-AC02-05CH11231. Additional support is provided by the U.S. Environmental Protection Agency (EPA), the Carbon Capture Project (CCP), and various other sources through the Berkeley Lab Work for Others program.

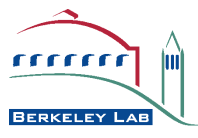




\title{
Large-Scale Hydrological Evaluation of $\mathrm{CO}_{2}$ InJeCtion-Storage and MODELING OF THE IMPACT ON GROUNDWATER SYSTEMS
}

\author{
Jens Birkholzer, Quanlin Zhou, Jonny Rutqvist, Keni Zhang, and Chin-Fu Tsang \\ Contact: Jens Birkholzer, 510/486-7134, JTBirkholzer@lbl.gov
}

\section{RESEARCH OBJECTIVES}

If carbon dioxide capture and storage (CCS) technologies are implemented, the amount of $\mathrm{CO}_{2}$ injected and sequestered underground will be extremely large. To date, the impact of large-scale injection and related brine displacement on regional multilayered groundwater systems has not been systematically assessed. The present project aims at enhancing understanding of the increase and extent of water pressure in the storage formation and in the shallower aquifers, due to injection of large amounts of supercritical $\mathrm{CO}_{2}$. In particular, the change in groundwater table level, the effect on discharge and recharge zones in the groundwater system, and the impact of these changes on the properties and characteristics of underground sources of drinking water (USDWs) will be investigated systematically.

\section{APPROACH}

A series of studies is being conducted with successive degrees of complexity, leading up to detailed modeling of one or two selected basin-scale groundwater systems in the United States with potential for large-scale $\mathrm{CO}_{2}$ sequestration. The first study is a systematic evaluation of storage capacity and pressure buildup in compartmentalized storage formations, where the native brine cannot easily escape because of closed boundaries. The second study assumes open multilayered groundwater systems and evaluates the potential impacts of brine displacement on shallow aquifers. Impact of coupled hydromechanical effects will be evaluated in the third study. Finally, the fourth study is an analysis and modeling of one or two representative basin-scale groundwater systems.

\section{ACCOMPLISHMENTS}

Results from a multiphase simulation model for $\mathrm{CO}_{2}$ injection into a compartmentalized saline formation were obtained as a function of storage formation volume and hydrogelogic characteristics. An example is shown in Figure 1. Based on comparison with simulation results, simple quick-assessment methods were developed for estimating the storage capacity in such pressure-constrained systems. We have also started a simulation study investigating $\mathrm{CO}_{2}$ injection into a multilayer system with open lateral boundaries as a function of the permeability of the upper and lower sealing units. It was found that the presence of low-permeability sealing layers may significantly affect fluid-pressure buildup and brine displacement for both laterally open and closed systems.

\section{SIGNIFICANCE OF FINDINGS}

Simple, quick-assessment methods for estimating storage capacity in pressure-constrained systems may prove to be valuable in practical applications, such as selection of an optimal site among several possibilities. The indicated significant influence of vertical brine movements through upper and lower confining layers on storage capacity may have been ignored in many large-scale studies to date. These will be further investigated. Papers summarizing results of the present research are being prepared for submission to journals.

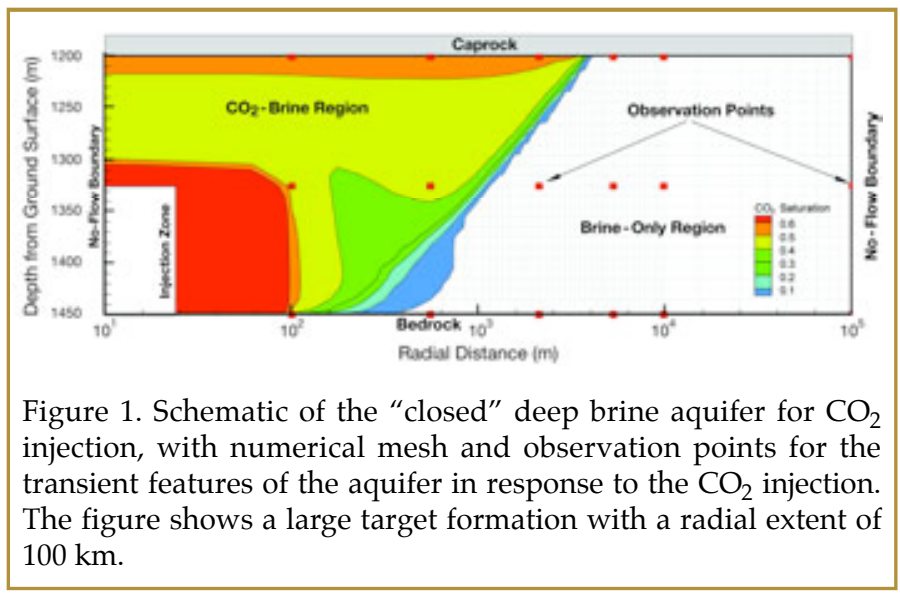

\section{RELATED PUBLICATION}

Zhou, Q., J. Birkholzer, J. Rutqvist, and C.-F. Tsang, Sensitivity study of $\mathrm{CO}_{2}$ capacity in brine aquifers with closed boundaries: Dependence on hydrogeologic properties. Abstract Submitted to 6th Annual Conference on Carbon Capture and Sequestration, Pittsburgh, PA, May 2007.

\section{ACKNOWLEDGMENTS}

This work was supported by the Assistant Secretary for Fossil Energy, Office of Coal and Power Systems, through the National Energy Technologies Laboratory (NETL), under the auspices of U.S. Department of Energy Contract No. DE-AC0205CH11231. 


\section{GeOCHEMicAl INVESTIGATIONS OF THE VulNERABILITY OF GROUNDWATER Resources in Case of $\mathrm{CO}_{2}$ Leakage from Deep Geologic Storage}

Jens T. Birkholzer, John Apps, Tianfu Xu, Yingqi Zhang, Liange Zheng, and Chin-Fu Tsang Contact: Jens Birkholzer, 510/486-7134, JTBirkholzer@lbl.gov

\section{RESEARCH OBJECTIVES}

If carbon dioxide stored in deep saline aquifers leaks into overlying sources of potable groundwater, the intruding $\mathrm{CO}_{2}$ would lower groundwater $\mathrm{pH}$ and thereby enhance the solubility of hazardous inorganic constituents (e.g., lead and arsenic). How and to what extent groundwater potability would be affected depend largely on the initial abundance and distribution of these constituents in the aquifers, as well as on the aquifer mineralogy and the oxidation state. The research objective is to understand in a general sense (1) which aquifer systems and regions of the United States might be vulnerable in case of $\mathrm{CO}_{2}$ intrusion, and (2) which inorganic constituents might adversely affect water quality and to what extent.

\section{APPROACH}

A database analysis is being conducted to evaluate almost 40,000 groundwater samples taken throughout the United States that report non-zero concentrations of selected hazardous constituents. Equilibrium models of aquifer chemistry are being developed to estimate the initial distribution of these hazardous constituents between the aqueous phase and adsorption and ion exchange sites, and in solid solution in primary and secondary minerals. The equilibrium models are then used as a starting point to (1) simulate the redistribution of hazardous constituents between the host rock and the groundwater in the case of $\mathrm{CO}_{2}$ intrusion with reactive transport models and (2) to predict the expected aqueous concentrations of such constituents for a range of relevant aquifer conditions. Laboratory experiments will also be conducted to validate and support the numerical results.

\section{ACCOMPLISHMENTS}

Data from almost 40,000 groundwater samples have been systematically evaluated in an automated procedure involving data management systems and distribution-of-species modeling. Results provide valuable information on the relevant geochemical conditions in aquifers defining the vulnerability of aquifers in case of $\mathrm{CO}_{2}$ intrusion. For example, we identified the fraction of groundwaters where minerals control the activity of hazardous constituents in the aqueous phase (which makes them vulnerable) and determined the number of samples that are saturated with respect to calcite (which makes them less vulnerable-Figure 1). In parallel to the database evaluation, a large number of reactive transport simulations have been conducted for modeling the mobilization of lead and arsenic in response to the intrusion of gaseous $\mathrm{CO}_{2}$ into a shallow aquifer. Various sensitivities have been evaluated related to aquifer conditions (e.g., $\mathrm{CO}_{2}$ intrusion rate, aquifer hydrogeology), geochemical parameters (e.g., rock mineralogy, thermodynamic data) and processes (e.g., adsorption/desorption, cation exchange, co-precipitation).

\section{SIGNIFICANCE OF FINDINGS}

Our preliminary results demonstrate that $\mathrm{CO}_{2}$-related dissolution may strongly increase concentrations of lead and arsenic in the groundwater, indicating the significance of this subject for geologic carbon sequestration. However, the simulations conducted to date arrive at water contamination above health-based limits only when very conservative assumptions are made with respect to the aquifer geochemical conditions. Our ongoing study will use more realistic scenarios and will be supplemented by laboratory experiments. Once completed, this research will provide a systematic evaluation of main aquifer types and determine their vulnerability in case of $\mathrm{CO}_{2}$ intrusion.

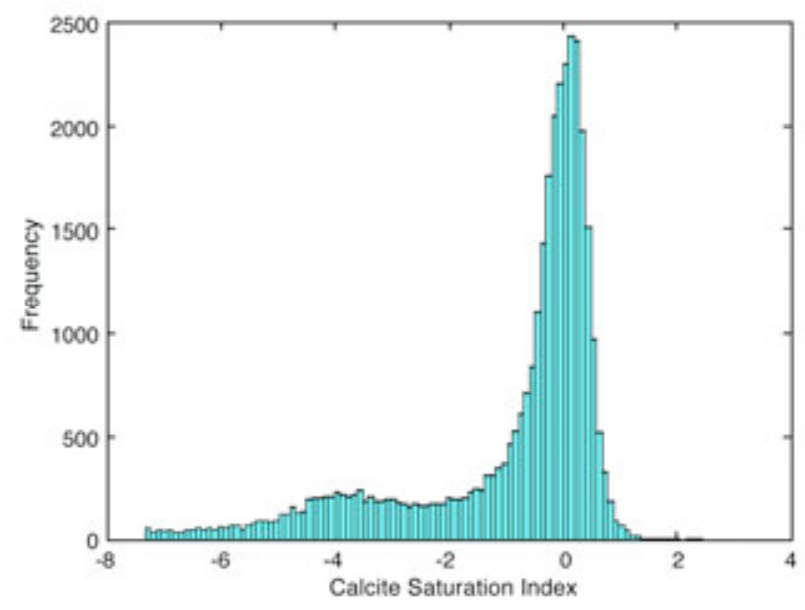

Figure 1. Calculated saturation index $(\log Q / K)$ distribution for calcite based on evaluation of almost 40,000 groundwater samples from aquifers throughout the United States

\section{RELATED PUBLICATIONS}

$\mathrm{Xu}, \mathrm{T} ., \mathrm{J}$. Apps, and J. Birkholzer, Contamination of groundwater by hazardous inorganic chemical constituents through induced acidification due to $\mathrm{CO}_{2}$ leakage from a storage formation. Abstract in Proceedings of the 6th Annual Conference on Carbon Capture and Sequestration, Pittsburgh, PA, May 2007.

Zhang, Y., J.A. Apps, J.A. Birkholzer, T. Xu, and C.-F. Tsang, A database analysis of the distribution of hazardous metals in groundwaters of the United States. Abstract in Proceedings 6th Annual Conference on Carbon Capture and Sequestration, Pittsburgh, PA, May 2007.

\section{ACKNOWLEDGMENTS}

This work was supported by the U.S. Environmental Protection Agency, Office of Water and Office of Air and Radiation, under an Interagency Agreement with the U.S. Department of Energy and Lawrence Berkeley National Laboratory.

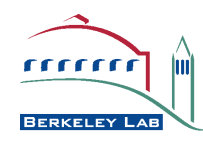




\section{Optimality in Characterizing $\mathrm{CO}_{2}$ Seepage from Geologic Carbon Sequestration Sites \\ Andrea Cortis, Curtis M. Oldenburg, and and Sally M. Benson ${ }^{1}$ \\ ${ }^{1}$ Stanford University, Stanford, CA \\ Contact: Andrea Cortis, 510/495-2507, acortis@lbl.gov}

\section{RESEARCH OBJECTIVES}

Surface monitoring to ensure safe and effective $\mathrm{CO}_{2}$ storage is complicated by the large area that may need to be monitored, and by large fluctuations in natural background $\mathrm{CO}_{2}$ fluxes and concentrations. These complications make seepage (surface $\mathrm{CO}_{2}$ leakage) monitoring potentially very expensive. We adopt the philosophy that monitoring is the activity involved in detecting and locating seepage areas, while measurements a re made to pinpoint and quantify seepage once it is detected. To be effective at detecting seepage, a monitoring program must be affordable enough to carry out and therefore requires optimization. In this work, we explore various optimization strategies for characterizing seepage using near-surface measurement approaches such as accumulation chambers and eddy covariance towers.

\section{APPROACH}

We are using a combination of theoretical and numerical approaches. Simulations of $\mathrm{CO}_{2}$ seepage in a 3-D system with variable topography were carried out using TOUGH2/EOS7CA to generate a virtual seepage signal. The resulting virtual $\mathrm{CO}_{2}$ concentrations were analyzed using MATLAB routines for artificial neural networks (ANNs) and particle swarm optimization (PSO) to develop a promising approach for optimizing seepage characterization.

\section{ACCOMPLISHMENTS}

Assuming no a priori knowledge of where seepage will occur, a static grid-based arrangement of monitoring stations (e.g., eddy covariance towers) is the only configuration suitable to ensure detection of seepage above some threshold value. The fixed-grid approaches needed to detect seepage are expected to require large numbers of eddy covariance towers for large-scale geologic $\mathrm{CO}_{2}$ storage. Once seepage has been detected and roughly located using a fixed grid approach, seepage zones and features can be optimally pinpointed through a dynamic search strategy, e.g., employing accumulation chambers and/or soil-gas sampling. Quantification of seepage rates can be done through measurements on a localized fixed grid once the seepage is pinpointed.

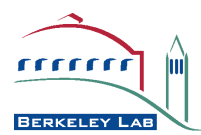

ANNs can be used as regression models for distinguishing natural system behavior from $\mathrm{CO}_{2}$ seepage without the need for detailed

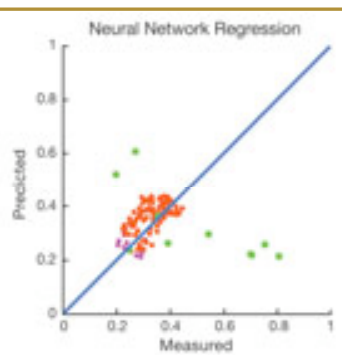

understanding of natural system processes. An example of the use of ANNs for seepage detection is shown in Figure 1. Because of natural variability, simple steepest-descent algorithms are not effective, and evolutionary computation algorithms are proposed as a paradigm for dynamic monitoring networks to pinpoint $\mathrm{CO}_{2}$ seepage areas. One such approach is PSO, in which a swarm of particles is assigned a random position and velocity to sample the search space. We used PSO to locate the $\mathrm{CO}_{2}$ seepage anomaly in the virtual landscape of Figure 1. Convergence to the global minimum was achieved in the majority of the runs for different random initializations. Additional analyses will need to be undertaken for a better understanding of convergence and convergence rates.

\section{SIGNIFICANCE OF FINDINGS}

The expense anticipated to carry out effective geologic $\mathrm{CO}_{2}$ storage monitoring motivates optimization. We find that ANNs and PSO are promising approaches for lowering the cost of $\mathrm{CO}_{2}$ seepage characterization.

\section{RELATED PUBLICATIONS}

Cortis, A., C.M. Oldenburg, and S.M. Benson, On the optimality of above-ground monitoring networks for carbon capture and storage. LBNL-62512 Abs. Sixth Annual Conference on Carbon Capture and Sequestration, Pittsburgh, PA, May 8-10,2007,

Oldenburg, C.M., A. Cortis, and S.M. Benson, Near-Surface Dispersion of $\mathrm{CO}_{2}$ Seepage from Geologic Storage Sites: Interplay of Process and Detection Strategy. LBNL-62875 Abs. American Geophys. Union Fall Meeting, San Francisco, CA, Dec. 2006.

\section{ACKNOWLEDGMENTS}

This work was conducted as part of the Zero Emissions Research and Technology (ZERT) project, supported by the Assistant Secretary for Fossil Energy, Office of Sequestration, Hydrogen, and Clean Coal Fuels, through the National Energy Technology Laboratory, U.S. Department of Energy, under Contract No. DE-AC02-05CH11231. Hydrogen and Clean Coal Fuels, through the National Energy Technology Laboratory, U.S. Department of Energy, under Contract No. DE-AC0205CH11231. 


\title{
Continuous Active-Source Seismic Monitoring (CASSM) for $\mathrm{CO}_{2}$ INJECTION IN A BRINE AQUIFER
}

\author{
Thomas M. Daley, Ray D. Solbau, Jonathan B. Ajo-Franklin, and Sally M. Benson ${ }^{1}$ \\ ${ }^{1}$ Stanford University, Stanford, CA \\ Contact: Tom Daley, 510/486-7316, tmdaley@lbl.gov
}

\section{RESEARCH OBJECTIVES}

Monitoring of subsurface $\mathrm{CO}_{2}$ storage is a key component of carbon sequestration. As part of a small-scale $\mathrm{CO}_{2}$ injection, known as the Frio-II experiment, we developed a novel crosswell seismic monitoring approach, which would allow continuous crosswell monitoring during injection through use of a borehole source deployed in the injection well. The Frio-II experiment had the goal of monitoring $\mathrm{CO}_{2}$ plume migration under conditions strongly affected by buoyancy of $\mathrm{CO}_{2}$ in the brine aquifer. Monitoring buoyancy required crosswell geophysical measurements because fluid sampling in the observation well could not be relied on to detect the depth of initial $\mathrm{CO}_{2}$ breakthrough within the reservoir interval.

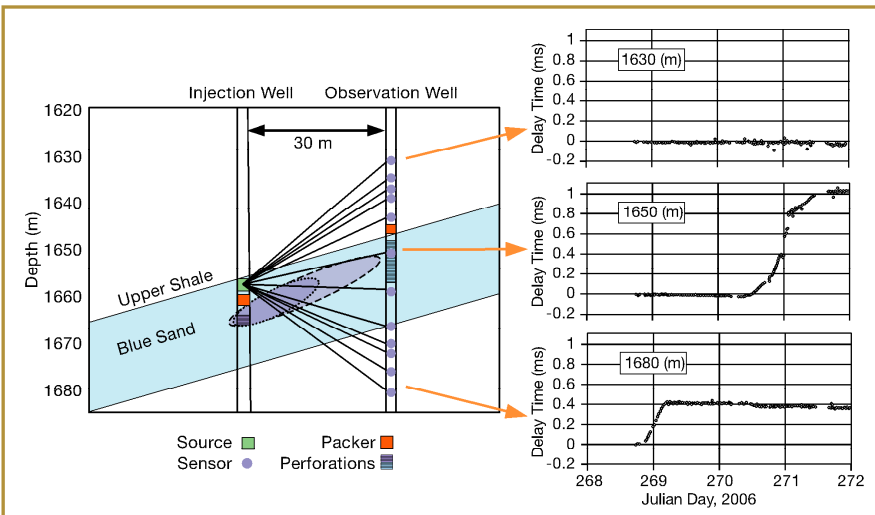

Figure 1. Schematic of Frio-II seismic monitoing experiment with conceptual $\mathrm{CO}_{2}$ plume after one day (inner short dash) and after 2 days (outer long dash), with measured delay times at three sensor depths over 3.5 days of $\mathrm{CO}_{2}$ injection shown at right.

\section{APPROACH}

Time-lapse crosswell tomographic imaging of the Frio- $\mathrm{ICO}_{2}$ plume demonstrated that large changes in seismic velocity (a 500 $\mathrm{m} / \mathrm{s}$ decrease within the plume) were caused by the injection of supercritical $\mathrm{CO}_{2}$ into the brine reservoir. The large seismic velocity change measured in the Frio-I test suggested that continuous monitoring of crosswell travel time during injection could detect $\mathrm{CO}_{2}$ saturation changes along a given raypath. Therefore, we designed a crosswell CASSM experiment for Frio-II in which data would be acquired continuously during injection along a set of fixed raypaths (Figure 1). Conceptually, if the $\mathrm{CO}_{2}$ saturation and / or plume thickness increased along a given raypath, the travel time would decrease, thereby allowing detection with some spatial resolution, especially in the vertical direction. Obtaining continuous crosswell seismic data required deploying the seismic source and sensors via production tubing concurrently with a geochemical fluid sampling system. The seismic source needed to be deployed on the outside of production tubing, within a small annular space. To address this limitation, we designed a novel "piezotube" source (Daley et al., patent pending). The source could then be deployed in the injection well for the duration of the injection.

\section{ACCOMPLISHMENTS}

Continuous crosswell seismic monitoring of a small-scale $\mathrm{CO}_{2}$ injection was accomplished with the development of a novel tubing-deployed piezoelectric borehole source. This "piezotube" source, deployed on the $\mathrm{CO}_{2}$ injection tubing near the top of the saline aquifer reservoir at 1,657 $\mathrm{m}$ depth, enabled acquisition of crosswell recordings at 15-minute intervals during the multiday injection. The change in travel time recorded at various depths in a nearby observation well allowed hourby-hour monitoring of the growing $\mathrm{CO}_{2}$ plume via the induced seismic velocity change (Figure 1 ).

\section{SIGNIFICANCE OF FINDINGS}

Our field-scale application of the CASSM methodology, along with the development of a tubing deployable source, demonstrates a novel approach for characterizing reservoir processes in situ. The travel-time measurements indicate that the $\mathrm{CO}_{2}$ plume reached the top of the reservoir sand before reaching the observation well, thus providing information about the in situ buoyancy of $\mathrm{CO}_{2}$. Potential applications include perimeter detection of migrating $\mathrm{CO}_{2}$ and detailed crosswell "movies" of reservoir fluid movement.

\section{RELATED PUBLICATIONS}

Daley, T.M., R.D. Solbau, J.B. Ajo-Franklin, and S.M. Benson, Continuous active-source monitoring of $\mathrm{CO}_{2}$ injection in a brine aquifer. Geophysics, 71(5), 33-41, 2007.

Daley, T.M., L.R. Myer, J.E. Peterson, E.L. Majer, and G.M. Hoversten, Time-lapse crosswell seismic and VSP monitoring of injected $\mathrm{CO}_{2}$ in a brine aquifer. Environmental Geology (in press), 2007.

\section{ACKNOWLEDGMENTS}

This work was conducted as part of the Geologic Carbon Sequestration (GeoSeq) Project, supported by the Assistant Secretary for Fossil Energy, Office of Sequestration, Hydrogen, and Clean Coal Fuels, through the National Energy Technology Laboratory, U.S. Department of Energy, under Contract No. DE-AC02-05CH11231.

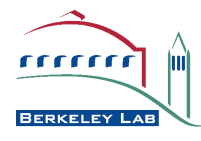




\section{Modeling Geologic Storage of Carbon Dioxide: Comparison of Nonhysteretic and Hysteretic Characteristic Curves \\ Christine Doughty \\ Contact: Christine Doughty, 510/486-6453, cadoughty@lbl.gov}

\section{RESEARCH OBJECTIVES}

Geologic storage of carbon dioxide $\left(\mathrm{CO}_{2}\right)$ in brine-bearing formations has been proposed as a means of reducing the atmospheric load of greenhouse gases. In the subsurface, supercritical $\mathrm{CO}_{2}$ forms an immiscible gas-like phase and partially dissolves in the brine. Numerical models of $\mathrm{CO}_{2} /$ brine systems use characteristic curves to represent the interactions of nonwetting-phase supercritical $\mathrm{CO}_{2}$ and wetting-phase brine. The simplest characteristic curves are nonhysteretic-the capillary pressure and relative permeabilities depend only on the local saturation at the current time. A more sophisticated approach is a hysteretic formulation, in which capillary pressure and relative permeabilities depend not only on the current value of the local saturation, but on the history of the local saturation and the process that is occurring: drainage (replacement of wetting phase with nonwetting phase) or wetting (replacement of nonwetting phase with wetting phase, also known as imbibition). The objective of this research is to investigate the impact of using nonhysteretic or hysteretic characteristic curves to model various aspects of $\mathrm{CO}_{2}$ geological storage.

\section{APPROACH}

Two example problems involving geologic $\mathrm{CO}_{2}$ storage are simulated with TOUGH2, a multiphase, multicomponent code developed at Berkeley Lab for flow and transport through geologic media. TOUGH2 considers all flow and transport processes relevant for a two-phase (liquid-gas), three-component $\left(\mathrm{CO}_{2}\right.$, water, dissolved $\left.\mathrm{NaCl}\right)$ system. Both a nonhysteretic and a newly developed hysteretic formulation are employed to represent characteristic curves, to illustrate the applicability and limitations of nonhysteretic methods. The first application considers leakage of $\mathrm{CO}_{2}$ from the storage formation to the ground surface, while the second examines the role of heterogeneity within the storage formation.

\section{ACCOMPLISHMENTS}

Simulation results show drastically different behavior for the long-term evolution of $\mathrm{CO}_{2}$ plumes for nonhysteretic and hys-

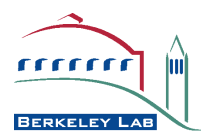
teretic formulations. For an idealized problem that involves only drainage or only wetting, a nonhysteretic formulation, in which capillary pressure and relative permeability depend only on the current value of the gridblock saturation, would be adequate. However, for a more realistic problem that includes both injection of $\mathrm{CO}_{2}$ (a drainage

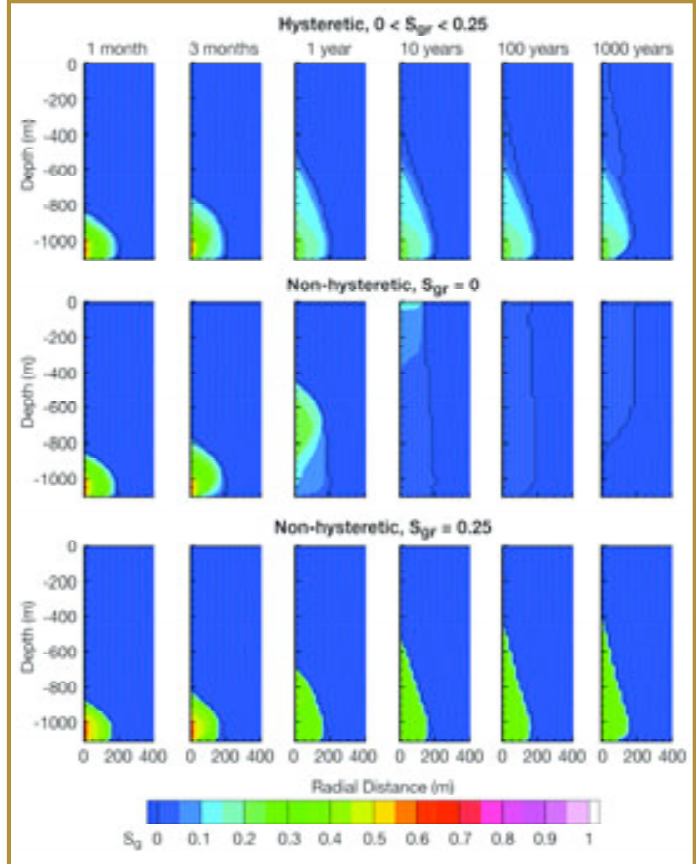

Figure 1. Free-phase $\mathrm{CO}_{2}$ distributions at a series of times for hysteretic (top) and nonhysteretic (middle and bottom) formulations for characteristic curves. $\mathrm{CO}_{2}$ injection at $1,000 \mathrm{~m}$ depth lasts for th. Neither nonhysteretic case can mode is more mobile at the top than at the bottom and a small amount of $\mathrm{CO}_{2}$ reaches the surface while the bulk of the plume remains trapped at depth. process) and its subsequent postinjection evolution (a combination of drainage and wetting), hysteretic characteristic curves are required to correctly capture the behavior of the $\mathrm{CO}_{2}$ plume. This finding is a consequence of the fact that residual gas saturation $\mathrm{S}_{\mathrm{gr}}$ (the saturation below which free-phase $\mathrm{CO}_{2}$ is trapped) is strongly process-dependent, with a zero value during drainage and a potentially large value during imbibition, a value that increases with the maximum local historical value of gas saturation.

\section{SIGNIFICANCE OF FINDINGS}

During post-injection periods, the leading edge of the $\mathrm{CO}_{2}$ plume undergoes drainage $\left(\mathrm{S}_{\mathrm{gr}}=0\right)$, while the trailing edge undergoes wetting (large $S_{g r}$ ). Thus, the plume is more mobile at the leading edge and less mobile at the trailing edge, so it elongates. In addition to trapping significant quantities of free-phase $\mathrm{CO}_{2}$ at the trailing edge of the plume, this elongation enables more $\mathrm{CO}_{2}$ to dissolve in the brine and greater interaction with rock minerals to occur, further immobilizing $\mathrm{CO}_{2}$. It is impossible to correctly model these processes using a nonhysteretic formulation for characteristic curves.

\section{RELATED PUBLICATION}

Doughty, C., Modeling geologic storage of carbon dioxide: comparison of non-hysteretic and hysteretic characteristic curves. Energy Conversion and Management 48(6), 1768-1781, 2007.

Related web site: http:/ / www-esd.lbl.gov/GEOSEQ/index.html

\section{ACKNOWLDGMENTS}

This work was conducted as part of the Geologic Carbon Sequestration (GeoSeq) and Zero Emissions Research and Technology (ZERT) projects, supported by the Assistant Secretary for Fossil Energy, Office of Sequestration, Hydrogen, and Clean Coal Fuels, through the National Energy Technology Laboratory, U.S. Department of Energy, under Contract No. DE-AC02-05CH11231. 


\title{
Site Characterization for $\mathrm{CO}_{2}$ Geologic Storage and Vice-Versa- The Frio Brine Pilot, Texas, as a Case Study
}

\author{
Christine Doughty, Barry M. Freifeld, and Robert C. Trautz \\ Contact: Christine Doughty, 510/486-6453, cadoughty@lbl.gov
}

\section{RESEARCH OBJECTIVES}

Geologic storage of carbon dioxide $\left(\mathrm{CO}_{2}\right)$ in brine-bearing formations has been proposed as a means of reducing the atmospheric load of greenhouse gases. Careful site characterization is critical for successful geologic storage of $\mathrm{CO}_{2}$, because of the many physical and chemical processes impacting $\mathrm{CO}_{2}$ movement and containment in the subsurface. Traditional site characterization techniques-such as geological mapping, geophysical imaging, and well logging and testing-provide the basis for judging whether or not a site is suitable for $\mathrm{CO}_{2}$ storage. However, only through the injection and monitoring of $\mathrm{CO}_{2}$ itself can the coupling between buoyancy flow, geologic heterogeneity, and history-dependent multiphase flow effects be observed and quantified. The purpose of this work is to demonstrate that $\mathrm{CO}_{2}$ injection and monitoring can provide a valuable addition to the site-characterization process.

\section{APPROACH}

At the Frio brine pilot, a researh project located in Dayton, Texas, 1,600 metric tons of $\mathrm{CO}_{2}$ were injected over a period of 10 days into a steeply dipping brine-saturated sand layer at a depth of $1,500 \mathrm{~m}$. The pilot is used as a case study to illustrate an iterative sequence in which traditional site characterization is used to prepare for $\mathrm{CO}_{2}$ injection, and then monitoring of the $\mathrm{CO}_{2}$ injection itself is used to further site-characterization efforts.

Numerical modeling plays a central role in integrating geological, geophysical, and hydrological field observations. In the subsurface, supercritical $\mathrm{CO}_{2}$ forms an immiscible gas-like phase that is strongly buoyant and partially dissolves in the brine. The numerical simulator TOUGH2 considers all flow processes relevant for a two-phase (liquid-gas), three-component $\left(\mathrm{CO}_{2}\right.$, water, dissolved $\left.\mathrm{NaCl}\right)$ system, including newly developed hysteretic formulations for capillary pressure and relative permeability functions.

\section{ACCOMPLISHMENTS}

Pre-injection activities included traditional site-characterization techniques, such as review of the regional geology, development of a local geological model, analysis of wireline logs, laboratory analysis of core samples, chemical analysis of brine samples, pressure-transient analysis of an interference well test, and breakthrough curve analysis for a two-well recirculation tracer test. Additionally, the $\mathrm{CO}_{2}$ injection itself served as an interference well test by monitoring downhole pressure in both wells and as a two-well tracer test by sampling fluid at the observation well using a novel U-tube configuration. Geophysical monitoring of $\mathrm{CO}_{2}$ movement in the subsurface during and after the injection period provided information on the spatial distribution of $\mathrm{CO}_{2}$ at different scales. $\mathrm{CO}_{2}$-saturation-sensitive well logs were run periodically in both injection and observation wells, crosswell seismic was conducted in the plane between the injection and observation wells before and after $\mathrm{CO}_{2}$ injection, and vertical seismic profiles (VSP) monitored the $\mathrm{CO}_{2}$ plume as it migrated beyond the immediate vicinity of the wells.

By comparing field observations to model results, we obtained both an improved understanding of the local geology and better-constrained values of multiphase flow parameters (see Figure 1).

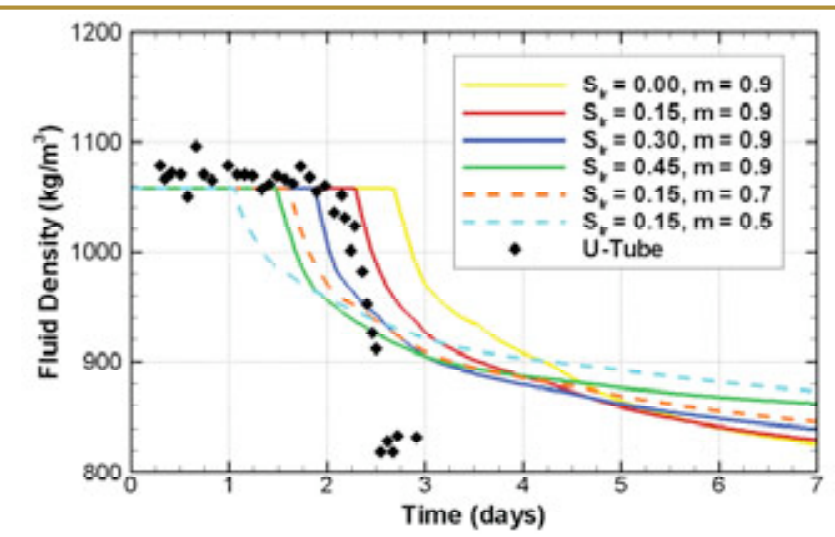

Figure 1. $\mathrm{CO}_{2}$ arrival at observation well as monitored with $\mathrm{U}$ tube sampling and model results for the $8 \mathrm{~m}$ sand model considering different two-phase flow parameters: $S_{1 \mathrm{r}}$ is residual liquid saturation, and $\mathrm{m}$ quantifies the interference between liquid and gas phases.

\section{SIGNIFICANCE OF FINDINGS}

Well thought-out site characterization is essential for successful geologic storage of $\mathrm{CO}_{2}$, and the site-characterization process greatly benefits from the addition of $\mathrm{CO}_{2}$ injection and monitoring.

\section{RELATED PUBLICATION}

Doughty, C., B.M. Freifeld, and R.C. Trautz, Site characterization for $\mathrm{CO}_{2}$ geologic storage and vice versa-The Frio brine pilot, Texas, USA, as a case study. Environmental Geology DOI 10.10007/s00254-007-0942-0, 2007.

\section{ACKNOWLEDGMENTS}

This work was conducted as part of the Geologic Carbon Sequestration (GeoSeq) Project, supported by the Assistant Secretary for Fossil Energy, Office of Sequestration, Hydrogen, and Clean Coal Fuels, through the National Energy Technology Laboratory, U.S. Department of Energy, under Contract No. DE-AC02-05CH11231.

\section{RELATED WEB SITE}

http:/ / www-esd.lbl.gov/GEOSEQ/index.html

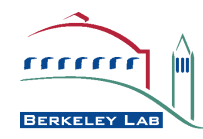




\section{Eddy Covriance Measurements of $\mathrm{CO}_{2}$ Emissions at MAMMOTH MOUNTAIN, CALIFORNia}

Jennifer L. Lewicki, Marc L. Fischer, and George E. Hilley

Contact: Jennifer L. Lewicki, 510/495-2818, jllewicki@lbl.gov

\section{RESEARCH OBJECTIVES}

The eddy covariance (EC) method, a micrometeorological technique traditionally used to measure $\mathrm{CO}_{2}$ fluxes across the interface between the atmosphere and a plant canopy, has been proposed as a viable technique to monitor for $\mathrm{CO}_{2}$ leakage from geologic storage sites. EC provides the benefit of an automated flux measurement that is averaged over both time and space. However, the theory that underlies EC assumes spatial homogeneity of surface fluxes and flat terrain, conditions that are not typically met at sites being considered for $\mathrm{CO}_{2}$ storage. The objective of this work is to evaluate the quality of $\mathrm{EC} \mathrm{CO}_{2}$ flux measurements made at Mammoth Mountain, California, a site that challenges the basic assumptions of EC with complex terrain and high, spatially heterogeneous $\mathrm{CO}_{2}$ emission rates.

\section{APPROACH}

An EC station was deployed on Mammoth Mountain from September 8, 2006, to October 24, 2006. EC CO fluxes were calculated by time averaging over half-hour periods the product of the time series of fluctuating vertical wind velocity and atmospheric $\mathrm{CO}_{2}$ concentration, measured at a fixed height above the ground surface. Surface $\mathrm{CO}_{2}$ fluxes were also measured at point locations on a grid using the chamber method, repeatedly over a ten-day period. EC fluxes were compared to chamber fluxes through the footprint model, a weighting function that describes the relative contribution of each point source surface flux to the EC flux. On any given day, the grid of chamber $\mathrm{CO}_{2}$ flux measurements was weighted by the halfhour EC footprint function (hereafter referred to as the footprint $\mathrm{CO}_{2}$ flux) and compared to the half-hour EC flux.

\section{ACCOMPLISHMENTS}

The mean and standard deviation of the relative difference between the EC and footprint $\mathrm{CO}_{2}$ fluxes, expressed as a percentage of the footprint flux, were -0.3 and $23 \%$, respectively, indicating that the measurements were nearly unbiased. A plot of EC versus footprint $\mathrm{CO}_{2}$ flux (Figure 1a) shows that the data are moderately correlated ( $\mathrm{R} 2=0.42$ for $1: 1$ line). The correlation increases ( $\mathrm{R} 2=0.70$ for 1:1 line) for average daily EC versus average daily footprint $\mathrm{CO}_{2}$ flux (Figure 1b).

\section{SIGNIFICANCE OF FINDINGS}

$\mathrm{EC} \mathrm{CO}_{2}$ fluxes were measured at a site that challenged the underlying assumptions of EC theory. Variability in the EC and footprint flux datasets was likely caused by our inability to completely characterize spatial-temporal variations in surface $\mathrm{CO}_{2}$ fluxes using the chamber method, as well as by inherent random errors associated with both the EC and chamber methods.

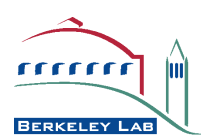
Average daily EC and footprint $\mathrm{CO}_{2}$ fluxes were well correlated, indicating that when random error is reduced by temporal averaging, the EC technique can perform well under certain challenging site conditions. EC has the potential to be reliably used for leakage monitoring at $\mathrm{CO}_{2}$ storage sites.

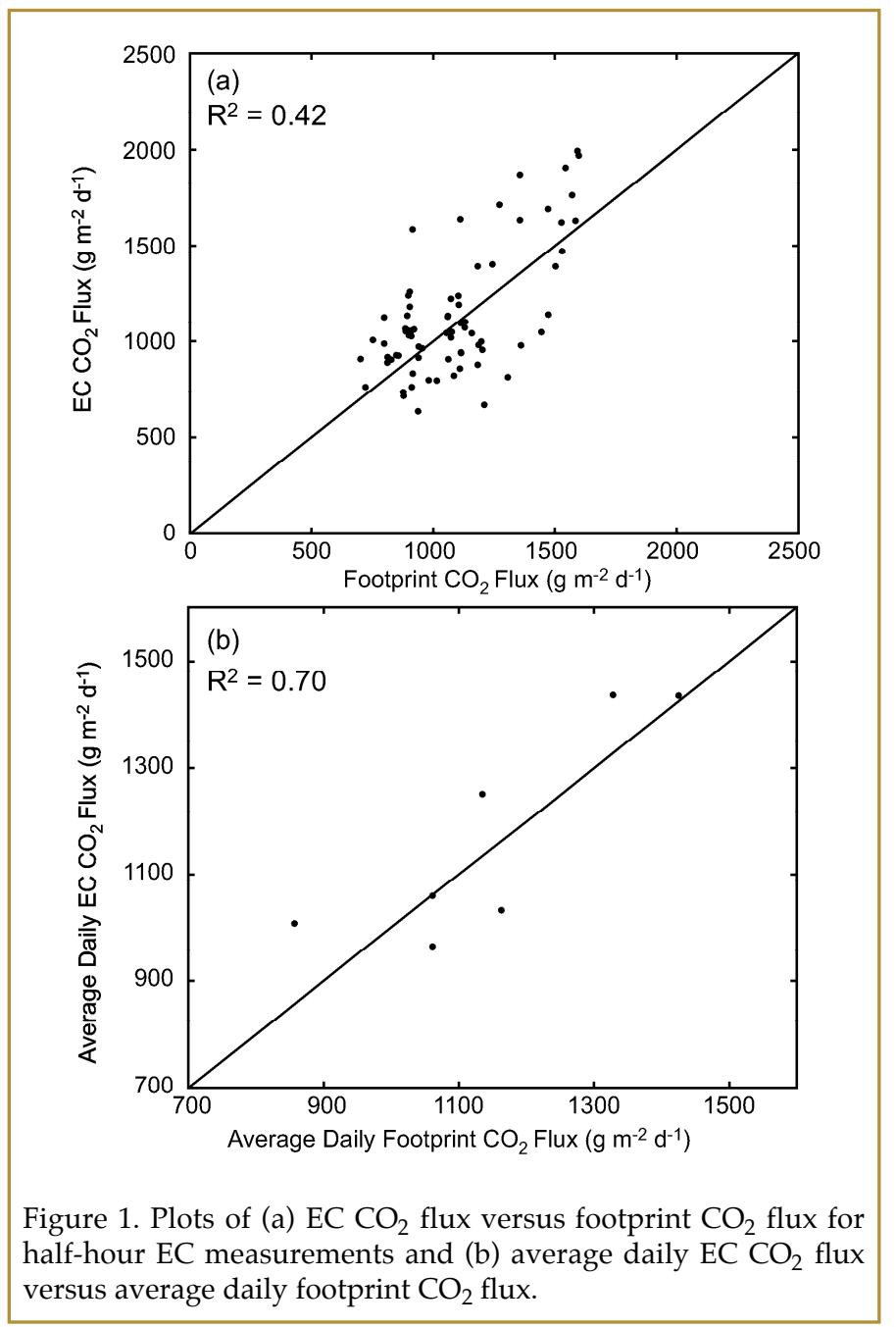

\section{RELATED PUBLICATION}

Lewicki, J.L., G.E. Hilley, T. Tosha, R. Aoyagi, K. Yamamoto, and S.M. Benson, Dynamic coupling of volcanic $\mathrm{CO}_{2}$ flow and wind at the Horseshoe Lake tree kill, Mammoth Mountain, California. Geophysical Research Letters, 34, L03401, doi:10.1029/2006GL028848, 2007.

\section{ACKNOWLEDGEMENTS}

This work was supported by the Assistant Secretary for Fossil Energy, Office of Sequestration, Hydrogen, and Clean Coal Fuels, through the National Energy Technology Laboratory, U.S. Department of Energy under Contract No. DE-AC02-05CH11231. 


\title{
Natural Analogues for $\mathrm{CO}_{2}$ Leakage from Geologic Storage Sites
}

\author{
Jennifer L. Lewicki, Jens Birkholzer, and Chin-Fu Tsang
}

Contact: Jennifer L. Lewicki, 510/495-2818, jllewicki@lbl.gov

\section{RESEARCH OBJECTIVES}

While the purpose of geologic carbon storage is to trap $\mathrm{CO}_{2}$ underground, $\mathrm{CO}_{2}$ could migrate away from the storage site into the shallow subsurface and atmosphere. Although limited $\mathrm{CO}_{2}$ leakage does not negate the net reduction of $\mathrm{CO}_{2}$ emissions to the atmosphere, adverse health, safety, and environmental risks associated with elevated $\mathrm{CO}_{2}$ concentrations must be evaluated. Cases of $\mathrm{CO}_{2}$ leakage from natural reservoirs to the near-surface environment serve as analogues for the potential release of $\mathrm{CO}_{2}$ from geologic storage sites. The objective of this research is to summarize and compare the features, events, and processes (FEPs) of $\mathrm{CO}_{2}$ leakage examples from natural geologic reservoirs. We describe causes and consequences of $\mathrm{CO}_{2}$ leakage, and discuss implications for geologic carbon storage and risk assessment work.

\section{APPROACH}

Carbon dioxide leakage cases were classified according to (1) key features of the $\mathrm{CO}_{2}$ accumulation, including site location, the source of the $\mathrm{CO}_{2}$, and the geologic model for $\mathrm{CO}_{2}$ accumulation; (2) the events leading to leakage from the reservoir; and (3) the processes by which $\mathrm{CO}_{2}$ was released at the surface, including the pathway(s) for leakage and the style of surface emission. Cases from volcanic, geothermal, and sedimentary basin settings were considered.

\section{ACCOMPLISHMENTS}

Key FEPs were identified for a wide range of $\mathrm{CO}_{2}$ leakage cases. Here, we show one example from each major geologic setting (Table 1).

\section{SIGNIFICANCE OF FINDINGS}

Four general FEPs were identified based on analysis of a range of natural analogues for $\mathrm{CO}_{2}$ leakage, from which lessons can be learned and should be applied to risk assessment associated with geologic carbon storage:

(1) $\mathrm{CO}_{2}$ can both accumulate beneath, and be released from, primary and secondary reservoirs with capping units located at a wide range of depths. Both primary and secondary reservoir entrapments for $\mathrm{CO}_{2}$ should be properly characterized at potential geologic carbon sequestration sites.

(2) Many $\mathrm{CO}_{2}$ releases have been correlated with a specific event that has triggered the release, such as magmatic or seismic activity. The potential for processes that could cause geomechanical damage to sealing cap rocks and trigger the release of $\mathrm{CO}_{2}$ from a storage reservoir should be evaluated.

(3) Unsealed fault and fracture zones can act as fast and direct conduits for $\mathrm{CO}_{2}$ flow from depth to the surface. Risk assessment should therefore emphasize determining the potential for and nature of $\mathrm{CO}_{2}$ migration along these structures.

(4) The style of $\mathrm{CO}_{2}$ release at the surface varies widely between and within different leakage sites. In rare circumstances, the release of $\mathrm{CO}_{2}$ can be a self-enhancing and/or eruptive process; this possibility should be assessed in the case of $\mathrm{CO}_{2}$ leakage from storage reservoirs.

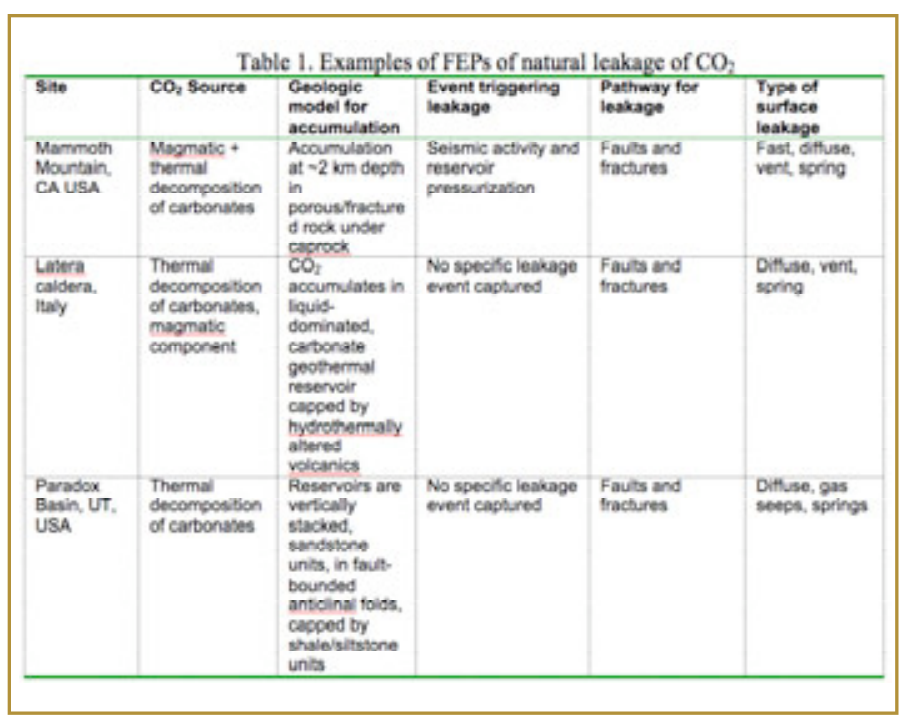

\section{RELATED PUBLICATION}

Lewicki, J.L., J. Birkholzer, and C.-F. Tsang, Natural and industrial analogues for leakage of $\mathrm{CO}_{2}$ from storage reservoirs: Identification of features, events, and processes and lessons learned. Environmental Geology, doi:10.1007/s00254-0060479-7, 52, 2007.

\section{ACKNOWLEDGEMENTS}

This work was supported by the U.S. Environmental Protection Agency, Office of Water and Office of Air and Radiation, under an Interagency Agreement with the U.S. Department of Energy at the Lawrence Berkeley National Laboratory, Contract No. DE-AC02-05CH11231.

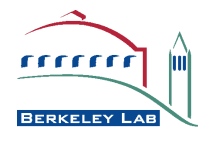




\section{Certification Framework (CF) for Geologic $\mathrm{CO}_{2}$ Storage}

Curtis M. Oldenburg, Steven L. Bryant ${ }^{1}$, Jean-Philippe Nicot ${ }^{2}$, Yingqi Zhang, and Preston Jordan

${ }^{1}$ University of Texas, Austin, TX, USA; ${ }^{2}$ Bureau of Economic Geology, Austin, TX, USA

Contact: Curtis M. Oldenburg, 510/486-7419, cmoldenburg@lbl.gov

\section{RESEARCH OBJECTIVES}

Critical to the large-scale deployment of carbon dioxide capture and storage (CCS) is a simple, transparent, and accepted basis for regulators and stakeholders to certify that the risks of geologic CCS projects to health, safety, and the environment (HSE) are acceptable. The objective of this effort is to develop a simple, transparent, and accepted framework for evaluating leakage risk for certifying operation and decommissioning of geologic $\mathrm{CO}_{2}$ storage sites.

\section{APPROACH}

The U.S. EPA's Underground Injection Control (UIC) program is used successfully to regulate deep injection of liquids. Under the most stringent set of regulations, injected liquid is required not to migrate away from the injection zone for 10,000 years. This is the so-called non-migration requirement. There are fundamental differences between liquids regulated under the UIC program and geologic $\mathrm{CO}_{2}$ storage, differences that make a nonmigration requirement inappropriate for CCS. For example, vastly larger volumes of $\mathrm{CO}_{2}$ will be injected relative to hazardous liquid-waste disposal, and the buoyancy of supercritical $\mathrm{CO}_{2}$ creates an upward driving force for migration. Under the conditions applicable to $\mathrm{CO}_{2}$ storage, we propose an effective trapping requirement analogous to the nonmigration requirement of the UIC program. In the $\mathrm{CF}$, effective trapping, based on $\mathrm{CO}_{2}$ leakage risk (CLR), is the overarching requirement for safety and effectiveness of a $\mathrm{CO}_{2}$ storage site. With user input on subsurface properties, wells, faults, vulnerable assets, and injection parameters, the CF calculates the CLR for a given site. In the $\mathrm{CF}, \mathrm{CO}_{2}$ leakage risk is defined as the product of impact and probability of impact $(C L R=I \times P)$. Impacts are assumed to occur when $\mathrm{CO}_{2}$ enters one or more of the following compartments at a rate above an agreed-upon limit:

ECA $=$ Emission Credits and Atmosphere

HSE $=$ Health, Safety, and Environment

USDW $=$ Underground Sources of Drinking Water

$\mathrm{HMR}=$ Hydrocarbon and Mineral Resources

Reservoir simulation is used to calculate $\mathrm{CO}_{2}$ fluxes with time into compartments. Fluxes and concentrations are either read from a catalog of pre-simulated results or computed on a site-specific basis. Probabilities of the injected $\mathrm{CO}_{2}$ plume intersecting wells and/or faults (Pwf), and of wells and/or faults intersecting compartments (Pic), are based on input data on well and fault density, along with computed $\mathrm{CO}_{2}$ plume geometry. $\mathrm{CF}$ is probabilistic in existence of a flow pathway and deterministic in flow along the pathway. If the CLR is below threshold values, then the $\mathrm{CO}_{2}$ is considered effectively trapped, meaning that the storage site is safe and effective. A flow chart of the CF approach is presented in Figure 1.

\section{ACCOMPLISHMENTS}

We have developed a framework for calculating $\mathrm{CO}_{2}$ leakage risk to various compartments. We have assembled a tenmember international advisory board from whom we get feedback and comments on our approach. We are currently carrying out a case study using the framework to evaluate a hypothetical $\mathrm{CO}_{2}$ storage project in the Texas Gulf Coast.

\section{SIGNIFICANCE OF FINDINGS}

The CF is a simple and transparent framework in which limited leakage is allowable, as long as the leakage risk is below agreed-upon thresholds.

\section{RELATED PUBLICATIONS}

Oldenburg, C.M., and S.L. Bryant, Certification framework for geologic $\mathrm{CO}_{2}$ storage. LBNL-63395 Abs. Sixth Annual Conference on Carbon Capture and Sequestration, Pittsburgh, PA, May 8-10, 2007

\section{ACKNOWLEDGMENT}

This work was supported in part by the $\mathrm{CO}_{2}$ Capture Project (CCP) of the Joint Industry Program (JIP), and by Lawrence Berkeley National Laboratory under Department of Energy Contract No. DE-AC02-05CH11231.

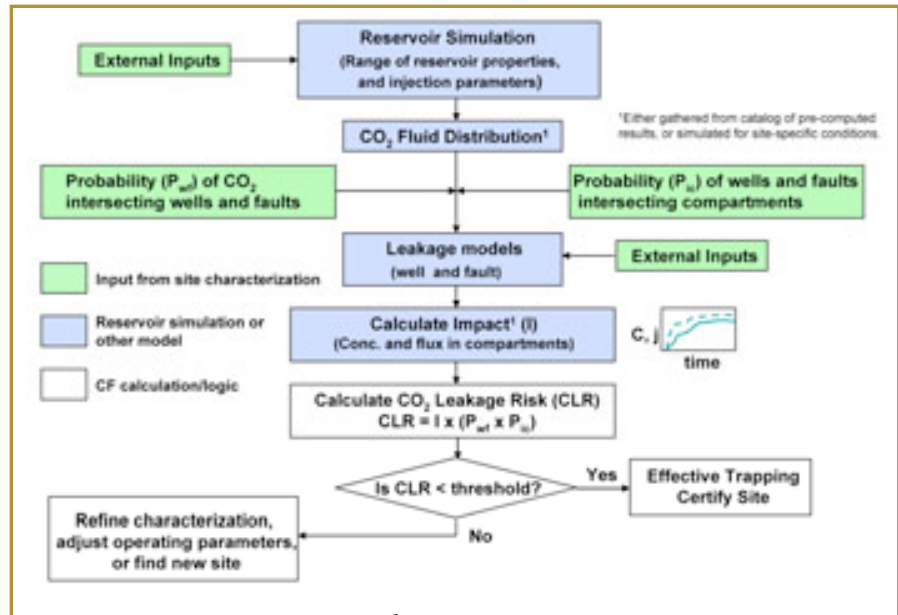

Figure 1. CF flow diagram showing inputs, outputs, and CF logic

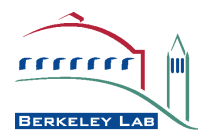




\section{Simulations OF $\mathrm{CO}_{2}$ RElEASE FOR \\ The ZERT Montana SHallow Release ExPERIMENT \\ Curtis M. Oldenburg \\ 510/486-7419, cmoldenburg@lbl.gov}

\section{RESEARCH OBJECTIVES}

Demonstrations by the technical community of the ability to detect, characterize, mitigate, and remediate $\mathrm{CO}_{2}$ seepage from geologic $\mathrm{CO}_{2}$ storage sites are needed to satisfy public concerns about the safety and environmental impact of geologic $\mathrm{CO}_{2}$ storage. To develop and demonstrate approaches for detection and characterization of $\mathrm{CO}_{2}$ seepage, the Zero Emissions Research Technology (ZERT) team constructed a shallow $\mathrm{CO}_{2}$ release facility in an agricultural field on the Montana State University (MSU) campus. The idea behind the facility is to emulate $\mathrm{CO}_{2}$ seepage through direct injection of $\mathrm{CO}_{2}$ at shallow depths. The facility consists of a $70 \mathrm{~m}$ long horizontal well approximately $2.5 \mathrm{~m}$ deep. The well is packed off into six sections, into which $\mathrm{CO}_{2}$ is injected independently to model leakage through a linear feature such as a fault or fracture. In the study presented here, numerical simulations of $\mathrm{CO}_{2}$ release are carried out to provide modeling support for experiment design and data interpretation.

\section{APPROACH}

The simulator TOUGH2/EOS7CA (Oldenburg and Unger, 2003; 2004) is used to model $\mathrm{CO}_{2}$ injections into the shallow subsurface at the MSU site for two sets of experiments: (1) shallow vertical-well injection using a 2-D radial grid; and (2) shallow horizontal-well injection using a 2-D cartesian grid. The shallow subsurface at the MSU site consists of $1.2 \mathrm{~m}$ of soil overlying a cobble formation, with water table at approximately $1.5 \mathrm{~m}$ depth. The shallow vertical-well injection consisted of a $1.6 \mathrm{~L} / \mathrm{min}\left(4.8 \times 10^{-5} \mathrm{~kg} / \mathrm{s}\right) \mathrm{CO}_{2}$ injection at a depth of approximately $3 \mathrm{~m}$. Seepage fluxes were measured in the field using an accumulation chamber around the injection well in the $\mathrm{N}, \mathrm{S}, \mathrm{E}$, and $\mathrm{W}$ directions. Measured $\mathrm{CO}_{2}$ seepage fluxes were used as constraints to fit appropriate model permeabilities to the soil and cobble layers. Subsequently, these fitted soil and cobble properties were used in forward models for design and prediction of the horizontal release experiment.

\section{ACCOMPLISHMENTS}

Presented in Figure 1 (top) is the simulated vertical-injection well seepage flux (solid lines) along with the measured data showing the fit obtained for $\mathrm{k}_{\text {soil }}=5 \times 10^{-11} \mathrm{~m}^{2}(50 \mathrm{D})$, and $\mathrm{k}_{\text {cobble }}=3.2 \times 10^{-12} \mathrm{~m}^{2}(3.2 \mathrm{D})$. The high inferred permeability of the soil likely arises from cracks and root casts that create macropores through which soil gas and atmospheric air readily flow. Figure 1 (bottom) shows simulated results for the horizontal well. Various injection rates were simulated. The 100 $\mathrm{kg} / \mathrm{d}$ rate predicted breakthrough after 1.5 days and seepage fluxes that were neither too easy nor too difficult to detect $\left(\mathrm{q}_{\max } \sim 100 \mu\right.$ moles $/\left(\mathrm{m}^{2} \mathrm{~s}\right)\left(380 \mathrm{~g} /\left(\mathrm{m}^{2} \mathrm{~d}\right)\right)$. The subsequent field experiment, run at an injection rate of $100 \mathrm{~kg} / \mathrm{d}$, resulted in the collection of field data using a variety of approaches that generally agreed with the predictive calculations.

\section{SIGNIFICANCE OF FINDINGS}

That shallow soils can be very permeable to upward $\mathrm{CO}_{2}$ seepage is a key finding of this research project. The implication is that $\mathrm{CO}_{2}$ in the shallow subsurface can easily migrate upwards and seep out of the ground, where various monitoring approaches may be used for characterizing the seepage.

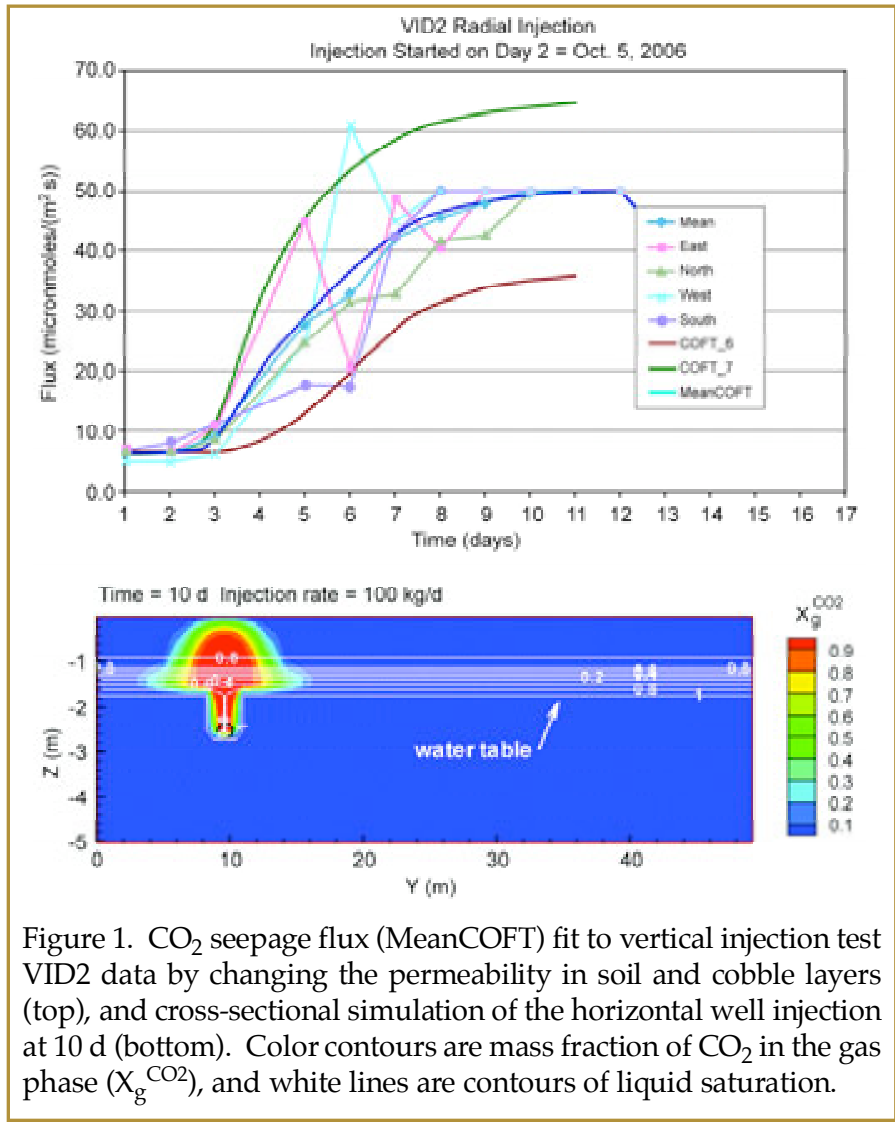

\section{RELATED PUBLICATIONS}

Oldenburg, C.M., and A.J.A. Unger, On leakage and seepage from geologic carbon sequestration sites: Unsaturated zone attenuation. LBNL-51928. Vadose Zone Journal, 2(3), 287-296, August 2003.

Oldenburg, C.M., and A.J.A. Unger, Coupled vadose zone and atmospheric surface-layer transport of $\mathrm{CO}_{2}$ from geologic carbon sequestration sites. LBNL-55510. Vadose Zone Journal, 3, 848-857, 2004.

\section{ACKNOWLEDGMENTS}

This work was conducted as part of the Zero Emissions Research and Technology (ZERT) Project, supported by the Assistant Secretary for Fossil Energy, Office of Sequestration, Hydrogen, and Clean Coal Fuels, through the National Energy Technology Laboratory, U.S. Department of Energy, under Contract No. DE-AC02$05 \mathrm{CH} 11231$.

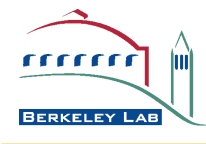




\section{Joule-Thomson CoOling due to $\mathrm{CO}_{2}$ Injection into Natural Gas Reservoirs \\ Curtis M. Oldenburg \\ 510/486-7419, cmoldenburg@lbl.gov}

\section{RESEARCH OBJECTIVES}

Depleted natural gas reservoirs are a promising target for the storage of anthropogenic $\mathrm{CO}_{2}$ as a greenhouse gas mitigation strategy. One effect of injecting $\mathrm{CO}_{2}$ into a low-pressure (depleted) gas reservoir is Joule-Thomson cooling. Potential problems that could arise from Joule-Thomson cooling include the formation of $\mathrm{CO}_{2}$ and/or $\mathrm{CH}_{4}$ hydrates, freezing of residual water and associated reduction in injectivity, and generation of thermal stresses that could fracture the formation. The purpose of this study is to use numerical simulation to investigate the magnitude of JouleThomson cooling that may arise during $\mathrm{CO}_{2}$ injection into depleted $\mathrm{CH}_{4}$ reservoirs.

\section{APPROACH}

We used a simple one-dimensional radial geometry to represent an idealized depleted gas reservoir (Figure 1, top). Production is idealized as being from the outermost gridblock at a distance of 1100 $\mathrm{m}$ in a five-spot pattern. We used the TOUGH2 module called EOS7C (Oldenburg et al., 2004), which models five components (water, brine, noncondensible gas, tracer, and methane) under
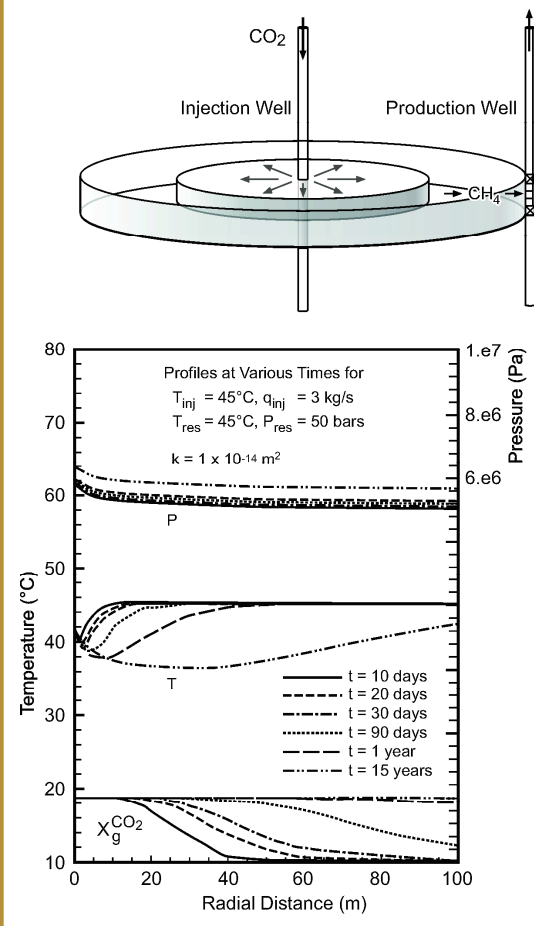

Figure 1. Radial injection geometry (top), and profiles $(0-100 \mathrm{~m})$ of pressure $(\mathrm{P})$, temperature $(\mathrm{T})$, and $\mathrm{CO}_{2}$ mass fraction $(\mathrm{XgCO})$ (bottom) at six different times for the case of radial injection into a reservoir with permeability equal to $1 \times 10^{-14} \mathrm{~m}^{2}(10 \mathrm{mD})$. showed that for a constant injection rate, lower permeability causes larger $\Delta P$ and correspondingly larger $\Delta T$, owing to Joule-Thomson cooling. We also confirmed that low-porosity allows more heat to be provided by the matrix grains to offset Joule-Thomson cooling. Therefore, the effects of permeability and porosity may partially compensate, although it appears the effect of permeability is larger than the effect of porosity.

\section{SIGNIFICANCE OF FINDINGS}

Joule-Thomson cooling during constant-rate $\mathrm{CO}_{2}$ injection is larger for lower permeability reservoirs and higher porosity reservoirs. The simulation results show that Joule-Thomson cooling is a minor effect $\left(<4^{\circ} \mathrm{C}\right)$ for low-injection rates and for permeabilities in the range expected in the Sacramento Valley. Overall, the conclusion from this study is that Joule-Thomson cooling is not expected to be a significant obstacle to successful $\mathrm{CO}_{2}$ storage in depleted or depleting gas reservoirs.

\section{RELATED PUBLICATIONS}

isothermal or nonisothermal conditions. The subroutines used in EOS7C are available as a web-based tool to calculate properties of gas mixtures (http:/ / esdtools.lbl.gov/gaseos/home.html). We verified the methods used in EOS7C for expansion-related cooling by modeling the classical Joule-Thomson experiment, in which gas flows at constant enthalpy from a bulb at high pressure to a bulb at lower pressure as the temperature change is recorded. Simulated results agreed well with reference values.

\section{ACCOMPLISHMENTS}

Here we present a single example simulation consisting of a constant-rate $\mathrm{CO}_{2}$ injection at $3 \mathrm{~kg} / \mathrm{s}$ with an injection temperature and initial reservoir temperature of $45^{\circ} \mathrm{C}$ (Figure 1, bottom). Production of $\mathrm{CH}_{4}$ occurs at a rate of $0.56 \mathrm{~kg} / \mathrm{s}$. Shown in Figure 1 are results for $k=10^{-14} \mathrm{~m}^{2}(10 \mathrm{mD})$. As shown, cooling is approximately $8^{\circ} \mathrm{C}$ over a $100 \mathrm{~m}$ radius from the well after 15 years of injection, not enough to cause any concerns for hydrates, freezing, or thermal stresses. The simulation study
Oldenburg, C.M., Joule-Thomson cooling due to $\mathrm{CO}_{2}$ injection into natural gas reservoirs. LBNL-60158. Energy Conversion and Management, 48, 1808-1815, 2007.

Oldenburg, C.M., G.J. Moridis, N. Spycher, and K. Pruess, EOS7C Version 1.0: TOUGH2 module for carbon dioxide or nitrogen in natural gas (methane) reservoirs. LBNL-56589, March 2004.

\section{ACKNOWLEDGMENT}

This work was conducted as part of the West Coast Regional Carbon Sequestration Partnership (WESTCARB) (specifically, the Sacramento Valley applications) and Zero Emissions Research and Technology (ZERT) (specifically, code development) projects, supported by the Assistant Secretary for Fossil Energy, Office of Sequestration, Hydrogen, and Clean Coal Fuels, through the National Energy Technology Laboratory, U.S. Department of Energy, under Contract No. DE-AC02-05CH11231. 


\section{ECO2N: A TOUGH2 Fluid Property Module FOR Mixtures of WATER, NACL, AND $\mathrm{CO}_{2}$}

Karsten Pruess

Contact: 510/486-6732, K_Pruess@lbl.gov

\section{RESEARCH OBJECTIVES}

Numerical simulation plays a key role in evaluating the feasibility of storing $\mathrm{CO}_{2}$ in geologic formations-identifying favorable as well as unfavorable hydrogeologic conditions at proposed storage sites, designing and analyzing tests, and optimizing storage design. The purpose of this work was to develop accurate and robust simulation capabilities, and to make such capabilities available to the technical community.

\section{APPROACH}

The desired simulation capability was developed as an enhancement of our existing multipurpose reservoir simulation code TOUGH2. Building on a fluid property module EWASG for mixtures of saline brine and $\mathrm{CO}_{2}$, and on previously developed research codes for $\mathrm{CO}_{2}$ storage, a new module ECO2N was developed as an add-on to TOUGH2.

\section{ACCOMPLISHMENTS}

The new ECO2N module includes a comprehensive description of the thermodynamics and thermophysical properties of $\mathrm{H}_{2} \mathrm{O}-\mathrm{NaCl}-\mathrm{CO}_{2}$ mixtures, one that reproduces fluid properties largely within experimental error for the temperature, pressure, and salinity conditions of interest $\left(10^{\circ} \mathrm{C} \leq \mathrm{T} \leq\right.$ $110^{\circ} \mathrm{C} ; \mathrm{P} \leq 600$ bar; salinity up to full halite saturation). Flow processes can be modeled isothermally or nonisothermally, and phase conditions represented may include a single (aqueous or $\mathrm{CO}_{2}$-rich) phase, as well as two-phase mixtures. Fluid phases may appear or disappear in the course of a simulation, and solid salt may precipitate or dissolve. ECO2N uses newly developed correlations that accurately predict the partitioning of water and $\mathrm{CO}_{2}$ between immiscible brine and $\mathrm{CO}_{2}$-rich phases. A detailed user's guide was written that gives technical specifications of ECO2N, includes instructions for preparing input data, and provides illustrative applications to several sample problems, including problems that had been previously investigated in a code intercomparison study.

As an example, Figure 1 presents an application of TOUGH2/ECO2N to a simplified version of the first industrial-scale $\mathrm{CO}_{2}$ aquifer storage project in the Norwegian sector of the North Sea.

\section{SIGNIFICANCE OF FINDINGS}

The ECO2N module was released to the public through DOE's Energy Science and Technology Software Center (ESTSC). It is available to licensees of TOUGH2 at no additional charge, and has been quickly adopted by many groups in academia and industry, both nationally and internationally.

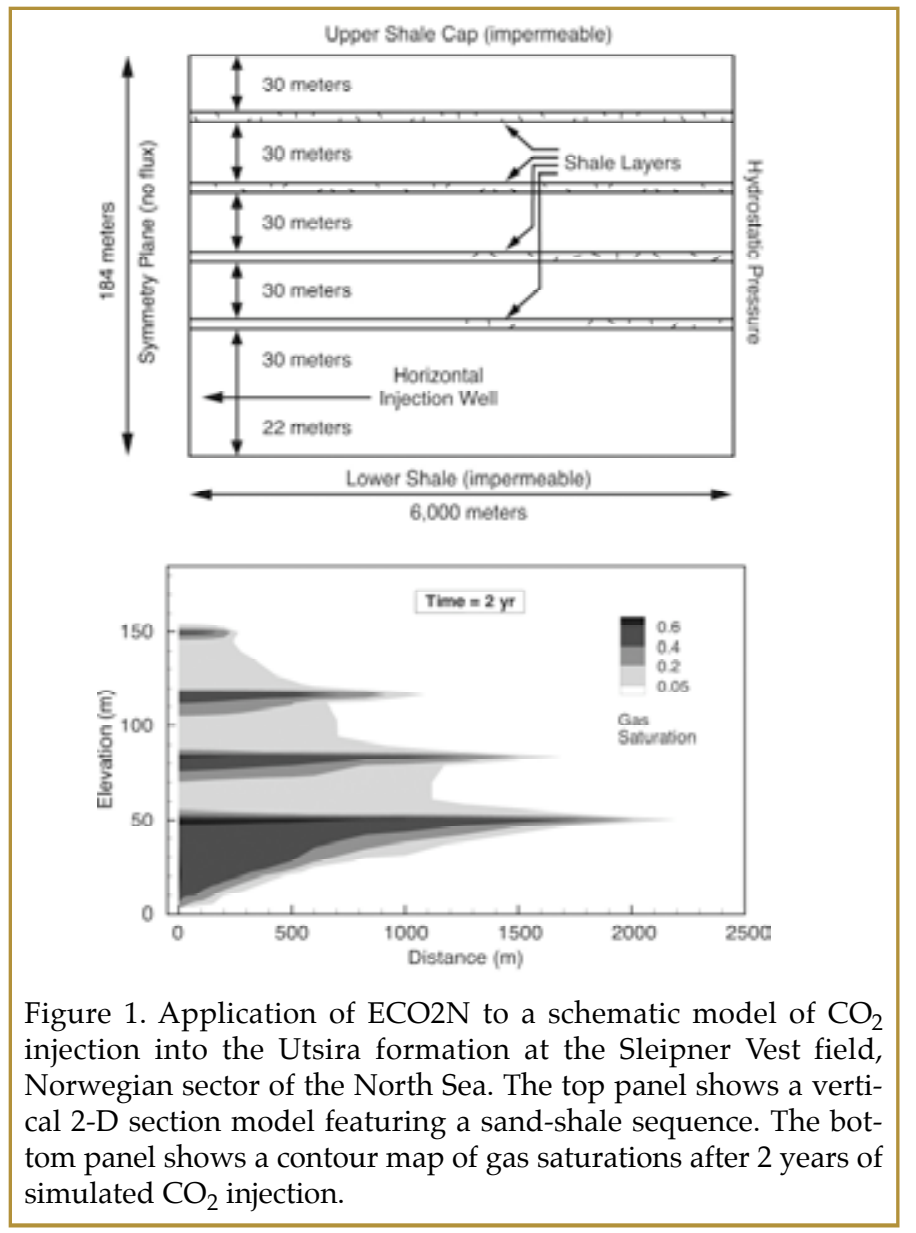

\section{RELATED PUBLICATIONS}

Pruess, K., ECO2N: A TOUGH2 fluid property module for mixtures of water, $\mathrm{NaCl}$, and $\mathrm{CO}_{2}$. LBNL-57592, Berkeley, CA, June 2005.

Pruess K., and N. Spycher. ECO2N-A fluid property module for the TOUGH2 code for studies of $\mathrm{CO}_{2}$ storage in saline aquifers. Energy Conversion and Management, 48(6), pp. 1761-1767, doi:10.1016/j.enconman.2007.01.016, 2007.

\section{ACKNOWLEDGMENTS}

This work was conducted as part of the Zero Emissions Research and Technology (ZERT) Project, supported by the Assistant Secretary for Fossil Energy, Office of Sequestration, Hydrogen, and Clean Coal Fuels, through the National Energy Technology Laboratory, U.S. Department of Energy, under Contract No. DE-AC02-05CH11231.

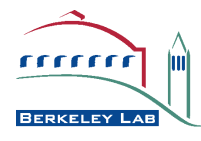




\title{
Analysis of Fault Rupture and $\mathrm{CO}_{2}$ UpWelling during the 1960s Matsushiro EarthQuake Swarm as a Natural Analogue of $\mathrm{CO}_{2}$ StOrage and Leakage
}

\author{
Jonny Rutqvist, Frederic Cappa, Jennifer Lewicki, and Sally Benson \\ Contact: Jonny Rutqvist, 510/486-5432, jrutqvist@lbl.gov
}

\section{RESEARCH OBJECTIVES}

This study is part of a collaborative research between Berkeley Lab and Japanese organizations on the Matsushiro Earthquake Swarm that occurred in central Japan in the mid1960s. During the five-year term of the swarm, between 1965 to 1970, approximately 60,000 earthquakes were felt, and ten million tons of $\mathrm{CO}_{2}$-bearing water appeared at the ground surface, flowing up through newly created surface ruptures. One part of this research project, described herein, was to use coupled reservoir-geomechanical modeling to understand the effects of a deep high-pressure $\mathrm{CO}_{2}$ source on the initiation and propagation of the fault ruptures that resulted in the Matsushiro Earthquake Swarm.

\section{APPROACH}

The coupled $\mathrm{CO}_{2}$ fluid flow and geomechanical processes, including fault motion and associated permeability changes, were modeled using the TOUGH-FLAC coupled reservoir-geomechanical simulator. A model for fault permeability changes with fault reactivation was implemented into the TOUGHFLAC simulator. Then a series of two and three-dimensional simulations were conducted to identify key parameters and investigate possible causes and mechanisms behind the Matsushiro Earthquake Swarm.

\section{ACCOMPLISHMENTS}

Figure 1 shows a three-dimensional model domain and the results of fault-rupture distribution associated with the upwelling $\mathrm{CO}_{2}$. The geomechanical analysis explains the cause of the Matsushiro Earthquake Swarm as overpressure caused by the upwelling $\mathrm{CO}_{2}$ fluid. The mechanisms of the earthquake are attributed to shear failure initiated by reduced effective stress on pre-existing fracture planes within and near the two main faults. It is concluded that the in situ th ree-dimensional stress regime, as well as fault strength and permeability, are likely the most important parameters that control the nucleation, propagation, arrest and occurrence of the earthquake swarm during its two years of migration through the seismogenic crust. Moreover, surface deformation and increased seismicity were found to be precursors to the $\mathrm{CO}_{2}$ surface release, because these mechanical responses were detected up to a year before any chemical changes were measured at the ground surface.

\section{SIGNIFICANCE OF FINDINGS}

The analysis of the Matsushiro Earthquake Swarm is an example of how natural analogues can provide a greater understanding of the factors controlling retention and leakage of $\mathrm{CO}_{2}$. Specifically, it was demonstrated that geomechanical

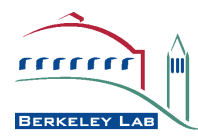

available today can help improve our understanding and quantifying of the factors leading to leakage of $\mathrm{CO}_{2}$. Such an understanding will be needed to avoid selecting sites where the probability of leakage is high, and to quantify leakage should it occur.

\section{RELATED PUBLICATION}

Yamamoto, K., R. Aoyagi, H. Koide, T. Tosha, S. Nakanishi, N. Todaka, S. Benson, J. Rutqvist, and J. Lewicki, Matsushiro earthquake swarm (1965-1967) as natural analogue of $\mathrm{CO}_{2}$ storage and leakage. Eos Trans. AGU, 87(52), Fall Meeting, Suppl., Abstract H21A-1359, (2006).

\section{ACKNOWLEDGMENTS}

The work presented in this paper was financed by the Ministry of Economy, Trade, and Industry (METI) of Japan through the Mizuho Information and Research Institute.

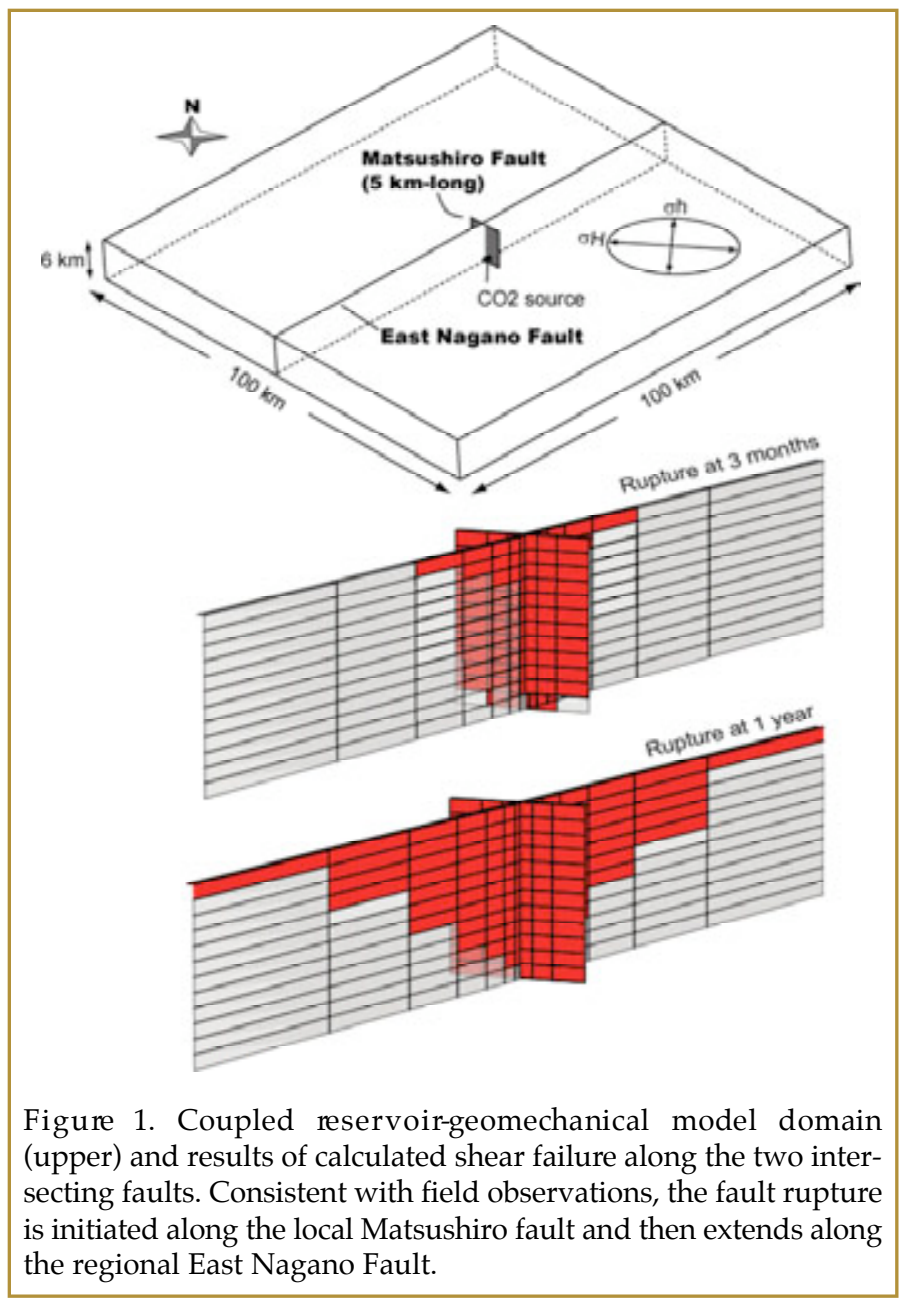




\title{
Coupled Reservoir-Geomechanical Analysis of Geomechanical Damage Associated With $\mathrm{CO}_{2}$ GeOlogic Storage
}

\author{
Jonny Rutqvist, Jens Birkholzer, and Chin-Fu Tsang \\ Contact: Jonny Rutqvist, 510/486-5432, jrutqvist@lbl.gov
}

\section{RESEARCH OBJECTIVES}

The objective of this research is to study factors affecting the maximum sustainable injection pressure and the potential for mechanical failure associated with deep underground injection of carbon dioxide $\left(\mathrm{CO}_{2}\right)$. Large-scale underground injection of $\mathrm{CO}_{2}$ may involve a substantial increase in reservoir pressure, which will induce mechanical stresses and deformations in and around the injection reservoir. If reservoir pressure becomes too large in the overlying caprock formations, new fractures or faults may be created there or existing ones reactivated. This could provide flow paths for $\mathrm{CO}_{2}$ to escape, thereby substantially reducing the effectiveness of sequestration.

\section{APPROACH}

Using the TOUGH-FLAC coupled reservoir-geomechanical simulator, we conducted a study of hydromechanical processes during injection of supercritical $\mathrm{CO}_{2}$ into deep brine formations. We investigated the possibility of rock-mechanical failure in the form of hydraulic fracturing or shear slip reactivation of existing faults.

\section{ACCOMPLISHMENTS}

We have analyzed mechanical stress changes and the potential for mechanical failure associated with underground injection of $\mathrm{CO}_{2}$. Figure 1 shows an example of simulation results for $\mathrm{CO}_{2}$ injection into an injection zone overlain by several aquifers and caprock units. In this case, a vertical fault zone allowed for limited upward migration of the $\mathrm{CO}_{2}$ through the two first caprock layers, affecting the potential for shear failure in overlying units. One important conclusion from this study is that the potential for mechanical failure, and the type and orientation of failure, depends to a large extent on the initial stress field (stress regime). As such, the stress field should be much more carefully measured and monitored than is typical in the current practice for oil and gas explorations. Furthermore, the potential for mechanical failure should be analyzed for the entire region affected by mechanical stress changes, which is generally more extensive than the region of fluid pressure change at depth.

\section{SIGNIFICANCE OF FINDINGS}

Results from this study may be important input when developing possible guidelines for site investigations and for estimating maximum sustainable injection pressure at a geological $\mathrm{CO}_{2}$ injection site. For example, potential mechanical failure at shallow levels above the injection zone may need to be assessed.

\section{RELATED PUBLICATIONS}

Rutqvist J., J. Birkholzer , F. Cappa , and C.F. Tsang, Estimating maximum sustainable injection pressure during geological sequestration of $\mathrm{CO}_{2}$ using coupled fluid flow and geomechanical fault-slip analysis. Energy Conversion and Management 48(6), 1798-1807, 2007.

Rutqvist, J., J.T. Birkholzer, and C.F. Tsang, Coupled reservoir-geomechanical analysis of the potential for tensile and shear failure associated with $\mathrm{CO}_{2}$ injection in multilayered reservoir-caprock systems. Int. J. Rock Mech. \& Min. Sci., 45(1), 132-142, 2007.

\section{ACKNOWLEDGMENTS}

This work was conducted with funding from the U.S. Environmental Protection Agency, Office of Water and Office of Air and Radiation, under an Interagency Agreement with the U.S. Department of Energy at the Lawrence Berkeley National Laboratory, and with funding from the Assistant Secretary for Fossil Energy, Office of Coal and Power Systems, through the National Energy Technologies Laboratory, under Department of Energy Contract No. DE-AC02-05CH11231.

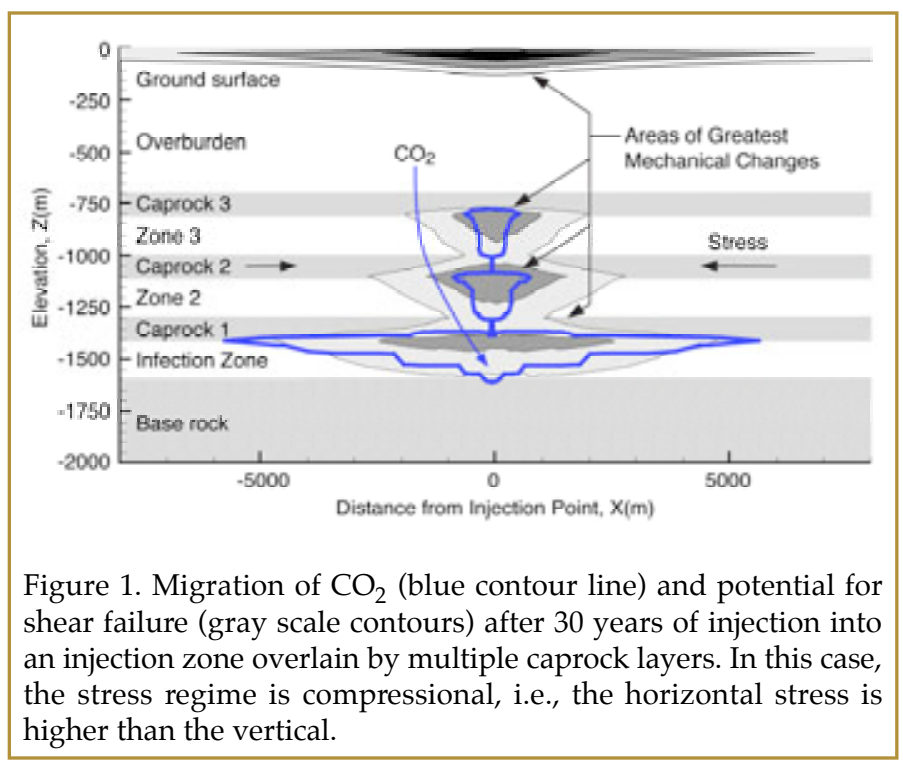

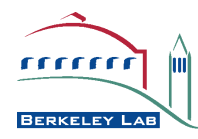




\title{
A Model of Buoyancy-Driven Evolution of a Carbon Dioxide Plume
}

\author{
Dmitriy Silin, Tad Patzek, and Sally M. Benson ${ }^{1}$ \\ ${ }^{1}$ Stanford University, Stanford, CA \\ Contact: Dmitriy Silin, 510/495-2215, dsilin@lbl.gov
}

\section{RESEARCH OBJECTIVES}

This work is motivated by the growing interest in injecting carbon dioxide into deep geologic formations as a means of avoiding its atmospheric emissions and consequent contribution to global warming. One of the key questions regarding the feasibility of this technology is the potential rate of leakage out of the primary storage formation. Here, we perform a modelbased study and theoretical prediction of evolution of a gas plume in an aquifer flowing upward under buoyancy.

\section{APPROACH}

We seek simple analytical solutions in a model of twophase flow of supercritical gas and brine. We focus on the role of interactions among buoyancy, viscous, and capillary forces. To achieve this objective, we assume homogeneous formation and neglect the heat and chemical transport and phase change processes. We consider vertical countercurrent flow, which takes place in the central part of a laterally spread plume or in a permeable fault.

\section{ACCOMPLISHMENTS}

Our principal finding is that the evolution of the saturation profile of a moving plume can be described as a sequence of traveling and rarefaction waves. Driven by buoyancy, the plume propagates by stretching vertically upward, unlike a gas bubble in water. It has been established that the Darcy velocity of each fluid reaches its maximum near the top of the original plume location. This maximum-flow point is characterized by a constant fluid saturation and does not move during evolution of the plume. The fluid displacement mechanism above this location is drainage, whereas below it, it is imbibition. The velocity of plume propagation and the fluid saturation in the leading part of the plume have been expressed through the properties of the formation and the fluids. The calculations suggest that the velocity of propagation for a plume of supercritical carbon dioxide can be on the order of tens of meters per year in an aquifer whose permeability is on the order of 100 millidarcies (Figure 1).

\section{SIGNIFICANCE OF FINDINGS}

Characterization of plume propagation is crucially important for development of optimal injection technologies to maximize greenhouse gas trapping and sequestration. Possible leakage of injected carbon dioxide to the surface could result in health and safety concerns. Development of monitoring policies in the areas near gas storage sites will benefit from theoretical estimates of plume propagation velocity.

\section{RELATED PUBLICATIONS}

Silin, D., T.W. Patzek, and S.M. Benson, Exact solutions in a model of vertical gas migration. SPE paper 103156. In: 2006 SPE Annual Technical Conference and Exhibition, SPE, San Antonio, TX, September 24-27, 2006.

Silin, D., T.W. Patzek, and S.M. Benson, A model of buoyancydriven two-phase countercurrent fluid flow. LBNL-62607. Transport in Porous Media (in press), 2007.

\section{ACKNOWLEDGMENTS}

This work was conducted as part of the Zero Emissions Research and Technology (ZERT) Project, supported by the Assistant Secretary for Fossil Energy, Office of Sequestration, Hydrogen, and Clean Coal Fuels, through the National Energy Technology Laboratory, U.S. Department of Energy, under Contract No. DE-AC02-05CH11231.

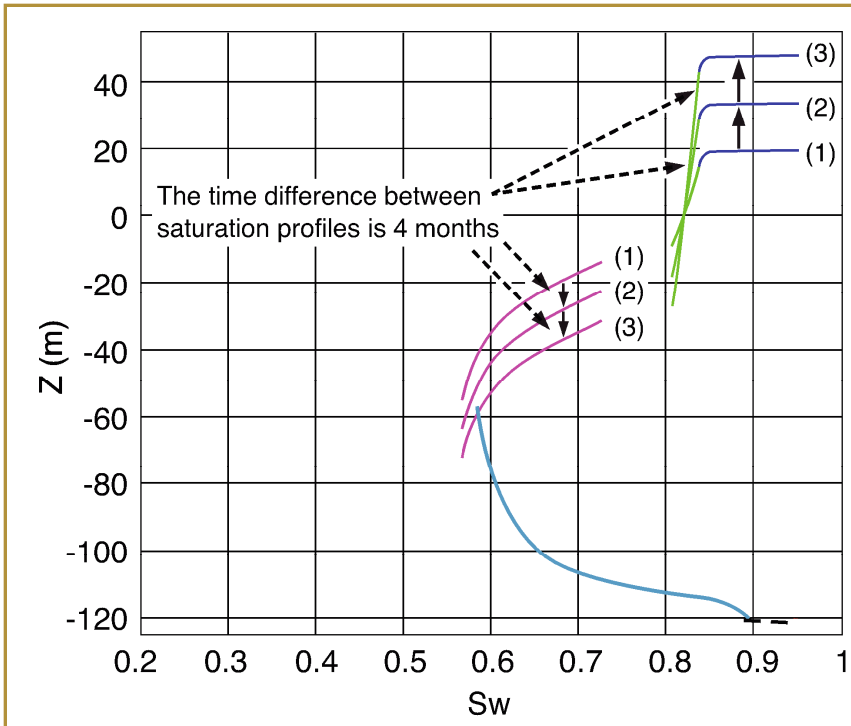

Figure 1. Evolution of vertical water saturation profile. The origin $z=0$, is associated with the top of the plume. The absolute permeability of formation is 100 millidarcy. The properties of the supercritical gas and water correspond to a depth near $1 \mathrm{~km}$. 


\title{
Development of an Efficient Parallel Simulator for Modeling $\mathrm{CO}_{2}$ Geologic Sequestration In Saline Aquifers
}

\author{
Keni Zhang, Christine Doughty, Yu-Shu Wu, and Karsten Pruess \\ Contact: Keni Zhang, 510/486-7393, kzhang@lbl.gov
}

\section{RESEARCH OBJECTIVES}

Carbon dioxide geologic sequestration in saline aquifers involves very complex multiphase flow processes. Numerical modeling can play an important role in evaluating the feasibility and reliability of $\mathrm{CO}_{2}$ disposal. However, modeling of these processes in general requires fine spatial and temporal discretization, and also may require coverage of a large simulation domain, all of which represent a large computational challenge. The objective of this study is to develop an efficient parallel simulator for these types of simulations.

\section{APPROACH}

The parallel simulation approach is based on the ECO2N module of the TOUGH2 code. The parallel simulator retains all the process-modeling capabilities of the original TOUGH2/ECO2N and parallel computation features of TOUGH2-MP. In this study, a domain decomposition approach is adopted for model parallelization and MPI (message passing interface) for parallel implementation. The code partitions the simulation domain, defined by an unstructured grid, using a partitioning algorithm from the METIS software package. In a parallel simulation, each processor handles one portion of the simulation domain for updating thermophysical properties, assembling mass and energy-balance equations, solving linear equation systems, and performing other local computations. Local linear-equation systems are solved in parallel by multiple processors with the Aztec linear solver package. The parallel simulator has been built with efficient communication schemes.

\section{ACCOMPLISHMENTS}

An efficient parallel simulator for large-scale, long-term $\mathrm{CO}_{2}$ geologic sequestration in saline aquifers has been developed. The parallel simulator is a three-dimensional, fully implicit model that solves large, sparse linear systems arising from discretization of the mass- and energy-balance equations in porous and fractured media. The simulator provides a comprehensive description of the thermodynamics and thermophysical properties of $\mathrm{H}_{2} \mathrm{O}-\mathrm{NaCl}-\mathrm{CO}_{2}$ mixtures, and models single and/or two-phase isothermal or nonisothermal flow processes, two-phase mixtures, appearance or disappearance of fluid phases, and salt precipitation or dissolution.

The code demonstrates excellent scalability. Test runs show that a linear or super-linear speedup can be obtained on Linux clusters as well as on supercomputers (See Figure 1). Because the parallel simulator was developed from an existing mature code, it inherits not only simulation functions from the original code, but also all other features, including input/ output format, error handling, and improvements for code stability. These features provide robustness for the parallel code and ease of use for the user community of the original code.

\section{SIGNIFICANCE OF FINDINGS}

The domain decomposition approach and parallel computation enhance model simulation capabilities, in terms of problem size and complexity, to a level that cannot be reached by singleCPU codes. By using the parallel simulator, multimillion gridblock problems can be run on a typical Linux cluster with several tens to hundreds of processors to achieve a ten to hundred times improvement in computational time or problem manageability.

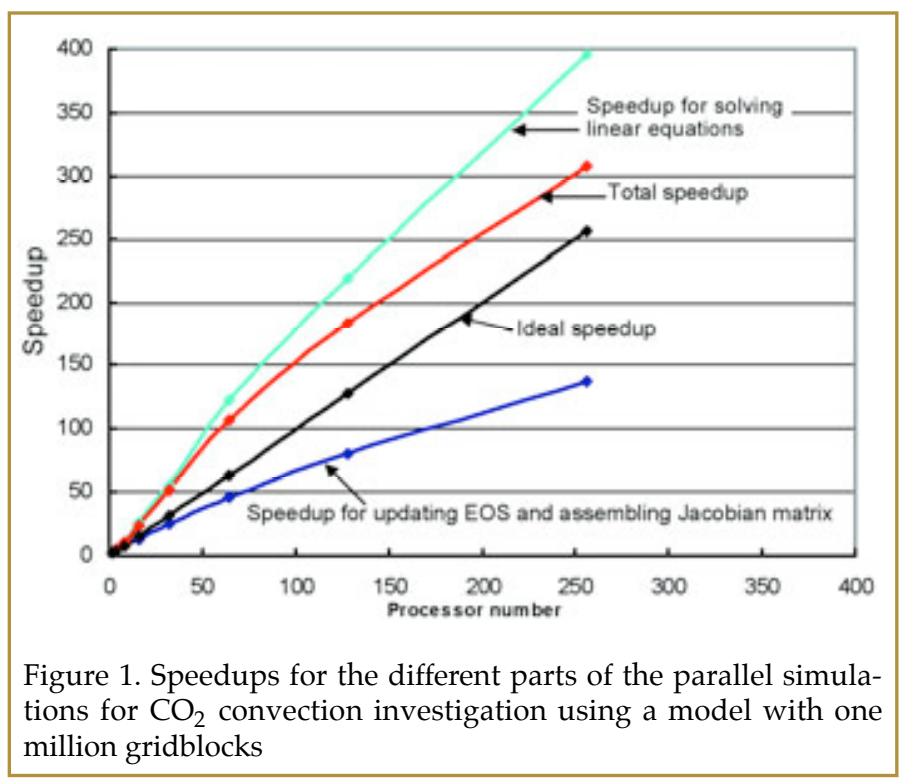

\section{RELATED PUBLICATION}

Zhang, K., C. Doughty, Y-S Wu, and K. Pruess, Efficient parallel simulation of $\mathrm{CO}_{2}$ geologic sequestration in saline aquifers. Paper SPE 106026, Proceedings of the 2007 SPE Reservoir Simulation Symposium, Houston, Texas, 2007.

\section{ACKNOWLEDGMENTS}

This work was conducted as part of the Zero Emissions Research and Technology (ZERT) project, supported by the Assistant Secretary for Fossil Energy, Office of Sequestration, Hydrogen, and Clean Coal Fuels, through the National Energy Technology Laboratory, U.S. Department of Energy, under Contract No. DE-AC02-05CH11231.

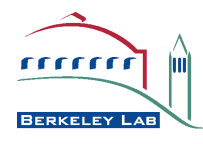




\section{SySTEM-LEVEL MODELING FOR ECONOMIC EVALUATION OF GeOlogic $\mathrm{CO}_{2}$ StORAGe in Gas Reservoirs \\ Yingqi Zhang, Curtis M. Oldenburg, Stefan Finsterle, and Gudmundur S. Bodvarsson Contact: Yingqi Zhang, 495-2983, yqzhang@lbl.gov}

\section{RESEARCH OBJECTIVES}

The objective of this study is to develop a system-level model that has the capability to evaluate the economic and environmental impacts of geological $\mathrm{CO}_{2}$ storage under uncertainty. This system model specifically evaluates the feasibility of carbon sequestration with enhanced gas recovery (CSEGR) in the Rio Vista region of California. Implemented within this model are $\mathrm{CO}_{2}$ capture and separation, compression, pipeline transportation to the storage site, and $\mathrm{CO}_{2}$ injection into and methane out of a gas reservoir. The subsurface component of the system is calculated based on simulations from a detailed process model of the gas reservoir.

\section{APPROACH}

A top-down method is used to build a hierarchical model for the studied CSEGR system. First, CSEGR subsystems are identified and connected to track $\mathrm{CO}_{2}$ and $\mathrm{CH}_{4}$ flows (Figure 1). Next, components of each subsystem are identified and connected. Finally, potential features, events and processes (FEPs) are identified, and corresponding feedbacks are incorporated within the system. The level of detail increases at lower levels of the system hierarchy. The advantage of this top-down method (over a bottom-up method) is that processes in different sectors can be properly coupled and their feedbacks properly considered.

The system-level analysis is performed using GoldSim (www.goldsim.com), a flexible probabilistic simulation platform. The reservoir simulations are performed using EOS7C (Oldenburg et al., 2004), a TOUGH2 module for multicomponent gas mixtures.

\section{ACCOMPLISHMENTS}

We have developed a system-level model for analyzing geological storage of $\mathrm{CO}_{2}$. Our system model includes detailed reservoir simulations of $\mathrm{CO}_{2}$ injection, $\mathrm{CH}_{4}$ displacement and production, and potential leakage through a fault or abandoned well. The associated environmental consequences are evaluated. Using process simulation embedded in a systemlevel model, the economic benefits of $\mathrm{CO}_{2}$ sequestration and enhanced gas recovery can be directly weighed against the costs of $\mathrm{CO}_{2}$ injection.

\section{SIGNIFICANCE OF FINDINGS}

There is much research effort being conducted to improve understanding of factors affecting particular aspects of geological $\mathrm{CO}_{2}$ storage (such as storage performance, storage capacity, and health, safety, and environmental [HSE] impacts), as well as to lower the cost of $\mathrm{CO}_{2}$ capture and related processes. However, there has been less emphasis to date on system-level analyses of geologic $\mathrm{CO}_{2}$ storage that consider geologic, economic, and environmental issues by linking detailed process models to representations of engineering components and associated economic models. This study provides a framework/capability for feasibility evaluations on geological $\mathrm{CO}_{2}$ storage under uncertainty, and can be extended to other waste management operations.

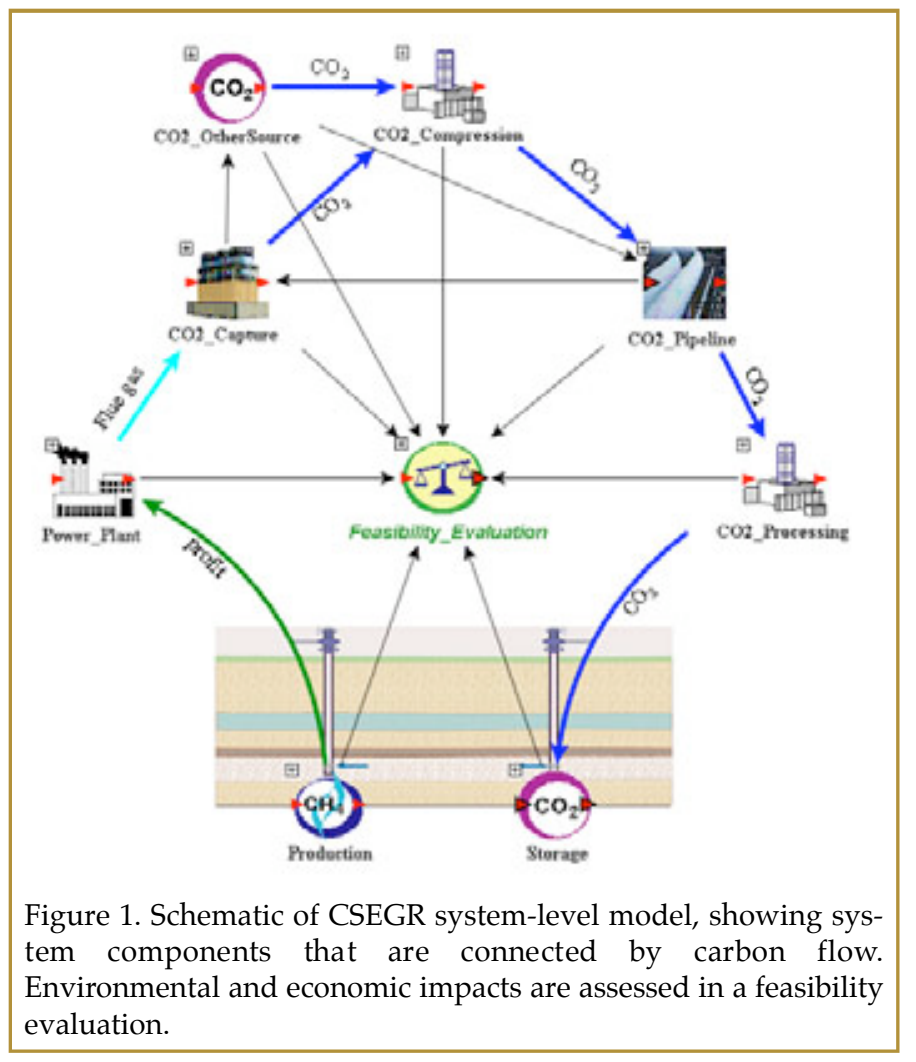

\section{RELATED PUBLICATION}

Zhang, Y., C.M. Oldenburg, S. Finsterle, and G.S. Bodvarsson, System-level modeling for economic evaluation of geological $\mathrm{CO}_{2}$ storage in gas reservoirs. Energy Conservation and Management, 48, 1827-1833, doi:10.1016/j.enconman.2007. 01.018, 2007.

\section{ACKNOWLEDGMENTS}

This work was supported, in part, by the West Coast Regional Carbon Sequestration Partnership (WESTCARB) through the Assistant Secretary for Fossil Energy, Office of Coal and Power Systems, National Energy Technology Laboratory, and by Lawrence Berkeley National Laboratory under Department of Energy Contract No. DE-AC02-05CH11231.

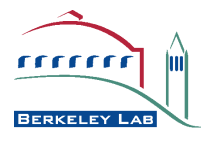




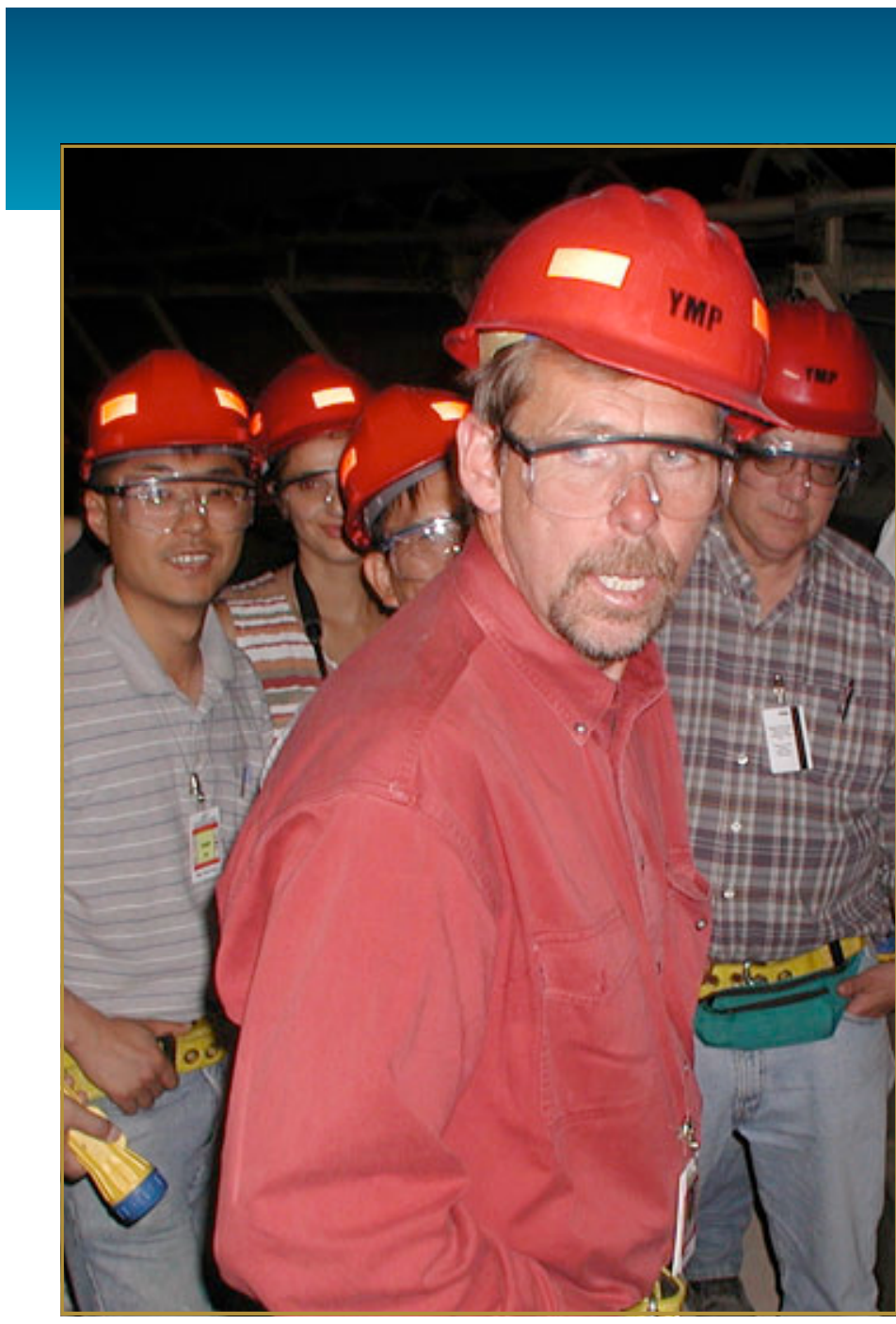

Geological isolation of spent nuclear fuels and high-level radioactive waste is currently the preferred means of disposal for many countries worldwide. The role of ESD's Nuclear Waste Program (NWP) is to assist the U.S. Department of Energy, the United States, and other countries in achieving the safe disposal of high-level radioactive waste-by means of high-quality scientific analyses that encompass modeling, laboratory and field experiments, and technology development. Research within NWP focuses on flow and transport, as thermally driven hydrological, chemical, and mechanical coupled processes. Many of the studies within NWP relate to Yucca Mountain, the proposed site for the permanent storage of highlevel nuclear waste in the USA; although NWP has also collaborated on nuclear-waste disposal issues with countries such as Japan, Finland, Switzerland, Spain, Sweden, and China.

\section{NUClEAR ENERGY AND Waste Program \\ Yvonne Tsang \\ (510) 486-6115 yttsang@lbl.gov}

\section{Jens Birkholzer \\ (510) 486-7134 jtbirkholzer@lbl.gov}

The geologic repository program in the United States is at a point where the Department of Energy (DOE) is close to completing the license application for repository construction at Yucca Mountain to the Nuclear Regulatory Commission (scheduled for June 2008. The safe performance of a high-level nuclear waste repository hinges on the multiple-barrier concept-namely, that the natural system and the engineered system would each contribute significantly to prevent radionuclides from leaving the repository and entering the biosphere. The proposed repository at Yucca Mountain, consisting primarily of fractured volcanic tuffs that vary in degree of welding, will be located about $350 \mathrm{~m}$ below ground surface within a thick unsaturated zone (UZ) above the water table. Over the last decade, NWP's work at Yucca Mountain consists of site characterization studies aimed at understanding the barrier function of the UZ, through field testing in an underground facility, an $8 \mathrm{~km}$ long underground tunnel known as the Exploratory Studies Facility (ESF). Complex numerical models have also been developed to simulate and understand the relevant processes related to multiphase, nonisothermal flow and transport through the UZ. Some of the key questions addressed by NWP scientists include:

- How much water percolates through the UZ to the repository at Yucca Mountain?

- What fraction of the water flows in fractures and what fraction flows through the rock matrix blocks?

- How much of this water will seep into the emplacement drifts (tunnels)?

- How will the radionuclide migration from the repository to the water table be retarded?

- How will coupled TH (thermal-hydrological), THC (thermal-hydrological-chemical), and THM (thermal-hydrological-mechanical) processes affect flow and transport?

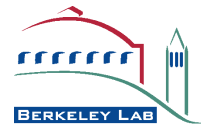


NWP scientists have also conducted studies as part of the Science and Technology (S\&T) Program within the DOE Office of Civilian Radioactive Waste Management (OCRWM). Distinct from, but in parallel to, the licensing effort at Yucca Mountain, the role of the Science and Technology program is to advance technologies not previously considered, to identify new or substantially revised scientific methods or tools that would provide a better understanding of the repository environment.

LBNL ESD is the Lead for the Natural Barriers Thrust within the S\&T Program. The goal for the Natural Barriers Thrust is to focus on research that would provide the essential scientific basis and demonstration of large contributions to repository performance by the unsaturated and saturated volcanic rocks at Yucca Mountain. The enhanced understanding of the different processes in the natural system would lead to reduction of uncertainty and obviate the need for overconservatism. NWP scientists are conducting studies pertaining to
- In-drift processes, integrating thermal-hydrologic-chemical-transport (THCM) models that simultaneously consider source term, corrosion, and the hydrological-chemical environment around waste package processes and conditions-and synthesize these complex processes into transparent, realistic, and defensible process models

- Near-drift processes, such as laboratory, field, and analogue studies to confirm the drift shadow concept, and the fact that it will lead to a large delay and sorption of radionuclides in the near-drift region

- Processes and conditions that will retard or mitigate flow and transport through the unsaturated and saturated volcanic rocks

\section{FUNDING}

Funding for research in the Nuclear Waste Program comes primarily from the Department of Energy, through the Director of the Office of Civilian Radioactive Waste Management.

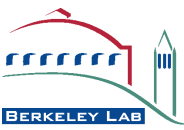




\title{
Geomechanical/Geochemical Modeling Studies Conducted with the International CoOperative DecovaleX-THMC Project
}

\author{
Jens Birkholzer, Jonny Rutqvist, Eric Sonnenthal, and Chin-Fu Tsang \\ Contact: Jens Birkholzer, 510/486-7134, jtbirkholzer@lbl.gov
}

\section{RESEARCH OBJECTIVES}

DECOVALEX-THMC is an international cooperative project managed by SKI, the Swedish Nuclear Power Inspectorate. The general goal is to encourage collaborative research on modeling coupled thermal-hydrological-mechanical-chemical (THMC) processes in geologic formations in support of the performance assessment for underground storage of radioactive waste. One of the ongoing research tasks within this project, initiated in 2004 by the U.S. Department of Energy (DOE), addresses the long-term impact of geomechanical and geochemical processes on flow conditions near waste emplacement drifts. The objective of this task is to (a) develop new insights into such processes and (b) to provide valuable peerreview of the respective models and their prediction results.

\begin{tabular}{|c|c|c|c|c|}
\hline Research Team & Simulator & Coupling & Mechanical/Chemical Model & Hydraulic and Transport Model \\
\hline DOE/LBNL & TOUGH-FLAC & THM & $\begin{array}{l}\text { Elastic, Elastoplastic, } \\
\text { Viscoplastic }\end{array}$ & $\begin{array}{l}\text { Discrete, single or dual continuum; } \\
\text { multiphase liquid and gas flow }\end{array}$ \\
\hline DOE/LBNL & ROCMAS & THM & $\begin{array}{l}\text { Elastic, Elastoplastic, } \\
\text { Viscoplastic }\end{array}$ & $\begin{array}{l}\text { Discrete or single continuum; } \\
\text { unsaturated liquid flow; } \\
\text { thermal vapor diffusion }\end{array}$ \\
\hline $\begin{array}{l}\text { BGR/Center } \\
\text { for Applied } \\
\text { Geosciences } \\
\text { (Germany) }\end{array}$ & Geosys/Rockflow & $v$ THM & $\begin{array}{l}\text { Elastic, Elastoplastic, } \\
\text { Viscoplastic }\end{array}$ & $\begin{array}{l}\text { Discrete or single continuum; } \\
\text { unsaturated liquid flow; } \\
\text { thermal vapor diffusion }\end{array}$ \\
\hline $\begin{array}{l}\text { CAS, Chinese } \\
\text { Academy } \\
\text { of Sciences }\end{array}$ & FRT-THM & THM & $\begin{array}{l}\text { Elastic, Elastoplastic, } \\
\text { Viscoplastic }\end{array}$ & $\begin{array}{l}\text { Discrete or single continuum; } \\
\text { unsaturated liquid flow; } \\
\text { thermal vapor diffusion }\end{array}$ \\
\hline $\begin{array}{l}\text { JAEA, Japan } \\
\text { Atomic Energy } \\
\text { Agency }\end{array}$ & THAMES & THM & $\begin{array}{l}\text { Elastic, Elastoplastic, } \\
\text { Viscoplastic }\end{array}$ & $\begin{array}{l}\text { Discrete or single continuum; } \\
\text { unsaturated liquid flow; } \\
\text { thermal vapor diffusion }\end{array}$ \\
\hline DOE/LBNL & TOUGHREACT & THC & $\begin{array}{l}\text { Equilibrium and kinetic } \\
\text { mineral-water-gas reactions } \\
\text { HKF activity model }\end{array}$ & $\begin{array}{l}\text { Discrete, single or dual continuum; } \\
\text { multiphase liquid and gas flow; } \\
\text { advection/ diffusion of } \\
\text { total concentrations (sequential) }\end{array}$ \\
\hline $\begin{array}{l}\text { BGR/Center } \\
\text { for Applied } \\
\text { Geosciences } \\
\text { (Germany) }\end{array}$ & $\begin{array}{l}\text { Geosys/Rockflow } \\
\text { with PHREEQC }\end{array}$ & THC & PHREEQC & $\begin{array}{l}\text { Discrete or single continuum; } \\
\text { unsaturated liquid flow; } \\
\text { thermal vapor diffusion; } \\
\text { advection/diffusion of total } \\
\text { concentrations (sequential) }\end{array}$ \\
\hline $\begin{array}{l}\text { JAEA, Japan } \\
\text { Atomic Energy } \\
\text { Agency }\end{array}$ & $\begin{array}{l}\text { THAMES with } \\
\text { Dtransu-3D-EL } \\
\text { and PHREEQC }\end{array}$ & THMC & PHREEQC & $\begin{array}{l}\text { Discrete or single continuum; } \\
\text { unsaturated liquid flow; } \\
\text { thermal vapor diffusion; } \\
\text { advection/ diffusion of total } \\
\text { concentrations (sequential) }\end{array}$ \\
\hline
\end{tabular}

\section{APPROACH}

The four research teams (from China, Germany, Japan, and USA) participating in DOE's task within the DECOVALEXTHMC project were asked to conduct predictive analysis of the long-term coupled processes in generic repositories with simplified conditions and geometry. Participating research teams model the THMC processes in the fractured rock close to a representative emplacement drift as a function of time, predict long-term changes in hydrological properties, and evaluate the impact on near-field flow and transport processes. Two generic repositories situated in different host rock types and featuring different emplacement conditions are analyzed for comparison. One is a simplified repository model of the United States' Yucca Mountain site, a deep unsaturated vol-

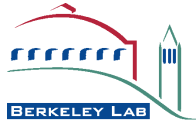
canic rock formation with emplacement in open gas-filled drifts. The second repository model is "located" in saturated crystalline rock, with emplacement drifts backfilled using a low-permeability buffer material such as bentonite (a concept considered in many European countries and in Japan).

\section{ACCOMPLISHMENTS}

As shown in Table 1, the research teams use different codes with different model approaches and characteristics. Since all teams simulate the same task configuration, research results from the participating teams can be directly compared. To date, good progress has been made in both model development and application. Comparison of geomechanical results indicates good overall agreement with respect to temperatures, stresses, and various hydrological parameters, despite the fact that different model approaches were used. Geochemical models show good quantitative agreement regarding aqueous species concentrations, while some differences with respect to mineral alterations still need to be worked out.

\section{SIGNIFICANCE OF FINDINGS}

The collaborative research conducted by international teams helps to develop a broader understanding of the complex THMC processes occurring near geologic repositories for radioactive waste. Good agreement between simulation results obtained with different model approaches provides enhanced confidence in their predictive capabilities when applied, for example, to the proposed Yucca Mountain repository.

\section{RELATED PUBLICATIONS}

Birkholzer, J., D. Barr, J. Rutqvist, and E.L. Sonnenthal, Motivation, description, and summary status of geomechanical and geochemical modeling studies in Task D of the International DECOVALEX Project. Proceedings, Geoproc 2006, China, May 2006.

Rutqvist, J., J. Birkholzer, M. Chijimatsu, O. Kolditz, Q. Liu, Y. Oda, W. Wang, and C. Zhang, Comparative simulation study on coupled THM processes near back-filled and open-drift nuclear waste repositories in Task D of the International DECOVALEX Project. Proceedings Geoproc 2006, China, May 2006.

Xie, M., E.L. Sonnenthal, W. Wang, O. Kolditz, J. Birkholzer, Y. Oda, and M. Chijimatsu, Geochemical predictions for a hypothetic repository located in saturated crystalline rock-Comparative evaluation of two different research teams. Proceedings Geoproc 2006, China, May 2006.

\section{ACKNOWLEDGMENTS}

This work was supported by the Director, Office of Civilian Radioactive Waste Management, Office of the Chief Scientist, of the U.S. Department of Energy (DOE) under DOE Contract No. DE-AC02-05CH11231. 


\section{CONTINUOUS-Time Random-WALK ANALYSis OF DuAl-PERMEABILITY Fractured MEDia}

Andrea Cortis and Jens Birkholzer

Contact: Andrea Cortis, 510-495-2507, acortis@lbl.gov

\section{RESEARCH OBJECTIVES}

Reliable prediction of fluid and solute movement in fractured porous formations is of paramount importance in many practical applications. Fracture networks and the surrounding porous (and permeable) matrix are not hydraulically independent domains - they interact by exchanging solutes at their interface. Solute particles traveling on fast flow paths in the fractures can be retarded by solute exchange with the porous matrix, a diffusive or advective process depending on matrix properties. These retardation effects are extremely difficult to model in a classical transport framework. The objective of this research is to apply the continuous-time random-walk (CTRW) transport theory to the analysis of dual-permeability fractured media, to characterize transport by means of probabilistic distributions of the solute retention times.

\section{APPROACH}

In the CTRW approach, the interaction between the fractured and porous rock domains is modeled by a transition time probability distribution function (pdf), which characterizes the retention time inside the fractured medium. In our research, this approach is validated numerically through the analysis of discrete numerical solutions of tracer transport in idealized dual permeability fractured media. A numerical inversion procedure identifies the transition time pdf from the analysis of the BTCs.

\section{ACCOMPLISHMENTS}

We applied the CTRW analysis to a series of synthetic breakthrough curves (BTC) obtained by means of a standard finite element code, on a finely gridded 2-D geometry consisting of a regular arrangement of fractures (oriented at a $45^{\circ}$ angle with respect to the direction of the mean flow) in a permeable porous domain (see Figure 1). By changing matrix permeability over a wide range (from representing rather permeable sandstone on one end of the spectrum to almost impermeable granites or shales on the other), we have considered various fracture-to-matrix permeability contrasts, which reflect typical values of densely fractured geological formations.

\section{SIGNIFICANCE OF FINDINGS}

Our results indicate that a CTRW analysis can completely characterize transport in dual-permeability fractured media by means of a probabilistic distribution function (pdf) of solute retention times. The pdf can be obtained from an analysis of the macroscopic (experimental) BTCs and contains all the necessary information for predicting the solute behavior at different times and sections. In the CTRW model, the transport velocity can be estimated from the composite porosity of the dual permeability medium. Furthermore, the classical dispersivity parameter, an essential component of any advection-dispersion based model, does not scale with the distance of the observation, but rather stays constant and relatively small. These findings indicate the CTRW method is an important and valuable alternative to more complex model approaches for fractured porous formations, such as discrete models or dual-permeability formulations.
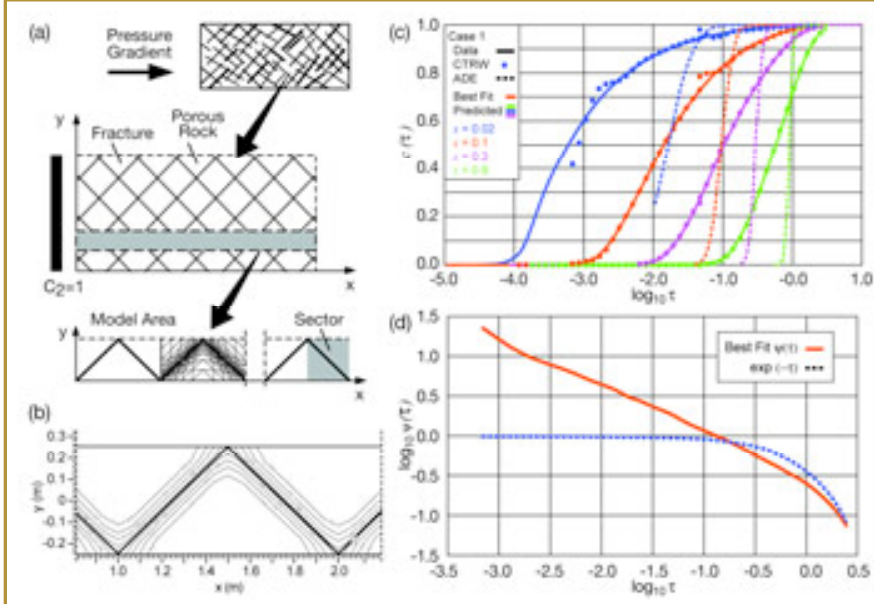

Figure 1. (a) Fractured domain under study (b) Discrete finite-element solute-transport simulations for a permeability contrast of approximately 12,000. (c) Breakthrough curves at four different sections. Discrete simulations are solid lines; CTRW predictions are circles; advection dispersion equation (ADE) predictions are dashed lines. (d) Probabilistic distribution of the solute residence times from CTRW analysis (red line), and comparison with the decaying exponential distribution (ADE model).

\section{RELATED PUBLICATIONS}

Cortis, A., Peclet-dependent memory kernels for transport in heterogeneous media. Phys Rev. E, 2007 (submitted).

\section{ACKNOWLEDGMENTS}

This work was supported by the Director, Office of Civilian Radioactive Waste Management, Office of the Chief Scientist, of the U.S. Department of Energy (DOE) under DOE Contract No. DE-AC02-05CH11231.

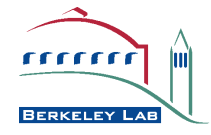




\title{
Heterogeneous Seepage at the Nopal I Uranium Mine, Chinuahua, Mexico
}

\author{
Patrick F. Dobson, Paul J. Cook, Teamrat Ghezzehei, J. Alfredo Rodríguez ${ }^{1}$, and Rodrigo de la Garza ${ }^{2}$ \\ ${ }^{1}$ Instituto de Ecología, A.C., and ${ }^{2} \mathrm{UACH}$ \\ Contact: Patrick F. Dobson, 510/486-5373, pfdobson@lbl.gov
}

\section{RESEARCH OBJECTIVES}

The primary objective of this analogue study is to evaluate flow and transport processes of relevance to the proposed Yucca Mountain repository. Seepage data obtained from this study will be used to constrain flow and transport models being developed for the Nopal I system.

\section{APPROACH}

A water collection system, consisting of 240 separate $30 \mathrm{~cm}$ $\times 30 \mathrm{~cm}$ compartments that are each connected to a $125 \mathrm{~mL}$ bottle, was installed in April 2005 within the $+00 \mathrm{~m}$ adit of the Nopal I mine, to collect water that had infiltrated from the +10 $\mathrm{m}$ level and seeped into the adit. This system was upgraded in November 2005, when instrumentation was added to six collector sites to measure seepage rates continuously. An automated weather station was installed at the site in March 2006 to permit correlation of local precipitation events with seepage.

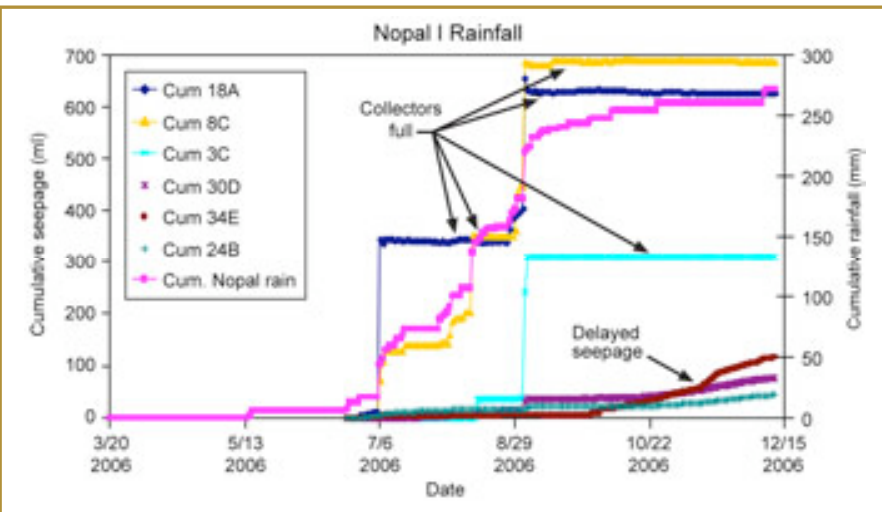

Figure 1. Cumulative rainfall and seepage amounts for instrumented collectors from Nopal I +00 m adit.

\section{ACCOMPLISHMENTS}

Rainfall in central Chihuahua is seasonal, with most precipitation occurring during the summer monsoon period. Initial modeling of infiltration and seepage through a series of planar, vertical fractures was conducted to evaluate flow transit times and seepage rates (Ghezzehei et al., 2006). Using a range of fracture apertures and frequencies, and assuming no fracturematrix interaction, infiltration through the $8 \mathrm{~m}$ high vertical fracture system and seepage into the adit was predicted to occur within 24 hours after a 6-hour rainfall event.

Monitoring of seepage within the adit between April 2005 and December 2006 indicates that seepage is highly heterogeneous in both time and space. Within the back adit area, there are a few zones where large volumes of water have been collected. These large volume seepage events (Figure 1) are linked to fast flow path fractures ( $<4 \mathrm{~h}$ transit times) and are associated with heavy rainfall $(>25 \mathrm{~mm})$. In most locations, however, there is a significant (1-6 month) time lag between major precipitation events and seepage within the adit, with longer water residence times observed for the front adit area.

\section{SIGNIFICANCE OF FINDINGS}

The wide variability in the location, timing, and amount of seepage occurring within the Nopal I adit suggests that a number of fast-flow fracture pathways are active immediately after large rainfall events. Flow focusing along these pathways may explain the heterogeneous seepage distribution. However, delayed seepage observed in many locations within the adit indicate that even a relatively thin $(8 \mathrm{~m})$ rock mass can exert a noticeable damping effect on infiltration, and that flow and transport models must incorporate fracture flow heterogeneity. The initial results of this work are consistent with the fast-flow-path model postulated for Yucca Mountain.

\section{RELATED PUBLICATIONS}

Ghezzehei, T.A., P.F. Dobson, J.A. Rodriguez, and P.J. Cook, Infiltration and seepage through fractured welded tuff. 2006 International High Level Radioactive Waste Management Conference, April 30-May 4, 2006, Las Vegas, NV, American Nuclear Society, La Grange Park, IL, pp. 105-110, 2006.

\section{ACKNOWLEDGMENTS}

This work was supported by the Director, Office of Civilian Radioactive Waste Management, Office of the Chief Scientist, of the U.S. Department of Energy (DOE) under DOE Contract No. DE-AC02-05CH11231. We thank the Instituto de Ecologia, A.C. and the Universidad Autónoma de Chihuahua for their assistance.

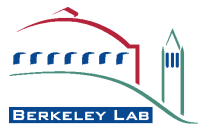




\section{Evaluation of UnCertainties due to Hydrogeological Modeling AND Groundwater Flow Analysis: Strategy for Characterizing a NeW Site}

Christine Doughty and Kenzi Karasaki

Contact: Kenzi Karasaki, 510/486-6759, KKarasaki@lbl.gov

\section{RESEARCH OBJECTIVES}

It is quite challenging to build a reliable model for simulating groundwater flow in a large body of rock mass, particularly when the rock is fractured. Largescale groundwater flow models are typically calibrated to the steady-state pressure head data. However, steady-state head data alone are not sufficient for building a reliable predictive model. The overall objective of this project is to develop methodologies for reducing the uncertainty and increasing the reliability of such a model. In the present work, we develop a strategy for characterizing a new site where a minimal number of wells are available.

\section{APPROACH}

We begin with a $9 \mathrm{~km} \times 9 \mathrm{~km} \times 2 \mathrm{~km}$ thick effective continuum model of the fractured rock of the Tono, Japan, area developed from regional geographic, geologic, geophysical, and surface and subsurface hydrological data. The model is calibrated simultaneously to data from 17 wells: steady-state head profiles in 11 wells, 21 pressure transients from 8 wells, and steady-state temperature profiles in 11 wells, which constrain the porosity and permeability of the granitic rock, overlying sediments, and a major subvertical fault that bisects the model. Also so constrained are the amount of surface infiltration that recharges the deep groundwater flow system and the lateral boundary conditions of the model. The resulting model is considered the best model possible of the Tono region, since it utilizes the full set of available data.

Next, we consider data from various subsets of these 17 wells and compare performance measures obtained from the resulting models to those of the best model, to investigate how many wells to use and how to optimally locate wells for a preliminary site characterization. Additionally, some of the key assumptions regarding model heterogeneity and boundary conditions are examined to assess the impact they have on recommended well locations.

\section{ACCOMPLISHMENTS}

We find that, for the most part, our understanding of the regional groundwater flow and advective tracer transport does not improve significantly as more and more wells are used for site
Dashed - open lateral boundaries Solid - closed lateral boundaries
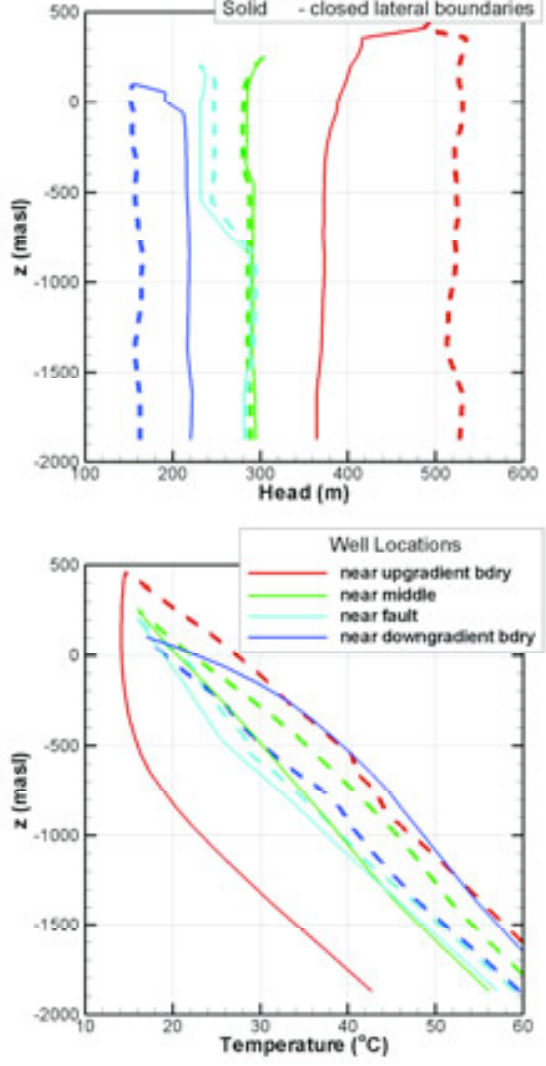

Figure 1. Head (top) and temperature (bottom) profiles at four hypothetical well locations colors), for closed and open lateral boundaries (different line styles). Profiles from wells located near the boundaries are useful for characterizing lateral boundary conditions. characterization. This is because these measures are controlled by surface topography, surface and lateral boundary conditions, and permeability and porosity distributions, and the present property distributions have short correlations, hence using one well for site characterization provides as much information about material properties as using many wells does.

On the other hand, observing head profiles in more wells increases the probability that large-scale features that do impact groundwater flow (such as a fault) can be identified. An additional caveat is that if the permeability distribution has longrange correlations, then a small number of wells are not as likely to provide a representative sample of rock properties, nor a true picture of the regional groundwater flow.

\section{SIGNIFICANCE OF FINDINGS}

Lateral boundary conditions have a large impact on all aspects of flow and transport. If existing studies of topography and regional groundwater flow do not provide this information, wells should be located near the presumed upgradient and downgradient boundaries of the site, and head and temperature profiles should be examined for the characteristics of closed and open groundwater flow systems.

\section{RELATED PUBLICATION}

Karasaki, K., J. Apps, C. Doughty, H. Gwatney, C.T. Onishi, R.C. Trautz, and C.F. Tsang, Feature Detection, Characterization and Confirmation Methodology-Final Report. NUMO-LBNL Collaborative Research Project Report, March 2007.

\section{ACKNOWLEDGMENTS}

This work is supported by the Nuclear Waste Management Organization of Japan (NUMO) and the Japan Atomic Energy Agency (JAEA) through a bi-national agreement between JAEA and the U.S. Department of Energy (DOE), and conducted at the Ernest Orlando Lawrence Berkeley National Laboratory under DOE Contract No. DEAC02-05CH1123.

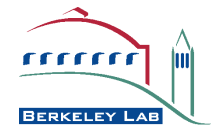




\title{
Analysis of Flowing Fluid-Electric-Conductivity Logs UNDER NONIDEAL CONDITIONS
}

\author{
Christine Doughty and Chin-Fu Tsang \\ Contact: Christine Doughty, 510/486-6453, cadoughty@lbl.gov
}

\section{RESEARCH OBJECTIVES}

In the study of flow and transport in the subsurface, knowledge of flow zones and their hydraulic properties is essential. Coring and geophysical methods in boreholes drilled deep into the rock may be able to identify the fractures themselves, but they are unlikely to provide information on fracture flow properties. Straddle-packer pump-testing yields fracture flow properties, but is very time-consuming. Flow-logging techniques are an attractive alternative- they are sensitive to fracture flow and are efficient to deploy in the field.

The flowing fluid-electric-conductivity (FFEC) logging method provides information on the depths, salinities, transmissivities, and pressure heads of individual conductive features intercepted by a borehole, without the need of specialized probes. The method has been successfully applied to deep boreholes in granitic formations. This summary presents the application of the method to two zones in a 1,000 $\mathrm{m}$ borehole in sedimentary rock at Horonobe, Japan. The data sets involve a number of complications, such as variable well diameter, free water table decline in the well, periods of time with unknown pumping rate, and effects of drilling mud. Our objective is to determine whether the method is robust enough to use under these nonideal conditions.

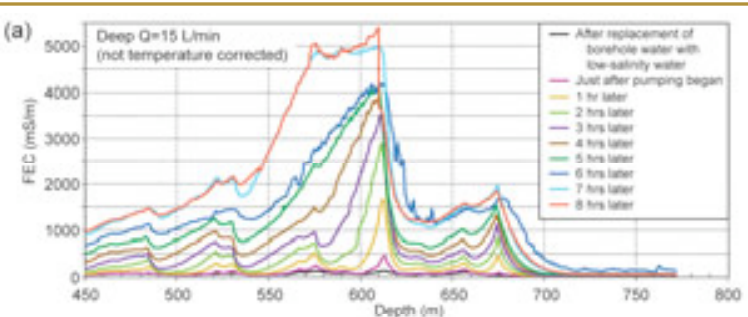

(b)

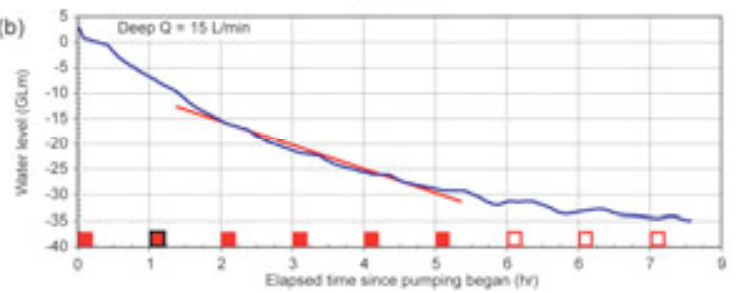

Figure 1. Sample of FFEC data, illustrating some of the complications: (a) Set of FFEC logs for a deep-zone test. Logs obtained 6, 7, and 8 hours after pumping began are not consistent with other logs, probably owing to slime from drilling mud adhering to the probe; (b) Water-level data during logging. Red boxes identify times at which logs were collected; open boxes indicate a problem with the FFEC log; the black-outlined box was used as initial conditions for model. A period of high, unknown pumping rate occurred early on, causing a sudden water-level decline. Thereafter, water-level declined steadily, but at a decreasing rate. Only logs collected while the decline is approximately linear (red line) are analyzed.
ACCOMPLISHMENTS

In spite of complications associated with the tests, analysis data is able to identify 44 hydraulically conducting fracFEC), and transmissivities and pressure heads (in dimensionless form) of these 44 features are obtained and found to vary significantly among one another. These results are compared with data from eight packer tests with packer intervals of $10-80 \mathrm{~m}$, which were conducted in this borehole over the same depth interval. They are found to be consistent with these independent packer-test data, thus demonstrating the robustness of the FFEC logging method under nonideal conditions.

\section{SIGNIFICANCE OF FINDINGS}

FFEC logging provides an efficient, affordable means of characterizing the hydraulically conductive features intersecting a borehole, with high vertical resolution and without the need of specialized probes. Such information is valuable for characterization of regional groundwater flow, design of nuclear waste storage facilities, remediation of subsurface contamination, and a host of other prob-

\section{APPROACH}

The FFEC logging method involves the replacement of wellbore water by de-ionized water, followed by pumping at a constant rate, during which a series of fluid electric conductivity logs are taken. The logs can be analyzed to identify depth locations of inflow, and evaluate inflow rate and electric conductivity (salinity) of the fluid at each inflow point. When the method is repeated with two or more pumping rates, a combined analysis of the multi-rate data allows an efficient means of also determining transmissivity values of all inflow points, as well as their inherent (so-called far-field) pressure heads.

For each of the two zones logged in the 1,000 m wellbore, three sets of logs were collected using different pumping rates, each set measured over a period of about one day (Figure 1). To analyze the data, we apply various techniques that have been developed

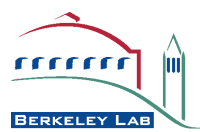
for analyzing FFEC logs: direct-fitting, massintegral, and the multi-rate method mentioned above. lems. Moreover, it can be very useful in conjunction with other subsurface site-characterization activities, such as providing highresolution monitoring during a tracer test, or providing ground truth at boreholes for crosshole geophysical imaging methods.

\section{RELATED PUBLICATIONS}

Doughty, C., C.-F. Tsang, K. Hatanaka, S. Yabuuchi and H. Kurikami, Application of direct-fitting, mass-integral, and multi-rate methods to analysis of flowing fluid electric conductivity logs from Horonobe, Japan. LBNL-63307. Water Resour. Res. (in press), July 2007.

\section{ACKNOWLEDGMENTS}

This work is supported by the Nuclear Waste Management Organization of Japan (NUMO) and the Japan Atomic Energy Agency (JAEA), through a bi-national agreement between JAEA and the U.S. Department of Energy (DOE), and conducted at Berkeley Lab under DOE Contract No. DE-AC02-05CH1123. 


\section{Climatic Forecasting of Net Infiltration at Yucca Mountain Using Analogue Meteorological Data}

Boris Faybishenko

510/486-4852, bafaybishenko@lbl.gov

\section{RESEARCH OBJECTIVES}

The objective is to develop a semi-empirical model and forecast average net infiltration rates, using the limited meteorological data from analogue meteorological stations, for interglacial (present-day), and future monsoon, glacial transition, and glacial climates over the Yucca Mountain region.

\section{APPROACH AND METHODS}

Net infiltration, aridity, and precipitation-effectiveness ( $\mathrm{P}$ E) indices were calculated using a modified Budyko's waterbalance model, with reference surface potential evapotranspiration determined from the radiation-based Penman formula. The computed net infiltration rates were corroborated by comparing them with the empirically and numerically determined groundwater recharge and percolation rates through the unsaturated zone from published data.

\section{ACCOMPLISHMENTS}

Net infiltration rates are forecasted to generally increase from the present-day climate to monsoon climate, to glacial transition (intermediate) climate, and then to the glacial climate, following a power law relationship between net infiltration and precipitation. The forecasting results indicate the overlap between the ranges of net infiltration for different climates (Figure 1). The calculated net infiltration rates have yielded a good match with other field and modeling study results pertaining to groundwater recharge and percolation flux through the unsaturated zone at Yucca Mountain. This comparison indicates the robustness of the simple water-balance approach used in this study.

\section{SIGNIFICANCE OF FINDINGS}

Computed present-day and potential future net infiltration can be used as a hydrologic parameter to assess the rate of deep percolation, groundwater recharge, radionuclide transport, and seepage into tunnels-all of which are, in turn, useful parameters for the performance assessment of the proposed nuclear waste repository at Yucca Mountain, Nevada.

\section{RELATED PUBLICATION}

Faybishenko, B., Climatic forecasting of net infiltration at Yucca Mountain using analogue meteorological data. Vadose Zone Journal, 6, 77-92, 2007.

\section{ACKNOWLEDGMENTS}

This work was supported by the Director, Office of Civilian Radioactive Waste Management, of the U.S. Department of Energy (DOE) under DOE Contract No. DE-AC02-05CH11231.

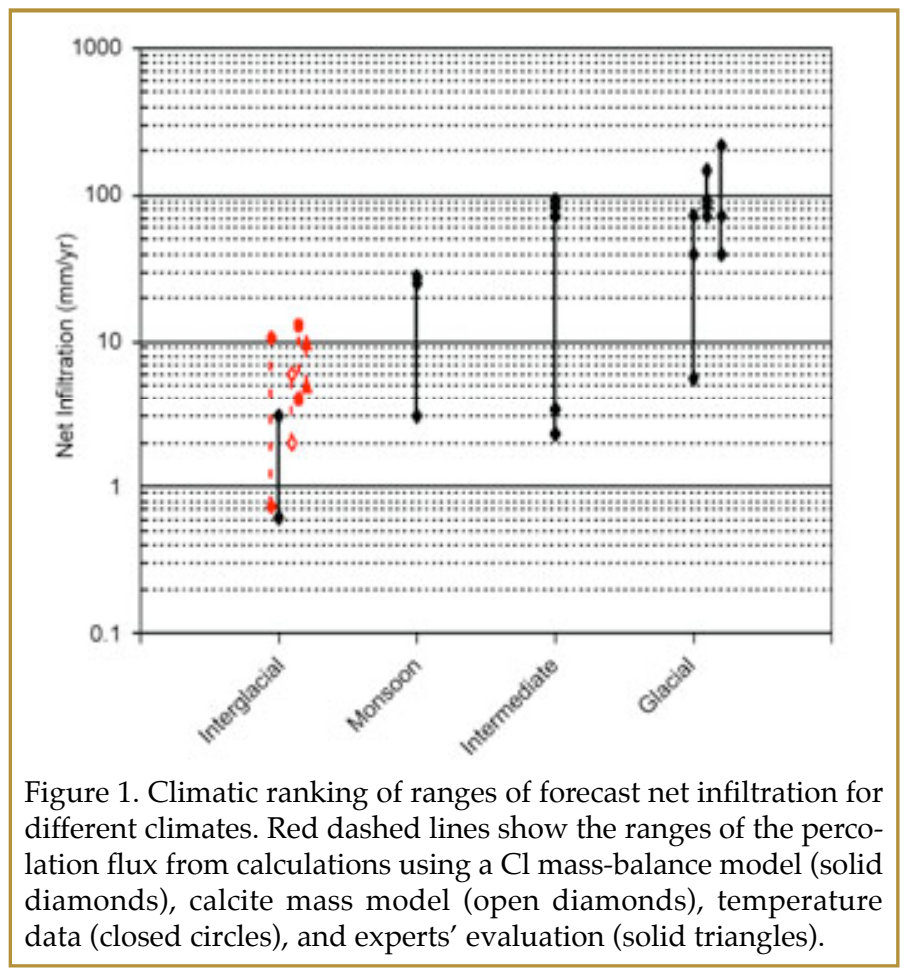

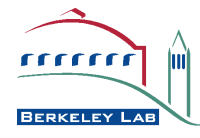




\title{
A Modeling Study on Natural Convection in Emplacement Drifts AND ITS IMPACT ON DRIFT SEEPAGE
}

\author{
N. Halecky, J. Birkholzer, G. Danko ${ }^{1}$, and D. Bahrami ${ }^{1}$; ${ }^{1}$ University of Nevada, Reno \\ Contact: Nicholaus Halecky, 510/486-5793, nehalecky@lbl.gov
}

\section{RESEARCH OBJECTIVES}

The decay heat outputs of the radioactive waste potentially to be emplaced at Yucca Mountain will strongly affect the thermal-hydrological (TH) conditions in and near the geologic repository. This decay heat elevates drift temperatures above ambient conditions. Pore formation water in the near-field rock mass evaporates into the open air spaces of the drift and is transported by natural convection processes from the hot drift center to the cool drift end (where no waste is emplaced) and condenses. Our goal is to better understand this reduction of moisture content in the near-drift fractured rock and its role in the potential reduction of seepage into the drift.
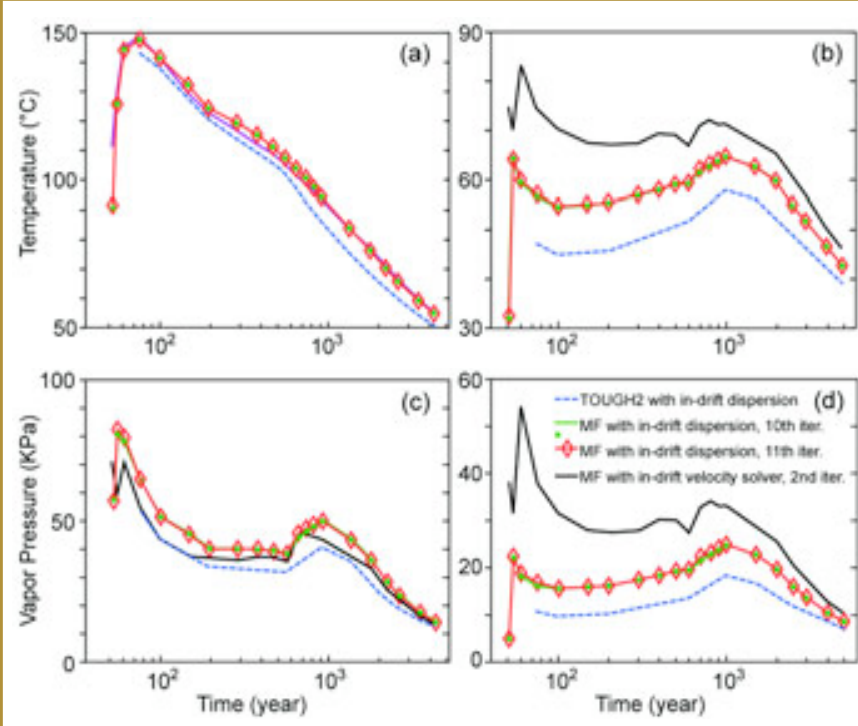

Figure 1. Drift wall temperature evolution at the hottest (a) and coldest (b) drift segments; and vapor-pressure evolution at the hottest (c) and coldest (d) drift segments.

\section{APPROACH}

A multiscale seepage modeling procedure was developed that accounts for transport processes in and between the fractured rock mass and the open air drift, two distinct domains. To fully account for 3-D effects, a first model (MF-T2) operates on a scale that encompasses the entire drift plus surrounding rock units. In MF-T2, the flow and transport processes in the rock mass are simulated with the multiphase, multicomponent simulator TOUGH2, and the in-drift heat and moisture flows are simulated with MULTIFLUX (MF), a lumped-parameter CFD (Computational Fluid Dynamics) code (Danko et al., 2007). MF provides an efficient iterative coupling technique for matching the mass and heat transfer between the rock mass and the drift.

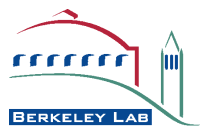

In addition, explicit modeling of the impact of in-drift moisture transport on seepage is being conducted with a separate high-resolution seepage model, with model inputs (in-drift temperature and relative humidity) provided by the full drift-scale MF-T2 model.

\section{ACCOMPLISHMENTS}

The new solution procedure has been applied to evaluate the heat-driven flow and transport processes in a representative emplacement drift at Yucca Mountain, embedded in a monolithic, three-dimensional rock mass. Two alternative approaches were tested to simulate in-drift natural convection: (1) a lumped-parameter CFD dispersion model and (2) a model explicitly simulating the air velocity distribution. Several iterations were completed, refreshing the MF results against TOUGH2 runs, with both solution procedures showing excellent convergence (Figure 1). Also, alternative results from a simplified in-drift model within TOUGH2 are shown (Birkholzer et al., 2006). High-resolution seepage model development is ongoing, with preliminary results indicating that strong natural convection processes can significantly reduce the near-field fracture moisture content, thereby reducing the seepage potential.

\section{SIGNIFICANCE OF FINDINGS}

The difficult task of coupling CFD models with porous media models, to understand moisture reduction in the near drift, is undertaken with a unique and efficient approach. Initial results from the coupled MF-T2 solution procedure show good convergence between the two domains for a full drift-scale representation. The ongoing efforts of applying results from these simulations into a high-resolution seepage model will allow for a better understanding of seepage reduction due to natural convection processes, thereby further characterizing potentially beneficial repository behavior.

\section{RELATED PUBLICATIONS}

Birkholzer, J., S.W. Webb, N. Halecky, P.F. Peterson, and G.S. Bodvarsson, Evaluating the moisture conditions in the fractured rock at Yucca Mountain-The impact of natural convection in heated emplacement drifts. Vadose Zone Journal, 5, 1172-1193, 2006.

Danko, G., D. Bahrami, and J.T. Birkholzer, The effect of unheated sections on moisture transport in the emplacement drift. Nuclear Technology (in press), 2007.

\section{ACKNOWLEDGMENTS}

This work was supported by the Director, Office of Civilian Radioactive Waste Management, Office of the Chief Scientist, of the U.S. Department of Energy (DOE) under DOE Contract No. DE-AC02-05CH11231. 


\section{Examining The Drift Shadow in Nature}

Timothy J. Kneafsey, Teamrat Ghezzehei, Paul Cook, and John Peterson

Contact: Timothy Kneafsey, 510/486-4414, tjkneafsey@lbl.gov

\section{RESEARCH OBJECTIVES}

The drift shadow is a region in the unsaturated zone beneath an underground opening (such as a cave or mined tunnel) that is partially sheltered from downward-percolating water, because the capillarity is not strong enough to draw water into the rock immediately below the drift. Modeling studies of the drift shadow suggest that transport in this region is controlled by diffusive rather than by advective processes. The drift shadow has not yet been observed in nature. Our research objective is to demonstrate the presence (or absence) of the drift shadow at a field location and compare our measurements to predictions.

\section{APPROACH}

To identify a drift shadow, we must find an appropriate field site where a drift shadow might be observed, obtain core samples from locations at different depths around the opening, and make measurements of water potential and water content that would indicate the presence or absence of the drift shadow. In addition, we would like to impose a water flux over a drift and observe its flow behavior and possible drift shadow formation. We must also compare our field results with modeling results of a detailed sitespecific model to gain confidence in modeling studies of the drift shadow.

\section{ACCOMPLISHMENTS}

We have performed extensive modeling to understand the theoretical definition and extent of the drift shadow for various sets of conditions. Based on our modeling, we selected and are investigating the presence of the drift shadow in the East Bay Regional Parks District Hazel-Atlas Mine, a former sand mine in Northern California that is now operated as a museum. Our study location, not currently in the museum, contains a driftover-drift configuration. We have retrieved core from core holes fanning the two drifts, x-ray-scanned them looking for density changes, and measured gravimetric moisture content of subsamples of the core. We have performed ground-penetrating radar studies using the core holes to look for saturation changes indicating the presence of a drift shadow and are constructing an active test to impose water flow around the lower drift.

\section{SIGNIFICANCE OF FINDINGS}

Demonstrating the presence of a drift shadow will provide another line of evidence to build confidence in the theory of flow and transport in unsaturated media and its numerical extension. In addition, it will allow the consideration of significantly reduced transport from waste emplacement drifts at the proposed high-level nuclear waste repository at Yucca Mountain, Nevada, in which waste packages are expected to be placed in near-horizontal drifts in the unsaturated zone.

\section{RELATED PUBLICATIONS}

Ghezzehei, T.A., T.J. Kneafsey, and G.W. Su, Correspondence of the Gardner and van Genuchten relative permeability function parameters. Water Resources Research (in press), 2007.

Houseworth, J.E., S.A. Finsterle, and G.S. Bodvarsson, Flow and transport in the drift shadow in a dual-continuum model. Journal of Contaminant Hydrology, 62-63: 133-156, 2003.

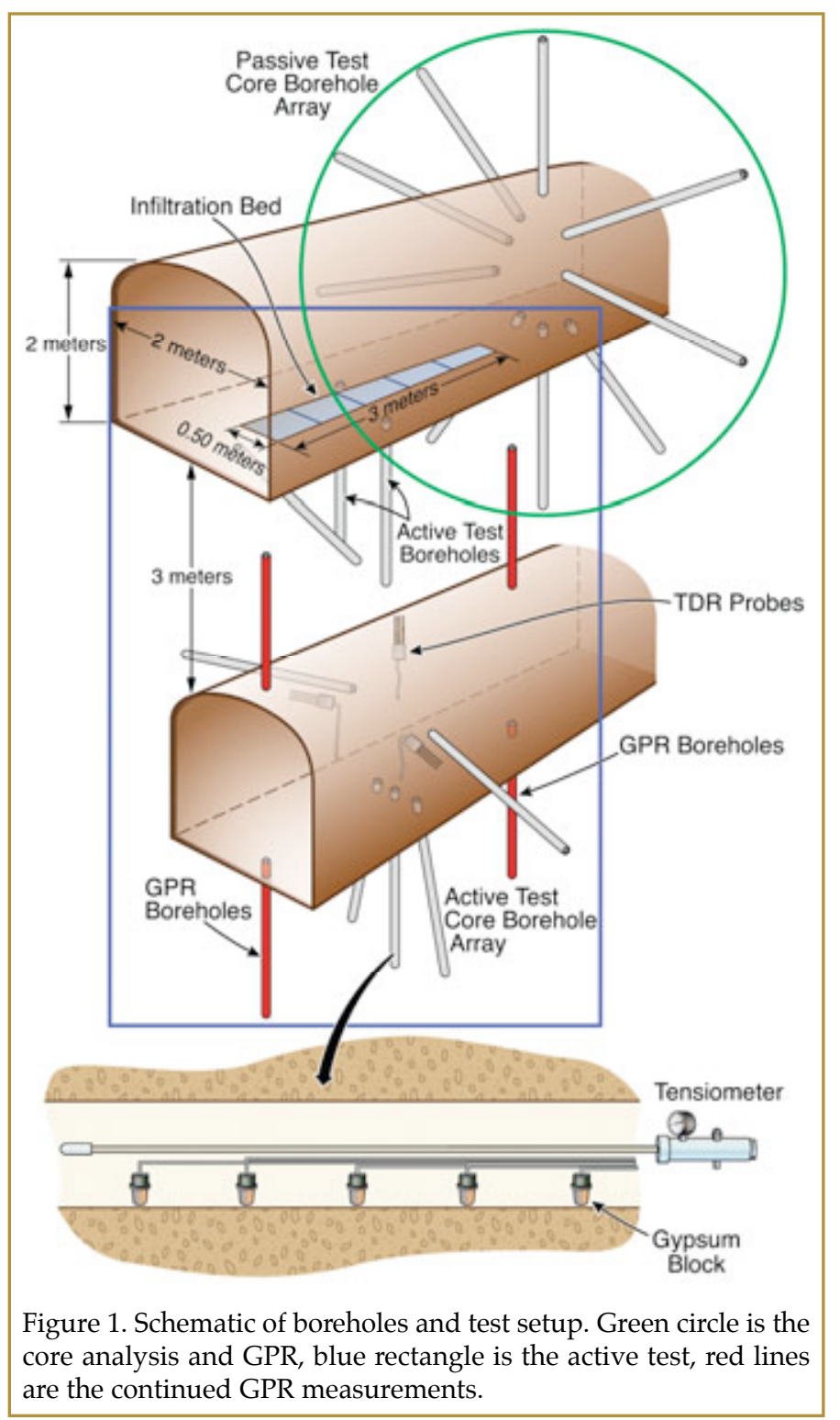

\section{ACKNOWLEDGEMENTS}

This work was supported by the Director, Office of Civilian Radioactive Waste Management, Office of the Chief Scientist, of the U.S. Department of Energy (DOE) under DOE Contract No. DE-AC02-05CH11231. 


\section{Sensitivity Analysis for Joint InVersion of Ground-Penetrating RADAR AND THERMAL-Hydrological Data From a LARge-ScAle UndERgROUND HEATER TeST}

Michael B. Kowalsky, Jens Birkholzer, John E. Peterson, Stefan Finsterle, Sumit Mukhopadhyay, and Yvonne Tsang Contact: Michael B. Kowalsky, 510/486-7314, mbkowalsky@lbl.gov

\section{RESEARCH OBJECTIVES}

To develop site-specific hydrological models, the joint analysis of hydrological and geophysical data has the potential to significantly improve the characterization of the subsurface, and thus increase the reliability of model predictions. The merit of any given data type depends on its usefulness in providing quantitative information about flow and transport properties (at a reasonable resolution). Since geophysical data offer valuable information on the subsurface structure, developing methods for integrating such data with hydrological data has the potential to significantly advance site characterization in complex, heterogeneous systems.

The objective of this research is to develop a joint inversion approach so that it can be applied to increasingly complex thermal-hydrological processes. These processes include the transport of water, water vapor, air and heat in fractured porous media, the transitions between the liquid and gaseous phases, and vapor-pressure lowering effects as a result of capillary pressure increases.
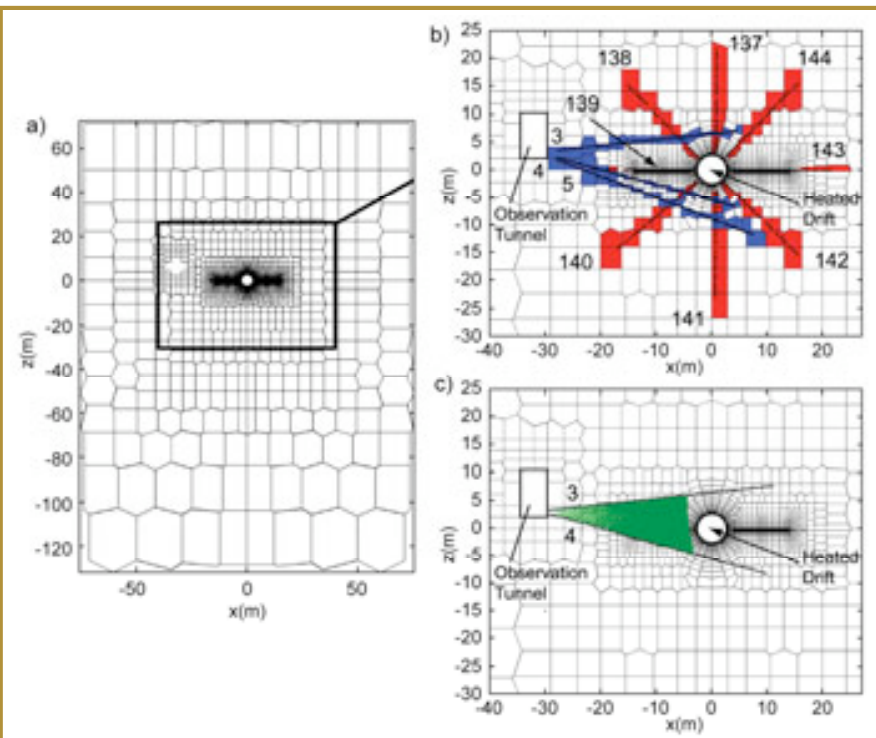

Figure 1. Thermal-hydrological model and measurement locations: (a) model grid; (b) locations of water content (measured by neutron probes) and temperature measurements (indicated by blue- and red-shaded gridblocks, respectively); and (c) locations for ground-penetrating radar data used for inversion (green lines connect transmitting and receiving antennas).

\section{APPROACH}

The approach integrates the coupled simulation of thermalhydrological and geophysical data-ground-penetrating radar (GPR) data-within the iTOUGH2 optimization framework to estimate (1) thermal-hydrological parameters (such as permeability, porosity, thermal conductivity, and parameters of the capillary pressure and relative permeability functions) that are needed for predicting the flow of fluids and heat in fractured porous media; and (2) parameters of the petrophysical function that relates water saturation, porosity, and temperature to the dielectric constant.

\section{ACCOMPLISHMENTS}

We applied the approach to a large-scale in situ heater test that was conducted at Yucca Mountain, Nevada, to better understand the coupled thermal, hydrological, mechanical, and chemical processes that occur in the fractured rock mass around a geologic repository for high-level radioactive waste. We examined the sensitivity of the most relevant thermalhydrological and petrophysical parameters to the time-lapse GPR data and thermal-hydrological data (temperature and water content) collected before and during the four-year heating phase of the test. To demonstrate the feasibility of the approach, and as a first step toward comprehensive inversion, we applied the approach to estimate the permeability of the rock matrix.

\section{SIGNIFICANCE OF FINDINGS}

Preliminary results indicate that estimation of thermalhydrological and petrophysical parameters is possible through the combination of geophysical, hydrological, and thermal measurements. The large-scale heater test provides a unique data set to which our approach can be further applied and tested.

\section{RELATED PUBLICATIONS}

Kowalsky, M.B., J. Birkholzer, J. Peterson, S. Finsterle, S. Mukhopadhyay, and Y. Tsang, Sensitivity analysis for joint inversion of GPR and thermal-hydrological data from a large-scale underground heater test. Nuclear Technology (submitted), 2007.

Kowalsky, M., S. Finsterle, J. Peterson, S. Hubbard, Y. Rubin, E. Majer, A. Ward, and G. Gee, Estimation of field-scale soil hydraulic parameters and dielectric parameters through joint inversion of GPR and hydrological data. Water Resour. Res. 41, W11425, doi:10.1029/2005WR004237, 2005.

\section{ACKNOWLEDGMENTS}

This work was supported by the Director, Office of Civilian Radioactive Waste Management, of the U.S. Department of Energy (DOE) under DOE Contract No. DE-AC02-05CH11231.

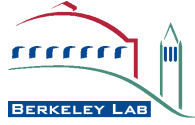




\title{
Mechanisms for Scale Dependence of the Effective Matrix Diffusion Coefficient
}

\author{
Hui-Hai Liu, Yingqi Zhang, Quanlin Zhou, and Fred J. Molz
}

Contact: Hui-Hai Liu, 510/486-6452, hhliu@lbl.gov

\section{RESEARCH OBJECTIVES}

Matrix diffusion denotes the exchange of solute mass (through molecular diffusion) between fluid in fractures and fluid in the rock matrix. Owing to the orders-of-magnitude slower flow velocity in the matrix compared to that in fractures, matrix diffusion can significantly affect solute transport in fractured rock, and therefore is an important process for a variety of problems, including remediation of subsurface contamination and geological disposal of nuclear waste. The effective matrix diffusion coefficient is a key parameter for describing this matrix diffusion process. Our previous studies have indicated that the effective matrix diffusion coefficient values, obtained from a large number of field tracer tests, are enhanced in comparison with local values and increase with test scale. The major objective of this study is to investigate the physical mechanisms behind this scale dependence.

\section{APPROACH}

Numerical experiments were performed to evaluate the effects of flow-path geometry in a fracture network (Liu et al., 2007a). The focus of the experiments was on solute transport in flow paths having geometries consistent with percolation theories and characterized by local flow loops formed mainly by small-scale fractures. Values for effective transport parameters were obtained by matching breakthrough curves from numerical experiments with an analytical solution for solute transport along a single fracture. To investigate the effects of property heterogeneity on the rock matrix, we also derived analytical expressions for the effective matrix-diffusion coefficient for two idealized fracture-matrix systems: a single fracture system associated with rock matrix heterogeneity along the water flow direction in the fracture, and a multiple fracture system with rock-matrix heterogeneity among different fractures (Liu et al., 2007b). These analytical results allow for direct demonstrations of relationships between the scale dependence and spatial variability of the rock-matrix diffusive properties.

\section{ACCOMPLISHMENTS}

Our study indicates that the observed scale-dependence of the effective matrix diffusion coefficient results from a combination of flow-path-geometry effects and the heterogeneity of rock-matrix diffusive properties. Numerical experiment results show that the matrix diffusion process associated with local flow loops formed by small-scale (or high-level) fractures (which is mostly ignored in current modeling practices) seems to be an important mechanism in causing the observed scale dependence (Figure 1). Our analytical results further demonstrate that different-scale heterogeneities of the rock-matrix diffusive properties also contribute to this scale dependence.

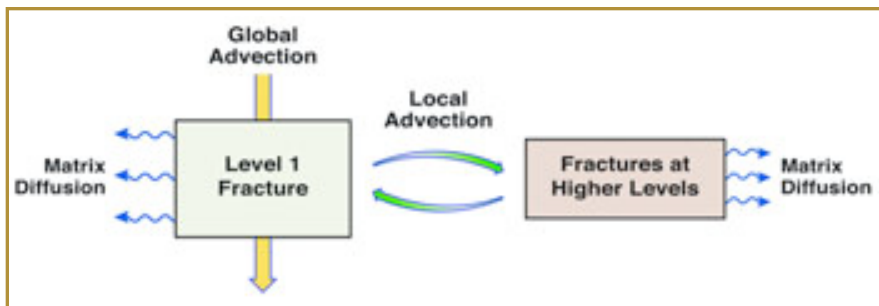

Figure 1. Effective matrix diffusion as a combination of local-scale advection and matrix diffusion in fractures at different scales.

\section{SIGNIFICANCE OF FINDINGS}

While the scale dependence of permeability and dispersivity has been an active research topic for many years, this study shows that the effective matrix diffusion coefficient, an important parameter controlling matrix diffusion processes, is also scale dependent. This finding has many important implications for problems involving matrix diffusion, including remediation of subsurface contamination in fractured rock and geological disposal of nuclear waste.

\section{RELATED PUBLICATIONS}

Liu, H.H., Y.Q. Zhang, Q. Zhou, and F. J. Molz, An interpretation of the potential scale dependence of the effective matrix diffusion coefficient. LBNL-60744. Journal of Contaminant Hydrology, 90(1-2), 41-57, 2007a.

Liu, H.H., Y. Zhang and F. J. Molz, Scale dependence of effective matrix diffusion coefficient: Some analytical results. LBNL-60746. Vadose Zone Journal (in press), 2007b.

\section{ACKNOWLEDGMENTS}

This work was supported by the Director, Office of Civilian Radioactive Waste Management, Office of the Chief Scientist, of the U.S. Department of Energy (DOE) under DOE Contract No. DE-AC02-05CH11231.

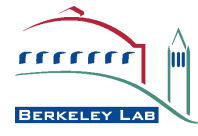




\title{
Feedback of Coupled Thermal-Hydrological-Chemical Processes on Seepage at Yucca Mountain, Nevada: Role of Permeability and Capillarity HeTEROGENEITY
}

\author{
Sumit Mukhopadhyay, Eric L. Sonnenthal, and Nicolas Spycher \\ Contact: Sumit Mukhopadhyay, 510-495-2440, SMukhopadhyay@lbl.gov
}

\section{RESEARCH OBJECTIVES}

In a recent publication (Mukhopadhyay et al., 2006), it was demonstrated that the coupled thermal-hydrological-chemical (THC) processes resulting from repository heating could alter the hydrological properties of the rock, which might in turn influence seepage into emplacement drifts. It was shown that seepage could occur when the THC processes were included in the predictive model, whereas none was predicted in the absence of these THC processes. These observations, however, were based on limited analysis: while heterogeneity in fracture permeability was included in the model, the corresponding heterogeneity in fracture capillarity (arising from the THC processes) was not accounted for. In this study, the influence of THC processes on seepage is investigated by accounting for heterogeneities in both fracture permeability and capillarity.

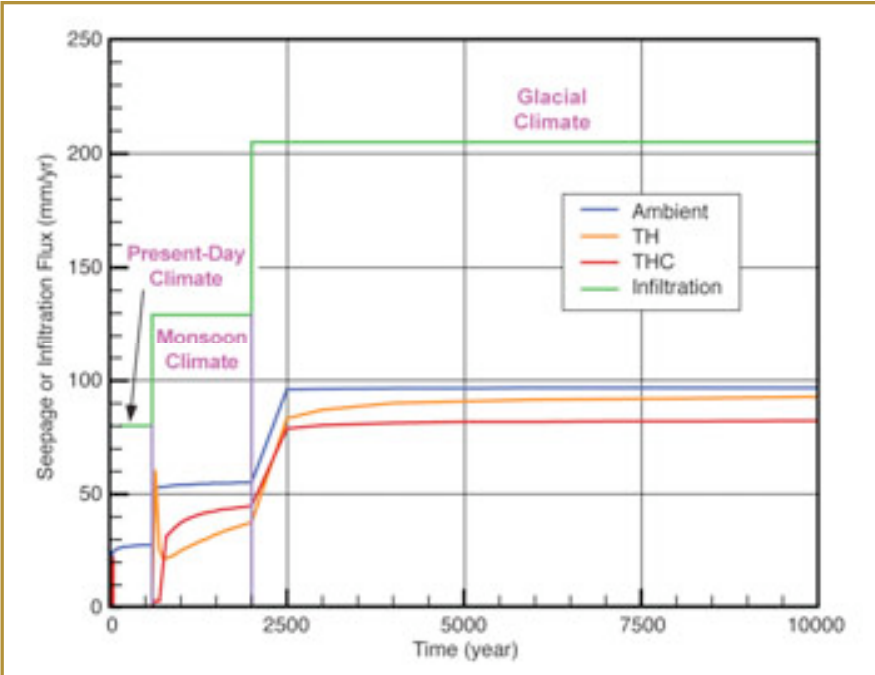

Figure 1. Comparison of ambient, $\mathrm{TH}$, and THC seepage

\section{APPROACH}

A detailed description of the expected thermal-hydrological (TH) and THC processes in the unsaturated near-field fractured rock can be found elsewhere (Mukhopadhyay et al., 2006; Mukhopadhyay et al., 2007). In this study, TH and THC processes are simulated with the TOUGHREACT reactive transport numerical code. Ambient, TH, and THC simulations are performed in a two-dimensional vertical model domain extending from the ground surface to the water table. Heterogeneous fracture-permeability distributions are generated using measured air-permeability data from the host rock. Multiple realizations of the heterogeneous fracture permeability distribution are included in the simulations. Capillarity is allowed to change with changes in porosity and permeability, according to the Leverett scaling law. The fractured rock is modeled as two separate but interacting continua, one for the rock matrix and the other for the fractures.

\section{ACCOMPLISHMENTS}

No seepage was observed when the mean infiltration fluxes expected at Yucca Mountain were used in the simulations. Figure 1 shows the amount of seepage from ambient, TH, and THC simulations, when ten times the mean infiltration fluxes were used. After the thermal period is over, ambient seepage is predicted to be the largest and THC seepage the least. This observation is contrary to the findings of Mukhopadhyay et al. (2006); capillarity heterogeneity was ignored in that study, resulting in overprediction of local flow channeling and seepage.

\section{SIGNIFICANCE OF FINDINGS}

The observation that ambient seepage is larger than $\mathrm{TH}$ or THC seepage means that, for the prediction of repository performance, the seepage at all times, including the period when the temperature is elevated above ambient temperature, can be assumed to be ambient seepage. Further details can be found in Mukhopadhyay et al. (2007).

\section{RELATED PUBLICATIONS}

Mukhopadhyay, S., E.L. Sonnenthal, E.L., and N. Spycher, Modeling coupled thermal-hydrological-chemical processes in the unsaturated rock of Yucca Mountain, Nevada: heterogeneity and seepage. Physics and Chemistry of the Earth, 31, 626-633, 2006.

Mukhopadhyay, S., N. Spycher, E.L. Sonnenthal, G. Zhang, and S. Finsterle, THC sensitivity study of heterogeneous permeability and capillarity effects. ANL-NBS-HS-000047 REV 01, Sandia National Laboratory_Lead Laboratory, Las Vegas, NV, 2007.

\section{ACKNOWLEDGMENTS}

This work was supported by the Director, Office of Civilian Radioactive Waste Management, of the U.S. Department of Energy (DOE) under DOE Contract No. DE-AC02 -05CH11231.

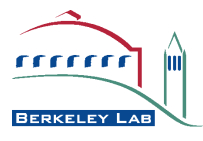




\title{
Simple Model Representations of Transport in a Complex Fracture AND Their Use in LONG-Term Predictions
}

\author{
Chin-Fu Tsang and Christine Doughty \\ Contact Mail: cftsang@lbl.gov or cadoughty@lbl.gov
}

\section{RESEARCH OBJECTIVES}

A complex fracture model for fluid flow and tracer transport was previously developed that incorporates all the important physical effects of a complex fracture zone. These effects include advection through a heterogeneous fracture plane, partitioning of flow into multiple subfractures in the third dimension, diffusion and sorption into fracture-filling gouge, small altered rock matrix blocks within the fracture zone, and the unaltered semi-infinite rock matrix on both sides of the fracture zone (Tsang and Doughty, 2003).

It is common, however, to represent the complex fracture by much simpler models consisting of a single fracture, without subfractures and with only the unaltered semi-infinite rock matrix for diffusion and sorption. The fracture may have a uniform (homogeneous) or heterogeneous transmissivity distribution over its plane, bounded on both sides by a homogeneous semi-infinite matrix. The parameters of the simple model can also be taken from laboratory data or calibration to short-term site-characterization (SC) data. The question posed by the present research is, how adequate are these simplified models for long-term performance assessment (PA) calculations that cover thousands of years?

\section{APPROACH}

We use a particle-tracking approach to calculate tracer transport in a complex fracture model, incorporating all the features described above, for a one-day SC tracer test and a 10,000-year PA prediction calculation. The results are considered the "realworld." Next, two simple fracture models, homogeneous and heterogeneous, are introduced. Properties for these simple models are taken either from laboratory data or found by calibration to one-day SC tracer-test breakthrough curves (BTC) obtained with the complex fracture model. Then, the simple models are used to simulate tracer transport at the PA time scale.

\section{ACCOMPLISHMENTS}

First, the results from laboratory-measured parameters are compared with those when data from the one-day SC tracer tests are used. The BTCs from the two cases are quite different, but by adjusting model properties, the simple models can reproduce peak arrival time and height. The overall match, however, is still poor (left frame of Figure 1).

Second, using simple models with SC-calibrated parameters for PA calculations causes order-of-magnitude errors in tracer BTCs: peak arrival time is 10 to 100 times too late and peak height is 100 to 1,000 times too small (right frame of Figure 1). On the other hand, using simple models with laboratory-measured properties of unfractured rock samples for PA calculations also produces erroneous results: peak arrivals and heights can be up to a factor of ten too early and high, respectively (right frame of figure). These are not general conclusions, since they depend on the parameter values assumed for the complex fracture model, and thus are more illustrative in nature, indicating the need for careful consideration.

\section{SIGNIFICANCE OF FINDINGS}

If a simple heterogeneous or homogeneous fracture model is used to predict tracer transport for a complex fracture at PA time scale, large errors may arise no matter what method is used to determine model properties. A remedy may be to determine properties by calibration to longer-term tracer tests (one to a few months rather than one to a few days), which will be sensitive to the detailed complex fracture features. A paper describing this work is in preparation.

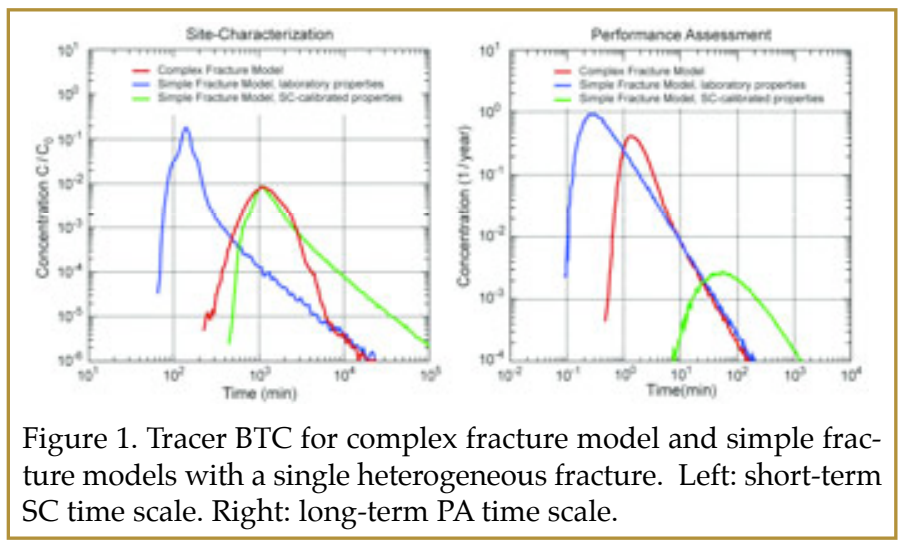

\section{RELATED PUBLICATIONS}

Tsang, C.-F. and C. Doughty, A particle-tracking approach to simulating transport in a complex fracture. Water Resour. Res., 39(7), 1174, doi:10.1029/2002WR001614, 2003.

\section{ACKNOWLEDGMENTS}

Work is supported by JAEA through a bi-national agreement between JAEA and the U.S. Department of Energy (DOE) and conducted under DOE Contract No. DE-AC02-05CH1123.

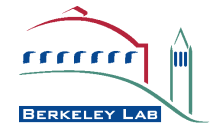




\title{
Estimating the Large-Scale Fracture Permeability of Unsaturated Rock Using Barometric Pressure Data
}

\author{
Yu-Shu Wu, Keni Zhang, and Hui-hai Liu \\ Contact: Yu-Shu Wu, 510/486-7291, yswu@lbl.gov
}

\section{RESEARCH OBJECTIVES}

Fracture permeability is a key parameter for flow and transport simulations using dual-continuum approaches. However, how to properly estimate fracture permeability at large spatial scales is still a challenging task. The large-scale model parameters for fractures are in general more difficult to measure at the site than those for the rock matrix. The main objective of this study is to develop an approach for estimating large-scale fracture permeability, using the changes in subsurface pneumatic pressure in response to barometric pressure changes at the land surface.

\section{APPROACH}

Large-scale fracture permeabilities for the Yucca Mountain site are estimated through a comprehensive modeling effort by using pneumatic data measured from boreholes at the site. The modeling approach, built on a three-dimensional (3-D) mountain-scale unsaturated zone (UZ) flow model, incorporates pneumatic data into a modeling analysis of two-phase liquid and gas flow under ambient geothermal conditions. The gas-flow modeling studies are performed under present-day infiltration conditions using the site-specific geological model and characterization data. Calibration of model-predicted gas pressures against field-measured pneumatic data leads to a methodology for estimating fracture permeability in the unsaturated fractured rock.

\section{ACCOMPLISHMENTS}

A three-dimensional model of gas flow in the unsaturated fractured rock of Yucca Mountain has been developed. Largescale fracture permeabilities of the site have been calculated through model calibration. This calibration was done through comparing gas-flow simulation results with the measured pneumatic data from underground boreholes. Fracture permeabilities, initially estimated by small-scale air-injection testing and 1-D model inversion, were adjusted to obtain an overall good match between the 3-D model predictions and pneumatic data (see Figure 1). The ability to match field pneumatic data observed from multiple sources, including pneumatic data over a long time, indicates the reliability of the numerical model in describing air and water flow processes within the Yucca Mountain UZ system, through better estimates of fracture flow properties.

\section{SIGNIFICANCE OF FINDINGS}

The results of this study indicate that using field-measured pneumatic data, in combination with numerical modeling analyses, provides a practical and powerful technique for estimating flow properties of vadose zone formations. Periodic responses of subsurface gas-pressure signals to surface barometric-pressure changes, which are easy to measure, may reveal invaluable information on gas mobility in unsaturated porous media. In addition, this work demonstrates that multidimensional effects on model-estimated permeability are significant when determining fracture permeability in heterogeneous fractured media. These effects can be captured only by 3-D modeling analyses on relevant model scales.

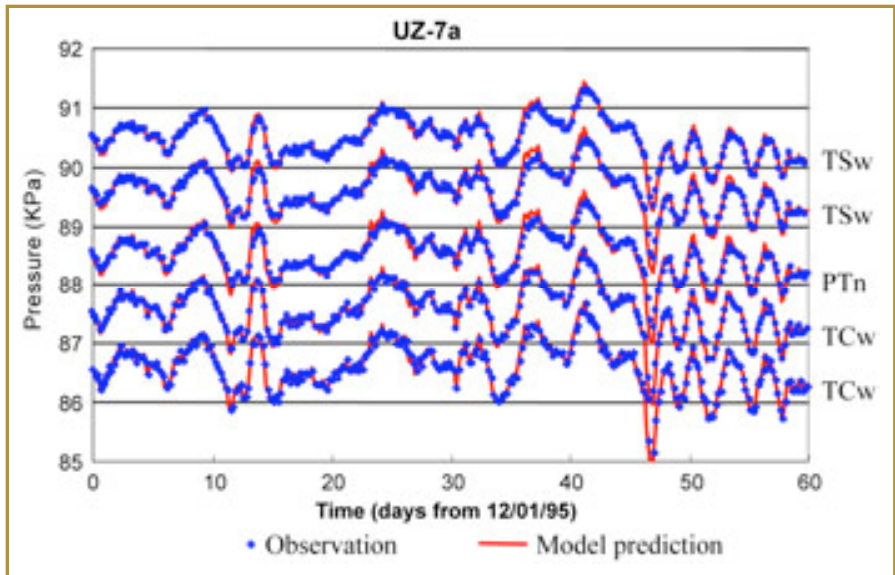

Figure 1. Comparison of simulated and observed gas pressures at borehole UZ-7a over a 60-day period, with 3-D model calibration.

\section{RELATED PUBLICATIONS}

Wu, Yu-Shu, Keni Zhang, and Hui-Hai Liu, Estimating largescale fracture permeability of unsaturated rock using barometric pressure data. LBNL-57614. Vadose Zone Journal, 5, 1129-1142, 2006.

\section{ACKNOWLEDGMENTS}

This work was supported by the Director, Office of Civilian Radioactive Waste Management, of the U.S. Department of Energy (DOE) under DOE Contract No. DE-AC02-05CH11231. 


\title{
Investigation of Unsaturated Flow Patterns in Fractured Rock Using an InTEgrated Modeling APPROACH
}

\author{
Yu-Shu Wu, Guoping Lu, Keni Zhang, and Lehua Pan \\ Contact: Yu-Shu Wu, 510/486-7291, yswu@lbl.gov
}

\section{RESEARCH OBJECTIVES}

Characterizing percolation patterns in unsaturated fractured rock poses a significant challenge to modeling investigations, because of the heterogeneous nature of unsaturated media and the many variables impacting unsaturated flow. The primary objective of this work is to quantitatively characterize percolation patterns in the fractured rock of the unsaturated zone (UZ) of Yucca Mountain, using an integrated modeling methodology.

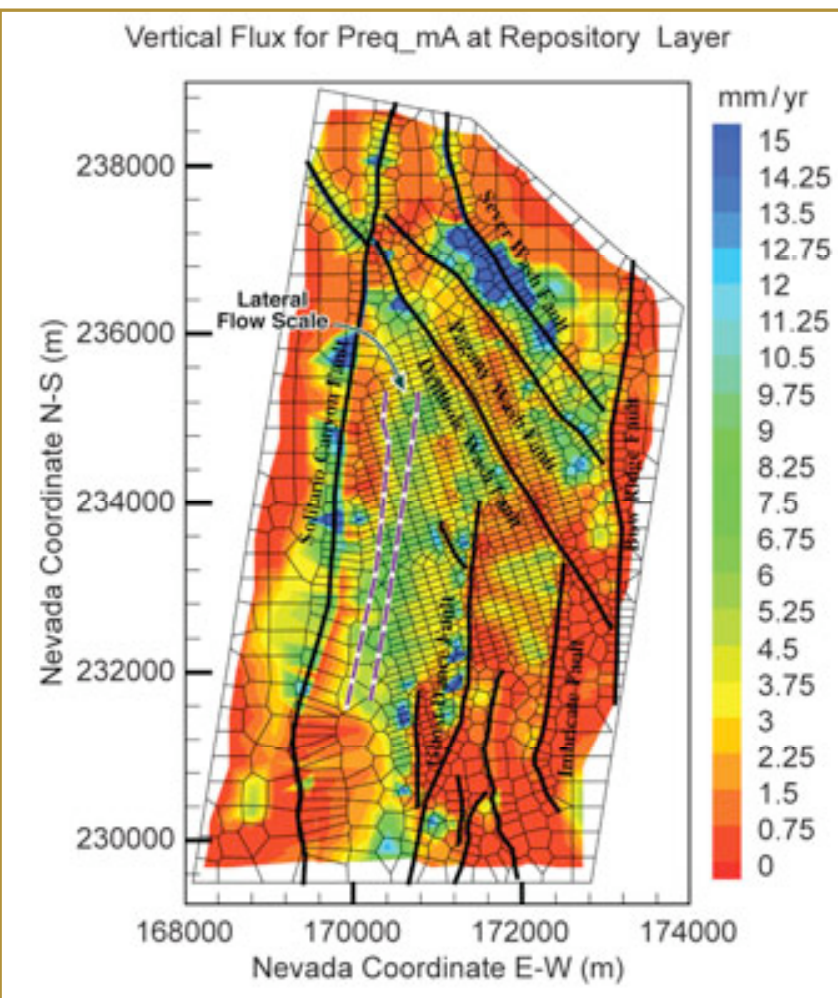

Figure 1. Simulated percolation fluxes at the repository horizon, using the present-day, mean infiltration scenario, base-case model results.

\section{APPROACH}

The integrated modeling approach combines a wide variety of field data into a comprehensive three-dimensional numerical model for flow pattern analyses. It takes into account the coupled processes of fluid and heat flow and chemical isotopic transport in Yucca Mountain's highly heterogeneous, unsaturated fractured tuffs. The fractured rocks are represented by a dual-permeability media. The main activities of study include (1) UZ model description; (2) model calibration using pneumatic, moisture, and geochemical data; (3) simulated percolation pattern analysis; and (4) assessment of percolation patterns and flow behavior using thermal and geochemical data.

\section{ACCOMPLISHMENTS}

An integrated modeling approach was used in a large-scale field study characterizing percolation patterns in the UZ of Yucca Mountain, The developed model integrated different fieldobserved data, such as moisture, gas pressure, chloride, and temperature data, into one single 3-D UZ flow and transport model. This combined model calibration provided a consistent crosscheck or verification of model results, as well as better insight into UZ flow patterns. The integrated modeling effort also provided consistent model predictions for different but interrelated hydrological, pneumatic, geochemical, and geothermal processes. Most importantly, such an integrated approach improved the capability and credibility of numerical models in characterizing subsurface flow and transport processes.

The important findings from this study were: (1) at Yucca Mountain, water may not flow directly downward in a thick, heterogeneous unsaturated zone, but rather may be diverted laterally towards the east, along the sloping layers, and focused into major faults; and (2) lateral flow diversion occurs mainly at the Calico Hills formation $(\mathrm{CHn})$, the stratigraphic unit below the repository horizon, resulting from the presence of perched water or thick lowpermeability layers. Under the current hydrogeological conceptualization, faults act as major flow paths through the $\mathrm{CHn}$ or below the repository horizon. In addition, the modeled percolation fluxes show that fracture flow is dominant in the welded tuff, both at the repository horizon and at the water table, while the matrix carries the majority of water percolation through the nonwelded tuff. Figure 1 shows the simulated flux distribution at the repository horizon.

\section{SIGNIFICANCE OF FINDINGS}

The integrated modeling approach provides a practical modeling tool for characterizing flow and transport processes in complex subsurface systems and results in better understanding of percolation patterns and flow behavior through the Yucca Mountain UZ.

\section{RELATED PUBLICATIONS}

Wu, Y.-S., G. Lu, K. Zhang, L. Pan, and G. S. Bodvarsson,

Analyzing unsaturated flow patterns in fractured rock using an integrated modeling approach. LBNL-54006. Hydrogeology Journal, 15, 553-572, 2007.

\section{ACKNOWLEDGMENTS}

This work was supported by the Director, Office of Civilian Radioactive Waste Management, of the U.S. Department of Energy (DOE) under DOE Contract No. DE-AC02-05CH11231.

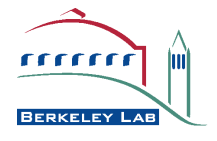




\title{
Coupling SEePage and RAdionuclide FATE/Transport in AND AROUND EMPLACEMENT DRIFTS AT YUCCA MOUNTAIN
}

\author{
Guoxiang Zhang, Nic Spycher, Eric Sonnenthal, and Carl Steefel \\ Contact: G. Zhang, 510-486-4980, gxzhang@lbl.gov
}

\section{RESEARCH OBJECTIVES}

The objectives of this project are to: (1) develop a quantitative model of coupled thermal, hydrological, and chemical (THC) processes potentially leading to brine formation on top of waste packages and/or a drip shield and (2) dynamically integrate such a model into the larger-scale models of processes within and around waste emplacement tunnels, as well as into the smaller-scale waste-package corrosion models.

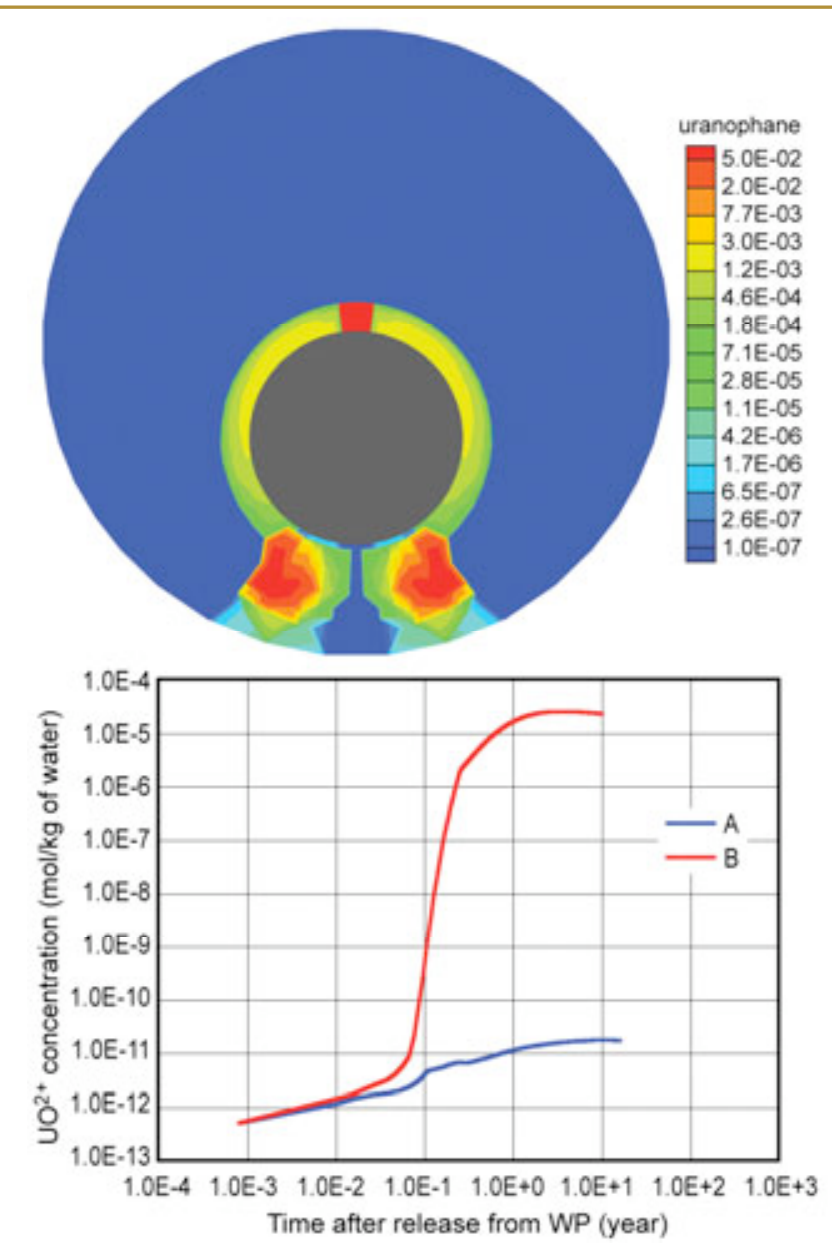

Figure 1. Simulated distribution of uranophane $\left(\mathrm{mol} / \mathrm{m}^{3}\right.$ of medium) precipitated after 25 years of continuous dripping (top) and simulated time evolution of $\mathrm{UO}_{2}{ }^{+2}$ concentration at the base of the drift with (A) and without (B) consideration of uranophane precipitation.

\section{APPROACH}

Process models were implemented into TOUGHREACT to allow modeling of (1) evaporative concentration to very high ionic strength, (2) boiling point elevation due to

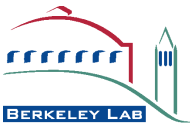
dissolved salts, (3) boiling/evaporation to dryness, and (4) salt deliquescence. An integrated near-field/in-drift THC simulation was run using a vertical 2-D grid extending from near the ground surface to the groundwater table, and covering a width equal to half the design drift spacing of $81 \mathrm{~m}$. The integrated model was then used to simulate a discrete dripping event within the drift. The model considered the release of radionuclides into seepage water as this water contacts the waste package and flows through the invert. The precipitation of uranophane and Np-uranophane was also considered. These minerals form in the invert from the neutralization of mildly acidic seepage water by clay minerals.

\section{ACCOMPLISHMENTS}

The main findings from this modeling effort are as follows: (1) the near-field and in-drift brine chemical evolution is dominated by the precipitation of $\mathrm{NaCl}, \mathrm{CaSO}_{4}$, and $\mathrm{CaCO}_{3}$; (2) the generation of acid gases at high evaporative concentration yields $\mathrm{P}_{\mathrm{HCl}} \sim 10^{-7}$ bar at boiling temperatures, with $\mathrm{pH}$ staying $>5$ in condensation areas; (3) the clay minerals in the invert neutralize the $\mathrm{pH}$ of seepage water, although this result is sensitive to assumptions regarding the kinetics of reactions with clays; (4) the drift invert may act as a $\mathrm{pH}$ buffer that promotes the precipitation of uranophane and impedes further downward migration of radionuclides at elevated concentrations (Figure 1). However, the pH buffer effect is subject to the content and composition of the clay minerals in the invert.

\section{SIGNIFICANCE OF FINDINGS}

The model captures some of the processes involved in salt formation and radionuclide transport, and can be further applied to capturing the details of radionuclide transport between the waste form and the rock through the invert.

\section{RELATED PUBLICATIONS}

Zhang, G., N. Spycher, E. Sonnenthal, and C. Steefel, Implementation of a Pitzer activity model into TOUGHREACT for modeling concentrated solutions. Proceedings, TOUGH2 Symposium, Lawrence Berkeley National Laboratory, Berkeley, CA, May 15-17, 2006.

Zhang, G., N. Spycher, T. Xu, E. Sonnenthal, and C. Steefel, Reactive geochemical transport modeling of concentrated aqueous solutions: Supplement to the TOUCHREACT Users's Guide for the Pitzer ion-interaction model. LBNL-62718, Lawrence Berkeley National Laboratory, Berkeley, CA, 2007.

\section{ACKNOWLEDGMENTS}

This work was supported by the Director, Office of Civilian Radioactive Waste Management, Office of the Chief Scientist, of the U.S. Department of Energy (DOE) under DOE Contract No. DE-AC02-05CH11231. 


\section{EVALUATION OF UNCERTAINTY IN INFILTRATION SCENARIOS at Yucca Mountain, Nevada}

Yingqi Zhang and Hui-Hai Liu

Contact: Yinqi Zhang, 510-495-2983, yqzhang@lbl.gov

\section{RESEARCH OBJECTIVES}

An accurate estimate of infiltration rates is critical for the performance assessment of geological disposal of the highlevel nuclear wastes at Yucca Mountain. The key components considered in the current infiltration model for Yucca Mountain include climate information, water transport and storage in the shallow zone, evapotranspiration, and surface runon and runoff. As a result of the uncertainty in the input parameters, 40 different infiltration maps were generated with the same probability of occurrence. However, the estimated infiltration maps are subject to some uncertainties due to the fact that the infiltration model was developed without consideration of subsurface data, such as temperature and chloride concentration measurements.

\section{APPROACH}

We developed an approach based on the generalized likelihood uncertainty estimate (GLUE) methodology (Beven and Binley, 1992). The GLUE procedure requires a definition of a likelihood measure to quantify how well each infiltration map reproduces the measured data. For each infiltration map, a process model is used to simulate water percolation, chloride transport, and heat transfer within the Yucca Mountain unsaturated zone. The simulation results are compared to the observed data to evaluate the likelihood function. A higher weighting factor is assigned to infiltration maps that more closely reproduce the data. Because of the inherent subjectivity in the choice of a likelihood function, the use of GLUE introduces a new type of uncertainty: the epistemic uncertainty in the analysis. To consider this uncertainty, we extended GLUE to use multiple likelihood measures and combine the results. The proposed likelihood functions cover a certain range of different functional types. The final weighting factor is an average of the weighting factors calculated from all the selected likelihood functions.

\section{ACCOMPLISHMENTS}

An effort was made in this study to handle the uncertainties in defining the likelihood function in the GLUE procedure. Specifically, four likelihood measures, each having a different evaluation focus and addressing a different aspect of model behavior, were chosen to assign weighting factors for the selected infiltration maps. Although the results from these likelihood functions varied, they consistently assign higher weights to the same infiltration maps. The final averaged weighting factors and their uncertainties were determined to weigh infiltration scenarios in the probabilistic performance assessment calculation.

\section{SIGNIFICANCE OF FINDINGS}

Net infiltration is a key hydrologic parameter for controlling percolation rate, groundwater recharge, potential seepage into waste emplacement drifts, and radionuclide transportand therefore a key parameter in evaluating repository performance at Yucca Mountain. The GLUE method was developed to constrain the infiltration-rate uncertainties by using additional subsurface data collected from the unsaturated zone. Although the study is done for a specific setting, it provides a general framework from which to consider different types of uncertainty in environmental applications.

\section{RELATED PUBLICATIONS}

Beven, K., and A. Binley, The future of distributed models: Model calibration and uncertainty prediction. Hydrological Processes, 6, 279-298, 1992.

Wu, Y-S., et al., UZ Flow Models and Submodels, MDL-NBSHS-000006, REV 03, June 2007.

\section{ACKNOWLEDGMENTS}

This work was supported by the Director, Office of Civilian Radioactive Waste Management, of the U.S. Department of Energy(DOE) under DOE Contract No. DE-AC02-05CH11231. 


\section{Earth Sciences Division Acronyms \& Abbreviations}

3-D—three dimensional

ACRF-Atmospheric Radiation Measurement Climate Research Facility (U.S. Southern Great Plains)

ADE—advection dispersion equation

AGU_American Geophysical Union

AHG—anterior hindgut

ALS—Advanced Light Source (Berkeley Lab)

ANN—artificial neural networks

AOGCM-Atmosphere-Ocean General Circulation Model

APS-Advanced Photon Source (Argonne National Laboratory)

ARM-Atmospheric and Radiation Measurements Program

ARM-SGP-Atmospheric and Radiation Measurements Program, Southern Great Plains (USA)

AVA—amplitude versus angles

BNL—Brookhaven National Laboratory

BTC—-breakthrough curve(s)

BUD—Berkeley Unexploded-ordnance Discriminator

BWC-Berkeley Water Center

C2VSIM-California Central Valley Simulation Model

CASSM-continuous-active-source seismic monitoring

$\mathrm{CCP}-\mathrm{CO}_{2}$ Capture Project

CCS—carbon dioxide capture and storage

CDAT—climate data analysis tool
$\mathrm{CDE}$ - convection dispersion equation

CDWR—California Department of Water Resources'

CEC-California Energy Commission

$\mathrm{CF}$-certification framework

CFD—Computational Fluid Dynamics

CLASIC—Cumulus-Land Surface Interaction Campaign

CLM—Community Land Model

$\mathrm{CLR}-\mathrm{CO}_{2}$ leakage risk

$\mathrm{CO}_{2}$ - carbon dioxide

CSEGR—carbon sequestration with enhanced gas recovery

CSEM—controlled source electromagnetic

CTRW—continuous-time random-walk

DCE—dichloroethylene

DECOVALEX—DEvelopment of COupled THM models and their VALidation against Experiments (an international cooperative project related to nuclear waste disposal)

DIC—dissolved inorganic carbon

DIRB—dissimilatory iron-reducing bacteria

DLS—dynamic light scattering

DoD—U.S. Department of Defense

DOE_U.S. Department of Energy

EC—eddy covariance

ECA—emission credits and atmosphere

EGS—enhanced geothermal systems

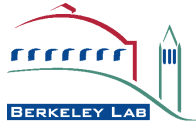


EM-electromagnetic

EPA-U.S. Environmental Protection Agency

ERDAS-Earth Resources Data Analysis System

ERSD_Environmental Remediation Sciences Division

ERSP_Environmental Remediation Sciences Program

ERT_electrical resistance tomography

ESTCP_Environmental Security Technology Certification Program (DoD)

ESTSC-Energy Science and Technology Software Center (DOE)

EXAFS-extended x-ray absorption fine structure spectroscopy

FEP_features, events, and processes

FFEC—flowing fluid electric conductivity

FG-foregut

FGA-functional gene array

FRC_Field Research Center (ERSD-ORNL)

FTIR—Fourier transform infrared

GCS-geologic carbon sequestration; Geologic Carbon Sequestration Program (ESD)

GCM-general circulation models (climate)

GeoSeq-Geologic Carbon Sequestration Project (long-term carbon sequestration project led by ESD)

GFDL_Geophysical Fluid Dynamics Laboratory climate model (NOAA)

GhG_-greenhouse gas

GHSZ_-gas-hydrate stability zone

GISAXS—grazing incidence small-angle x-ray scattering
GLUE_-generalized likelihood uncertainty estimate

GPR - ground-penetrating radar

GPS—global positioning system

GTL-Genomes to Life (DOE)

HBS-hydrate-bearing sediments

HMR — hydrocarbon and mineral resources

HRCTM-hydrogen release compound (a slow-release polylactate compound)

HSE-health, safety, and environment(al)

$\mathrm{IP}$-induced polarization (bioremediation)

IPCC - Intergovernmental Panel on Climate Change

ITS—internal transcribed spacer

JAEA-Japan Atomic Energy Agency

JIP—Joint Industry Program

JJAS-June through September (June, July, August, September)

JOI-Joint Oceanographic Institutions, Inc.

LAI-leaf area index

LB-lattice-Boltzmann

LDRD-Laboratory Directed Research and Development (Berkeley Lab)

MCMC-Markov-chain Monte Carlo

MDA—multiple displacement amplification

METI-Ministry of Economy, Trade, and Industry (Japan)

MG-midgut

MEQ—-micro-earthquake

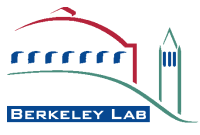


MPI—message passing interface

MSA - multiple sequence alignment

MSU-Montana State University

MTBE-methyl tert-butyl ether

NASA-National Aeronautic and Space Administration

NCAR—National Center for Atmospheric Research

NERSC-National Energy Research Scientific Computing Center (Berkeley Lab)

NETL-National Energy Technology Laboratory

NCDC - National Climatic Data Center

NCEP—National Center for Environmental Prediction

NEE-net ecosystem exchanges

NOAA-National Oceanic \& Atmospheric Administration (U.S.)

NSF-National Science Foundation

NSLS-National Synchrotron Light Source (BNL)

NTA—nitrylotriacetate

NUMO_Nuclear Waste Management Organization of Japan

OA-oriented attachment

OC-organic carbon

OCRWM-Office of Civilian Radioactive Waste Management (DOE)

ODP_Ocean Drilling Program (NSF)

ORNL—Oak Ridge National Laboratory

PA_-performance assessment
PCAP_Protein Complex Analysis Project

PCE-perchloroethylene

PCM-Parallel Climate Model

PCR_polymerase chain reaction

PDF (pdf) - probability density function

PHG-posterior hindgut (PHG)

PI-principal investigator

P-E-precipitation-effectiveness

PIER_Public Interest Energy Research

PM-particulate matter

PNNP_Pacific Northwest National Laboratory

PSO_particle swarm optimization

QM-quantum mechanical

REU-Research Experiences for Undergraduates

RCMs-regional climate models

RSM-Regional Spectral Model (NOAA)

RUC_rapid update cyle

SAO_-Santa Ana Occurrence

SAXS-small-angle x-ray scattering

SC-site-characterization

SCCM-Single Column Climate Model (NCAR)

SCUC - shaped-core uniaxial compression

SEG - Society of Exploration Geophysicists

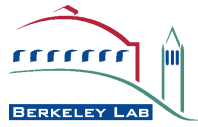


SERDP—Strategic Environmental Research and Development Program

SJR — San Joaquin River

SP_self-potential (bioremediation)

SRES-Special Report on Emission Scenarios (IPCC)

SSRL—Stanford Synchrotron Radiation Laboratory

SST—sea surface temperature

STD—soil thermal diffusion

STXM-scanning transmission x-ray microscopy

SWE-snow water equivalent

SZ-saturated zone

TAN-Test Area North (Idaho National Laboratory)

TCE-trichloroethylene

TEP_triethyl phosphate

TH-thermal-hydrological

THC_-thermal-hydrological-chemical

THM-thermal-hydrological-mechanical

THMC—-thermal-hydrological-mechanical-chemical
TOUGH-Transport of Unsaturated Groundwater and Heat (ESD computer code)

TSPA-Total System Performance Assessment (Yucca Mountain Project)

UCSC_-University of California at Santa Cruz

UCSD_University of California at San Diego

UIC-Underground Injection Control program

UMTRA-Uranium Mill Tailings Remedial Action

USDW-underground sources of drinking water

$\mathrm{UXO}$ - unexploded ordnance

UZ-unsaturated zone

VIMSS-Virtual Institute for Microbial Stress and Survival

VSP_vertical seismic profiling

WAXS—-wide angle x-ray scattering

WESTCARB-West Coast Regional Carbon Sequestration Partnership

WRF-Weather Research and Forecasting Model

ZERT-Zero Emissions Research and Technology Program

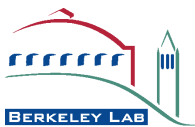




\section{Earth Sciences Division Publications 2006-2007}

Abulencia, C.B. et al., Environmental whole-genome amplification to access microbial diversity in contaminated sediments. LBNL-60416. Applied and Environmental Microbiology, 72 (5), 3291-3301, 2006.

Aciego, S., D. DePaolo, B.M. Kennedy, M.P. Lamb, K. W.W. Sims, and W.E. Dietrich, Combining [ $\left.{ }^{3} \mathrm{He}\right]$ cosmogenic dating with $\mathrm{U}-\mathrm{Th} / \mathrm{He}$ eruption ages using olivine in basalt. LBNL-62379. Earth and Planetary Science Letters, 254, 288-302, 2007.

Ajo-Franklin, J.B., J.T. Geller, and J.M. Harris, A survey of the geophysical properties of dense chlorinated solvents. LBNL-56061. Journal of Applied Geophysics, 59 (3), 177-189, 2006.

Ajo-Franklin, J.B., J.T. Geller, and J.M. Harris, Ultrasonic properties of granular media saturated with DNAPL/water mixtures. LBNL-63041. Geophysical Research Letters, 34 (L07404), 2007.

Ajo-Franklin, J.B., B.J. Minsley, and T.M. Daley, Applying compactness constraints to differential traveltime tomography. LBNL-60057. Geophysics, 72 (4), 2007.

Amodei, D., H. Keers, D.W. Vasco, and L. Johnson, Computation of uniform wave forms using complex rays. LBNL-61349. Physical Review E, 73 (036704), 1-14, 2006.

Anderson, M., N. Miller, B. Heiland, J. King, B. Lek, S. Nemeth, T. Pranger, and M. Roos, Climate change impacts on flood management. LBNL-60674. In: Progress on Incorporating Climate Change into Management of California's Water Resources. F. Chung (Editor) State of California - Dept. of Water Resources, Sacramento, CA, pp. 6.1-6.38, 2006.

Andre, L., N. Spycher, T. Xu, F.-D. Vuataz, and K. Pruess, Modeling brine-rock interaction in the deep, enhanced geothermal system at Soultz-Sous-Forêts (France) - A Joint approach using two geochemical codes: FRACHEM and TOUGHREACT. LBNL-62357. LBNL, Berkeley, CA. 2006.

Andrews, J.L., S. Finsterle, and M.O. Saar, Mass- and temperature dependent diffusion coefficients of light noble gases for the TOUGH2 module. LBNL-62595. Lawrence Berkeley National Laboratory, Berkeley, Calif., April 2007.

Apps, J.A., J. Birkholzer, T. Xu, and C.-F. Tsang, A database analysis of the distribution of hazardous metals in groundwaters of the United States. 6th Annual Conference on Carbon Capture and Sequestration, DOE/NETL, Pittsburgh, Pennsylvania. 2007.

Apps, J.A., and G.D. Redden, Nucleation, precipitation, and dissolution processes. LBNL-61679. Vadose Zone Journal (in press), 2007.

Arthur, R., W. Zhou, T. Xu, K. Pruess, and B. Stromberg, Experimental calibration of a reactive-transport model of buffer cementation. Chinese J. of Rock Mechanics and Engineering, 25 (4), 2006.
Assaf, H., N.W.T. Quinn, et al., Generic simulation models for facilitating stakeholder involvement in water resources planning and management. LBNL-63386. In: Environmental Modelling, Software and Decision Support (in press), 2007.

Audigane, P., I. Gaus, I. Czernichowski-Lauriol, K. Pruess, and T. Xu, Two-dimensional reactive transport modeling of $\mathrm{CO}_{2}$ injection in a saline aquifer at the Sleipner Site, North Sea. LBNL-61584. American Journal of Science, 307, 974-1008, DOI 10.2475/07.2007.02, September 2007.

Battaglia, M., and D.W. Vasco, The search for magma reservoirs in Long Valley Caldera: Single versus distributed sources. LBNL-61353. In: Mechanisms of Activity and Unrest at Large Calderas. C. D. N. Troise, G.; Kilburn, C.R. (Editors) The Geological Society of London, London, England, pp. 173-180, 2006.

Bear, J., and Q. Zhou, Sea water intrusion into coastal aquifers. LBNL-63047. In: The Handbook of Groundwater Engineering, Second Edition. J. Delleur (Editor) CRC Press, Taylor \& Francis Group, Boca Raton, FL, pp. 12.1-12.29, 2007.

Bender, K.S. et al., Analysis of a ferric uptake regulator (Fur) mutant of Desulfovibrio vulgaris Hildenborough. Appl. Environ. Microbiology, 73 (AEM), 00276-07v1, 2007.

Benson, S., Monitoring geological storage of carbon dioxide. LBNL-59420. In: Carbon Capture and Sequestration: Integrating Technology, Monitoring, and Regulation. E. Wilson and D. Gerard (Editors) Blackwell Publishing, Ames, Iowa, USA, 2007.

Benson, S., and T. Surles, Carbon dioxide capture and storage: An overview with emphasis on capture and storage in deep geological formations. LBNL-59429. Proceedings of the IEEE, 94 (10), 1795-1805, 2006.

Berhe, A.A., J. Harte, J.W. Harden, and M.S. Torn, The significance of the erosion-induced terrestrial carbon sink. LBNL63322. Bioscience, 57 (4), 337-346, 2007.

Berryman, J. G., Elastic and transport properties in polycrystals of cracked grains: Cross-property relations and microstructure. LBNL-63502. International Journal of Engineering Science, 2007 (in press).

Berryman, J.G., Exact seismic velocities for TI media and extended Thomsen Formulas for stronger anisotropies. LBNL-62907. Geophysics (in press), 2007.

Berryman, J.G., Seismic waves in rocks with fluids and fractures. LBNL-62925. Geophysical Journal International, 171 (2), 954-974, 2007.

Berryman, J.G., and V. Grechka, Random polycrystals of grains containing cracks: Model of quasistatic elastic behavior for fractured systems. LBNL-61135. Journal of Applied Physics, 100 (Paper No. 113527), 2007.

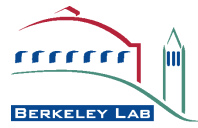


Bird, J.A., and M.S. Torn, Fine roots versus needles: A comparison of ${ }^{13} \mathrm{C}$ and ${ }^{15} \mathrm{~N}$ dynamics in a Ponderosa Pine Forest soil. LBNL-60055. Biogeochemistry, 79 (3), 53-67, 2007.

Birkholzer, J., N. Halecky, S.W. Webb, P. Peterson, and G.S. Bodvarsson, A modeling study evaluating the thermal hydrological conditions in and near waste emplacement tunnels at Yucca Mountain. LBNL-61359. Nuclear Technology (in press), 2007.

Birkholzer, J., K. Pruess, J.L. Lewicki, J. Rutqvist, C.-F. Tsang, and A. Karimjee, Large releases from $\mathrm{CO}_{2}$ storage reservoirs: Analogues, scenarios, and modeling needs. LBNL-63338. International Conference on Greenhouse Gas Control Technologies, Trondheim, Norway, June 19-22, 2006.

Birkholzer, J., and C.-F. Tsang (Guest Editors), Special Issue on Site Characterization for $\mathrm{CO}_{2}$ Storage. Springer Verlag, Germany (in press) 2007.

Birkholzer, J., and C.-F. Tsang, Introduction to the special issue on site characterization for geological storage of $\mathrm{CO}_{2}$. LBNL62673. Journal of Environmental Geology (in press), 2007.

Birkholzer, J., and Y. Zhang, The impact of fracture-matrix interaction on thermal-hydrological conditions in heated fractured rock. LBNL-57667. Vadose Zone Journal, 5 (2), 657-672, 2006.

Birkholzer, J., Q. Zhou, J. Rutqvist, P. Jordan, K. Zhang, and C.F. Tsang, Research project on $\mathrm{CO}_{2}$ geological storage and groundwater resources: Large-scale hydrological evaluation and modeling of impact on groundwater systems. LBNL-63544. Lawrence Berkeley National Laboratory, Berkeley, CA. 2007.

Birkholzer, J.T., A temperature-profile method for estimating flow processes in geologic heat pipes. LBNL-56716. Journal of Contaminant Hydrology, 85 (3-4), 89-117, 2006.

Birkholzer, J.T., S.W. Webb, N. Halecky, P.F. Peterson, and G.S. Bodvarsson, Evaluating the moisture conditions in the fractured rock at Yucca Mountain: The impact of natural convection in heated emplacement drifts. LBNL-59334. Vadose Zone Journal, 5, 1172-1193, 2006.

Block, G., M.B. Rubin, J. Morris, and J.G. Berryman, Simulations of dynamic crack propagation in brittle materials using nodal cogseive forces and continuum damage mechanics in the distinct element code LDEC. LBNL-61404. International Journal of Fracture, 144, 131-147, 2007.

Bodvarsson, G.S., Thermohydrological effects and interactions. LBNL-48294. In: Uncertainty Underg round: Yucca Mountain and the Nation's High-Level Nuclear Waste. A. Macfarlane and R. C. Ewing, eds., MIT Press, Cambridge, MA, pp. 257-270, 2006.

Bodvarsson, G.S., Earth Sciences Division Research Summaries 2004-2005. LBNL-59633. Lawrence Berkeley National Laboratory, Berkeley, CA. 2006.

Bourg, I.C., G. Sposito, and A.C.M. Bourg, Tracer diffusion in compacted water-saturated bentonite. LBNL-59826. Clays and Clay Minerals, 54 (3), 363-374, 2006.
Bourg, I.C., G. Sposito, and A.C.M. Bourg, Modeling the acidbase surface chemistry of montmorillonite. LBNL-62408. Journal of Colloid and Interface Science, 312 (2), 297-310, 2007.

Bourg, I.C., and G. Sposito, Molecular dynamics simulations of kinetic isotope fractionation during the diffusion of ionic species in liquid water. LBNL-61833. Geochimica et Cosmochimica Acta, 71 (23), 5583-5589, 2007.

Bourg, I.C., and G. Sposito, Isotopic fractionation of noble gases by diffusion in liquid water: Molecular dynamics simulations and hydrologic applications. LBNL-63458. LBNL-63461. Geochimica et Cosmochimica Acta (in press), 2007.

Bourg, I.C., G. Sposito, and A.C.M. Bourg, Modeling cation diffusion in compacted water-saturated Na-bentonite at low ionic strength. LBNL-63446. Environmental Science and Technology 41 (23), 8118-8122, 2007.

Brodie, E.L., T.Z. DeSantis, Y.M. Piceno, and G. L. Andersen, High-density DNA microarray analysis for monitoring microbial community composition and dynamics. In: Molecular Microbial Ecology Manual. $3^{\text {rd }}$ edition, G.A.Kowalchuk, ed., Kluwer Academic Publishers, Amsterdam, The Netherlands, 2007.

B rodie, E.L. et al., Application of a high-density oligonucleotide microarray approach to study bacterial population dynamics during uranium reduction and reoxidation. LBNL-59761. Appl. Environ. Microbiol., 72 (9), 6288-6298, 2006.

Brodie, E.L., T.Z. DeSantis, J.P. Moberg-Parker, I.X. Zubietta, Y.M. Piceno, and G.L. Andersen, Urban aerosols harbor diverse and dynamic bacterial populations. LBNL-62199. PNAS-Proceedings of the National Academy of Sciences of the United States of America, 104 (1), 299-304, 2007.

Brodie, E.L. et al., Phylogenetic and functional gene microarray analysis demonstrates direct and indirect mechanisms for sustained chromium bio-immobilization. LBNL-62428. Annual Meeting of the American Society for Microbiology, American Society for Microbiology, Toronto, Canada. 2007.

Brunnel, M. S., G. M. Litton, and S. E. Borglin, San Joaquin River up-stream DO TMDL Project ERP-02D-P63 Task: 9 Zooplankton abundance and diversity in the Lower San Joaquin River above the stock. LBNL-63243. Lawrence Berkeley National Laboratory, Berkeley, CA. 2007.

Bryant, J.A., G.M. Yogodzinski, M.L. Hall, J.L. Lewicki, and D.G. Bailey, Geochemical constraints on the origin of volcanic rocks from the Andean Northern Volcanic Zone, Ecuador. LBNL-55805. Journal of Petrology, (61), doi:10.1093/ petrology / eg1006, 2006.

Buesseler, K.O., C. H.Lamborg, P.W. Boyd, P. J. Lam, T. W. Trull, R. R. Bidigare, J.K.B. Bishop, K. L. Casciotti, F. Dehairs, M. Elskens, M. Honda, D. M. Karl, D. Siegel, M. W. Silver, D. K. Steinberg, J. Valdes, B. Van Mooy and S. Wilson (2007). Revisiting carbon flux through the oceans twilight zone. LBNL-63076. Science, 316, 567-570, 2007.

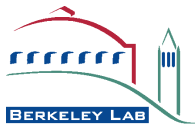


Cappa, F., Y. Guglielmi, J. Rutqvist, C.-F. Tsang, and A. Toraval, Hydromechanical modeling of pulse tests that measure both fluid pressure and fracture-normal displacement of the Coaraz Laboratory site, France. LBNL-60442. International Journal of Rock Mechanics \& Mining Sciences, 43 (7), 1062-1082, 2006.

Cappa, F., Y. Guglielmi, J. Rutqvist, C.-F. Tsang, and A. Thoraval, A new in situ test for determination of rock-fracture hydromechanical properties: The hydromechanical pulse injection test (HMPIT). Proceedings of the GEOPROC2006 International symposium, pp. 428-433, $2^{\text {nd }}$ International Conference on Coupled Thermo-HydroMechanical-Chemical Processes in Geosystems and Engineering, HoHai University, Nanjing, China, May 22-25, 2006.

Chabra, S. R. et al., Global analysis of heat shock response in Desulfovibrio vulgaris Hildenborough. LBNL-60298. Journal of Bacteriology, 188 (5), 1817-1828, 2006.

Chain, P.S.G., P. Hu, S.A. Malfatti, L. Radnedge, F. Larimer, L.M. Vergez, P.L. Worsham, M.C. Chu, and G.L. Andersen, Complete genome sequence of Yersinia pestis strains Antiqua and Nepal516: Evidence of gene reduction in an emerging pathogen. LBNL-60248. Journal of Bacteriology, 188 (12), 4453-4463, 2006.

Chambers, D.H., and J.G. Berryman, Target characterization using decomposition of the time-reversal operator: Electromagnetic scattering from small ellipsoids. LBNL61607. Inverse Problems, 22, 2145-2163, 2006.

Chen, B., B. Gilbert, H. Zhang, and J. Banfield, Mechanism of inhibition of nanoparticle growth and phase transformation by surface impurities. LBNL-63060. Physical Review Letters, 98, 106103, 2007.

Chen, J., and T. Dickens, Effects of uncertainty in rock-physics models on reservoir parameter estimation using seismic variation with angle and controlled-source electromagnetic data. LBNL-62608. J. Art. Geophysical Prospecting (in press), 2007.

Chen, J., G.M. Hoversten, D. Vasco, Y. Rubin, and Z. Hou, A sampling-based Bayesian model for gas saturation estimation using seismic AVA and marine CSEM data. LBNL60249. Geophysics, 72 (2), WA85-WA95, 2007.

Chen, J., S. Hubbard, J. Peterson, M. Fienen, P.M. Jardine, K. Williams, and D.B. Watson, Development of a joint hydrogeophysical inversion approach and application to a contaminated fractured aquifer. LBNL-60758. Water Resources Research, 42 (W06425), 1-13, 2006.

Chen, J.S., A. Kemna, and S.S. Hubbard, A comparison between deterministic and stochastic methods for inverting spectral induced polarization data for Cole-Cole parameters. Geophysics (in press), 2007.
Chen, J., M. van der Laan, M. Smith, and A.E.. Hubbard, A comparison of methods to control Type-I errors in microarray studies. LBNL-63457. Statistical Applications in Genetics and Molecular Biology, 6 (1), Art. 28, 2007.

Christensen, J.N., S.S. Cliff, R.A. VanCuren, K.D. Perry, and D.J. DePaolo, Tracking intercontinental dust transport with radiogenic isotopes: Hefei, China to California, Spring 2002. LBNL-63387 Abs. American Geophysical Union (AGU) Fall Meeting 2006, American Geophysical Union (AGU), San Francisco, CA. 2006.

Christensen, J.N., M.E. Conrad, D.J. DePaolo, and P.E. Dresel, Isotopic studies of contaminant transport at the Hanford Site, WA. LBNL-61935. Vadose Zone Journal, 6, 1018-1030, 2007.

Christensen, L., W. Riley, and I. Ortiz-Monasterio, Nitrogen cycling in an irrigated wheat system in Sonora, Mexico: Measurements and modeling. LBNL-60806. Nutrient Cycling in Agroecosystems, doi: 10.1007/s10705-006-9025-y, 2006.

Christenson, B.W., B.M. Kennedy, M.C. Adams, S.C. Bjornstad, and C. Buck, Chemical and isotopic characteristics of the COSO East Flank hydrothermal fluids: Implications for the location and nature of the heat source. LBNL-62359. 32nd Workshop on Geothermal Reservoir Engineering, Stanford, CA. 2007.

Chung, F. et al., Progress on incorporating climate change into management of California's water resources. LBNL-61292. California Department of Water Resources, Sacramento, CA., 338, 2006.

Clark, M.E. et al., Temporal transcriptomic analysis of Desulfovibrio vulgaris Hildenborough transition into stationary phase growth during electron donor depletion. LBNL60466. Applied and Environmental Microbiology, 72 (8), 5578-5588, 2006.

Clavijo, R.I., C. Loui, G.L. Andersen, W. Riley, and S.W. Lu, Identification of genes associated with survival of Salmonela enterica Serovar enteridis in chicken egg albumen. LBNL60807. Applied and Environmental Microbiology, 72 (2), 1055-1064, 2006.

Codd, R., P. Lay, N.Y. Tsibakhashvili, T.L. Kalabegishvili, I.G. Murusidze, and H.-Y.N. Holman, Chromium(V) complexes generated in Arthrobacter oxydans by simulation analysis of EPR spectra. LBNL-61264. J. Inorg. Biochem, 100 (11), 1827-1833, 2006.

Collins, W., R. Colman, J. Haywood, M.R. Manning, and P. Mote, The physical science behind climate change. Scientific American, 297 (2), 64-73, 2007.

Commer, M., S. L. Helwig, A. Hordt, C. Scholl, and B. Tezkan, New results on the resistivity structure of Merapi Volcano (Indonesia), derived from 3D restricted inversion of longoffset transient electromagnetic data. LBNL-60493. Geophysical Journal International, 167, 1172-1187, 2006.

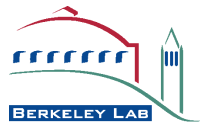


Commer, M., and G. A. Newman, New advances in three-dimensional controlled-source electromagnetic inversion. LBNL63010. Geophysical Journal International (in press) 2007.

Commer, M., G. A. Newman, J. J. Carazzone, T. A. Dickens, K. E. Green, L. A. Wahrmund, and D. E. Willen, Massively parallel electrical-conductivity imaging of hydrocarbons using the BlueGene/L supercomputer. LBNL-63009. IBM Journal of Research and Development (in press), 2007.

Conrad, M.E., D.J. DePaolo, K. Maher, G.W. Gee, and A.L. Ward, Field evidence for strong chemical separation of contaminants in the Hanford vadose zone. LBNL-62598. Vadose Zone Journal, 6 (4), 1031-1041, 2007.

Cortis, A., C.M. Oldenburg, and S.M. Benson, The role of optimality in characterizing $\mathrm{CO}_{2}$ seepage from geological carbon sequestration sites. EOS Transactions of the American Geophysical Union 2007 Fall Meeting, San Francisco, December 10-14, 2007.

Cortis, A., P.F. Dobson, and H.H. Liu, Review of "Understanding the micro to macro behaviour of rock-fluid systems." LBNL-63004. Journal of Sedimentary Research, ONLINE EDITION, 3, 2007.

Cortis, A., and T. Ghezzehei, On the transport of emulsions in porous media. LBNL-63364. Journal of Colloid and Interface Science, 313, 1-4, 2007.

Cortis, A., and C. Knudby, A continuous time random walk approach to transient flow in heterogeneous porous media. LBNL-59885. Water Resources Research, 42 (W10201), 2006.

Cox, M.H., J. Constantz, and G.W. Su, Comparison of waterquality parameters with heat as groundwater tracers along the Russian River, CA. LBNL-59430. Ground Water, 45 (2), 187-195, 2007.

Daley, T. M., B.M. Freifeld, J.B. Ajo-Franklin, C. Doughty, S.M. Benson, Frio II Brine Pilot: Report on GEOSEQ Activities. LBNL-63613. Lawrence Berkeley National Laboratory, Berkeley, CA. 2007.

Daley, T.M., E.L. Majer, G.M. Hoversten, and J.E. Peterson, Insitu borehole seismic monitoring of injected $\mathrm{CO}_{2}$ at the Frio Site. International CO2SC Symposium, LBNL/ESD, Berkeley, CA. 2006.

Daley, T.M., L.R. Myer, J.E. Peterson, E.L. Majer, and G.M. Hoversten, Time-lapse crosswell seismic and VSP monitoring of injected $\mathrm{CO}_{2}$ in a brine aquifer. LBNL-62396. Environmental Geology, DOI 10.1007/ s00254-007-0943-z, 2007.

Daley, T.M., F. Niu, P.G. Silver, and E.L. Majer, Acquisition of crosswell seismic monitoring data. LBNL-62400. Special Issue on Active Source Monitoring (in press), 2007.

Daley, T.M., M.A. Schoenberg, J. Rutqvist, and K.T. Nihei, Fractured reservoirs: An analysis of coupled elasto-dynamic and permeability changes due to pore pressure variation. LBNL-50697. Geophysics, 71 (5), 033-041, 2006.

Daley, T.M., R.D. Solbau, J.B. Ajo-Franklin, and S.M. Benson, Continuous active-source seismic monitoring of $\mathrm{CO}_{2}$ injection in a brine aquifer. LBNL-63325. Geophysics, 72 (5), A57-A61, 2007.
Daley, T. M., R. D. Solbau, J. B. Ajo-Franklin, and S. M. Benson, Continuous crosswell seismic during $\mathrm{CO}_{2}$ injection: A new monitoring technology deployed at the Frio-II Experiment. LBNL-63325. Sixth Annual Conference on Carbon Capture and Sequestration, SEG, 2007.

Daley, T., L. Myer, G. M. Hoversten, J. E. Peterson, Jr., and V. Korneev, Borehole seismic monitoring of injected $\mathrm{CO}_{2}$ at the Frio Site. LBNL-60160. 8th International Conference on Greenhouse Gas Control Technologies Congress, Trondheim, Norway. 2006.

Danko, G., J. Birkholzer, and D. Bahrami, Coupled in-rock and in-drift hydrothermal model study for Yucca Mountain. LBNL-61360. Nuclear Technology (in press) 2007.

DePaolo, D., Isotopic effects in fracture-dominated reactive fluid-rock systems. LBNL-60809. Geochimica et Cosmochimica Acta, 70 (5), 1077-1096, 2006.

DePaolo, D., K. Maher, J. N. Christensen, and J. McManus, Sediment transport time measured with U-Series isotopes: Results from ODP North Atlantic Drill Site 984. LBNL60856. Earth and Planetary Science Letters, 248 (1-2), 394410, 2006.

DeSantis, T., E. Brodie, J.P. Moberg, I.X. Zubieta, Y.M. Piceno, and G.L. Andersen, High-density universal 16S rRNA microarray analysis reveals broader diversity than typical clone library when sampling the environment. LBNL59763. Microbial Ecology, Published online http: / / dx.doi.org/10.1007/ s00248-006-9134-9, 2007.

DeSantis, T., et al., Greengenes: Chimera-checked 16S rRNA gene database and workbench compatible with ARB. LBNL-59764. Applied and Environmental Microbiology, 72 (7), 5069-5072, 2006.

DeSantis, T.Z., P. Hugenholtz, K. Keller, E.L. Brodie, N. Larsen, Y. M. Piceno, R. Phan, and G. L. Andersen, NAST: A multiple sequence alignment server for comparative analysis of 165 rNRA genes. LBNL-59765. Nucleic Acids Research, 34 (Supplement 2), W394-W399, 2006.

Dobson, P., S. Maruyama, J. Blank, and J. G. Liou, Petrology and geochemistry of boninite series volcanic rocks, Chichijima, Bonin Islands, Japan. LBNL-57671. International Geology Review, 48, 669-701, 2006.

Doughty, C., Modeling geologic storage of carbon dioxide: Comparison of hysteretic and non-hysteretic curves. LBNL61176. Energy Conversion and Management (Special Issue with Papers from TOUGH Symposium), 48 (6), 1768-1871, 2007.

Doughty, C., Site characterization for $\mathrm{CO}_{2}$ geologic storage and vice versa-The Frio Brine Pilot as a case study. LBNL517E. Environmental Geology, doi:10.1007/s00254-0070942-0, 2007.

Doughty, C., C.-F. Tsang, K. Hatanaka, S. Yabuuchi, and H. Kurikami, Application of direct-fitting, mass-integral, and multi-rate methods to analysis of flowing fluid electric conductivity logs from Horonobe, Japan. LBNL-63307. Water Resources Research (in press), 2007. 
Doughty, C. and L.R. Myer, Scoping calculations on leakage of $\mathrm{CO}_{2}$ in geologic storage. In: Science and Technology of Carbon Sequestration, B. McPherson and E. Sundquist, eds., American Geophysical Union, Washington DC, 2007.

Doughty, C., K. Karasaki, and K. Ito, Evaluation of uncertainties due to hydrogeological modeling and groundwater flow analysis: Progress Report on Complete $9 \times 9 \mathrm{~km}$ Model of the Tono Site, model from a subset of wells, and strategy for characterizing a new site. NUMO-LBNL Collaborative Research Project Report to JAEA, K. Karasaki, J. Apps, C. Doughty, H. Gwatney, C. Tiemi Onishi, R. Trautz, and C.-F. Tsang, eds., May 2006.

Doughty, C., K. Karasaki, and K. Ito, Evaluation of uncertainties due to hydrogeological modeling and groundwater flow analysis: Complete $9 \times 9 \mathrm{~km}$ model of the Tono site, models from different subsets of wells, and strategy for characterizing a new site. In: Feature Detection, Characterization, and Confirmation Methodology: Final Report, NUMO-LBNL Collaborative Research Project Report to JAEA, K. Karasaki, J. Apps, C. Doughty, H. Gwatney, C. Tiemi Onishi, R. Trautz, and C.-F. Tsang, eds., March 2007.

Dressel, P. E., K. B. Olsen, J. C. Hayes, J. I. McIntyre, S. R. Waichler, B. D. Milbrat, M. Cooper, and B. M. Kennedy, Environmental application of stable xenon and radioxenon monitoring. LBNL-61563. Journal of Radioanalytical and Nuclear Chemistry (in press), 2007.

Dunphy Guzman, K.A., M. Taylor, and J.F. Banfield, Environmental risks of nanotechnology: National nanotechnology initiative funding, 2000-2004. LBNL-59431. Environmental Science \& Technology, 40 (5), 1401-1407, 2006.

Evans, W. C., D. Bergfeld, M. van Soest, M. A. Huebner, J. Fitzpatrick, and K. M. Revesz, Geochemistry of low-temperature springs northwest of Yellowstone caldera: Seeking the link between seismicity, deformation, and fluid flow. LBNL-59813. Journal of Volcanology \& Geothermal Research, 154 (3-4), 169-180, 2006.

Faybishenko, B., Climatic forecasting of net infiltration at Yucca Mountain using analogue meteorological data. LBNL59279. Vadose Zone Journal, 6 (1), 77-92, 2007.

Faybishenko, B. et al., A conceptual model of coupled biogeochemical and hydrogeological processes affected by in situ $\mathrm{Cr}(\mathrm{VI})$ bioreduction in groundwater at Hanford $100 \mathrm{H}$ site of the geometry and physics of water flow in a fractured basalt vadose zone. LBNL-63362. EOS Transactions, 88 (23), H32B-03, 2007.

Fields, M., C.E. Bagwell, S.L. Carroll, T. Yan, X. Liu, P.M. Jardine, C.S. Criddle, T.C. Hazen, and J. Zhou, Polyphasic characterization of microbial communities under stressful conditions and the identification of different relationships based upon geochemical data or multiple gene sequences. LBNL-60460. Environmental Science \& Technology, 40 (8), 2601-2607, 2006.
Finsterle, S., Demonstration of optimization techniques for groundwater plume remediation using iTOUGH2. LBNL56624. Environmental Modeling and Software, 21 (5), 665-680, 2006.

Finsterle, S., Comment on "Seepage into drifts and tunnels in unsaturated fractured rock" by Dani Or, Markus Tuller, and Randall Fedors. LBNL-60409. Water Resources Research, 42 (W07603), doi:10.1029/2005WR004777, 2006.

Finsterle, S., and M.B. Kowalsky, Joint hydrological-geophysical inversion for soil structure identification. LBNL-60088. Vadose Zone Journal (in press), 2007.

Finsterle, S., M. Conrad, B.M. Kennedy, K. Pruess, T. Kneafsey, R. Salve, G. Su., and Q. Zhang, Mobility of tritium in engineered and earth materials at the NuMI Facility, Fermilab. LBNL-61798. Lawrence Berkeley National Laboratory, Berkeley, Calif., March 2007.

Finsterle, S., C. Doughty, M.B. Kowalsky, G.J. Moridis, L. Pan, T. Xu, Y. Zhang, and K. Pruess, Advanced vadose zone simulation using TOUGH. LBNL-63367. Vadose Zone Journal (in press), 2007.

Finsterle, S., and M.B. Kowalsky, iTOUGH2-GSLIB User's Guide. LBNL/PUB-3191. Lawrence Berkeley National Laboratory, Berkeley, CA. 2007.

Fischer, M.L., D.P. Billesbach, J.A. Berry, W.J. Riley, and M.S. Torn, Spatiotemporal variations in growing season exchanges of $\mathrm{CO}_{2}, \mathrm{H}_{2} \mathrm{O}$, and sensible heat in agricultural fields of the Southern Great Plains. LBNL-63014. Earth Interactions, 11, 2007.

Flanagan, J. L. et al., Loss of bacterial diversity during antibiotic treatment of intubated patients colonized with Pseudomonas aeruginosa. Journal of Clinical Microbiology, 45 (6), 1954-1962, 2007.

Forster, P., V. Ramaswamy, P. Artaxo, T. Berntsen, R. Betts, D. Fahey, J. Haywood, J. Lean, D. Lowe, G. Myhre, J. Nganga, R. Prinn, G. Raga, M. Schulz, R. Van Dorland, G. Bodeker, G. Boer, O. Boucher, W.D. Collins, et al., Changes in atmospheric constituents and in radiative forcing. Chapter 2 in: Climate Change 2007: The Physical Science Basis. Contribution of Working Group I to the Fourth Assessment Report of the Intergovernmental Panel on Climate Change, S. Solomon, D. Qin, M. Manning, Z. Chen, M. Marquis, K.B. Averyt, M. Tignor and H.L. Miller, eds., Cambridge University Press, Cambridge, United Kingdom, and New York, NY, USA, 996 pp., 2007.

Foxall, B., and D. Vasco, Inversion of synthetic aperture radar interferograms for sources of subsidence at the Dixie Valley geothermal field. LBNL-63546. Journal of Volcanology and Geothermal Research (in press), 2007.

Fransson, A., C.-F. Tsang, J. Rutqvist, and G. Gustafson, A new parameter to assess hydromechanical effect in single-hole hydraulic testing and grouting. International Journal of Rock Mechanics and Mineral Science, 44 (7), 1011-1021, 2007.

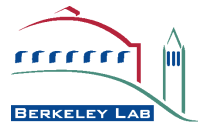


Fried, J.S., J.K. Gilless, W.J. Riley, T.J. Moody, C. Simon de Blas, K. Hayhoe, M. Moritz, S. Stephens, and M.S. Torn, Predicting the effect of climate change on wildfire behavior and initial attack success. Climatic Change (in press), 2007.

Freda, C., M. Gaeta, D. Karner, F. Mavra, P. Renne, J. Taddeucci, P. Scarlato, J. Christensen, and L. Dallai, Eruptive history and petrologic evolution of the Albano Multiple Maar (Alban Hills, Central Italy). LBNL-58203. Bulletin of Volcanology, 68 (6), 567-591, 2006.

Freifeld, B., and R.C. Trautz, Real-time quadrupole mass spectrometer analysis of gas in borehole fluid samples acquired using the U-Tube sampling methodology. LBNL-61091. Geofluids, 6 (3), 217-224, 2006.

Freifeld, B.M., T.J. Kneafsey, and F. Rack, On-site geologic core analysis using a portable $\mathrm{x}$-ray computed tomographic system. LBNL-55698. Journal of the Geological Society, (267), 165-178, 2006.

Freifeld, B.M., C. Doughty, and S. Finsterle, Preliminary estimates of specific discharge and transport velocities near Borehole NC-EWDP-24PB. LBNL-60740. Lawrence Berkeley National Lab., Berkeley, Calif., 2006.

Gaeta, M., C. Freda, J. Christensen, L. Dallai, F. Mavra, D. Karner, and P. Scarlato, Coherent time-dependent co-variation at $875 \mathrm{r} / 865 \mathrm{r}$ and trace elements in Clinopyroxenes from the Alban Hills Volcanic District (Central Italy): Clues to the evolution of the source of ultrapotassic magma. LBNL58204. Lithos, 86 (3-4), 330-346, 2006.

Garczarek, F., M. Dong, D. Typke, H.E. Witkowska, T.C. Hazen, E. Nogales, M.D. Biggin, and R.M. Glaeser, Octomeric pyruvate-ferredoxin oxidoreductase from Desulfovibrio vulgaris. LBNL-62439. Journal of Structural Biology, 159, 9-18, 2007.

Gasperikova, E., and L.P. Beard, Special issue on geophysics applied to detection and discrimination of unexploded ordnance. LBNL-146E. Journal of Applied Geophysics, 61, 165-167, 2007.

Gasperikova, E., A. Becker, H.F. Morrison, and J.T. Smith, A multisensor system for the detection and characterization of UXO. LBNL-58327. ESTCP 59, 2006.

Gasperikova, E., and G. M. Hoversten, A feasibility study of non-seismic geophysical methods for monitoring geologic $\mathrm{CO}_{2}$ sequestration. LBNL-61307. The Leading Edge, 25 (10), 1282-1288, 2006.

Gasperikova, E., J. T. Smith, F. Morrison, and A. Becker, Berkeley UXO Discriminator (BUD). LBNL-62263. SAGEEP 2007, Environmental and Engineering Geophysical Society, Denver, Colorado. 2007.

Gasperikova, E., J. T. Smith, H. F. Morrison, and A. Becker, UXO detection and characterization using new Berkeley UXO Discriminator (BUD). Partners in Environmental Technology Technical Symposium \& Workshop, SERDP / ESTCP / NAOC, Washington, D.C., 2006.
Gasperikova, E., J.T. Smith, H.F. Morrison, and A. Becker, UXO detection and discrimination with Berkeley UXO Discriminator (BUD). UXO Forum, Dept. of Army, Orlando, FL. 2007.

Gherardi, F., T. Xu, and K. Pruess, Numerical modeling of selflimiting and self-enhancing caprock alteration induced by $\mathrm{CO}_{2}$ storage in a depleted gas reservoir. Chem. Geol., 244, 103-129, 2007.

Ghezzehei, T., T. Kneafsey, and G. Su, Correspondence of the Gardner and van Genuchten/Mualem relative permeability function parameters. LBNL-62433. Water Resources Research, 43, 2007.

Gilbert, B., F. Huang, H. Zhang, G. Waychunas, and J.F. Banfield, Surface chemistry controls crystallinity of ZnS nanoparticles. LBNL-59040. Nanoletters, 6 (4), 605-610, 2006.

Gilbert, B., C. S. Kim, C.-L. Dong, J. Guo, P.S. Nico, and D.K. Shuh, Oxygen K-edge emission and absorption spectroscopy of iron oxyhydroxide nanoparticles: X-ray absorption fine structure-XAFS 13. LBNL-62225. $13^{\text {th }}$ International American Institute of Physics Conference American Institute of Physics, Stanford, CA., 2006.

Gilbert, B., G. Lu, and C.S. Kim, Stable nanoparticle clusters formed under environmentally relevant conditions. LBNL60208. Journal of Colloid and Interface Science, 313, 152-159, 2007.

Gilbert, B., H. Zhang, B. Chen, M. Kunz, H. Huang, and J.F. Banfield, The compressibility of zinc sulfide nanoparticles. LBNL-59574. Physical Review B, 74 115405, 2006.

Glatfelter, A., N. Stephenson, S. Bai, C. Dybowski, and D.L. Perry, Quantitative determination of lead in mixtures of lead(ii) halides using solid-state 207Pb NMR spectroscopy. LBNL-63086. The Analyst, 131, 2006.

Glatfelter, A., S. Bai, C. Dybowski, D. Martinez, and D.L. Perry, Solid-state nmr studies of lead(II) thiourea materials. LBNL-63077. University of Delaware, 2006.

Glatfelter, A., C. Dybowski, S. Bai, and D.L. Perry, Syntheses of photo-active lead(II) - 1,10 - phenanthroline materials. LBNL-63094. Materials Letters, 61 (2), 437-439, 2007.

Glover, D.M., C.L. Chandler, S.C. Doney, K.O. Buesseler, J.K. Bishop, and G.R. Flierl, The U.S. JGOFS data management experience. LBNL-59804. Deep Sea Research II, 53, 793-802, 2006.

Goloshubin, G., D. Silin, V. Vingalov, G. Takkand, and M. Latfullin, Reservoir permeability from seismic attribute analysis. LBNL-305E. The Leading Edge (in press), 2007.

Goloshubin, G. M., V. Korneev, D. Silin, V. Vingalow, and C. VanSchuyer, Reservoir imaging using low frequencies of seismic reflections. LBNL-60853. The Leading Edge, 25 (5), 527-531, 2006. 
Goodell, C. M., B. Gilbert, S. J. Weigang, and J. Banfield, Kinetics of the water adsorption driven structural transformation of ZnS nanoparticles. LBNL-63448. Journal of Physical Chemistry C (in press), 2007.

Guan, J., F. J. Molz, Q. Zhou, H.-H. Liu, and C. Zheng, Behavior of the mass transfer coefficient during the MADE-2 experiment: New insights. LBNL-63023. Water Resources Research (in press) doi:10.1029/2007WR006120, 2007.

Guglielmi, Y., F. Cappa, J. Rutqvist, C.-F. Tsang, and A. Thoraval, Mesoscale characterization of coupled hydromechanical behavior of a fractured-porous slope in response to free water-surface movement. International Journal of Rock Mechanics and Mining Sciences (in press), online November 2007.

Guglielmi Y., F. Cappa, J. Rutqvist, C.-F. Tsang, and A. Thoraval, Field and numerical investigations of free-water surface oscillation effects on rock slope hydromechanical behaviour-Consequences for rock slope stability analyses. Proceedings of the GEOPROC2006 International Symposium: $2^{\text {nd }}$ International Conference on Coupled Thermo-Hydro-Mechanical-Chemical Processes in Geosystems and Engineering, HoHai University, Nanjing, China, May 22-25, 2006. p. 174-182, 2006.

Gupta, A., T.J. Kneafsey, G.J. Moridis, Y. Seol, M.B. Kowalsky, and E.D. Sloan Jr., Methane hydrate thermal conductivity in a large heterogeneous porous sample. LBNL-59088. J. Phys. Chem. B; ASAP Web Release Date: August 2, 2006; DOI: 10.1021/jp0619639, 2006.

Gupta, A., T.J. Kneafsey, G.J. Moridis, Y. Seol, M. B. Kowalsky, and E.D. Sloan, Composite thermal conductivity in a large heterogeneous porous methane hydrate sample. LBNL61865. Journal of Phys. Chem B., 110 (3), 16384-16392, 2006.

Haines, S.S., S.R. Pride, S.L. Klemperer, and B. Brondi, Seismoelectric imaging of shallow targets. LBNL-61968. Geophysics, 72 (2), G9-G20, 2007.

Hame, T. et al., Kioto + mission : Global and accurate monitoring of forest, land cover, and carbon. LBNL-61409. VTTTechnical Research Center of Finland, Helsinki, Finland. 43, 2006.

Hammersley, L., and D. DePaolo, Isotopic and geophysical constraints on the structure and evolution of the Clear Lake volcanic system. LBNL-60926. Journal of Volcanology and Geothermal Research, 153 (3-4), 331-356, 2006.

Hausrath, E.M., A.K. Navarre-Sitchler, P.B. Sak, C.I. Steefel, and S.L. Brantley, Basalt weathering rates on Earth and the duration of liquid water on the plains of Gusev Crater, Mars. Geology (in press), 2007.

Holman, H.-Y., K. Bjornstad, M. Martin, E.A. Blakely, and F.G. Blankenberg, Midinfrared reflection-absorption spectromicroscopy (MIRAS) of Atherosclerosis in ApoE(-l-) knockout mice. LBNL-61263. Nature Medicine, 2007.

Hovorka, S.D., S.M. Benson. C. Doughty, B.M. Freifeld, S. Sakurai, T.M. Daley, Y.K. Kharaka, M.H. Holtz, R.C. Trautz,

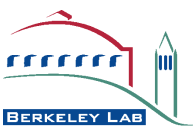

Measuring permanence of $\mathrm{CO}_{2}$ storage in saline formations: The Frio experiment. Environmental Geosciences, 13 (2), 1-17, DOI:10.1306/eg.11210505011, 2006.

Hazen, T.C., and D. Stahl, Process control in natural and engineered systems by monitoring microbial stress response. LBNL-60297. Current Opinions in Biotechnology, 17, 285-290, 2006.

He, Q., K. Huang, Z. He, E. Alm, M. Fields, T. Hazen, A. Arkin, J. Wall, and J. Zhou, Energetic consequences of nitrite stress in Desulfovibrio vulgaris Hildenborough inferred from global transcriptional analysis. LBNL-60422. Applied and Environmental Microbiology, 72 (6), 4370-4381, 2006.

He, Y. T., J. Wan, and T. K. Tokunaga, Kinetic stability of hematite nanoparticles: The effect of particle sizes LBNL63366. J. Nanopart. Res., 10, 321-332, 2007.

He, Z., A. Datta Gupta, and D. W. Vasco, Rapid inverse modeling of pressure interference tests using trajectory-based travel time and ampltude matching. LBNL-61355. Water Resources Research, 42 (W03419), 1-15, 2006.

Heal, K., C. Valeo, T. Oki, and S. Hubbard, Intersection of hydrology and other disciplines. LBNL-60883. In: Hydrology 2020: An Integrating Science to Meet Water Challenges. T. Oki, C. Valeo and K. Heal,eds., IAHS, Oxfordshire, UK, 2006.

Hegerl, G., F. Zwiers, P. Braconnot, N. Gillett, Y. Luo, J. Marengo, N. Nicholls, J. Penner, P. Stott, M. Allen, C. Ammann, N. Andronova, R. Betts, A. Clement, W.D. Collins, et al., Understanding and attributing climate change. Chapter 9 in: Climate Change 2007: The Physical Science Basis. Contribution of Working Group I to the Fourth Assessment Report of the Intergovernmental Panel on Climate Change, S. Solomon, D. Qin, M. Manning, Z. Chen, M. Marquis, K.B. Averyt, M. Tignor and H.L. Miller, eds., Cambridge University Press, Cambridge, United Kingdom, and New York, NY, USA, 996 pp., 2007.

Henderson-Sellers, A., M. Fischer, I. Aleinov, K. McGuffie, W.J. Riley, G.A. Schmidt, K. Sturm, K. Yoshimura, and P. Irannejad, Stable water isotope simulation by current landsurface schemes: Results of IPILPS phase 1. LBNL-60932. Global and Planetary Change, 51 (1-2 Special Issue), 34-58, 2006.

Hengpraprom, S., C. M. Lee, and J. T. Coates, Sorption of humic acids and alpha-endosulfan by lay minerals. LBNL-60933. Environmental Toxicology And Chemistry, 25 (1), 11-17, 2006.

Hidalgo, H. G., L. D. Brekke, N. L. Miller, N. W. T. Quinn, J. Keyantash, and J. A. Dracup, Assessment of the impacts of climate change on the water allocation, water quality and salmon production in the San Joaquin River Basin. LBNL60617. In: Climate Change and Variability: Consequences and Responses, M. Ruth, K. Donaghy, and P. Kirshen, eds., pp. 30-57, 2006.

Hillson, N.J., P. Hu, G.L. Andersen, and L. Shapiro, Caulobacter crescentus as a whole-cell uranium biosensor. Applied and Environmental Microbiology, 73 (23), 7615-7621, 2007. 
Holman, H.-Y. N., and M. C. Martin, Synchrotron radiation infrared spectromicroscopy: A non-invasive molecular probe for biogeochemical processes. LBNL-60323. Advances in Agronomy, 90, 79-127, 2006.

Holtzman, R., D.B. Silin, T.W. Patzek, Mechanical properties of granular materials: a variational approach to grain-scale simulations. Int. J. Numer. Anal. Meth. Geomech. (in press), 2007.

Hou, Z., Y. Rubin, G.M. Hoversten, D. Vasco, and J. Chen, Reservoir parameter identification using minimum relative entropy-based Bayesian inversions of seismic AVA and marine CSEM data. LBNL-60931. Geophysics, 71 (6), O77-O88, 2006.

Houseworth, J.E., An analytical model for solute transport in unsaturated flow through a single fracture and porous rock matrix. LBNL-56342. Water Resources Research, 42 (1), W01416, 2006.

Hoversten, G. M., F. Cassassuce, E. Gasperikova, G. A. Newman, J. Chen, Y. Rubin, H. Zhangshuan, and D. Vasco, Direct reservoir parameter estimation using joint inversion of marine seismic AVA \& CSEM data. Geophysics, 71, C1C13, 2006.

Hoversten, G. M., G. Newman, N. Geier, and G. Flanagan, 3D modeling of a deepwater EM exploration survey. LBNL60837. Geophysics, 71 (5), G239-G248, 2006.

Hovorka, S.D., S.M. Benson, C. Doughty, B.M. Freifeld, S. Sakurai, T.M. Daley, Y.K. Kharaka, M.H. Holtz, R.C. Trautz, S.H. Nance, L.R. Myer, and K.G. Knauss, Measuring permanence of $\mathrm{CO}_{2}$ storage in saline formations: The Frio experiment. LBNL-59434. Environmental Geosciences, 13 (2), 1-17, DOI:10.1306/eg. 11210505011, 2006.

Hubbard, S., Introduction to special section on Hydrologic Synthesis. LBNL-59433. Water Resources Research, 42 (3), W03S01, 2006.

Hubbard, S., I. Lunt, K. Grote, and Y. Rubin, Vineyard soil water content: Mapping small scale variability using ground penetrating radar. LBNL-60860. In: Fine Wine and Terroir-The geoscience perspective: Geoscience Canada Reprint Series Number 9. R.W. Macqueen and L.D. Meinert, eds., St. John's Press, Newfoundland, pp. 193-202, 2006.

Hubbard, S., and Y. Rubin, Hydrogeological characterization using geophysical methods. LBNL-59126. In: The Handbook of Groundwater Engineering. J. W. Delleur, ed., CRC Press, A. Taylor \& Francis Group Company, pp. 14.1-14.52, 2006.

Hubbard, S., C. Valeo, and S. Uhlenbrook, Chapter 7: Hydrological challenges: Scientific, technological and organizational bottlenecks. LBNL-59762. In: Hydrology 2020: An Integrating Science to Meet Water Challenges. T. Oki, C. Caleo and K. Heal, eds., IAHS, Paris, France, Oxfordshire, UK, pp. 141-154, 2006.
Hubbard, S.S., K. Williams, M. Conrad, B. Faybishenko, J. Peterson, J. Chen, P. Long and T. Hazen, Geophysical monitoring of hydrological and biogeochemical transformations associated with $\mathrm{Cr}(\mathrm{VI})$ Biostimulation. LBNL-145E. Environmental Science and Technology (in press), 2007.

Jensen, M.P., A.M. Vogelmann, W.D. Collins, G.J. Zhang, and E. Luke, Investigation of regional and seasonal variations in marine boundary layer cloud properties from MODIS observations. J. Climate (in press), 2007.

Jin, J., and N. Miller, Analysis of the impact of snow on daily weather variability in mountainous regions. LBNL-53845. Journal of Hydrometeorology, 8, 245-258, 2006.

Jin, J., and N.L. Miller, Regional climate simulations to quantify the range of land use change and irrigation impacts on hydroclimate in the California Central Valley. LBNL-62625. Journal of Geophysical Research (in press), 2007.

Jin, J., N.L. Miller, and N. Schegel, Understanding the role of land surface processes in the Regional Climate System: A WRF modeling study. Proceedings of the Annual WRF Workshop, Boulder, Colorado, 2007.

Jordan, P., and I. Javandel, Hydrogeology and tritium transport in Chicken Creek Canyon, Lawrence Berkeley National Laboratory, Berkeley, California. LBNL-63557. Lawrence Berkeley National Laboratory, Berkeley, CA. 2007.

Joslin, J.D., J.B. Gaudinski, M.S. Torn, W.J. Riley, and P.J. Hanson, Unearthing live fine root turnover times in a hardwood forest: The roles of root diameter, soil depth, and root branching order. LBNL-57347. New Phytologist, 172, 523-535, 2006.

Kalscheuer, T., M. Commer, S.L. Helwig, A. Hoerdt, and B. Tezkan, Electromagnetic evidence for an ancient avelanche caldera rim on the south flank of Mount Merapi, Indonesia. LBNL-63020. Journal of Volcanology and Geothermal Research, 162, 81-97, 2007.

Kang, Z., Y.-S. Wu, J. Li, Y. Wu, J. Zhang, and G. Wang, Modeling multiphase flow in naturally fractured vuggy petroleum reservoirs. LBNL-60852. SPE-102356, Presented at the SPE Annual Technical Conference and Exhibition, San Antonio, TX, USA, September 24-27, 2006.

Kang, Z., Y.-S. Wu, J. Li, Y. Wu, J. Zhang, and G. Wang, A triplecontinuum numerical model for simulating multiphase flow in vuggy fractured reservoirs. LBNL-58676. Presented at CMWR XVI-Computational Methods in Water Resources XVI International Conference, Copenhagen, Denmark, June 19-22, 2006.

Keers, H., L.R. Johnson, and D. Vasco, W., Determination of porosity and saturation using seismic waveform inversion. Studia Geophysica and Geodetica, 51 (1), 119-140, 2007.

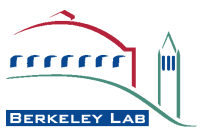


Kennedy, B.M., and M.C. van Soest, Flow of mantle fluids through the ductile lower crust: Helium isotope trends. Science, 318, 1433-1436, 2007.

Kennedy, B.M., and M. C. van Soest, A helium isotope perspective on the Dixie Valley, Nevada hydrothermal system. LBNL-60930. Geothermics, 35, 26-43, 2006.

Kennedy, N., E. L. Brodie, J. Connolly, and C. N., Seasonal influences on fungal community structure in unimproved and improved upland grassland soils. LBNL-59760. Canadian Journal of Microbiology, 52 (7), 689-694, 2006.

Kharaka, Y., D. Cole, S.D. Hovorka, W.D. Gunter, K.G. Knauss, and B.M. Freifeld, Gas-water-rock interactions in Frio formation following $\mathrm{CO}_{2}$ injection: Implications for the storage of greenhouse gases in sedimentary basins. LBNL61279. Geology, 34 (7), 577-580, 2006.

Kim, C.S., C.J. Lentini, and G.A. Waychunas, Associations between iron oxyhydroxide nanoparticle growth and metal adsorption/structural incorporation. In: Geomaterials II, D. Kent and M. Barnett, eds., Elsevier (in press), 2007.

Kirukhin, A.V., V.A. Korneev, and A.Y. Poliakov, On a possible connection of strong earthquakes with anomalous pressure changes in a two-phase geothermal reservoir. LBNL-61072. Volcanology and Seismology (in Russian), 2006.

Kiryukhin, A. V., N. P. Asaulova, S. Finsterle, T. V. Rychkova, and N. Obora, Modeling the Pauzhetsky Geothermal Field, Kamchatka, Russia, using iTOUGH2. LBNL-60087. TOUGH Symposium 2006, Lawrence Berkeley National Laboratory, Berkeley, CA. 2006.

Kiryukhin, A.V., N.P. Asaulova, and S. Finsterle, Inverse modeling and forecasting for the exploitation of the Pauzhetsky geothermal field, Kamchatka, Russia. Geothermics (in press), doi:10.1016/j.geothermics.2008.04.003, 2007.

Kneafsey, T., L. Tomutsa, G. Moridis, Y. Seol, B. Freifeld, C.E. Taylor, and A. Gupta, Methane hydrate formation and dissociation in a core-scale partially saturated sand sample. LBNL-59087. Journal of Petroleum Science and Engineering, 56, 108-126, 2007.

Korneev, V., and A. Bakulin, On the fundamentals of the virtual source method. LBNL-61085. Geophysics, 71 (3), A13-A17, 2006.

Korneev, V.A., Slow waves in fractures filled with viscous fluid. Geophysics (in press), 2007.

Kowalsky, M.B., J. Birkholzer, J.E. Peterson, S. Finstele, S. Mukhopadhyay, and Y.T. Tsang, Joint inversion of groundpenetrating radar and thermal-hydrological data from a large-scale heater test. LBNL-63358. Nuclear Technology (in press), 2007.

Kowalsky, M.B., J. Chen, and S.S. Hubbard, Joint inversion of geophysical and hydrological data for improved subsurface characterization. LBNL-59995. The Leading Edge, 5 (5), 730-734, 2006.
Kowalsky, M.B., and G.J. Moridis, Comparison of kinetic and equilibrium reactions in simulating the behavior of gas hydrates. LBNL-63357. Energy Conversion and Management, 48, 1850-1863, 2007.

Kueppers, L.M, M.A. Snyder, L.C. Sloan, D Cayan, J. Jin, H. Kanamaru, M. Kanamitsu, N.L. Miller, Mary Tyree, H. Du, and B. Weare, Seasonal temperature responses to land-use change in the western United States. CEC-500-2006-031, Global and Planetary Change (in press), 2007.

Kwon, K. D., K. Refson, and G. Sposito, Defect-induced photoconductivity in layered manganese oxides: A density functional theory study. LBNL-63294. Physical Review Letters (in press) 2007.

Lai, C., W. Riley, C. Owensby, J. Ham, A. Schauer, and J. Ehleringer, Seasonal and interannual variations of carbon and oxygen isotopes of respired $\mathrm{CO}_{2}$ in a tallgrass prairie: Measurements and modeling results from three years with contrasting water availability. LBNL-60741. Journal Geophysical Research-Atmospheres, 111 D08S06, 2006.

Lam, P.J., and J.K.B. Bishop, High biomass low export regimes in the Southern Ocean. LBNL-59452. Deep Sea Research II, 54 (5-7), 601-638, 10.1016/j.dsr2.2007.01.013. 2007.

Lam, P.J., J.K.B. Bishop, C.C. Henning, M.A. Marcus, G.A. Waychunas, and I.Y. Fung, Wintertime phytoplankton bloom in the Subarctic Pacific supported by continental margin iron. LBNL-55467. Global Biogeochemical Cycles, 20 (GB1006), doi:10.1029/2005GB002557, 2006.

Lambot, S., A. Binley, E. Slob, and S. Hubbard, Ground-penetrating radar in hydrogeophysics. Vadose Zone Journal (in press), 2007.

Lehikoinen, A., S. Finsterle, A. Voutilainen, L. M. Heikkinen, M. Vauhkonen, and K.J. Kaipio., Approximation errors and truncation of computational domains with application to geophysical tomography. LBNL-60042. Inverse Problems and Imaging, 1 (2), 371-389, 2007.

Lehikoinen, A., S. Finsterle, A. Voutilainen, M. B. Kowalsky, and J. P. Kaipio, A nonstationary inversion approach for imaging fluid flow in unsaturated porous media. LBNL63095. 5th World Congress on Industrial Process Tomography, World Congress on Industrial Process Tomography, Bergen, Norway. 2007.

Lehikoinen, A., J. M. J. Huttunen, S. Finsterle, M. B. Kowalsky, and K. J.P., An extension of a nonstationary inversion method with approximation error analysis applied to hydrological p rocess monitoring. LBNL-63093. AGU Joint Assembly, American Geophysical Union, Acapulco, Mexico. 2007.

Lehikoinen, A., A. Voutilainen, K. J.P., S. Finsterle, and M. B. Kowalsky, A nonstationary inversion approach for imaging fluid flow in unsaturated porous media. LBNL-63095 Conf. Inverse Problems, Design, and Optimization Symposium, AIM Sciences, Miami, Florida. 2007.

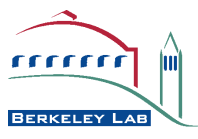


Lei, Q., W. Xiong, C. Yuan, and Y.-S. Wu, Analysis of stress sensitivity and its influence on oil production from tight reservoirs. LBNL-63410. SPE-111148, Presented at the 2007 SPE Eastern Regional Meeting held in Lexington, Kentucky, U.S.A., October 17-19, 2007.

Lewicki, J.L., J. Birkholzer, and C.-F. Tsang, Natural and industrial analogues for leakage of $\mathrm{CO}_{2}$ from storage reservoirs: Identification of features, events, and processes and lessons learned. LBNL-59784. Environmental Geology, 52 (3), 457-467, doi: 10.1007/ s00254-006-0479-7, 2007.

Lewicki, J.L., G.E. Hilley, T. Tosha, R. Aoyagi, H. Yamamoto, and S.M. Benson, Dynamic coupling of volcanic $\mathrm{CO}_{2}$ flow and wind at the Horseshoe Lake tree kill, Mammoth Mountain, CA. LBNL-62375. Geophysical Research Letters, 34, L03401, doi:10.1029/2006GL028848, 2007.

Lewicki, J. L., C. M. Oldenburg, L. Dobeck, and L. Spangler, Surface $\mathrm{CO}_{2}$ leakage during two shallow subsurface $\mathrm{CO}_{2}$ releases. LBNL-63528. Geophysical Research Letters, 34, L24402, doi:101029/2007GL032047, 2007.

Lewicki, J. L., M.L. Fischer, and G.E. Hilley, Six-week time series of eddy covariance $\mathrm{CO}_{2}$ flux at Mammoth Mountain, California: performance evaluation and role of meteorological forcing. LBNL-181E. Journal of Volcanology and Geothermal Research (in press), 2007.

Lewicki, J.L., G.E. Hilley, and C.M. Oldenburg, An improved strategy to detect $\mathrm{CO}_{2}$ leakage for verification of geologic carbon sequestration. LBNL-58885. 8th International Conference on Greenhouse Gas Control Technologies, Trondheim, Norway, 2006.

Li, L., C.A. Peters, and M.A. Celia, Effects of mineral spatial distributions on their reaction rates in porous media. LBNL-61974. Water Resources Research, 43 (W01419), doi: 10.1029/2005WR004848, 2007.

Li, L., C.A. Peters, and M.A. Celia, Reply to "Comments on upscaling geochemical reaction rates using pore-scale network modeling" by Peter C. Lichtner and Qinjun Kang. LBNL-61973. Advances in Water Resources, 43 (3), 691-695, 2007.

Li, L., C.A. Peters, and M.A. Celia, Upscaling geochemical reaction rates using pore-scale network modeling. LBNL-61972. Advances in Water Resources, 29 (9), 1351-1370, 2006.

Li, L., C.I. Steefel, and L. Yang, Mineral dissolution kinetics at the pore scale. LBNL-63303. Geochimica et Cosmochimica Acta (in press), 2007.

Li, L., C.A. Peters, and M.A. Celia, Applicability of averaged concentrations in determining geochemical reaction rates in heterogeneous porous media. LBNL-63304. American Journal of Science, 307, 1146-1166, 2007.

Li, W., A. Englert, O.A. Cirpka, J. Vanderborght, and H. Vereecken, Two-dimensional characterization of hydraulic heterogeneity by multiple pumping tests. Water Resour. Res., 43 (4), W04433, doi:10.1029/2006WR005333, 2007.
Li, W., A. Englert, O.A. Cirpka, and H. Vereecken, Threedimensional geostatistical inversion of flowmeter and pumping-test data. Ground Water (in press), 2007.

Lin, L.-H., E. Brodie, T.C. Hazen, G.L. Andersen, T. DeSantis, et al., Long term biosustainability in a high energy, low diversity crustal biotome. LBNL-60468. Science, 314, 479-482, 2006.

Lin, Z., B. Gilbert, Q. Liu, G. Ren, and F. Huang, A thermodynamically stable nanophase material. LBNL-60742. Journal of the American Chemical Society, 128 (18), 6126-6131, 2006.

Lin, Z., Y. Zhu, T.L. Kalabegishvili, N.Y. Tsibakhashvili, and H.Y. Holman, Effect of chromate action on morphology of basalt-inhabiting bacteria. LBNL-61266. Materials Science and Engineering, 26 (4), 610-612, 2006.

Linde, N., J. Chen, M.B. Kowalsky, and S. Hubbard, Hydrogeophysical parameter estimation approaches for field scale characterization. LBNL-56739. Chapter 2 In: Hydrogeophysics, H. Verecken, ed., Springer, pp. 9-44, 2006.

Linde, N., S. Finsterle, and S. Hubbard, Inversion of tracer test data using tomographic constraints. LBNL-54603. Water Resources Research, 42 (W04410), doi:10.1029/2004WR003806, 2006.

Linde, N., A. Tryggvason, J. Peterson, and S. Hubbard, Joint inversion of crosshole radar and seismic traveltimes. LBNL331E. J. of Geophysics (in press), 2007.

Liu, H.H., Y.Q. Zhang, and F.J. Molz, Scale dependence of the effective matrix diffusion coefficient: Some analytical results. LBNL-60746. Vadose Zone Journal, 6 (3), 679-683, 2007.

Liu, H.H., Y.Q. Zhang, Q. Zhou, and F.J. Molz, An interpretation of potential scale dependence of the effective matrix diffusion coefficient. LBNL-60744. Journal of Contaminant Hydrology, 90 (1-2), 41-57, 2007.

Liu, H.-H., G.S. Bodvarsson, and J.H. Dane, Temperature dependence of a large-scale water retention curves: A case study. LBNL-49075. Hydrogeology Journal, 14 (8), 1408-1408, 2006.

Lo, W.-C., G. Spsoito, and E. Majer, Low-frequency dilatational wave propagation through fully-saturated poroelastic media. LBNL-58091. Advances in Water Resources, 29, 408-416, 2006.

Lo, W.-C., G. Sposito, and E. Majer, Low-frequency dilatational wave propagation through unsaturated porous media containing two immiscible fluids. Transport in Porous Media, 68, 91-105, 2007.

Lu, G., E.L. Sonnenthal, and G.S. Bodvarsson, Multiple end-member mixing model of dilution: Hydrochemical effects of construction water at Yucca Mountain, Nevada. LBNL-54514. Hydrogeology Journal, (HJ-2007-0599) (in press), 2007. 
Lu, G., and C. Zheng, Novel direct propagation neural network and application to parameter estimation. LBNL-56485. Journal of Contaminant Hydrology (in press), 2007.

Lynch, S.V., L. Dixon, M.R. Benoit, E.L. Brodie, M. Keyhan, P. $\mathrm{Hu}$, D.F. Ackerley, G.L. Andersen, and A. Matin, Role of the rapA gene in controlling antibiotic resistance of Escherichia coli biofilms. Antimicrobial Agents and Chemotherapy, 51 (10), 3650-3658, October 2007.

Machavaram, M.V., D.O. Whittemore, M.E. Conrad, and N.L. Miller, Precipitation induced stream flow: An event based chemical and isotopic study of a small stream in the Great Plains region of the USA. LBNL-60897. Journal of Hydrology, 333 (3-4), 470-480, 2006.

Maggi, F., C. Gu, W.J. Riley, G.M. Hornberger, R.T. Venterea, T. $\mathrm{Xu}$, N. Spycher, C. Steefel, N.L. Miller, and C.M. Oldenburg, Mechanistic modeling of biogeochemical nitrogen cycling: Model development and application in an agricultural system, Journal of Geophysical Research (in press), 2007.

Maher, K., D. DePaolo, and J. N. Christensen, U-Sr isotopic speedometer fluid flow and chemical weathering rates in aquifers. LBNL-60859. Geochimica et Cosmochimica Acta, 70 (17), 4417-4435, 2006.

Maher, K., C. I. Steefel, D. DePaolo, and B. Viani, The mineral dissolution rate conundrum: Insights from reactive transport modeling of $U$ isotopes and pore fluid chemistry in marine sediments. LBNL-59802. Geochimica et Cosmochimica Acta, 70 (2), 337-363, 2006.

Majer, E., T. Daley, V. Korneev, D. Cox, J.E. Peterson Jr., and J.H. Queen, Cost effective imaging of $\mathrm{CO}_{2}$ injection with borehole seismic methods. LBNL-61293. The Leading Edge, 25 (10), 1290-1302, 2006.

Majer, E., and J.E. Peterson, The impact of injection on seismicity at The Geysers California geothermal field. LBNL-61693. International Journal of Rock Mechanics \& Mining Sciences, 44, 1079-1090, 2007.

Majer, E.L., Barrier systems for environmental contaminant containment and treatment In: Airborne and Surface Geophysical Method Verification. C.C. Chein, H.I. Inyang and L.G. Everett, eds., Taylor \& Francis Group, Boca Raton, FL, 2006.

Majer, E.L., R. Baria, M. Stark, S. Oates, J. Bommer, B. Smith, and H. Asanuma, Induced seismicity associated with enhanced geothermal systems. LBNL-61681. Geothermics, 36 (3), 185-222, 2007.

Masson, Y., and S.R. Pride, Poroelastic finite-difference modeling of seismic atenuation and dispersion due to mesoscopic heterogeneity. LBNL-61830. Journal of Geophysical Research, 112 (B03204), 2007.

Masson, Y., S.R. Pride, and K.T. Nihei, Finite-difference modeling of Biot's poroelastic equations at seismic frequencies. LBNL-61829. Journal of Geophysical Research, 111 (B10305), 1-13, 2006.
McDowell, N., D. Baldocchi, M. Barbour, C. Bickford, M. Cuntz, D. Hanson, A. Knohl, H. Powers, T. Rahn, J.T. Randerson, W.J. Riley, C.J. Still, K. Tu, and A. Walcroft, Measuring and modeling the stable isotope composition of biosphere atmosphere $\mathrm{CO}_{2}$ exchange: Where are we and where are we going? EOS (in press), 2007.

McGrath, P.T., H. Lee, L. Zhang, A.A. Iniesta, A.K. Hottes, N.J. Hillson, P. Hu, L. Shapiro, and H.H. McAdams, Highthroughput identification of transcription start sites, conserved promoter motifs and predicted regulons. LBNL63029. Nature Biotechnology, 25 (5), 584-592, 2007.

McIntosh, B. S. et al., Bridging the gaps between design and use: Developing tools to support environmental management and policy. LBNL-63343. Environmental Modelling and Software (in press), 2007.

McKinley, J.P., J.M. Zachara, J. Wan, D.E. McCready, and S.M. Heald, Geochemical controls on contaminant uranium in vadose Hanford formation sediments at the 200 Area and 300 Area, Hanford Site, Washington. Vadose Zone Journal, 6, 1004-1017. 2007.

Meehl, G.A., J.M. Arblaster, and W.D. Collins, Effects of black carbon aerosols on the South Asian Monsoon. J. Climate (in press), 2007.

Meehl, G.A., T.F. Stocker, W.D. Collins, et al., Global climate projections. Chapter 10 in: Climate Change 2007: The Physical Science Basis. Contribution of Working Group I to the Fourth Assessment Report of the Intergovernmental Panel on Climate Change, S. Solomon, D. Qin, M. Manning, Z. Chen, M. Marquis, K.B. Averyt, M. Tignor, and H.L. Miller, eds., Cambridge University Press, Cambridge, United Kingdom, and New York, NY, USA, 996 pp., 2007.

Mikutta, R., M. Kleber, M. S. Torn, and R. Jahn, Stabilization of soil organic matter: Association with minerals or chemical recalcitrance? LBNL-59045. Biogeochemistry, 77, 25-56, 2006.

Miller, N. L., K. Hayhoe, J. Jin, and M. Auffhammer, Climate, extreme heat, and energy demand in California. LBNL61979. Journal of Applied Meteorology and Climatology, 47 (6), 1834-1844, 2007.

Miller, N.L., L.L. Dale, S. Vicuna, C. Brush, J. Dogrul, and T. Kadir, Drought analysis of the California Central Valley surface-groundwater-conveyance system. Report to the Department of Water Resources, Presentation at the Fall 2006 AGU, J. American Water Resources Association. (in press), 2007.

Miller, N.L., and N.J. Schlegel, Climate change projected fire weather sensitivity: California Santa Ana wind occurrence. LBNL-61004. Geophysical Research Letters, 33 (L15711), 1-5, 2006.

Monaselidze, J. et al., Characterization of chromium-induced apoptosis in cultured mammalian cells: A differential scanning calorimetry study. LBNL-60739. Thermochimica Acta, 441 (1), 8-15, 2006.

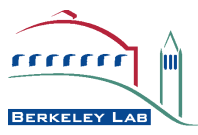


Montlor-Curley, C., E. L. Brodie, M. G. Lechner, and A. H. Purcell, Exploration for facultative endosymbionts of glassy-winged sharpshooter (Hemiptera: Cicadellidae). LBNL-63568. Annals of the Entomological Society of America, 100 (3), 345-349, 2007.

Moore, J. R., and S. D. Glaser, Self-potential observations during hydraulic fracturing. LBNL-63455. Journal of Geophysical Research, 112 (B02204), doi: 10.1029/2006JB004373, 2007.

Moreau, J. W., P. K. Weber, M. C. Martin, B. Gilbert, I. D. Hutcheon, and J. F. Banfield, Extracellular proteins limit the dispersal of biogenic nanoparticles. LBNL-62685. Science, 316 (5831), 1600-1603, 2007.

Moridis, G., and Y. Seol, Three-dimensional radionuclide transport through the unsaturated zone of the Yucca Mountain site: 1 . Nonsorbing $\mathrm{TcO}_{4}$ - solutes. LBNL-60639. Transport in Porous Media (in press), 2007.

Moridis, G., and Y. Seol, Three-dimensional radionuclide transport through the unsaturated zone of the Yucca Mountain site: 2. Sorbing solutes. LBNL-60640. Transport in Porous Media (in press), 2007.

Moridis, G., and Y. Seol, Three-dimensional radionuclide transport through the unsaturated zone of the Yucca Mountain site: 3. Colloids. LBNL-60641. Transport in Porous Media (in press), 2007.

Moridis, G. J., and M. Kowalsky, Gas production from unconfined Class 2 hydrate accumulations in the oceanic subsurface. LBNL-57299. In: Economic Geology of Natural Gas Hydrate. A.M.J. Max, W. Dillon, and T.S. Collett (eds.), Kluwer Scientific, Dordrecht, Netherlands, 2006.

Moridis, G.J., and M.B. Kowalsky, Response of oceanic hydrate-bearing sediments to thermal stresses. LBNL60150. SPE Journal, 12 (2), 253-268, 2007.

Moridis, G.J., M.B. Kowalsky, S. Finsterle, and K. Pruess, TOUGH+: The new generation of object-oriented family of codes for the solution of problems of flow and transport in the subsurface. LBNL-60087. TOUGH Symposium 2006, Lawrence Berkeley National Laboratory, Berkeley, CA. 2007.

Moridis, G.J., M.B. Kowalsky, and K. Pruess. Depressurizationinduced gas production from Class 1 hydrate deposits. LBNL-59780. SPE Reservoir Evaluation and Engineering, 10(5), 458-481, 2007.

Moridis, G. J., and E. D. Sloan, Gas production potential of disperse low-saturation hydrate accumulations in oceanic sediments. LBNL-61446. Energy Conversion and Management, 48 (6), 1834-1849, 2007.

Moridis, G., M. T. Reagan, S.-J. Kim, Y. Seol, and K. Zhang, Evaluation of the gas production potential of marine hydrate deposits in the Ulleung Basin of the Korean East Sea, SPE-110859, 2007 SPE Asia Pacific Oil \& Gas Conference and Exhibition. Society of Petroleum Engineers, Jakarta, Indonesia, 10/30-11/01, 2007.

Mukhopadhyay, A. et al., Salt stress in Desulfovibrio vulgaris Hildenborough: An integrated genomics approach. LBNL59862. J. Bacteriol., 188, 4068-4078, 2006.
Mukhopadhyay, A. et al., Cell wide responses to low oxygen exposure in Desulfovibrio vulgaris Hildenborough. LBNL63534. Journal of Bacteriology, 189 (16), 5996-6010, 2007.

Mukhopadhyay, S., E.L. Sonnenthal, and N. Spycher, Modeling coupled thermal-hydrological-chemical processes in the unsaturated fractured rock of Yucca Mountain, Nevada: Heterogeneity and seepage. LBNL-60163. Physics and Chemistry of the Earth, 31 (10-14), 626-633, 2006.

Mukhopadhyay, S., E.L. Sonnenthal, and N. Spycher, Modeling of coupled heat transfer and reactive transport processes in porous medium: Application to seepage studies at Yucca Mountain, Nevada. LBNL-63459. Journal of Porous Media (in press), 2007.

Mukhopadhyay, S., Y. Tsang, and J. Birkholzer, Estimation of field-scale thermal conductivities of unsaturated rocks from in situ temperature data. LBNL-60834. Water Resources Research, 43 (W09418), doi:10.1029/2006WR005283, 2007.

Myšjak, J., J.D. Brown, J.M. Jansen, and N.W.T. Quinn, Environmental policy aid under uncertainty. LBNL-63333. Environmental Modeling and Software (in press), 2007.

Nakagawa, S., and T.M. Daley, Modeling wave generation by borehole orbital vibrator source. LBNL-55828. Geophysics, 71 (1), F1-F11, 2006.

Nakagawa, S., and M. Schoenberg, Poroelastic modeling of seismic boundary conditions across a fracture. LBNL-60862. Journal of Acoustical Society of America, 122 (2), 831-847, 2007.

Narasimhan, T.N., Central ideas of Buckingham (1907): A century later. LBNL-62678. Vadose Zone Journal, 6 (4), 687-693, doi:10.2136/vzj2007.0080, 2007.

Narasimhan, T. N., Limitations of science and adapting to nature. LBNL-62152. Environmental Research Letters, 2 (3) (July-September 2007) doi:10.1088/1748-9326/2/3/034003, 2007.

Narasimhan, T. N., Water, law, science. LBNL-63536. Journal of Hydrology (in press), 2007.

Nash, B. P., M. E. Perkins, J. N. Christensen, D.-C. Lee, and A. N. Halliday, The Yellowstone hotspot in space and time: $\mathrm{Nd}$ and Hf isotopes in silici magmas. LBNL-60528. Earth \& Planetary Science Letters, 247 (1-2), 143-156, 2006.

Newman, G.A., E. Gasperikova, G.M. Hoversten, and P.E. Wannamaker, 3D magnetotelluic characterization of the Coso Geothermal Field. LBNL-58328 Geothermics (in press), 2007.

Neiss, J., B.D. Stewart, P.S. Nico, and S. Fendorf, Speciation dependent microbial reduction of uranium within ironcoated sands under dynamic flow. LBNL-63022. Environmental Science and Technology (in press), 2007.

Nico, P.S., M.V. Ruby, Y.W. Lowney, and S.E. Holm, Chemical speciation and bioaccessibility of arsenic and chromium in chromated copper arsenate-treated wood and soils. LBNL59097. Environmental Science \& Technology, 40 (1), 402-408, 2006.

Niu, F., P.G. Silver, T.M. Daley, E.L. Majer, and X. Cheng, Prerupture dilantancy observed from active source monitoring at the Parkfield SAFOD Drill Site, Nature (in press), 2007.

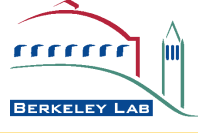


Oden, M., A. Niemi; C.-F. Tsang, and J. Öhman, Regional channelized transport in fractured media with matrix diffusion and linear sorption. Water Resour. Res. (in press), 2007.

Oldenburg, C., Migration mechanism and potential impacts of $\mathrm{CO}_{2}$ leakage and seepage. LBNL-58872. In: Carbon Capture and Geologic Sequestration: Integrating Technology, Monitoring, and Regulation, E. Wilson and D. Gerard, eds., Blackwell Publishing, pp. 127-146, 2007.

Oldenburg, C., Joule-Thomson cooling due to $\mathrm{CO}_{2}$ injection into natural gas reservoirs. LBNL-60158. Energy Conversion and Management, 48, 1808-1815, 2007.

Oldenburg, C., Screening and ranking framework (SRF) for geologic $\mathrm{CO}_{2}$ storage site selection on the basis of HSE risk. LBNL-63306. Environmental Geology (in press), 2007.

Oldenburg, C., and J. L. Lewicki, On leakage and seepage of $\mathrm{CO}_{2}$ from geologic storage sites into surface water. LBNL59225. Environmental Geology, 50 (5), 691-705, 2006.

Oldenburg, C. M., Geologic carbon sequestration: $\mathrm{CO}_{2}$ transport in depleted gas reservoirs. LBNL-63337. In: Gas Transport in Porous Media, C.K. Ho and S. Webb, eds., Springer, Dordrecht, Netherlands, pp. 419-426, 2007.

Oldenburg, C.M., L. Dobeck, and L. Spangler, Modeling nearsurface carbon dioxide migration from a shallow horizontal well, EOS Transactions of the American Geophysical Union 2007 Fall Meeting, San Francisco, December 10-14, 2007.

Or, D., and T. Ghezzehei, Comment on "Computer simulation of two-phase immiscible fluid motion in unsaturated complex fractures using a volume of fluid method" by Hai Huang, Paul Meakin, and Moubin Liu. LBNL-60673. Water Resources Research, 43 (W07601), 1-2, 2006.

Pan, F., M. Ye, Y.-S. Wu, B. X. Hu, J. Zhu, and Z. Yu, Simulation of radionuclide transport in the randomly heterogeneous, unsaturated media of Yucca Mountain. LBNL-63335. Advances in Water Resources (in press), 2007.

Pan, L., J. Jin, N.L. Miller, Y.-S. Wu, and G.S. Bodvarsson, Modeling hydraulic responses to meteorological force: From canopy to aquifer. LBNL-61018. Vadose Zone Journal (in press), 2007.

Personna, Y., D. Ntarlagiannis, L. Slater, N. Yee, M. O’Brien, and S. Hubbard, Spectral induced polarization monitoring and modeling of microbial-mediated iron sulfide transformations. JGR Biosciences (in press), 2007.

Pride, S.R., and E.G. Flekkey, Seismic stimulation for enhanced oil recovery. LBNL-61828. Geothermics (in press), 2007.

Pride, S.R., and Y. Masson, Acoustic attenuation in self-affine porous structures. LBNL-61827. Physical Review Letters, 97 (184301), 1-184301.4, 2006.

Pruess, K., Leakage of $\mathrm{CO}_{2}$ from geologic storage: Role of secondary accumulation at shallow depth. LBNL-63354. Int. J. Greenhouse Gas Control (in press), 2007.

Pruess, K., On the production behavior of enhanced geothermal systems with $\mathrm{CO}_{2}$ as working fluid. LBNL-63355. Energy Conversion and Management (in press), 2007.
Pruess, $\mathrm{K}$., On $\mathrm{CO}_{2}$ fluid flow and heat transfer behavior in the subsurface, following leakage from a geologic storage reservoir. LBNL-60076. Environmental Geology, doi: 10.1007/ s00254-007-0945-x, 2007.

Pruess, K., Role of fluid pressure in the production behavior of enhanced geothermal systems with $\mathrm{CO}_{2}$ as working fluid. Transactions of the Geothermal Resources Council, 31, 307-311, October 2007.

Pruess, K., and N. Spycher, ECO2N-A fluid property module for the TOUGH2 code for studies of $\mathrm{CO}_{2}$ storage in saline aquifers. LBNL-61077. Energy Conversion and Management, 48 (6), 1761-1767, 2007.

Pruess, K., N. Spycher, and T. Kneafsey, Water injection as a means for reducing non-condensible and corrosive gases in steam produced from vapor-dominated reservoirs. LBNL62376. Thirty-Second Workshop on Geothermal Reservoir Engineering, Stanford University, Stanford, CA. 2007.

Pruess, K., Enhanced geothermal systems (EGS): Comparing water and $\mathrm{CO}_{2}$ as heat transmission fluids. LBNL-63627. New Zealand Geothermal Workshop 2007, University of Auckland, Auckland, New Zealand, 2007.

Pruess, $\mathrm{K}$., $\mathrm{On} \mathrm{CO}_{2}$ behavior in the subsurface, following leakage from a geologic storage reservoir. LBNL-59628. Lawrence Berkeley National Laboratory, Berkeley, CA, 2006.

Pruess, K., On leakage from geologic storage reservoirs of $\mathrm{CO}_{2}$. LBNL-59732. International Symposium on Site Characterization for $\mathrm{CO}_{2}$ Geologic Storage (CO2SC), LBNL, Berkeley, CA. 2006.

Pruess, K., Enhanced geothermal systems (EGS) using $\mathrm{CO}_{2}$ as working fluid-A novel approach for generating renewable energy with simultaneous sequestration of carbon. LBNL60397. Geothermics, 35 (4), 351-367, August 2006.

Pruess, K., Numerical modeling of $\mathrm{CO}_{2}$ sequestration in geologic formations - Recent results and open challenges. LBNL-59888. Computational Methods in Water Resources (CMWR XVI), Technical University of Denmark, Copenhagen, Denmark. 2006.

Pruess, K., and M. Azaroual, On the feasibility of using supercritical $\mathrm{CO}_{2}$ as heat transmission fluid in an engineered hot dry rock geothermal system. Proceedings, Thirty-First Workshopon Geothermal Reservoir Engineering, Stanford University, Stanford, CA, January 30-February 1, 2006.

Qin, L., W. Xiong, J. Yuan, Y. Cui, and Y.-S. Wu, Analysis of stress sensitivity and its influence on oil production from tight reservoirs. LBNL-63410. SPE-111148, Presented at the 2007 SPE Eastern Regional Meeting held in Lexington, Kentucky, U.S.A., October 17-19, 2007.

Quinn, N.W.T., Hydrogeologic assessment of the 4-S Land and Cattle Company Ranch. LBNL-60011. Lawrence Berkeley National Lab, Berkeley, CA. 2006.

Quinn, N.W.T., Hydrogeologic assessment of the East Bear Creek Unit, San Luis National Wildlife Refuge. LBNL63212. Lawrence Berkeley National Lab, Berkeley, CA, 2007.

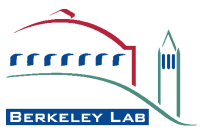


Quinn, N.W.T., and K.C. Jacobs, Design and implementation of an emergency environmental response system to protect migrating salmon in the lower San Joaquin River, California. LBNL-60622. Rev. Environmental Modelling and Software, 22 (4), 416-422, 2007.

Quinn, N.W.T., J.C. Linneman, and K.C. Tanji, The San Joaquin Valley westside perspective. World Environmental and Water Resources Congress 2006. LBNL-60613. World Environmental and Water Resources Congress, 2006.

Reagan, M.T. and G.J. Moridis, Oceanic gas hydrate instability and dissociation under climate change scenarios. LBNL62999. Geophys. Res. Lett., 34, L22709, doi: 10.1029/2007 GL031671, 2007.

Reagan, M.T., WebGasEOS 1.0 User Guide. LBID-3188. LBNL, Berkeley, CA. 2006.

Rebscher, D., F. May, and C. Oldenburg, Sequestration of CO2 in the Altmark natural gas field, Germany: Mobility control to extend enhanced gas recovery. LBNL-60157. TOUGH Symposium 2006, LBNL, Earth Sciences Division, Berkeley, CA. 2006.

Redding, A., A. Mukhopadhyay, D. Joyner, T. C. Hazen, and J. Keasling, Study of nitrate stress in Desulfovibrio Vulgaris Hildenborough using iTRAQ proteomics. LBNL-59867. Briefings in Functional Genomics and Proteomics, 5 (2), 133-143, 2006.

Revil, A., N. Linde, A. Cerepi, D. Jougnot, S. Matthai, and S. Finsterle, Electrokinetic coupling in unsaturated porous media. LBNL-63028. Journal of Colloid and Interface Science, 313, 315-327, doi:10.1016/j.jcis.2007.03.037, 2007.

Richter, F., R. Mendybaev, J. Christensen, I. Hutcheson, R. Williams, N. Starchio, and A. Beloso Jr., Kinetic isotopic fractionation during diffusion of ionic species in water. LBNL-58268. Geochimica et Cosmochimica Acta, 70 (2), 277-289, 2006.

Rizzoli, A. E. et al., Integrated modelling frameworks for environmental assessment and decision support. LBNL-63317. Environmental Modeling and Software (in press), 2007.

Rutqvist, J., J. Birkholzer, F. Cappa, and C.-F. Tsang, Estimating maximum sustainable injection pressure during geological sequestration of $\mathrm{CO}_{2}$ using coupled fluid flow and geomechanical fault-slip analysis. LBNL-61786. Energy Conversion Management, 48 (6), 1798-1807, 2007.

Rutqvist, J., J. Birkholzer, M. Chijimatsu, O. Koklitz, Q.-S. Liu, Y. Oda, W. Wang, and C.-Y. Zhang, Results from an international simulation study on coupled thermal, hydrological, and mechanical (THM) processes near geological nuclear waste repositories LBNL-59056. Nuclear Technology (in press), 2007.

Rutqvist, J., J.T. Birkholzer, and C.F. Tsang, Coupled reservoirgeomechanical analysis of the potential for tensile and shear failure associated with $\mathrm{CO}_{2}$ injection in multilayered reservoir-Caprock systems. LBNL-62675. International Journal of Rock Mechanics and Mineral Science, 45 (1), 132-142, 2007.
Rutqvist, J., and C.M. Oldenburg, Development of fluid injection strategies for optimizing steam production at The Geysers Geothermal Field, California-Task 2.3 coupled thermal-hydrological-mechanical analysis of induced seismicity. LBNL-62577. Lawrence Berkeley National Laboratory, Berkeley, CA. 53, 2007.

Rutqvist, J. and C. Oldenburg, Analysis of cause and mechanism for injection-induced seismicity at the Geysers geothermal field, California Geothermal Research Council, Annual Meeting, Sparks, Nevada, September 30-October 3, 2007.

Rutqvist, J. and G. Moridis, Numerical studies on the geomechanical stability of hydrate-bearing sediments. OTC-18860, $P$ resented at the 2007 Offshore Technology Conference held in Houston, Texas, U.S.A., April 30-May 3, 2007.

Rutqvist, J., J.T. Birkholzer and C.-F. Tsang, Modeling of geomechanical processes during injection in a multilayered reservoir-caprock system and implications on site characterization. Proceedings CO2SC 2006, International Symposium on Site Characterization for CO2 Geological Storage, Berkeley CA, March 20-22, 2006.

Rutqvist, J., J. Birkholzer, F. Cappa, C. Oldenburg, and C.-F. Tsang, Shear slip analysis in multi-phase fluid flow reservoir engineering applications using TOUGH-FLAC. LBNL63353. Proceedings of the TOUGH Symposium 2006, Earth Sciences Division, Lawrence Berkeley National Laboratory, Berkeley, CA., May 15-17, 2006.

Rutqvist, J., E. Majer, C. Oldenburg, J. Peterson J., and D. Vasco, Integrated modeling and field study of potential mechanisms for induced seismicity at The Geysers Geothermal Field, California. GRC Transactions, 30, 629-633, 2006.

Rutqvist, J., J.T. Birkholzer, and C.-F.Tsang, Modelling hydrological and geomechanical processes related to $\mathrm{CO}_{2}$ injection in a faulted multilayer system. 8th International Conference on Greenhouse Gas Control Technologies, Trondheim, Norway, June 19-22, 2006.

Salve, R., T. Ghezzehei, and R. Jones, Infiltration into fractured bedrock. LBNL-63628. Water Resources Research (in press), 2007.

Salve, R., and P.J. Cook, A sensor array for monitoring moisture dynamics in unsaturated soil. LBNL-63351. Lawrence Berkeley National Laboratory, 2007.

Salve, R., N.Y. Krakauer, M.B. Kowalsky, and S.A. Finsterle, A qualitative assessment of microclimate perturbations in a tunnel. LBNL-63049. International Journal of Climatology, (in press), 2007.

Samper, J., G. Zhang, and L. Montenegro, Coupled microbial and geochemical reactive transport models in porous media: formulation and application to synthetic and in situ experiments. LBNL-60900. Journal of Iberian Geology 32 (2): 215-231. 2006.

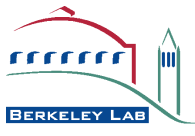


Scheibe, T.D., Y. Fang, C.J. Murray, E.E. Roden, J. Chen, Y.-J. Chien, S.C. Brooks, and S.S. Hubbard, Transport and biogeochemical reaction of metals in a physically and chemically heterogeneous aquifer. LBNL-55886. Geosphere, 2 (4), doi: 10.1130/GES00029.1, 2006.

Schoenberg, M., and S. Nakagawa, Poroelastic modeling of seismic boundary conditions across a fracture. LBNL-60862. Meeting of the Society Exploration Geophysicists, Society Exploration Geophysicists, New Orleans, LA. 2991-2995, 2006.

Schwarz, K., T. Patzek, and D. Silin, On prediction of windborne plumes with simple models of turbulent transport. LBNL-59656. Vadose Zone Journal, 312, 1747-1748, 2006.

Sengör, S.S., S.N.F., T.R. Ginn, J. Moberly, B. Peyton, and R.K. Sani, Reductive dissolution and metal transport in Lake Coeur D'Alene sediments. LBNL-63045. In: Water-Rock Interaction, WRI-12, T. Bullen and Y. Wang, eds., Balkema Publishers, New York, NY (in press), 2007.

Sengör, S.S., N. Spycher, T.R. Ginn, R.K. Sani, and B. Peyton, Biogeochemical reactive-diffusive transport of heavy metals in Lake Coeur d'Alene sediments. LBNL-63036. Applied Geochemistry, 22, 2569-2594, 2007.

Sengör, S.S., N. Spycher, T.R. Ginn, R.K. Sani, B.M. Peyton, J. Moberly, and S. Barua, Reactive diffusive transport of heavy metals in Lake Coeur d'Alene sediments. LBNL63352. Fall 2006 AGU Meeting, American Geophysical Union, San Francisco, CA. 2006.

Shan, C., An analytical solution for transient gas flow in a multi-well system. LBNL-60294. Water Resources Research, 42 (W10401), doi:10.1029/2005WR004737, 2006.

Shen, P., B. Zhu, X.-B. Li, and Y.-S. Wu, The influence of interfacial tension on water-oil two-phase relative permeability. LBNL-55640. SPE-95405, Presented at the 2006 SPE/DOE Symposium on Improved Oil Recovery, held in Tulsa, Oklahoma, U.S.A., April 22-26, 2006.

Silin, D., T. Patzek, and S.M. Benson, A model of buoyancydriven countercurrent two-phase fluid flow. LBNL-62607. Transport in Porous Media (in press), 2007.

Silin, D., T. Patzek, and S. M. Benson, Modeling evolution of leaking gas plume migration. LBNL-63445. 2007 Ground Water Protection Council Annual Forum, Ground Water Protection Council (GWPC), San Diego, CA. 2007.

Silin, D.B., V.A. Korneev, G.M. Goloshubin, and T.W. Patzek, Low-frequency asymptotic analysis of seismic reflection from a fluid-saturated medium. LBNL-54955. Transport in Porous Media, 62 (3), 283-305, 2006.

Silin, D., and T. Patzek, Pore space morphology analysis using maximal inscribed spheres. LBNL-59335. Physica A, 371 (2), 336-360, 2006.

Silin, D., T.W. Patzek, and S.M. Benson, Exact solutions in a model of vertical gas migration. SPE Paper 103156. In: 2006 SPE Annual Technical Conference and Exhibition, SPE, San Antonio, TX, September 24-27, 2006.
Silver, P.G., T.M. Daley, F. Niu, and E.L. Majer, Active source monitoring of crosswell seismic travel time for stress induced changes. LBNL-62398. Bulletin of the Seismological Society of America, 97 (18), 281-293, 2007.

Singleton, M.J., K. Maher, D.J. DePaolo, M.E. Conrad, and P.E. Dresel, Dissolution rates and vadose zone drainage from strontium isotope measurements of groundwater in the Pasco Basin, WA, unconfined aquifer. LBNL-55679. Journal of Hydrology, 321 (1-4), 39-58, 2006.

Slater, L., D. Ntarlagniannis, Y. Personna, and S. Hubbard, Pore-scale spectral induced polarization (SIP) signatures associated with FeS biomineral transformations. Geophysical Research Letters, 34, L21404, doi:10.1029/2007GL031840, 2007

Smith, J.T., and H.F. Morrison, Approximating spheroid inductive responses using spheres. LBNL-54602. Geophysics, 71 (2), G21-G25, 2006.

Smith, J.T., H.F. Morrison, L.R. Doolittle, and H.-W. Tseng, Multi-transmitter multi-receiver null coupled systems for inductive detection and characterization of metallic objects. LBNL-59020. Journal of Applied Geophysics, 61 (3-4), 227-234, 2007.

Snieder, R., S. Hubbard, M. Haney, G. Bawden, P. Hatchell, and A. Revil, Advanced non-invasive geohysical monitoring techniques. LBNL-61478. Annual Review of Earth and Planetary Sciences, 35, 653-683, 2007.

Solomon, C.T., P.K. Weber, J.J. Cech, B.L. Ingram, M.E. Conrad, M.V. Machavaram, A.R. Pogodina, and R.L. Franklin, Experimental determination of the sources of otolith carbon and associated isotopic fractionation. LBNL-60726. Canadian Journal of Fish. Aquat. Sci., 63 (1), 79-89, 2006.

Song, K.-P., Y.-S. Wu, and B.-Y. Ji, A $\Phi$-function method for estimating residual iil Saturation Distributions in Waterflooding Reservoirs. LBNL-60896. Journal of Acta Petrolei Sinica, 27 (3), 91-95, Chinese Petroleum Society, 2006.

Sonnenthal, E., In-drift flow. LBNL-61128. Workshop on Development of Radionuclide Getters for the Yucca Mountain Waste Repository, Sandia National Laboratories, Albuquerque, NM. 21-22, 2006.

Spycher, N., S. Mukhopadhyay, D. Sheilds, J. Leem, C. Bryan, and E. Sonnenthal, THC sensitivity study of repository edge and heterogeneous permeability effects REV00. LBID2587. BSC, Las Vegas, NV. 221, 2006.

Spycher, N., E. Sonnenthal G. Zhang, and S. Mukhopadyay, Drift-Scale THC Seepage Model. LBID-2612. MDL-NBSHS-000001 REV 05. Las Vegas, Nevada: Sandia National Laboratories. ACC: DOC.20071010.0004, 2007.

Steefel, C.I., Geochemical kinetics and transport. LBNL-62362. In: Kinetics of Water-Rock Interaction, S.L. Brantley, J.D. Kubicki, and A.F. White, eds., Springer, New York, pp. 545589, 2007.

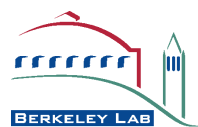


Stringfellow, W., San Joaquin River up-stream DO TMDL Project Task 4: Monitoring study interim Task Report \# 3. LBNL-63243. Lawrence Berkeley National Laboratory, Berkeley, CA. 2007.

Su, G., N.W.T. Quinn, P.J. Cook, and W. Shipp, Miniaturization of the flowing fluid electric conductivity logging technique. LBNL-59032. Ground Water, 44 (5), 754-757, 2006.

Su, G.W., J. Jasperse, D. Seymour, J. Constantz, and Q. Zhou, Simulation analysis of pumping-induced unsaturated regions beneath a perennial river. LBNL-63048. Water Resources Research, 43 (W08421), doi:10.1029/2006WR005389, 2007.

Tang, Y., R. Chakraborty, H. Garcia-Martin, J. Chu, T.C. Hazen, and J.D. Keasling, Flux analysis of central metabolic pathways in Geobacter metallireducens during reduction of soluble Fe (III)-NTA. LBNL-63533. Appl. Environ. Microbiology, 73 (12), 3859-3864, 2007.

Tang, Y., F. Pingitore, A. Mukhopadhyay, R. Phan, T.C. Hazen, and J.D. Keasling, Pathway confirmation and flux analysis using ${ }^{13} \mathrm{C}$ isotopic labeling of metabolites in Desulfovibrio vulgaris Hildenborough via FT-ICR mass spectrometry. LBNL-61053. Journal of Bacteriology, 189 (3), 940-949, 2007.

Teh, Y.A., W.L. Silver, and M. Conrad, Effects of soil structure destruction on methane production and carbon partitioning between methanogenic pathways in tropical rain forest soils. LBNL-60694. Journal of Geophysical Research, 111 (1-8), doi:10.1029/2005JG000020, 2006.

Teh, Y.A., W.L. Silver, M.E. Conrad, S. Borglin, and C.M. Carlson, Carbon isotope fractionation by methane-oxidizing bacteria in tropical rain forest soils. LBNL-60692. Journal of Geophysical Research, 111, 1-8, 2006.

Templeton, A. S., K.-H. Chu, L. Alvarez-Cohen, and M. E. Conrad, Variable carbon isotope fractionation expressed by aerobic CH4-oxidizing bacteria. LBNL-60690. Geochimica et Cosmochima Acta, 70 (7), 1739-1752, 2006.

Tillmann, A., A. Englert, Z. Nyari, I. Fejes, J. Vanderborght, and H.Vereecken, Characterization of subsoil heterogeneity, estimation of grain size distribution and hydraulic conductivity at the Krauthausen test site using Cone Penetration Test. Jour. of Contaminant Hydrol., 95 (1-2), doi:10.1016/j.jconhyd.2007.07.013, 2007.

Tokunaga, T., J. Wan, A. Lanzirotti, S. R. Sutton, and M. Newville, Long-term stability of organic carbon-stimulated chromate reduction in contaminated soils, and its relation to manganese redox status. LBNL-62676. Environmental Science and Technology, 41 (12), 4326-4331, 2007.

Tomutsa, L., D. Silin, and V. Radmilovich, Analysis of chalk petrophysical properties by means of submicron-scale pore imaging and modeling. LBNL-59822. SPE Reservoir Evaluation Journal, 10 (3), 285-293, 2007.

Torn, M.S. and J. Harte. 2006. Missing feedbacks, asymmetric uncertainties, and the underestimation of future warming. Geophys. Res. Lett., 33, L10703, doi:10.1029/2005GL025540, 2006.
Trautz, R., S. M. Benson, L. R. Myer, C. M. Oldenburg, E. Seeman, H. E., and B. Funderburk, The Rosetta resources $\mathrm{CO}_{2}$ storage project-A WESTCARB geologic pilot test. LBNL-60222. Fifth Annual Conference on carbon Capture and Sequestration, DOE-NETL, Alexandria, VA. 2006.

Trautz, R., L. Myer, S. Benson, C. Oldenburg, T. Daley, and J. Seeman, A combined saline formation and gas reservoir $\mathrm{CO}_{2}$ injection pilot in Northern California. LBNL-60169. 8th International Green House Gas Control Technology, Research Council of Norway, Trondheim, Norway. 2006.

Treseder, K.K., M.S. Torn, and C.A. Masiello, An ecosystemscale radiocarbon tracer to test use of litter carbon by ectomycorrhizal fungi. LBNL-59047. Soil Biology and Biochemistry, 38 (5), 1077-1082, 2006.

Tsang, C.-F., and J. Birkholzer, Special issue on site characterization for $\mathrm{CO}_{2}$ storage. Journal of Environmental Geology (in press), 2007.

Tsang, C.-F., J. Birkholzer, and J. Rutqvist, A comparative review of hydrologic issues involved in geologic storage of $\mathrm{CO}_{2}$ and injection disposal of liquid waste. LBNL-62674. Journal of Environmental Geology (in press), online October 2007.

Tsang, C.-F. and C. Doughty, Some insights from simulations of SWIW Tests on a complex fracture. LBNL-63564. Lawrence Berkeley National Lab., Berkeley, Calif., 2007. Also available as Rep. SKI-INSITE TRD-07-06, Swedish Nuclear Power Inspectorate, Stockholm, Sweden, 2007.

Tsang, C.-F., C. Doughty, J. Rutqvist, and T. Xu, Modeling to understand and simulate physico-chemical processes of $\mathrm{CO}_{2}$ geological storage. LBNL-59068. In: Carbon Capture and Sequestration-Integrating Technology, Monitoring, Regulation. E. Wilson and D. Gerard, eds., Blackwell Publishing, Ames, Iowa, pp. 35-72, 2007.

Tsang, C.-F., J. Rutqvist, and K.-B. Min, Fractured rock hydromechanics: From borehole testing, solute transport, to $\mathrm{CO}_{2}$ storage. LBNL-63460. In: Rock Physics and Geomechanics in the Study of Reservoirs and Repositories, C. David and M. LeRavalec-Dupin, eds., Geological Society of London Special Publication 284, pp. 15-34, London, UK, 2007.

Tsang, C.-F., C. Doughty, and M. Uchida, Simple model representations of transport in a complex fracture and their use in long-term predictions. Water Resour. Res. (in press), 2007.

Tsang, C.-F., Coupled hydro-mechanical processes in fractured rock and in indurated and plastic clays: A comparative discussion. Invited Opening Keynote Lecture, Second International GeoProc2006 Symposium, Nanjing, China, May 2006.

Tsang, Y.Y.W., Yucca Mountain heater tests. LBNL-51614. In: Gas Transport in Porous Media. C. Ho, S.W. Webb, and J. Wilson, eds., Academic Kluwer/Plenum, United Kingdom, pp. 371-384, 2006.

Tsang, Y., Effects of coupled processes on a proposed high-level waste repository at Yucca Mountain, Nevada. LBNL-63305. In: Characterizing the Proposed Geologic Repository at Yucca Mountain, Nevada-Hydrology and Geochemistry. J. S. Stuckless, ed., The Geological Society of America (in press), 2007.

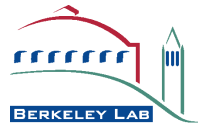


Tsibakhashvili, N.Y., M.V. Frontasyeva, T.I. Kirkesali, N.G. Aksenova, T.L. Kalabegishvili, I.G. Murusidze, L.M. Mosulishvili, and H.-Y.N. Holman, Epithermal neutron activation analysis of $\mathrm{Cr}(\mathrm{VI})$-reducer basalt-inhabiting bacteria. LBNL-60810. Analytical Chemistry, 78 (18), 6285-6290, 2006.

Uhlenbrook, S., S. Franks, K. Heal, S. Hubbard, H. Karambiri, T. Oki, and C. Valeo, Key messages, recommendations, and concluding remarks. LBNL-60881. In: Hydrology 2020: An Integrating Science to Meet Water Challenges, T. Oki, C. Valeo and K. Heal, eds., IAHS, Oxfordshire, UK, pp. 155-158, 2006.

Van Bramer, S. E., A. Glatfelter, S. Bai, C. Dybowksi, G. GNeue, and D. L. Perry, Solid-state 207Pb NMR studies of leadgroup 16 and mixed transition-metal-lead-group 16 element-containing materials. LBNL-60681. Magnetic Resonance Chemistry, 44 (3), 357-365, 2006.

Vasco, D.W., Modeling quasi-static poroelastic propagation using an asymptotic approach. LBNL-63547. Geophysical Journal International, 142, 2007.

Vasco, D.W., Invariance, groups, and non-uniqueness: The discrete case. LBNL-57367. Geophysical Journal International, 168, 473-490, 2007.

Vasco, D.W., Trajectory-based modeling of broadband electromagnetic wavefields. LBNL-61362. Geophysical Journal International, 168, 949-963, 2007.

Vasco, D.W., On the evaluation of asymptotic expressions for tracer transport in a heterogeneous medium. Water Resources Research (in press), 2007.

Vasco, D.W., Trajectory-based methods for modeling and characterization. LBNL-63531. In: Quantitative Information Fusion for Hydrological Sciences. C. Xing and T.-C.J. Yeh, eds., Springer-Verlag (in press), 2007.

Vasco, D.W., Zeroth order inversion of transient head observations. LBNL-63537. Water Resources Research (in press), 2007.

Vasco, D.W., and H. Keers, Seismic imaging of reservoir flow properties: Resolving water influx and reservoir permeability. LBNL-63532. Geophysics (in press), 2007.

Vasco, D. W., C. M. Puskas, R. B. Smith, and C. M. Meertens, Crustal deformation and source models of the Yellowstone volcanic field from geodetic data. LBNL-61514. Journal of Geophysical Research, 112, doi:10.1029/2006JB004641, 2007.

Vasco, D.W., and K. Karasaki, Interpretation and inversion of low-frequency head observations. LBNL-61275. Water Resources Research, 42 (W05408), 1-18, 2006.

Verma, S.P., I.S. Torres-Alvarado, M. Satir, and P.F. Dobson, Hydrothermal alteration effects in geochemistry and $\mathrm{Sr}$, $\mathrm{Nd}, \mathrm{Pb}$, and $\mathrm{O}$ isotopes of magmas from the Los Azufres geothermal field (Mexico): A statistical approach. LBNL61864. Geochemical Journal, 39 (2), 141-163, 2006.
Villalobos, M., B. Lanson, A. Manceau, B. Toner, and G. Sposito, Structural model for the biogenic Mn oxide produced by Pseudomonas putida. American Mineralogist, 91, 489-502, 2006.

Wan, J., T.K. Tokunaga, Y. Kim, Z. Wang, A. Lanzirotti, E. Saiz, and R. J. Serne, Effect of saline waste solution infiltration rates on uranium retention and spatial distribution in Hanford sediments. LBNL-63501. Environmental Science and Technology (in press), 2007.

Wan, J, T. Tyliszczak, and T. K. Tokunaga, Organic carbon distribution, speciation, and elemental correlations within soil microaggregates: Applications of STXM microscopy and NEXAFS spectroscopy. LBNL-63422. Geochimica et Cosmochimica Acta, 71, 5439-5449, 2007.

Wang, J.S.Y., Earth science collaborations for deep underground science and engineering laboratory. In: Rock Mechanics: Meeting Society's Challenges and Demands. D. S. E. Eberhardt, and T. Morrison, eds., Taylor \& Francis Group, London, U.K., pp. 1105-1114, 2007.

Waychunas, G.A., Y.-S. Jun, P. J. Eng, S. Ghose, and T. P. Trainor, Anion sorption topology on hematite: Comparison of arsenate and silicate. LBNL-61150. Geomaterials II (chapter) (in press), 2007.

Wenk, H.R., I. Lonardelli, H. Franz, K. Nihei, and S. Nakagawa, Preferred orientation and elastic anisotropy of illite-rich shale LBNL-63376. Geophysics, 72 (2), E69-E75, 2007.

Werner, M., P.S. Nico, B. Gau, I. Kennedy, and C. Anastasio, Atmospheric transformations of chromium in ultrafine combustion aerosol particles. LBNL-59104. Aerosol Science and Technology, 40 (7), 545-556, 2006.

Werner, M. L., P.S. Nico, M.A. Marcus, and C. Anastasio, Use of Micro-XANES to speciate of chromium in airborne fine particles in the Sacramento Valley. LBNL-62779. Environmental Science and Technology, 41 (14), 4914-4924, 2007.

Williams, K.H., S. Hubbard, and J. Banfield, Galvanic interpretation of anomalous SP accompanying microbial sulfate reduction. LBNL-63396. Journal of Geophysical ResearchBiogiosciences, 112 (G03019), doi:10.1029/2007JG000440, 2007.

Woods, K.N., H.-Y.N. Holman, and H. Wiedemann, Solvent induced fluctuations and the collective librational dynamics of lysozyme studied by THz spectroscopy. LBNL-61692. Biophysical Journal, 2007.

Wozei, E., S.W. Hermanowicz, and H.-Y. Holman, Developing a biosensor for estrogens in water samples: Study of the real-time response of live cells of the estrogen-sensitive yeast strain RMY / ER-ERE using fluorescence microscopy. LBNL-58194. Biosensors and Bioelectronics, 21 (8), 1654-1658, 2006.

$\mathrm{Wu}, \mathrm{Y} .-\mathrm{S} ., \mathrm{An}$ integrated methodology for characterizing flow and transport processes in fractured rock. LBNL-63411. Journal of China University of Geosciences, 18 (special issue), 2007.

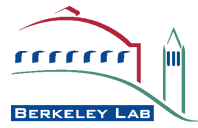


Wu, Y.-S., G. Lu, K. Zhang, L. Pan, and G. S. Bodvarsson, Analyzing flow patterns in unsaturated fractured rock of Yucca Mountain using an integrated modeling approach. LBNL-54006. Hydrogeology Journal, 15 (3), 553-272, 2007.

Wu, Y.-S., S. Mukhopadhyay, K. Zhang, and G. Bodvarsson, A mountain-scale thermal hydrologic model for simulating fluid flow and heat transfer in unsaturated fractured rock. LBNL-57921. Journal of Contaminant Hydrology, 86 (1-2), 128-159, 2006.

Wu, Y.-S., K. Zhang, and H.-H. Liu, Estimating large-scale fracture permeability of unsaturated rock using barometric pressure data. LBNL-57614. Vadose Zone Journal, 5 (4), 1129-1142, 2006.

Wu, Y.-S., and P.A. Forsyth, Efficient schemes for reducing numerical dispersion in modeling multiphase transport through porous and fractured media. LBNL-60056. Vadose Zone Journal (in press), 2007.

Wu, Y.-S., C. Ehlig-Economides, G. Qin, Z. Kang, W. Zhang, B. Ajayi, and Q. Tao, A triple continuum pressure transient model for a naturally fractured vuggy reservoir. LBNL63412. SPE-110044, Presented at the 2007 SPE Annual Technical Conference and Exhibition held in Anaheim, California, November 11-14, 2007.

Wu, Y.-S., G. Lu, K. Zhang, and G.S. Bodvarsson, An integrated modeling approach for characterizing multiphase flow, chemical transport and heat transfer in Fractured Reservoirs. SPE-106996, Presented at the SPE Europec/EAGE Annual Conference and Exhibition held in London, United Kingdom, June 11-14, 2007.

Wu, Y.-S., G. Qin, R.E. Ewing, Y. Efendiev, Z. Kang, and Y. Ren, A multiple-continuum approach for modeling multiphase flow in naturally fractured vuggy petroleum reservoirs. LBNL-59785. SPE-104173, Presented at the 2006 SPE International Oil \& Gas Conference and Exhibition in Beijing, China, 2006.

Wu, Y.-S., S. Mukhopadhyay, K. Zhang, and G.S. Bodvarsson, The influence of proposed repository thermal load on multiphase flow and heat transfer in the unsaturated zone of Yucca Mountain. LBNL-59783. Presented at the 2006 International High Level Radioactive Waste Management Conference, Las Vegas, NV, April 30-May 4, 2006.

$\mathrm{Xu}, \mathrm{M} ., \mathrm{Y}$. Ma, and G. Lu, Slope seepage resulting from torrential precipitation during a flood discharge event at a large hydroelectric station. Hydrogeology Journal (in press), 2007.

$\mathrm{Xu}, \mathrm{T} ., \mathrm{R}$. Senger, and S. Finsterle, Water-steel canister interaction and $\mathrm{H}_{2}$ gas pressure buildup in a nuclear waste repository. LBNL-62246. The Twelfth International Symposium on Water-Rock Interaction, China University of Geoscience (Wuhan, China), Kumming, China. 2007.

$\mathrm{Xu}$, T., Incorporation of aqueous reaction kinetics and biodegradation into TOUGHREACT: Application of a multi-region model to hydrobiogeochemical transport of denitrification and sulfate reduction. LBNL-61086. Vadose Zone Journal, (in press), 2007.
Xu, T., J.A. Apps, K. Pruess, and H. Yamamoto, Numerical modeling of injection and mineral trapping of $\mathrm{CO}_{2}$ with $\mathrm{H}_{2} \mathrm{~S}$ and $\mathrm{SO}_{2}$ in a sandstone formation. LBNL-57426. Chemical Geology, 242 (3-4), 319-346, 2007.

$\mathrm{Xu}, \mathrm{T} ., \mathrm{E}$. Sonnenthal, N. Spycher, and K. Pruess, TOUGHREACT: A simulation program for non-isothermal multiphase reactive geochemical transport in variably saturated geologic media. LBNL-56740. Computer \& Geosciences, 32 (2), 145-165, 2006.

Yang, L., and R.J. Donahoe, The form, distribution and mobility of arsenic in soils contaminated by arsenic trioxide, at sites in southeast USA. LBNL-63380. Applied Geochemistry, 22, 320-341, 2007.

Yang, L., R.J. Donahoe, and J.C. Redwine, In situ chemical fixation of arsenic-contaminated soils: An experimental study. LBNL63522. Science of the Total Environment, 387 (1-3), 28-41, 2007.

Yang, L., and C.I. Steefel, Kaolinite dissolution and precipitation kinetics at $22^{\circ} \mathrm{C}$ and $\mathrm{pH}$ 4. LBNL-63301. Geochimica et Cosmochimica Acta (in press), 2007.

Ye, M., F. Pan, Y.-S. Wu, B. Hu, C. Shirley, and Z. Yu, Assessment of radionuclide transport uncertainty in the unsaturated zone of Yucca Mountain. LBNL-59118. Advances in Water Resources, 30, 118-137, 2007.

Yoshioka, M., N.M. Mahowald, A.J. Conley, W.D. Collins, D.W. Fillmore, C.S. Zender, and D.B. Coleman, Impact of desert dust radiative forcing on Sahel precipitation: Relative importance of dust compared to sea surface temperature variations, vegetation changes and greenhouse gas warming. J. Climate, 20 (8), 1445-1467, 2007.

Zhang, G., N. Spycher, T. Xu, E. Sonnenthal, and C. Steefel, Reactive geochemical transport modeling of concentrated aqueous solutions: Supplement to TOUGHREACT User's Guide for the Pitzer Ion-Interaction Model. LBNL-62718. Lawrence Berkeley National Laboratory Report, 2006.

Zhang, G., N. Spycher, E. Sonnenthal, C. Steefel, and T. Xu, Modeling reactive multiphase flow and transport of concentrated solutions. LBNL-60016. Journal of Nuclear Technology (in press), 2007.

Zhang, G., J. Samper, and L. Montenegro, Coupled thermohydro-bio-geochemical reactive transport model of CERBERUS heating and radiation experiment in Boom clay. LBNL-60901. Applied Geochemistry (in press), 2007.

Zhang, H., B. Chen, B. Gilbert, and J. Banfield, Kinetically controlled formation of a novel nanoparticulate ZnS with mixed cubic and hexagonal stacking. LBNL-63061. J. Mater. Chem., 16, 249-254, 2006.

Zhang, K., C. Doughty, Y.-S. Wu, and K. Pruess, Efficient parallel simulation of $\mathrm{CO}_{2}$ geologic sequestration in saline aquifers. LBNL-63316. SPE-106026, Presented at SPE Reservoir Simulation Symposium, Society of Petroleum Engineers (SPE), Houston, TX. February 26-28, 2007.

Zhang, K., Y.-S. Wu, and J. Houseworth, Sensitivity analysis of hydrological parameters in modeling flow and transport in the unsaturated zone for Yucca Mountain. LBNL-59819. Hydrogeology Journal, 14 (8), 1599-1619, 2006.

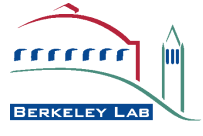


Zhang, K., Y.-S. Wu, and L. Pan, Temporal damping effect of the Yucca Mountain fractured unsaturated rock on transient infiltration pulses. LBNL-57539. Journal of Hydrology, 327 (1-2), 235-248, 2006.

Zhang, Y., H.-H. Liu, Q. Zhou, and S. Finsterle, Effects of diffusive property heterogeneity on effective matrix diffusion coefficient for fractured rock. LBNL-58695. Water Resources Research, 42 (W04405), 1-8, 2006.

Zhang, Y., C. M. Oldenburg, S. Finsterle, and G. S. Bodvarsson, System-level modeling for geological storage of $\mathrm{CO}_{2}$. LBNL-60401. Proceedings of TOUGH Symposium, Lawrence Berkeley National Laboratory, Berkeley, Calif., May 15-17, 2006.

Zhang, Y., C.M. Oldenburg, S. Finsterle, and G.S. Bodvarsson, System-level modeling for economic evaluation of geological $\mathrm{CO}_{2}$ storage in gas reservoirs. LBNL-62617. Energy Conversion and Management, 48, 1827-1833, 2007.

Zheng, Z., J. Wan, and T.K. Tokunaga, Sodium meta-autunite colloids: Synthesis, characterization, stability. LBNL-54563. Journal of Colloid and Interface Sciences, 274 (1), 48-55, 2006.

Zheng, Z., G. Zhang, and J. Wan, Reactive transport modeling and sensitivity analysis of column experiments for the behavior of alkaline-Saline tank waste solutions in variably saturated media. LBNL-55879. Journal of Contaminant Hydrology (in press), 2007.
Zhou, Q., J. Birkholzer, J. Rutqvist, and C.-F. Tsang, Sensitivity study of $\mathrm{CO}_{2}$ storage capacity in brine aquifers with closed boundaries: Dependence on hydrogeologic properties. LBNL-63388. The Sixth Annual Conference on Carbon Capture and Sequestration, DOE/NETL, Pittsburgh, PA. 2007.

Zhou, Q., J. Birkholzer, C.-F. Tsang, and J. Rutqvist, A method for quick assessment of $\mathrm{CO}_{2}$ storage capacity in closed and semi-closed saline aquifers. LBNL-63820. International Journal of Greenhouse Gas Control (in press), 2007.

Zhou, Q., H.-H. Liu, F. J. Molz, Y. Zhang, and G. S. Bodvarsson, Field-scale effective matrix diffusion coefficient for fractured rock: Results from literature survey. LBNL-57368. Journal of Contaminant Hydrology, 93, 161-187, 2007.

Zhou, Q., H.-H. Liu, G. Bodvarsson, and F.J. Molz, Evidence of multi-process matrix diffusion in a single fracture from a field tracer test. LBNL-58198. Transport in Porous Media, 63 (3), 473-487, 2006.

Zhou, Q., R. Salve, H. H. Liu, J. S. Wang, and D. Hudson, Analysis of a meso-scale infiltration and water seepage test in unsaturated fractured rock: Spatial variabilities and discrete fracture patterns. LBNL-55489. Journal of Contaminant Hydrology, 87, 96-122, 2006. 


\section{EARTH SCIENCES Division StAFF 2006-2007}

Special Note: This list is a snapshot of ESD's staffing for the 2006 and 2007 years.

Staffing list may not accurately reflect the department assignments for "Climate Science" as of the 2007 year. A more detailed description of Department changes can be found in the "Operations and Facilities Overview" section.

\section{DiVISION DIRECTORS}

\section{Donald J. DePaolo}

Gudmundur S. (Bo) Bodvarsson

(2001-2006)

In Memoriam November 11, 1952 - November 29, 2006

\section{SCIENTISTS / ENGINEERS}

\begin{tabular}{l} 
ClimATE SCIENCE \\
\hline Collins, William Drew \\
Jin, Jiming \\
Kleber, Markus \\
Mambelli, Stefania \\
Maxwell, Reed \\
Miller, Norman L. \\
Riley, William J. \\
Sharif, Hatim O. \\
Torn, Margaret S. ${ }^{* *}$ \\
\\
${ }^{*}$ Department Head \\
${ }^{* *}$ Program Head
\end{tabular}

\section{ECOLOGY}

Andersen, Gary Beller, Harry R. Brodie, Eoin L.

Hazen, Terry C. *

Holman, Hoi-Ying

Hunter-Cevera, Jennie C.

Jansson, Janet R.

Peters, Charlotte G.

Quinn, Nigel

Trevelyan, William

Sapra, Rajat

Stolyar, Sergey

Stringfellow, William T.

Zuniga, Efrain

\section{GEOCHEMISTRY}

Apps, John A.

Carroll, Susan A.

Christensen, John Neil

Conrad, Mark S. *

Dixit, Suvasis

Dobson, Patrick F.

Finnin, Lawrence

Gilbert, Benjamin

Kennedy, Burton M.

Knauss, Kevin

Lu, Guoping

Nico, Peter S.

Nishiizumi, Kunihiko

Perry, Dale L.

Sonnenthal, Eric

Spycher, Nicolas

Steefel, Carl I.

Waychunas, Glenn A.

Yang, Andrew

Zhang, Guoxiang

\section{GEOPHYSICS}

Ajo-Franklin, Jonathan B.

Alumbaugh, David L.

Berryman, James G. *

Budnitz, Robert Jay

Carazzone, James J.

Chen, Jinsong

Clymer, Richard

Commer, Michael

Gasperikova, Erika

Goldman, Mark

Gritto, Roland

Henshaw, Paul C.

Hill, Ross

Hoversten, Gary M.

Hubbard, Susan S. **

Hutchings, Lawrence John

Korneev, Valeri A.

Lee, Ki H.

Lindwall, Dennis A.

Lo, Wei-Cheng

Majer, Ernest L. **

Mazzella, Aldo T.

Myer, Larry R. **

Nadeau, Robert

Nakagawa, Seiji

Newman, Gregory A.

Nihei, Kurt T.

Pride, Steven R.

Schoenberg, Michael A.

Smith, Jeremy T.

Swift, Charles Jr.

Tseng, Hung-Wen

Vasco, Donald W. 


\section{SCIENTISTS / ENGINEERS}

\section{HYDROGEOLOGY}

Benson, Sally M.

Birkholzer, Jens T. **

Cappa, Frederic

Cortis, Andrea

Doughty, Christine A.

Faybishenko, Boris A.

Finsterle, Stefan A. *

Freifeld, Barry M.

Garcia, Julio

Ghezzehei, Teamrat A.

Javandel, Iraj

Karasaki, Kenzi

Kneafsey, Timothy J.

Kowalsky, Michael

Lewicki, Jennifer L.

Lippmann, Marcelo J.
Liu, Hui-Hai
Moridis, George J.
Oldenburg, Curtis M. **
Pruess, Karsten
Reagan, Matthew T.
Rebscher, Dorothee
Rutqvist, Jonny
Salve, Rohit
Seol, Yongkoo
Shan, Chao
Shvidler, Mark I.
Silin, Dmitriy
Su, Grace W.

Tokunaga, Tetsu K.

Truesdell, Alfred

Tsang, Chin-Fu

Tsang, Yvonne T. **

Wan, Jiamin

Wang, Joseph S.

Wang, Yuan

$\mathrm{Wu}, \mathrm{Yu}-\mathrm{Shu}$

$\mathrm{Xu}$, Tianfu

Zhang, Keni

Zhang, Yingqi

Zhou, Quanlin

Zimmerman, Robert W.

* Department Head

** Program Head

\section{FACULTY}

\section{Climate Science}

Bishop, James K.

Dietrich, William

Farrell, Alexander E. (In Memoriam

January 1, 1962-April 2008)

Fung, Inez

\section{ECOLOGY}

Ackerly, David

Alvarez-Cohen, Lisa

Buchanan, Bob B.

Chiang, John Chun Hong

Coates, John D.

Dreger, Douglas S.

Firestone, Mary

Glaser, Steven

Rhew, Robert

Zhou, Jizhong

\section{GEOCHEMISTRY}

Banfield, Jillian

Boering, Kristie A.

DePaolo, Donald J. ***

Ingram, B. Lynn

Kyriakidis, Phaedon C.

Myneni, Satish

Sposito, Garrison

\section{GEOPHYSICS}

Becker, Alex

Brimhall, George

Cooper, George A.

Doyle, Fiona M.

Goldstein, Norman E

Hornberger, George M.

Johnson, Lane R.

Morrison, Huntly F.

Rector, James W.

Romanowicz, Barbara A.

Rubin, Yoram

Wenk, Hans R.

\section{HydROGEOLOGY}

Manga, Michael Narasimhan, T. N. Patzek, Tadeusz W. Radke, Clayton J. Witherspoon, Paul A.

*** Division Director 


\section{Postdoctoral Fellows}

\section{Climate Science}

Berhe, Asmeret A.

Bird, Jeffrey

Gaudinski, Julia B.

Lam, Phoebe J.

Maggi, Federico

Swanston, Christopher

Von Kiparski, Guntram R.

\section{ECOLOGY}

Bernard, Stephanie M. Chakraborty, Romy

Weber, Karrie A.

Woods, Kristina N.

Wozei, Eleanor

$\mathrm{Wu}$, Cindy $\mathrm{H}$.

\section{GEOCHEMISTRY}

Bourg, Ian C.

Cervini-Silva, Javiera

Duckworth, Owen W.

Dunphy-Guzman, Katherine

$\mathrm{Gu}$, Chuanhui

Jourdan, Frederic

Jun, Young-Shin

Katz, Jordan E.

Kwon, Kideok

$\mathrm{Li}, \mathrm{Li}$

Ostroverkhov, Victor

Shaw, Stephanie

Simon, Justin I.

Singleton, Michael J.

Spagnoli, Dino D.

Thompson, Aaron

Wilkins, Michael J.

Yang, $\mathrm{Li}$

\section{GeOPHYSICS}

Englert, Andreas Ludwig

Moore, Jeffrey

\section{HydRogeOLOGY}

Alessandro, Uccelli

He, Yongtian

Zheng, Liange

- Scientific Engineering Associates, Research Associates/Technicians, Technical Managers, and Specialists

\section{Climate Science}

Biraud, Sebastien

Castanha, Cristina

Cooley, Heather S.

Nguyen, Mai T.

Weintraub, Samantha R.

Wood, Todd J.

\section{GEOCHEMISTRY}

Boyle, Katie

Brown, Shaun Tyler

Cox, Dale

Davis, Simon

Kormi, Albert

Larsen, Joern T.

Mendieta, Renata N.

Owens, Thomas L.

van Soest, Matthijs C.

Woods, Katharine N.

\section{ECOLOGY}

Alusi, Thana

Borglin, Sharon E.

Burns, Josephine R.

Camp, Lauren E.

DeSantis, Todd

Engelage, Samantha K.

Fortney, Julian

Geller, Jil T.

Goldfarb, Katherine Coperine

Hanlon, Jeremy

Hansen, Drew J.

Herman, Donald J.

$\mathrm{Hu}$, Ping

Hulls, John R.

Hutchison, Kathleen A.

Joyner, Dominique C.

Kain, Schuyler

Osman, Shariff R.

Phan, Richard

Piceno, Yvette M.

Rogers, Brendan M.

Singer, Mary E.

\section{GEOPHYSICS}

Clyde, John Richard

Daley, Thomas M.

Friday, John

Haught, John R.

Lippert, Donald R.

Morales, Alejandro

Peterson, John E.

Solbau, Ray D.

Tomutsa, Liviu

Williams, Kenneth H.

\section{HydRogeOLOGY}

Arias, Jose G.

Beyer, John Henry

Cook, Paul J.

Frye, George A.

Gonzalez Jr., Emilio

Houseworth, James E.

Jordan, Preston D.

Kim, Yongman

Mukhopadhyay, Sumit

Pan, Lehua

Trautz, Robert C.

Wang, Duo 


\section{- Post-Baccalaureate Fellows, Student and Graduate Student Research Assistants}

\section{Climate Science}

Allen, Lucy $\mathrm{H}$.

Barker, Marc A.

Currey, Jovauna

Cusack, Daniela F.

Harrison, Julie

Hopkins, Francesca M.

Huang, April Y.

Huppert, Laura A.

Keck, Daniel C.

Koteen, Laurie E.

Madrigal, Oriana

Marin-Spiotta, Erika

Ong, Sufey

Parvizian, Zoha A.

Pecot, Katrina J.

Picetti, Michael A.

Porras, Rachel C.

Rohilla, Aditya K.

Schlegel, Nicole-jeanne

Tang-Smith, Eliot

West, Kimberlee

\section{GeOCHEMISTRY}

Aciego, Sarah

Aradottir, Edda S.P.

Goodell, Carmen M.

Hoag, Katherine J.

Lee, Patrick

Lee, Victoria E.

Legg, Benjamin A.

Mahendra, Shaily

Pena, Jasquelin

Peterson, Brook T.

Ryan, Cristina S.

Teh, Yit Arn

Topp, Erik K.

Weaver, Karrie L.

\section{ECOLOGY}

Ball, Gregory Ian

Belnap, Chris

Bilir, Teresa E.

Bister, John M.

Bradbury, Damon C.

Chan, Lydia Suet Fan

Chiang, Catherine $\mathrm{H}$.

Chou, Joyce

Crago, Melissa

Daly, Rebecca

Downey, Michael A.

Dubinsky, Eric A.

Esmaili, Laleh

Granadosin, Arlene P.

Hillson, Nathan J.

Huckelbridge, Katherine $\mathrm{H}$.

Hunt, Richard N.

Johnson, David R.

Kam, Winnie

Kapoor, Trishul

Karpuzcu, Ekrem

Katsuura, Yoshihiro

Kim, Eun Hye

Lam, Siu Pan

Leung, Chin Man

Long, Darcie H.

Lundquist, Tryg

Ma, Byron

Maas, Talia M.

Mattson, Jolene K.

Merkel, Jesse

Moshier, Emily

Nguyen, Nhu

Nuccio, Erin E.

Placella, Sarah

Rogers, Mathew

Shapland, Elaine

Shelby, Megan

Shetty, Amala

Simmons, Sheri L.

Sokolovskaya, Natalya

Sun, Christine L.

Wong, Tiffany G.

Wrighton, Kelly C.

\section{GeOPHysics}

Anderson, Heidi L.

Dai, Bo

Hou, Zhangshuan

Kappler, Karl

McLaskey, Gregory C.

Masson, Yder Jean

\section{Hydrogeology}

Arias, Jose G.

Benito, Pascual H.

Cha, Minsu

Grover, Tarun

Gwatney, Hope

Halecky, Nicholaus

Holtzman, Ran

Jin, Guodong

Kuhlmann, Sonja

Lehikoinen, Anssi T.

Miljkovic, Ljubinko

Shen, David

Walsh, Matthew R.

$\mathrm{Xu}$, Suying 


\section{Technical, Administrative, and Management Staff}

Atkinson, Maria Fink

Avina, Kryshna

Butson, Marie Louise

Chappell, Darren

Denn, Walter

Espinoza-Ross, Valarie M.

Fissekidou, Vassiliki A

Harris, Stephen D.

Hawkes, Daniel S.

Jackson, June D.
Kelly, Lisa M.

Kooyman, Yingying (Nieves) T. Kramer, Bridget

Lau, Peter K.

McClung, Ivelina A.

Miller, Grace

Nodora, Donald N.

Pollard, Theresa A.

Prieto (Strohmeier), Helen G.

Rasar, Janis E.
Saarni, Marilyn E. Seybold, Sherry A. Swantek, Diana M. Taylor, Bryan E. Templer, Jason Valladao, Carol A. Villavert, Maryann Wong, Johnny Wright, Sam A. 


\section{Photo Captions and Credits for ESD Research Summaries 2006-2007}

\section{FRONT COVER}

(Top) Climate model simulation of sunlight reflected by aerosols for 2001 (courtesy of David Fillmore and Bill Collins) (Lower Left) U-tube apparatus, Frio Brine pilot project, TX

(Lower Right) Tunnel boring machine for Yucca Mountain Project, Yucca Mountain, NV

\section{BACK COVER}

(Top) Three-dimensional resistivity image derived from Coso (CA) Geothermal Field from magnetotelluric data sets (courtesy of Greg Newman)

(Lower Left) The Berkeley Unexploded Ordnance Detector (BUD) and its developers—winner of a 2007 R\&D 100 Award

(Lower Right) Simulation of Yucca Mountain experiment using 3-D unsaturated flow code (courtesy of Stefan Finsterle)

\section{DIVISION DIRECTOR'S PERSPECTIVE}

(page 1) Earth Sciences Division, Building 90, LBNL (aerial view)

(page 3) Earth Sciences Division Council

(page 4) Geosciences Measurement Facility (GMF).

\section{RESOURCE DEPARTMENTS}

(page 7 ) John Peterson collecting surface electromagnetic data at the uranium-contaminated Rifle, CO, field study site, to delineate subsurface heterogeneity

Hydrogeology

(page 9 ) Barry Freifeld and Tom Daley at the Otway Basin Pilot Project, Victoria, Australia

Geophysics

(page 11) Seismic vibroseis source truck at borehole geophysical project site in West Texas. Field personnel (left to right): Dale Cox (Berkeley Lab), Ernie Majer (Berkeley Lab), Cecil Hoffpauir (Univ. of Texas), Tom Daley (Berkeley Lab), John Queen (Hi-Q Geophysics). Not pictured: Alex Morales (LBNL)

Geochemistry

(page 13 ) Tom Owen in the Center for Isotope Geochemistry

Ecology

(page15) Terry Hazen (left) and Gary Andersen, ESD microbiologists, with the Berkeley Lab PhyloChip, a microarray that can analyze a sample for the unique DNA signatures of all known bacterial species. The PhyloChip won a 2008 R\&D 100 Award.

\section{Climate Sciences}

(page 17) The Carbon Explorer transmitting data from the Southern Ocean. The Carbon Explorer, a "smart" telemetryenhanced ocean float with advanced optical sensors for characterizing carbon in the ocean, won a 2006 R\&D 100 Award. 


\section{RESEARCH PROGRAMS}

(page 19) Oil drilling program to deploy an x-ray diffraction apparatus

\section{Energy Resources}

(page 21) Emplacement of geophones in microwells for monitoring enhanced oil recovery with $\mathrm{CO}_{2}$

Fundamental and Exploratory Research

(page 37) Computer simulation of isotopic fractionation during aqueous diffusion (courtesy of Ian Bourg) Photography by Barry Freifeld

Environmental Remediation and Water Resources

(page 53) Installation of shallow electrode holes by ESD's Kenneth H. Williams and Phillip Rizzo at DOE's Rifle Integrated Field Challenge (IFC) site near Rifle, Colorado. Nonpolarizing electrodes are embedded in each hole and used for induced polarization monitoring of stimulated subsurface bioremediation activities under investigation at the Rifle IFC site.

\section{Climate and Carbon Sciences}

(page 87) Pat Dowell (ARM technician) collecting a pair of flask for trace gas analysis (including: $\mathrm{CO}_{2}, \mathrm{CO}, \mathrm{CH}_{4}, \mathrm{~N}_{2} \mathrm{O}$, ${ }^{13} \mathrm{CO} 2, \mathrm{CO}^{18} \mathrm{O}$, carbonyl sulfide, and many trace hydrocarbon species) at the Atmospheric Radiation Measurement (ARM) Climate Research Facility (ACRF) in the Southern Great Plains. Note the DOE aircraft in the upper left corner, flying vertical profile over the site.

\section{Geologic Carbon Sequestration}

(page 103) As part of an Australia-U.S. collaboration under the GeoSeq project, ESD researchers designed, built, and deployed the downhole monitoring and fluid sampling assembly shown here being installed by crane into the Otway Basin Pilot Project observation well in Victoria, Australia.

\section{Nuclear Waste}

(page 125) Gudmundur (“Bo") Bodvarsson (1952-2006):

Leader, mentor, and guiding force for ESD and its Nuclear Waste Program

"Bo lived his life 48 hours a day"

-Odette Lockwoord-Stewart, Pastor of the Epworth Methodist Church, Berkeley, California

\section{PRODUCTION CREDITS}

Design and Production: Walter Denn, ESD

Editor: Dan Hawkes, ESD

Production Management: Kryshna Avina, Maryann Villavert, ESD

Publication List: Dan Hawkes, ESD

Photography: Roy Kaltschmidt (Creative Services Office, LBNL), except where noted 
CELEBRATING 30 YEARS...

July 21, 2007 marked the commencement of the Earth Sciences Division 30 years ago.

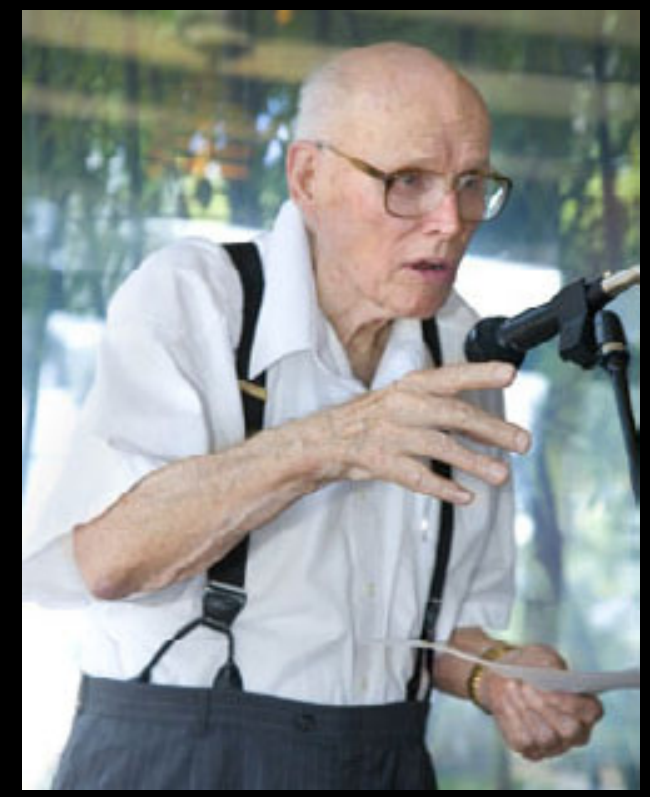

On July 20, 2007, the Division marked the occasion with a casual gathering with cake and story telling.

Happy Anniversary!
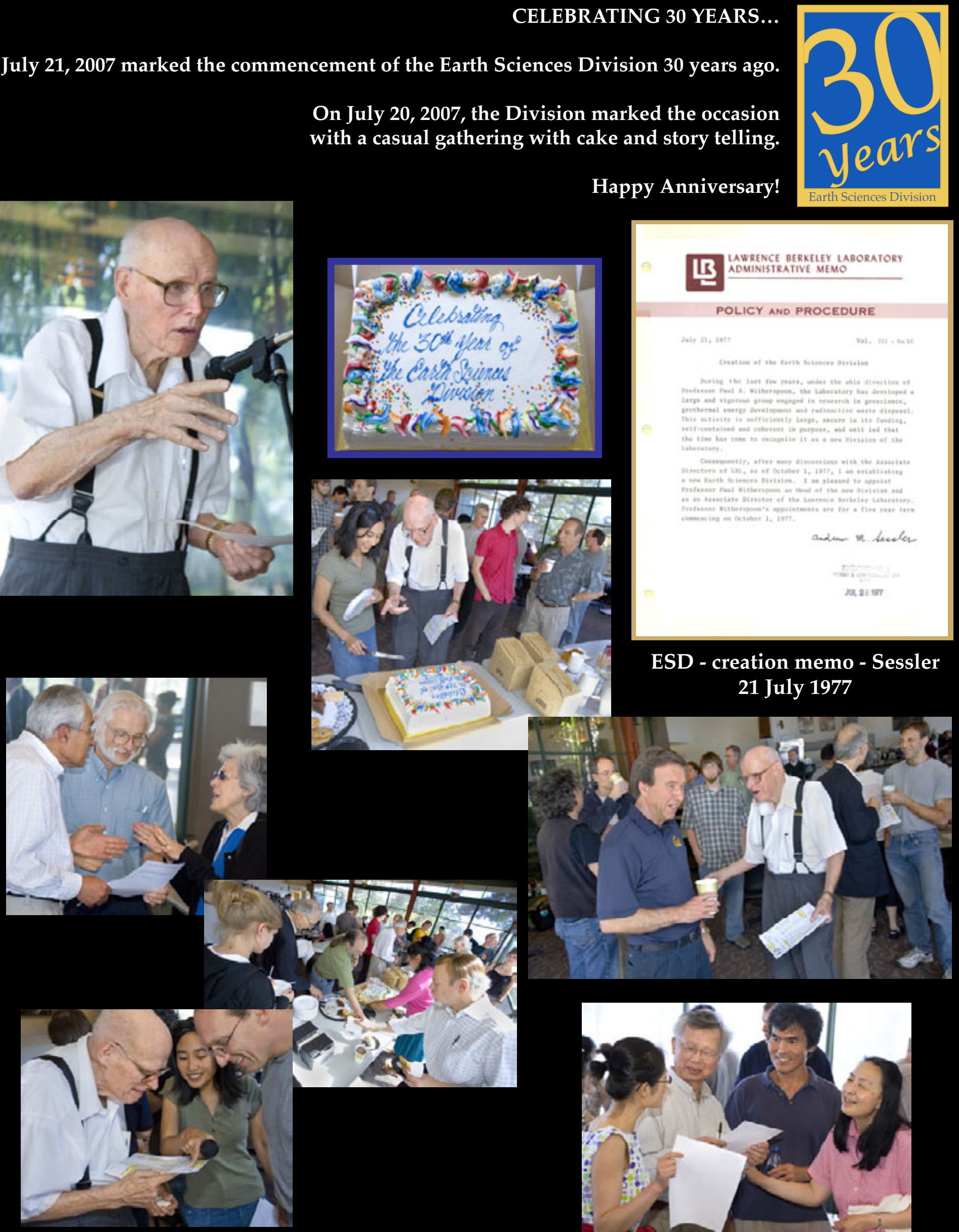

ESD - creation memo - Sessler 21 July 1977
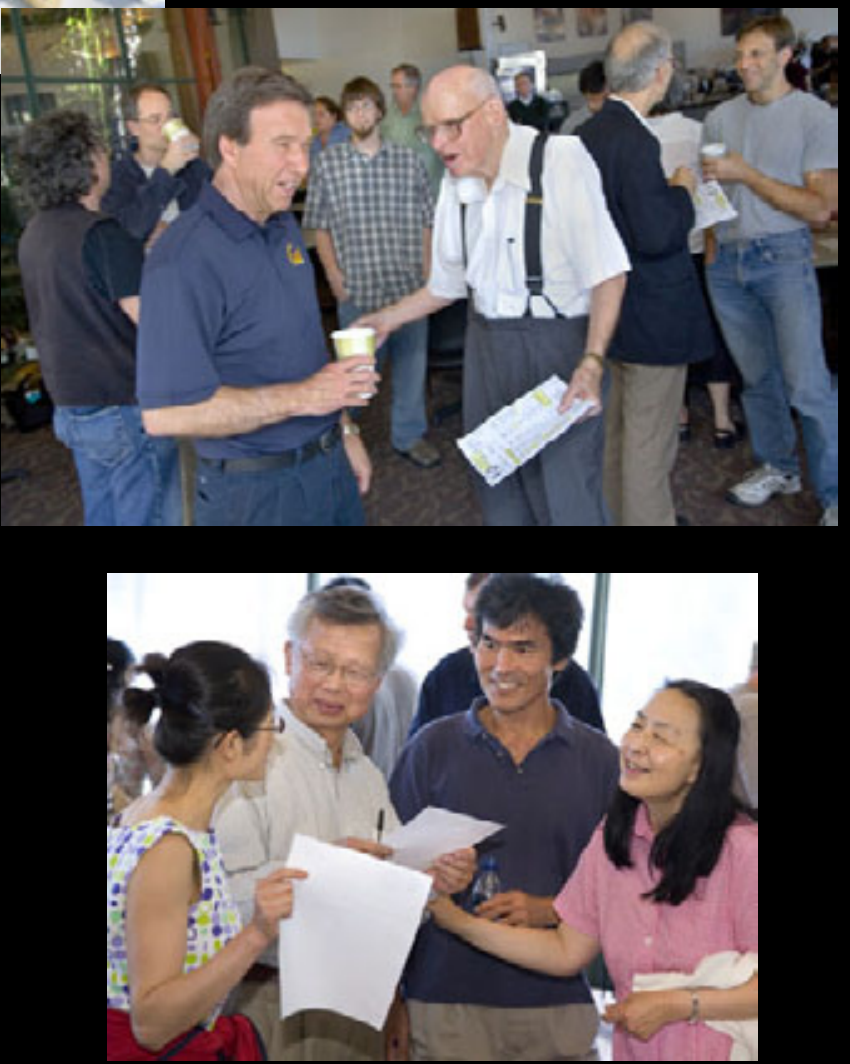

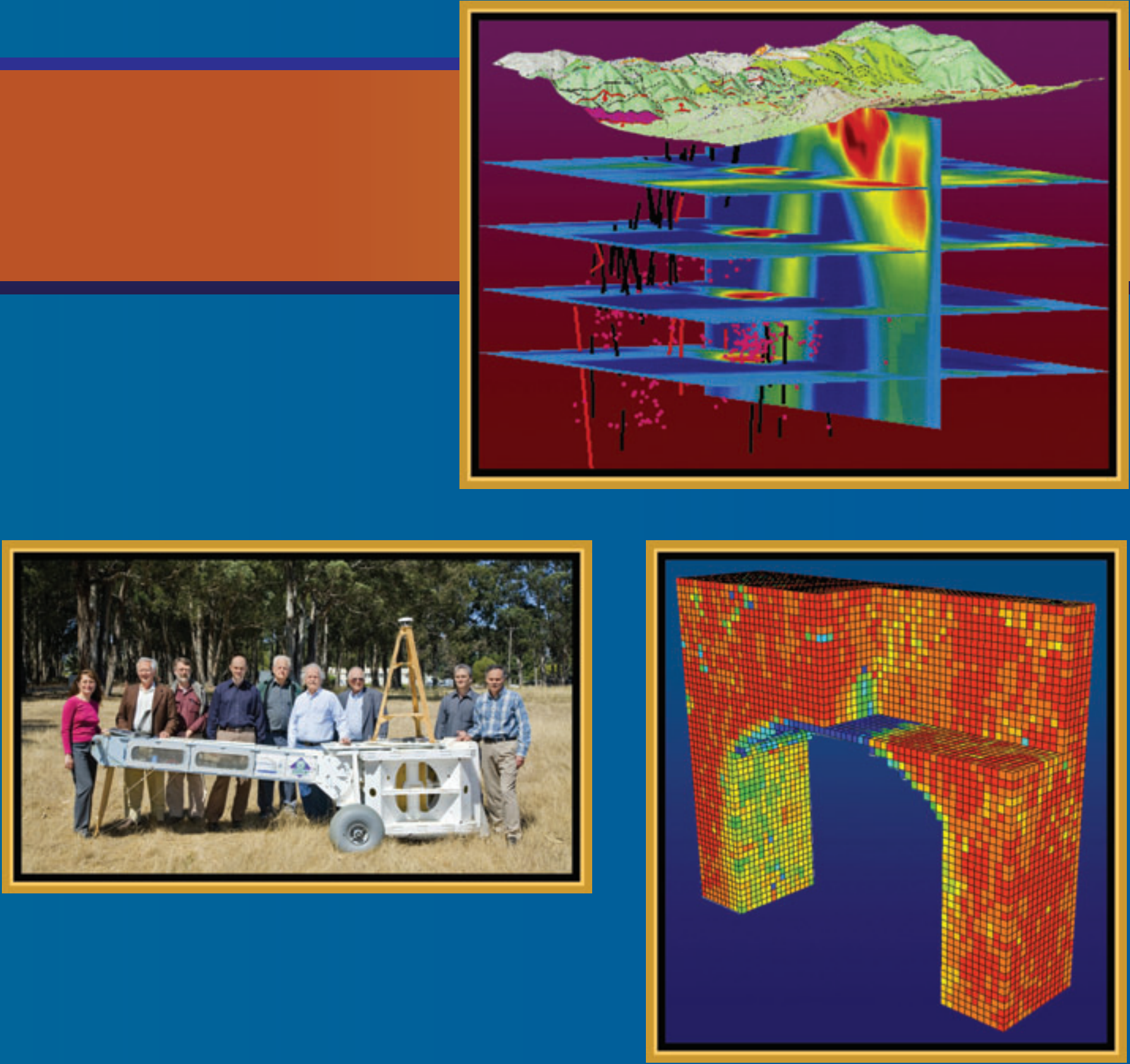

\section{6-2007}

\section{EARTH SCIENCES DIVISION RESEARCH SUMMARIES}

